Portland State University

PDXScholar

\title{
UHF and Microwave Phase-Modulated Scattering
}

\section{Array}

Nasr Nomas Hussein Alkhafaji

Portland State University

Follow this and additional works at: https://pdxscholar.library.pdx.edu/open_access_etds

Part of the Electrical and Computer Engineering Commons Let us know how access to this document benefits you.

\section{Recommended Citation}

Alkhafaji, Nasr Nomas Hussein, "UHF and Microwave Phase-Modulated Scattering Array" (2019).

Dissertations and Theses. Paper 4998.

https://doi.org/10.15760/etd.6874

This Dissertation is brought to you for free and open access. It has been accepted for inclusion in Dissertations and Theses by an authorized administrator of PDXScholar. Please contact us if we can make this document more accessible: pdxscholar@pdx.edu. 
UHF and Microwave Phase-Modulated Scattering Array

by

Nasr Nomas Hussein Alkhafaji

A dissertation submitted in partial fulfillment of the requirements for the degree of

Doctor of Philosophy

in

Electrical and Computer Engineering

Dissertation Committee:

Richard Campbell, Chair

Branimir Pejcinovic

Martin Siderius

John Acken

Erik Sanchez

Portland State University

2019 


\begin{abstract}
This dissertation investigates the use an array of active nonlinear elements, with particular emphasis on controlling distortion products generated by nonlinear elements in space rather than using conventional ways such as transmission lines, waveguides, and power dividers and combiners. The nonlinear elements are made of assemblies of antennas and electronic switches, called modulated scatterers (MSs). These so-called MSs elements are utilized in a wide variety of applications such as radio frequency identification (RFID) systems, microwave imaging, Internet-of-Things sensors, etc. However, no research work has been reported in the literature regarding exploiting and controlling several distortion products generated by MSs at the same time according to the best of authors' knowledge. To facilitate controlling distortion products which means suppressing or enhancing distortion products in space, we present a nonlinear array with elements that are MSs instead of conventional antennas. MSs are switched ON-OFF at different times by modulation signals having the same frequency. The time delay of the switching process between array elements represents a relative phase shift difference in the frequency domain. Thus, the presented structure is called the phase-modulated scattering array (PMSA). The PMSA has a similar layout of phased arrays, but it does not have a feeding network and is fed by an external source called the illuminating source. Because our system does not need a feeding network and phase shifters, it is potentially easier to implement with low cost. Two different signals which are the illuminating (incident) and modulation signals interact inside switches to generate a huge number of distortion products due to the nonlinearity of switches and the periodic nature of the presented system. Distortion products then leave the presented PMSA to space again (i.e., scattering
\end{abstract}


distortion products). The PMSA is able to treat distortion products and achieve beamforming functions.

Phase and frequency modulation combined with modulated scattering arrays offer a variety of new functionalities and electromagnetic properties not discovered yet. Thus, some unique functionalities of the presented PMSA will be disclosed here. Leveraging unique properties of the PMSA, suppression, and enhancement of distortion products in space can be simply realized.

The operation mechanism of the PMSA is explained by developing two different mathematical models. Communication signal processing perspectives are the basis of the first mathematical model developed to show the spatial characteristics of distortion products generated by our presented PMSA. Its root is originated from a mathematical model of the widely-used polyphase multipath technique in $\mathrm{RF}$ communication circuits. However, the adopted technique is suitable only for communication circuits with a single output and parameters prescribed in advance. Thus, the model is further developed to circumvent all the problems mentioned above and to be able to detect the spatial characteristics of distortion products at any point in space. Static impacts of the measurement environment, real radiation patterns of actual antennas utilized in prototypes, and phase and gain errors among paths have been taken into account as well. In the model, every single scatterer is represented by a single separate path. Furthermore, the modified model is extended to include single, two, and multi tones modulation signals. Simulation results have been obtained before and after the modification for a different number of paths and modulation signals with different tones. Results show that the modified model can quantify spatial characteristics of distortion products at any point in space where specific distortion products are enhanced, and others are canceled. Because distortion products are independent in their nature (i.e., each single distortion product has 
different frequency and phase), they have independent radiation patterns (scattered beams). Therefore, the second mathematical model based on phased antenna array perspectives is developed. The relationship between the two models states that a distortion product which is enhanced at a certain point in space has a maximum scattered beam at that point. Also, the second mathematical model being similar to mathematical models of phased arrays considers effects of all distortion products resulting from single, two, and multi tones modulation signals, and it states that each single distortion component has its particular scattered beam.

Next, sub-models for some properties and applications of the presented PMSA such as a diffraction grating-like behavior, nonreciprocity, beamforming, a tool for distortion product analysis of phased arrays and multi-input multi-output (MIMO systems), a reconfigurable-spatial harmonic generator, and a direction finding technique are derived depending on the two main mathematical models. All parts are simulated and results validate all proposed functionalities.

Single antennas, antenna arrays, electronic switches (modulators), and a 4-to-8 phase transformer kit using only resistors have been designed, simulated, fabricated, assembled, and tested.

Eventually, different structures of the presented PMSAs working at $432 \mathrm{MHz}$ and 2.3GHz are tested inside the anechoic chamber. Both frequencies are downconverted to the band $2-22 \mathrm{kHz}$. Modulation signals used in the experimental setups are single and two tones. Data are measured using the commercial software SigView running on a laptop and a spectrum analyzer. Both spatial characteristics and scattered beams of distortion products are measured. Comparisons have been made between measured received responses of scattered signals and theoretical results. They are in good agreement although limitations and challenges are encountered with each round of measurement. Measured results confirm practically that as a number of 
scatterers increases, more distortion products are controlled at the same time. The distortion product rejection ratio DPRR is more than $15 \mathrm{~dB}$ for all measured distortion products supposed to be canceled. Directions of scattered beams are found at expected locations with errors less than $3 \%$. Furthermore, directions of illuminating signals $\theta_{o}$ or distances $d$ separating between PMSA elements are varied to change directions of scattered beams when prescribed values of parameters governing the overall performance are being broken. In other words, the beamforming functionality has been validated practically. Different elements of $8 \times 1$-PMSA are turned-off at measurements in order to find fault tolerances of the presented system. Measured results show that when two elements are failed simultaneously, responses can be accepted to some extent. 


\section{Dedication}

I dedicate this work to the memory of my brother, Ryadh Nomas.

To my wife (Hawraa), and my kids Dimah, Abbas, and Layan 


\section{Acknowledgments}

First of all, I would like to express my deepest gratitude to Allah Almighty for his blessing and for giving me the strength and faith to finish this research work and my ongoing education. I would like to extend my thanks and gratitude to my dissertation advisor Dr. Richard Campbell, who introduced me for the first time to the field of modulated scattering techniques. He has offered all advice, guidance, encouragement, and motivation. Also, I want to thank Dr. Branimir Pejcinovic, Dr. Martin Siderius, Dr. John Acken, and Dr. Erik Sanchez for serving on my dissertation committee, and for their valuable comments.

I am also extremely grateful for Dr. Branimir Pejcinovic for reviewing my dissertation to be in good shape and for his outstanding classes on microwave theory. I want to say thank you again to Dr. John Acken for his help with some editing. Furthermore, my thanks go to Mr. Glenn LaBrasseur, the RF anechoic chamber lab expert, for his help with experimental and testing parts. Special thanks to Madeleine Roche, a graduate student in the RAD lab.

I am very grateful for my parents, sisters, and brother for their prayers. Special thanks to my amazing wife for her support, encouragement, and patience and thanks to my delightful children Dimah, Abbas, and Layan.

Finally, I want to express my sincere gratitude for the Higher Committee of Education Development (HCED) in Iraq for sponsoring me during my Ph.D. program study at Portland State University (PSU), and my gratitude is also sent to my

university, Alfurat Al-Awsat University, in my home country, Iraq, for their support. 


\section{Table of Contents}

Abstract $\quad$ i

Dedication $\quad$ v

Acknowledgments $\quad$ vi

List of Tables $\quad$ xi

List of Figures xii

Chapter 1: Introduction 1

1.1 Motivation . . . . . . . . . . . . . . . . . 1

1.2 Phase-Modulated Scattering Array (PMSA) Paradigm . . . . . . . . 2

1.3 Literature Review . . . . . . . . . . . . . . . . . . . . 4

1.3.1 Modulated Scattering Techniques (MST) . . . . . . . . . . 4

1.3.1.1 Single MST Scatterers in Literature (RFID and IoT Sensors) . . . . . . . . . . . . . 6

1.3.1.2 Arrays of MST Scatterers in Literature (Microwave Imaging) . . . . . . . . . . . . . . . . . . . 8

1.3.2 Time Modulated Linear Array (TMLA) . . . . . . . . . . . . 9

1.3.3 Time Modulated Metasurfaces (TMMS) . . . . . . . . . . . . 12

1.3.4 Distortion Products in 5G Phased Arrays . . . . . . . . . . . . 13

1.4 Research Objectives . . . . . . . . . . . . . . . . . 14

1.5 Dissertation Outline . . . . . . . . . . . . . . . 15

Chapter 2: $\quad$ The Basic Theoretical Background 18

2.1 Introduction . . . . . . . . . . . . . . . . . . 18

2.2 The Study of Modulated Scattering Technique (MST) . . . . . . . . . 18

2.2.1 Mathematical Basics of Modulated Scattering Techniques (MST) 19

2.2.2 Far-field Based Modulated Scattering Techniques (MST)

Interpretation . . . . . . . . . . . . . . . . 24

2.2.3 Modulated Scatterer (MS) as an Analog Signal Processor (ASP) 30

2.3 The Study of Array Signal Processing (Phased Antenna Array) . . . 32

2.3.1 A Uniform Linear Array ULA Beam-forming based on Delay and Sum . . . . . . . . . . . . . . . . . 33

2.3.2 Steering Phase, Grating Lobes, and Element Weighting . . . 35

2.3.2.1 Steering Phase . . . . . . . . . . . . . 35

2.3.2.2 Grating Lobes . . . . . . . . . . . . . . . . . . 38

2.3.2.3 Array Weighting . . . . . . . . . . . . . . . 39

2.3.3 Two Dimensional Antenna Array (Planar Array) . . . . . . . 40

2.4 The Study of Image-Rejection Mixers . . . . . . . . . . . . . . . . . . 41 
Chapter 3: The Mathematical Model of the Proposed Design under a Communication Signal Processing Perspective

3.1 Introduction . . . . . . . . . . . . . . . . . . 45

3.2 The General Representation of the Phase Modulated Scattering Array (PMSA) . . . . . . . . . . . . . . . . 46

3.2.1 The Derivation of the Mathematical Model . . . . . . . . . . 48

3.2.1.1 A Single Scatterer Model . . . . . . . . . . . . . . 48

3.2.1.2 Scattering Array Model . . . . . . . . . . . . . . 62

3.2.1.3 The Proposed System Model under the Conventional Polyphase Multipath Technique . . . . . . . . . . 70

3.2.1.4 Handling of Intermodulation Distortion IMD Products of Multi-Tone Modulation Sources . . . . . 81

3.3 Simulation Results . . . . . . . . . . . . . . . . . . . . . . . 84

3.4 The PMSA Model under the Modified Polyphase Multipath Technique (Realistic-Model) . . . . . . . . . . . . . . . . . . . . . 91

3.5 Non-idealities of the Phase-Modulated Scattering Array (PMSA) . . . 106

3.5 .1 Example . . . . . . . . . . . . . . . . . . . 110

Chapter 4: The Mathematical Model of the Proposed Design under a Phased Antenna Array Perspective

4.1 Introduction . . . . . . . . . . . . . . . . . . . . . . 113

4.2 General Mathematical Model based on Phased Antenna Array Theory 116

4.2.1 Single Frequency-Modulated Element (Scatterer) . . . . . . 116

4.2.2 An Array of Frequency-Modulated Scatterers

(Phase-Modulated Scattering Array) . . . . . . . . . . . . 122

Chapter 5: Features and Some Applications of the PMSA 134

5.1 Introduction . . . . . . . . . . . . . . . . . . 134

5.2 The Diffraction Grating-Like Behavior . . . . . . . . . . . 135

5.2.1 The Equation of Spatial Characteristics of Distortion Products (Modes) . . . . . . . . . . . . . . . . 136

5.2.2 Cutoff Modes and the Total Number of Real Scattered Modes 141

5.2 .3 Incident Frequency Sensitivity . . . . . . . . . . . . . . . 143

5.2 .4 Retrodirective Modes . . . . . . . . . . . . . . . . . . . . . . 144

5.3 The Non-Reciprocity . . . . . . . . . . . . . . . . . . . . 146

5.3.1 Half-Duplex Non-Reciprocal Operations . . . . . . . . . . 146

5.3.2 Full-Duplex Non-Reciprocal Device . . . . . . . . . . . . . . 149

5.4 Beamforming of Distortion Products _ . . . . . . . . . 150

5.4 .1 Numerical Examples . . . . . . . . . . . . . . . . . . . . 151

5.4.1.1 Example 1: 4-element PMSA with $d=0.7 \lambda_{o} \ldots \ldots .152$

5.4.1.2 Example 2: an 8-element PMSA with $d=0.5 \lambda_{o} \ldots 160$

5.5 The PMSA as an Analysis Tool of Distortion Products in Phased Arrays161

5.5.1 The Mathematical Analysis . . . . . . . . . . . . . . . . 165

$5.5 .2 \quad$ Simulation Example . . . . . . . . . . . . . . . . . . 172 
5.6 The PMSA Based Tunable Spatial Harmonic Generator . . . . . . . . 175

5.7 Direction Finding Based on the PMSA . . . . . . . . . . . . . . . . . 178

Chapter 6: Preliminary Works 185

6.1 Introduction . . . . . . . . . . . . . . . . . . . . 185

6.2 Design of Antennas for the PMSA . . . . . . . . . . . . . 186

6.2.1 Single Antenna Design . . . . . . . . . . . . . . . . . . 187

6.2.1.1 Printed Broadband Yagi dipole Antenna . . . . . . . 187

6.2.1.2 Inset-fed Microstrip Patch Antenna . . . . . . . . . . 190

6.2.1.3 Monopole Antenna over a Finite Rectangular Ground 192

6.2.1.4 Conventional Folded Yagi-Uda Antenna . . . . . . . 193

6.2 .2 Arrays . . . . . . . . . . . . . . . . . . . . . 194

6.2.2.1 Design of Nominated Arrays . . . . . . . . . . . . . . 197

6.3 Design of RF Reflective-Type Switch (RF-RTS) . . . . . . . . . . . 205

6.3.1 RTS Power Signal Analysis . . . . . . . . . . . . . . . 206

6.3.2 RTS Design . . . . . . . . . . . . . . . . 210

6.4 Multi-Phase Modulation Signal Generator . . . . . . . . . . . . 216

$\begin{array}{lll}\text { Chapter 7: } & \text { Experimental Setup } & 226\end{array}$

7.1 Introduction . . . . . . . . . . . . . . . 226

7.2 Testing Environment . . . . . . . . . . . . . . . 226

7.3 Testing Apparatus . . . . . . . . . . . . . . . . 226

7.4 Limitations and constraints . . . . . . . . . . . . . . . . 231

Chapter 8: Experimental Results 233

8.1 Introduction . . . . . . . . . . . . . . . . . 233

8.2 Experimental Results of the Phase Modulated Scattering Array (PMSA) 234

8.2.1 432MHz Measurement Results (Initial Experiments) . . . . . . 236

8.2.2 2.3GHz Measurement Results . . . . . . . . . . . . . . 241

8.2.2.1 Single-tone Experimental Results . . . . . . . . . . . 242

8.2.2.2 Two-tone Experimental Results . . . . . . . . . . . 263

8.3 Parametric Investigations . . . . . . . . . . . . . . . . . . . . . . 272

8.3.1 Investigating the Effects of Varying $\theta_{o}$ and $d$ on the Overall

Performance . . . . . . . . . . . . . . 275

8.3.2 Investigations of the Failure of Scatterers on the Overall Performance of the PMSA . . . . . . . . . . . . . 278

8.4 Potential Applications of the Proposed Phase-Modulated Scattering

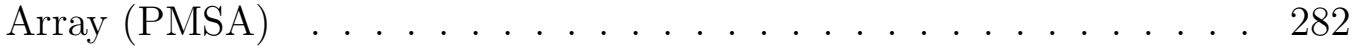

8.4.1 Tunable Spatial Distortion Product Generator . . . . . . . . . 282

8.4.2 PMSA based Direction Finding . . . . . . . . . . . . . 282

Chapter 9: Conclusion and Future Work 286

9.1 Conclusion . . . . . . . . . . . . . . . . 286

9.2 Accomplished Objectives . . . . . . . . . . . . . . . . . . . 292

9.3 Future Work . . . . . . . . . . . . . . . . . . 293 
$\begin{array}{ll}\text { Bibliography } & 296\end{array}$

Appendix A: Nonlinear Circuits $\quad 320$

A.1 Single-tone Analysis . . . . . . . . . . . . . . . . . . 320

Appendix B: Three Paths Polyphase Circuit 322 


\section{List of Tables}

5.1 Types of distortion products (modes) for $L=4$ with two different incident angles . . . . . . . . . . . . . . . . . . . . . . . . . . 142

5.2 Illustration of the retordirective modes for $L=4$ with five different incident angles . . . . . . . . . . . . . . . . . . . . 145

5.3 Frequencies and phases of some IMDs and HDs generated in a 4-element PMSA . . . . . . . . . . . . . . . . . . . . . 161

5.4 Apparent angles of IMDs generated from three interferers incident at three different angles $\theta_{i}, \theta_{j}$, and $\theta_{l} \ldots \ldots \ldots \ldots$. . . . . . . . 173

5.5 IMD3 and IMD5 components for interferers in Figure 5.13 . . . . . . 175

5.6 Calculation and simulation results . . . . . . . . . . . . . . 181

5.7 Calculation and simulation results of direction finding for five unknown non-coherent signals . . . . . . . . . . . . . . . 183

6.1 Dimensions of the printed quasi-Yagi dipole antenna printed on the Rogers 4003C substrate with a thickness of 60mil, shown in Figure 6.1a 188

6.2 Properties of nominated arrays . . . . . . . . . . . . . . . . . 198

6.3 Errors in amplitudes and phases of $1004 \mathrm{~Hz}$ modulation signals passing through phase transformer kit . . . . . . . . . . . . . . . 223

6.4 Errors in amplitudes and phases of $899 \mathrm{~Hz}$ modulation signals passing through phase transformer kit . . . . . . . . . . . . . . . . 223

8.1 Description of harmonic terms . . . . . . . . . . . . . . . . 245

8.2 DPRRn of distortion products obtained from the six experimental setups of the $4 \times 4$-element PMSA shown in Figure 8.11a . . . . . 263 


\section{List of Figures}

1.1 The general representation of the proposed phase-modulated scattering array $(\mathrm{PMSA}) \ldots \ldots \ldots \ldots \ldots \ldots$

2.1 General scenario of MST systems a) bistatic b) monostatic . . . . . 21

2.2 Different representations for differential reflection coefficients on the Smith chart . . . . . . . . . . . . . . . . . . . . 31

2.3 General representation of the Uniform Linear Array ULA of $L$ elements with a single-frequency RF signal incident at an angle $\theta \ldots$. . . . .

2.4 Radiation patterns of 10-element ULA at the desired directions a) $\theta_{o}=$ $0^{\circ}$ b) $\theta_{o}=15^{\circ}$ c) $\theta_{o}=30^{\circ}$ d) $\theta_{o}=45^{\circ} \ldots \ldots \ldots 37$

2.5 Comparison of the radiation patterns of two different array weightings a) 10-element ULA array pattern b) 10-element -28dB triangular-weight array pattern _. . . . . . . . . . . . 39

2.6 Two-dimensional planar array . . . . . . . . . . . . . . . . . . . 40

2.7 3D Radiation patterns and their top views of a $10 \times 10$-element uniform planar array at directions a) broadside-3D b) broadside-top view c) $\theta_{o}=25^{\circ}$ and $\left.\phi_{o}=40^{\circ}-3 \mathrm{D} \mathrm{d}\right) \theta_{o}=25^{\circ}$ and $\phi_{o}=40^{\circ}$-top view $\ldots .42$

2.8 Image-reject mixer architecture . . . . . . . . . . . . . . . 44

3.1 General representation of the PMSA where its distortion products are scattered in space . . . . . . . . . . . . . . . . . . . 47

3.2 a) Antenna-signal source equivalent model b) an array of spatial sources (antenna array) . . . . . . . . . . . . . . . .

3.3 Representation of the modulation sources in the model when having a) single-tone signals b) two-tone signals . . . . . . . . . . . . 52

3.4 RTS ON and OFF states . . . . . . . . . . . . . . . . 54

3.5 Equivalent circuit of the RTS . . . . . . . . . . . . . 54

3.6 Nonlinear circuits with single-tone and two-tone input signals $\ldots . \quad 55$

3.7 Mixer used as a phase shifter . . . . . . . . . . . . . . . 59

3.8 Equivalent circuits of a single scatterer when using a) single-tone modulation source b) two-tone modulation source . . . . . . . . . . 60

3.9 Polyphase multipath circuit . . . . . . . . . . . . . . . . . . . 63

3.10 Two-path polyphase circuit . . . . . . . . . . . . . . 68

3.11 a) Three-path polyphase circuit b) its phasor and magnitude plots . . 69

3.12 Polyphase multipath model of PMSA when using a) single-tone modulation sources b) two-tone modulation sources . . . . . . . 72

3.13 Types of the PMSA model a) A b) B c) C d) D e) E f) F . . . . . 75

3.14 Block diagram of the polyphase multipath technique in a) communication circuits b) the PMSA . . . . . . . . . . . 76 
3.15 Simulated output spectra of signals scattered normally (broadside) for a single path PMSA model when using modulation sources with a) single-tone b) two-tone c) three-tone . . . . . . . . . . . . .

3.16 Simulated output spectra of signals scattered normally (broadside) for a two-path PMSA model when using single-tone modulation sources a) odd-Balanced Type b) even-Balanced Type c) USB image-reject mixer d) LSB image-reject mixer . . . . . . . . . . . . . . . . . .

3.17 Simulated output spectra of signals scattered normally (broadside) when using single-tone modulation sources for a) 3-path PMSA for (A and $\mathrm{C}$ types) b) 4-path PMSA for (A and C types) c) 6-path PMSA for (A and $\mathrm{C}$ types) d) 8-path for (A and $\mathrm{C}$ types) e) 3-path PMSA for (B and D types) f) 4-path PMSA for (B and D types) g) 6-path PMSA for (B and D types) h) 8-path PMSA for (B and D types) . .

3.18 Simulated output spectra of signals scattered normally (broadside) for an 8-path PMSA model (types A and C) when using modulation sources with a) two-tone b) three-tone . . . . . . . . . . .

3.19 Simulated output spectra of signals scattered normally (broadside) when using prescribed or non-prescribed values of the second phase shifters a) 4-Path $90^{\circ}$ (prescribed) b) 4-Path $60^{\circ}$ (non-prescribed) c) 8-path $45^{\circ}$ (prescribed) d) 8-path $30^{\circ}$ (non-prescribed) . . . . . . 91

3.20 Realisitc-modified polyphase multipath model of the proposed PMSA

3.21 Relative phase shift differences of the second phase shifters vs. a) $\theta_{o}$ for different values of $d \mathrm{~b}$ ) $d$ for different values of $\theta_{o}$ c) $\theta_{o}$ and $d$ (3D view) d) $\theta_{o}$ and $d$ (3D-top view) . . . . . . . . . . .

3.22 Direction of the spatial outputs (scattered distortion products, harmonics n) generated by the PMSA with a) 4-path (element) and $d=0.5 \lambda_{o}$ b) 4-path (element) and $d=0.7 \lambda_{o}$ c) 8-path (element) $d=0.5 \lambda_{o}$ d) 8-path (element) and $d=0.7 \lambda_{o} \ldots \ldots \ldots$

3.23 Angular locations of the spatial outputs (scattered angles) of distortion products (harmonics $n$ ) generated by the proposed PMSA vs. a number of harmonic and a relative phase shift difference of second phase shifters with a) 4-path (element) and $d=0.5 \lambda_{o}$ b) 8-path (element) and $d=$ $0.5 \lambda_{o} \ldots \ldots \ldots \ldots \ldots \ldots \ldots \ldots$

3.24 Angular locations of the spatial outputs (scattered angles) of distortion products (harmonics $n$ and IMDs $k w_{1}+m w_{2}$ ) generated by the proposed PMSA vs. a distortion product term number $k$ and $m$ a) 4-path (element) with $90^{0}$ relative phase shift difference of second phase shifters and $d=0.5 \lambda_{o}$ b) 8-path (element) with $45^{0}$ relative phase shift difference of second phase shifters and $d=0.5 \lambda_{o} \quad \ldots \ldots \ldots$ 
3.25 Angular locations of the spatial outputs (scattered distortion products, harmonics $n$ ) generated by the proposed PMSA vs. a distortion product term numbers $n$ for different relative phase shift differences of the second phase shifter a) 4-path (elements) type A circuit with $d=0.5 \lambda_{o}$ b) 4-path (elements) type $\mathrm{D}$ circuit with $d=0.5 \lambda_{o}$ c) 8-path (elements) type A circuit with $d=0.5 \lambda_{o} \ldots . . . . . . . .$.

3.26 Simulated scattered power from the 4-path type A PMSA model when a relative phase shift difference of the second phase shifter is $120^{\circ}$ a) single-tone at a spatial output $\left.\left(\theta_{r}=56.44^{\circ}\right) \mathrm{b}\right)$ single-tone at a spatial output $\left.\left(\theta_{r}=19.47^{\circ}\right) \mathrm{c}\right)$ single-tone at a spatial output $\left(\theta_{r}=-9.594^{\circ}\right)$ d) single-tone at a spatial output $\left(\theta_{r}=-41.8^{\circ}\right)$ e) two-tone at a spatial output $\left.\left(\theta_{r}=56.44^{\circ}\right) \mathrm{f}\right)$ two-tone at a spatial output $\left.\left(\theta_{r}=19.47^{\circ}\right) \mathrm{g}\right)$ two-tone at a spatial output $\left.\left(\theta_{r}=-9.594^{\circ}\right) \mathrm{h}\right)$ two-tone at a spatial output $\left.\left(\theta_{r}=-41.8^{\circ}\right) \mathrm{i}\right)$ three-tone at a spatial output $\left.\left(\theta_{r}=56.44^{\circ}\right) \mathrm{j}\right)$ three-tone at a spatial output $\left.\left(\theta_{r}=19.47^{\circ}\right) \mathrm{k}\right)$ three-tone at a spatial output $\left(\theta_{r}=-9.594^{\circ}\right)$ l) three-tone at a spatial output $\left(\theta_{r}=-41.8^{\circ}\right)$

3.27 Simulated scattered power from the 4-path type D PMSA model when a relative phase shift difference of the second phase shifter is $45^{\circ}$ captured at spatial outputs a) $\theta_{r}=48.59^{\circ}$ b) $\theta_{r}=14.48^{\circ}$ c) $\theta_{r}=-14.48^{\circ}$ and d) $\theta_{r}=-48.59^{\circ} \ldots \ldots \ldots$ a relative phase shift difference of the second phase shifter is $-30^{\circ}$ captured at spatial outputs a) $\theta_{r}=66.44^{\circ}$ b) $\theta_{r}=41.81^{\circ}$ c) $\theta_{r}=-24.62^{\circ}$ d) $\theta_{r}=9.594^{\circ}$ e) $\theta_{r}=-4.78^{\circ}$ f) $\theta_{r}=-19.47^{\circ}$ g) $\theta_{r}=-35.69^{\circ}$ and h) $\theta_{r}=9.594^{\circ} 107$

3.29 Polyphase multipath model of the PMSA with errors in phases and amplitudes of signals . . . . . . . . . . . . . . . . 111

4.1 General representation of scattered beams of distortion products generated by the PMSA . . . . . . . . . . . . . . . . 115

4.2 General representation of the PMSA with potential locations of the illuminating source and directions of progressive phase shift distributions of the modulation sources . . . . . . . . . . . . . . . . 121

4.3 Stages of signal manipulation by the PMSA . . . . . . . . . . . . . 124

4.4 Spatial representation of scattered beams of distortion products generated by the PMSA when incident signals are normal . . . . . . . 133

5.1 An illustrative comparison between the diffraction grating and the PMSA136

5.2 Wavefronts of modes at normal incident signals a) the zero mode (specular) b) the first LSB mode(propagating) c) the first USB mode (propagating) d) the second USB mode (cutoff) . . . . . . . . . . . . 140

5.3 Wavefronts of modes at $-30^{\circ}$ incident signals a) the zero mode (specular) b) the first LSB mode (cutoff) c) the first USB mode (propagating) d) the second USB mode (propagating) . . . . . . . . . 141 
5.4 Retrodirective modes in the PMSA . . . . . . . . . . . . 145

5.5 Nonreciprocal half-duplex system a) upconversion part b) downconversion part c) equivalent schematic of the upconversion d) equivalent schematic of the downconversion . . . . . . . . . .

5.6 Scattered beams of distortion products for the 4-element PMSA for the incident angles a) $-21^{\circ}$ b) $0^{\circ}$ c) $21^{\circ}$ and d) $0^{\circ}$ reversed-type ...

5.7 Scattered beams of distortion products for the 4-element PMSA with

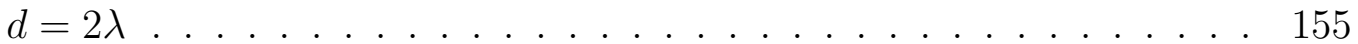

5.8 Plot of frequency varying scattered beams versus angle for different incident angles a) $\left.-21^{\circ} 3 \mathrm{D} \mathrm{b}\right)-21^{\circ} 2 \mathrm{D}$ c) $0^{\circ} 3 \mathrm{D}$ and d) $0^{\circ} 2 \mathrm{D}$ e) $21^{\circ}$ $\left.3 \mathrm{D} \mathrm{f}) 21^{\circ} 2 \mathrm{D} \mathrm{g}\right) 0^{\circ} 3 \mathrm{D}$ inverted-type h) $0^{\circ} 2 \mathrm{D}$ inverted-type $\ldots . .157$

5.9 a) Plots of the 1USB and the 1LSB scattered beams of the 4-element PMSA for different modulation phase shifts b) scattered beams of a 4-element PMSA with $\emptyset_{m}=0$ and $\theta_{o}=0^{\circ}$ c) scattered beams patterns of a 4-element PMSA with $\emptyset_{m}=0$ and $\theta_{o}=-21^{\circ} \ldots \ldots \ldots 158$

5.10 Scattered beams of distortion products for different incident angles a) 1USB b) 1LSB . . . . . . . . . . . . . . . . . . . 159

5.11 Potential directions of scattered beams of harmonics a) 1USB b) 1LSB c) 2USB and d) 2LSB 160

5.12 Plot of the frequency varying scattered beams of distortion products versus angle for different incident angles generated in the 8-element PMSA a) $\left.\left.-35^{\circ} 3 \mathrm{D} \mathrm{b}\right)-35^{\circ} 2 \mathrm{D} \mathrm{c}\right)-15^{\circ} 3 \mathrm{D}$ and d) $-15^{\circ} 2 \mathrm{D}$ e) $0^{\circ} 3 \mathrm{D}$ f) $\left.\left.0^{\circ} 2 \mathrm{D} \mathrm{g}\right) 15^{\circ} 3 \mathrm{D} \mathrm{h}\right) 15^{\circ} \ldots \ldots \ldots \ldots \ldots$

5.13 Receive-mode phased array with three interferers in the YZ cut-plane incident on each single element in the array . . . . . . . . . .

5.14 Simulation patterns of IMD3 and IMD5 components along with their distortion products at the output of the phased array in Figure 5.13 a) array pattern of $\mathrm{IMD} 5=28.6 \mathrm{GHz}$ b) distortion characterstics of IMD $5=28.6 \mathrm{GHz}$ c) array pattern of IMD $5=28.7 \mathrm{GHz}$ d) distortion characterstics of IMD $5=28.7 \mathrm{GHz}$ e) array pattern of IMD $3=\mathrm{IMD} 5=28.8 \mathrm{GHz}$ f) distortion characterstics of IMD $3=28.8 \mathrm{GHz}$ g) distortion characterstics of IMD $5=28.8 \mathrm{GHz}$ h) array pattern of IMD $3=\mathrm{IMD} 3=28.9 \mathrm{GHz}$ i) distortion characterstics of IMD3 $=28.9 \mathrm{GHz}$ j) distortion characterstics of IMD $3=28.9 \mathrm{GHz} \mathrm{k}$ ) array pattern of IMD $3=29.3 \mathrm{GHz}$ l) distortion characterstics of $\mathrm{IMD} 3=29.3 \mathrm{GHz} \mathrm{m}$ ) array pattern of $\mathrm{IMD} 3=29.4 \mathrm{GHz}$ n) distortion characterstics of IMD $3=29.4 \mathrm{GHz}$ o) array pattern of IMD $5=29.6 \mathrm{GHz}$ p) array pattern of IMD $5=29.6 \mathrm{GHz} \ldots \ldots \ldots \ldots \ldots \ldots \ldots$

5.15 Simulated patterns of IMDs with the main signals when nine interferers are incident on the phased array for a) different frequencies coming from different incident angles b) different frequencies coming from the same incident angles $0^{\circ} \mathrm{c}$ ) same frequencies coming from different incident angles . . . . . . . . . . . . . . . 
5.16 Simulation results of the tunable spatial harmonic generator a) spatial characteristics of the 1USB distortion product b) scattered beams of the 1USB distortion product directed toward angles $0^{\circ} \ldots$. . . . . .

5.17 Scenario of the direction finding based on the PMSA . . . . . . . . .

5.18 Scattering angles of harmonics vs. incident angles of unknown illuminating signals for the 8-element PMSA with $0.5 \lambda \ldots \ldots$

5.19 Scattering angles of the 1USB and 1LSB harmonics vs. incident angles of the five unknown illuminating signals for the 8-element PMSA with $0.5 \lambda \ldots \ldots \ldots \ldots \ldots$

5.20 Distortion characteristics at the moving detector output recorded at spatial angles a) $71^{\circ}$ b) $-53^{\circ}$ c) $18^{\circ}$ d) $4^{\circ}$ e) $-17^{\circ} \ldots . . . . . . .184$

6.1 Printed Yagi dipole antenna a) geometry b) 3D exploded view c) equivalent circuit d) current distribution over an antenna aperture at 2.3GHz e) fabricated top-view f) fabricated bottom-view . . . . . . .

6.2 Input impedance of the printed Yagi dipole antenna for $l s=11 \mathrm{~mm}$ short dashed red line, $l s=21 \mathrm{~mm}$ long dashed red line, and $l s=31 \mathrm{~mm}$ solid red line. . . . . . . . . . . . . . . . .

6.3 a) Simulated and measured S11 of the printed quasi-Yagi dipole antenna b) simulated E-cut and $\mathrm{H}$-cut planes of the gain radiation pattern at $2.3 \mathrm{GHz}$ c) simulated $3 \mathrm{D}$ gain radiation pattern at $2.3 \mathrm{GHz}$ d) simulated gain . . . . . . . . . . . . . . . . .

6.4 Microstrip patch inset-fed antenna a) geometry $w_{s}=l_{s}=64 \mathrm{~mm} w_{p}=$ $41 \mathrm{~mm} l_{p}=30.25 \mathrm{~mm} w_{f}=3.4 \mathrm{~mm} l_{\text {inset }}=10 \mathrm{~mm}$ and $s_{\text {inset }}=1.4 \mathrm{~mm}$ b) fabricated c) simulated S11 d) simulated real and imaginary parts of Zin e) simulated electric field distribution over an antenna aperture f) simulated transmission coefficient . . . . . . . . . . . .

6.5 Properties of microstrip patch inset-fed antenna a) E-cut plane radiation pattern b) H-cut plane radiation pattern c) $3 \mathrm{D}$ view of

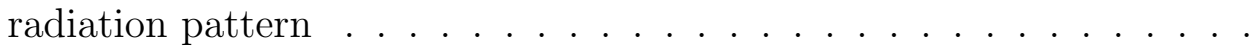

6.6 Monopole antenna over a rectangular ground a) 3D geometry b) 3D exploded view c) fabricated d) simulated S11 e) 3D radiation pattern f) 3D representation of electric field distribution . . . . . . . . .

6.7 Conventional folded Yagi-Uda antenna working at a frequency of a) $432 \mathrm{MHz}$ b) $2.3 \mathrm{GHz}$. . . . . . . . . . . . . . . . .

6.8 Conventional folded Yagi-Uda antenna arrays working at a frequency of $432 \mathrm{MHz}$ and performing inside the anechoic chamber a) 2-element array b) 4-element array . . . . . . . . . . . . . . . . .

6.9 Impact of mutual coupling on antenna properties a) gain radiation patterns of the first element for four different scenarios b) S11 for four different scenarios c) Snn of the fourth scenario only d) Tn of the fourth scenario only . . . . . . . . . . . . . . . . . . 201

6.10 Two-element H-coupling type printed quai-Yagi antenna array . . . . 201 
6.11 a) A $4 \times 4$ quasi-Yagi printed antenna array and its $3 \mathrm{D}$ radiation patterns directed to b) $\theta=-45^{\circ}, \phi=0^{\circ}$ c) $\theta=-30^{\circ}, \phi=0^{\circ}$ d) $\theta=-15^{\circ}, \phi=0^{\circ}$ e) $\theta=0^{\circ}, \phi=0^{\circ}$ f) $\theta=15^{\circ}, \phi=0^{\circ}$ g) $\theta=30^{\circ}, \phi=0^{\circ}$ h) $\theta=45^{\circ}, \phi=0^{\circ} \ldots \ldots \ldots \ldots$

6.12 a) An $8 \times 2$ quasi-Yagi printed antenna array and its $3 \mathrm{D}$ radiation patterns directed to b) $\theta=-45^{\circ}, \phi=0^{\circ}$ c) $\theta=-30^{\circ}, \phi=0^{\circ}$ d) $\theta=-15^{\circ}, \phi=0^{\circ}$ e) $\theta=0^{\circ}, \phi=0^{\circ}$ f) $\theta=15^{\circ}, \phi=0^{\circ}$ g) $\theta=30^{\circ}, \phi=0^{\circ}$ h) $\theta=45^{\circ}, \phi=0^{\circ} \ldots \ldots \ldots \ldots \ldots$

6.13 a) An $8 \times 1$ printed quasi-Yagi antenna array b) an $4 \times 1$ microstrip inset-fed patch antenna array c) an $8 \times 1$ quarter-wavelength monoples over ground antenna array . . . . . . . . . . . . . . . . . . . . 204

6.14 RTS switch a) general schematic b) open-closed states . . . . . . . . . 208

6.15 a) RTS schematic design using the ADS b) cross-section of a low barrier Schottky diode c) equivalent circuit of a Schottky diode . . . . . . . .

6.16 a) Simulated S11 with a DC sweep from -3v to 3v on the Smith chart b) simulated factor $M$ for different values of modulation voltages $c$ ) simulated RF incident and RF reflected signals in the time domain d) simulated $\mathrm{RF}$ incident and $\mathrm{RF}$ reflected signals in the frequency domain (spectral analysis) . . . . . . . . . . . . . .

6.17 a) Photograph of the RTS prototype using the HSMS2860 Schottky diode b) photograph of the RTS prototype using the HP5200-2835 Schottky diode c) comparison between the simulated and measured S11 of the RTS using HSMS2860 Schottky diode for the reversed and forward biasing on the Smith chart d) comparison between the simulated and measured factor M of RTS using a HSMS2860 Schottky diode e) RTS using HSMS2860 Schottky diode under test connected to a modulating source and a digital oscilloscope f) simulated response of the RTS with single input signal (absence of the RF signals and only presence of the modulation signal) g) measured response of the RTS with single input signal (Modulation signal) . . . . . . . . . . .

6.18 a) $700 \mathrm{~Hz}$ quadrature signal generator b) $899 \mathrm{~Hz}$ and $1004 \mathrm{~Hz}$ quadrature signal generator c) measured waveforms of $700 \mathrm{~Hz}$ quadrature signal generator d) measured waveforms of $899 \mathrm{~Hz}$ quadrature signal generator e) measured waveforms of $1004 \mathrm{~Hz}$ quadrature signal generator . . . .

a) Auxiliary phase shift generator type-A b) its phasor diagram c) its input and output waveforms d) main phase shift generator type-B e) its phasor diagram . . . . . . . . . . . . . . 222

6.20 Modified output phase according into the two input phases combination223

6.21 a) Schematic of the phase transformer kit using the ADS b) schematic of the phase kit transformer using LTspice c) simulated input and output waveforms using the ADS d) simulated input and output waveforms using the LTspice . . . . . . . . . . . . . 
6.22 a) Photograph of the phase transformer kit prototype top-view b) photograph of the phase transformer kit prototype bottom-view c) measured input and output waveforms . . . . . . . . . . . 225

7.1 Inside view of the anechoic chamber with an air-jet [9] . . . . . . . 227

7.2 Block diagram of the experiemental setup inside and outside the anechoic chamber . . . . . . . . . . . . . . . . . 227

7.3 a) Testing apparatus outside the anechoic chamber b) testing apparatus inside the anechoic chamber . . . . . . . . . . . . . 228

7.4 a) In-house signal source working at $2.304 \mathrm{GHz}$ b) in-house receiver working at $2.304 \mathrm{GHz}$. . . . . . . . . . . . . . . . . . . . . . . . . . . 228

7.5 Screenshot of the interface panel of the SigView commercial software that used to acquire the measured data . . . . . . . . . . . . . . . . . 230

7.6 Ranges of angles where the measured data are taken depending on the total length of the proposed PMSA and its representative $d_{f}$. . . . . 231

8.1 a) $432 \mathrm{MHz}(70 \mathrm{~cm})$ folded dipole Yagi scatterer b) Measured received spectrum of a single scatterer . . . . . . . . . . . . . . .

8.2 a) 2-element PMSA working at $432 \mathrm{MHz}$ b) measured received spectrum with 1USB suppression c) measured received spectrum with 1LSB

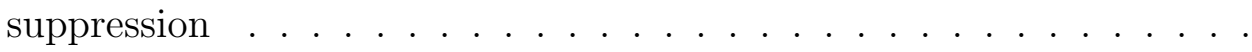

8.3 a) 4 -Element $\mathrm{PMSA}$ working at $432 \mathrm{MHz}$ b) measured received response of scattered signals with 1USB, 2USB, 2LSB, and 3LSB suppression c) measured received response of scattered signals with 1LSB, 2LSB, 2USB, and 3USB suppression . . . . . . . . . . . .

8.4 E-cut plane radiation pattern gain of the printed quasi-Yagi dipole

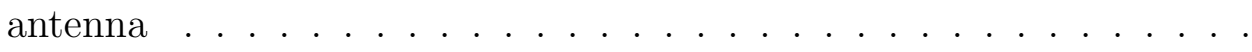

8.5 a) $2.3 \mathrm{GHz}$ printed quasi-Yagi scatterer b) received spectrum of scattered signals at $\theta=-90^{\circ}$ c) received spectrum of scattered signals at $\theta=-45^{\circ} \mathrm{d}$ ) received spectrum of scattered signals at $\theta=0^{\circ} \mathrm{e}$ ) received spectrum of scattered signals at $\theta=+45^{\circ}$ f) received spectrum of scattered signals at $\theta=+90^{\circ} \ldots \ldots$. . . . . . . . . .

8.6 a) Measured received spectrums of scattered signals generated by a single scatterer for both $\mathrm{ON}$ and OFF states b) simulated scattered beams of some selected harmonics components generated in a single scatterer c) measured scattered beams of some selected harmonics components generated in a single scatterer . . . . . . . . . . . . . . 246

8.7 2.3GHz 2-element PMSA using printed quasi-Yagi scatterers . . . . . 249 
8.8 Measured and simulated performances of the 2-element PMSA performing as a spatial image-reject mixer for three different setups a) received scattered beams of harmonics for the first setup b) received spectrum of scattered signals at $\theta=0^{\circ}$ for the first setup c) received spectrum of scattered signals at $\theta=-44^{\circ}$ for the first setup d) received scattered beams of harmonics for the second setup e) received spectrum of scattered signals at $\theta=+21^{\circ}$ for the second setup f) received spectrum of scattered signals at $\theta=-21^{\circ}$ for the second setup g) received scattered beams of harmonics for the third setup $h$ ) received spectrum of scattered signals at $\theta=0^{\circ}$ for the third setup i) received spectrum of scattered signals at $\theta=-44^{\circ}$ for the third setup . . . . .

8.9 A comparison between measured received spectrums of a 2-element PMSA in the first round and a single scatterer captured at an angle $\theta=0^{\circ} \ldots \ldots \ldots \ldots \ldots \ldots$

8.10 Measured and simulated performances of the 2-element PMSA performing as a spatial balanced-modulator for an illuminating signal $\theta_{o}=+30^{\circ}$ and $d=\lambda_{o}$ a) scattered beams of distortion products (harmonics) b) received spectrum of scattered signals at $\theta=0^{\circ}$ c) received spectrum of scattered signals at $\theta=-30^{\circ} \ldots$. . . . . . . . 252

8.11 Continued . . . . . . . . . . . . . . . . 253

8.11 a) Photographs of the $4 \times 4$-element PMSA b) photographs of the $8 \times 2$-element PMSA (bottom view) c) Photographs of the $8 \times 2$-element PMSA (top view) d) received spectrum of scattered signals when the $8 \times 2$-element PMSA is OFF e) received spectrum of scattered signals when the $8 \times 2$-element PMSA is ON (higher bandwidth resolution) $\mathrm{f}$ ) received spectrum of scattered signals when the $8 \times 2$-element PMSA is $\mathrm{ON}$ (lower bandwidth resolution) . . . . . . . . . . . .

8.12 Scattered beams of distortion products generated by the $4 \times 4$-element PMSA from (solid-line simulated and dotted-line measured) a) 1st experimental round b) the 2nd experimental round c) the 3rd experimental round $\mathrm{d}$ ) the 4 th experimental round e) the 5 th experimental round $\mathrm{f}$ ) the 6 th experimental round . . . . . . . . .

8.13 Measured and simulated spectrums of the $4 \times 4$-element PMSA when the first element on the left in Figure 8.11a considered as a reference for the direction of progressive phase shift distribution a) taken at $\theta=+30^{\circ}$ for $\theta_{o}=+30^{\circ} \mathrm{b}$ ) taken at $\theta=0^{\circ}$ for $\theta_{o}=+30^{\circ} \mathrm{c}$ ) taken at $\theta=-30^{\circ}$ for $\theta_{o}=+30^{\circ} \mathrm{d}$ ) taken at $\theta=+30^{\circ}$ for $\theta_{o}=0^{\circ}$ e) taken at $\theta=0^{\circ}$ for $\theta_{o}=0^{\circ} \mathrm{f}$ ) taken at $\theta=-30^{\circ}$ for $\left.\theta_{o}=0^{\circ} \mathrm{g}\right)$ taken at $\theta=+30^{\circ}$ for $\theta_{o}=-30^{\circ} \mathrm{h}$ ) taken at $\theta=0^{\circ}$ for $\theta_{o}=-30^{\circ} \mathrm{i}$ ) taken at $\theta=-30^{\circ}$ for $\theta_{o}=-30^{\circ} \ldots \ldots \ldots \ldots \ldots$ 
8.14 Measured and simulated spectrums of the $4 \times 4$-element PMSA when the first element on the right in Figure 8.11a considered as a reference for the direction of progressive phase shift distribution a) taken at $\theta=+30^{\circ}$ for $\theta_{o}=+30^{\circ} \mathrm{b}$ ) taken at $\theta=0^{\circ}$ for $\theta_{o}=+30^{\circ} \mathrm{c}$ ) taken at $\theta=-30^{\circ}$ for $\left.\theta_{o}=+30^{\circ} \mathrm{d}\right)$ taken at $\theta=+30^{\circ}$ for $\left.\theta_{o}=0^{\circ} \mathrm{e}\right)$ taken at $\theta=0^{\circ}$ for $\theta_{o}=0^{\circ} \mathrm{f}$ ) taken at $\theta=-30^{\circ}$ for $\theta_{o}=0^{\circ} \mathrm{g}$ ) taken at $\theta=+30^{\circ}$ for $\theta_{o}=-30^{\circ} \mathrm{h}$ ) taken at $\theta=0^{\circ}$ for $\left.\theta_{o}=-30^{\circ} \mathrm{i}\right)$ taken at $\theta=-30^{\circ}$ for $\theta_{o}=-30^{\circ}$. . . . . . . . . . . . . . .

8.15 A comparison between the measured received spectrums of the $4 \times$ 4-element PMSA and a single scatterer . . . . . . . . . . . .

8.16 Measured and simulated scattered beams and spectrums of the $8 \times$ 2-element PMSA when the first element on the right in Figure 8.11b considered as a reference for the direction of progressive phase shift of modulation signals and the illuminating angle $\theta_{o}=+15^{\circ}$ a) scattered beams of LSB distortion products (solid-line simulated and dotted-line measured) b) scattered beams of USB distortion products (solid-line simulated and dotted-line measured) c) received spectrum taken at $\theta=+30^{\circ} \mathrm{d}$ ) received spectrum taken at $\theta=+15^{\circ}$ e) received spectrum taken at $\theta=0^{\circ} \mathrm{f}$ ) received spectrum taken at $\left.\theta=-15^{\circ} \mathrm{g}\right)$ received spectrum taken at $\theta=-30^{\circ} \ldots \ldots$. . . . . . . . . . . .

8.17 Measured and simulated scattered beams and spectrums of the $8 \times$ 2-element PMSA when the first element on the left in Figure 8.11b considered as a reference for the direction of progressive phase shift and the illuminating angle $\theta_{o}=0^{\circ}$ a) scattered beams of LSB distortion products (solid-line simulated and dotted-line measured) b) scattered beams of USB distortion products (solid-line simulated and dotted-line measured)c) received spectrum taken at $\theta=+30^{\circ} \mathrm{d}$ ) received spectrum taken at $\theta=+15^{\circ}$ e) received spectrum taken at $\theta=0^{\circ} \mathrm{f}$ ) received spectrum taken at $\theta=-15^{\circ} \mathrm{g}$ ) received spectrum taken at $\theta=-30^{\circ}$

8.18 Measured responses taken at an angle of $\theta=0^{0}$ when a $2 \times 1$-element PMSA beahves like a) a two-tone USB image reject mixer b) a two-tone LSB image reject mixer c) a balanced modulator . . . . . . . . . . .

8.19 Measured spectrums of a single scatterer and a $8 \times 2$-element PMSA, when the first element on the right in Figure 8.11b considered as a reference for the direction of progressive phase shifts distributions of the two-tone modulation signals and the illuminating signal source located at $\theta_{o}=+15^{\circ}$, taken at a) a single scatterer $\theta=0^{\circ}$ b) $\theta=-45^{\circ}$ c) $\theta=-30^{\circ}$ d) $\theta=-15^{\circ}$ e) $\theta=0^{\circ}$ f) $\theta=+15^{\circ}$ g) $\theta=+30^{\circ}$ h) $\theta=+45^{\circ} 270$

8.20 Measured and simulated scattered beams of IMDs and HDs generated by the $8 \times 2$-element PMSA a) scattered beams of LSBs (1st setup) b) scattered beams of USBs (1st setup) c) scattered beams of LSBs (2nd setup) d) scattered beams of USBs (2nd setup) e) scattered beams of LSBs (3rd setup) f) scattered beams of USBs (3rd setup) . . . . . . . 273 
8.21 Measured spectrums of the $8 \times 2$-element PMSA, when the first element on the left in Figure 8.11b considered as a reference for the direction of progressive phase shifts of the two-tone modulation signals and the illuminating signal source located at $\theta_{o}=+15^{\circ}$, taken at a) $\theta=-45^{\circ}$ b) $\theta=-30^{\circ}$ c) $\theta=-15^{\circ}$ d) $\theta=0^{\circ}$ e) $\theta=+15^{\circ}$ f) $\theta=+30^{\circ}$ g) $\theta=+45^{\circ} 274$

8.22 A photograph of the $8 \times 1$-element PMSA prototype performing inside the anechoic chamber . . . . . . . . . . . . . . . 275

8.23 Scattered beams of distortion products generated by the $8 \times 1$-element PMSA with fixed $d$ and varying $\theta_{o}$ of a) the 2USB b) the 1USB c) the 1LSB d) the 2LSB, (solid-line simulated, dotted-line measured) . . . .

8.24 Scattered beams of distortion products generated by the $8 \times 1$-element PMSA with varying $d$ of a) the 2 USB b) the 1 USB c) the 1 LSB $d$ ) the 2LSB, (solid-line simulated, dotted-line measured) . . . . . . . . .

8.25 Measure received spectrums of scattered distortion products when some elements of the $8 \times 1$ PMSA turned-off a) all elements turned-on b) 2 nd c) 4 th d) 4 th +5 th e) 2 nd +6 th f) 3 rd +7 th g) $3 r d+5$ th +7 th h) 1 st +5 th +8 th i) 2 nd +4 th +6 th . . . . . . . . . . . . . . . 280

8.26 Scattered beams of distortion products (2LSB, 1LSB, 1USB, and 2USB) when a number of elements in the $8 \times 1$ PMSA turned-off a) single element b) two elements c) three elements (solid-line simulated, dotted-line measured) . . . . . . . . . . . . . . .

8.27 Measured results of the frequency tunable PMSA captured at an angle of $\theta=0^{\circ}$ for a) $700 \mathrm{~Hz}$ b) $899 \mathrm{~Hz}$ c) $1004 \mathrm{~Hz}$. . . . . . . . . . . . . 283

8.28 A photograph of the $8 \times 1$-element PMSA prototype using quarter-wave monopoles antennas, performing inside the anechoic chamber . . . . .

8.29 Measured results along with theoretical results of the angles of the incident RF signals versus the captured angles of the 1LSB and 1USB distortion products . . . . . . . . . . . . . . . 285 


\section{Chapter 1: Introduction}

\subsection{Motivation}

In the realm of microwave and radio frequency $R F$ circuit design, the nonlinear term refers to elements that distort RF signals when passing through the nonlinear elements. Frequency spectrums of distorted signals consist of a massive number of distortion products. The distortion product term refers to harmonic distortion (HD) products or intermodulation distortion (IMD) products when single-tone or multi-tone signals are exposed to impacts of nonlinear elements, respectively. Although the generation of distortion products is a persistent problem with operation of active devices such as mixers, low noise amplifiers (LNAs), and especially power amplifiers (PAs) [166] [81] [162] [182] [35], passive devices such as transmission-lines (TLs), filters, antennas, and connectors could also distort signals and generate distortion products called passive intermodulation (PIM) distortions, but with less marked effects [179] [152] [183] [33] [68] [126]. In essence, as the power of signal increases, influences of distortion products become prominent, leading to a degradation in overall performances of communication systems.

For decades, tremendous efforts have been made by researchers to alleviate impacts of distortion products on receiving and transmitting circuits, and this topic is still of great interest, especially for new emerging technologies such as massive multi-input multi-ouput (MIMO) and 5th generation communication systems. To remove unwanted distortion products, conventionally, designers use fixed-band filters as frequency-selective devices. Filters should have responses with steeper roll-offs to dismiss or at least highly attenuate unwanted distortion products to acceptable levels. Using filters is feasible only in receivers while power amplifiers which consider the main 
source of distortion products are the last part in transmitters before launching RF signals into space. As a consequence, only IMDs which lie within the frequency band of interest that cannot be removed by filters exist at outputs of receivers, whereas all harmonics and IMDs are emitted into space in transmitters. Generally, distortion products are unavoidable, but they can be mitigated to some extent being acceptable in some applications if some techniques are utilized.

If either receivers or transmitters consist of sub-circuits and each sub-circuit has its own antenna and amplifier as in active arrays, sub-circuits are independent sources of distortion products [29] [121] [19]. These distortion products are subject to rules of phased antenna arrays, being constructively or destructively added relying on phases of distortion products generated by different sub-circuits. Hence, analyzing distortion products produced by an array of nonlinear elements and controlling them in space rather than using conventional ways such as waveguides, TLs, and power dividers and combiners, considering only the transmitting side in our analysis, is the main theme of this research work.

\subsection{Phase-Modulated Scattering Array (PMSA) Paradigm}

The proposed phase-modulated scattering array (PMSA) consists of an array of modulated scatterers (MSs). Each single MS consists of an antenna and an electronic switch (typically, it is a diode or a transistor). Switches are controlled by modulation signals, and they are not switched $\mathrm{ON} / \mathrm{OFF}$ at the same time. The time delay in the switching process across an array of scatterers can be interpreted as a phase shift distribution in the frequency domain analysis as will be seen later in theoretical models of the presented structure in Chapters 3 and 4 . It can be leveraged from this property to obtain the functionality of phase shifters without using real ones. This makes our proposed structure is simple, affordable, and easy to reconstruct. 
To function properly, the PMSA should be illuminated by an external signal called the incident signal as shown in Figure 1.1 which ranges from acoustic to terahertz bands depending on an application and a resonant frequency of scatterers, making it as a scalable design. Moreover, the modulation frequency should be much smaller than the illuminating frequency for reasons illustrated throughout this dissertation. The incident signals experienced modulation by a process of the switching ON-OFF reflect back (scatter) toward space again. The incident signals in our proposed system are single-frequency components, but the scattered signals are multi frequency components (i.e., distortion products). As will be seen later on, each single-frequency component (i.e., single distortion product) of scattered signals emerges at a different location in space. In other words, this means that different distortion products generated by different MSs combine constructively or destructively at different spatial locations over-the-air, considered as a substantial feature of the proposed system. The process of directing different scattered distortion products into different spatial locations resembles the functional operation of the spatio-temporal technique. In fact, each scattered signal represents a single distortion component generated by active switches as a result of mixing of the illuminating signals with modulation signals.

Because the incident signals coming from a particular direction arrive at array elements at different times, they have the same frequency and different phases when induced over elements. Thus, Phases of the incident signals mainly depend on the spatial location of the illuminating source, which in turn affect phases of the scattered distortion products. As a consequence, directions of scattered signals (distortion products) are changed. We can deduce that by changing locations of the illuminating source, switching times of the modulation signals, or both, locations of distortion products will change in space. The resulting PMSA is a 
reconfigurable or tunable structure without modifying the main structure of the PMSA. As we will see later, because each single distortion product generated from different scattering elements has an independent phase shift distribution and a frequency from other distortion products generated from the same PMSA at the same time, it is associated with an independent scattered beam (i.e., radiation pattern). The scattered beams can be scanned in our proposed structure. The PMSA can

provide the beamforming functionality without using incorporated feeding network (transmission-lines) and incorporated phase shifters. Our proposed PMSA is simpler to design and build compared to conventional phased arrays. Also it can be considered as a spatially-reconfigurable distortion products canceler. This considers as a new paradigm shift for processing distortion products (signals) in space.

\subsection{Literature Review}

Because this is the first time where handling of distortion products occurs in space based on the modulated scattering techniques (MST), the directly related literature is sparse. Thus, we will address some topics that are either included in the basic structure of the PMSA or somewhat similar in their underlying work. They are as following:

\subsubsection{Modulated Scattering Techniques (MST)}

The modulated scatterer (MS) which considers the main brick in our proposed systems is one of the main parts in the MST. Thus, this subsection briefly summarizes the state of the art of the research work exploiting the features of the MST to distinguish signals scattered from targeted objects surrounded by other obstacles or non-intended objects. 

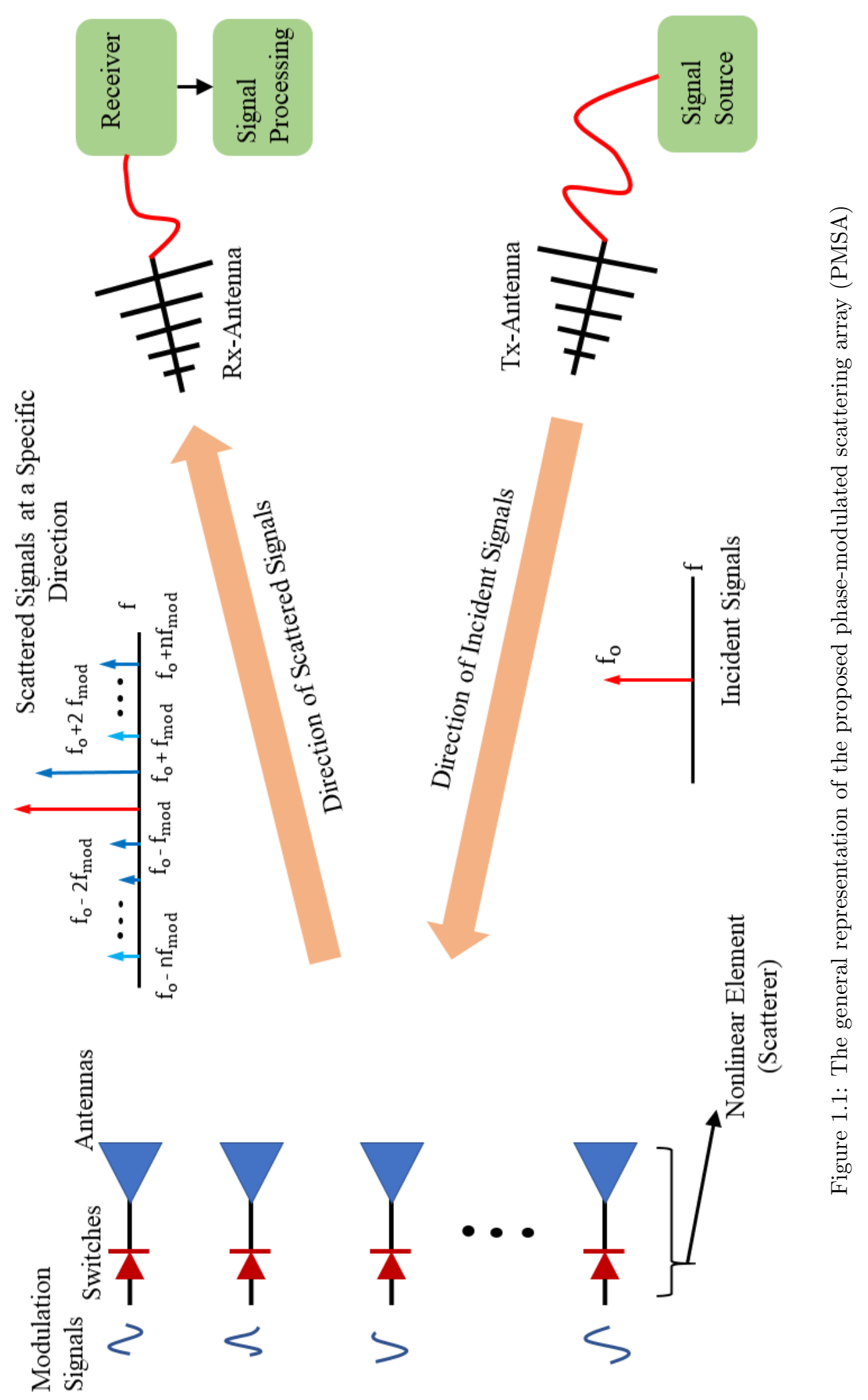


\subsubsection{Single MST Scatterers in Literature (RFID and IoT Sensors)}

Radio frequency identification (RFID) tag is one of the most common applications of the MST, interrogated by readers using electromagnetic waves [39]. The scattering of transmitted signals using a single scatterer (tag) enables the communication between a reader and a tag by means of the power reflection, and it is considered as the basis of the RFID technology. Data embedded inside a tag can be retrieved from fluctuations of scattered signals. Furthermore, the recent evolution of IC-manufacturing and embedded systems have assisted in developing multifunctional scatterers able to receive, collect, analyze, store, and re-transmit the related information [39]. In [43] [41], authors developed a guideline to optimize an RFID sensor based on the MST, demonstrating that the new optimization technique enables an extended communication range, while in [23] [15], an analytically-mathematical procedure to exploit the MST as a basis to design an RFID tag is presented. Authors used two different analytical scenarios which are the far-field FF scenario and the reciprocity scenario to obtain a compromise between the scavenged and scattered powers as a step to energize internal circuits of a tag by the requirement of the lowest power level and to achieve the longest scattering range by reflecting the highest available power level. In the X-band, at $10 \mathrm{GHz}$, an RFID tag sensor has been implemented to work at a distance of up to $10 \mathrm{~m}$, thanks to the use of an antenna array and a rectifying multiplier circuit [41]. To increase the reading range at distances of up to $20 \mathrm{~m}$, a low-power switching circuit used in the design has been obtained [42]. Furthermore, two identical antennas connected to outputs of a 3dB hybrid coupler working as a temperature sensor is proposed. Inputs of a coupler are modulated utilizing a frequency modulator incorporated with a thermal capacitance to sense the surrounding temperature. It is shown that the proposed system was able to connect 
with readers at distances of about $10 \mathrm{~m}$ [27].

All works presented above have limitations in a number of the transmitted bits which is only a single bit, so in [24] [170] [167], authors were working to find a way that can increase a number of transmitted bits that can also be useful to reduce the power wasted inside internal circuits of tags which leads to increase the scattered power which in turn increases the reading range at the cost of the design complexity. In [24], authors present some new configurations that use a bank of loads (at least three different loads) rather than using two loads as in the conventional binary-MST (B-MST). Using more than two loads aids to send more than one bit at a time. Also, in [24], a thermistor acting as an extra load is proposed to realize a useful application for their proposed work. CMOS technology has been utilized to build new small foot-print underlying circuits of tags with low power consumption to achieve high data-rate connection such as 4QAM, 8QAM, and 16QAM schemes [170] [167].

Without using sensors, Internet-of-things (IoT) will not exist. Therefore, looking for sensors working effectively with low power consumption, sensors based on the MST are the most suitable to perform this task. Moreover, sensors play a vital role to make things simultaneously connected to the infrastructure of the Internet, observing and measuring the environment periodically. Many sensors based on the MST probes buried inside soils to monitor plants are reported in [38] [37]. Each sensor has its own frequency band exploiting frequency multiplexing techniques to enable a robust data exchange without disturbing the monitored and measured information. In [30], frequency doubling technique based an IoT sensor is presented to avoid the scattering from undesired objects and the direct coupling between a receiver and a transmitter of a base station (reader), thereby mitigating the unwanted influences.

Some research efforts have been directed to increase the reading range regardless the use of proposed designs as an RFID tag or an IoT sensor. All research works 
presented up to now, with different technologies of manufacturing, have distance limitations of no more than $20 \mathrm{~m}$, so finding out a way to increase a reading range is necessary. In [4] [7] [6], reflection amplifiers, which are tunnel diode amplifiers, are proposed to be used instead of conventional RF switches. Authors demonstrated that their work does not consume high energy which is only $2.9 \mathrm{pJ} /$ bit compared to Bluetooth Low Energy (BLE) transmitters which need 10nJ/bit. A 35dB return gain has been achieved in [6] to enhance the reading range which was above $1.2 \mathrm{~km}$ achieved on the campus of Georgia Institute of Technology (Gatech) while consuming power as low as $20.4 \mathrm{uW}$. In [49], a $17 \mathrm{~dB}$ tunnel diode amplifier has obtained at input power $-30 \mathrm{~dB}$, while the $\mathrm{DC}$ voltage bias is $200 \mathrm{mV}$ with power consumption equal to 0.2mW. These designs help MST probes to interact with low power incident signals. Also, active amplifiers incorporating 3dB hybrid couplers are proposed in [48] [80] to perform as two functional devises which are retro-directive systems and MST scatterers. In [48] low power FET transistors are employed to work as dual-band reflection amplifiers (i.e., dual-band active MST scatterers) using synthesized artificial transmission lines (metamaterial) to building matching circuits and $3 \mathrm{~dB}$ hybrid couplers.

\subsubsection{Arrays of MST Scatterers in Literature (Microwave Imaging)}

In this part, applications using arrays of MST scatterers are presented to show that they can work as a whole system to handle some issues that could be encountered if a single MST scatterer is used to perform the same task. Although a mechanically scanned single scatter is used to measure the spatial distributions of electric fields of objects, it takes long times to examine a whole object where this time is undesirable in real-time imaging applications [14]. To circumvent this problem, researchers used MST arrays in different real-world applications to provide measures of electric fields 
at locations of scattering array elements [1]. The measuring times can be incredibly reduced, but the new challenge is a number of scatterers in arrays especially when working at high frequencies (e.g., millimeter frequencies). In [16], the MST based imaging systems using such a spatial multiplexing technique has been proposed. By spatially controlling the MST scatterers at known locations of fields of interest, we can extract an important information by mapping fields according to their proper locations [125]. One single-frequency receiver located away from an object being imaged is adequate to retrieve the required fields. To accelerate the imaging process, a system, consisting of an array of elements where each single element associated with a different frequency modulator to increase the system resolution, has been implemented. In [45] [54], authors proposed a two-dimensional millimeter array with focused scattered fields using lens at the front of the scattering array.

The measure of antenna patterns using the MST array as a basis of the scheme is widely used [53], while it was first presented in [138]. There are several types of scatterers proposed in the literature to map electric fields [53], but the small linear dipole scatterers are the most used type. In [51] [103], sub-resonant dipoles arranged in one-dimensional arrays have been designed and fabricated at $12 \mathrm{GHz}$. The proposed MST imaging systems have some advantages which are compact and robust since each scatterer in imaging arrays works at a different frequency, thereby no need to use a multiplexing control circuit. Thus, this frequency multiplexing scheme allows the use of a single RF chain and it aids the modulation and demodulation processes to distinguish and localize the measured signals according to their frequencies.

\subsubsection{Time Modulated Linear Array (TMLA)}

The concept of TMLA is similar to a phased antenna array but with simple hardware implementation. Thus, the TMLA considers as an unconventional phased array, being 
increasingly researched during the last two decades [139]. In its simplest configuration, it is a phased array whose radiation patterns are governed by periodically switching ON/OFF excitations of array elements not at the same time. Switches are also considered here the main parts of a design. Using time to control directions of radiation patterns is first proposed by [88] [150], providing an additional degree of freedom. Authors formalized a theoretical model for their new idea. Afterward, they introduced several antenna array configuration using the TMA technique such as a linear array controlled by a square wave. By using a common parabolic dish antenna with a moving horn antenna working at a frequency $133 \mathrm{MHz}$, they succeeded to achieve a multi-beam radiation pattern to simultaneously obtain a constructively added beam at the main frequency and destructively added beam at the the first sideband harmonic. In 1963, an 8-element waveguide slotted antenna array operating at the $10 \mathrm{GHz}$ (X-band) with a set of RF switches integrated into the incorporated feeding network is proposed as the first use of the fourth dimension in realizing phased antenna array [88]. They stated that sidelobes levels (SLL) have been highly reduced, and sidelobes levels can also be changed by only reprogramming duration times of switches. Some optimization algorithms such as a differential evolution algorithm are proposed in [180] to synthesize radiation beams at the main and harmonic frequencies. Other different optimization algorithms such as genetic algorithm, simulating annealing, particle swarm optimization (PSO), and artificial bee colony are introduced in the subsequent works [50] [181] [129] [109].

Later on, the time dimension is divided into two parts which are switching-ON instants of controlling signals and signal durations as illustrated in [164] [3]. Radiation beams of harmonics can be reduced if switches integrated with antennas have different switch-ON instants as illustrated in [185]. Likewise, sub-arraying techniques, narrow-band antennas, and pulse shaping are also used to mitigate 
impacts of harmonics generated by the switching process. To reduce the power lost during switch-OFF times, in [184], authors suggested to use a single-pole double-throw(SPDT) switches in such a way that each single SPDT switch can govern a pair of adjacent antennas, thereby reducing the switching-OFF time to zero.

Some researchers decided to profitably exploit harmonics generated by TMAs instead of ignoring them by means of mitigation [96] [128] [173]. As a matter of fact, radiation patterns associated with each single harmonic can endow smart-antenna capabilities if they are exploited properly. Although the beamforming capabilities are theoretically introduced by the seminal work of Shank in 1961 [149], such a feature is efficiently demonstrated in an experimental work using a $4 \times 2$-element patch time modulated array [13]. Algorithms of adaptive beamforming associated with TMA capabilities are originally investigated from theoretical and practical points of view by [97], synthesizing radiation patterns of harmonics in [172], and even discussing benefits of the time redundancy. A different scenario for beamforming of radiation patterns of harmonics via the pulse splitting approach was demonstrated in detail in [128]. In such an approach, with the PSO, authors controlled two patterns simultaneously: the first pattern at the main frequency and the second pattern at any preselected harmonic, keeping other harmonics below the desired level. In [11], an example of how beamforming capabilities of TMA is profitably applied with two different sets of coefficients of beamforming, in the case of the sidelobes blanking in radar and spectrometery, was proposed. Direction of arrival or direction finding is also demonstrated employing the TMA beamforming capabilities as introduced in [65] [98] [165]. 


\subsubsection{Time Modulated Metasurfaces (TMMS)}

Owing to their capabilities to manipulate wavefronts of electromagnetic signals, metasurfaces have been extensively used to build ultrathin structures. Generally, they consist of an array of patches, rods, or any other shapes arranged in a periodic manner with spacing between any centers of two successive elements less than a quarter wavelength at a design frequency. In this condition, whole structures are considered as homogeneous, and their impacts on waves become prominent [117]. Although they are used in a wide range of wave manipulations, we address only the time modulated metasurfaces for their similarities in somehow to our proposed work in their underlying functional work.

Metasurfaces are also utilized widely in optical applications because of using the electro-optical material such as indium tin oxide ITO and graphene that have quick responses for biasing voltages. They can be modulated by frequencies up to several gigahertz [144] [145] [176] [72], so that such structures can be modulated using the conventional RF biasing circuits. Also here, introducing the time to modulate the properties of metasurfaces by changing biasing voltages leads to generation of harmonics due to the periodical behavior of time modulation. Due to generation of high order harmonics distributed about the main illuminating frequency, the time reversal symmetry is broken. Breaking the reversal symmetry has been exploited in several research efforts to design magnet-less nonreciprocal devices [161] [154] [61]. This occurs by utilizing a periodic space-time (spatiotemporal) modulation along the direction of modulation wave propagation which enables the circulation and isolation of the electromagnetic waves (light) by isolating different momentums to propagate in forward and backward directions. In [61], researchers show that the guided-modes in space-time photonic metasurfaces are transited into leaky-modes to enable a nonreciprocal dynamic scanning-beam leaky-wave antenna. However, a bandwidth of 
their design structure is narrowband, so in [144], authors used metasurfaces modulated with a temporal phased delay to circumvent the narrowband issue. Time modulated Huygens metasurface is also presented in [102], consisting of unit cells of magnetic and electric dipoles.

\subsubsection{Distortion Products in 5G Phased Arrays}

Because arrays of nonlinear elements (active phased arrays) are a key part of the next generation of communication systems, we here briefly review some recent literature about impacts of distortion products generated in the millimeter-wave 5G phased arrays on the overall performances. Using beamforming techniques is necessary to ensure high data rate at higher frequencies to compensate free-space losses [141]. In [143] [142], some 5G phased array prototypes have been demonstrated for ranges of several hundred meters with gigabit per second communication links. 5G phased arrays serve a big number of users, so interference becomes a major problem and decreases sensitivity of $5 \mathrm{G}$ phased arrays. All nonlinear elements in each branch of phased antenna arrays generate distortion products, and these distortion products could be added constructively or destructively depending on phases of distortion products at different elements of $5 \mathrm{G}$ phased arrays.

In [146] [66] [74] [75], transmit phased arrays with fixed multi-beam characteristics have been analyzed under impacts of intermodulation distortion IMD products, while in [77], authors proposed a technique to alleviate impacts of third-order IMD (IMD3). However, these studies focused only on fixed beams transmit arrays, but beams should be scanned to fulfill requirements of the $5 \mathrm{G}$ systems. Analyzing distortion products generated in receive phased arrays has been achieved in [127] [105] [106]. In [127], an experimental setup with two-interferer has been demonstrated. A phased array in the receive mode working at $28 \mathrm{GHz}$ using 
three-interferer scenarios has been analyzed to show influences of distortion products on the entire proposed system. Their work demonstrates that distortion products can be beamformed relying on directions of interferers, and thus radiation beams of distortion products, especially IMD3, could be added destructively or constructively at an array output. In summary, dynamic ranges of receivers are highly reduced if patterns of distortion products are added constructively with the patterns of desired signals.

\subsection{Research Objectives}

In this research work, the modulated scattering technique (MST) based analysis and control of distortion products generated by a nonlinear array in space is presented. The presented design which is the phase-modulated scattering array (PMSA), is introduced for the first time. Thus, the main objectives of this research work are as in the following:

1. We aim to develop a comprehensive mathematical model used to analyze distortion products generated by our presented PMSA over-the-air rather than using conventional physical transmission lines under communication signal processing perspectives as in RF and microwave communication circuits.

2. Because each single distortion component is independent in frequency and phase and it may or may not have the same phase compared to other replicas generated by other nonlinear elements (i.e., scatterers), we aim to develop a mathematical model that is able to deal with beam patterns (scattered beams) of different distortion products simultaneously under phased antenna array perspectives.

3. We seek to shed light on some features, parametric studies, and potential applications of the proposed PMSA. 
4. We aim to physically implement prototypes of the proposed PMSA at two different frequencies and with different number of elements to validate that the PMSA is scalable.

5. We aim to evaluate single-element, 2-element, 4-element, and 8-element PMSAs at two different frequencies which are $432 \mathrm{MHz}$ and $2.3 \mathrm{GHz}$ using different types of antennas and nonlinear elements.

6. We aim to experimentally validate some parametric studies.

7. We also aim to practically validate some features and applications of the proposed PMSA such as retrodirective scattered beams, beamforming, tunable spatial harmonic generator, and direction finding.

In general, in the theoretical parts which are the objectives 1,2 , and 3 , we seek to develop appropriate and convenient mathematical models to analyze distortion products generated by not only our proposed PMSA but also any array of nonlinear elements such as conventional phased arrays, massive MIMO arrays, time-modulated arrays, and time-modulated metasurfaces. The mathematical models are usually the most tedious as no systematic and universal mathematical models exist so far connecting between the distortion products generated in active arrays and their locations in space and such mathematical analysis is usually performed via different simulations of different structures for our PMSA.

\subsection{Dissertation Outline}

This dissertation has nine chapters, as following:

1. Chapter One presents a general motivation, a paradigm of the proposed PMSA, a literature review, objectives, and a general structure of the dissertation. 
2. Chapter Two introduces background basic theories of some related topics.

3. Chapter Three presents the first mathematical model derived under communication signal processing perspectives exploiting the well-known polyphase multipath technique without and with gain and phase errors and it also presents some simulation results supporting the derived mathematical model.

4. Chapter Four gives the second mathematical model derived under phased antenna array theory perspectives to investigate scattered beams of different distortion products simultaneously.

5. Chapter Five discusses some features of the presented design such as diffraction grating-like behavior, types of scattered beams, nonreciprocal property, beamforming, and it also discusses some applications such as beamforming, spatial distortion product generator, and direction finding.

6. Chapter Six presents designs of different single antennas and arrays using the software ADS and HFSS with preliminary measurements performed outside the anechoic chamber to characterize antennas before testing the whole PMSAs. Also, this chapter includes designs and fabrications of nonlinear elements (RF diode switches) and a 4-to-8 phase transformer kit using only resistors.

7. Chapter Seven discusses the environment with some apparatus (experimental setup) required to perform measurements of the presented system.

8. Chapter Eight presents the experimental program to obtain the measured results conducted to validate our presented ideas along with the theoretical results. Different structures of PMSAs have been tested. 
9. Chapter Nine summarizes the theoretical and experimental works and conclusion drawn based on previous chapters. Also, it provides the future works which can be potentially obtained using our mathematical model, proposed structures, or both. 


\section{Chapter 2: The Basic Theoretical Background}

\subsection{Introduction}

This chapter covers the most basic concepts and underlying theories that are useful to the study of the work done in this dissertation which is the phase-modulated scattering array (PMSA). This study is rooted in the combination of three different study fields: (1) the study of modulated scattering technique (MST) at any arbitrary frequency band ranging from audio to terahertz signals in the vicinity of electromagnetic radiators, (2) the study of phased antenna array theory as a spatial filtering system, and (3) the study of image-rejection mixer process. Thus, the goal of this chapter is to narrow our focus on these three essential subjects to offer a useful context for the proposed structure that will be thoroughly deliberated in the next three chapters. However, slight deviations can be found in some places in the background narrative to include all necessary background, leading to a complete understanding.

\subsection{The Study of Modulated Scattering Technique (MST)}

The detection of spatial scattered beams of distortion products departing an array of modulated scatterers (i.e., PMSA) in a periodical dynamic environment is nothing more than the characterization of phased arrays that their elements constructing a whole system are frequency-modulated antennas (i.e., modulated scatterers MSs) rather than using conventional antennas. MSs are the main bricks of modulated scattering techniques (MST). Generally, the modulated scattering technique MST is widely used to accurately characterize electric field distributions of objects being measured or imaged in vicinity of radiating sources [76] [138]. In antiquated field measurement and detection ways, a reciprocal antenna is placed where interesting 
locations are to measure intended scattered responses due to illuminating signals hitting objects. Those methods require systems in optimal conditions to yield a precise field acquisition. Consequently, many factors can influence field distributions such as antennas, cables, uncontrolled reflections from the surrounding background, and probe movements, so a measurement setup is a bit challenging to reproduce due to unpredictable surroundings. All these limitations, previously mentioned, motivated to develop a technique capable of removing or substantially mitigating the severity of these problems. This technique is the MST [138] [69] [156] [36] [175] [52] [174] [71]. The MST which is an alternative method to the former measurement manner plays a vital role in the study and investigation of the core of this dissertation.

The following subsection aims to introduce an underlying mathematical model describing the mechanism of operation of MSs which is optically or electrically driven by modulation sources.

\subsubsection{Mathematical Basics of Modulated Scattering Techniques (MST)}

Figure 2.1 shows a general MST communication scenario where a transmitter Tx and a receiver Rx communicate with an a scatterer (MST probe, transponder, or tag) in a specific small area. The $\mathrm{Tx} / \mathrm{Rx}$ and the scatterer can be used any type of antennas to avoid loss of generality. Scatterers can be placed in free-space or embedded in some materials or structures to monitor surrounding regions. The Tx antenna is fed via transmission lines such as coaxial cables, waveguides, microstrip lines and so on. The transmitted power from the Tx antenna is $P_{a}=1 / 2|a|^{2}$ and the reflected power at the $\mathrm{Rx}$ antenna input is $P_{b}=1 / 2|b|^{2}$, where $a$ and $b$ are the normalized incident and reflected waves, respectively [89]. An input impedance of a scatterer antenna is $Z_{a}=R_{a}+j X_{a}$ connected to a load with an impedance $Z_{L}=R_{L}+j X_{L}$. The origin of the MST paradigm came from altering the load 
impedance, leading to changing properties of scattered signals. For conventional binary modulated scattering techniques, impedance loads have two prescribed values, switched from $Z_{L 1}=R_{L 1}+j X_{L 1}$ to $Z_{L 2}=R_{L 2}+j X_{L 2}$, enabling the ASK or PSK communication over the scattering channel [87]. A transmitter sends signals and propagate toward a scatterer; a part of the sent signals is reflected off a scatterer toward a signal source again when working in a receiving mode, for the mono-static type. The normalized reflected power wave is denoted as $b$, including various kinds of reflections. The first reflection comes from the self-coupling between the transmitter and receiver when using the same antenna(monostatic case), which is given as a.S11. The second type which represents all scattered fields (reflections) from different static obstacles surrounding the intended scatterer is $b_{\text {diff }}$. The last type of reflections, playing a vital role in the performance of the MST, comes from the scatterer itself $b_{\text {tran }}$

$$
b=a . S_{11}+b_{d i f f}+b_{t r a n}
$$

where all terms in (2.1) represent the first-order interaction between the scatterer and the Tx/Rx antenna [56] [57], neglecting all high order interactions since they are very weak. The reflections $b_{\text {tran }}$ arise from a scatterer antenna itself, denoting the antenna mode (AM) resulting from the mechanism of antenna radiation, and the structural mode (ST) resulting from the scattering mechanism due to the metal structure of an antenna:

$$
b_{\text {tran }}=b_{A M}+b_{S T}
$$

The first and second terms of the right-side in (2.2) have different definitions [56], and they can be explained as follows: $b_{A M}$ is totally related to the mechanism of antenna radiation (i.e., received, interacted with a load, and retransmitted again). As a consequence the load impedance directly affects the amount of retransmitted (i.e., 


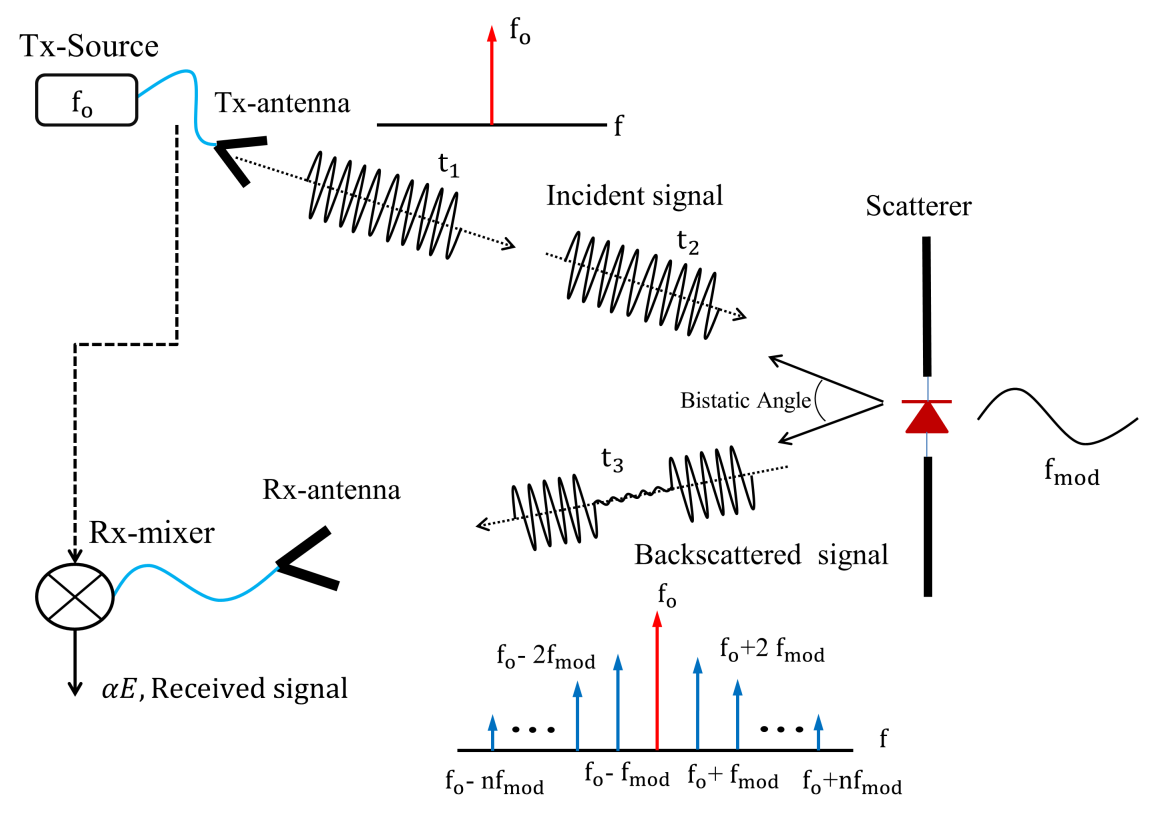

(a)

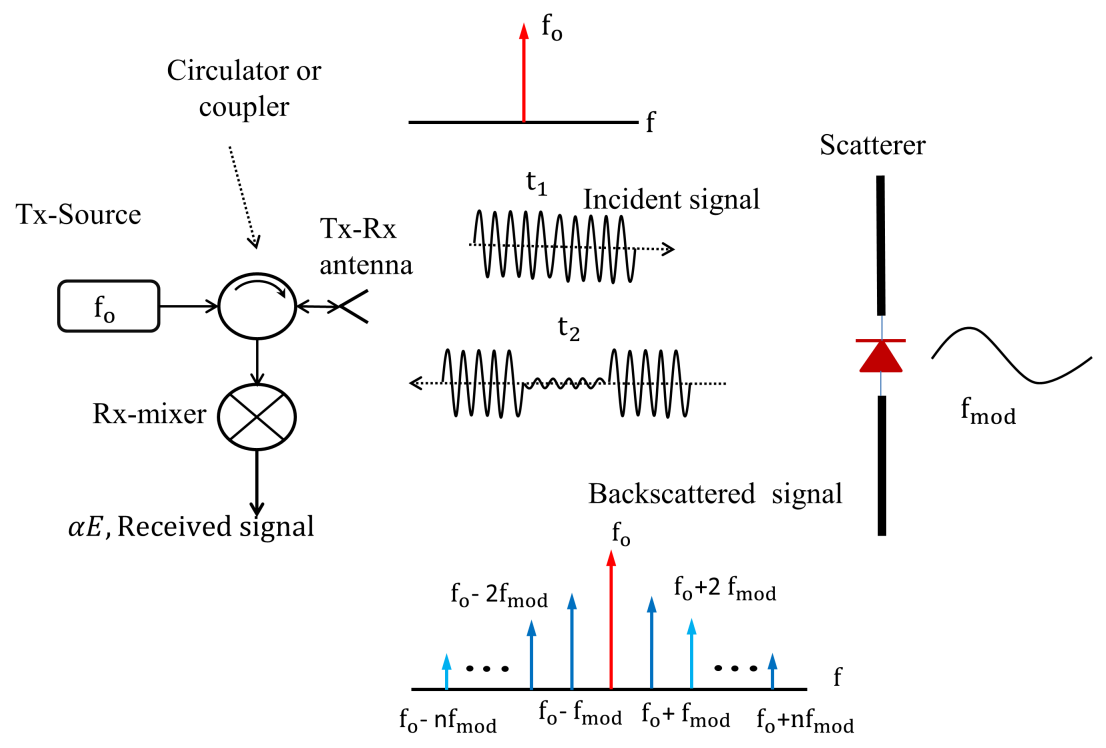

(b)

Figure 2.1: General scenario of MST systems a) bistatic b) monostatic 
scattered) signal power $b_{A M}$, called the load-dependent term. In contrast to that, the load-independent load term $b_{S T}$ has a different mechanism of scattering, depending on an antenna structure (the metallic surface), being scattered back regardless of the load impedance.

To differentiate between signals generated by these two mechanisms, when the incident signal has $90^{\circ}$ polarization rotation (cross-polar) with respect to the scatterer antenna, only the $b_{S T}$ term will exist due to the absence of excited voltage at the input port of the scatterer antenna, as such scattered signals are independent of the antenna load impedance. Moreover, the dependent load term $b_{A M}$ exists only when the co-polar component is available, depending on the ratio of the polarization mismatch between of the incident and scattered signals. The voltage induced by the co-polar component at the antenna input port relies directly on the load impedance, resulting in a current induced over the scatterer antenna related to the load value. Due to difficulty of distinguishing between the two terms of (2.2), there will be an urgent need to find a way able to separate those two terms.

In [155], a point to point communication depended on modulating the reflected power was achieved, resulting in isolating the load-dependent term $b_{A M}$ from the rest of non-modulated and unwanted terms. This process requires a load connected into a scatterer being variable. Changes of load impedance can be carried out either mechanically or electrically. Rotating or displacing scatterers falls under the umbrella of mechanical techniques [16], while, in the electrical methods, a variable load is utilized. There are many ways to realize variable loads such as PIN or Schottky diodes with different biasing states [138], RFID-ICs with variable input impedance [83], or a phototransistor [112]. In this research work, we will focus mainly on the electrical modulation techniques using only Schottky diodes as modulator switches.

Eq.(2.1) can be simplified and grouped into two distinct terms. The first 
term includes all non-modulated contributions from metallic geometrical shapes of scatterers, obstacles and clutters, and the direct coupling between the receiver and transmitter into a one term called $b_{N O N}$, while the modulated term based on the variable load is the second term. Thus, it can be rewritten as

$$
b=b_{N O N}+b_{A M}
$$

For conventional binary scattering systems when having electrical switches (RF diodes as in our PMSA), Eq.(2.3) becomes

$$
b 1=b_{N O N}+b_{A M, 1} \quad\left(\text { for } Z_{L 1}\right)
$$

or

$$
b 2=b_{N O N}+b_{A M, 2} \quad\left(\text { for } Z_{L 2}\right)
$$

We can see that only the second term varies when the load switches between its two states (from $Z_{L 1}$ to $Z_{L 2}$ ), taking the difference between the two states, resulting in a differential reflected wave $\Delta b$

$$
\Delta b=b 2-b 1=b_{A M, 2}-b_{A M, 1}
$$

As a consequence, this process aids to isolate the first term $b_{N O N}$ of $(2.3)$, considering only the second term $b_{A M}$ although both effects present in the measurements, see Chapter 8. In the following part, the MST based on the far-field viewpoint will be explained. 


\subsubsection{Far-field Based Modulated Scattering Techniques (MST) Interpretation}

As described before, a scatterer reflects a portion of the incident signals, constituting a target for $\mathrm{Tx} / \mathrm{Rx}$ transceiver (reader). The radar (scattering) cross-section (RCS) technique and the radar power equation are exploited to introduce an initial analysis of the scattered electric field $E$ from a scatterer, assuming the scatterer (target) stationary and placed in the far-field area of the Tx/Rx system. Thus, the RCS can control how strongly a target (scatterer) scatters electromagnetic signals in a specific direction when illuminated by a given incident signal. There are two kinds of RCSs which are the bistatic RCS and the monostatic RCS. The monostatic RCS is a special case of the bistatic RCS when both of Tx and Rx antennas are located at the same position. On the contrary, they are spatially separated. Figure 2.1 shows both the bistatic and the monostatic MST scenarios. The bistatic angle depends on a distance separating between Tx and Rx antennas if we look to them from the scatterer side, as illustrated in Figure 2.1a. It is equal to zero in the monostatic case and can be any value between 0 and 180 in the bistatic case [100]. The RCS can be expressed mathematically as

$$
\sigma=\lim _{r \rightarrow \infty}\left[4 \pi r^{2} \frac{S_{s}}{S_{i}}\right]
$$

or

$$
\sigma=\lim _{r \rightarrow \infty}\left[4 \pi r^{2} \frac{\left|E^{s}\right|^{2}}{\left|E^{i}\right|^{2}}\right]
$$

where $r$ denotes a distance separating between a scatterer and a receiver, $E^{s, i}$ are the incident and scattered electric fields, and $S_{s, i}$ are the scattered and incident power densities. Predicting the existence of scatterers depends totally on an amount of power received by a receiver. The received power $P_{R x}$ based on the Friis transmission model equation represented by the two-way path loss and the RCS of an object (scatterer) 
is given by [169]

$$
P_{R x}=\frac{P_{T} G_{R} G_{T} \lambda_{o}^{2} \sigma}{(4 \pi)^{2} r_{1}^{2} r_{2}^{2}}
$$

in which $P_{T}$ denotes the transmitted power by an illuminating source, $G_{R}$ and $G_{T}$ are gains of receiving and transmitting antennas, and they can be replaced by $G^{2}$ in the monostatic case, $r_{1}$ and $r_{2}$ are distances between a transmitter and a scatterer and a scatterer and a receiver, respectively, and they can be replaced by $r^{2}$ in the monostatic scenario, and $\lambda_{o}$ is the free-space wavelength at a design frequency $f_{o}$. Bistatic angle and polarization mismatch are not taken into account for the sake of simplicity.

The maximum detectable distance can be recognized if the minimum detectable power at the receiver is known by rearranging (2.9). In military applications, it is useful to reduce or minimize the RCS parameter, thus making a detection process of targets very difficult. However, in [155], the author demonstrated for the first time that the scattered signals from objects can be employed to deliver information over the scattering channel rather than the communication channel, exploiting the change in the RCS of objects to represent data. Fluctuations in the RCS are sensed and demodulated at a receiver to retrieve information or to distinguish a scatterer response from responses of surrounding obstacles. In our research work, the basic idea is to modulate the incident signals hitting our scattering array to generate a spectra of scattered distortion products as will be seen in the next chapters.

Similar to the reflected waves in (2.3), the scattered (reflected) electrical fields, $E_{S C A T}$, is composed of two parts, being independent-load scattered electrical fields $E_{S T}$ and dependent-load scattered electrical fields $E_{A M}$. They can be expressed as

$$
E_{S C A T}=E_{S T}+E_{A M}
$$


The term which is relevant for the transponder (scatterer) response is the second, and the load directly influences it. The RCS will follow the same process, given as

$$
\sigma_{S C A T}=\sigma_{S T}+\sigma_{A M}
$$

where $\sigma_{S C A T}, \sigma_{S T}$, and $\sigma_{A M}$ are the total RCS, the structural RCS, and the scatterer RCS, respectively. The scatterer RCS $\sigma_{A M}$ is easily calculated by taking into accounts the power at a load delivered by a scatterer as [171]

$$
P_{\text {Load }}=P_{a v a} \cdot r_{m i s s}=S_{i} A_{e f f} r_{m i s s}=\frac{\left|E^{i}\right|^{2}}{\eta} A_{e f f} r_{m i s s}
$$

where $A_{\text {eff }}$ is the effective aperture of scatterer antenna, $S_{i}$ represents the power density of an illuminating signal at the scatterer antenna, $\eta$ is the free-space impedance, and $r_{\text {miss }}$ denotes the impedance mismatch factor between the input impedance of the scatterer antenna and the load impedance given by

$$
r_{m i s s}=\frac{4 R_{L} R_{A N T}}{\left|Z_{L}+Z_{A N T}\right|^{2}}
$$

The factor $r_{\text {miss }}$ has a significant influence on the performance of a scatterer, due to its impact on the overall amounts of the scattered power. The scattered power $P_{S C A T}$ also has two components, being the structural $P_{S T}$ and antenna $P_{A M}$ components

$$
P_{S C A T}=P_{S T}+P_{A M}
$$

The second term of the right side in (2.14) represents the scattered load-dependent power that is reradiated back by the scatterer antenna to reach a receiver when a scatterer works in its transmitting mode with a current $I_{\text {ind }}$ induced over it by 
illuminating signals

$$
P_{A M}=\left|I_{i n d}\right|^{2} R_{A N T}=P_{\text {Load }} \frac{R_{A N T}}{R_{L}}
$$

The scattered power density $S_{s}$ is related into the scattered antenna-mode power term through

$$
S_{s, A M}=\frac{P_{A M} G_{s c a t}}{4 \pi r^{2}}
$$

where $G_{\text {scat }}$ represents the gain of scatterer antenna. Eventually, to obtain RCS, we substitute (2.16) in (2.7)

$$
\sigma_{A M}=\lim _{r \rightarrow \infty}\left[4 \pi r^{2} \frac{S_{s, A M}}{S_{i}}\right]=\frac{\lambda_{0}^{2} G_{s c a t}^{2} \mathrm{R}_{A N T}^{2}}{\pi\left|Z_{A N T}+2_{L}\right|^{2}}
$$

The antenna at the receiver side captures a series of scattered signals having different amplitudes, phases, or both due to a variable load (changing between $Z_{L 1}$ and $Z_{L 2}$ in conventional binary modulated scattering systems). Thus, it is required to obtain the effective RCS which is the difference between the two states, differential RCS, $\Delta \sigma_{A M}[64][131][119]$

$$
\Delta \sigma_{A M}=\frac{\lambda_{0}^{2} G_{s c a t}^{2}}{4 \pi}\left(\left|\frac{2 R_{A N T}\left(Z_{L 2}-Z_{L 1}\right)}{\left(Z_{A N T}+Z_{L 1}\right)+\left(Z_{A N T}+Z_{L 2}\right)}\right|^{2}\right)
$$

Substituting (2.18) in (2.9) to obtain the differential received power, $\Delta P_{R x}$

$$
\begin{aligned}
& \Delta P_{R x}=\frac{P_{T} G_{R} G_{T} \lambda^{2} \Delta \sigma_{A M}}{(4 \pi)^{2} r_{1}^{2} r_{2}^{2}}(\text { bistatic }) \\
& \Delta P_{R x}=\frac{P_{T} G^{2} \lambda^{2} \Delta \sigma_{A M}}{(4 \pi)^{2} r^{4}}(\text { monostatic })
\end{aligned}
$$

The last two equations implicitly establish the relationship between s load impedance and the amount of received power, so they can be exploited to recognize the 
scattered data at a receiver end. The MST systems do not need to have complicated circuits. They have only two main parts which are antennas (passive elements) and impedance modulators, so they do not consume high power compared to RF front-end communication systems, having power-Hungary circuits.

Moreover, in order to make use of the Smith chart to demonstrate load impedance impacts on the amount of the scattered and received power, the expression of differential RCS must be related to the differential reflection coefficient $\Delta \Gamma_{\text {tran }}$ of the scatterer. To do so, we can rewrite $(2.18)$ as [168]

$$
\Delta \sigma_{A M}=\frac{\lambda^{2} G_{s c a t}^{2}}{4 \pi}\left|\Gamma_{L 1}^{*}-\Gamma_{L 2}^{*}\right|^{2}
$$

where $\Gamma_{L 1,2}^{*}$ are the conjugate reflection coefficients of two different loads at a scatterer input. Now, we can say that the received power is obviously related to the transponder load reflection coefficients, as illustrated below

$$
\begin{aligned}
& \Delta P_{R x}=\frac{P_{T} G_{R} G_{T} G_{S c a t}^{2} \lambda_{o}^{4}}{(4 \pi)^{3} r_{1}^{2} r_{2}^{2}}\left|\Gamma_{L 1}^{*}-\Gamma_{L 2}^{*}\right|^{2}(\text { bistatic }) \\
& \Delta P_{R x}=\frac{P_{T} G^{2} G_{s c a t}^{2} \lambda_{o}^{4}}{(4 \pi)^{3} r^{4}}\left|\Gamma_{L 1}^{*}-\Gamma_{L 2}^{*}\right|^{2} \text { (monostatic) }
\end{aligned}
$$

It is worth noting that the modulated received power is directly related to the square of the differential conjugate load reflection coefficients of the scatterer. Therefore, we can straightforwardly consider it as the modulation index of the scatterer.

To represent $\Delta \Gamma_{S C}=\left(\Gamma_{2}-\Gamma_{1}\right)$ geometrically on the Smith chart where $\Gamma_{2,1}$ are input reflection coefficients of scatterer inputs with two different loads, this requires doing some modifications on scatterer reflection coefficients. The modification is carried out by grouping an imaginary part of input impedance of scatterer antenna into a load 
impedance as [89] [119]

$$
Z_{t}=Z_{L}+Z_{A N T}=Z_{L}+R_{A N T}+j X_{A N T}=\left(Z_{L}+j X_{A N T}\right)+R_{A N T}=Z_{L}^{\prime}+R_{A N T}
$$

The modified reflection coefficient is

$$
\bar{\Gamma}=\frac{Z_{L}^{\prime}-R_{A N T}}{Z_{L}^{\prime}+R_{A N T}}
$$

For binary scattering, there are two loads, so the modified reflection coefficients are

$$
\bar{\Gamma}_{1,2}=\frac{Z_{L 1,2}^{\prime}-R_{A N T}}{Z_{L 1,2}^{\prime}+R_{A N T}}=\left.\Gamma_{\operatorname{tran} 1,2}\right|_{R_{A N T}=Z_{o}}
$$

where $Z_{o}$ is the reference impedance of a system. Four different differential reflection coefficients are plotted on the Smith chart as shown in Figure 2.2 to demonstrate all potential states of differential reflection coefficients. The first state is when both reflection coefficients of the two loads located inside the Smith chart arbitrarily. The squared distance from points 1 to point 2 is equal to the modulation index of the $\operatorname{MST},\left|\Delta \Gamma_{s c}\right|$. We can notice that $\Delta \Gamma_{s c}$ is bounded by 2 , (i.e., $0 \leq \Delta \Gamma_{s c} \leq 2$ ). To reach the maximum value, the two reflection coefficients must be placed on the outer circle of the Smith chart in the opposite direction to each other, representing the state 2. In state 2, the modulation index of the MST is equal to $6 \mathrm{~dB}$. One of the possibilities to achieve state 2 is obtained when short and open circuits are chosen as two different loads.

However, some applications like RFID want to harvest energy from the incident signals, making state 2 is undesirable or impossible to establish connections between readers and tags. Thus, one of the impedance loads should be matched to an antenna input impedance in order to deliver power into the RFID-IC. RFID systems lie on the 
state 3, and the maximum MST modulation index is equal to 1. All modulators in the previous states are passive. However, increasing the received power or increasing distances separating between sources and scatterers requires increasing one of the parameters given in (2.22) and (2.23). The most candidate parameter is the MST modulation index, so the idea is to make it larger than 2, employing an active modulator instead of passive ones. State 4 is the active MST modulation index, and reflection coefficients are located outside of the Smith chart because they are larger than one. In [5], authors succeeded to achieve a connection between the source and the transponder at distances larger than 1000m. Also, some research efforts have been made to enhance reflection coefficients and make them larger than two. In [48] [22], transistors as active components are used exploiting the oscillator design principles, but in [22], authors employed two transistors and a 3dB hybrid coupler to be a part of an active retro-directive array. Tunnel diodes also have been used in many works because of their negative resistances at specific voltages [5]. Authors exploit this property to design reflection amplifiers in order to improve power of the scattered signals [80] [6].

\subsubsection{Modulated Scatterer (MS) as an Analog Signal Processor (ASP)}

The periodic variations in an impedance of loads attached a scatterer antenna results in a periodic reflection coefficient given as [92]

$$
\Delta \Gamma_{\text {tran }}(t)=\Delta \Gamma_{a v g}+\Delta \Gamma_{\text {tran }} \cos \left(w_{m} t\right)
$$

where $w_{m}$ is the modulation frequency. Figure 2.1 depicts an illuminating wave incident on a periodic time-modulated scatterer. The incident single-tone signal, represented as a plane wave and polarized along the y-axis with a frequency $w_{o}$, is 


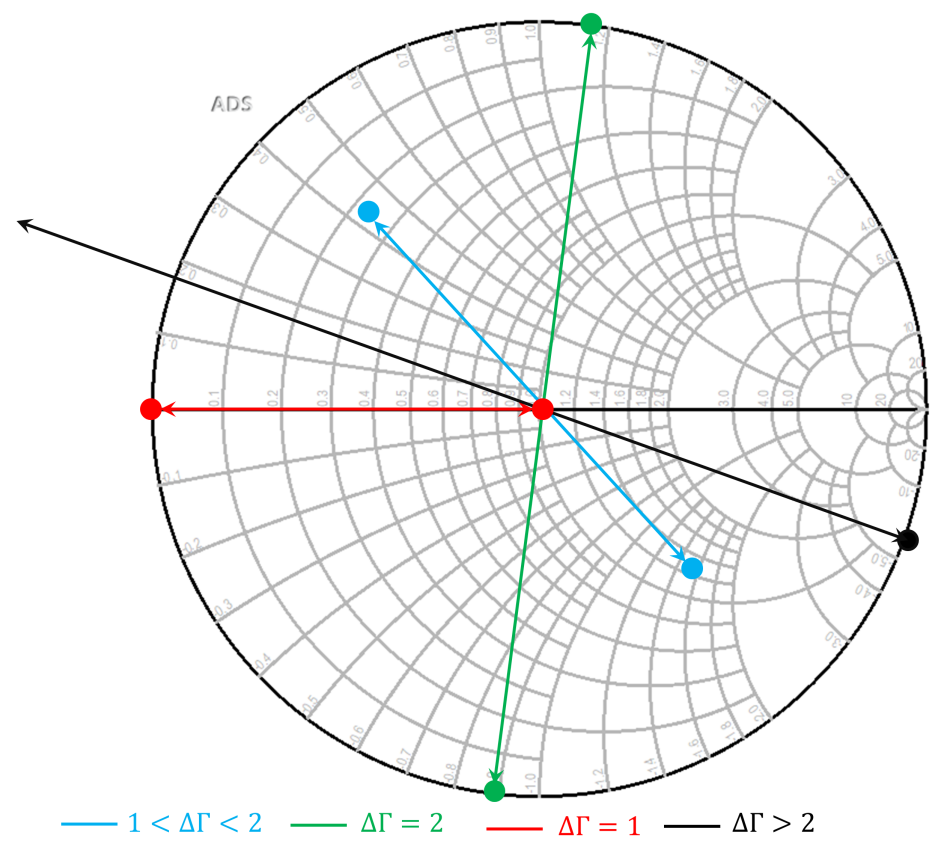

Figure 2.2: Different representations for differential reflection coefficients on the Smith chart

given as

$$
E_{\text {inc }}(z, t)=\hat{y} E_{o} e^{-j\left(K z-w_{o} t\right)}
$$

and impinges on a time-modulated scatterer. The scatterer introduces $n$ terms of harmonics products, i.e. $w_{o} \pm n w_{m}$, distributed about the illuminating frequency. $K$ is the free-space wave number and is equal to $w_{o} / c, E_{o}$ is the amplitude of incident fields, and $c$ is the free-space wave velocity. The scattered electric fields reflected back from a scatterer may be formulated as [92] [91]

$$
E_{\text {scat }}(z, t)=\hat{y} \sum_{n=-\infty}^{\infty} E_{o} \Delta \Gamma_{n} e^{-j\left(\left(K+n k_{m}\right) z-\left(w_{o}+n w_{m}\right) t\right.}
$$

or

$$
E_{\text {scat }}(z, t)=\hat{y} \sum_{n=-\infty}^{\infty} \Delta E_{n} e^{-j\left(\left(K+n k_{m}\right) z-\left(w_{o}+n w_{m}\right) t\right.}
$$


where $E_{o} \Delta \Gamma_{n}$ is replaced by $\Delta E_{n}$. The periodic nature of scattered fields from a single modulated scatterer (transponder) given in (2.29) and (2.30) ensures that scattered fields consists of an infinite number of frequency components (i.e., distortion products), so we will exploit this property by using an ensemble of scatterers. Thus, presenting a method to control distortion products (e.g. harmonics HDs and intermodulation distortions IMDs) in space rather than using conventional ways such as transmission lines, waveguides, $\cdots$, etc is the main focus of this research study. This process requires that each mode (i.e., harmonic or IMD) has replicas generated in other modulated scatterers (MSs) as found in the operations of distortion products enhancement and suppression in the conventional RF communication circuits [136].

To this end, we introduced a comprehensive mathematical model for a single modulated scatterer (MS), considering all parameters affecting its functionality. Its mathematical derivations can be considered as a useful background to start deriving mathematical models of our work presented later.

\subsection{The Study of Array Signal Processing (Phased Antenna Array)}

Since our proposed system consists of an array of modulated scatterers, this section presents the essential background for array signal processing, such that the interested readers can completely recognize and understand the fundamentals related to our

theoretical and experimental models in the subsequent chapters. The concepts of array signal processing were first used in research about earthquake direction finding using seismic transducer arrays, in the 1950s [133]. In the 1960s, researchers applied the array processing in applications called sound navigation and ranging (SONAR) [18]. The desired array response has been obtained based on the summation of weighted inputs for these early research works [25] [26] [17].

There are two of an element-level weighting, being either deterministic or 
adaptive (statistical optimization). In the former one, it depends on the geometry as in the delay and sum beamforming technique, while in the later one, it changes its elements weights adaptively (randomly) to improve the signal to noise ratio (SNR) according to directions of desired signals. Most of earlier research work before emerging the analog-to-digital (ADC) converters are not relevant to adaptive antenna arrays. In the 1980s, adaptive (phased) antenna arrays based on beamforming techniques witnessed good deployments and emergence of new concepts.

\subsubsection{A Uniform Linear Array ULA Beam-forming based on Delay and Sum}

Figure 2.3 depicts the simple graphical representation of a linear array LA. It consists of a number of identical elements $L$, separating by the same distance $d$, so this kind of arrays is uniform LA, ULA. Distances $d$ separating array elements have a non-trivial influence on the overall performances of antenna arrays. It follows the same Nyquist sampling fundamentals as illustrated in the communication theory [124]. When we want arrays to cover (scan) the entire front view (i.e., \pm 90$), d$ separating elements should obey (2.31)

$$
d \leq \frac{\lambda_{o}}{1+\sin (\theta)}
$$

where $\theta$ denotes the desired spatial direction, and $\lambda_{o}$ is the free-space wavelength that should be determined at the highest frequency of interest. Proper spatial locations of array elements are important to avoid aliasing effects and thereby sampling phases of plane waves at an array aperture is unambiguously obtained. When a plane wave hits an array, it reaches elements at different times according to $d \sin \theta$ that could be approximated as a phase difference for narrow-band signals. Thus, the phase difference at the $l-t h$ element can be given as

$$
\Delta \psi_{l}=l K d \sin \theta ; \quad 0 \leq l \leq L-1
$$


where $K=2 \pi / \lambda_{o}$ is free-space wavenumber. In the receiving mode, signals reaching elements are added at an output of an array using transmission lines, resulting in an array factor given by

$$
A F(\theta)=\sum_{l=0}^{L-1} A_{l} e^{-j \Delta \psi_{l}}=\sum_{l=0}^{L-1} A_{l} e^{-j l K d \sin \theta}
$$

where $A_{l}$ is the $l-t h$ element excitation factor. In the transmitting mode, signals departing array elements are added constructively or destructively in space rather than transmission-lines wich are used in receiving-mode antenna arrays.

The maximum array factor value in (2.33) is $L$ when signals reach elements from an angle of $\theta=0^{\circ}$. However, normalizing the array factor may be useful when we want to make a relative comparison between main and side lobes levels. The normalization is carried out by dividing the original array factor by a number of elements $L$. The ULA array factor is simplified as given in (2.34) after a simple math manipulation using the geometric series principles [34]

$$
\begin{aligned}
A F(\theta) & =\frac{\sin \left(\frac{L}{2} K d \sin \theta\right)}{\sin \left(\frac{1}{2} K d \sin \theta\right)} \approx \frac{\sin \left(\frac{L}{2} K d \sin \theta\right)}{\left(\frac{1}{2} K d \sin \theta\right)} \\
& =L \frac{\sin \left(\frac{L}{2} K d \sin \theta\right)}{\left(\frac{L}{2} K d \sin \theta\right)}=L \operatorname{sinc}\left(\frac{L}{2} K d \sin \theta\right)
\end{aligned}
$$

The reduced sinc form in (2.34) cab be normalized if it is divided by a number of elements $L$ as

$$
A F_{l}(\theta)=\operatorname{sinc}\left(\frac{L}{2} K d \sin \theta\right)
$$


Eq. (2.35) presents the voltage response pattern of an $L$-element ULA, while the power pattern response of the same $L$-element ULA is presented in (2.36)

$$
A F(d B)=10 \log _{10}\left(\overline{|A F(\theta)|^{2}}\right)=10 \log _{10}\left(\left|\operatorname{sinc}\left(\frac{L}{2} K d \sin \theta\right)\right|^{2}\right)
$$
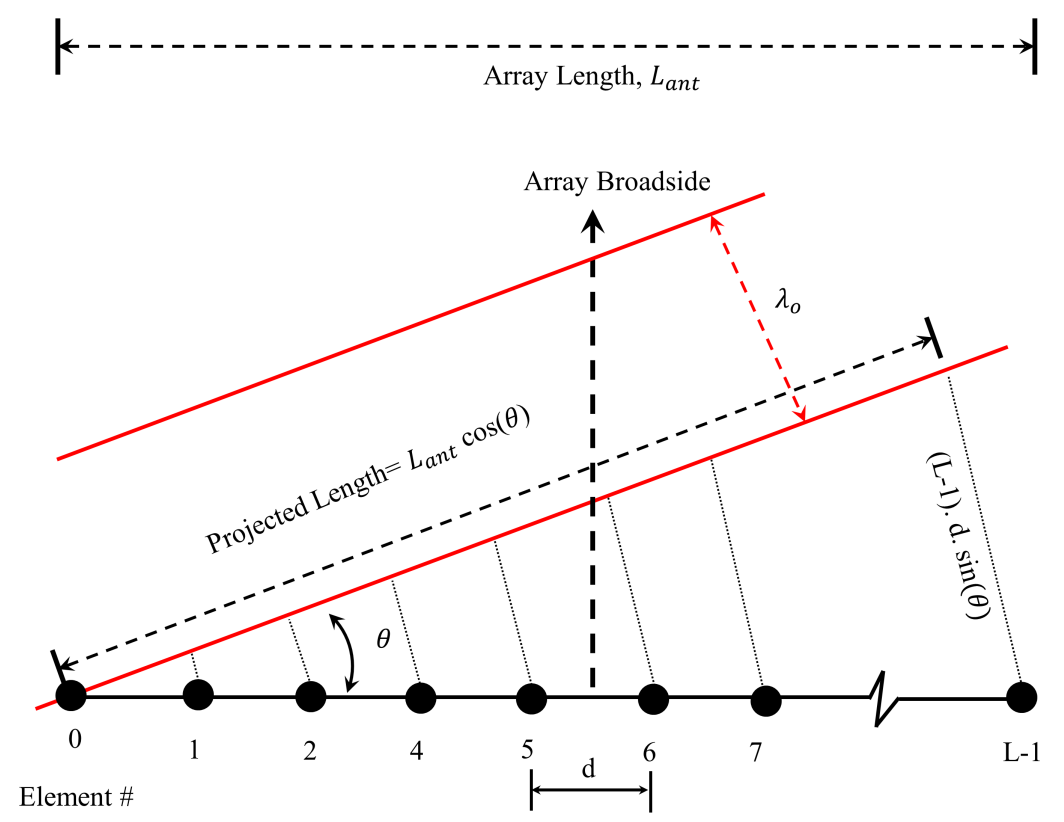

Figure 2.3: General representation of the Uniform Linear Array ULA of $L$ elements with a single-frequency RF signal incident at an angle $\theta$

\subsubsection{Steering Phase, Grating Lobes, and Element Weighting}

\subsubsection{Steering Phase}

In real-world applications, it is not necessary to receive or transmit signals from normal directions, so tilted signals creating angles with perpendicular trajectory produce phase shifts distributed across array elements. To compensate phase shifts due to the tilted signals, phase shifters having opposite values to phases of incident signals at the $l-t h$ element in the receiving mode must be associated with each 
element to align and add signals constructively at an array output. Typically, this process is called the beamsteering or adaptive beamforming if phase shifters are able to continuously adjust their values to follow directions of the desired signals. To accommodate phases offsets into (2.33), it can be rewritten as

$$
A F(\theta)=\sum_{l=0}^{L-1}\left|A_{l}\right| e^{j \Delta \psi_{l}} e^{-j l \Delta \psi_{o}}=\sum_{l=0}^{L-1} A_{l} e^{j l K d\left(\sin \theta-\sin \theta_{o}\right)}
$$

where $\theta_{o}$ represents an angle of the desired signals. Figure 2.4 depicts radiation patterns for a 10-element ULA at angles $\theta_{o}=0^{\circ}, 15^{\circ}, 30^{\circ}$, and $45^{\circ}$, to demonstrate beamforming capabilities. As can be seen, a beamwidth of radiation patterns become wider as a beam gets off the normal (broadside) direction. The reason behind beam broadening is the array projected length which varies with the cosine of the radiation angle as illustrated in Figure 2.3. Thus, this aperture length decreases toward zero when an angle of the scanned beam makes $90^{\circ}$ off from the broadside (i.e., endfire direction). In the previous explanations, we assumed that array elements are isotropic, equally radiated in all direction, but actual arrays have no isotropic radiators. Hence, some modification must be carried out to take into account impacts of elements patterns on the overall performance of an array [34]. For example, a common response of element pattern is given by

$$
A(\theta)=\cos ^{\frac{\xi}{2}}(\theta)
$$

where $\xi$ is a factor that controls the response roll-off of an element pattern with an angle $\theta$. Its values range from 1.4 to 1.6 , but 1.5 is the most appropriate value. Using the concept of pattern multiplication to find the total actual array response must be performed for a given element pattern. This multiplication takes place between the 


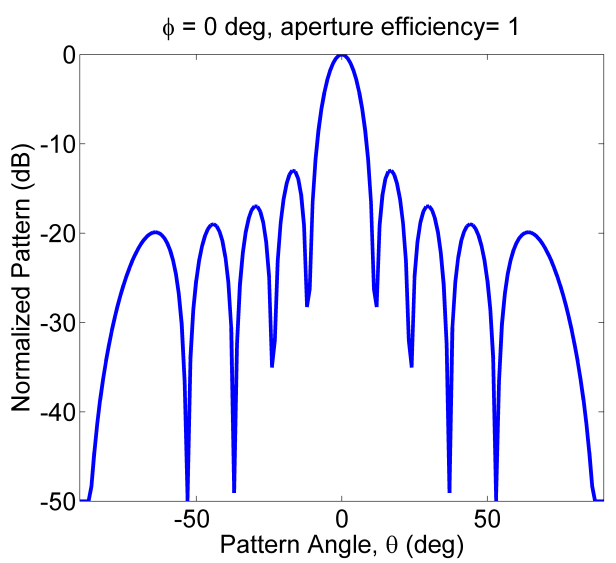

(a)

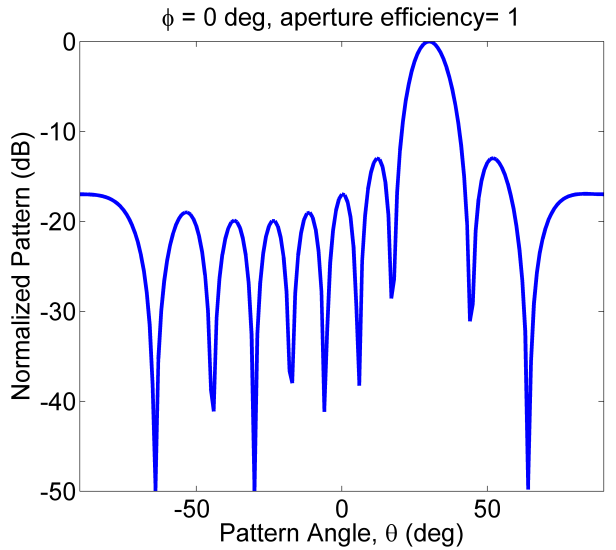

(c)

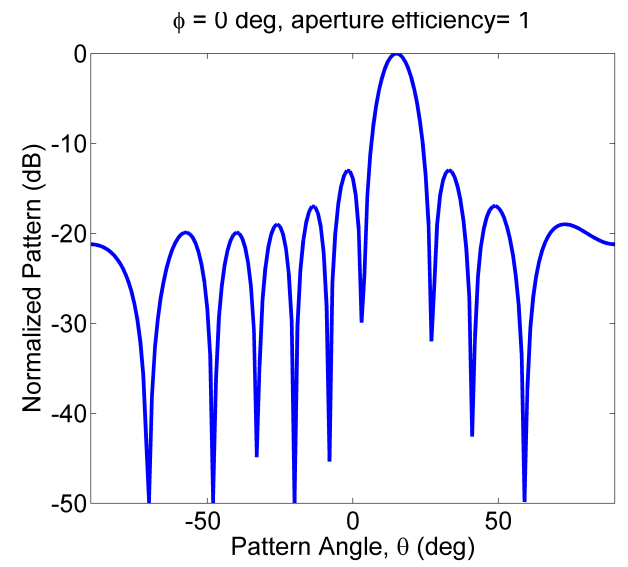

(b)

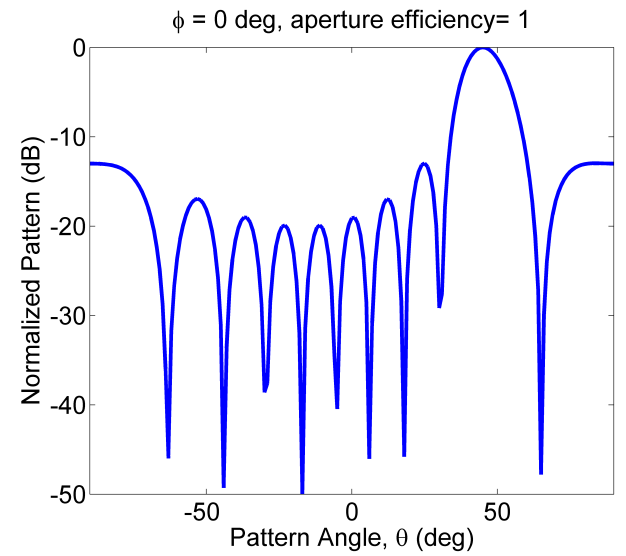

(d)

Figure 2.4: Radiation patterns of 10-element ULA at the desired directions a) $\theta_{o}=0^{\circ}$ b) $\theta_{o}=15^{\circ}$ c) $\theta_{o}=30^{\circ}$ d) $\theta_{o}=45^{\circ}$

array factor in (2.37) and the element pattern in (2.38), thus

$$
A F_{T}(\theta)=A(\theta) * A F(\theta)=\cos ^{\frac{3}{2}}(\theta) * \sum_{l=0}^{L-1} A_{l} e^{j n K d\left(\sin \theta-\sin \theta_{o}\right)}
$$

Assuming that all elements are identical having the same contribution, this means that we ignore the mutual coupling among elements. 


\subsubsection{Grating Lobes}

The concept of grating lobes in responses of antenna arrays is an undesirable phenomenon relating directly to spaces separating elements that must be chosen properly to avoid ambiguous sampled phases of desired signals. Also, grating lobes are unintentionally radiation beams which are similar to main radiation beams locating in uncontrolled directions. The physical interpretation behind this unwanted phenomena is that because arrays are spatially periodic structures leads into periodic array factors. Therefore, to ensure grating lobes (periodic copies) being located outside of visible regions of arrays (i.e., Field of View, FoV), factors causing the emergence of grating lobes should be carefully chosen. The mathematical analysis given below illustrates potential spatial locations of grating lobes. To show the mechanism of grating lobes, we start an array factor as given below

$$
A F(\theta)=\frac{\sin \left(L d \frac{\pi}{\lambda_{0}}\left[\sin \theta_{o}-\sin \theta\right]\right)}{\sin \left(d \frac{\pi}{\lambda_{o}}\left[\sin \theta_{o}-\sin \theta\right]\right)}
$$

It can be seen that the array factor is maximum whenever the denominator of $(2.40)$ approaches zero, so it can be expressed as

$$
d \frac{\pi}{\lambda_{o}}\left[\sin \theta_{o}-\sin \theta\right]=p \pi, p=0, \pm 1, \pm 2, \pm 3, \ldots
$$

From Eq.(2.41), the element spacing $d$ and the angle $\theta_{o}$ govern grating lobes, so the equation can be rearranged in a straightforward form to easily understand it.

$$
\sin \theta_{G}=\sin \theta_{o}-p \frac{\lambda_{o}}{d}
$$


where $\theta_{G}$ represents an angle of the grating lobes at the $p-t h$ repetition term of the array $A F(\theta)$ in space. Eventually, when distances $d$ separating elements are less than or equal to a half wavelength $\lambda_{o} / 2$, the grating lobes always fall outside of the visible region of the array for any desired signal angle $\theta_{o}$. In contrast, problems appear once distances $d$ get larger than a half wavelength $\lambda_{o} / 2$, requiring special considerations when building arrays.

\subsubsection{Array Weighting}

This topic is so important in the ULA antenna array design because in the uniform linear array, all elements are equally excited. Thus, the ULA array has large sidelobes. Reducing sidelobes requires using tapering excitations across array elements. This process is similar to the windowing process in digital signal processing [108]. In the ULA array, a relative difference between the main beam and the first sidelobe levels is about $-13.4 \mathrm{~dB}$ but in the tapering aperture excitation, sidelobes can be highly mitigated at the cost of the width of the main beamwidth. Figure 2.5 shows a comparison between a uniform linear array radiation pattern and $-28 \mathrm{~dB}$ triangular-weight array pattern.

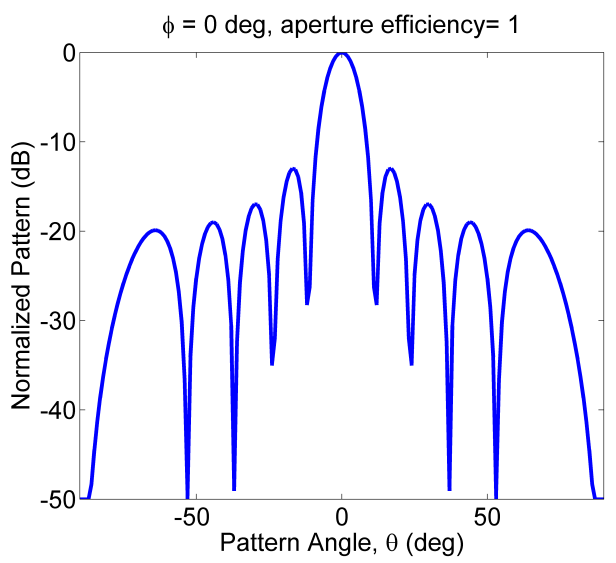

(a)

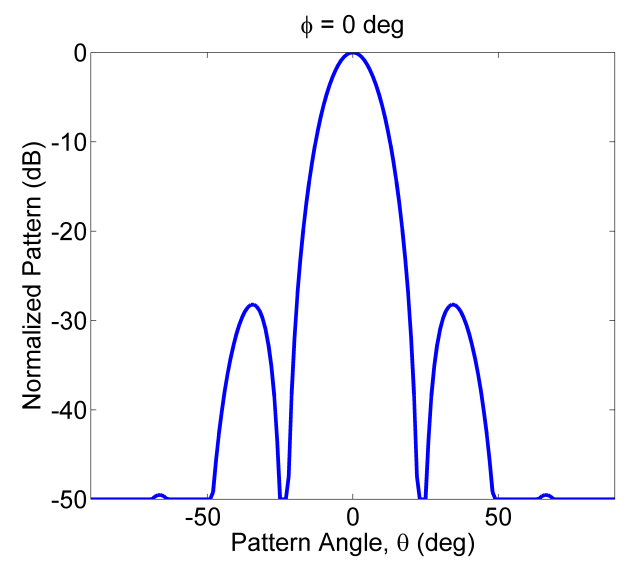

(b)

Figure 2.5: Comparison of the radiation patterns of two different array weightings a) 10-element ULA array pattern b) 10-element $-28 \mathrm{~dB}$ triangular-weight array pattern 


\subsubsection{Two Dimensional Antenna Array (Planar Array)}

If a group of $M$ of linear arrays is placed in parallel, they will form a rectangular grid of antennas in the $\mathrm{x}-\mathrm{y}$ plane, with an element spacing $d_{x}$ in the $\mathrm{x}$-direction and $d_{y}$ in the y-direction. Figure 2.6 shows a 2D planar array. The array factor expression of $2 \mathrm{D}$ arrays can be expressed as

$$
A(\theta, \emptyset)=\sum_{m=0}^{M-1} \sum_{l=0}^{L-1} A_{m l} e^{j K\left(m d_{x} \sin (\theta) \cos (\emptyset)+l d_{y} \sin (\theta) \sin (\emptyset)\right.}
$$

Here $A_{m n}$ represents the $m n-t h$ element excitation in the array, being a complex number.

To make the array factor separable for the two different radiation planes along $\mathrm{x}$

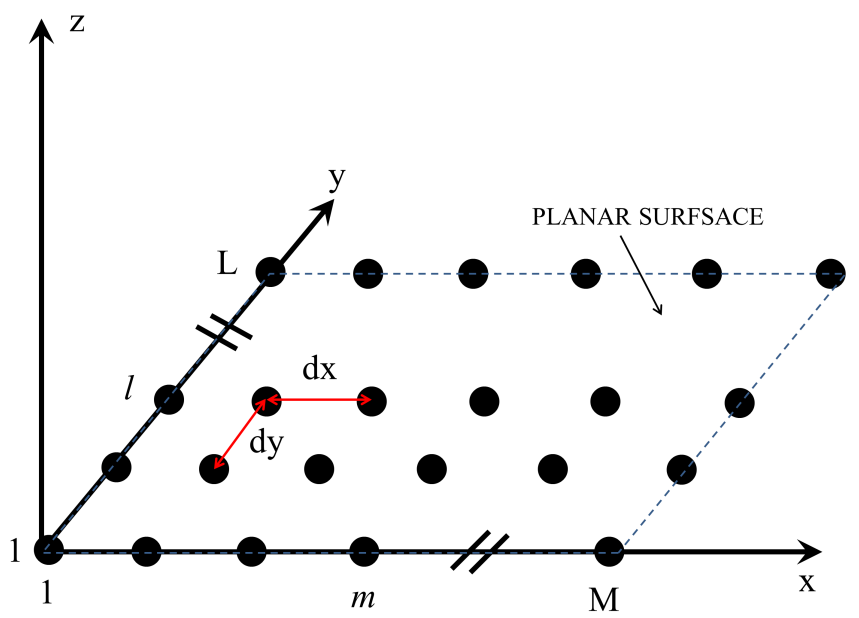

Figure 2.6: Two-dimensional planar array

and $y$, excitations are such that

$$
A_{m l}=A_{x m} A_{y l}
$$


These two different excitations, being along the $\mathrm{x}$ and the $\mathrm{y}$ directions independently, consist of uniform amplitudes and progressive phases as

$$
\begin{gathered}
A_{x m}=A_{o} e^{j m \beta x} \\
A_{y l}=A_{o} e^{j l \beta y}
\end{gathered}
$$

where $\beta_{x, y}$ are phases gradient distributed across the array perspective directions. Then, the array factor can be rewritten as

$$
A F(\theta, \emptyset)=\sum_{l=0}^{L-1} e^{j l\left(K d_{y} \sin (\theta) \sin (\phi)+\beta y\right)} \sum_{m=0}^{M-1} A_{o} e^{j m\left(K d_{x} \sin (\theta) \cos (\emptyset)+\beta x\right)}
$$

It is clearly seen that the $2 \mathrm{D}$ array factor is simply the product of two uniform linear array ULA factors. Figure 2.7 shows three dimensional array factors of a $10 \times 10$-element uniform planar array when the main beam is directed for two different directions being $\left(\theta_{o}=0^{\circ}\right.$ and $\phi_{o}=0^{\circ}$ broadside $)$ and $\left(\theta_{o}=25^{\circ}\right.$ and $\left.\phi_{o}=40^{\circ}\right)$.

\subsection{The Study of Image-Rejection Mixers}

In this part, although the proposed phase-modulated scattering array (PMSA) deals with distortion products in space, it has the same underlying behavior of the image-reject mixer. In other words, instead of processing signals over transmission lines as in communication circuits, these processes occur in space leveraging from the simple math of image-reject mixers to formulate our mathematical model that supports the research work in this dissertation. Furthermore, an image-reject mixer has a fixed design to cancel either a down-converted signal or an up-converted signal relying on a sign of a phase shifter placed after the Q-mixer as shown in Figure 2.8. However, the presented structure is flexible to choose either band depending on several 


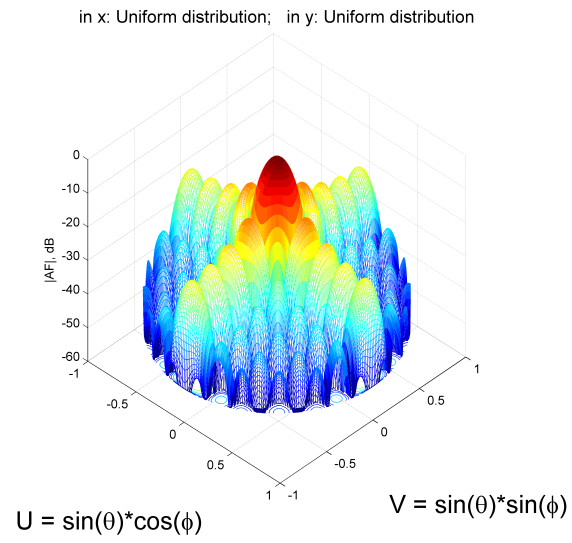

(a)

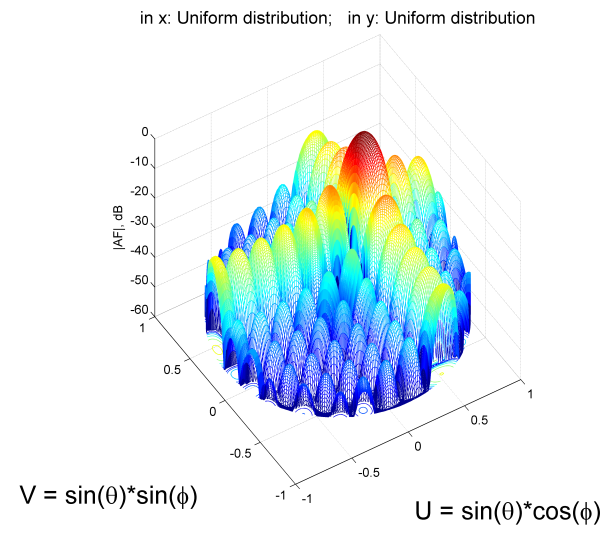

(c)

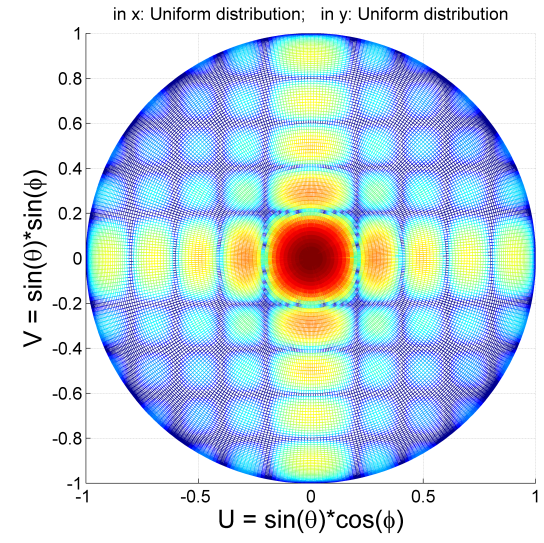

(b)

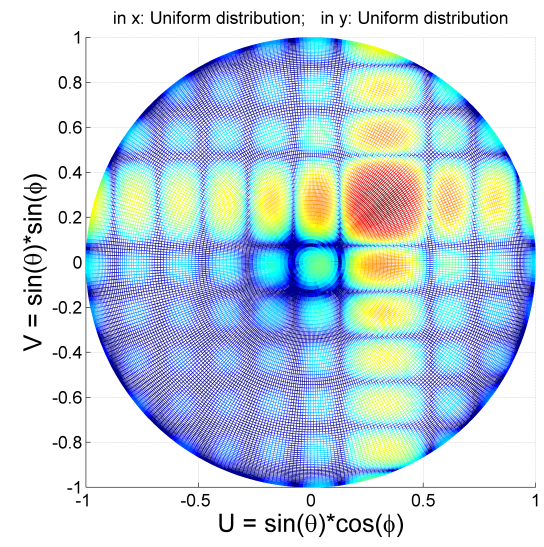

(d)

Figure 2.7: 3D Radiation patterns and their top views of a $10 \times 10$-element uniform planar array at directions a) broadside-3D b) broadside-top view c) $\theta_{o}=25^{\circ}$ and $\phi_{o}=40^{\circ}-3 \mathrm{D} \mathrm{d}$ ) $\theta_{o}=25^{\circ}$ and $\phi_{o}=40^{\circ}$-top view

factors such as an incident angle of the illuminating signals and angles of scattered beams, where more deliberated information will be provided in Chapter 3. Figure 2.8 shows an image-reject mixer consisting of two input signals with $90^{\circ}$ phase shift, two oscillators, two mixers, two low pass filters LBF, and one power combiner. To understand how it works, the mathematical description of operational principles is 
given below. The signal at the point $\mathrm{A}$ is

$$
\begin{aligned}
A(t) & =A_{L O} \cos \left(w_{L O} t+\varphi_{I}\right) \times \cos \left(w_{I F} t+\varphi_{I}\right) \\
& =\frac{A_{I F}}{2} \cos \left(w_{L O} t-w_{I F} t+\varphi_{L O}-\varphi_{I}\right)+\frac{A_{I F}}{2} \cos \left(w_{L O} t+w_{I F} t+\varphi_{L O}+\varphi_{I}\right)
\end{aligned}
$$

and the signal at the point $\mathrm{C}$ is

$$
\begin{aligned}
C(t) & =C_{L O} \sin \left(w_{L O} t+\varphi_{L O}\right) \times \cos \left(w_{I F} t+\varphi_{Q} \pm 90\right) \\
& =\mp \frac{C_{I F}}{2} \cos \left(w_{L O} t-w_{I F} t+\varphi_{L O}-\varphi_{Q}\right) \pm \frac{C_{I F}}{2} \cos \left(w_{L O} t+w_{I F} t+\varphi_{L O}+\varphi_{Q}\right)
\end{aligned}
$$

The addition of (2.48) and (2.49) yields either up-converted signal $\left(w_{L O} t+w_{I F} t\right)$ or down-converted signal $\left(w_{L O} t-w_{I F} t\right)$ depending on the sign of the phase shifter (i.e. lead $(+)$ or lag $(-))$.

If $C_{I F}=A_{I F}$, then the output is given as

$$
\begin{aligned}
& S_{\text {out }}=A_{I F} \cos \left(w_{L O} t+w_{I F} t+\varphi_{L O}+\varphi_{I}\right) \quad \text { phase }=90^{\circ} \\
& S_{\text {out }}=A_{I F} \cos \left(w_{L O} t-w_{I F} t+\varphi_{L O}-\varphi_{I}\right) \quad \text { phase }=-90^{\circ}
\end{aligned}
$$

We can deduce from the simple image-rejection scheme that the chosen signal component at the output changes between the two bands if the phase shifter can obtain its value (i.e., $\pm 90^{\circ}$ ), leading to an adaptive or a tunable image-reject mixer. This property triggers and aids to develop a mathematical derivation being able to predict the general operation of the presented system and initial design calculations before fabricating the phase-modulated scattering array (PMSA). The mathematical model explained above will be extended to include a large number of input signals and a large number of output signals. Chapter 3 presents the extended mathematical model, and it also illustrates its advantageous features of the PMSA. 


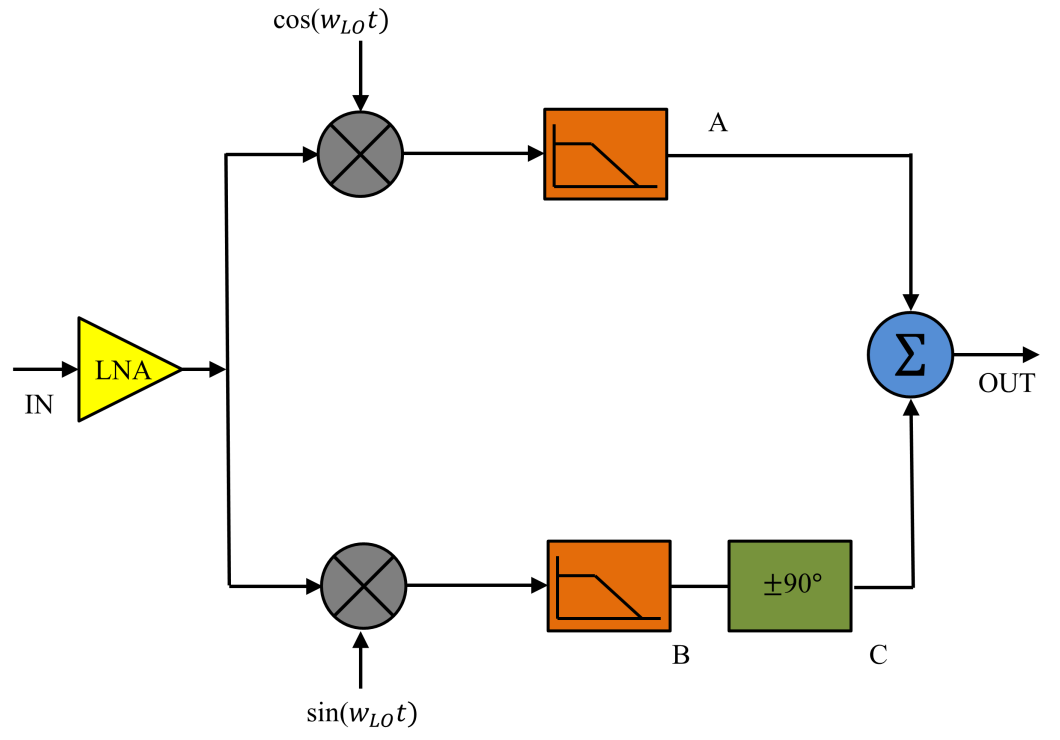

Figure 2.8: Image-reject mixer architecture 


\section{Chapter 3: The Mathematical Model of the Proposed Design under a Communication Signal Processing Perspective}

\subsection{Introduction}

In the previous chapter, some basic theories were provided which are necessary for the analysis of our presented system. Here, the investigations are developed to derive a mathematical approach being required to verify and validate the functionality of the PMSA. The mathematical model is developed and investigated using RF communication circuit and signal processing theories. The mathematical model that will be developed here should be capable of predicting spatial characteristics of distortion products resulting from the nonlinear nature of our presented system at any point in space.

In this chapter, we start with the approach using the extended version of the image reject-mixer being the polyphase modulator as an equivalent representation for the PMSA. The polyphase modulator consists of multiple independent branches, known as a polyphase multipath modulator, which has the capability to generate same signals with different phases, thus granting the ability to handle signals (distortion products) relying on their phases at ends of paths. Each scatterer in the PMSA is represented by a single path in the polyphase multipath circuit. Thus, a number of paths in the mathematical model is equal to a number of scatterers in the PMSA, to make a whole representation. In communication circuits, a function of the polyphase modulator is limited to enhancing a single distortion product (i.e., single harmonic or intermodulation distortion IMD product) and eliminating others. Here despite the similarity between the functional work of the PMSA and the polyphase multipath technique, there is a substantial difference that our PMSA 
supports different outputs, being elaborated in detail in the following section. Hence, we will add some extra terms for the conventional polyphase circuit to make it compatible with our presented model to become more realistic. Although we modify the conventional polyphase multipath modulator, the equivalent model based on the polyphase multipath technique is used for the first time to represent distortion products generated in active antenna arrays on the best of the authors knowledge. Finally, non-idealities in the proposed systems are considered by adding amplitude and phase errors into the ideal presented model.

\subsection{The General Representation of the Phase Modulated Scattering Array (PMSA)}

In this section, we derive a mathematical model that enables us to treat and handle distortion products generated inside the PMSA with the help of the polyphase multipath modulation technique. The adopted technique is widely used in communication circuits due to its ability to effectively cancel distortion products [113] [153]. Also, its advantages are twofold: on the one hand, it can easily demonstrate the enhanced and suppressed distortion products in space especially when we make modifications on the adopted technique, and on the other hand, it shows the factors governing enhancement and cancellation of distortion products in space. The distortion product term includes one or both of harmonics HDs and intermodulation distortions IMDs, and we will use the distortion product term in some places to express about them.

Now, consider an arrangement of our novel phase-modulated scattering array PMSA, depicted in Figure 3.1. Elements that are non-conventional antennas are uniformly placed along the x-axis where $L$ denotes a number of scattering array elements. Each element consists of an antenna, an RF switch (i.e., diode or transistor), and a modulation signal source driving a switch. Our discussion focuses on two 
dimensional problem and three dimensional systems are out of the this research scope, a future work. The mutual coupling between elements is ignored for the sake of brevity although it influences the measurement results that can be tolerated to some extent as shown in Chapter 8. In addition, the study focuses only on the backscattering side (front view of the array aperture, $z>0$ ), and will leave the forwardscattering (back-view of the array aperture, $\mathrm{z}<0$ ) as a future work.

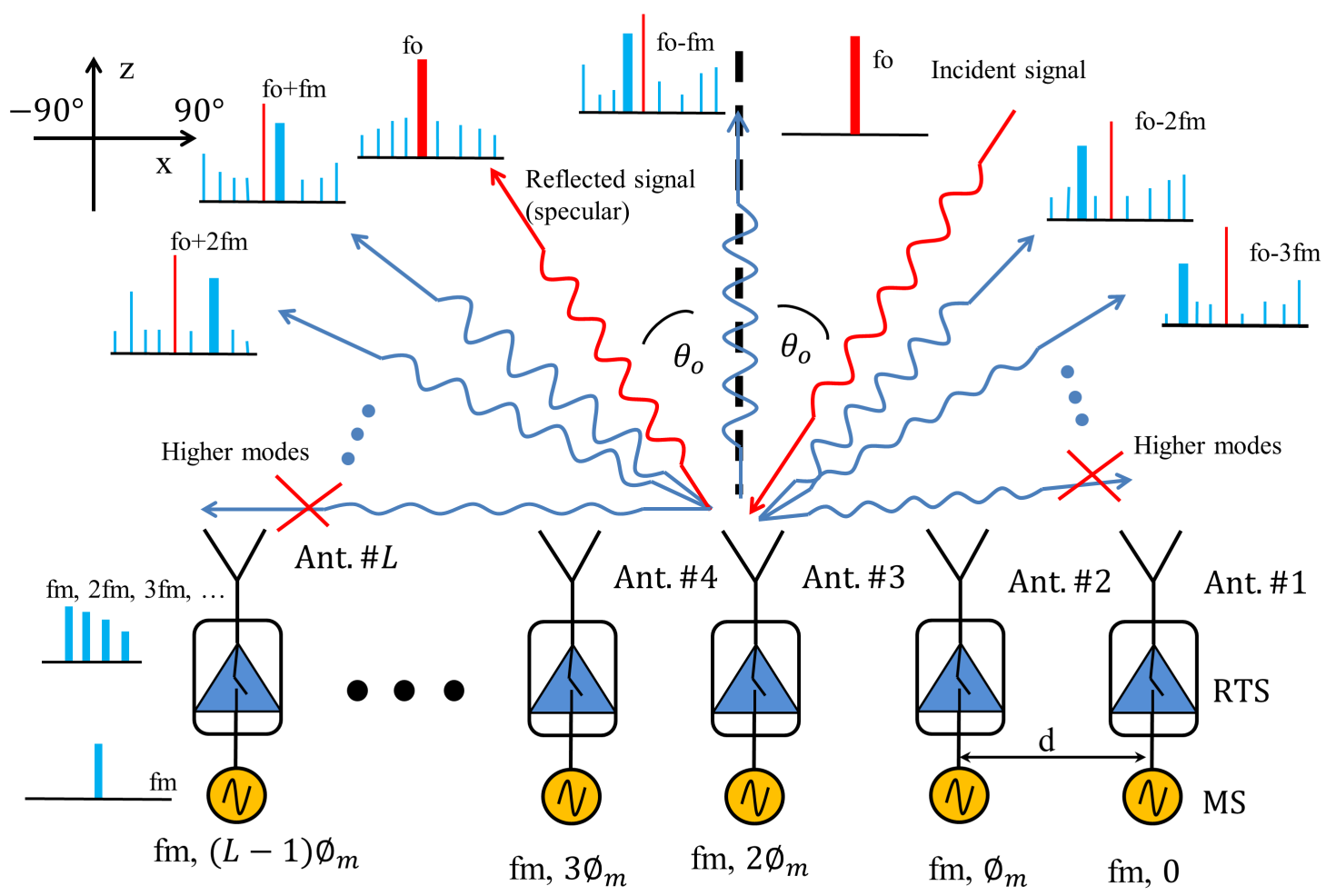

MS = Modulation source $\quad$ RTS $=$ Reflective - type switch,$\quad$ Ant. $=$ Antenna

Figure 3.1: General representation of the PMSA where its distortion products are scattered in space

Before starting the investigation, it is so important to make an equivalence relationship between the PMSA system and the adopted technique (i.e., polyphase multipath technique) to conduct the investigation efficiently and effectively. To do this, it requires finding the equivalent circuit for every single scatterer. Thus, dividing the scatterer into its main parts will ease the process of a scatterer conversion to its 
counterparts which is a path in the polyphase multipath circuit. In the following discussion, we study parts of a scatterer separately and we then combine them to introduce a mathematical model and an equivalent circuit for a whole single scatterer as given below.

\subsubsection{The Derivation of the Mathematical Model}

\subsubsection{A Single Scatterer Model}

\section{Antennas}

As shown in Figure 3.1, the incident signals reach an array aperture from a particular angle of $\theta_{o}$ with respect to the the perpendicular axis (z-axis) on the the array axis (x-axis). Thus, RF signals induced on the $l-t h$ element can be expressed as

$$
E_{R, l}=S_{R, l} \cdot e_{R, l}
$$

where $S_{R, l}$ is the electric field intensity of the incident signal at the $l-t h$ element and $e_{R, l}$ represents the element pattern of the same $l-t h$ element. Since there is more than one element to form the PMSA, the incident signals reach elements at different times. A time delay between elements is represented by a phase shift in the frequency domain. If distances separating array elements are equal (i.e., uniform array UA), this makes a similar phase shift difference between any two successive elements. The relative phase shift difference can be defined as

$$
\emptyset_{o}=K d \sin \theta_{o}
$$

where $K$ is the wave number of the RF incident signal and $d$ denotes a distance separating between any two successive elements. In our design, two values have been selected for the $d$, which are $0.5 \lambda_{o}$ and $0.7 \lambda_{o}$ where $\lambda_{o}$ is the wavelength of the RF 
incident signal in free-space. Now, each antenna can be considered as a signal source, where its mathematical expression is

$$
E_{o, l}=A_{l} \cos \left(w_{o} t-\emptyset_{o, l}\right)
$$

in which $A_{l}$ represents an amplitude of the incident signal at the $l-t h$ element, $w_{o}$ is an angular frequency of the RF incident signal, and $\emptyset_{o, l}$ is a phase of the incident signal at the $l-t h$ element where the negative sign in the argument of (3.3) indicates the delay time (lag phase) for the incident signals to reach array elements with respect to a reference element.Figure $3.2 \mathrm{a}$ illustrates the analogy between an antenna and an equivalent signal source. Thus, it can be considered as a fictitious spatial source, and it provides significant information about the incident signals where they are sampled in space compared to the first element in the PMSA. This process can

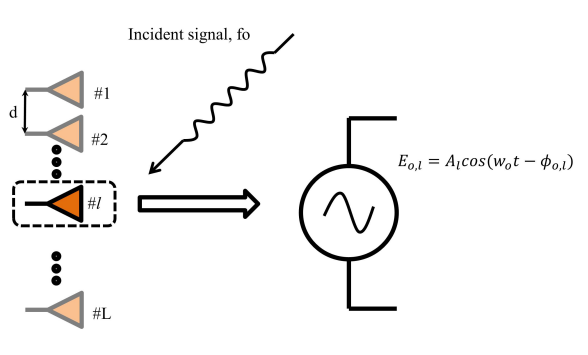

(a)

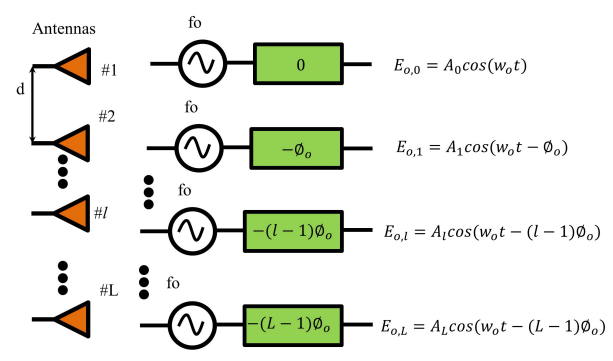

(b)

Figure 3.2: a) Antenna-signal source equivalent model b) an array of spatial sources (antenna array)

be repeated as many antennas as we have in the PMSA. The spatial sources have the same frequencies, but their amplitudes and phases are different. We can use a vector notation to represent amplitudes and phases of spatial sources as given below

$$
\left[A_{0}, A_{1}, A_{2}, \ldots, A_{l}, \ldots, A_{L-1}\right]
$$




$$
\left[\emptyset_{o, 0}, \emptyset_{o, 1}, \emptyset_{o, 2}, \ldots, \emptyset_{o, l}, \ldots, \emptyset_{o, L-1}\right]
$$

Because antennas, representing the spatial sources of RF signals in the model, are located close to each other compared to a distance between a source of the incident signals and array elements, differences in amplitudes of spatial sources can be ignored, and they will be equal $A_{0}=A_{1}=A_{2}=\cdots=A_{l}=A_{L-1}$. Also, from (3.2), (3.3), and (3.5), we can see that their phases are not equal, and they vary if either $d$ or $\theta_{o}$, or both change. Figure $3.2 \mathrm{~b}$ shows an array of the spatial RF sources.

\section{Modulation Sources}

Figure 3.1 shows that each antenna element has its particular modulation source. The modulation sources may produce single, two, or multi tones signals. In this research study, we will use single-tone and two-tone modulation sources in our experimental tests. Modulation signals have equal frequencies, equal amplitudes, and different phases. Frequencies of modulation signals should be much smaller than frequencies of RF incident signals $w_{m h}<<w_{o}$

$$
\left(w_{m 1}, w_{m 2}, \ldots, w_{m h}, w_{m H}\right) \ll w_{o}
$$

where $w_{m h}$ represents a modulation frequency of the $h-t h$ tone and $H$ represents the total number of modulation tones. Phases of different tones applied to the same scatterer element may or may not be equal. A vectors notation for phases of single-tone modulation sources is

$$
\left[\emptyset_{m, 0}, \emptyset_{m, 1}, \emptyset_{m, 2}, \ldots, \emptyset_{m, l}, \ldots, \emptyset_{m, L-1}\right]
$$


for two-tone modulation sources:

$$
\left[\emptyset_{m 1,0}, \emptyset_{m 1,1}, \emptyset_{m 1,2}, \ldots, \emptyset_{m 1, l}, \ldots, \emptyset_{m 1, L-1}\right]
$$

and

$$
\left[\emptyset_{m 2,0}, \emptyset_{m 2,1}, \emptyset_{m 2,2}, \ldots, \emptyset_{m 2, l}, \ldots, \emptyset_{m 2, L-1}\right]
$$

and for H-tone modulation sources:

$$
\left[\emptyset_{m 1,0}, \emptyset_{m 1,1}, \emptyset_{m 1,2}, \ldots, \emptyset_{m 1, l}, \ldots, \emptyset_{m 1, L-1}\right]
$$

$$
\left[\emptyset_{m 2,0}, \emptyset_{m 2,1}, \emptyset_{m 2,2}, \ldots, \emptyset_{m 2, l}, \ldots, \emptyset_{m 2, L-1}\right]
$$

$$
\left[\emptyset_{m h, 0}, \emptyset_{m h, 1}, \emptyset_{m h, 2}, \ldots, \emptyset_{m h, l}, \ldots, \emptyset_{m h, L-1}\right]
$$

and

$$
\left[\emptyset_{m H, o}, \emptyset_{m H, 1}, \emptyset_{m H, 2}, \ldots, \emptyset_{m H, l}, \ldots, \emptyset_{m H, L-1}\right]
$$

where $\emptyset_{m h, l}$ is the modulation source phase of the $h-t h$ tone at the $l-t h$ scatterer element. Phases of modulation sources, having $\mathrm{H}$ tones, can be combined in a matrix for convenient representation 


$$
\left(\begin{array}{c}
\emptyset_{m 1, o}, \emptyset_{m 1,1}, \emptyset_{m 1,2}, \ldots, \emptyset_{m 1, l}, \ldots, \emptyset_{m 1, L-1} \\
\emptyset_{m 2, o}, \emptyset_{m 2,1}, \emptyset_{m 2,2}, \ldots, \emptyset_{m 2, l}, \ldots, \emptyset_{m 2, L-1} \\
\vdots \\
\emptyset_{m h, o}, \emptyset_{m h, 1}, \emptyset_{m h, 2}, \ldots, \emptyset_{m h, l}, \ldots, \emptyset_{m h, L-1} \\
\vdots \\
\emptyset_{m H, o}, \emptyset_{m H, 1}, \emptyset_{m H, 2}, \ldots, \emptyset_{m H, l}, \ldots, \emptyset_{m H, L-1}
\end{array}\right)
$$

where $\mathrm{H}$ could be one, two, or any positive integer value. Figure 3.3 shows an array of modulation sources, having single and two tones signals.

\section{Nonlinear Active Switches or Reflective-Type Switches (RTS)}

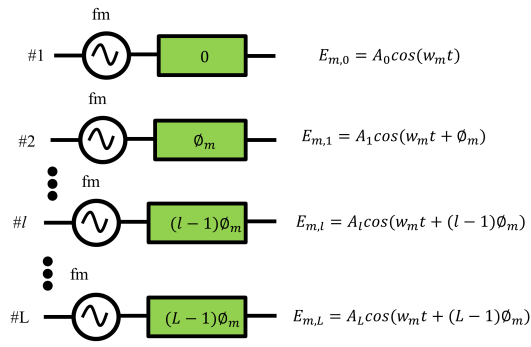

(a)

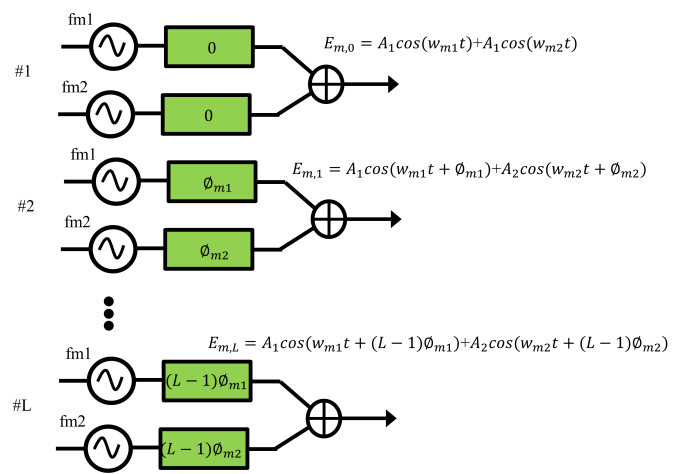

(b)

Figure 3.3: Representation of the modulation sources in the model when having a) single-tone signals b) two-tone signals

Referring to Figure 3.1, there is a switch associated with each single scatterer. Two different signals are applied on both sides of a switch simultaneously. One of the signals works to turn ON/OFF the switch, produced by the modulation sources, while the second one is the incident signal. Figure 3.4 shows the switch in its two states (i.e., open and short). Each switch operates to reflect back induced signals coming from an antenna (spatial source) toward it again in both short and open states, so it is called as a reflective-type switch (RTS). More details about the switch 
design will be given in Chapter 6. Although this process is similar to the function performed by mixers [107], the difference here is that the modulation and induced signals are applied to the two sides of RTS separately, while they may be applied to the same side in mixers. Phases of the incident signals will be inverted due to the processes of reflections [59]. Furthermore, the RTS is periodically turned ON/OFF by the modulation source to change the polarity of reflected signals. Then as a consequence, reflected signals are modulated. Reflected signals will not only contain a spectral component of the main frequency $w_{o}$ of incident signals but also other infinite components distributed about the main frequency at multiple integers of the modulation frequency $\pm n w_{m}$. If the modulation source is a two-tone signal generator, there will be both HDs and IMDs products. We can depict the explanation given above using an equivalent circuit for the RTS, as illustrated in Figure 3.5. It consists of two nonlinear circuits, one phase inverter, and one ideal mixer. The phase inverter is added only to a path of the incident signals because the RTS inverts phases of the incident signals. In real-world applications, no ideal mixer exists, but in the equivalent circuit model, mixer nonlinearities are split into different two blocks to ease understanding and following steps of the mathematical process as shown below. To mathematically describe nonlinear behaviors of the RTS with two input signals, the situation will be complicated. Therefore, we will divide the process into two main tasks. First, each signal will pass through a different nonlinear circuit, but the same mathematical model is applied to both. Second, the resulting signals from both nonlinear circuits enter an ideal mixer to perform the multiplication (mixing) process. Resulting spectrum contains sums and differences products of both multiple integers of the two signals.

If we assume the nonlinear circuits being memoryless and weakly nonlinear, the power series expansion seems a perfect mathematical model to describe their behaviors [113]. 

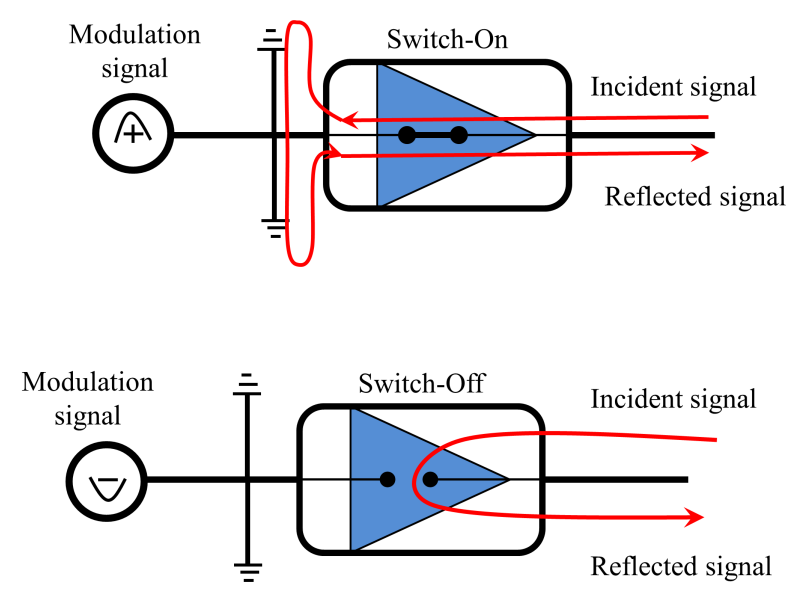

Figure 3.4: RTS ON and OFF states

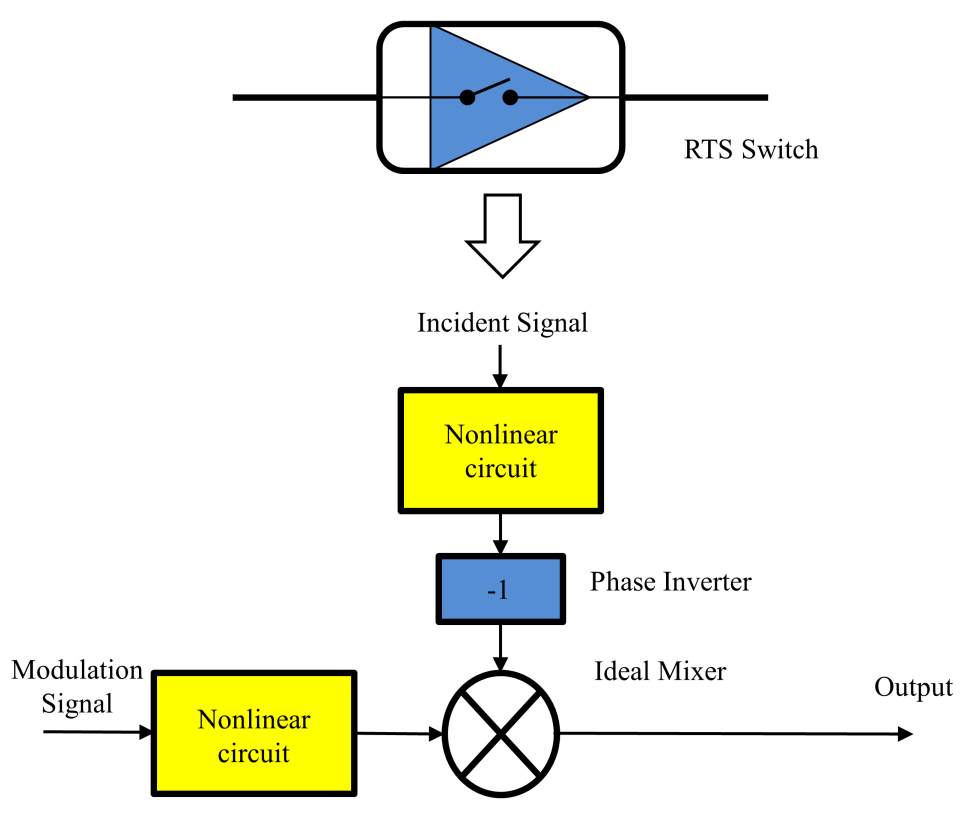

Figure 3.5: Equivalent circuit of the RTS

Figure 3.6 displays the nonlinear circuits when excited by single and two tones signals, producing harmonics and harmonics and IMDs from the first and second circuits, 
respectively. Harmonics and IMDs generated in the RTS can be tailored with other replicas in space generated in other RTSs as will be seen later.

If the input is $x(t)$, the output of the nonlinear circuit is

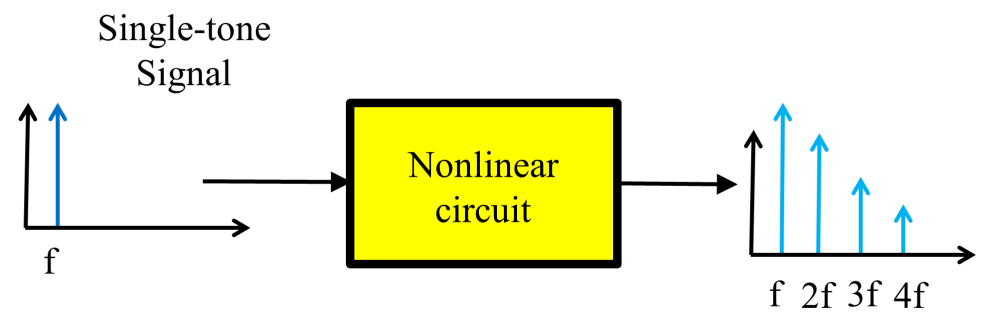

Two-tone

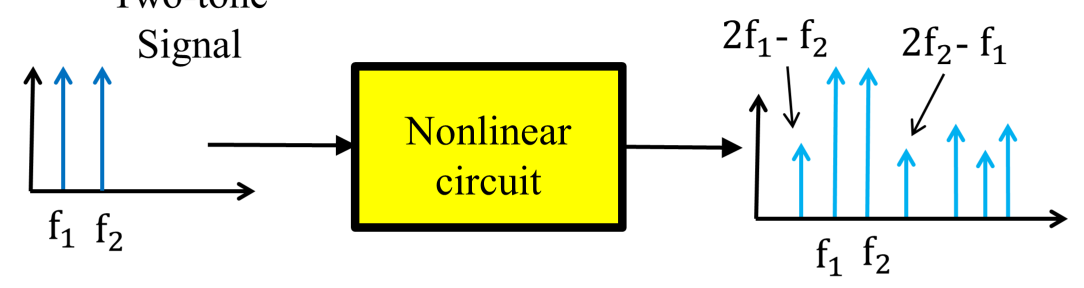

Figure 3.6: Nonlinear circuits with single-tone and two-tone input signals

$$
y(t)=a_{o}+a_{1} x(t)+a_{2} x^{2}(t)+a_{3} x^{3}(t)+\cdots
$$

where $a_{0}, a_{1}, a_{2}, a_{3}, \cdots$ are the power series coefficients. If the input signal is single-tone $x(t)=A \cos (w t+\emptyset)$, then the output is

$$
y(t)=a_{o}+a_{1} A \cos (w t+\emptyset)+a_{2} A^{2} \cos ^{2}(w t+\emptyset)+a_{3} A^{3} \cos ^{3}(w t+\emptyset)+\cdots
$$


where $w, A$, and $\emptyset$ are frequency, amplitude, and phase of the input signal, respectively. Also the input signal could be a two-tone signal $x(t)=$ $A_{1} \cos \left(w_{1} t+\emptyset_{1}\right)+A_{2} \cos \left(w_{2} t+\emptyset_{2}\right)$, so the output is

$$
\begin{aligned}
y(t) & =a_{0}+a_{1}\left(A_{1} \cos \left(w_{1} t+\emptyset_{1}\right)+A_{2} \cos \left(w_{2} t+\emptyset_{2}\right)\right) \\
& +a_{2}\left(A_{1} \cos \left(w_{1} t+\emptyset_{1}\right)+A_{2} \cos \left(w_{2} t+\emptyset_{2}\right)\right)^{2} \\
& +a_{3}\left(A_{1} \cos \left(w_{1} t+\emptyset_{1}\right)+A_{2} \cos \left(w_{2} t+\emptyset_{2}\right)\right)^{3}+\cdots
\end{aligned}
$$

These two equations can be simplified using some trigonometric identities and algebraic manipulations, see Appendix-A.

After making simplifications, Eqs.(3.16) and (3.17) can be rewritten as

$$
\begin{aligned}
y(t)= & b_{0}+b_{1} \cos (w t+\emptyset)+b_{2} \cos (2(w t+\emptyset))+b_{3} \cos (3(w t+\emptyset))+\cdots+b_{n} \cos (n(w t+ \\
& +\emptyset))+\cdots=\sum_{b=0}^{\infty} b_{n} \cos (n(w t+\emptyset))
\end{aligned}
$$


and

$$
\begin{aligned}
y(t) & =b_{00}+b_{10} \cos \left(w_{1} t+\emptyset_{1}\right)+b_{20} \cos \left(2\left(w_{1} t+\emptyset_{1}\right)\right)+b_{30} \cos \left(3\left(w_{1} t+\emptyset_{1}\right)\right) \\
& +\cdots+b_{k 0} \cos \left(k\left(w_{1} t+\emptyset_{1}\right)\right)+\cdots+b_{01} \cos \left(w_{2} t+\emptyset_{2}\right) \\
& +b_{02} \cos \left(2\left(w_{2} t+\emptyset_{2}\right)\right)+b_{03} \cos \left(3\left(w_{2} t+\emptyset_{2}\right)\right)+\cdots \\
& +b_{0 m} \cos \left(m\left(w_{2} t+\emptyset_{2}\right)\right)+\cdots+b_{11} \cos \left[\left(w_{1}-w_{2}\right) t+\left(\emptyset_{1}-\emptyset_{2}\right)\right] \\
& +b_{11} \cos \left[\left(w_{1}+w_{2}\right) t+\left(\emptyset_{1}+\emptyset_{2}\right)\right]+b_{21} \cos \left[\left(2 w_{1}-w_{2}\right) t+\left(2 \emptyset_{1}-\emptyset_{2}\right)\right] \\
& +b_{21} \cos \left[\left(2 w_{1}+w_{2}\right) t+\left(2 \emptyset_{1}+\emptyset_{2}\right)\right]+b_{12} \cos \left[\left(2 w_{2}-w_{1}\right) t+\left(2 \emptyset_{2}-\emptyset_{1}\right)\right] \\
& +b_{12} \cos \left[\left(2 w_{2}+w_{1}\right) t+\left(2 \emptyset_{2}+\emptyset_{1}\right)\right]+\cdots \\
& =\sum_{\substack{k=0 \\
b_{k 0}}}^{\infty} \cos \left(k\left(w_{1} t+\emptyset_{1}\right)\right)+\sum_{m=0}^{\infty} b_{0 m} \cos \left(m\left(w_{2} t+\emptyset_{2}\right)\right) \\
& +\sum_{\substack{k=-\infty \\
k \neq 0}}^{\infty} \sum_{\substack{m=-\infty \\
m \neq 0}}^{\infty} b_{k m} \cos \left[\left(k w_{1}+m w_{2}\right) t+\left(k \emptyset_{1}+m \emptyset_{2}\right)\right]
\end{aligned}
$$

Eq.(3.19) can also be written as

$$
y(t)=\sum_{k=-\infty}^{\infty} \sum_{m=-\infty}^{\infty} b_{k m} \cos \left[\left(k w_{1}+m w_{2}\right)+\left(k \emptyset_{1}+m \emptyset_{2}\right)\right]
$$

where $b_{n}$ and $b_{k m}$ represent the Taylor series coefficients when the modulation signals are single and two tones, respectively.

We can conclude here three essential facts based on Eqs.(3.18), (3.19), and (3.20)

1. When using input signals having more than one tone, there are not only harmonics products of individual tones $\left(k w_{1}\right.$ and $\left.m w_{2}\right)$ but also cross products of multiple integers of tones (intermodulation distortions IMDs, $\left( \pm k w_{1} \mp m w_{2}\right)$.

2. There is an infinite discrete number of harmonics and IMDs.

3. Phases of harmonics and IMDs products are phases of input signals multiplied 
by the harmonic or IMD term order (i.e., $n \emptyset$ or $\left.\left(k \emptyset_{1}+m \emptyset_{2}\right)\right)$.

From the above remarks, harmonics and IMDs are non-coherent in their nature, and they have their particular phases, so that they can be treated independently. Thus, these properties provide a powerful tool to deal with harmonics and IMDs, regardless of their original signals.

The remaining part that should be mathematically modeled is the mixing process between the incident and modulation signals where the modulation signals could be either single or two tones. The mixing action represents the last step in the whole mathematical representation for the stand-alone RTS. In addition, the mixing is one of the most useful processes in radio frequency $\mathrm{RF}$ communication circuits [94]. A mixer multiplies signals together to generate signals with new frequencies. The new frequencies are sums and differences of multiplied (input) frequencies. Figure 3.7 shows the conventional schematic of multiplication process (mixing) with two sinusoidal inputs having different frequencies. The output signal follows the identity, the multiplication of two trigonometric signals

$$
\cos (A) \cos (B)=\frac{1}{2}[\cos (A+B)+\cos (A-B)]
$$

If we replace $A$ and $B$ by $\left(2 \pi f_{1} t+\emptyset_{1}\right)$ and $\left(2 \pi f_{2} t+\emptyset_{2}\right)$, the resulting output is

$$
\begin{aligned}
y(t) & =\cos \left(2 \pi f_{1} t+\emptyset_{1}\right)+\cos \left(2 \pi f_{2} t+\emptyset_{2}\right) \\
& =\frac{1}{2}\left[\cos \left(2 \pi\left(f_{1}+f_{2}\right) t+\left(\emptyset_{1}+\emptyset_{2}\right)\right)+\cos \left(2 \pi\left(f_{1}-f_{2}\right) t+\left(\emptyset_{1}-\emptyset_{2}\right)\right)\right]
\end{aligned}
$$

In actual mixers, outputs contain not only multiplications between the fundamental frequencies of two input signals but also multiplications of their distortion products. In our proposed system the nonlinearities of mixers are combined with nonlinear circuits. Furthermore, whatever phases are added to the input signals, they will 


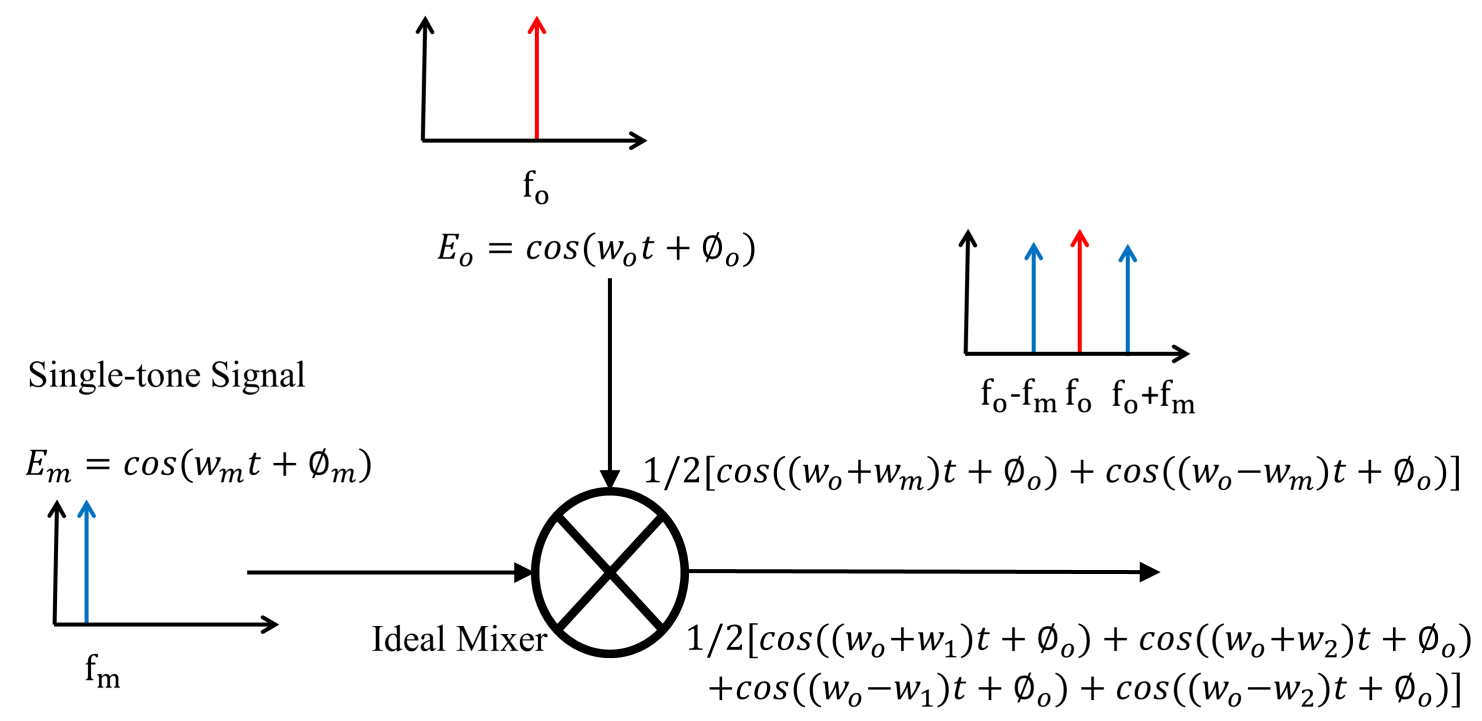

Two-tone Signal

$E_{m}=\cos \left(w_{m 1} t+\emptyset_{m 1}\right)+\cos \left(w_{m 2} t+\emptyset_{m 2}\right)$
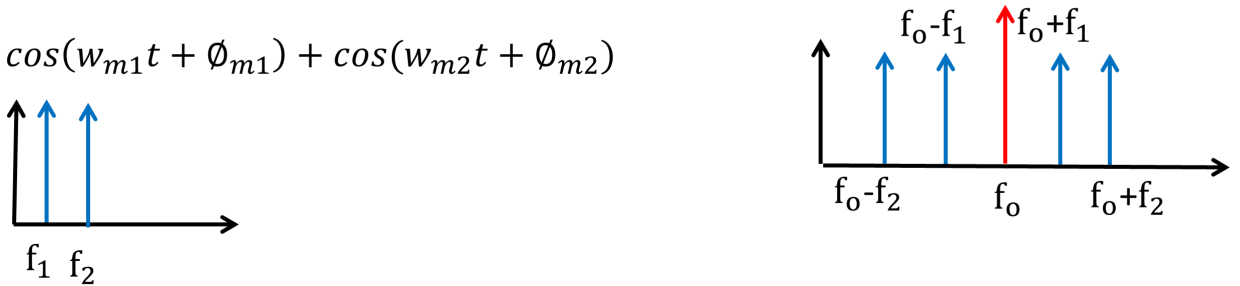

Figure 3.7: Mixer used as a phase shifter

appear at the mixer output. This means that any change happening in phases of the input signals will result in changing phases of the output signals regardless of their frequencies. This property enables the proposed system to work with all frequencies, making it as a scalable structure.

Up to now, we have only presented the mathematical models for all separate parts forming a whole scatterer as well as their equivalent circuits independently. Thus, these parts must be combined to characterize a scatterer in one block diagram. Figure 3.8 exhibits the whole equivalent circuits of a scatterer for both single-tone and two-tone modulation sources. Signals at input ports of the mixer are outputs of the nonlinear circuits. The final resulting output signals of a single scatterer when using single-tone and two-tone modulation sources are 


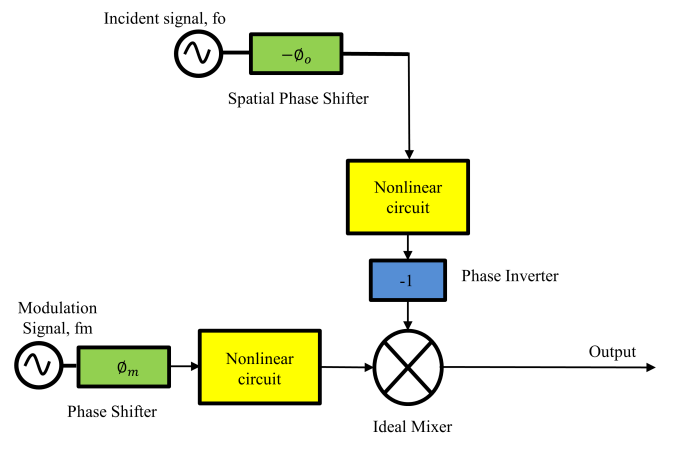

(a)

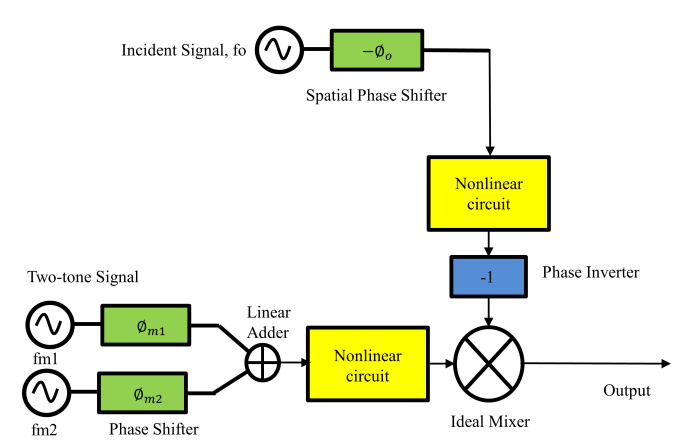

(b)

Figure 3.8: Equivalent circuits of a single scatterer when using a) single-tone modulation source b) two-tone modulation source

$$
\begin{aligned}
y(t) & =\sum_{h=0}^{\infty} b_{h} \cos \left(h\left(w_{o} t+\emptyset_{o}\right)\right) \times \sum_{n=0}^{\infty} b_{n} \cos \left(n\left(w_{m} t+\emptyset_{m}\right)\right) \\
& =\sum_{h=0}^{\infty} \sum_{n=0}^{\infty} \frac{1}{2} B\left(\cos \left(\left(h w_{o}+n w_{m}\right) t+h \emptyset_{o}+n \emptyset_{m}\right)\right. \\
& \left.+\cos \left(\left(h w_{o}-n w_{m}\right) t+h \emptyset_{o}-n \emptyset_{m}\right)\right) \\
& =\sum_{h=0}^{\infty} \sum_{n=-\infty}^{\infty} \frac{1}{2} B\left(\cos \left(\left(h w_{o}+n w_{m}\right) t+h \emptyset_{o}+n \emptyset_{m}\right)\right)
\end{aligned}
$$

and

$$
\begin{aligned}
\mathrm{y}(\mathrm{t})= & \left.\sum_{h=0}^{\infty} b_{h} \cos \left(h\left(w_{o} t+\emptyset_{o}\right)\right) \times \sum_{k=-\infty}^{\infty} \sum_{m=-\infty}^{\infty} b_{k m} \cos \left(\left(k w_{1}+m w_{2}\right) t+k \emptyset_{1}+m \emptyset_{2}\right)\right) \\
= & \sum_{h=0}^{\infty} \sum_{n=-\infty}^{\infty} \sum_{m=-\infty}^{\infty} \frac{1}{2} C\left[\cos \left(\left(h w_{o}+k w_{1}+m w_{2}\right) t+h \emptyset_{o}+k \emptyset_{1}+m \emptyset_{2}\right)\right. \\
& \left.+\cos \left(\left(h w_{o}-k w_{1}-m w_{2}\right) t+h \emptyset_{o}-k \emptyset_{1}-m \emptyset_{2}\right)\right]
\end{aligned}
$$

where $B$ and $C$ are constants. In Eq.(3.24), both sum and difference terms contribute into the same harmonics or IMDs components, so we can ignore either the sums term or the difference term with multiplying the non-ignored term by a factor 2 to 
compensate impacts of the ignored terms. Thus, Eq.(3.24) can be rewritten as

$$
y(t)=\sum_{h=0}^{\infty} \sum_{k=-\infty}^{\infty} \sum_{m=-\infty}^{\infty} C\left[\cos \left(\left(h w_{o}+k w_{1}+m w_{2}\right) t+h \emptyset_{o}+k \emptyset_{1}+m \emptyset_{2}\right)\right]
$$

To this end, we have established a mathematical model for a single scatterer as well as its equivalent block diagram. The mathematical model and block diagram will be extended in the following subsection to include an array of scatterers. The polyphase multipath technique based the scattering array model can predict which distortion products appear at the final circuit output relying on phases of the incident and modulation signals distributed across a scattering array aperture. Although this technique provides a significant tool to quickly find out the enhanced and suppressed distortion products at the circuit output, it loses its effectiveness once phases of the modulation and incident signals deviate form their prescribed values. This forces our adopted model based on the polyphase multipath technique to deal only with phases defined in advance. In other words, one of our phase shift types is related to angles of the incident signals and distances between scattering array elements, so if arrival angles of the incident signals are changed intentionally or unintentionally, resulting phases of the incident signals induced over antennas elements will be changed too, leading to the emergence of all wanted and unwanted distortion products at the circuit output.

Because we treat distortion products in space, we do not have an actual circuit output. Each point in space has different spatial characteristics of distortion products since distortion products traveling in space experience extra added phases. In addition, the mathematical model depended on the conventional polyphase multipath technique is incapable to predict where locations of enhancement and suppression of 
distortion products, so finding a way to overcome this deficit will be our substantial goal. Thus, we will address this problem by adding extra terms to make the polyphase multipath method more powerful, robust, and compatible. The modified form of the polyphase multipath model can work with any phase distribution for spatial (incident) and modulation sources and can define angles of the incident and scattered signals as well. We start explaining the proposed scattering array using the conventional polyphase multipath method, and then we move to describe the proposed modified model as a smooth transition between the modified model and its predecessor.

\subsubsection{Scattering Array Model}

\section{Conventional Polyphase Multipath Circuit}

The polyphase multipath technique is widely used in RF front-end designs due to its ability to cancel harmonics and IMDs and to make the output spectrum clean. Thus, it relaxes high-performance filter requirements. Its efficacy increases as a number of

paths increases. It is used in both receiving and transmitting sides [84] [157] [40]. The underlying idea behind the design is to use more than one channel carrying the same signals with different phases chosen in such a way that the undesired harmonics and IMDs products are suppressed, see Figure 3.9. Most designs in the communication field prefer either the first upper sideband 1USB or the first lower sideband 1LSB harmonic and dismiss others, but in our PMSA, we will deal with several harmonics and IMDs, leading to a substantial difference between the PMSA and the conventional polyphase multipath technique. We can say that our design is similar to the polyphase multipath circuit but with multiple outputs. However, signals generated in our system are added in space rather than the use of physical connections such as transmission-lines, waveguides, and power combiners and dividers. Therefore, this property makes the design feasible for high frequencies applications when size is 
matter.

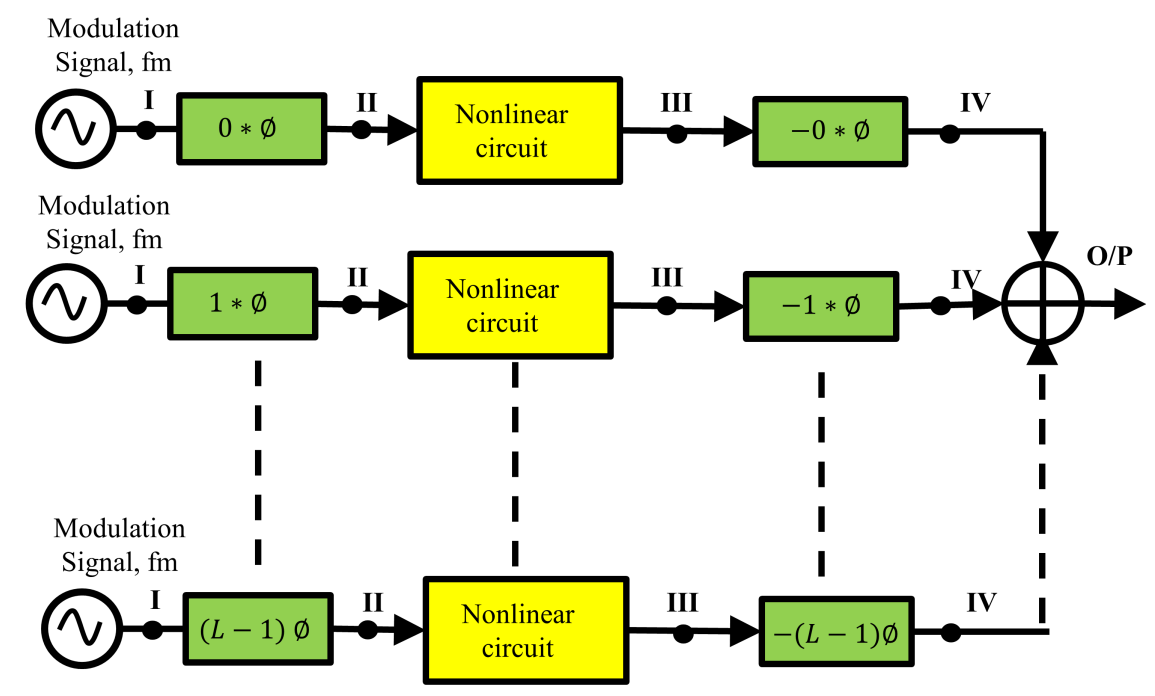

Figure 3.9: Polyphase multipath circuit

In every path, phases of first and second phase shifters are equal but opposite for the desired distortion product. If we take a path with its first phase equal to $(l-1) \times \emptyset$, where $\emptyset$ is equal to $360^{\circ} / L$, the second phase shift must be $-(l-1) \times \emptyset$. Thus, distortion products having this phase shift will be aligned back to zero at ends of paths in a circuit, leading to constructive interference. Other harmonics products may or may not be canceled at the final output relying on their phase distributions at ends of paths. Mathematically, this can be expressed using the Taylor series expansion in (3.18). Assuming an input signal $x(t)=A \cos (w t)$ applied to inputs of paths is illustrated in Figure 3.9, and signals at different points on a path can be expressed as;

signals at the point I is

$$
x(t)=A \cos (w t)
$$


signals at the point II is

$$
x^{\prime}(t)=A \cos (w t+(l-1) \times \emptyset)
$$

signals at the point III is the output of a nonlinear circuit, represented by the Taylor series,

$$
\begin{aligned}
x^{\prime \prime}(t) & =b_{o}+b_{1} \cos (w t+(l-1) \times \emptyset)+b_{2} \cos (2 w t+2(l-1) \times \emptyset) \\
& +b_{3} \cos (3 w t+3(l-1) \times \emptyset)+\cdots
\end{aligned}
$$

and signals at the point IV, where the second phase shifter is set into $-(l-1) \times \emptyset$, is

$$
x^{\prime \prime \prime}(t)=b_{o}+b_{1} \cos (w t)+b_{2} \cos (2 w t+(l-1) \times \emptyset)+b_{3} \cos (3 w t+2(l-1) \times \emptyset)+\cdots
$$

It can be seen that all paths have identical phases for the fundamental component which means that they will be added constructively at the circuit output. Also, we can select any distortion product component, for example, the second harmonic can be selected if we set a value of the second phase shifter is equal into $-2(l-1) \times \emptyset$, so Eq.(3.29) will be rewritten as

$$
x^{\prime \prime \prime}(t)=b_{o}+b_{1} \cos (w t-(l-1) \times \emptyset)+b_{2} \cos (2 w t)+b_{3} \cos (3 w t+(l-1) \times \emptyset)+\cdots
$$

Now, the second distortion products (harmonics) have equal phases at ends of paths and they are added constructively at the circuit output. Other distortion components in (3.29) and (3.30), except the desired ones, have different phases. The circuit can cancel all these distortion products if the relative phase difference between any two successive paths is equal to $\emptyset=360^{\circ} / L$. In general, the circuit can cancel all 
harmonics product except those obeying (3.31)

$$
n=p \times L+g
$$

where $p=0,1,2,3, \cdots, g=1,2,3, \cdots$ (i.e., is the desired distortion product term number), $L$ is a number of paths, and $n$ is the enhanced distortion product number. After subtracting values of second phase shifters from phases of distortion products (harmonics), phases of harmonics at ends of paths are $-(l-1) \times(n-g) \times \emptyset$. For $n \neq p \times L+g$, phases at ends of paths are balanced so that distortion products are suppressed. In contrast, for $n=p \times L+g$, harmonics will have the same phases at ends of paths, resulting in constructive addition. Handling distortion products is periodic with $p L$.

Mathematically speaking, adding distortion products in (3.29) and (3.30) at ends of paths is given in (3.32) and (3.33) as

$$
\begin{aligned}
y(t) & =L b_{o}+L b_{1} \cos (w t)+\sum_{l=1}^{L} b_{2} \cos (2 w t+(l-1) \times \emptyset) \\
& +\sum_{l=1}^{L} b_{3} \cos (3 w t+2(l-1) \times \emptyset)+\cdots
\end{aligned}
$$

and

$$
\begin{aligned}
y(t)= & L b_{o}+\sum_{l=1}^{L} b_{1} \cos (w t-(l-1) \times \emptyset)+L b_{2} \cos (2 w t)++\sum_{l=1}^{L} b_{3} \cos (3 w t+ \\
& 2(l-1) \times \emptyset)+\cdots
\end{aligned}
$$

Because some terms with $n \neq p \times L+g$ have balanced phases, they will be added 
destructively at the final output as mathematically shown in (3.34)

$$
\sum_{l=1}^{L} \cos (n w t+(n-g) \times(l-1) \times \emptyset)=0
$$

From Eqs.(3.33) and (3.34), all distortion products do not appear at the final output except those with $n=p \times L+g$, so the resulting output is

$$
\begin{aligned}
y(t) & =L b_{0}+L b_{g} \cos (g w t)+\sum_{l=1}^{L} b_{L+g} \cos ((L+g) w t) \\
& +\cdots+\sum_{l=1}^{L} b_{p L+g} \cos ((p L+g) w t) \\
& =L b_{0}+\sum_{p=0}^{\infty} \sum_{l=1}^{L} b_{L+g} \cos ((p L+g) w t)
\end{aligned}
$$

If we use a sufficient number of paths (i.e., a large number of scatterers) such that the high order distortion products are weak at the circuit output, we can deal with all other strong distortion products. To demonstrate distortion products handling (i.e., cancellation and enhancement) employing the adopted manner, two different instances are presented in the following part.

\section{Examples}

\section{2-Path Circuit}

Figure 3.10 displays a polyphase circuit having two paths. A phase shift of the upper path is zero, so all distortion products (harmonics) resulting from the nonlinear circuit at the same path as well as the fundamental frequency have zero phase shift at an output port of the nonlinear circuit. The lower path has an opposite phase with the upper path (i.e., $180^{\circ}$ ). Distortion products generated in the nonlinear circuit of the lower path have two different phase shifts. In other words, even order distortion 
products have $0^{\circ}$ or multiples of $360^{\circ}$ phase shifts while phase shifts of odd order distortion products are multiples of $180^{\circ}$. This contrast in phase shifts of distortion products between the two paths is exploited to cancel either odd or even distortion products depending on the second phase shifts placed after the nonlinear circuits. Canceling even order distortion products requires to make the phase of the second phase shifter equal to $180^{\circ}$ in the lower path, but suppressing the odd order distortion products, the phase shift of the second phase shifter in the upper path must be $180^{\circ}$. In the first case, odd distortion products are in-phase; leading to constructive addition, while even distortion products as well as the fundamental component, arriving in anti-phase and they are suppressed. In the second case, even distortion products are enhanced, and odd distortion products are canceled. We can see all distortion products with $n=p \times L+g$ being added constructively at the final output, where $L=$ $2, p=0,1,2$, and $g=1$ and 2 for the first and second cases, respectively. Furthermore, the first case circuit is well-known in the realm of communication theory as a balanced modulator.

\section{3-Path Circuit}

A circuit with three paths is exhibited in Figure 3.11a. It is similar to the two paths circuit, but the difference is that phases of the first phase shifters in paths 1 , 2 , and 3 are $0^{\circ}, 120^{\circ}$, and $240^{\circ}$, respectively. The relative phase difference between any two successive paths is $360 / L$, being equidistant. Phases of the 1 st, 2 nd, and 3rd distortion products of paths 1,2 , and 3 are $\left[\begin{array}{lll}0^{\circ} & 0^{\circ} & 0^{\circ}\end{array}\right],\left[\begin{array}{lll}0^{\circ} & 120^{\circ} & 240^{\circ}\end{array}\right]$, and $\left[\begin{array}{ll}0^{\circ} & 240^{\circ}\end{array}\right.$ $120^{\circ}$, respectively after the nonlinear circuits of paths 1,2 , and 3 . The mathematical illustration of the polyphase 3-path circuit is given in Appendix-B. Figure 3.11b shows how distortion products at end of each path combine. The 1st harmonic components are added constructively, while phases of the second and third harmonics are evenly balanced at the output, ensuring harmonic cancellation at the output. Although the 


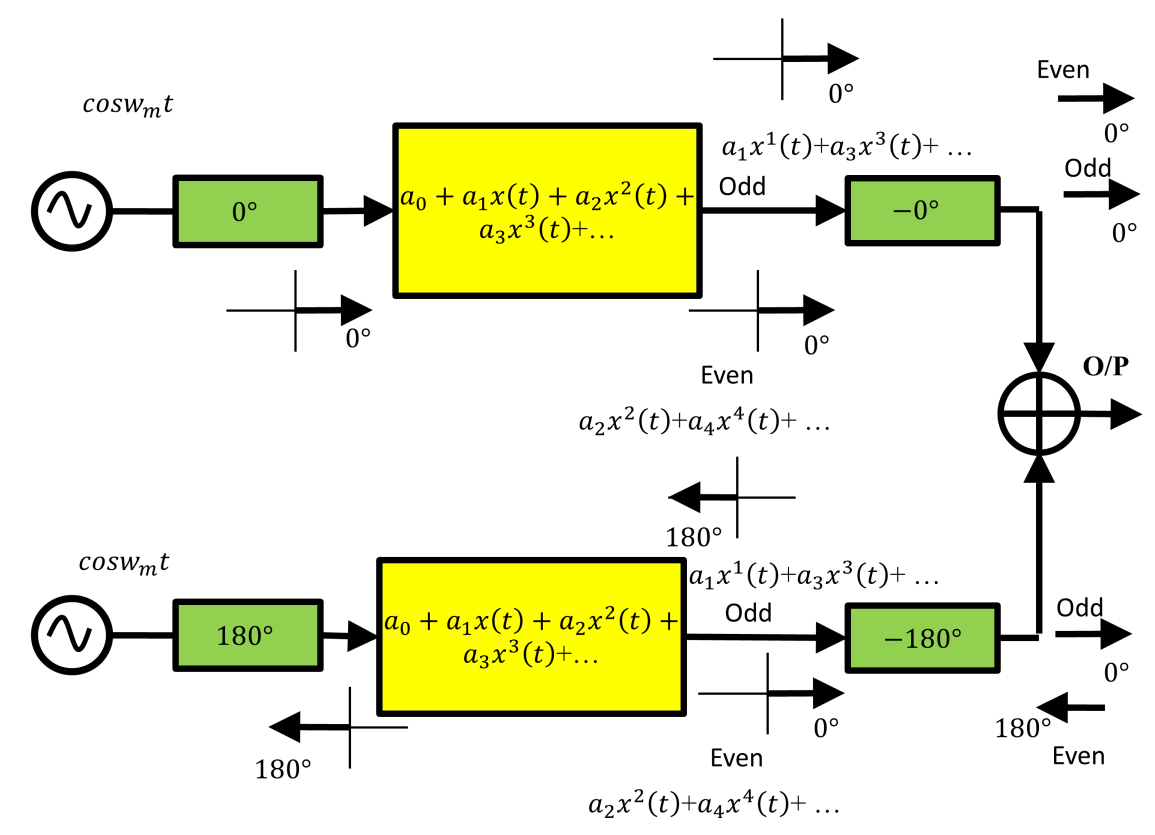

Figure 3.10: Two-path polyphase circuit

second and third harmonics are canceled, the fourth one is enhanced due to its phases at ends of paths being aligned in-phase again like the fundamental component. Thus, each harmonic obeying $n=p \times L+1(p=0,1,2, \cdots)$ is added constructively, but the circuit suppresses other harmonics. The constructive harmonics are the 1st, 4th, 7th, etc.

This can be generalized to any number of paths. For instance, non-canceled harmonics of a 4-path circuit are 1st, 5th, 9th, etc [113]. We can deduce here that as a number of paths increases, more distortion products can be treated. Theoretically, to handle all distortion products, we need an infinite number of paths, but in practice, this is impossible for many reasons such as design complexity, cost, mismatches between paths, and weakness of higher order distortion products. Furthermore, because the proposed system has antennas in its underlying structure, limited bandwidths of antennas will not allow us to treat all harmonics, which is another reason why we cannot treat all distortion products generated by our presented 
system.

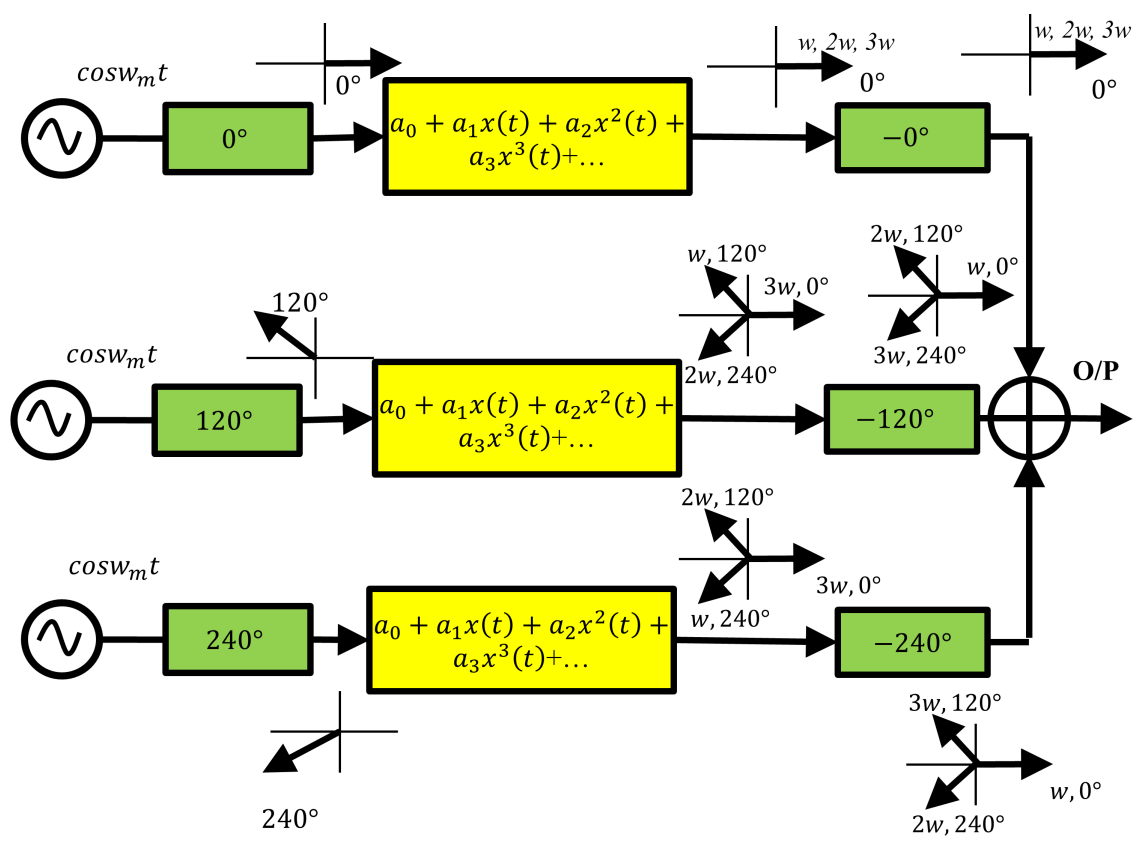

(a)

\section{$\because$ PHASE}

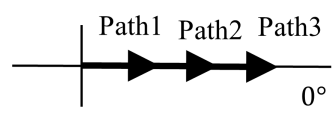

Fundmental, w

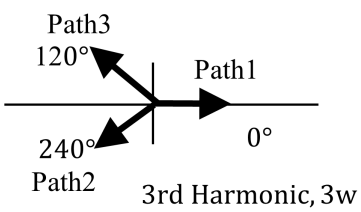

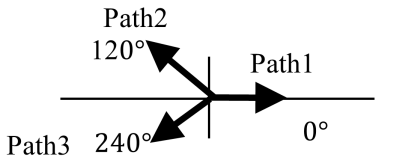

2nd Harmonic, 2w

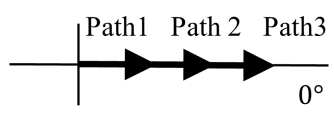

4th Harmonic, 4w

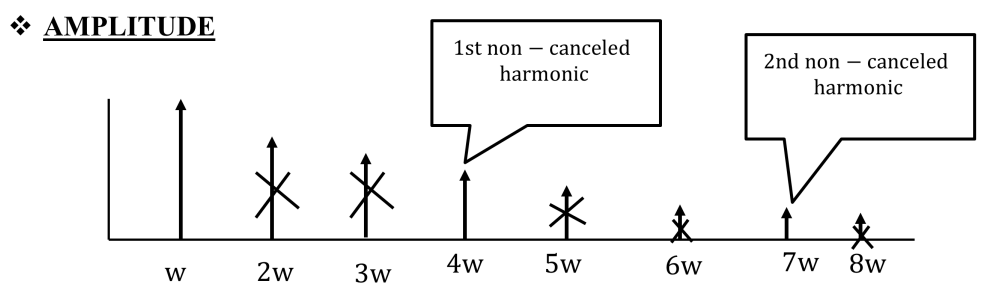

(b)

Figure 3.11: a) Three-path polyphase circuit b) its phasor and magnitude plots 


\subsubsection{The Proposed System Model under the Conventional Polyphase Multipath Technique}

In this section, we will continue in deriving and development a mathematical model used to study, analyze, and control distortion products generated by the PMSA. The derivation aims to focus on finding a mathematical approach for determining presence and absence of distortion products at an output of the PMSA under the ideal conditions (i.e., no mismatches between paths). The process of derivation highly relies on the preceding section. However, circuits in the previous part have a single signal source in every path, but the PMSA has two different signal sources which are the modulation and incident signals as illustrated above. Second phase shifters at end of each path in Figure 3.9 are replaced by mixers, which are one part of equivalent representations of RF-RTS switches, and spatial phase shifters. Thus, a model of a single scatterer presented in Figure 3.8 is used to form a polyphase multipath model of the PMSA, see Figure 3.12. Both single and two tones modulation sources are considered in this section to pave the way for multi-tone modulation sources based the PMSA. The usefulness of this approach lies within its strength to investigate and analyze harmonics and IMDs generated in the PMSA. A mechanism of distortion products generated in scatterers and conventional RF circuits such as power amplifiers, mixers, and low noise amplifiers (LNA) is similar. Most antenna arrays contain $\mathrm{RF}$ circuits, and these RF circuits are integrated into each antenna element in the array [146]. Thus, our mathematical model can be considered as a powerful tool to figure out spectral outputs of multi-user active phased arrays and massive MIMO systems, as will be described later.

Moreover, a polyphase multipath circuit deals with specific phase shifts for the first and second phase shifters that should be equal and opposite at the desired 
distortion product. This feature makes the polyphase multipath analysis method is not harmonious with requirements of the PMSA. If an angle of the incident signals varies, values of the second (spatial)phase shifters in the PMSA are deviated from their prescribed values, leading to failure of the adopted model to predict canceled and non-canceled distortion products. However, it is a good start point to develop a robust model satisfying requirements of the PMSA features as will be seen later. 


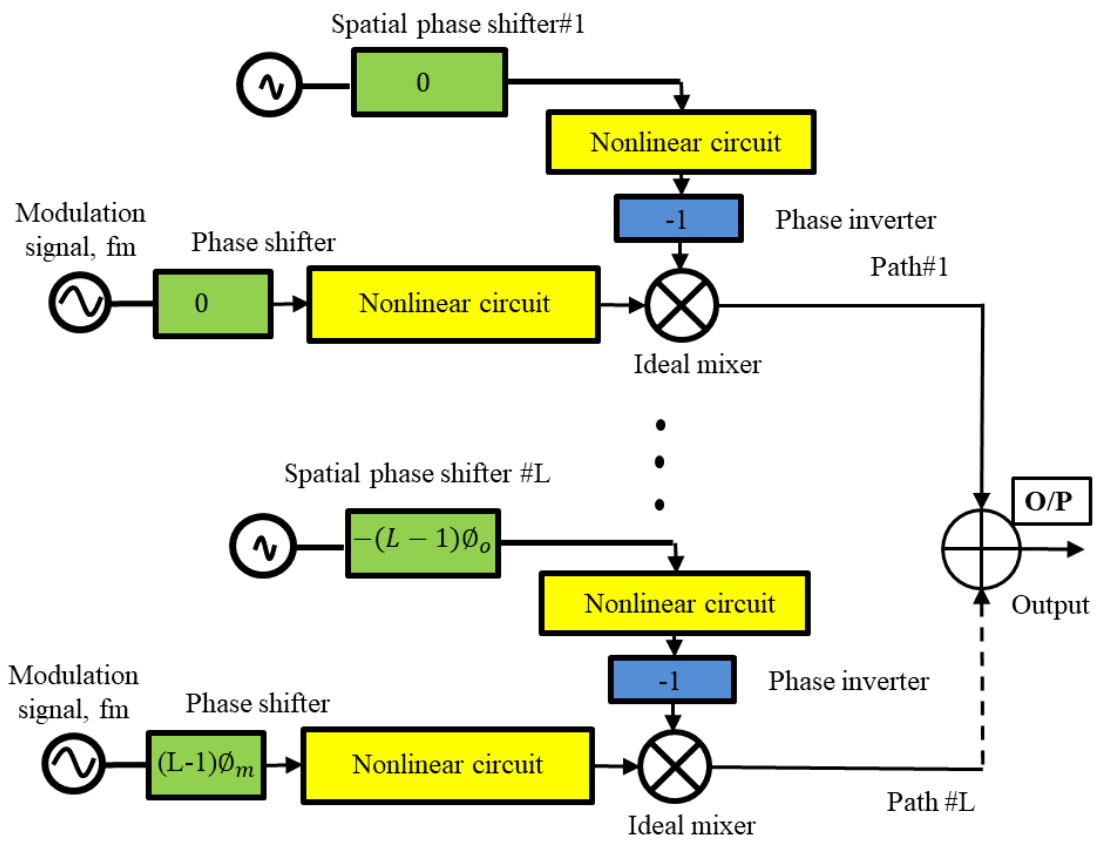

(a)

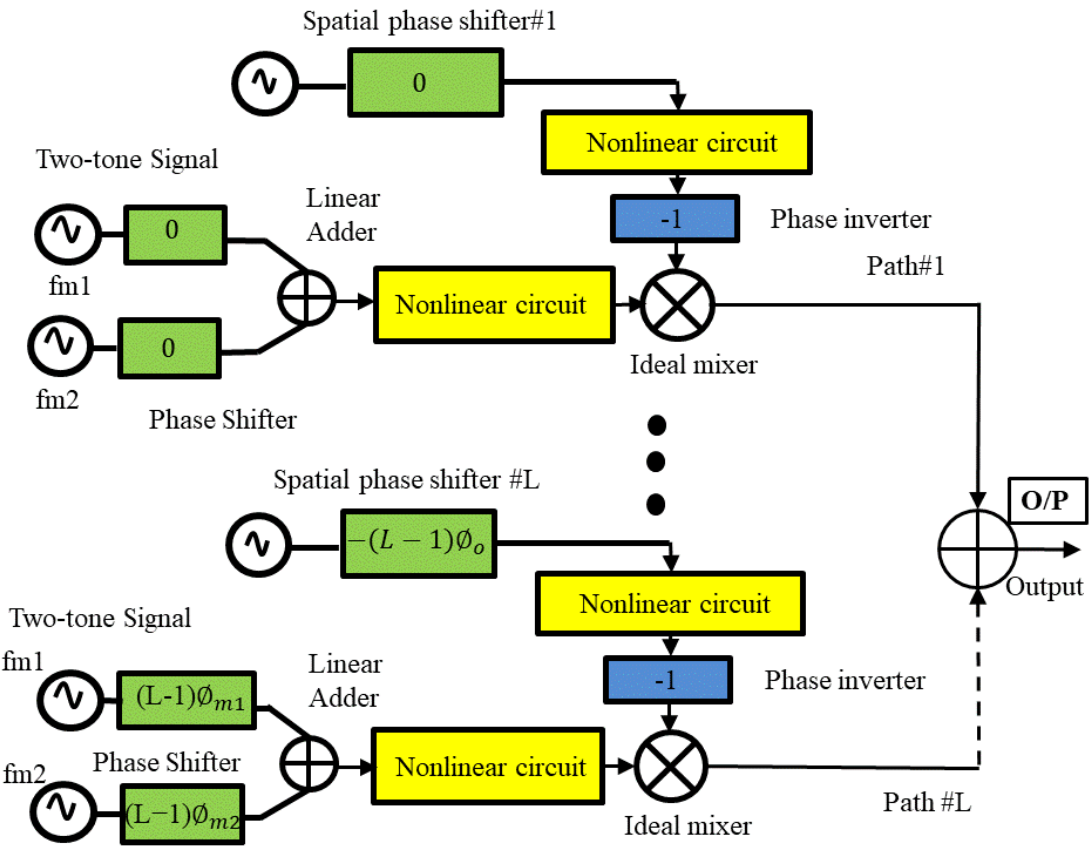

(b)

Figure 3.12: Polyphase multipath model of PMSA when using a) single-tone modulation sources b) two-tone modulation sources

Consider an array of scatterers replaced by their equivalent models as shown in Figure 3.12. Depending on a reference element which is either the most right or the 
most left element in the PMSA structure, directions of phase shift distributions of the modulation sources and incident signals induced between array elements (model paths) are used to classify types of the PSMA because directions of phase shift distributions not only control spatial locations of distortion products but also play a vital role in determining directions of negative and positive distortion products in space. Although this separation between negative and positive distortion products is similar to what happens in diffraction grating systems, our PMSA is able to dynamically change locations of distortion products and directions of negative and positive distortion products. Consequently, beamforming functionality of distortion products is also another feature of the PMSA as will be illustrated in Chapters 4 and 5.

Figure 3.13 displays all the possible types of the PMSA. In this regard, we name them as type A, B, C, and D. There are also other two secondary types when we all spatial sources are in-phase. The secondary types can be called as a type $\mathrm{E}$ and $\mathrm{F}$, and they will be more elaborated in the next section. In type $\mathrm{E}$, positive distortion products are distributed from $-90^{\circ}$ to $0^{\circ}$ in space, while negative distortion products are distributed from $0^{\circ}$ to $90^{\circ}$. These distortion products replace their locations in type F. Besides, other types follow the same process, but some of their negative distortion products are partially distributed in locations of positive distortion products or vice versa.

In each path, two outputs of nonlinear circuits are inserted into mixer inputs to produce sums and differences of original signals and their distortion products. All paths in a model circuit perform the same process knowing that they have different signal sources with same frequencies and different phases. Output signals resulting from each path are added at the last part in the model, being a summation circuit. However, distortion products of our PMSA are added in space, as in phased antenna 
arrays [34]. Different spatial locations in space represent different fictitious summation outputs of the PMSA, leading to a circuit with multiple outputs and each output has its particular characteristics of distortion products. This opposes the same model when used in RF communication circuits due to having one output. This feature is of significant interest in our ongoing research, being a polyphase multipath circuit with multiple-output. Figure 3.14 compares between the polyphase multipath technique used in RF-frontend designs and our PMSA.

Mathematically, summation of distortion products at outputs of all paths for type A can be expressed as

: for single-tone modulation sources

$$
\begin{aligned}
F(t) & =\sum_{l=1}^{L}\left[\sum_{h=0}^{\infty} b_{h} \cos \left(h\left(w_{o} t+(l-1) \emptyset_{o}\right)\right) \times \sum_{n=0}^{\infty} b_{n} \cos \left(n\left(w_{m} t+(l-1) \emptyset_{m}\right)\right)\right] \\
& =B B \sum_{l=1}^{L}\left[\sum_{h=0}^{\infty} \sum_{n=-\infty}^{\infty}\left[\cos \left(\left(h w_{o}+n w_{m}\right) t+h(l-1) \emptyset_{o}+n(l-1) \emptyset_{m}\right)\right]\right.
\end{aligned}
$$

where $B B=1 / 2 a_{h} b_{n}$, and for two-tone modulation sources is

$$
\begin{aligned}
F(t) & =\sum_{l=1}^{L}\left[\sum_{h=0}^{\infty} a_{h} \cos \left(h\left(w_{o} t+(l-1) \emptyset_{o}\right)\right) \times \sum_{k=-\infty}^{\infty} \sum_{m=-\infty}^{\infty} b_{k m} \cos \left(\left(k w_{1}+m w_{2}\right) t\right.\right. \\
& \left.\left.\left.+k(l-1)+\emptyset_{1}+m(l-1) \emptyset_{2}\right)\right)\right] \\
& =C C \sum_{l=1}^{L} \sum_{h=0}^{\infty} \sum_{k=-\infty}^{\infty} \sum_{m=-\infty}^{\infty} \cos \left(\left(h w_{o}+k w_{1}+m w_{2}\right) t\right. \\
& \left.+(l-1)\left(h \emptyset_{o}+k \emptyset_{1}+m \emptyset_{2}\right)\right)
\end{aligned}
$$

where $C C=1 / 2 a_{h} b_{k m}$. Now, from mathematical equations mentioned above, we can readily generalize the equations to include modulation signals with any number of tones, making the mathematical model of the PMSA compatible with analysis of distortion products created in multi-user large antenna arrays and MIMO systems. 


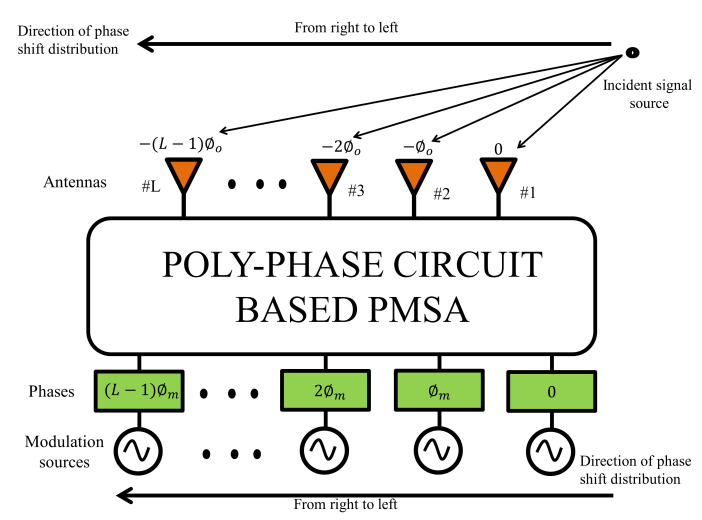

(a)

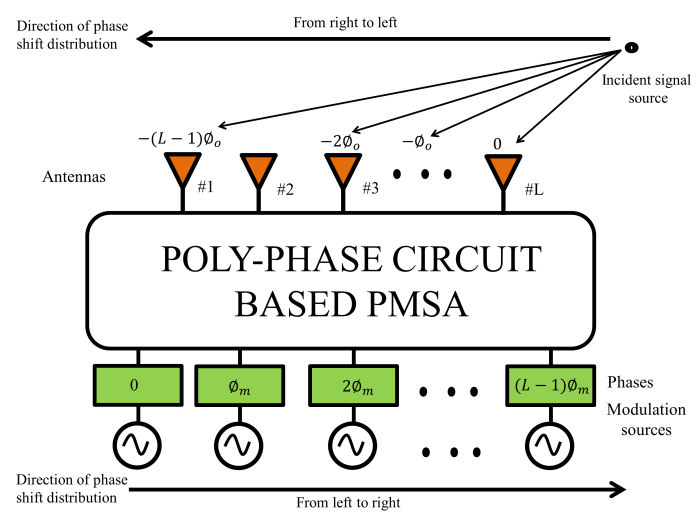

(c)

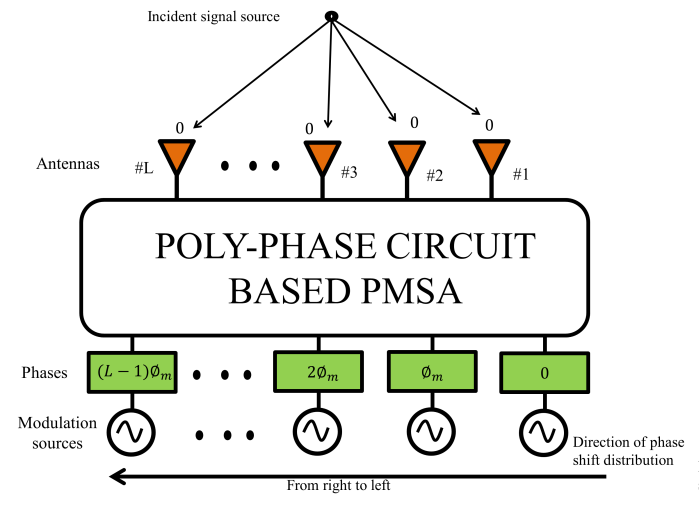

(e)

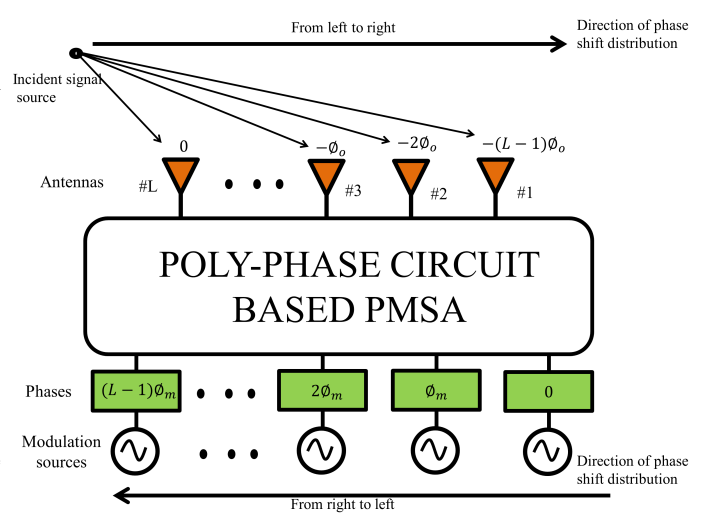

(b)

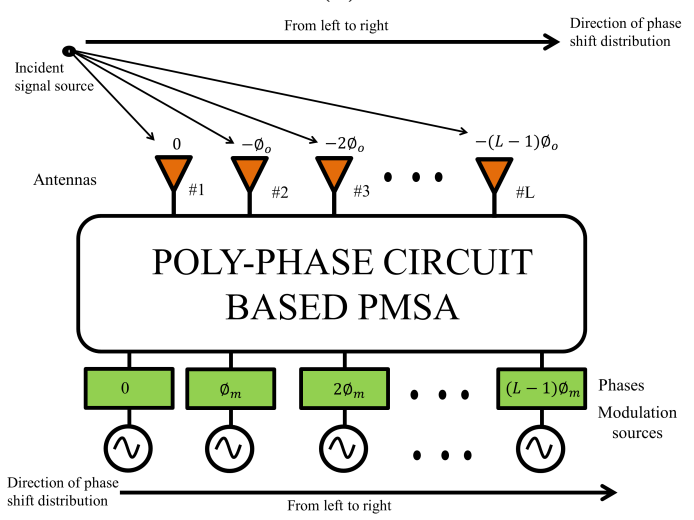

(d)

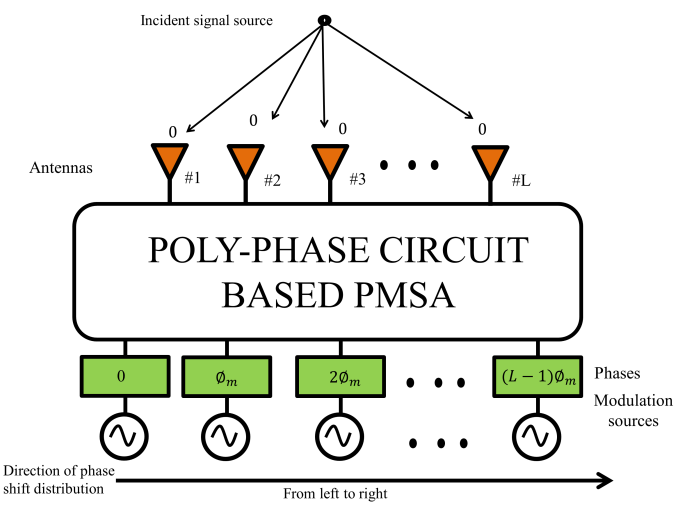

(f)

Figure 3.13: Types of the PMSA model a) A b) B c) C d) D e) E f) F

Thus, an output of the multi-tone PMSA is

$$
\begin{aligned}
F(t) & =D D \sum_{l=1}^{L} \sum_{h=0}^{\infty} \sum_{k=-\infty}^{\infty} \sum_{m=-\infty}^{\infty} \sum_{s=-\infty}^{\infty} \cdots \sum_{j=-\infty}^{\infty} \cos \left(\left(h w_{o}+k w_{1}+m w_{2}+s w_{3}\right.\right. \\
& \left.\left.+\cdots+j w_{x}\right) t+(l-1)\left(h \emptyset_{o}+k \emptyset_{1}+m \emptyset_{2}+s \emptyset_{3}+\cdots+j \emptyset_{x}\right)\right)
\end{aligned}
$$




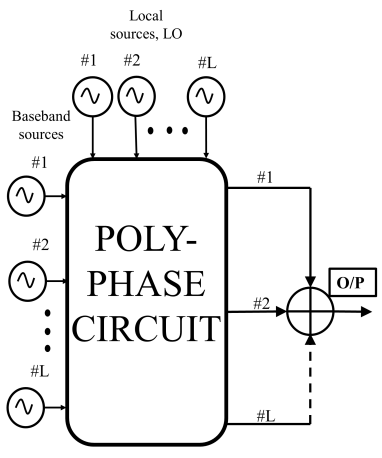

(a)

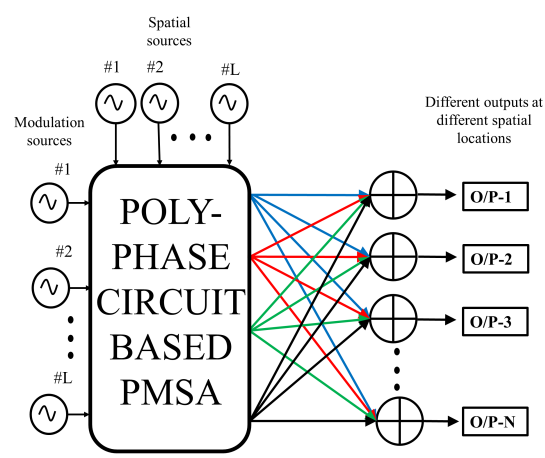

(b)

Figure 3.14: Block diagram of the polyphase multipath technique in a) communication circuits b) the PMSA

where $D D=1 / 2 a_{h} b_{k m s \cdots j}$.

One simplification that can be made before pursuing the derivation procedure is to neglect higher orders harmonics of the incident signal frequencies and leave only the fundamental component due to antennas in the PMSA structure being matched only at the main frequencies. We will take into account only distortion products of the modulation signals because their frequencies are much smaller than frequencies of the incident signals $w_{m}<<w_{o}$. Thus, Eqs.(3.36), (3.37) and (3.38) can be rewritten as

$$
\begin{gathered}
F(t)=B B \sum_{l=1}^{L}\left[\sum_{n=-\infty}^{\infty} \cos \left(\left(w_{o}+n w_{m}\right) t+(l-1) \emptyset_{o}+n(l-1) \emptyset_{m}\right)\right] \\
F(t)=C C \sum_{l=1}^{L}\left[\sum_{k=-\infty}^{\infty} \sum_{m=-\infty}^{\infty} \cos \left(\left(w_{o}+k w_{1}+m w_{2}\right) t+(l-1)\left(\emptyset_{o}+k \emptyset_{1}+m \emptyset_{2}\right)\right)\right] \\
F(t)=D D \sum_{l=1}^{L} \sum_{k=-\infty}^{\infty} \sum_{m=-\infty}^{\infty} \sum_{s=-\infty}^{\infty} \cdots \sum_{j=-\infty}^{\infty} \cos \left(\left(w_{o}+k w_{1}+m w_{2}+s w_{3}\right.\right. \\
\left.\left.+\cdots+j w_{x}\right) t+(l-1)\left(\emptyset_{o}+k \emptyset_{1}+m \emptyset_{2}+s \emptyset_{3}+\cdots+j \emptyset_{x}\right)\right)
\end{gathered}
$$

Distortion products of the modulation sources are evenly distributed about the 
incident signal frequency, creating double-sided signals. Distortion products have new frequencies and different phase shifts so that these properties enable us to control distortion products independently.

Typically, signs of the second phase shifters in the polyphase multipath circuit are negative (retarded phases) to align back phases of the first upper sideband (1USB) in all paths to zero, but signs of the second phase shifters in the PMSA are positive (advanced phases) because phases of the incident signals are inverted inside the RTSs [101] [28]. Due to the process of phase inversion, this makes enhancement of the first lower sideband (1LSB) instead of the 1USB. As a consequence, we add a term (-1) between the spatial sources and mixers in all paths.

The cosine models expressed in (3.39), (3.40), and (3.41), are only applicable to the PMSA when values of first and second phase shifters are equidistant $\left|360^{\circ} / L\right|$. If it is not so, we need to modify the model by adding some extra terms, as will be described in the next section. For the single-tone modulation sources, phases between scatterers are chosen as $\left(\emptyset_{m}=\emptyset_{o}=l \times \frac{360^{\circ}}{L}\right)$

$$
\emptyset_{m}=\left[0 \times \frac{360^{\circ}}{L}, 1 \times \frac{360^{\circ}}{L}, 2 \times \frac{360^{\circ}}{L}, \ldots,(L-1) \times \frac{360^{\circ}}{L}\right]
$$

Then, phases after the second phase shifters are

$$
\emptyset_{l, n}=(n+1) \emptyset_{m}
$$

or

$$
\emptyset_{l, n}=\left(\frac{n+1}{L}\right)\left[0 \times 360^{\circ}, 1 \times 360^{\circ}, 2 \times 360^{\circ}, \ldots,(L-1) \times 360^{\circ}\right]
$$

where $\emptyset_{l, n}$ is phase shift of the $n-t h$ distortion component at an end of the $l-t h$ path. Eq.(3.44) indicates that whenever the $(n+1)$ term is equal to a multiple of $L$ 
(i.e., $p L, p=0, \pm 1, \pm 2, \cdots)$, the phase shift in every path will be equal to a multiple of $360^{\circ}$, resulting in a constructive addition. This can be expressed as

$$
n=p \times L-1
$$

The term -1 in (3.45) represents the 1LSB distortion product being enhanced when $p=0$. If we replace $(-1)$ by the term $g$, this generalizes (3.45), making the equation applicable for enhancement of any distortion product at the normal direction in space. So, it becomes

$$
n=p \times L-g
$$

Also, Eq.(3.44) becomes

$$
\emptyset_{l, n}=\left(\frac{n+g}{L}\right)\left[0 \times 360^{\circ}, 1 \times 360^{\circ}, 2 \times 360^{\circ}, \ldots,(L-1) \times 360^{\circ}\right]
$$

The difference between (3.31) and (3.46) stems from that phases of the incident signals representing the second phase shifters in the PMSA are inverted due to the re-transmission (scattering) process, while in conventional RF frontends, phases are not subject to these effects. We can recognize that when seeing the sign before the term $g$. Again, when distortion products are $n \neq p \times L-g$, phase shifts of all paths are equidistantly distributed over $360^{\circ}$ causing the cancellation of those distortion products. Therefore, for a circuit with $L$ paths, all distortion products are suppressed except the $(p \times L-g)-t h$ distortion products. The output of the type-A PMSA 
model can be expressed mathematically as

$$
\begin{aligned}
F(t) & =\cdots+B B_{-2} \sum_{l=1}^{L} \cos \left(\left(w_{o}-2 w_{m}\right) t+(g(l-1)-2(l-1)) \frac{360^{\circ}}{L}\right) \\
& +B B_{-1} \sum_{l=1}^{L} \cos \left(\left(w_{o}-w_{m}\right) t\right. \\
& \left.+(g(l-1)-(l-1)) \frac{360^{\circ}}{L}\right)+B B_{0} \sum_{l=1}^{L} \cos \left(\left(w_{o}\right) t+(g(l-1)) \frac{360^{\circ}}{L}\right) \\
& +B B_{1} \sum_{l=1}^{L} \cos \left(\left(w_{o}+w_{m}\right) t+(g(l-1)+(l-1)) \frac{360^{\circ}}{L}\right) \\
& +B B_{2} \sum_{l=1}^{L} \cos \left(\left(w_{o}+2 w_{m}\right) t+(g(l-1)+2(l-1)) \frac{360^{\circ}}{L}\right)+\cdots
\end{aligned}
$$

Eq.(3.48) has both canceled and non-canceled terms, and each term satisfying the condition in (3.49) will be canceled

$$
\left.B B_{n} \sum_{l=1}^{L} \cos \left(\left(w_{o}+n w_{m}\right) t+(n+g) \times(l-1)\right) \times \frac{360^{\circ}}{L}\right)=0 \text { for } n \neq p \times L-g
$$


Thus, residual terms can be expressed as

$$
\begin{aligned}
F(t) & =\cdots+B B_{-2 L-g} \sum_{l=1}^{L} \cos \left(\left(w_{o}+(-2 L-g) w_{m}\right) t+(g(l-1)+(-2 L-g)\right. \\
& \left.\times(l-1)) \frac{360^{o}}{L}\right) \\
& +B B_{-L-g} \sum_{l=1}^{L} \cos \left(\left(w_{o}+(-L-g) w_{m}\right) t+(g(l-1)+(-L-g)(l-1)) \frac{360^{\circ}}{L}\right) \\
& +B B_{-g} \sum_{l=1}^{L} \cos \left(\left(w_{o}+(-g) w_{m}\right) t+(g(l-1)+(-g)(l-1)) \frac{360^{\circ}}{L}\right) \\
& +B B_{L-g} \sum_{l=1}^{L} \cos \left(\left(w_{o}+(L-g) w_{m}\right) t+(g(l-1)+(L-g)(l-1)) \frac{360^{\circ}}{L}\right) \\
& +B B_{2 L-g} \sum_{l=1}^{L} \cos \left(\left(w_{o}+(2 L-g) w_{m}\right) t+(g(l-1)+(2 L-g)(l-1)) \frac{360^{\circ}}{L}\right) \\
& +\cdots
\end{aligned}
$$

or

$$
\begin{aligned}
F(t) & =\sum_{p=-\infty}^{\infty} B B_{p L-g} \sum_{l=1}^{L} \cos \left(\left(w_{o}+(p L-g) w_{m}\right) t\right. \\
& \left.+(g(l-1)+(p L-g)(l-1)) \frac{360^{\circ}}{L}\right)
\end{aligned}
$$

Eq.(3.51) represents the mathematical model of distortion products for the type-A PMSA circuit model. In addition, it has only the non-suppressed terms and is only valid if both phase shifts of the modulation and spatial sources are chosen equidistantly (i.e., $360^{\circ} / L$ ). However, if anyone of these phases of either the modulation sources or spatial sources are not equidistant, the adopted model based on the conventional polyphase multipath technique fails to predict where desired distortion products are placed in space.

The same procedure can be repeated with the other types of the PMSA, but here 
we will write only their final formulas with the help of Eq.(3.51). The mathematical model is

for type-B PMSA circuit model

$$
\begin{aligned}
F(t) & =\sum_{p=-\infty}^{\infty} B B_{p L+g} \sum_{l=1}^{L} \cos \left(\left(w_{o}+(p L+g) w_{m}\right) t\right. \\
& \left.+(g(L-l)+(p L+g)(l-1)) \frac{360^{\circ}}{L}\right)
\end{aligned}
$$

for type-C PMSA circuit model

$$
\begin{aligned}
F(t) & =\sum_{p=-\infty}^{\infty} B B_{p L+g} \sum_{l=1}^{L} \cos \left(\left(w_{o}+(p L+g) w_{m}\right) t\right. \\
& \left.+(g(l-1)+(p L+g)(l-1)) \frac{360^{\circ}}{L}\right)
\end{aligned}
$$

and for type-D PMSA circuit model

$$
\begin{aligned}
F(t) & =\sum_{p=-\infty}^{\infty} B B_{p L-g} \sum_{l=1}^{L} \cos \left(\left(w_{o}+(p L-g) w_{m}\right) t\right. \\
& \left.+(g(L-l)+(p L-g)(l-1)) \frac{360^{\circ}}{L}\right)
\end{aligned}
$$

In equations representing the PMSA types given above, we considered only single-tone modulation sources. However, they can be extended to include sources with any number of tones as shown in the subsequent part.

\subsubsection{Handling of Intermodulation Distortion IMD Products of Multi-Tone Modulation Sources}

When the modulation sources used in the PMSA are multi-tone sources, other different frequencies will appear as a mix of frequencies of these tones called 
intermodulation distortion (IMD) products and will also distribute evenly about a frequency of the incident signals. Thus, output spectra of the PMSA not only has multiple integers of tones (harmonics: $k w_{1}, m w_{2}, s w_{3}, \cdots, j w_{x}$ ) but also has their cross products (IMD: $\pm k w_{1} \pm m w_{2} \pm s w_{3} \pm \cdots \pm j w_{x}$ ), where $k, m, s$, and $j$ can be positive and negative integers. The same way can be employed to handle IMDs (i.e., cancellation and enhancement). It can be shown that IMDs at $\pm k w_{1} \pm m w_{2} \pm s w_{3} \pm \cdots \pm j w_{x}$ will have phases of $\pm k \emptyset_{1} \pm m \emptyset_{2} \pm s \emptyset_{3} \pm \cdots \pm j \emptyset_{x}$ after nonlinear circuits and phases of $\pm k \emptyset_{1} \pm m \emptyset_{2} \pm s \emptyset_{3} \pm \cdots \pm j \emptyset_{x}+g \emptyset_{o}$ after mixing parts in the PMSA.

If all tones have the same phases, resulting phases are $( \pm k \pm m \pm s \pm \cdots \pm$ $j) \emptyset$ and $(k \pm m \pm s \pm \cdots \pm j) \emptyset+g \emptyset_{o}$ before and after mixing parts, respectively. The main difference between single-tone and multi-tone systems is that $n$ in (3.46) is replaced by $k+m+s+\cdots+j$. Therefore, if a system cancels the $n-t h$ distortion product, it is able to cancel $k+m+s+\cdots+j$ when $k+m+s+\cdots+j=n$. For instance, a system with two-tone modulation sources can produce an intermodulation product $k w_{1}+m w_{2}$. This intermodulation product can be canceled if $k+m=n$.

Analogous to the explanation in the previous section, only IMDs that cannot be suppressed are $k+m+s+\cdots+j=n=p \times L-g$. This means any IMD product with $k+m+s+\cdots+j=p \times L-g$ can never be suppressed. In other words, a two-tone system aims to enhance the 1LSB, it will never cancel IMDs with $k+m=-1$ unless the system cancels the harmonic product at $n=-1$. Unfortunately, this causes a big problem in RF communication systems especially the third order intermodulation products $-2 w_{1}+w_{2}$ and $w_{1}-2 w_{2}$. In our PMSA model, we can leverage from this to generate new signals with their own phase shift distributions across paths (scatterers). Outputs of the PMSA model for two-tone modulation sources with phase shifts which may or may not be equal can be expressed mathematically in a general form as 
for type-A PMSA circuit model

$$
F(t)=C C \sum_{l=1}^{L}\left[\sum_{k=-\infty}^{\infty} \sum_{m=-\infty}^{\infty} \cos \left(\left(w_{o}+k w_{1}+m w_{2}\right) t+(l-1)\left(\emptyset_{o}+k \emptyset_{1}+m \emptyset_{2}\right)\right)\right]
$$

for type-B PMSA circuit model

$F(t)=C C \sum_{l=1}^{L} \sum_{k=-\infty}^{\infty} \sum_{m=-\infty}^{\infty} \cos \left(\left(w_{o}+k w_{1}+m w_{2}\right) t+\left((L-l) \emptyset_{o}+(l-1)\left(k \emptyset_{1}+m \emptyset_{2}\right)\right)\right.$

for type-C PMSA circuit model

$F(t)=C C \sum_{l=1}^{L} \sum_{k=-\infty}^{\infty} \sum_{m=-\infty}^{\infty} \cos \left(\left(w_{o}+k w_{1}+m w_{2}\right) t+\left((l-1) \emptyset_{o}+(L-l)\left(k \emptyset_{1}+m \emptyset_{2}\right)\right.\right.$

and for type-D PMSA circuit model

$F(t)=C C \sum_{l=1}^{L} \sum_{k=-\infty}^{\infty} \sum_{m=-\infty}^{\infty} \cos \left(\left(w_{o}+k w_{1}+m w_{2}\right) t+\left((L-l) \emptyset_{o}+(L-l)\left(k \emptyset_{1}+m \emptyset_{2}\right)\right.\right.$

Outputs of the PMSA model for multi-tone modulation sources with phase shifts which may or may not be equal can be expressed mathematically in a general form as

for type-A PMSA circuit model

$$
\begin{aligned}
F(t)= & D D \sum_{l=1}^{L}\left[\sum _ { k = - \infty } ^ { \infty } \sum _ { m = - \infty } ^ { \infty } \sum _ { s = - \infty } ^ { \infty } \cdots \sum _ { j = - \infty } ^ { \infty } \operatorname { c o s } \left(\left(w_{o}+k w_{1}+m w_{2}+s w_{3}+\cdots+j w_{x}\right) t\right.\right. \\
& \left.\left.+(l-1)\left(\emptyset_{o}\right)+(l-1)\left(k \emptyset_{1}+m \emptyset_{2}+s \emptyset_{3}+\cdots+j \phi_{x}\right)\right)\right]
\end{aligned}
$$


for type-B PMSA circuit model

$$
\begin{aligned}
F(t)= & D D \sum_{l=1}^{L}\left[\sum _ { k = - \infty } ^ { \infty } \sum _ { m = - \infty } ^ { \infty } \sum _ { s = - \infty } ^ { \infty } \ldots \sum _ { j = - \infty } ^ { \infty } \operatorname { c o s } \left(\left(w_{o}+k w_{1}+m w_{2}+s w_{3}+\cdots\right.\right.\right. \\
& \left.\left.\left.+j w_{x}\right) t+(L-l)\left(\emptyset_{o}\right)+(l-1)\left(k \emptyset_{1}+m \emptyset_{2}+s \emptyset_{3}+\cdots+j \emptyset_{x}\right)\right)\right]
\end{aligned}
$$

for type-C PMSA circuit model

$$
\begin{aligned}
F(t) & =D D \sum_{l=1}^{L}\left[\sum _ { k = - \infty } ^ { \infty } \sum _ { m = - \infty } ^ { \infty } \sum _ { s = - \infty } ^ { \infty } \ldots \sum _ { j = - \infty } ^ { \infty } \operatorname { c o s } \left(\left(w_{o}+k w_{1}+m w_{2}+s w_{3}+\cdots\right.\right.\right. \\
& \left.\left.\left.+j w_{x}\right) t+(l-1)\left(\emptyset_{o}\right)+(L-l)\left(k \emptyset_{1}+m \emptyset_{2}+s \emptyset_{3}+\cdots+j \emptyset_{x}\right)\right)\right]
\end{aligned}
$$

and for type-D PMSA circuit model

$$
\begin{aligned}
F(t) & =D D \sum_{l=1}^{L}\left[\sum _ { k = - \infty } ^ { \infty } \sum _ { m = - \infty } ^ { \infty } \sum _ { s = - \infty } ^ { \infty } \ldots \sum _ { j = - \infty } ^ { \infty } \operatorname { c o s } \left(\left(w_{o}+k w_{1}+m w_{2}+s w_{3}+\cdots\right.\right.\right. \\
& \left.\left.\left.+j w_{x}\right) t+(L-l)\left(\emptyset_{o}\right)+(L-l)\left(k \emptyset_{1}+m \emptyset_{2}+s \emptyset_{3}+\cdots+j \emptyset_{x}\right)\right)\right]
\end{aligned}
$$

\subsection{Simulation Results}

This section provides an adequate amount of simulation results that support the mathematical model derived above for different numbers of paths and tones. Although the model is incapable of predicting spatial locations (i.e., angles of scattered distortion products) as well as its failure when phase shifts deviate from their prescribed values, its simulation results give readers the first impression about the system performance. Distortion products that show up at the model output are in-phase, so they are scattered normally according to phased antenna principles. This means that our model developed up to now is able to find only these distortion 
products (i.e., desired and unavoidable distortion products).

Figure 3.15-3.18 illustrate simulation results of the PMSA model when employing the conventional polyphase multi-path technique. The PMSA model is analyzed several times for different numbers of paths and circuit types (i.e., A, B, $\mathrm{C}$, and $\mathrm{D}$ ) using the single-tone modulation sources. In addition, the 1-path and the 8-path type $\mathrm{A}$ and $\mathrm{C}$ circuits are simulated using two and three tones modulation sources. The model in Figure 3.12 was implemented in Matlab assuming ideal phase shifters, identical signal sources, identical nonlinear circuits, and ideal mixers. Powers of final outputs of the chosen circuits are calculated using the fast Fourier transform (FFT) algorithm where the horizontal axis shows a frequency of distortion products and the vertical axis represents the scattered signal power in dBs. Although our proposed designs work at two different frequencies $432 \mathrm{MHz}$ and $2.3 \mathrm{GHz}$, the simulated results are achieved at a frequency range of $2-22 \mathrm{kHz}$ because both design frequencies are down-converted to this range of frequencies. In addition, dynamic ranges of simulated results are chosen from $-60 \mathrm{~dB}$ to $0 \mathrm{~dB}$ in order to match the measured results as will be seen in Chapter 8 .

Figures demonstrate that more distortion products can be controlled when a circuit employed to represent the PMSA model has more paths. For $L$-path circuits, the $(n=p \times L-g)-t h$ distortion products are not suppressed for $\mathrm{A}$ and $\mathrm{C}$ types and the $(n=p \times L+g)-t h$ distortion products are not canceled for $\mathrm{B}$ and D types. For instance, if the model circuit has $L=4$, the circuit output has the $(\cdots,-5 t h,-1 s t, 3 r d, 7 t h, \cdots)$ - th distortion products for $\mathrm{A}$ and $\mathrm{C}$ types while being the $(\cdots,-7 t h,-3 t h, 1 s t, 5 t h, \cdots)$ - th distortion products for $\mathrm{B}$ and D types (the negative signs stands for the distortion products appear at the lower sideband LSB). Distortion products that appear at outputs of circuits in all figures in this part represent the ones scattered at the angle zero (i.e., normal on the array 
axis,broadside) because these distortion products at ends of paths have the same phases which are equal to zero for the desired distortion product or integer multiples of $360^{\circ}$ for unavoidable distortion products. Also, incident angles selected here should satisfy phase shift distribution conditions of the conventional systems (i.e., $360^{\circ} / L$ ).

Communication circuits using the polyphase multi-path technique maintain desired distortion products and eliminate other distortion products permanently, but in the PMSA model distortion products will not be canceled forever and the PMSA directs them into different directions in space because distortion products have different phase shift distributions at array elements. Thus, our MPSAm considers as a polyphase multi-path multi-output circuit. However, the spatial diversity of proposed system cannot be demonstrated now due to the model insufficiency.

Figure 3.15 shows simulation results of single path circuits (i.e., single scatterer) when using modulation sources with different tones. Simulation results obtained from single path circuits are useful to compare with results of circuits consisting of more than one path. In Figure 3.16, simulation results of two-path circuits (2-element phased-modulated scattering array) are presented. When a relative phase shift difference between elements is $180^{\circ}$, all even and odd distortion products are suppressed as shown in Figure 3.16a and 3.16b, respectively. The figures show that both (3.31) and (3.46) can be applied since models are symmetrical, so they can be called even-balanced and odd-balanced systems, respectively. Types A and D support the odd-balanced model, while types B and C support the even-balanced model. However, if we replace the $180^{\circ}$ relative phase shift difference by $90^{\circ}$, we will mimic the performance of image-reject mixers, see Figure 3.16c and 3.16d. Also, we can obtain 1USB or 1LSB image-reject mixers relying on directions of phase distributions of sources (i.e., types A, B, C, and D). Image-reject models are special cases of two-path circuits because their phase distributions do not follow a rule of thumb 


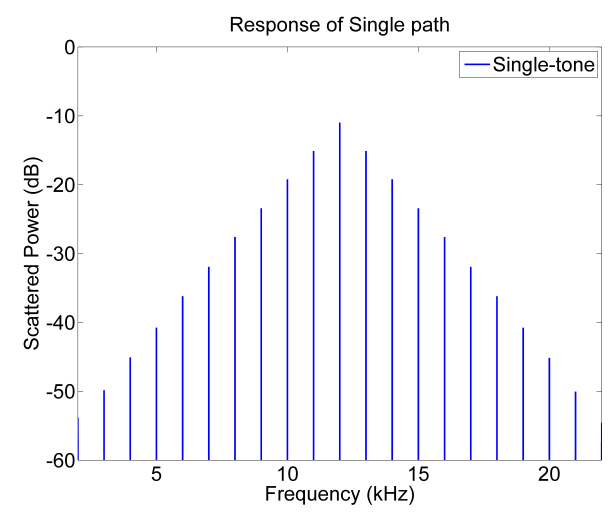

(a)

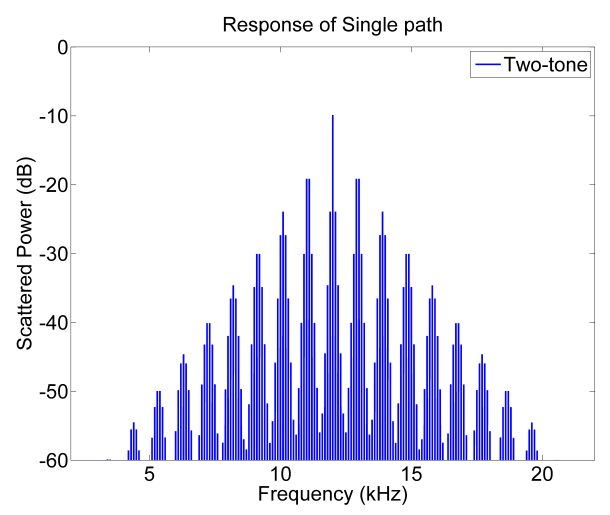

(b)

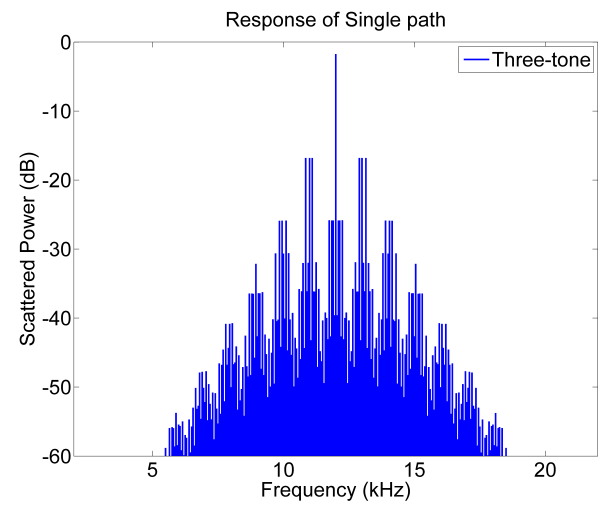

(c)

Figure 3.15: Simulated output spectra of signals scattered normally (broadside) for a single path PMSA model when using modulation sources with a) single-tone b) two-tone c) three-tone

which is $360^{\circ}$ divided by a number of paths $L$, so their distortion products at outputs are also not matched with (3.31) and (3.46). Figure 3.17 shows simulation results of 3, 4, 6, and 8 paths polyphase circuits when using single-tone modulation sources. Outputs represent all distortion products whose phases at ends of paths are zero or multiple integers of $360^{\circ}$. Figure 3.18 shows simulation results of 8-path polyphase circuits when using two and three tones modulation sources. Furthermore, some intermodulation distortion IMD products appear along with the desired harmonics since phases of these IMDs products are similar to phases of the desired harmonics. All PMSA models with more than two paths are simulated for all circuit types. Thus, as we can see in Figure 3.17, the 1LSB harmonic is enhanced in all plots along with 


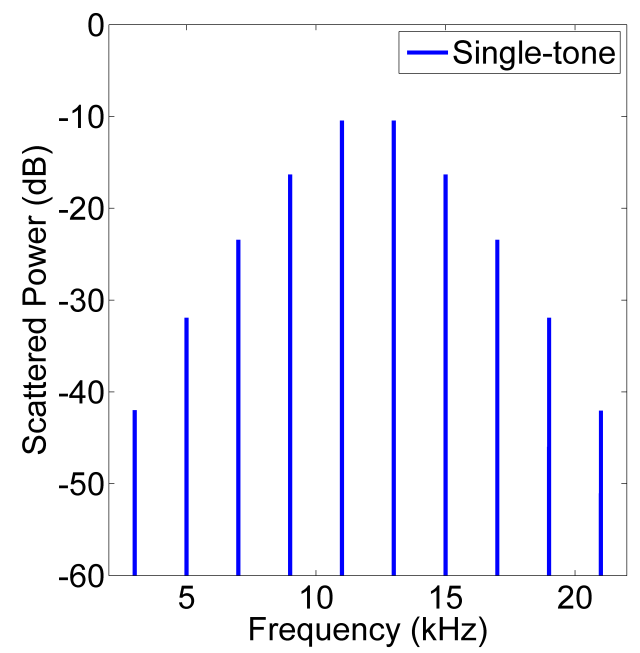

(a)

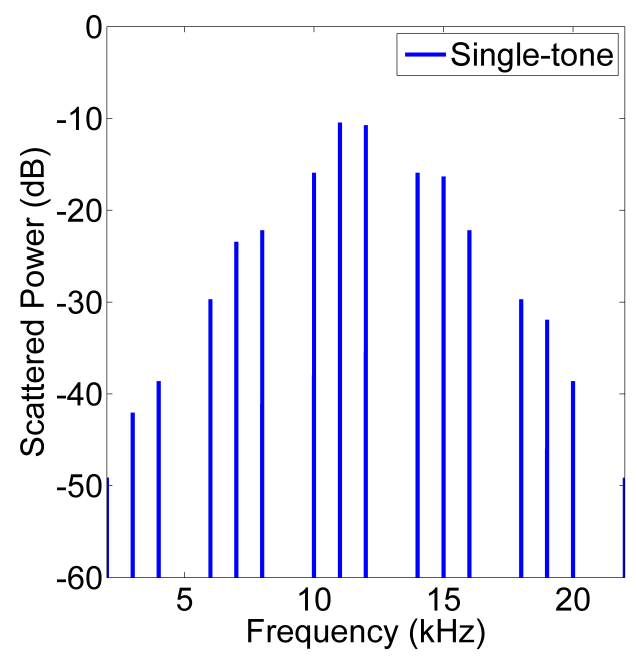

(c)

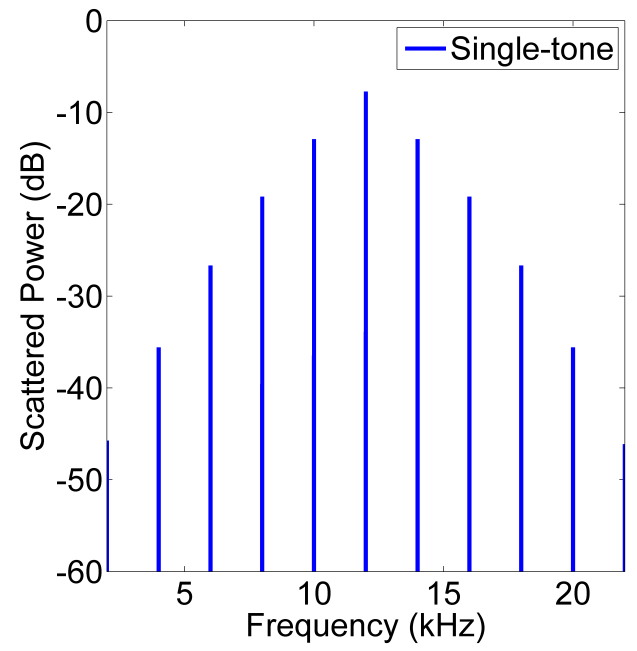

(b)

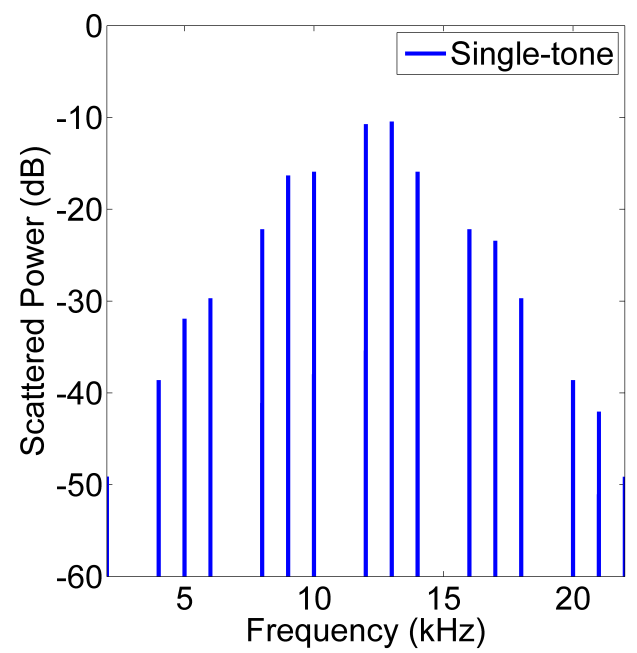

(d)

Figure 3.16: Simulated output spectra of signals scattered normally (broadside) for a two-path PMSA model when using single-tone modulation sources a) odd-Balanced Type b) even-Balanced Type c) USB image-reject mixer d) LSB image-reject mixer

non-canceled terms in A and C circuits types, whereas in type B and D,the 1USB harmonic is enhanced in all plots along with non-canceled terms.

To this end, we clarified that the proposed system works correctly if all its parts are appropriately chosen. However, crucial parts in the proposed system are phase shifters. In conventional fixed RF-front ends, phase shifters have fixed values to meet the design requirements. In contrast, in the PMSA, some factors governing 


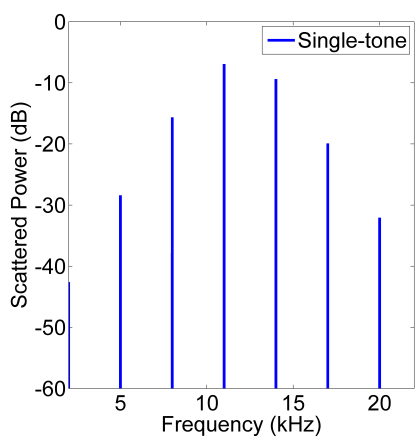

(a)

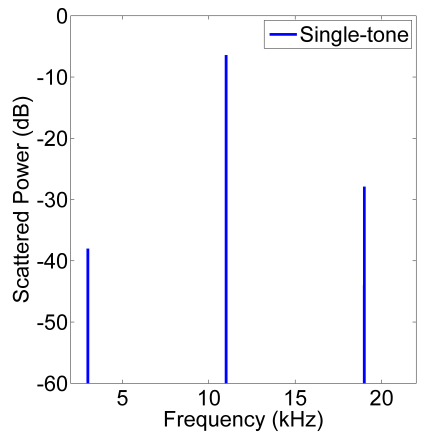

(d)

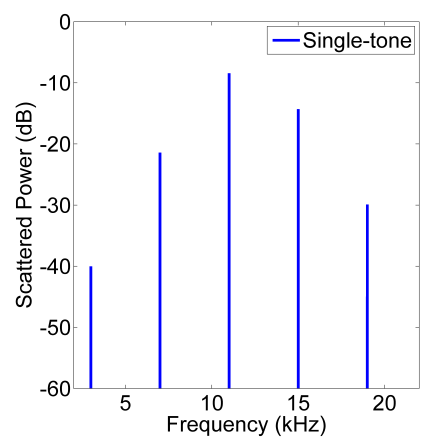

(b)

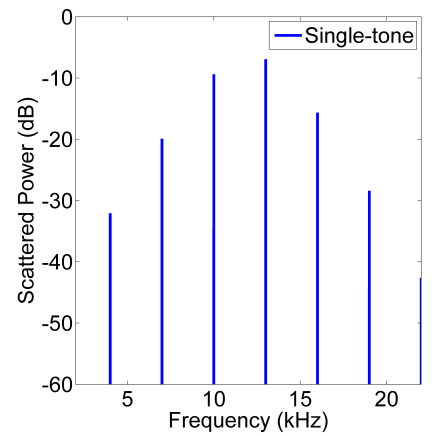

(e)

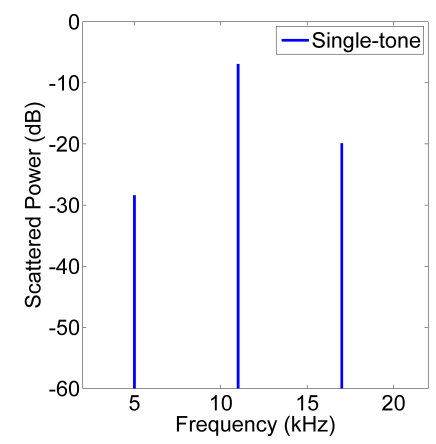

(c)

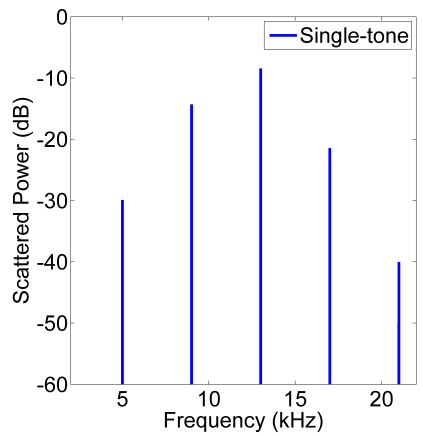

(f)

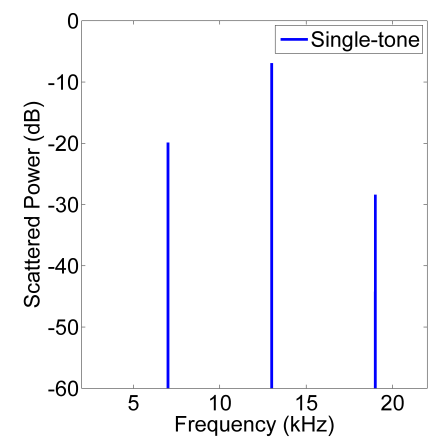

(g)

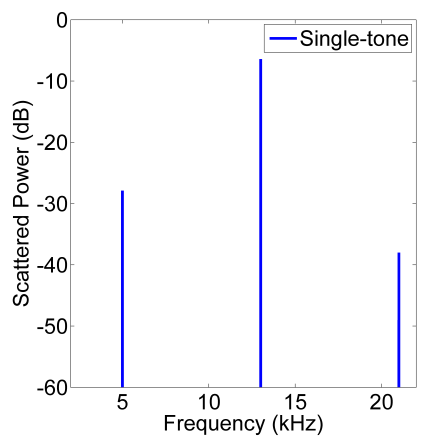

(h)

Figure 3.17: Simulated output spectra of signals scattered normally (broadside) when using single-tone modulation sources for a) 3-path PMSA for (A and C types) b) 4-path PMSA for (A and $\mathrm{C}$ types) c) 6-path PMSA for (A and $\mathrm{C}$ types) d) 8-path for (A and $\mathrm{C}$ types) e) 3-path PMSA for (B and D types) f) 4-path PMSA for (B and D types) g) 6-path PMSA for (B and D types) h) 8-path PMSA for (B and D types)

phase shifters such distances separating elements and angles of the incident signals are possibly changed during the operation. Thus, any deviations from their assigned values intentionally or unintentionally causes problems in the overall performance of the PMSA model based on the conventional polyphase multi-path technique. To 


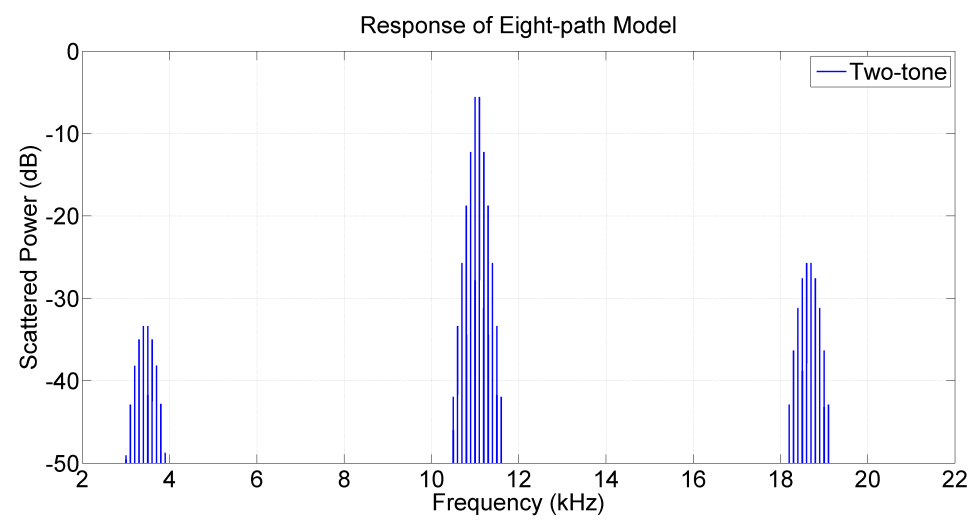

(a)

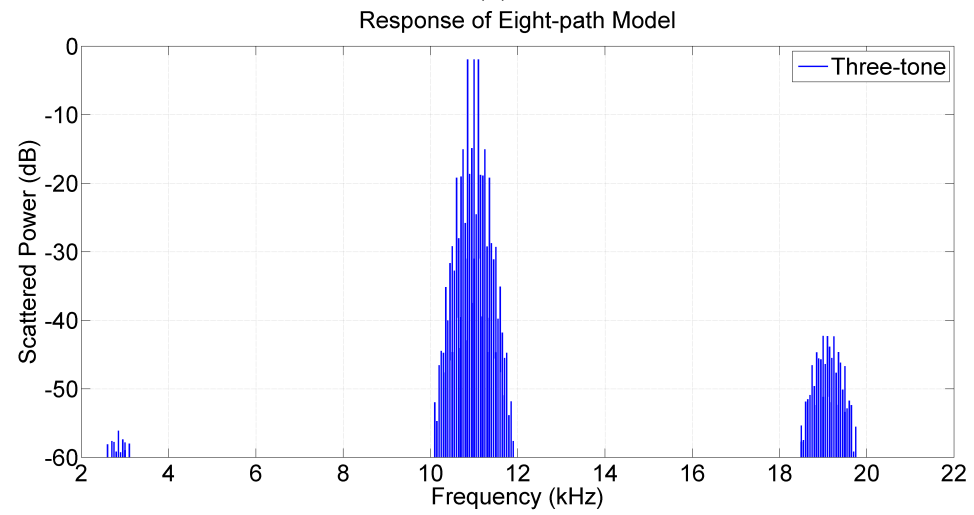

(b)

Figure 3.18: Simulated output spectra of signals scattered normally (broadside) for an 8-path PMSA model (types A and C) when using modulation sources with a) two-tone b) three-tone

demonstrate these effects, values of the second phase shifters have been changed in somehow (i.e., changing angles of the incident signals or distances between elements) from $90^{\circ}$ to $60^{\circ}$ and from $45^{\circ}$ to $30^{\circ}$ for 4 -path and 8-path circuits, respectively. Simulation results associated with the new phase shifts are illustrated in Figure 3.19. Outputs are meaningless. This meaningless is true in communication systems, but in our proposed, this will be considered as one of the proposed design advantages. As a consequence, distortion products will be redirected into different positions in space because the resulting phase shifts are different this time. Hence, to circumvent this model deficiency and to be adaptable with new circumstances, we will develop a model being able to work with any combination of phase shifts. 


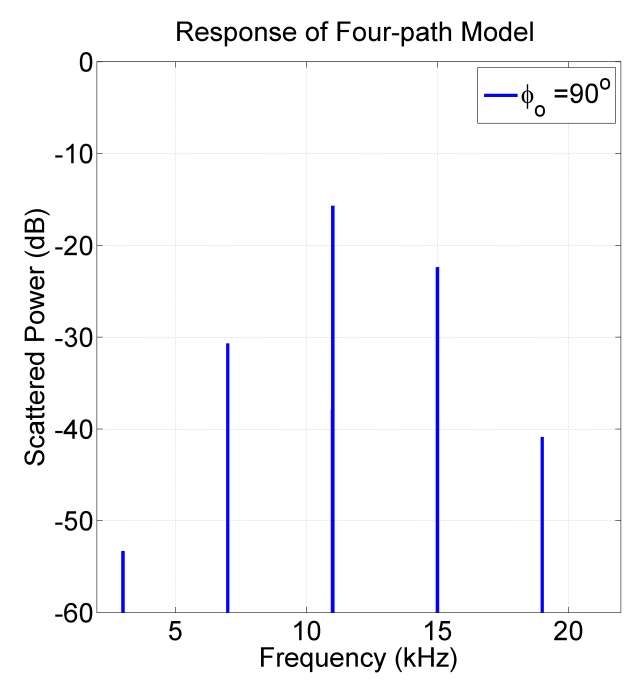

(a)

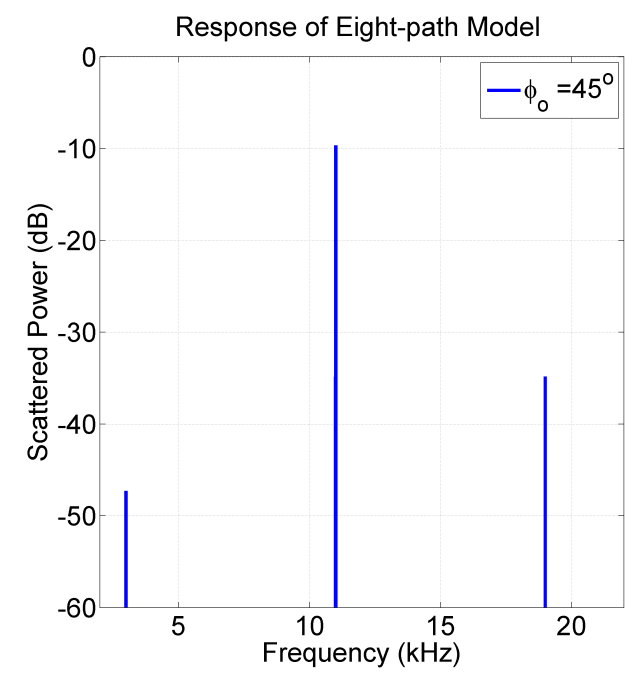

(c)

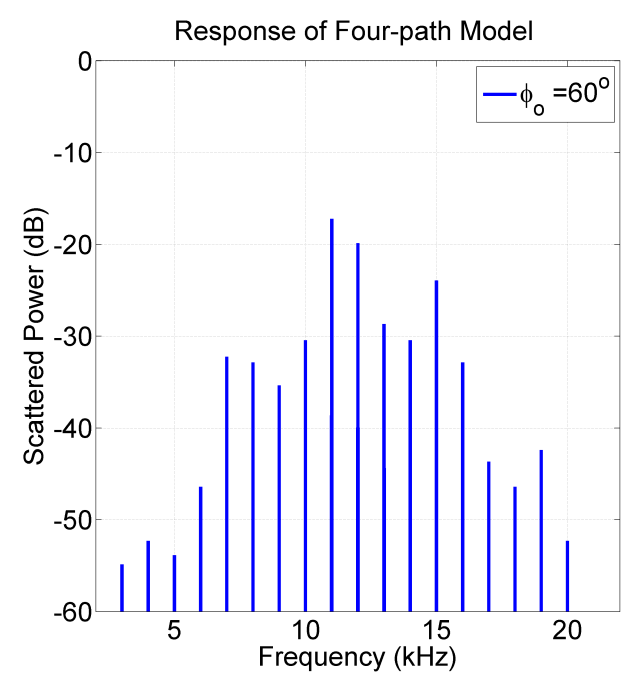

(b)

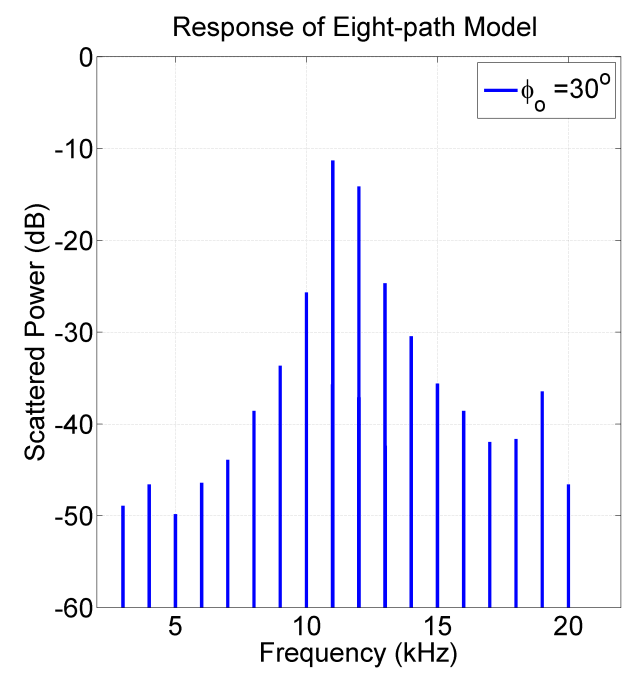

(d)

Figure 3.19: Simulated output spectra of signals scattered normally (broadside) when using prescribed or non-prescribed values of the second phase shifters a) 4-Path $90^{\circ}$ (prescribed) b) 4-Path $60^{\circ}$ (non-prescribed) c) 8-path $45^{\circ}$ (prescribed) d) 8-path $30^{\circ}$ (non-prescribed)

\subsection{The PMSA Model under the Modified Polyphase Multipath Technique (Realistic-Model)}

In this section, the limitations of using the conventional polyphase multipath technique encountered above are addressed here as a step toward a complete model being able to predict how and where the PMSA enhances and suppresses different 
distortion products at different angular locations in space. As we said, issues that will be taken into account are

1. Making the PMSA model working with any phase shift distribution for both the modulation and spatial sources (antennas) regardless of their values

2. Defining exact spatial outputs (i.e., angular locations) of distortion components Moreover, to make the model more realistic, we will add some other terms such as

1. Including impacts of real scatterers elements (patterns of non-isotropic elements) on the overall performance of the PMSA

2. Adding static responses and $\mathrm{Tx} / \mathrm{Rx}$ coupling of an experimental setup (load-independent term) as mentioned in Chapter 2.

The first issue in its both parts is addressed using extra terms of compensated phases added at ends of model paths. The purpose of extra terms is to align back deviations in resulting phase shifts as well as to define spatial outputs (angular locations) of distortion products. The extra terms are hypothetical too (i.e., not real components) like first and second phase shifters. Fortunately, the two parts are strongly connected to each other because any change in resulting phase shifts of distortion products before the extra terms results in a change in angular locations of spatial outputs of distortion products. In other words, deviations of phase shifts from their prescribed values cross model paths (scattering array elements) lead to having a superposition of signals having approximately the same amplitudes and different phase shifts. This phenomenon is similar to the process of radiation beamforming in reflect-arrays [12] [132], so corrected phase values of extra terms must be determined and applied to each path (scatterer element).

To find out values of the corrected phase shifts and to determine spatial outputs of the 
PMSA for different distortion products at their specific spatial directions, terms that will be added should contain explicit information about both of these two demands. These terms can be defined as

$$
(l-1) \emptyset_{c o}=(l-1) K d \sin \theta_{r}=-(l-1)\left(\emptyset_{o}+n \emptyset_{m}\right)
$$

where $\emptyset_{c o}$ denotes a value of the corrected phase shift that must be hypothetically added at each path end to correct and align back phases of distortion products, and $\theta_{r}$ is the spatial outputs (angular locations) of different scattered distortion products. The term $\emptyset_{c o}$ is governed by $\emptyset_{o}$ and $n \emptyset_{m}$. Additionally, as can be seen, when high order distortion products $(|n|>1)$ are considered, added terms also help to define their scattered angular locations in space. The second issue that also should be considered is effects of elements patterns on the model performance since scatterers are assumed as isotropic elements (scattering power equally in all directions) in the PMSA model thus far. All high gain antennas are non-isotropic (directive), and they are preferred over isotropic antennas. Thus, we will face a problem that an antenna field of view $(\mathrm{AFoV})$ does not support the whole scattering array field of view (SFoV). AFoV is less than $180^{\circ}$ and in most times it does not exceed $90^{\circ}$, while $\mathrm{SFoV}$ is $180^{\circ}$. In this research study, we will consider scattering arrays consisting of both quasi-isotropic and non-isotropic elements. Mathematically, this issue can be addressed by multiplying the mathematical model of the PMSA derived so far by an antenna element pattern as

$$
F\left(t, \emptyset_{o, m, c o}, \theta\right)=F\left(t, \emptyset_{o, m, c o}\right) \times E P(\theta)
$$

where $\operatorname{EP}(\theta)$ is an antenna element pattern which is equal to one in the case of isotropic elements, leading to $F\left(t, \emptyset_{o, m, c o}, \theta\right)=F\left(t, \emptyset_{o, m, c o}\right)$. The last issue is non-modulated signals scattered by the PMSA structure itself and other obstacles 
around our design from one side, and the coupling between a receiver and transmitter from another side. These signals fall under the same category which is the static scattered signals [16] [169]. These signals as described before, are non-modulated, so they can be mitigated but not entirely removed. Mathematically speaking, their impacts can be added to our model as

$$
F_{s}\left(t, \emptyset_{o, m, c o}, \theta\right)=F\left(t, \emptyset_{o, m, c o}, \theta\right)+S(t)
$$

where $S(t)$ denotes the non-modulated scattering term and is represented by $G \cos \left(w_{o} t\right)$ where $G$ is constant.

After explaining all terms making the model more realistic, it becomes necessary to integrate these terms into the previous model before the modifications to be rewritten as:

for the single-tone type-A PMSA circuit model

$$
\begin{aligned}
F_{s}\left(t, \emptyset_{o, m, c o}, \theta\right) & =B B \sum_{l=1}^{L} \sum_{n=-\infty}^{\infty} \cos \left(\left(w_{o}+n w_{1}\right) t+(l-1)\left(\emptyset_{o}+n \emptyset_{m}+\emptyset_{c o}\right)\right) \times E P(\theta) \\
& +G \cos \left(w_{o} t\right)
\end{aligned}
$$

for the two-tone type-A PMSA circuit model

$$
\begin{aligned}
F_{s}\left(t, \emptyset_{o, m, c o}, \theta\right) & =C C \sum_{l=1}^{L} \sum_{k=-\infty}^{\infty} \sum_{m=-\infty}^{\infty} \cos \left(\left(w_{o}+k w_{1}+m w_{2}\right) t+(l-1)\left(\emptyset_{o}+k \emptyset_{m 1}\right.\right. \\
& \left.\left.+m \emptyset_{m 2}+\emptyset_{c o}\right)\right) \times E P(\theta)+G \cos \left(w_{o} t\right)
\end{aligned}
$$


for the multi-tone type-A PMSA circuit model

$$
\begin{aligned}
F_{s}\left(t, \emptyset_{o, m, c o}, \theta\right) & =D D \sum_{l=1}^{L} \sum_{k=-\infty}^{\infty} \sum_{m=-\infty}^{\infty} \sum_{s=-\infty}^{\infty} \cdots \sum_{j=-\infty}^{\infty} \cos \left(\left(w_{o}+k w_{1}+m w_{2}\right.\right. \\
& \left.+s w_{3}+\cdots+j w_{x}\right) t+(l-1)\left(\emptyset_{o}+k \emptyset_{m 1}+m \emptyset_{m 2}+s \emptyset_{m 3}\right. \\
& \left.\left.+\cdots+j \emptyset_{m x}+\emptyset_{c o}\right)\right) \times E P(\theta)+G \cos \left(w_{o} t\right)
\end{aligned}
$$

The same terms can be added to all other types B, C, and D to obtain their realistic

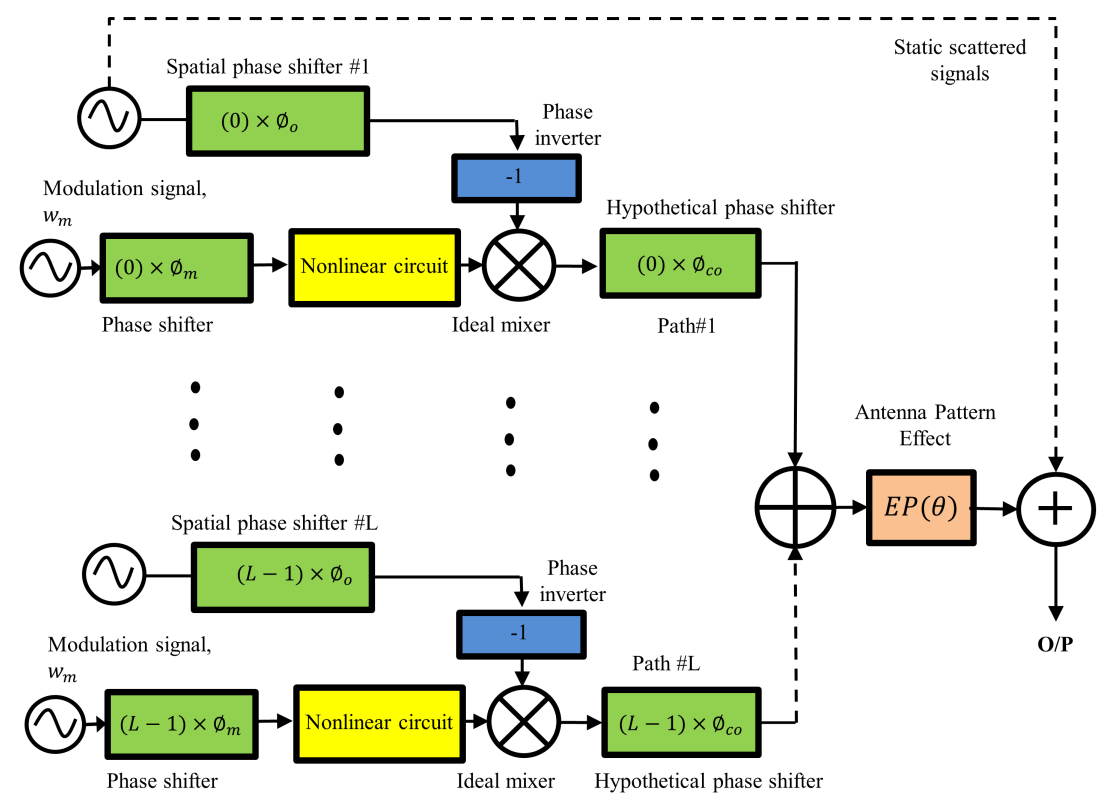

Figure 3.20: Realisitc-modified polyphase multipath model of the proposed PMSA

mathematical models, but here we will not mention them for the sake of simplicity. The block diagram of the realistic modified model is depicted in Figure 3.20. It has single-tone modulation sources, but, in general, these modulation sources may have more than one tone at the same time.

When second (spatial) phase shifts become $0^{\circ}$ which means that incident signals normally arrive at the PMSA aperture, spatial outputs of negative and positive 
distortion products are enhanced and suppressed (i.e., located) symmetrically about the normal-axis, separating by a spatial output of the zero-order distortion product. In all circuits types, scattered signals of the zero order (non-modulated) distortion products always separate between scattered signals of negative and positive distortion products. In types $\mathrm{E}$ and $\mathrm{F}$ circuits, zero order distortion products are located at a center of the PMSA front of view (SFoV), but locations of negative and positive outputs of distortion products are placed at specific angular locations (spatial outputs) depending on a term number (i.e., $n$ ) and a circuit type (i.e., A-F). In type E, negative and positive distortion products are located on right and left of zero order distortion products, respectively, while being vice versa for the type F.

Essential goals attained in (3.66), (3.67), and (3.68) are ability to deal with any phase shifts combinations and are also able to detect and recognize angular directions of the spatial outputs of distortion products. It becomes, therefore, necessary to demonstrate the relationship between relative corrected phase shifts and angular directions of the spatial outputs of distortion products. Substitute (3.2) in (3.63), so angular locations of the spatial outputs associated with $\theta_{r}$ can be calculated as, for the single-tone type-A PMSA circuit model

$$
\theta_{r_{n}}=-\arcsin \left(\frac{\emptyset_{o}+n \emptyset_{m}}{K d}\right)
$$

for the two-tone type-A PMSA circuit model

$$
\theta_{r_{k+m}}=-\arcsin \left(\frac{\emptyset_{o}+k \emptyset_{m 1}+m \emptyset_{m 2}}{K d}\right)
$$

for the multi-tone type-A PMSA circuit model

$$
\theta_{r_{k+m+s \ldots+j}}=-\arcsin \left(\frac{\emptyset_{o}+k \emptyset_{m 1}+m \emptyset_{m 2}+s \emptyset_{m 3}+\cdots+j \emptyset_{m x}}{K d}\right)
$$


Figure 3.21a shows relative phase shift differences of the second phase shifters vs. the incident angles for different values of $d$, while Figure 3.21b shows relative phase shift differences of the second phase shifters vs. $d$ for different values of the incident angles. Figure 3.21c and 3.21d show a three dimensional plot of relative phase shift differences of the second phase shifters and its top view.

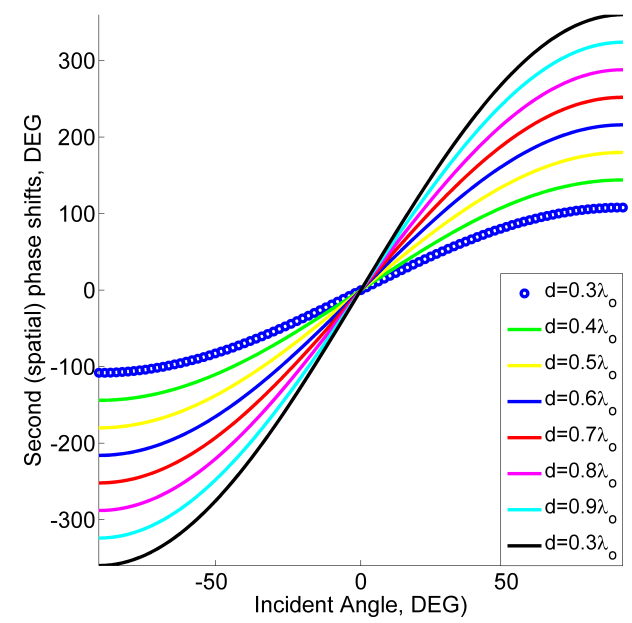

(a)

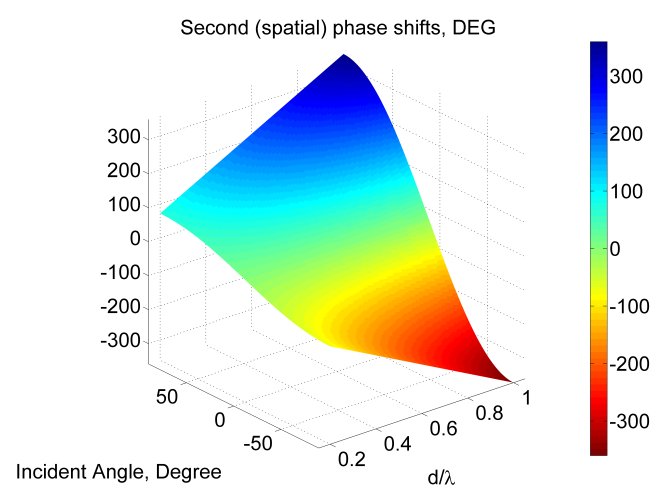

(c)

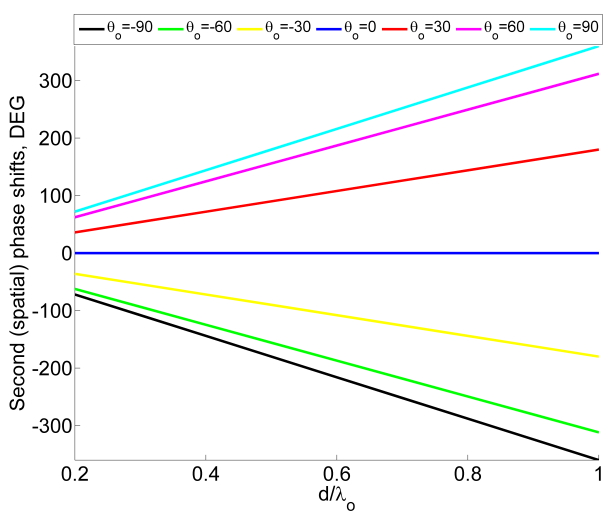

(b)

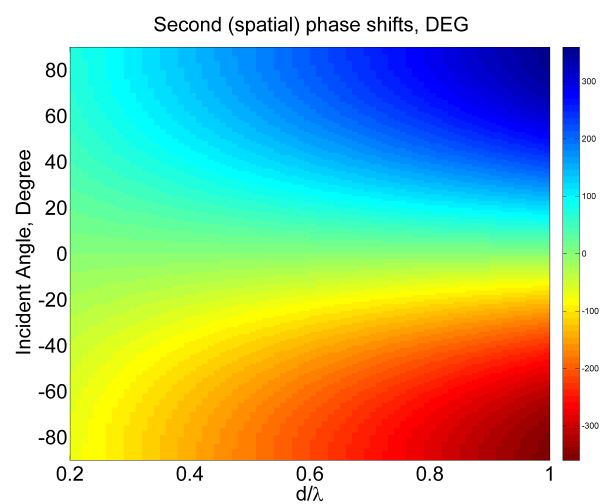

(d)

Figure 3.21: Relative phase shift differences of the second phase shifters vs. a) $\theta_{o}$ for different values of $d \mathrm{~b}$ ) $d$ for different values of $\theta_{o}$ c) $\theta_{o}$ and $d$ (3D view) d) $\theta_{o}$ and $d$ (3D-top view)

In some applications, it is convenient to control $d$, but it is not easy with a direction of the incident signals $\theta_{o}$, especially for unknown angles of the incident signals in direction finding applications. Thus, Eqs.(3.69), (3.70), and (3.71) can 
predict any change in angular locations of the spatial outputs (scattered angles) of distortion products.

Figure 3.22 illustrates angular locations of scattered distortion products vs. a relative phase shift of the second phase shifters for different distortion products orders. Also, the model is simulated with two different numbers of paths $(L=4$ and 8) with two different spacing distances $\left(d=0.5 \lambda_{o}\right.$ and $\left.d=0.7 \lambda_{o}\right)$. It can be seen that as $d$ increases, more spatial outputs can be obtained (i.e., more different distortion products are enhanced at different spatial locations for the PMSA; compare Figure 3.22a and 3.22c with Figure 3.22b and 3.22d.

Figure 3.23 exhibits angular locations of the spatial outputs (scattered angles) of distortion products vs. their product number $n$ and a relative phase shift difference of the second phase shifters $\emptyset_{o}$. Furthermore, the spatial outputs only support either negative or positive distortion products if an angle of the incident signals reaches its maxima $\left[-90^{\circ}, 90^{\circ}\right]$ provided the PMSA can deal with these angles (i.e., elements should be isotropic or omni-directional). More details about the parameters controlling the PMSA and a number of distortion products supported by the PMSA model will be more elaborated in Chapters 4 and 5. Figure 3.24 shows plots of angular locations of the spatial outputs (scattered angles) of IMDs in (3.70) where two-tone modulation sources are utilized in our PMSA. The $\mathrm{x}$ and $\mathrm{y}$ axes represent a term number of distortion products including $k w_{1}, m w_{2}$, and $k w_{1}+m w_{2}$ (harmonics and IMDs) and z-axis represents angular locations of the spatial outputs (scattered angle) of distortion products for 4-path and 8-path circuits, while relative phase shift differences of the second phase shifters used in simulations are $90^{\circ}$ for the 4-path PMSA model and $45^{\circ}$ for the 8-path PMSA model, respectively. From Figure 3.24, any IMD product with a term, $k+m=1$, has a similar spatial output to a harmonic term $n=1$. The same thing can happen with any IMDs products in which their 


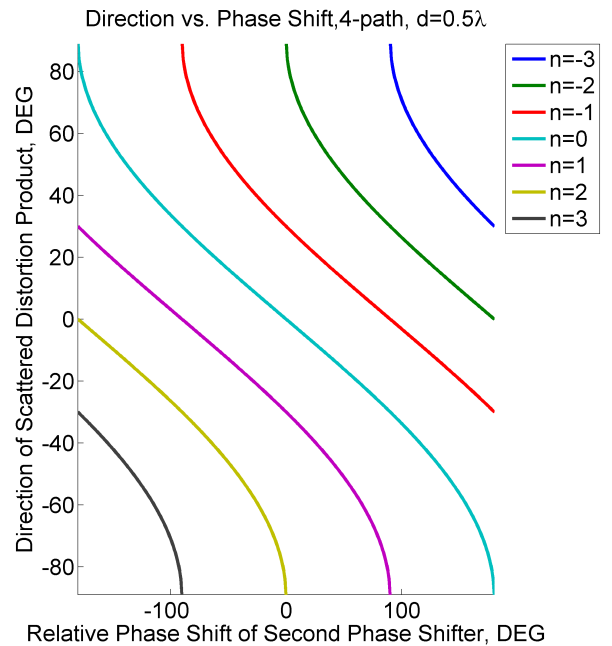

(a)

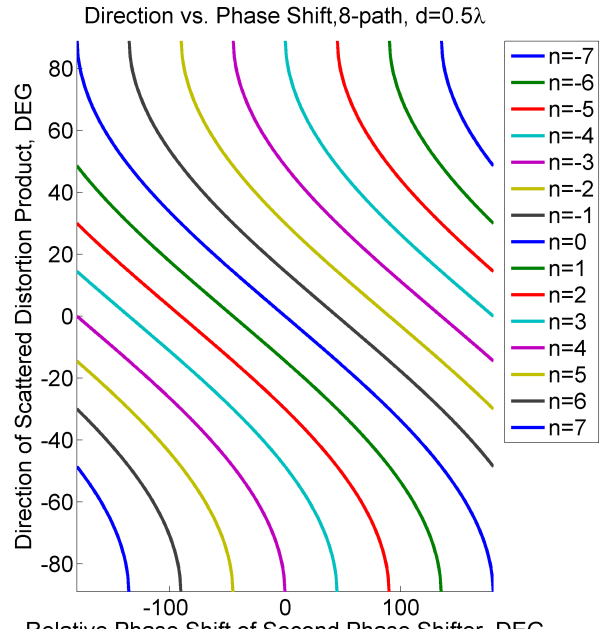

(c)

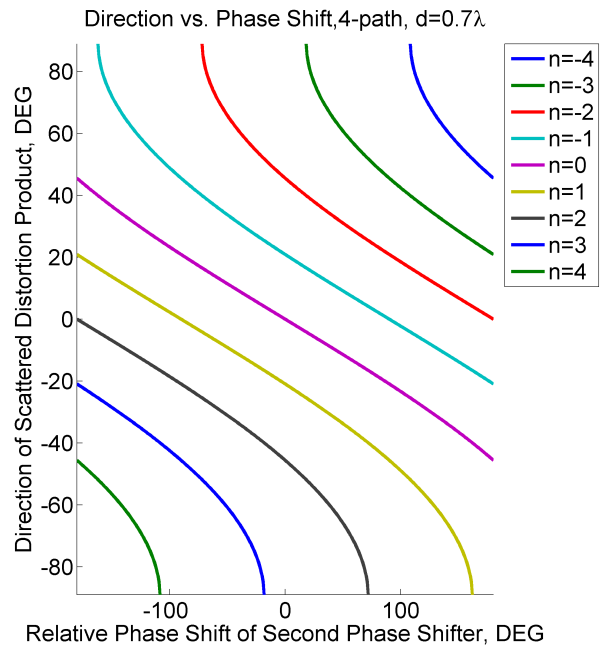

(b)

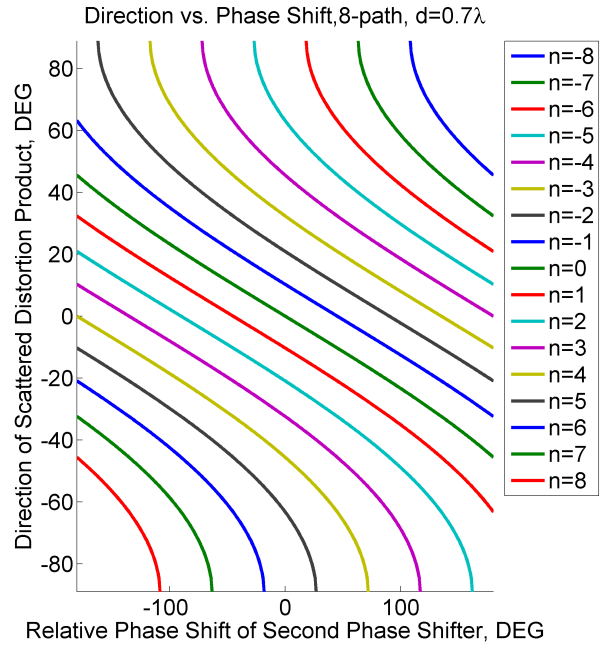

(d)

Figure 3.22: Direction of the spatial outputs (scattered distortion products, harmonics $n$ ) generated by the PMSA with a) 4 -path (element) and $d=0.5 \lambda_{o}$ b) 4 -path (element) and $d=0.7 \lambda_{o}$ c) 8-path (element) $d=0.5 \lambda_{o}$ d) 8-path (element) and $d=0.7 \lambda_{o}$

terms are equal to a harmonic term $n$ (i.e., $k+m=n$ ). This stems from the fact that because IMD $(k+m)$ and harmonic $(n)$ components have the same phase distributions across model paths, so they emerge at the same spatial output (i.e., they are enhanced and directed into the same angular location).

In figures given below, simulation results of the modified PMSA system are presented for both 4-path and 8-path circuits. Single-tone, two-tone, and three-tone modulation 


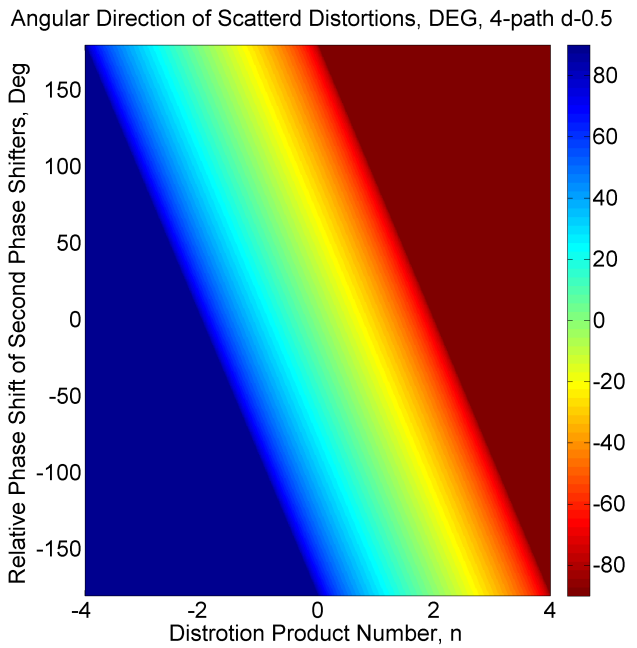

(a)

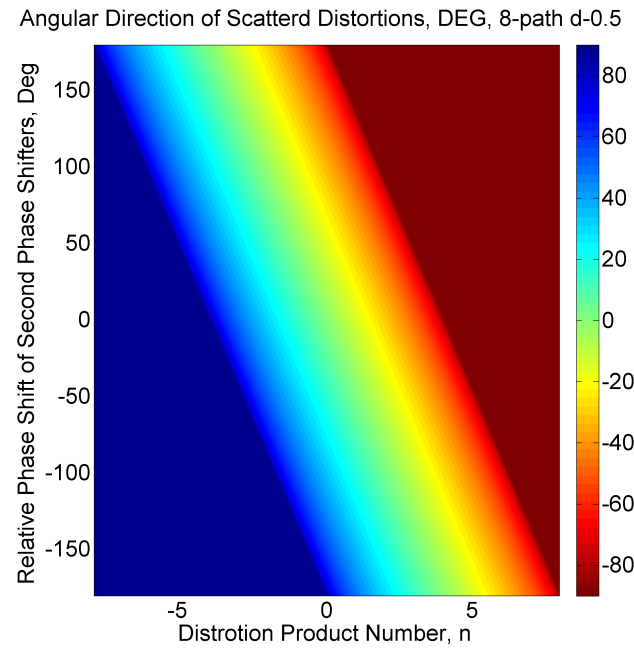

(b)

Figure 3.23: Angular locations of the spatial outputs (scattered angles) of distortion products (harmonics $n$ ) generated by the proposed PMSA vs. a number of harmonic and a relative phase shift difference of second phase shifters with a) 4-path (element) and $d=0.5 \lambda_{o}$ b) 8-path (element) and $d=0.5 \lambda_{o}$

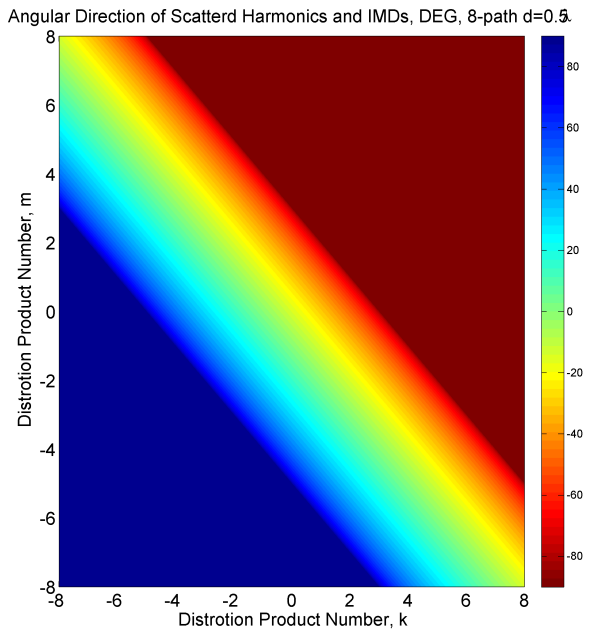

(a)

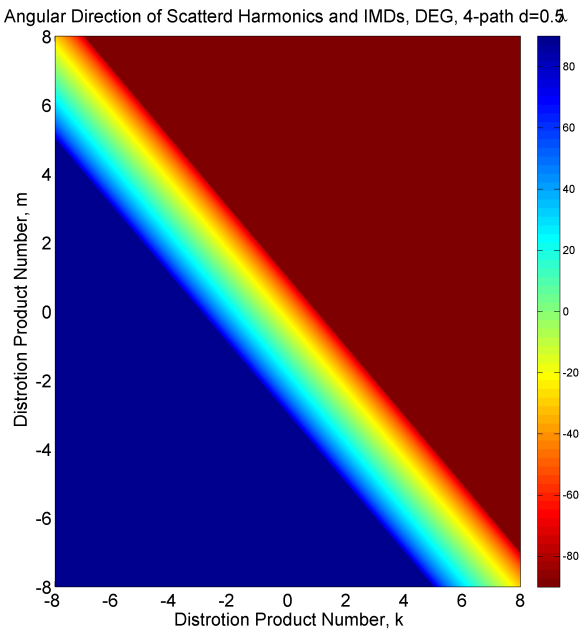

(b)

Figure 3.24: Angular locations of the spatial outputs (scattered angles) of distortion products (harmonics $n$ and IMDs $k w_{1}+m w_{2}$ ) generated by the proposed PMSA vs. a distortion product term number $k$ and $m$ a) 4-path (element) with $90^{\circ}$ relative phase shift difference of second phase shifters and $d=0.5 \lambda_{o}$ b) 8 -path (element) with $45^{0}$ relative phase shift difference of second phase shifters and $d=0.5 \lambda_{o}$

sources are used separately to prove that the modified PMSA model supports all systems regardless numbers of paths and tones. This property enables our system 
to be as a robust tool to analyze even those distortion products generated in large arrays such as multi-user active phased arrays and massive MIMO systems.

To examine the effectiveness of our modified mathematical model, we will apply phase shift distributions, which either obey the formula $\pm 360^{\circ} / L$ or not, to paths of the modified PMSA model. In other words, in the 4-path PMSA model, a relative phase shift difference typically is $\pm 90^{\circ}$, but in simulations, we examine the model with different values which are $\left(120^{\circ}, 90^{\circ}, 45^{\circ}\right.$ and $\left.-60^{\circ}\right)$ for type $\mathrm{A}$, and $\left(-120^{\circ},-90^{\circ},-45^{\circ}\right.$, and $\left.60^{\circ}\right)$ for type $\mathrm{D}$, respectively. Knowing that the change in phases is applied to the second (spatial) phase shifters only in this example, but the first (modulation) phase shifters keep their prescribed values. In the second example which is the 8-path PMSA model case, we follow the same procedure, but relative phase shift differences of the second phase shifters are $\left(90^{\circ}, 45^{\circ}\right.$, and $\left.-30^{\circ}\right)$ using the mathematical model of the type A PMSA circuit model. Results given in Figure 3.25 identify angular locations of the spatial outputs of scattered distortion products and state the existence status of distortion products as well.

Some significant points can be gleaned immediately from Figure 3.25. The first point is that each phase distribution assigned for the second phase shifters with the same numbers of paths results in emergence of different distortion products under an assumption that the modulation sources keep their phase distributions the same in all cases. For instance, when using $120^{\circ}$ as a relative phase shift difference, resulting distortion products terms at the PMSA circuit model output are $(n=-3,-2,-1$, and 0 ), while distortion products are $(n=-1,0,1$, and 2$)$ for the $-60^{\circ}$ relative phase shift difference as seen in Figure 3.25a. Moreover, these distortion products do not have the same spatial outputs for both different relative phase shift differences. In addition to the change in phases of the second phase shifters, this is true if phases of the modulation sources subject to change too. Another point that cannot be 
overlooked is those distortion products $(n=-4,-3,-2,-1,0$, and 1) change their spatial outputs (angular locations) from $\left(90^{\circ}, 56.44^{\circ}, 19.47^{\circ},-9.594^{\circ},-41.81^{\circ}\right.$, and $\left.-90^{\circ}\right)$ into $\left(-90^{\circ},-56.44^{\circ},-19.47^{\circ}, 9.594^{\circ}, 41.81^{\circ}\right.$, and $\left.90^{\circ}\right)$ when its counterpart type D replaces the type A PMSA model, symmetrical replacement of the spatial outputs. This feature gives rise to exploit the PMSA model in beamforming (scanning) of the spatial outputs (angular locations of distortion products), as we will see in Chapters 5 and 6.

As we illustrated above, when using multi-tone modulation sources, any resulting IMDs, with orders equal to harmonic term numbers $(k+m+s+\cdots+j=n)$, will experience what harmonics have experienced. In our design, associated IMDs with harmonics are enhanced too if harmonics are enhanced and are directed into the same spatial locations. However, this description is true if all phase distributions of tones are equal. In large arrays serving a vast number of users, each tone in our PMSA mathematical model represents a user, and it has its particular phase distribution, so resulting IMDs will not follow spatial locations of harmonics. However, fortunately, the proposed model is still robust and can sense enhanced distortion products and detect their scattered angles even if phase distributions of tones are not equal.

With the help of Figure 3.25, the PMSA model is simulated according to the spatial outputs (scattered angles) found in the figure. Figure 3.26 presents simulation results of the 4-path type A PMSA circuit model when a relative phase shift difference of the second phase shifter is $120^{\circ}$. The model is simulated at the spatial outputs of $\left(56.44^{\circ}, 19.47^{\circ},-9.594^{\circ},-41.81^{\circ}\right)$ for single-tone, two-tone and three-tone modulation sources. Repeating simulation analysis for the same circuit given in Figure 3.26 using modulation sources with different tones is necessary to provide a comprehensive analysis tool. It is seen that associated IMDs appear along with the same terms numbers of harmonics in Figure 3.26. The same circuit 


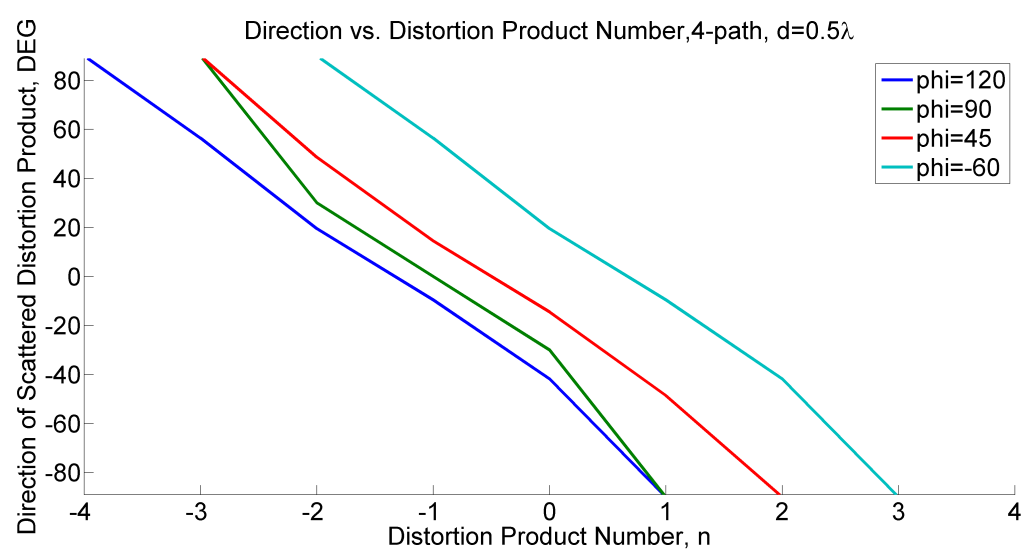

(a)

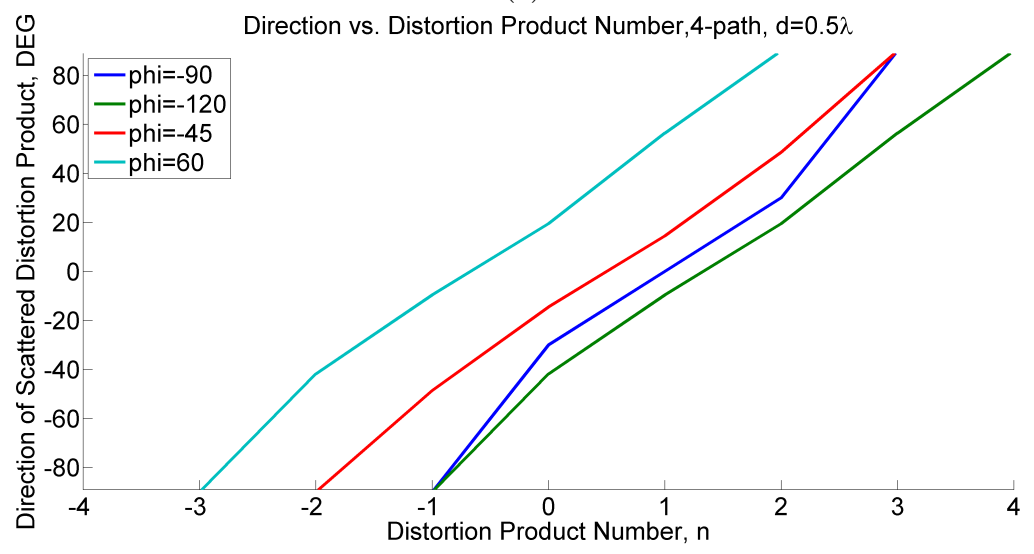

(b)

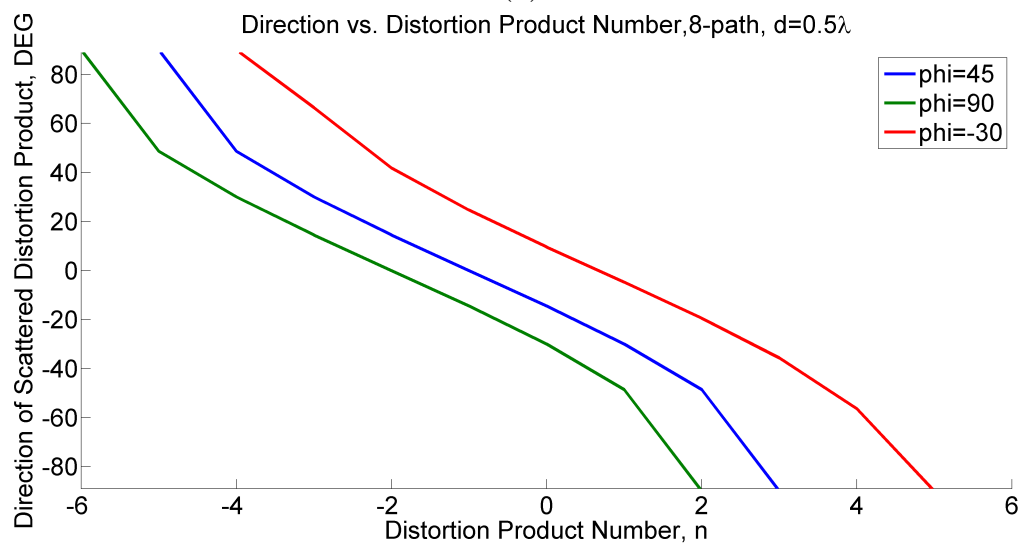

(c)

Figure 3.25: Angular locations of the spatial outputs (scattered distortion products, harmonics $n$ ) generated by the proposed PMSA vs. a distortion product term numbers $n$ for different relative phase shift differences of the second phase shifter a) 4-path (elements) type A circuit with $d=0.5 \lambda_{o}$ b) 4-path (elements) type D circuit with $d=0.5 \lambda_{o}$ c) 8-path (elements) type A circuit with $d=0.5 \lambda_{o}$ 
is analyzed again with a different phase shift distribution for the second phase shifters which is $45^{\circ}$ at the spatial outputs of $\left(48.59^{\circ}, 14.48^{\circ},-14.48^{\circ},-48.59^{\circ}\right)$, using only single-tone modulation sources, see Figure 3.27. The procedure is extended to increase a number of the PMSA model paths. The 8-path type A PMSA circuit model is analyzed at a relative phase shift difference of $-30^{\circ}$. Harmonics $(n=-3,-2,-1,0,1,2,3$, and 4$)$ are found at the spatial outputs of $\left(66.44^{\circ}, 41.81^{\circ}, 24.62^{\circ}, 9.594^{\circ},-4.78^{\circ},-19.47^{\circ},-35.69^{\circ}\right.$, and $\left.-56.44^{\circ}\right)$, respectively, as shown in Figure 3.28.

As a consequence, the modified model can adapt and deal with any value of phase shifts and can detect the spatial outputs of any order of distortion products. Distortion products at the spatial outputs of the PMSA model change their angular locations in space with any change in phases associated with each path in the model. This adaption feature makes the PMSA a sensitive structure to phases of the second phase shifters (i.e., angular directions of the incident signals), and phases of the modulation sources (i.e., time delay of switching ON/OFF between scatterers) under circumstances of ideal system parts. The results confirm that distortion products resulting from nonlinear activities in large arrays are emerged at different spatial outputs (i.e., pointed into different directions in space in the transmitting side and may or may not be added up with the desired signal in the receiving side, thereby leading to an improvement in the signal to noise ratio $\mathrm{S} / \mathrm{N}$ of the desired signal) [143]. Furthermore, when a number of tones of the modulation source increases and each tone has its particular phase shift, the resulting spectrum will cover most of the SFoV. 


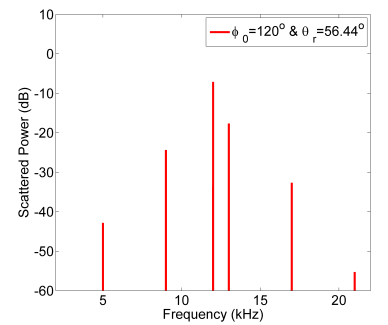

(a)

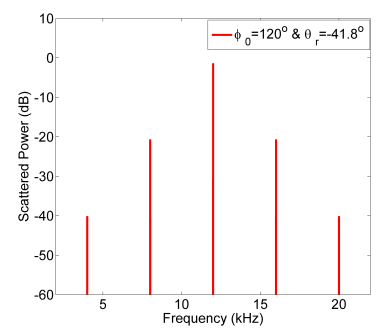

(d)

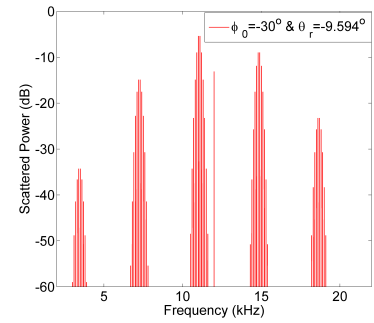

(g)

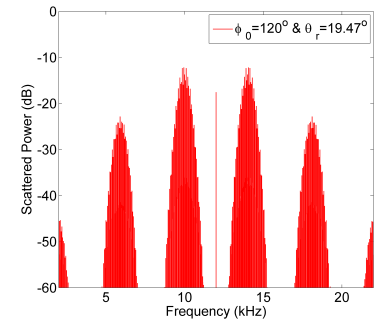

(j)

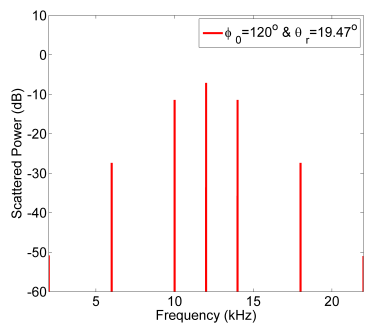

(b)

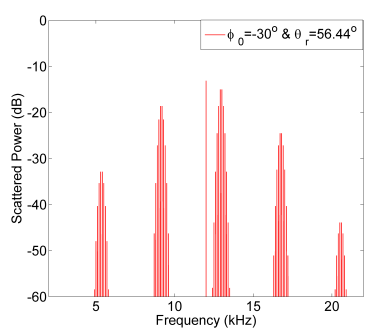

(e)

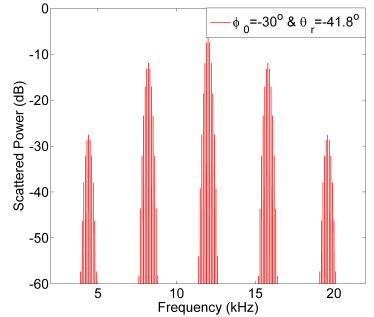

(h)

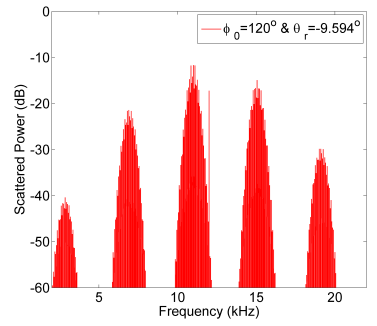

(k)

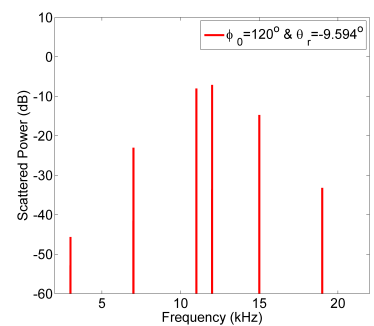

(c)

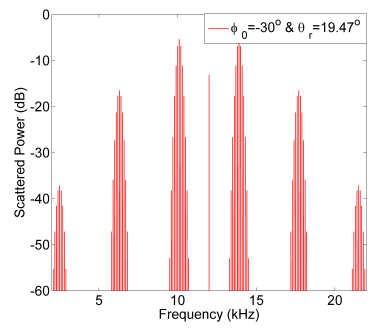

(f)

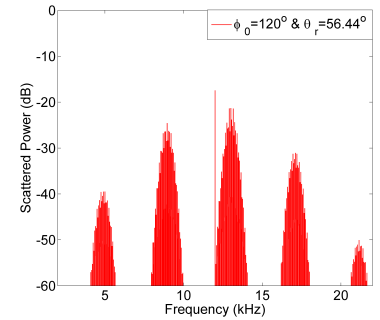

(i)

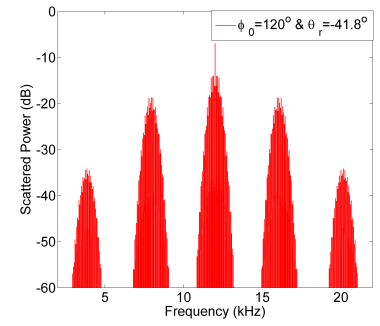

(l)

Figure 3.26: Simulated scattered power from the 4-path type A PMSA model when a relative phase shift difference of the second phase shifter is $120^{\circ}$ a) single-tone at a spatial output $\left(\theta_{r}=56.44^{\circ}\right)$ b) single-tone at a spatial output $\left(\theta_{r}=19.47^{\circ}\right)$ c) single-tone at a spatial output $\left(\theta_{r}=-9.594^{\circ}\right)$ d) single-tone at a spatial output $\left(\theta_{r}=-41.8^{\circ}\right)$ e) two-tone at a spatial output $\left.\left(\theta_{r}=56.44^{\circ}\right) \mathrm{f}\right)$ two-tone at a spatial output $\left.\left(\theta_{r}=19.47^{\circ}\right) \mathrm{g}\right)$ two-tone at a spatial output $\left.\left(\theta_{r}=-9.594^{\circ}\right) \mathrm{h}\right)$ two-tone at a spatial output $\left(\theta_{r}=-41.8^{\circ}\right)$ i) three-tone at a spatial output $\left.\left(\theta_{r}=56.44^{\circ}\right) \mathrm{j}\right)$ three-tone at a spatial output $\left.\left(\theta_{r}=19.47^{\circ}\right) \mathrm{k}\right)$ three-tone at a spatial output $\left.\left(\theta_{r}=-9.594^{\circ}\right) \mathrm{l}\right)$ three-tone at a spatial output $\left(\theta_{r}=-41.8^{\circ}\right)$ 


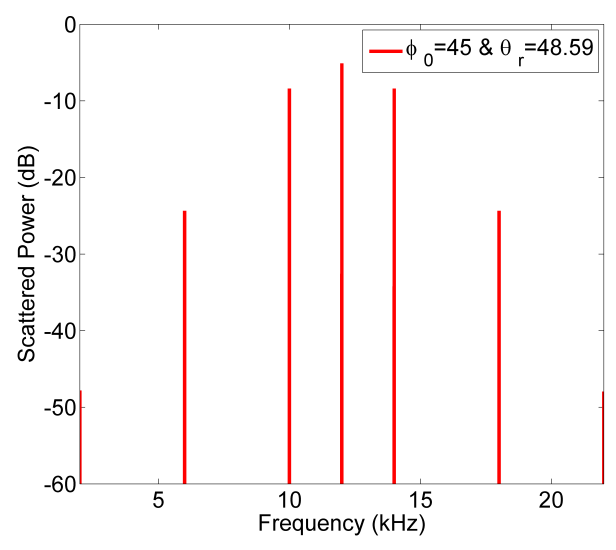

(a)

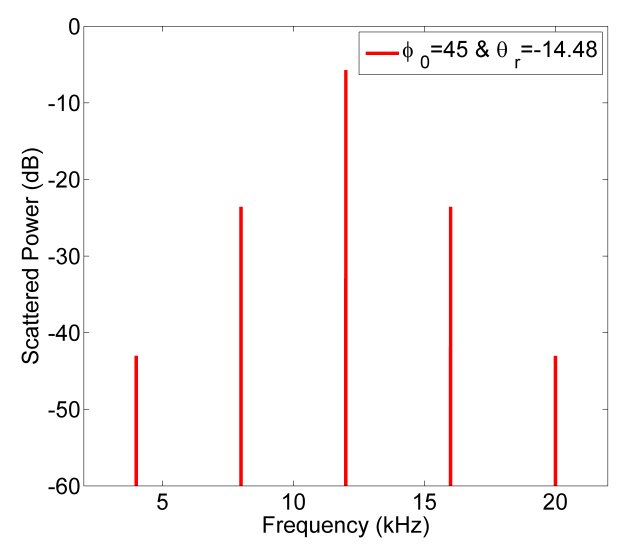

(c)

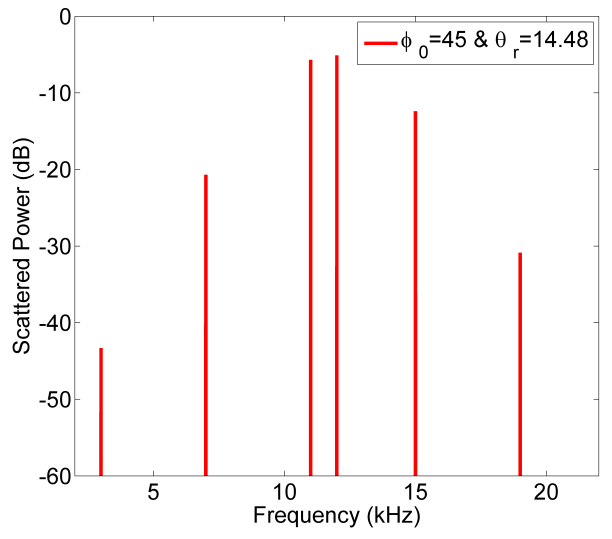

(b)

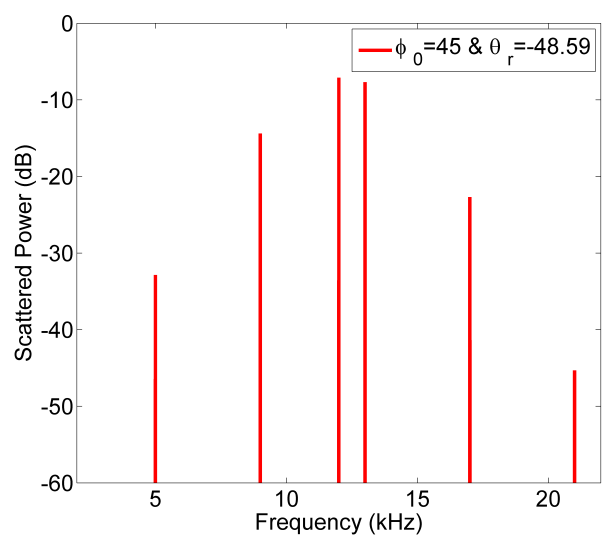

(d)

Figure 3.27: Simulated scattered power from the 4-path type D PMSA model when a relative phase shift difference of the second phase shifter is $45^{\circ}$ captured at spatial outputs a) $\theta_{r}=48.59^{\circ}$ b) $\theta_{r}=14.48^{\circ}$ c) $\theta_{r}=-14.48^{\circ}$ and d) $\theta_{r}=-48.59^{\circ}$

\subsection{Non-idealities of the Phase-Modulated Scattering Array (PMSA)}

Of course, the emergence of some distortion products and absence others at different specific spatial outputs (angular locations) described in the previous sections primarily rely on the matching between the PMSA model paths (i.e., scatterers). Note that some factors are crucial to determine the ability of the PMSA model to deal with distortion products generated by the processes of underlying switching. These factors are phases and amplitudes of the modulation and spatial sources. We cannot guarantee that the practical PMSA have identical elements. Therefore, errors 


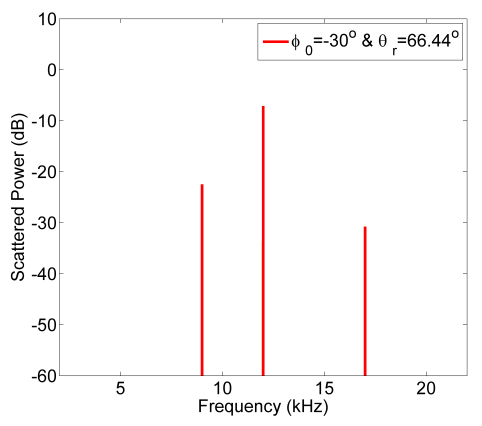

(a)

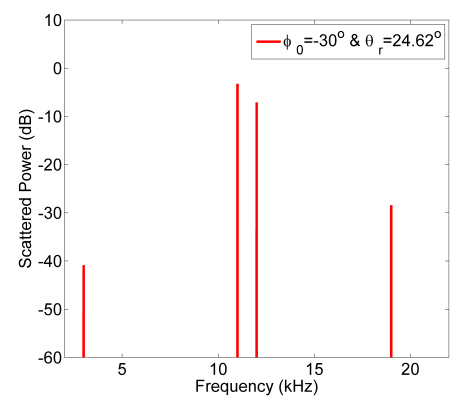

(c)

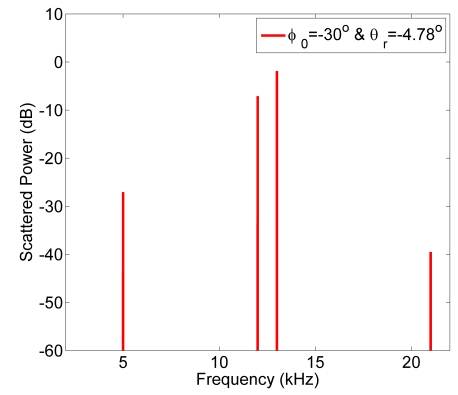

(e)

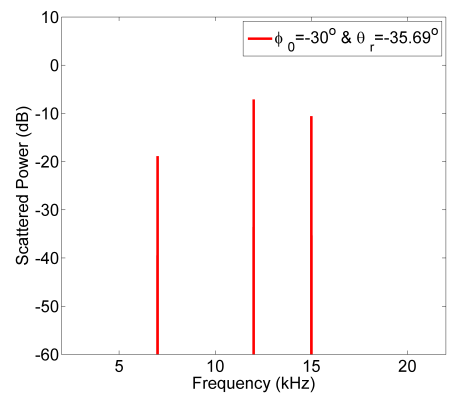

(g)

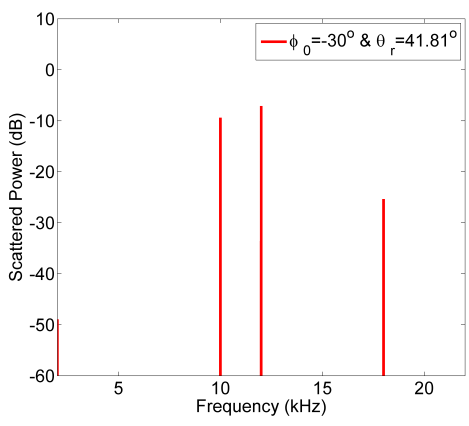

(b)

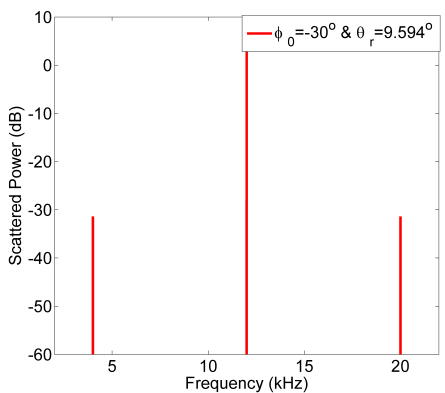

(d)

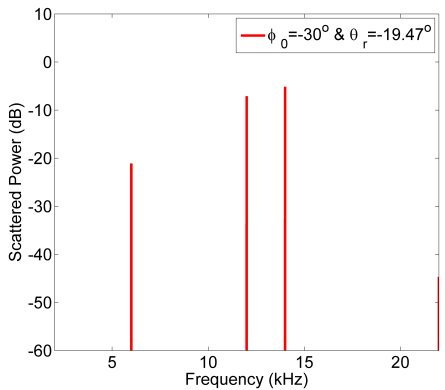

(f)

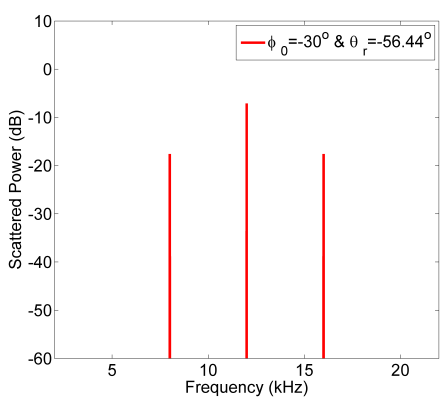

(h)

Figure 3.28: Simulated scattered power from the 8-path type A PMSA model when a relative phase shift difference of the second phase shifter is $-30^{\circ}$ captured at spatial outputs a) $\theta_{r}=66.44^{\circ} \mathrm{b}$ ) $\theta_{r}=41.81^{\circ}$ c) $\theta_{r}=-24.62^{\circ}$ d) $\theta_{r}=9.594^{\circ}$ e) $\theta_{r}=-4.78^{\circ}$ f) $\theta_{r}=-19.47^{\circ}$ g) $\theta_{r}=-35.69^{\circ}$ and h) $\theta_{r}=9.594^{\circ}$ 
in phase shifters of the modulations and spatial sources are $\vartheta_{l}$ and $\delta_{l}$, respectively. Besides, modulation sources have amplitude errors denoted by $\varepsilon_{l}$, while amplitude errors of the spatial sources are ignored due to the weakness of the RF incident (illuminating) signals. All these errors in phases and amplitudes are integrated into the former model and portrayed in Figure 3.29. The errors stem from non-idealities of electronics devices [147] [148], mutual coupling between scatterers, active switches, and multipath ray reflections. Mathematically, these errors can be integrated into the mathematical model as for the single-tone type-A PMSA circuit model

$F(t)=\sum_{l=1}^{L}\left[\cos \left(\left(w_{o} t+(l-1) \emptyset_{o}+\delta_{l}\right)\right) \times \sum_{n=0}^{\infty}\left(1+\varepsilon_{l}\right)^{n} * \cos \left(w_{m} t+(l-1) \emptyset_{m}+\vartheta_{l}\right)^{n}\right]$

and for the two-tone type-A PMSA circuit model

$$
\begin{aligned}
F(t)= & \sum_{l=1}^{L}\left[\cos \left(\left(w_{o} t+(l-1) \emptyset_{o}+\delta_{l}\right)\right) \times \sum_{n=0}^{\infty}\left[\left(1+\varepsilon_{1 l}\right) \times \cos \left(w_{1} t+(l-1) \emptyset_{1}\right.\right.\right. \\
& \left.\left.\left.+\vartheta_{1 l}\right)+\left(1+\varepsilon_{2 l}\right) \times \cos \left(w_{2} t+(l-1) \emptyset_{2}+\vartheta_{2 l}\right)\right]^{n}\right]
\end{aligned}
$$

Some algebraic manipulations are carried out and the corrected phases and realistic terms are added into (3.72) and (3.73), resulting in for the single-tone type-A PMSA circuit model

$$
\begin{aligned}
F(t) & =\sum_{l=1}^{L} \sum_{n=-\infty}^{\infty}\left(B B+\rho_{l}\right) \cos \left(\left(w_{o}+n w_{m}\right) t+(l-1)\left(\emptyset_{o}+n \emptyset_{m}+\emptyset_{c o}\right)\right. \\
& \left.+n \vartheta_{l}+\delta_{l}\right) \times E P(\theta)+G \cos \left(w_{o} t\right)
\end{aligned}
$$


and for the two-tone type-A PMSA circuit model

$$
\begin{aligned}
F(t) & =\sum_{l=1}^{L} \sum_{n=-\infty}^{\infty}\left(C C+\rho_{l}\right) \cos \left(\left(w_{o}+k w_{1}+m w_{2}\right) t+(l-1)\left(\emptyset_{o}+k \emptyset_{1}+m \emptyset_{2}+\emptyset_{c o}\right)\right. \\
& \left.+k \vartheta_{1 l}+m \vartheta_{2 l}+\delta_{l}\right) \times E P(\theta)+G \cos \left(w_{o} t\right)
\end{aligned}
$$

The performance of a certain distortion product cancellation is called as the distortion product rejection ratio $(D P R R)[153]$, and is defined as

$$
\operatorname{DPRR}_{n}=\frac{\text { The refrence power at a certain distortion product }}{\text { The rejected power at a certain distortion product }}=\frac{P_{\mathrm{n}, \text { reference }}}{P_{\mathrm{n}, \mathrm{rejected}}}
$$

This ratio considers as a measure of distortion product cancellations.

In the PMSA circuit model, it always uses the first path as a reference path, so all errors associated with that path are ignored. The process can be carried out by firstly calculating output powers of a specific distortion product if all $L$ paths are similar to the first path. In other words, the same phase shifts are applied to all paths. Thus, outputs of paths contain the same in-phase distortion products, resulting in power called $P_{n, \text { reference }}$. $P_{n, \text { reference }}$ tells us how strong a specific distortion product is at the circuit output if we do not use the polyphase multipath method. Then, we calculate the output power of that specific distortion product when applying the phase shifts differences to circuit paths. Phase shifts between paths depend on a number of circuit paths $L$, spatial phase shifters associated with incident angles $\theta_{o}$, and the spatial outputs of distortion products $\theta_{r_{n}}$. However, phases and amplitudes errors should be taken into account among the paths. The output power of a specific distortion product $P_{n, \text { rejected }}$, identifies its strength due to phases and amplitudes errors. In ideal conditions, this power is zero. Eq.(3.76) which is the ratio between the two powers tells us about the suppression amount achieved by the non-ideal 
PMSA compared to the same circuit but without applying phase shifts between the paths.

\subsubsection{Example}

If we want to calculate the $D P R R$ of a harmonic with $n=3$ in the PMSA circuit model with four paths, this can be achieved as follows. As a modulation signal, $A \cos \left(w_{m} t\right)$ is selected. The output voltage of the third harmonic $\left(w_{o}+3 w_{m}\right)$ at the end of first path is equal to $1 / 8 a_{3} A^{3} \cos \left(w_{o}+3 w_{m}\right) t$. The final output voltage after combining all four paths will be to $4 / 8 a_{3} A^{3} \cos \left(w_{o}+3 w_{m}\right) t$ if all paths are identical (no-polyphase applied). The resulting power of third harmonic $P_{3, \text { reference }}$ is $16 /\left(128 A^{6} R\right)$ where $R$ is an internal impedance of the polyphase circuit. To find $P_{3, \text { rejected }}$, the output power of $\left(w_{o}+3 w_{m}\right)$ product in Figure 3.29 should be calculated as:

at the end of the first path, the output voltage is

$$
V 1=1 / 8 A^{3}\left(1+\rho_{1}\right) \times \cos \left(\left(w_{o}+3 w_{m}\right) t+0 \times\left(\emptyset_{o}+3 \emptyset_{m}+\emptyset_{r}\right)+3 \vartheta_{1}+\delta_{1}\right)
$$

at the end of the second path, the output voltage is

$$
V 2=1 / 8 A^{3}\left(1+\rho_{2}\right) \times \cos \left(\left(w_{o}+3 w_{m}\right) t+1 \times\left(\emptyset_{o}+3 \emptyset_{m}+\emptyset_{r}\right)+3 \vartheta_{2}+\delta_{2}\right)
$$

at the end of the third path, the output voltage is

$$
V 3=1 / 8 A^{3}\left(1+\rho_{3}\right) \times \cos \left(\left(w_{o}+3 w_{m}\right) t+2 \times\left(\emptyset_{o}+3 \emptyset_{m}+\emptyset_{r}\right)+3 \vartheta_{3}+\delta_{3}\right)
$$




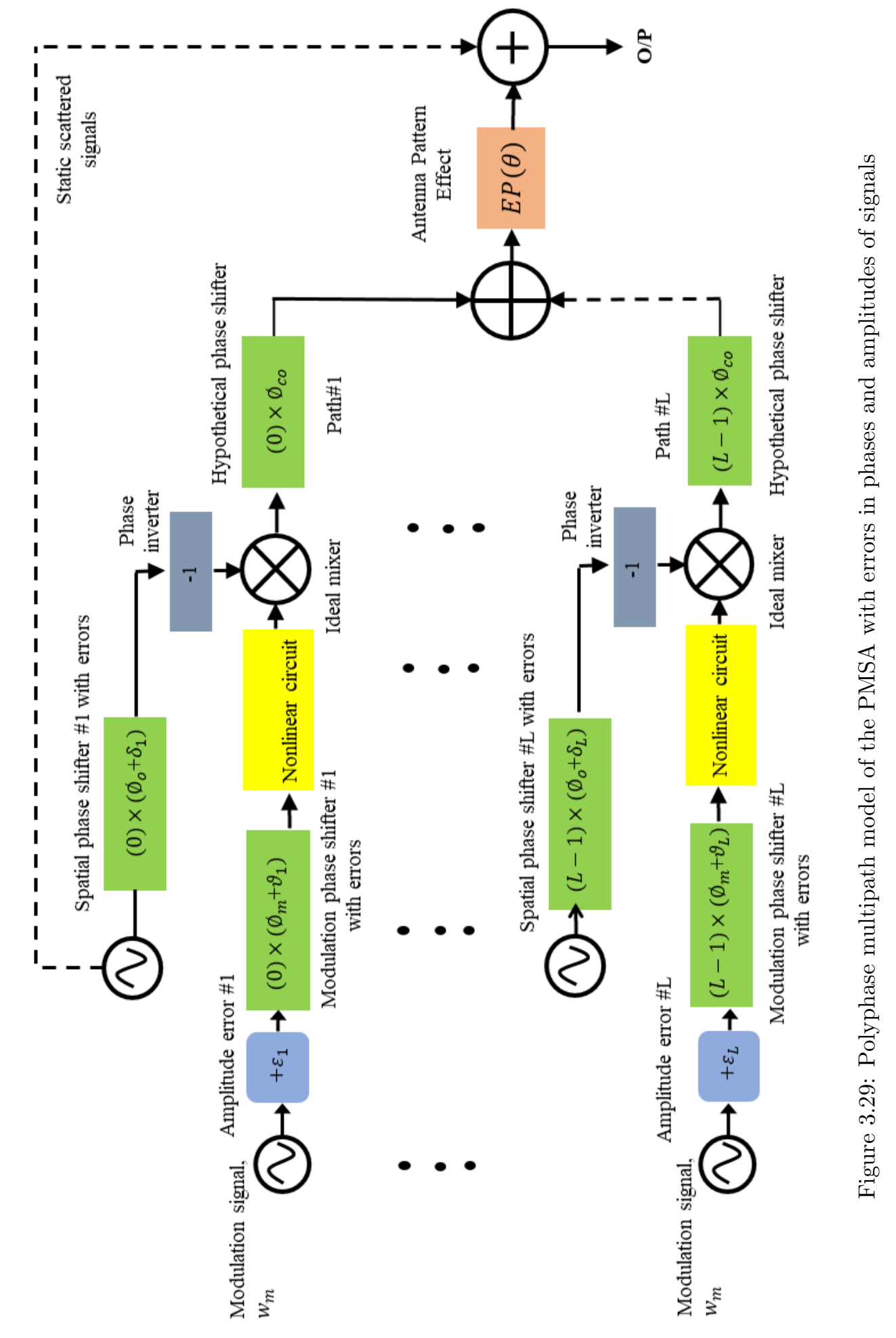


and at the end of the fourth path, the output voltage is

$$
V 4=1 / 8 A^{3}\left(1+\rho_{4}\right) \times \cos \left(\left(w_{o}+3 w_{m}\right) t+3 \times\left(\emptyset_{o}+3 \emptyset_{m}+\emptyset_{r}\right)+3 \vartheta_{4}+\delta_{4}\right)
$$

After adding these four voltages signals, squaring, and then dividing them by $R$, we will obtain $P_{3, \text { rejected. }}$ Thus, the $D P R R_{3}$ can be written as

$$
\mathrm{DPRR}_{3}=\frac{16 / 128 A^{6}}{\frac{1}{2}[V 1+V 2+V 3+V 4]^{2}}
$$

This expression can be analyzed using the Matlab software to quickly obtain results. More generally, calculations of $D P R R$ for $w_{o}+n w_{m}$ in a system with $L$ paths can be given as

$$
\operatorname{DPRR}_{\mathrm{n}}=\frac{L^{2} /\left(2^{2 L} A^{2 n}\right)}{[V 1+V 2+\cdots+V L]^{2}}=\frac{L^{2}}{(2 A)^{2 n}[V 1+V 2+\cdots+V L]^{2}}
$$

It can be seen that when using more paths $L$, the $D P R R_{n}$ becomes larger. However, the higher order harmonics are weaker, so the less suppression is sufficient. The term $n$ in (3.82) can be replaced by an intermodulation distortion IMD product term $(k+m)$ for two-tone modulation signals and $(k+m+\cdots+x+j)$ for multi-tone modulation signals, so it is valid to work with any kind of distortion products (i.e., either harmonics or IMDs). 


\section{Chapter 4: The Mathematical Model of the Proposed Design under a Phased Antenna Array Perspective}

\subsection{Introduction}

Having discussed the process of generation of distortion products and how to control them in Chapter 3, we now examine associated retransmitted (scattered) beams or radiation patterns of these distortion products. Knowing that directions of scattered beams here represent locations of the spatial outputs in Chapter 3. Because each single distortion product has independent and non-coherent frequency and phase properties, this enables the PMSA to govern several distortion products independently and concurrently. The mathematical model developed here in this chapter based on phased antenna array concepts will take into account all these factors governing spatial locations of distortion products to guarantee that the mathematical model is compatible with features of the PMSA. However, in phased antenna arrays, phase shifters must be integrated with each element of antenna arrays to enable beamforming functionality that there is no need to use them in our PMSA. This feature leads to a simple design structure.

In this chapter, we consider a narrowband signal source connected to a single directional antenna, illuminating the PMSA from a particular direction. At the PMSA, we assume elements as omnidirectional (i.e., antennas with isotropic-like radiation behavior) that we can use them as a starting point for the future derivatives based on directional antennas. The blue dotted line in Figure 4.1 represents a radiation pattern of the illuminating antenna while the red ones denote radiation patterns of array elements when working individually under the circumstances of no mutual coupling between elements. However, the PMSA has more than one scattered 
beam tagged with different frequencies in both back-scattering and forward-scattering directions as will be seen later in this chapter. Moreover, each single frequency associated with a single scattered beam denotes a single distortion product generated due to the nonlinear behavior of the PMSA.

Elements utilized in the PMSA are nonconventional antennas (nonlinear elements) which are frequency-modulated antennas (i.e., modulated scatterers MSs), and the entire PMSA is a phase-modulated structure. These two types of modulations can be considered as a domestic-modulation and a global-modulation for former and latter types, respectively. Modulations in the presented design give rise to the emergence of scattered radiation beams associated with different frequencies (distortion products) at different spatial locations. Moreover, spatial locations of scattered beams of the PMSA are governed by some factors as demonstrated in Figure 4.1, given by:

1. Angular directions of incident signals $\theta_{o}$

2. ON/OFF switching time delays between scatterers(considered as phases in the frequency domain)

3. Reference elements for the progressive ON/OFF switching times (considered as advanced or lag phases distributed across elements $\pm \emptyset_{m}$ )

4. Distances $d$ between array elements

5. Distortion product term numbers $n, k+m, k+m+s+\cdots+j$ for single-tone, two-tone, and multi-tone modulation sources,respectively.

Because each scattered radiation beam directed into a different spatial location is tagged with a different frequency, this property is like the frequency-diversity into spatial-diversity transformation (FDSDT) as in [110] [150] [129]. Also, determining a 


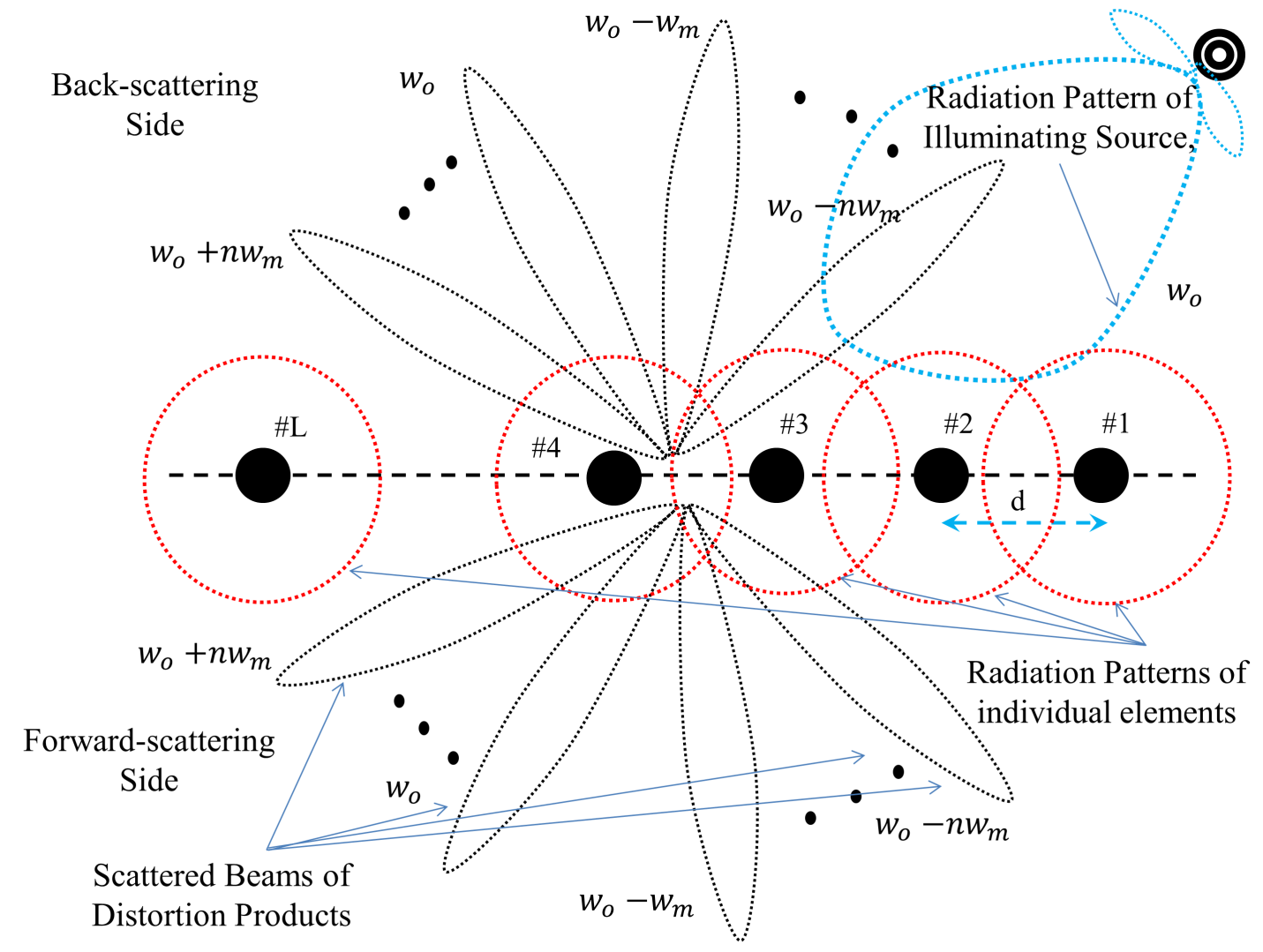

Figure 4.1: General representation of scattered beams of distortion products generated by the PMSA

source of distortion products and their particular spatial beams, it is useful in large arrays, especially that will perform in the next generation of communication systems 5G. In active antenna arrays, alleviating influences of distortion products requires to know a source of distortion products and how to control their radiation patterns being useful to increase the overall signal to noise ratio SNR [77] [127] [78] [75].

Thus, controlling directions, numbers, and types of beams of distortion products is of interest to us throughout this study. In this chapter, we only focus on a mathematical model based on phased antenna array theory to provide a comprehensive analysis tool for the PMSA. The model developed here will support the last model presented before in Chapter 3 although the underlying perspectives are different. We then look at some factors affecting the performance of PMSA from 
the phased antenna array standpoint.

\subsection{General Mathematical Model based on Phased Antenna Array Theory}

\subsubsection{Single Frequency-Modulated Element (Scatterer)}

The problem of interest is exhibited in Figure 4.1. An illuminating plane wave $E_{i}=$

$A e^{j w_{o} t}$, where $\mathrm{A}$ is the amplitude and $w_{o}$ is the illuminating frequency, impinges from a particular direction with an incident angle $\theta_{o}$ on a periodic phase-modulated scattering array of $L$ isotropic-elements. Because the incident signal reaches the array elements at different times, this leads to induce the same signals over elements with different phases. In case of non-modulation, induced signals over the $l-t h$ element can be expressed mathematically as

$$
E_{\text {ind,l }}=a_{l} e^{j\left(w_{o} t-\emptyset_{o, l}\right)}
$$

where $a_{l}$ represents an amplitude of the induced signal at the $l-t h$ element, $w_{o}$ is a frequency of the incident signal, $\emptyset_{o, l}$ denotes a phase of the induced signal at the $l-t h$ element. If an array is uniform (i.e., equal distances between elements), induced phases can be written as

$$
\emptyset_{o, l}=l \times \emptyset_{o}=l \times K d \sin \left(\theta_{o}\right)
$$

where $l=0,1,2, \cdots, L-1, K$ is the free-space wave number, and $d$ is a space between any successive two elements. The description given above is valid only for signals induced over non-modulated antennas. To take into account modulation effects, signals in (4.1) must be modified. Since modulations are periodic in their nature, the induced signals on modulated antennas apertures are periodic too and can be 
represented as

$$
\begin{aligned}
E\left(\theta, w_{m}\right) & =\left(b_{0, l}+b_{1, l} \cos \left(w_{m} t+\emptyset_{m, l}\right)+b_{2, l} \cos \left(2\left(w_{m} t+\emptyset_{m, l}\right)\right)\right. \\
& \left.+\cdots+b_{n, l} \cos \left(n\left(w_{m} t+\emptyset_{m, l}\right)\right)\right) \\
& =\sum_{n=0}^{\text {infty }}+b_{2, l} \cos \left(2\left(w_{m} t+\emptyset_{m, l}\right)\right) \cdot e^{j\left(w_{o} t-\emptyset_{o, l}\right)}
\end{aligned}
$$

where $b_{n, l}$ is the $n-t h$ Fourier series coefficient term at the $l-t h$ element, $\theta$ is a spatial representation of signals, and $w_{m}$ and $\emptyset_{m, l}$ are radian frequency and phase of modulation sources at the $l-t h$ element, respectively. A frequency of the incident signals is much higher than a frequency of the modulation signals. The difference between (4.1) and (4.3) is that the induced signal on a periodic element has an infinite number of frequencies (distortion products) in contrast to an unmodulated element which has a single frequency component. Recall that each single distortion component has its particular frequency and phase.

Using Euler identity to replace cosines in (4.3), it makes the model compatible with phased antenna array equations. Thus, each cosine term can be represented by

$$
\cos x=\frac{1}{2} e^{-j x}+\frac{1}{2} e^{+j x}
$$

Now, Eq.(4.3) becomes 


$$
\begin{aligned}
E\left(\theta, w_{m}\right) & =\sum_{n=0}^{\infty} b_{n, l}\left(\frac{1}{2} e^{-j n\left(w_{m} t+\emptyset_{m, l}\right)}+\frac{1}{2} e^{j n\left(w_{m} t+\emptyset_{m, l}\right)}\right) e^{j\left(w_{o} t-\emptyset_{o}\right)} \\
& =\frac{1}{2}\left(\sum_{n=-\infty}^{0} b_{-n, l} e^{j n\left(w_{m} t+\emptyset_{m, l}\right)}+\sum_{n=0}^{\infty} b_{n, l} e^{j n\left(w_{m} t+\emptyset_{m}\right)}\right) e^{j\left(w_{o} t-\emptyset_{o, l}\right)} \\
& =\frac{1}{2} \sum_{n=-\infty}^{\infty} b_{n, l} e^{j n\left(w_{m} t+\emptyset_{m, l}\right)} e^{j\left(w_{o} t-\emptyset_{o, l}\right)} \\
& =\frac{1}{2} \sum_{n=-\infty}^{\infty} b_{n, l} e^{j\left(\left(w_{o}+n w_{m}\right) t+n \emptyset_{m, l}-\emptyset_{o, l}\right)}
\end{aligned}
$$

where $b_{-n, l}=b_{n, l}$, because magnitudes of distortion products are symmetrical about the main component $b_{0, l}$. In the situation just described, where distortion components of modulation sources are added to the unmodulated frequency component of the incident signal, it is known as the frequency modulation, and an infinite set of new resulting frequencies is called as the Fourier series signals (distortion products). Although there is an infinite number of distortion products which could be spatially scattered, only a few of those distortion products terms can exist physically. Also, we can see that a phase for each single distortion product $\left(n \emptyset_{m, l}-\emptyset_{o, l}\right)$ is different from other distortion products. This holds true if our proposed system has an infinite number of elements, but because this is impossible and the phase is a periodic function with $2 \pi$, some distortion products will have the same phase shift. From the point of view of phased antenna array theory, scattered beams of distortion products (harmonics) have different spatial characteristics if phases of modulation sources working within the proposed PMSA are chosen in such a way as to guarantee that each single distortion product has its particular relative phase shift difference across the PMSA. Therefore, phases of the incident signal and modulation sources at 
the $l-t h$ element will be given as

$$
\begin{gathered}
\emptyset_{o, l}=l \times \emptyset_{o} \\
\emptyset_{m, l}=l \times \emptyset_{m}
\end{gathered}
$$

As a consequence, the final resulting phase of each harmonic at the $l-t h$ antenna terminals is given by

$$
\emptyset_{n}=l \times\left(n \emptyset_{m}-\emptyset_{o}\right)
$$

Eq.(4.8) provides clear evidence about phases of distortion products which are not similar at the same $l-t h$ antenna element except at the reference element which are equal to zero, and also states that the same distortion products at different elements do not have the same phases, leading into the spatial diversity of distortion products. In the scattering situation, phases of the incident signals are inverted due to reflection processes as in the retrodirective antenna arrays [59] [130], so Eq.(4.8) becomes

$$
\emptyset_{n}=l \times\left(n \emptyset_{m}+\emptyset_{o}\right)
$$

Eq.(4.9) is only valid when being the first element on right considered as a reference element as shown in Figure 4.1 for the progressive phase distributions of modulation and incidental signals. In other words, directions of phase distributions for both signals start from right to left. However, this is not the case since these phases distributions could start from left to right and also these two signals may not have the same direction for their phases distributions. Recall that, phases of the incident signals are attained because array elements receive the incident signals at different locations, so there will be spatial phase differences between elements. Moreover, Modulation signals turn $\mathrm{ON} / \mathrm{OFF}$ elements at different times, so in the frequency 
domain, there will phase difference between elements. The phase shift distribution from right to lift is called forward, whereas the phase shift distribution from left to right is called backward (i.e., reversed)

To generalize the mathematical model for any type of phase distributions, we can rewrite (4.5) and (4.9) as

for the single-tone modulation sources

$$
\emptyset_{n}=l \times\left( \pm n \emptyset_{m} \pm \emptyset_{o}\right)
$$

and

$$
E\left(\theta, w_{m}\right)=\frac{1}{2} \sum_{n=-\infty}^{\infty} b_{n, l} e^{j\left(l \times\left( \pm n \emptyset_{m} \pm \emptyset_{o}\right)\right)} \cdot e^{j\left(\left(w_{o}+n w_{m}\right) t\right.}
$$

The signs (-) and (+) in (4.10) and (4.11) represent the backward and forward directions of progressive phase shift distributions, respectively, see Figure 4.2. To this end, we assumed elements modulated by single-tone signals in our derivations. A comprehensive model must treat elements modulated by signals with an arbitrary number of tones (i.e., single-tone, two-tone, and multi-tone signals), so to derive their mathematical models, we will extend (4.10) and (4.11) instead of deriving again for the sake of simplicity to be as for the two-tone modulation signals

$$
\emptyset_{k+m}=l \times\left( \pm k \emptyset_{m 1} \pm m \emptyset_{m 2} \pm \emptyset_{o}\right)
$$

and

$$
\begin{aligned}
E\left(\theta, k w_{m 1}+m w_{m 2}\right) & =\frac{1}{2} \sum_{k=-\infty}^{\infty} \sum_{m=-\infty}^{\infty} c_{m+k, l} e^{j\left(l \times\left( \pm k \emptyset_{m 1} \pm m \emptyset_{m 2} \pm \emptyset_{o}\right)\right)} \\
& \times e^{j\left(\left(w_{o}+k w_{m 1}+m w_{m 2}\right) t\right.}
\end{aligned}
$$




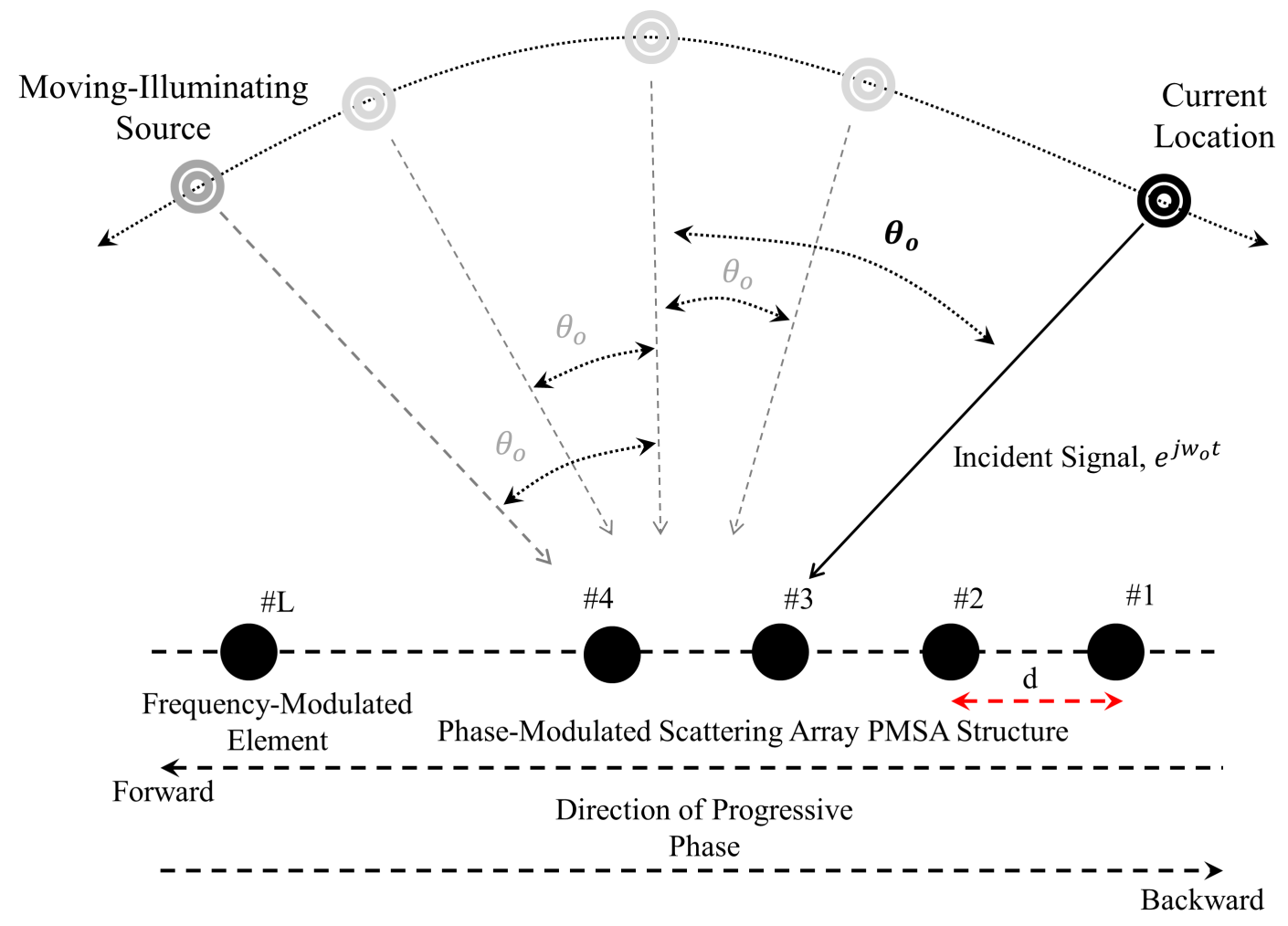

Figure 4.2: General representation of the PMSA with potential locations of the illuminating source and directions of progressive phase shift distributions of the modulation sources

for the multi-tone modulation signals

$$
\emptyset_{k+m+s+\cdots+x}=l \times\left( \pm k \emptyset_{m 1} \pm m \emptyset_{m 2} \pm s \emptyset_{m 3} \pm \cdots \pm j \emptyset_{m x} \pm \emptyset_{o}\right)
$$

and

$$
\begin{aligned}
E\left(\theta, k w_{m 1}+m w_{m 2}+\cdots+j w_{m x}\right) & =\frac{1}{2} \sum_{k=-\infty}^{\infty} \sum_{m=-\infty}^{\infty} \sum_{s=-\infty}^{\infty} \cdots \sum_{j=-\infty}^{\infty} d_{k+m+s+\cdots+j, l} \\
& \times e^{j\left(l \times\left( \pm k \emptyset_{m 1} \pm m \emptyset_{m 2} \pm s \emptyset_{m 3} \pm \cdots \pm j \emptyset_{m x} \pm \emptyset_{o}\right)\right)} \\
& \times e^{j\left(w_{o}+k w_{m 1}+m w_{m 2}+s w_{m 3}+\cdots+j w_{m x}\right) t}
\end{aligned}
$$




\subsubsection{An Array of Frequency-Modulated Scatterers (Phase-Modulated Scattering Array)}

Having derived and defined all field quantities induced over a single frequency-modulated element (scatterer) using single-tone, two-tone, and multi-tone modulations signals, separately, Now consider an array of frequency-modulated elements is placed uniformly along the $\mathrm{x}$-axis where individual elements are modulated by sinusoidal signals having the same frequency and different phases. As shown in Figure 4.2, directions of progressive phase shift distributions play a vital role in determining spatial locations of scattered beams associated with distortion products. Thus, this property paves the way for exploring potential functions of the proposed system, including beamforming capabilities. Other capabilities will also be illustrated in detail in Chapter 5.

For the case of plane wave signals arriving from an angle $\theta_{o}$, signals experience three different processes during their journey from the illuminating source (transmitter) side into the PSMA elements and gets back to the receiver side. First, inducing signals on elements apertures, they result from interactions between individual elements of the PMSA and the incident signals. Next, modulating the higher frequency (illuminating) signals by the lower frequency (modulation) signals is the second process called the modulation process. Eventually, re-transmitting (scattering) modulated signals into space happens due to the establishment of standing waves of distortion products signals over antennas apertures. Frequencies of induced distortion products must lie within bandwidths of antennas utilized in the PMSA to ensure scattering the most power of effective distortion products.

Fortunately, the previous section explained mathematically the first two processes as a preparation for the last process of signals manipulations. Figure 4.3 shows these three steps of processing that the incident signals experience throughout 
their journey.

Now, in the last step, we have multiple frequency-modulated elements, and each element produces the same distortion products. Besides, distortion products are non-coherent in their nature (i.e., different frequencies and different phases) hence every single product has its particular radiation beam that could be directed into different spatial location from radiation beams of other distortion products. However, this holds true if phases of signal sources modulating antennas are not equal. If this happens, it makes the distortion products directed into different spatial locations. In other words, we can say that a specific distortion product emerging at a specific location in the field of view of the PMSA (SFoV) may not show up again in other different locations relying on some factors as will be seen later. This process resembles the functionality of radio frequency $R F$ circuits that support specific distortion products and eliminate others. However, RF circuits work to enhance a single distortion product at a time, but our PMSA enhances different distortion products at different locations in space simultaneously. This feature is of significant interest in the PMSA, being summation (i.e., enhancement and cancellation) of distortion products in space at multiple different locations.

Mathematically speaking, addition of distortion products generated by different scatterers in space can be expressed as 


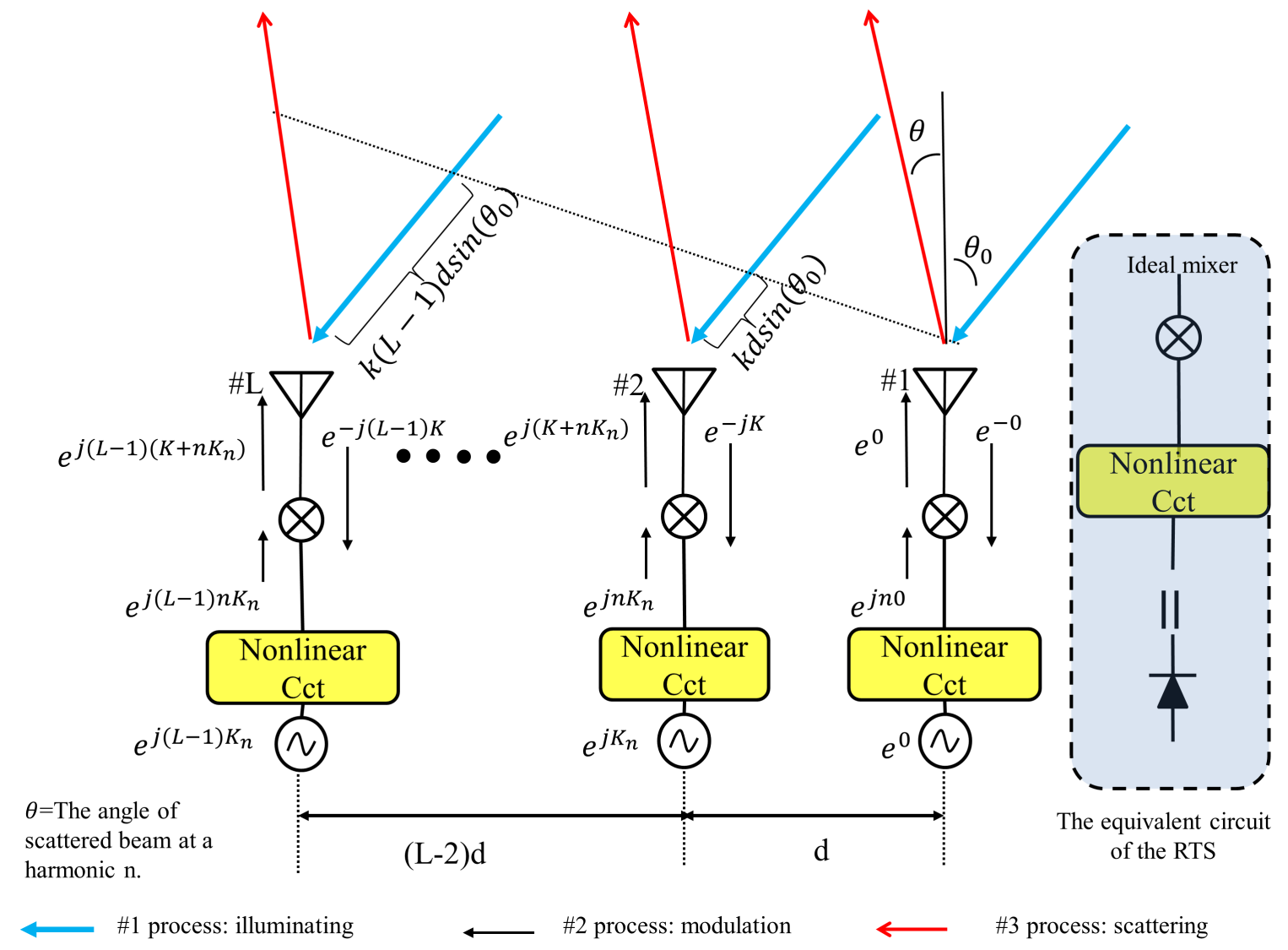

Figure 4.3: Stages of signal manipulation by the PMSA

for the single-tone modulation sources

$$
\begin{aligned}
E_{s}\left(\theta, n w_{m}\right) & =\frac{1}{2} \sum_{n=-\infty}^{\infty} b_{n, 0} e^{j\left(0 \times\left( \pm n \emptyset_{m} \pm \emptyset_{o}\right)\right)} \times e^{j\left(w_{o}+n w_{m}\right) t} \times e^{\left(0 \times j K_{n} d \sin (\theta)\right)} \\
& +\frac{1}{2} \sum_{n=-\infty}^{\infty} b_{n, 1} e^{j\left(1 \times\left( \pm n \emptyset_{m} \pm \emptyset_{o}\right)\right)} \times e^{j\left(w_{o}+n w_{m}\right) t} \times e^{\left(1 \times j K_{n} d \sin (\theta)\right)} \\
& +\frac{1}{2} \sum_{n=-\infty}^{\infty} b_{n, 2} e^{j\left(2 \times\left( \pm n \emptyset_{m} \pm \emptyset_{o}\right)\right)} \times e^{j\left(w_{o}+n w_{m}\right) t} \times e^{\left(2 \times j K_{n} d \sin (\theta)\right)}+\cdots \\
& +\frac{1}{2} \sum_{n=-\infty}^{\infty} b_{n, L-1} e^{j\left((L-1) \times\left( \pm n \emptyset_{m} \pm \emptyset_{o}\right)\right)} \times e^{j\left(w_{o}+n w_{m}\right) t} \\
& \times e^{\left((L-1) \times j K_{n} d \sin (\theta)\right)}
\end{aligned}
$$


or

$$
E_{s}\left(\theta, n w_{m}\right)=\frac{1}{2} \sum_{n=-\infty}^{\infty} \sum_{l=0}^{L-1} b_{n, l} e^{j\left(l\left(K_{n} d \sin (\theta) \pm n \emptyset_{m} \pm \emptyset_{o}\right)\right)} e^{j\left(w_{o}+n w_{m}\right) t}
$$

Each term in the right side of (4.16), as can be seen, consists of an infinite number of distortion products. The same distortion products repeat themselves as many as scatterers in the PMSA. As conventional phased antenna arrays have a closed mathematical form exploiting the geometric series formula [34], Eq. (4.16) can be expressed using the same closed mathematical form to be written as

$$
\begin{aligned}
& E_{S}\left(\theta, n w_{m}\right) \\
& =\frac{1}{2} \sum_{n=-\infty}^{\infty} b_{n} e^{j\left(\frac{L-1}{2}\right)\left(K_{n} d \sin (\theta) \pm n \emptyset_{m \pm} \emptyset_{o}\right)}\left\{\frac{\sin \left(\frac{L}{2}\left(K_{n} d \sin (\theta) \pm n \emptyset_{m} \pm \emptyset_{o}\right)\right.}{\sin \left(\frac{1}{2}\left(K_{n} d \sin (\theta) \pm n \emptyset_{m} \pm \emptyset_{o}\right)\right.}\right\} \cdot e^{j\left(w_{o}+n w_{m}\right) t}
\end{aligned}
$$

for the single-tone modulation sources.

Ideally speaking, Eq.(4.18) consists of an infinite number of array factors and each array factor supports a specific distortion product term $n$. However, most of these array factors do not bear real power (evanescent fields). The few residual array factors bearing real power (propagation fields) have maximum values when their arguments inside the brackets are equal to zero.

$$
K_{n} d \sin (\theta) \pm n \emptyset_{m} \pm \emptyset_{o}=0
$$

where $K_{n}$ are wave vectors of new frequencies (distortion products) generated from the nonlinear operations. It can be given as

$$
K_{n}=K+n K_{m}=\frac{w_{o}}{c}+n \frac{w_{m}}{c}
$$

where $c$ is the velocity of light in free-space. The term $\emptyset_{o}$ which is $K d \sin \theta_{o}$ and (4.20) 
are substituted in (4.19) to obtain

$$
\left(K+n K_{m}\right) d \sin (\theta) \pm K d \sin \left(\theta_{o}\right) \pm n \emptyset_{m}=0
$$

Then, angles, where array factors are maximized, can be established from (4.21) as

$$
\theta_{n}=\arcsin \frac{\mp K d \sin \left(\theta_{o}\right) \mp n \emptyset_{m}}{\left(K+n K_{m}\right) d}
$$

where each $\theta$ associated with separate distortion product term $n$ is replaced by $\theta_{n}$.If values of $\emptyset_{m}$ are equidistant (i.e., $\left.2 \pi / L\right)$, Eq.(4.22) becomes as

$$
\theta_{n}=\arcsin \frac{\mp K d \sin \left(\theta_{o}\right) \mp n \frac{2 \pi}{L}}{\left(K+n K_{m}\right) d}=\arcsin \frac{\mp L K d \sin \left(\theta_{o}\right) \mp 2 n \pi}{L\left(K+n K_{m}\right) d}
$$

Here in our proposed design, we assume $w_{m}<<w_{o}$, resulting in $K_{m}<<K$. Thus, angles of scattered beams given in (4.23) are turned out into

$$
\theta_{n}=\arcsin \frac{\mp L K d \sin \left(\theta_{o}\right) \mp 2 n \pi}{L K d}
$$

Eq.(4.24) confirms that directions of scattered beams are related to Fourier coefficients or distortion product term orders $n$. To extend this study to include modulation sources with any number of tones, their derivation would be cumbersome, so we will restrict ourselves only to the final results, taking advantages of the previous equations of the single-tone modulation sources for the sake of simplicity. 
For the two-tone modulation sources, Eqs.(4.18) and (4.22) can be rewritten as

$$
\begin{aligned}
E_{S}\left(\theta, k w_{m 1}+m w_{m 2}\right) & =\frac{1}{2} \sum_{k=-\infty}^{\infty} \sum_{m=-\infty}^{\infty} c_{k+m} e^{j\left(\frac{L-1}{2}\left(K_{k+m} d \sin (\theta) \pm k \emptyset_{m 1} \pm m \emptyset_{m 2} \pm \emptyset_{o}\right)\right)} \\
& \times \frac{\sin \left(\frac{L}{2}\left(K_{k+m} d \sin (\theta) \pm k \emptyset_{m 1} \pm m \emptyset_{m 2} \pm \emptyset_{o}\right)\right)}{\sin \left(\frac{1}{2}\left(K_{k+m} d \sin (\theta) \pm k \emptyset_{m 1} \pm m \emptyset_{m 2} \pm \emptyset_{o}\right)\right)} \\
& \times e^{j\left(w_{o}+k w_{m 1}+m w_{m 2}\right) t}
\end{aligned}
$$

and

$$
\theta_{k+m}=\arcsin \frac{\mp K d \sin \left(\theta_{o}\right) \mp k \emptyset_{m 1} \mp m \emptyset_{m 2}}{\left(K+(k+m) K_{m 1, m 2}\right) d}
$$

where $K_{m+k}$ is equal to

$$
K_{k+m}=K+(k+m) K_{m 1, m 2}=\frac{w_{o}}{c}+\frac{k w_{m 1}+m w_{m 2}}{c}
$$

and for the multi-tone modulation sources, Eqs.(4.18) and (4.22) become as

$$
\begin{aligned}
E_{S}\left(\theta, w_{t o t}\right) & =\frac{1}{2} \sum_{k=-\infty}^{\infty} \sum_{m=-\infty}^{\infty} \sum_{s=-\infty}^{\infty} \cdots \sum_{j=-\infty}^{\infty} \\
& \times d_{t o t} e^{j\left(\frac{L-1}{2}\left(K_{t o t} d \sin (\theta) \pm k \emptyset_{m 1} \pm m \emptyset_{m 2} \pm s \emptyset_{m 3} \pm \cdots \pm j \emptyset_{m x} \pm \emptyset_{o}\right)\right)} \\
& \times \frac{\sin \left(\frac{L}{2}\left(K_{t o t} d \sin (\theta) \pm k \emptyset_{m 1} \pm m \emptyset_{m 2} \pm s \emptyset_{m 3} \cdots \pm j \emptyset_{m x} \pm \emptyset_{o}\right)\right)}{\sin \left(\frac{1}{2}\left(K_{t o t} d \sin (\theta) \pm k \emptyset_{m 1} \pm m \emptyset_{m 2} \pm s \emptyset_{m 3} \cdots \pm j \emptyset_{m x} \pm \emptyset_{o}\right)\right)} \\
& \times e^{j\left(w_{o}+w_{t o t}\right) t}
\end{aligned}
$$

and

$$
\theta_{k+m+s+\cdots+j}=\arcsin \frac{\mp K d \sin \left(\theta_{o}\right) \mp k \emptyset_{m 1} \mp m \emptyset_{m 2} \mp s \emptyset_{m 3} \mp \cdots \mp j \emptyset_{m x}}{\left(K+(k+m+s+\cdots+j) K_{m 1, m 2, m 3, \ldots, m x}\right) d}
$$

where $w_{t o t}=k w_{m 1}+m w_{m 2}+s w_{m 3}+\cdots+j w_{m x}$, and tot $=k+m+s+\cdots+j$. 
$K_{k+m+s+\cdots+j}$ is equal to

$$
\begin{aligned}
K_{k+m+s+\cdots+j} & =K+(k+m+s+\cdots+j) K_{m 1, m 2, m 3, \ldots, m x} \\
& =\frac{w_{o}}{c}+\frac{k w_{m 1}+m w_{m 2}+s w_{m 3}+\cdots+j w_{m x}}{c}
\end{aligned}
$$

If we set $d=0.5 \lambda_{o}, K_{n} d$ will be equal to

$$
K_{n} d=\left(\frac{2 \pi}{\lambda_{n}}\right) \cdot 0.5 \lambda_{o}=\pi \frac{\lambda_{o}}{\lambda_{n}}
$$

where $\lambda_{n}$ are wavelengths of distortion products $n$ and they are given by for the single-tone modulation sources

$$
\lambda_{n}=\frac{c}{f_{o}+n f_{m}}
$$

for the two-tone modulation sources

$$
\lambda_{k+m}=\frac{c}{f_{o}+k f_{m 1}+m f_{m 2}}
$$

for the multi-tone modulation sources

$$
\lambda_{k+m+s+\cdots+j}=\frac{c}{f_{o}+k f_{m 1}+m f_{m 2}+s f_{m 3}+\cdots+j f_{m x}}
$$

Because, in our measurements, we will use very low modulation frequency $f_{m}\left(f_{m}<<\right.$ $f_{o}$ ) which is about $1 \mathrm{kHz}$ and $f_{o}$ about $2.3 \mathrm{GHz}$, then $\lambda_{n}, \lambda_{k+m}$, and $\lambda_{k+m+s+\cdots+j}$ in (4.32), (4.33), and (4.34) will be equal to

$$
\lambda_{o}=\frac{c}{f_{o}}
$$


Moreover, to plot scattered beams of distortion products, it is suitable to normalize their beams. Typically, this can be carried out by dividing scattered beams by their maximum values. In the presented designs, maximum values of scattered beams of distortion products are given by $L B_{n}$ for the single-tone modulation sources. As a result, all normalized scattered beams have equal amplitudes ( 1 or $0 \mathrm{~dB})$, so we cannot easily distinguish between scattered beams of different distortion products. Hence, we will divide scattered beams given in (4.18), (4.25), and (4.28), by values $L B_{0}, L C_{0}$, and $L D_{0}$, respectively. In other words, scattered beams are divided by maximum values of the fundamental distortion products in order to preserve variations between amplitudes of different distortion products.

We obtain the normalized scattered beams of distortion products after substituting $d=0.5 \lambda_{o}, \emptyset_{o}=K d \sin \left(\theta_{o}\right)=\pi \sin \left(\theta_{o}\right)$, equal phases for all modulation tones $\emptyset_{m 1, m 2, m 3, \cdots}=2 \pi / L, \lambda_{o}=\lambda_{n}=\lambda_{k+m}=\lambda_{k+m+s+\cdots+j}$ in (4.18), (4.25), and (4.28), separately, to yield as for the single-tone modulation sources

$$
\begin{aligned}
\left|E_{S, \text { norm }}\left(\theta, n w_{m}\right)\right| & =\frac{1}{L B_{0}} \sum_{n=-\infty}^{\infty} B_{n} e^{j\left(w_{o}+n w_{m}\right) t} \\
& \times\left[\frac{\sin \left(\frac{\pi L}{2}\left(\sin (\theta) \pm \sin \left(\theta_{o}\right) \pm \frac{2}{L}(n)\right)\right)}{\sin \left(\frac{\pi}{2}\left(\sin (\theta) \pm \sin \left(\theta_{o}\right) \pm \frac{2}{L}(n)\right)\right)}\right]
\end{aligned}
$$

for two-tone modulation sources

$$
\begin{aligned}
\left|E_{S, \text { norm }}\left(\theta, k w_{m 12}+m w_{m 2}\right)\right| & =\frac{1}{L C_{0}} \sum_{k=-\infty}^{\infty} \sum_{m=-\infty}^{\infty} C_{k+m} e^{j\left(w_{o}+k w_{m 1}+m w_{m 2}\right) t} \\
& \times\left[\frac{\sin \left(\frac{\pi L}{2}\left(\sin (\theta) \pm \sin \left(\theta_{o}\right) \pm \frac{2}{L}(k+m)\right)\right)}{\sin \left(\frac{\pi}{2}\left(\sin (\theta) \pm \sin \left(\theta_{o}\right) \pm \frac{2}{L}(k+m)\right)\right)}\right]
\end{aligned}
$$


and for multi-tone modulation sources

$$
\begin{aligned}
\left|E_{S, \text { norm }}\left(\theta, w_{\text {tot }}\right)\right| & =\frac{1}{L D_{0}} \sum_{k=-\infty}^{\infty} \sum_{m=-\infty}^{\infty} \sum_{s=-\infty}^{\infty} \cdots \sum_{j=-\infty}^{\infty} D_{t o t} e^{j\left(w_{o}+w_{t o t}\right) t} \\
& \times\left[\frac{\sin \left(\frac{\pi L}{2}\left(\sin (\theta) \pm \sin \left(\theta_{o}\right) \pm \frac{2}{L}(k+m+s+\cdots+j)\right)\right)}{\sin \left(\frac{\pi}{2}\left(\sin (\theta) \pm \sin \left(\theta_{o}\right) \pm \frac{2}{L}(k+m+s+\cdots+j)\right)\right)}\right]
\end{aligned}
$$

The integers $n, k, m, s$, and $j$ could be positive or negative numbers.

As mentioned above, sings $( \pm)$ in (4.36), (4.37), and (4.38) represent directions of progressive phase shift distributions across PMSA elements. The $(+)$ denotes a direction of phase shift distribution when the right-most element (the first element) is chosen as a reference element and the phase shift distribution increases gradually until the left-most element (the last element), and when a direction of progressive phase shift is reversed, we should use the sign $(-)$. When modulation sources of the PMSA have the first type of phase shift distribution, it is called the forward (FWD) PMSA whereas the backward (reversed, RVD) PMSA belongs to the second type of the phase distribution. In chapter 8, modulation signals having the same phase distribution will be used, but in the real-world applications especially in active arrays, this is impossible since each modulation source considers as a user having its own direction in the spatial environment.

Moreover, spatial phase shift distributions of incident signals depend on directions of their arrival angles which can be represented by signs $( \pm)$. Thus we can have four combinations of phase shift distributions. However, phase shifts of incident signals can be ignored if signals arrive at an array aperture normally. Thus, the term $\sin \left(\theta_{o}\right)$ will be zero, and Eqs.(4.36), (4.37), and (4.38) are simplified to be as 
for the single-tone modulation sources

$$
\left|E_{S, n o r m}\left(\theta, n w_{m}\right)\right|=\frac{1}{L B_{0}} \sum_{n=-\infty}^{\infty} B_{n}\left\{\frac{\sin \left(\frac{\pi L}{2}\left(\sin (\theta) \pm n \frac{2}{L}\right)\right.}{\sin \left(\frac{\pi}{2}\left(\sin (\theta) \pm n \frac{2}{L}\right)\right.}\right\} \cdot e^{j\left(\left(w_{o}+n w_{m}\right) t\right.}
$$

for the two-tone modulation sources

$$
\begin{aligned}
\left|E_{S, n o r m}\left(\theta, k w_{m 12}+m w_{m 2}\right)\right| & =\frac{1}{L C_{0}} \sum_{k=-\infty}^{\infty} \sum_{m=-\infty}^{\infty} C_{k+m} e^{j\left(w_{o}+k w_{m 1}+m w_{m 2}\right) t} \\
& \times\left[\frac{\sin \left(\frac{\pi L}{2}\left(\sin (\theta) \pm \frac{2}{L}(k+m)\right)\right)}{\sin \left(\frac{\pi}{2}\left(\sin (\theta) \pm \frac{2}{L}(k+m)\right)\right)}\right]
\end{aligned}
$$

and for multi-tone modulation sources

$$
\begin{aligned}
\left|E_{S, \text { norm }}\left(\theta, w_{\text {tot }}\right)\right| & =\frac{1}{L D_{0}} \sum_{k=-\infty}^{\infty} \sum_{m=-\infty}^{\infty} \sum_{s=-\infty}^{\infty} \cdots \sum_{j=-\infty}^{\infty} D_{t o t} e^{j\left(w_{o}+w_{t o t}\right) t} \\
& \times\left[\frac{\sin \left(\frac{\pi L}{2}\left(\sin (\theta) \pm \frac{2}{L}(k+m+s+\cdots+j)\right)\right)}{\sin \left(\frac{\pi}{2}\left(\sin (\theta) \pm \frac{2}{L}(k+m+s+\cdots+j)\right)\right)}\right]
\end{aligned}
$$

Here, the PMSA scatters beams of negative and positive distortion products symmetrically about the broadside direction due to their conjugate phase shifts. Figure 4.4 shows the spatial representation of the scattered beams of distortion products generated by the PMSA when incident signals are normal.

To this end, all explanations made above were about the backscattering (reflection). Thus, in order to generalize the explanation, we will extend the mathematical models to take into account the forward-scattering (transmission). The forward-scattering is nothing more than flipping directions of scattered beams around the array axis. Thus, exploiting mathematical models of backscattering derived above facilitates the procedure. In the forward-scattering, angles of scattered beams of distortion products are images of their counterparts in the backscattering. In 
the backscattering, both incident and scattered signals exist, while being only the scattered signals in the forward-scattering. Models presented in (4.18), (4.25), and (4.28) represent the backscattered signals for single-tone, two-tone, and multi-tone, respectively. These models can be denoted by $E_{B S}$, and the forward-scattering can be denoted by $E_{F S} . E_{F S}$ is equal to $E_{B S} \cdot e^{j \pi}$ provided we omit incident signals, where the term $e^{j \pi}$ comes from that scattered beams are flipped around an array axis. For instance, when a scattered beam of the 1USB harmonic product appears at the left-side of FoV of the PMSA in the backscattering, it will emerge at the right-side in the forward-scattering. Figure 4.1 exhibits locations of scattered beams of the same distortion products in both regions.

Mathematically, fields in both regions can be related as

$$
\left[\begin{array}{c}
E_{B S} \\
E_{F S}
\end{array}\right]=\left[\begin{array}{cc}
R(w) & 1 \\
0 & T(w)
\end{array}\right]\left[\begin{array}{c}
A e^{j w_{o} t} \\
A e^{j w_{o} t}
\end{array}\right]
$$

where $R(w)$ and $T(w)$ are reflection and transmission properties of the PMSA. The relationship between $R(w)$ and $T(w)$ is

$$
T(w)=R(w) \cdot e^{j \pi}
$$

The reflection and transmission coefficients are expressed as

$$
R(w)=\sum_{n=0}^{\infty} b_{n, l} \cos \left(n\left(w_{m}+\emptyset_{m, l}\right)\right)
$$

and

$$
T(w)=\sum_{n=0}^{\infty} b_{n, l} \cos \left(n\left(w_{m}+\emptyset_{m, l}+\pi\right)\right)
$$

Eqs.(4.44) and(4.45) stand only for the PMSA when driven by the single-tone 
modulation sources.

$U S B$

$L S B$

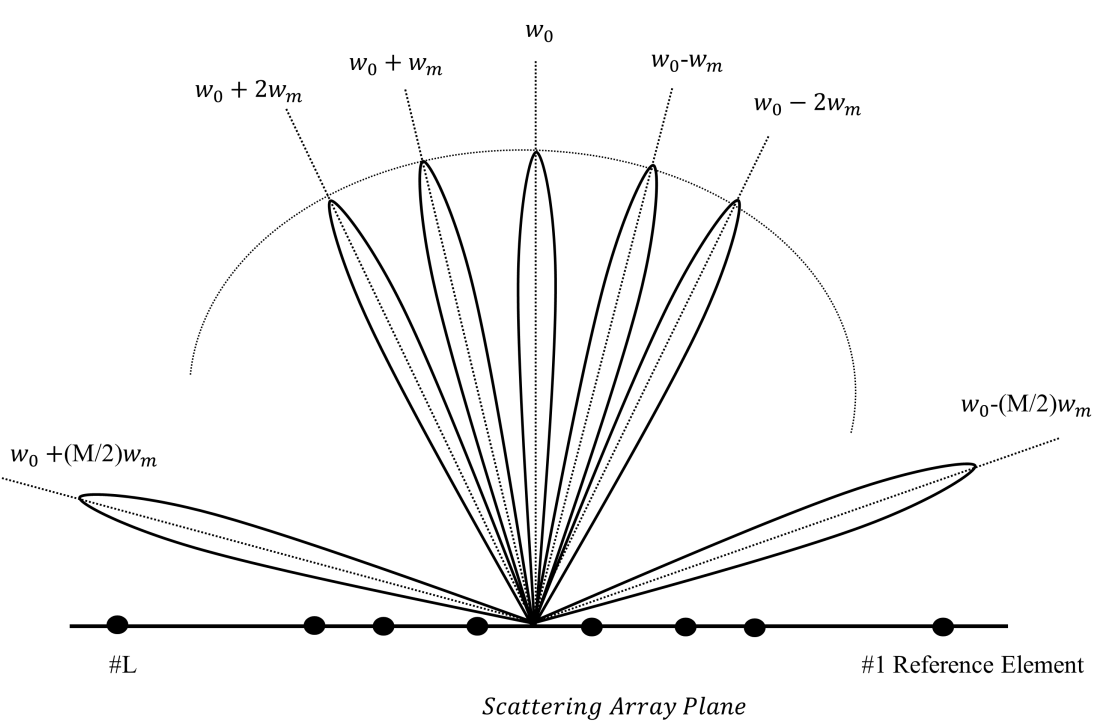

Figure 4.4: Spatial representation of scattered beams of distortion products generated by the PMSA when incident signals are normal 


\section{Chapter 5: Features and Some Applications of the PMSA}

\subsection{Introduction}

This chapter will mainly focus on exploring some features and applications of the PMSA which are not described in Chapters 3 and 4 . The scope of the previous two chapters was about how to derive mathematical models based on two different perspectives. These two different derived mathematical models open up the door to a plethora of curious features and applications that are not disclosed in the literature yet to the best of authors knowledge.

The diffraction grating-like behavior is one of the substantial features of the PMSA that is demonstrated in section 5.2. Generalizing a law for decomposition modes (distortion products), one can easily find out the spatial characteristics of modes, physical numbers of existing modes, and cutoff and retrodirective modes and how to control them. In section 5.3, another substantial feature of the PMSA being the non-reciprocity is established. This feature aids to build a structure able to deal with transmitting-receiving signals simultaneously without using particular devices such as circulators which are expensive and bulky. It is a promising feature of our presented structure. Subsequently, in section 5.4, we present a model function combining between the property and the application called the beamforming. The beamforming is the main and common feature of phased arrays. The PMSA may find its way in some beamforming applications for being a simple design, thereby reducing the cost. Then, the mathematical models of the proposed design will be utilized as a tool to analyze the distortion products generated in active arrays with an illustrative example supporting the model in section 5.5. Next, we will utilize the design as a tunable spatial harmonic generator in section 5.6. The tunability feature may make 
the PMSA useful in different applications such as radars. Eventually, in section 5.7, the wide angle performance of the direction finding application is described.

\subsection{The Diffraction Grating-Like Behavior}

In this section, we present one of the most important features of the PMSA model which is the diffraction grating-like behavior. The process of scattering of modes (i.e., distortion products) generated by the PMSA is somehow similar to the process of diffracting modes by diffraction grating structures. The PMSA is able to scatter decomposed modes spatially in different directions depending on the design properties and a mode number. Figure 5.1 illustrates a comparison between a conventional diffraction grating and the PMSA. The diffraction grating is made of a series of grooves etched over a glass or a metal surface while the PMSA is an array of scatterers, antennas integrated with modulators, placed linearly on a one axis. The diffraction grating is an optical device, whereas the PMSA model can be scaled up from low frequencies (acoustic band) to higher frequencies (terahertz $\mathrm{THz}$ band). In fixed diffraction grating devices, design properties cannot be altered [104] [122]. In contrast, parameters governing spatial properties of scattered modes (distortion products) generated by the PMSA can be readily changed, leading into a system with reconfigurable spatial properties. Relative phase shift differences $\emptyset_{m}$ of the modulations sources and their progressive directions (i.e., \pm ), distances separating elements $d$, directions of the incident signals, and frequencies are the major factors determining the spatial properties of distortion products (modes) of the PMSA. The parameters that have control over the spatial characteristics of modes make our PMSA

distinct bucause they can be varied without the need to change the design. Bear in mind, the word "mode" is interchangeable with the word "distortion product" wherever you find it in this section. Moreover, when using multi-tone modulation 
sources, newly mixed frequencies will appear in the spatial response of the PMSA due to the nonlinearities of active devices where it is impossible in conventional diffraction grating devices when shined by several incident signals at the same time.

In the following subsection, generalized law for the spatial scattered modes of the PMSA is presented.

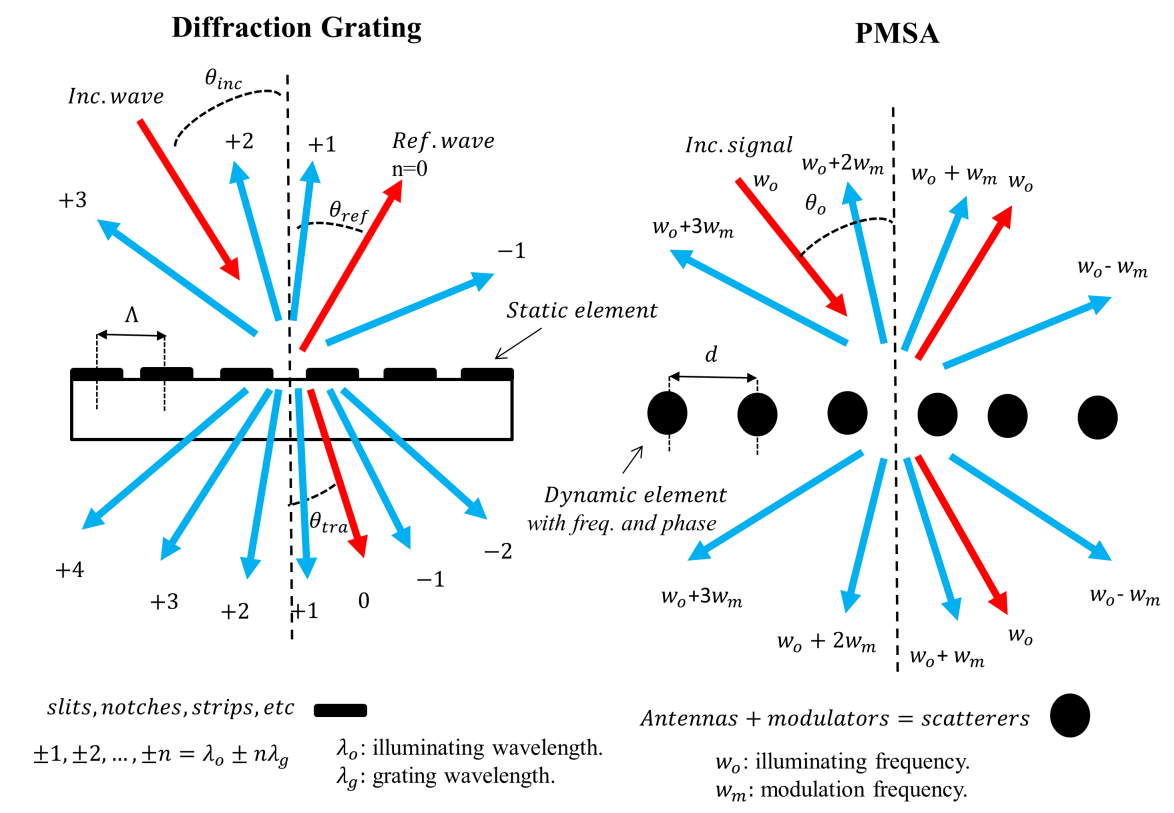

Figure 5.1: An illustrative comparison between the diffraction grating and the PMSA

\subsubsection{The Equation of Spatial Characteristics of Distortion Products (Modes)}

Referring to (4.21), an equation representing spatial characteristics of scattered modes of the PMSA when using the single-tone modulation sources can be written as

$$
K_{n} \sin \left(\theta_{n}\right)=K_{o} \sin \left(\theta_{o}\right) \mp n \frac{\emptyset_{m}}{d}
$$


Eq.(5.1) resembles the principal equation of the diffraction grating which is given by $[104]$

$$
K_{m} \sin \left(\theta_{m}\right)=K_{i n c} \sin \left(\theta_{i n c}\right)-m \frac{2 \pi}{\Lambda}
$$

where $K_{m}, K_{i n c}, \theta_{m}, \theta_{i n c}, \Lambda$, and $m$ are the wave number of the $m-t h$ diffracted mode, the wave number of incident mode, the diffracted angle of $m-t h$ mode, the angle of incident mode, the spacing between grating pitches, and the $m$ - th mode term number, respectively. Because (5.1) is similar to (4.21), we will not define its terms for the sake of simplicity. We can see that both equations (5.1) and (5.2) are similar, but there are two explicit differences. The first difference is the sign before the second terms in the right-side of both equations. In the diffraction grating equation, the sign cannot be altered unless we build a different diffraction grating. Whereas we can easily change the sign of the PMSA equation once we reverse a direction of the phase shift distribution of the modulation sources. The second difference is that we have terms $\emptyset_{m}$ and $d$ in (5.1) which are variable, while being constant terms $2 \pi$ and $\Lambda$ in (5.2). Thus, the terms $\emptyset_{m}$ and $d$ aid to build a reconfigurable (real-time) PMSA, making a substantial difference compared to conventional diffraction grating devices.

Each $\theta_{n}$ denotes a scattered angle associated with its own $n-t h$ mode term. Ideally, there is an infinite number of modes (spatial distortion products), but only few of them are actually propagating modes. Only these modes can carry real power. To differentiate between the propagating and non-propagating modes (evanescent), Eq.(5.1) must be solved for $\theta_{n}$. In case of the modulation frequencies being much smaller than the incident frequencies, $K_{n}$ will be almost equal to $K_{o}\left(K_{n} \approx K_{o}\right)$, which results in a dispersion-less structure.

As an example, we set $\emptyset_{m}=2 \pi / L$ as the standard values for phases of the modulation sources, where $L$ is the total number of scatterers in the PMSA, the elements spacing $d$ is a half wavelength $\lambda_{o} / 2$ at the incident frequency $f_{o}$, and $K_{n}=K_{o}=2 \pi / \lambda_{o}$. 
In addition, we consider only the forward-progressive phase distribution so that only the sign (-) in the second part of the right-side in (5.1) is taken into account. After applying all these substitutions and doing few simplifications, Eq.(5.1) becomes

$$
\sin \left(\theta_{n}\right)=\sin \left(\theta_{o}\right)-\left(\frac{2 n}{L}\right)
$$

When the incident signal in Figure 5.1 becomes normal, $\theta_{o}=0^{\circ}$, the term $\sin \left(\theta_{o}\right)$ in (5.3) will be dropped, leading into

$$
\sin \left(\theta_{n}\right)=-\left(\frac{2 n}{L}\right)
$$

For $L=4$, the first scattered modes to appear are $n= \pm 1$. These modes bend toward $\theta_{ \pm 1}= \pm \arcsin (1 / 2)= \pm 30^{\circ}$. The following scattered modes bend toward $\theta_{ \pm 2}= \pm \arcsin (1)= \pm 90^{\circ}$, and the third order scattered modes are directed toward $\theta_{ \pm 3}= \pm \arcsin (3 / 2)=$ imaginary angles (not exist). Moreover, the zero-order mode is reflected back into the same incident direction $\theta_{o}=0^{\circ}$, because $\theta_{0}=\arcsin (0)=0^{\circ}$. As a consequence, the different modes generated from the PMSA are directed into different angles, so it would be better if we classify them and determine which mode can propagate, relying on their spatial scattered angles of modes. These modes can be classified as specular, propagating, cutoff, and evanescent modes. The specular mode is reflected back into the opposite direction of the incident angle $-\theta_{0}$ due to effects of the phase inversion and absence of modulation effects. Here in this example $\theta_{0}=0^{\circ}$ so that the angles of incident and specular modes are not different. Furthermore, this mode carries real power, and most times is dominant because of its highest energy. Other modes which also carry real power are the scattered propagating modes, and they are placed between the normal-axis $\theta=0^{\circ}$ and off-axis $\theta= \pm 90^{\circ}$ directions. Other modes being the cutoff and the evanescent modes are located at $\pm 90^{\circ}$ angles and 
beyond, respectively. Powers of the cutoff modes are highly attenuated by patterns of the PMSA antennas, while being reactive powers (not real) in the evanescent modes. Figure 5.2 displays wavefronts of scattered modes in both reflection and transmission regions. It can be seen that the fundamental mode does not experience any phase shift as seen in Figure 5.2a. Also, the 1LSB mode is bent toward the angles $30^{\circ}$ and $-30^{\circ}$ in regions above (backscattering) and under (forward-scattering) of the PMSA axis, respectively, as seen in Figure 5.2b. In Figure 5.2c, the 1USB mode is similar to the 1LSB mode but it propagates in the opposite direction. The $\pm 1^{\text {st }}$ modes have symmetrical bending angles leading us to conclude that all the higher order modes $\pm n$ are distributed symmetrically when incident signals are normal. This symmetry between the positive and negative modes disappears once the incident signals depart the normal trajectory. Thus, in order to validate the context of the model with any incident angle, repeating the former example with an incident angle of $-30^{\circ}$ is given in Figure 5.3. The phase gradient introduced by the oblique incidence is altered or canceled out by varying phases of the modulation sources. The same mode for both incident angles has different wavefront directions. For example, the 1LSB mode directs toward the angle $+30^{\circ}$ in Figure $5.2 \mathrm{~b}$, while it directs toward $+90^{\circ}$ in Figure 5.3b. Hence, directions of the incident signals play a vital role in determining directions of scattered modes. Realization of the mode beamforming can be carried out just by changing an angle of the incident signals, and the real-time direction tunability of scattered modes has become possible without using real phase shifters. A functionality of beamforming will be described later in this chapter. As can be seen, some modes exchanged their status (i.e., types of modes). For example, the cutoff mode in Figure 5.2d becomes a propagating mode in Figure 5.3d, and the propagating mode in Figure 5.2b becomes a cutoff mode in Figure 5.3b. To generalize the discussion given above when utilizing multi-tone modulation sources, 


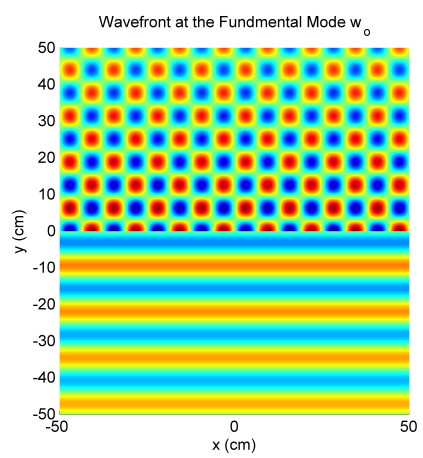

(a)

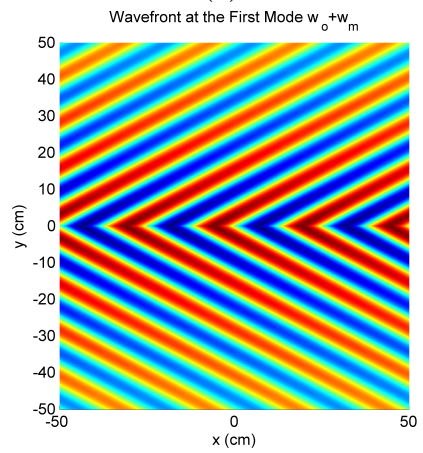

(c)

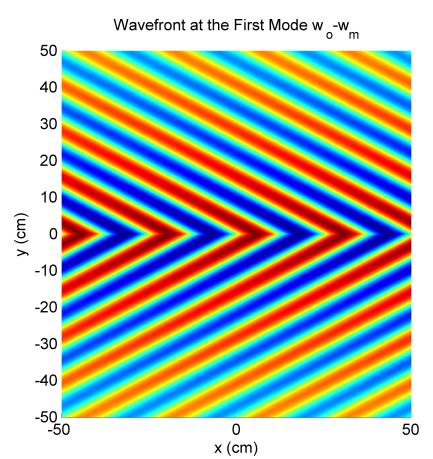

(b)

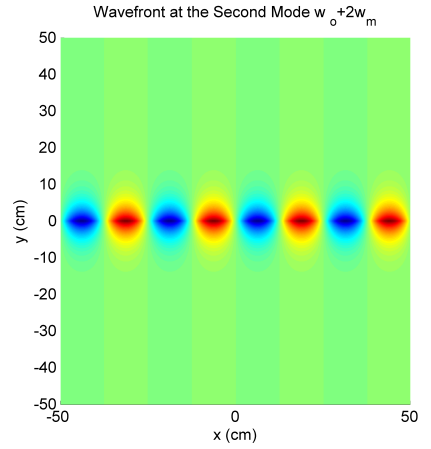

(d)

Figure 5.2: Wavefronts of modes at normal incident signals a) the zero mode (specular) b) the first LSB mode(propagating) c) the first USB mode (propagating) d) the second USB mode (cutoff)

it just adds phase contributions resulting from extra tones into (5.1).

For the two-tone modulation sources, the scattering equation becomes

$$
K_{k+m} \sin \left(\theta_{k+m}\right)=K_{o} \sin \left(\theta_{o}\right) \mp k \frac{\emptyset_{m 1}}{d} \mp m \frac{\emptyset_{m 2}}{d}
$$

For the multi-tone modulation sources, the scattering equation becomes

$$
K_{k+m+s+\cdots+j} \sin \left(\theta_{k+m+s+\cdots+j}\right)=K_{o} \sin \left(\theta_{o}\right) \mp\left(\frac{k \emptyset_{m 1}+m \emptyset_{m 2}+s \emptyset_{m 3}+\cdots+j \emptyset_{m x}}{d}\right)
$$




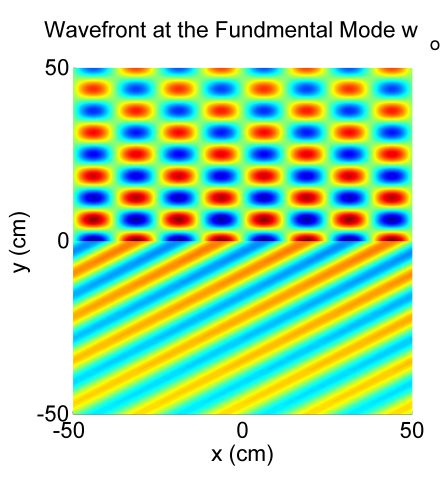

(a)

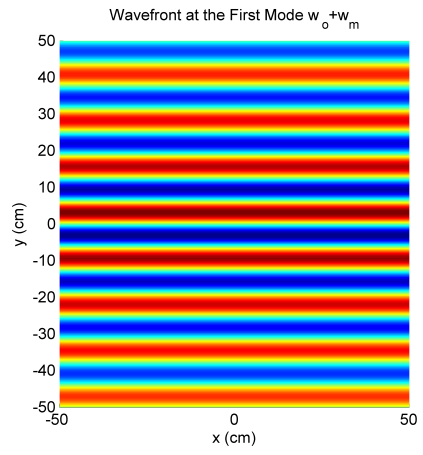

(c)

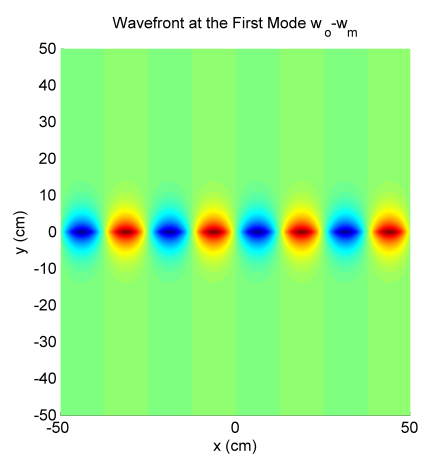

(b)

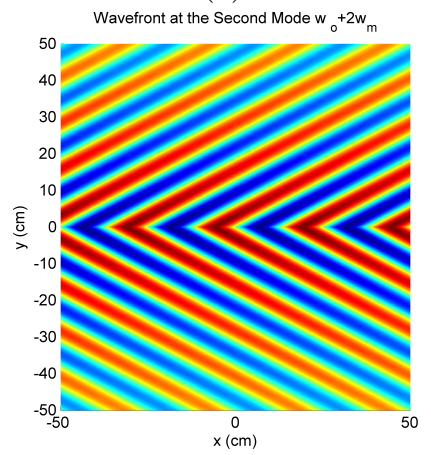

(d)

Figure 5.3: Wavefronts of modes at $-30^{\circ}$ incident signals a) the zero mode (specular) b) the first LSB mode (cutoff) c) the first USB mode (propagating) d) the second USB mode (propagating)

\subsubsection{Cutoff Modes and the Total Number of Real Scattered Modes}

When the scattered angle $\theta_{n}$ becomes imaginary, the $n$-th mode will not transfer real power, being an evanescent mode. The mode separating between the propagating and evanescent modes is called the cutoff mode. If this mode exists, it always appears at an angles $\pm 90^{\circ}$. Mathematically, to figure out the cutoff modes, $\theta_{n}$ should be replaced by $\pm 90^{\circ}$ in (5.1), and after doing some mathematical simplifications, the cutoff mode equation can be expressed as

$$
n_{\text {cutoff }}=\frac{-K_{o} d\left(\sin \left( \pm 90^{\circ}\right)+\sin \left(\theta_{o}\right)\right)}{\emptyset_{m}}
$$


where $\sin \left( \pm 90^{\circ}\right)= \pm 1$, and the sign $(-)$ in the onset of the right side of the equation helps to distinct between modes of forward and the backward phase distributions of the modulation sources. The same assumptions given above in the example are utilized again here, $L=4, d=\lambda_{o} / 2, \emptyset_{m}=2 \pi / L$, and two values for $\theta_{o}=0^{\circ}$ and $30^{\circ}$. Therefore, Eq.(5.7) becomes

$$
n_{\text {cutoff }}= \pm\left( \pm 2+2 \sin \left(\theta_{o}\right)\right)
$$

If the forward phase distribution across the PMSA is only considered, we can rewrite (5.8) as

$$
n_{\text {cutoff }}=-\left( \pm 2+2 \sin \left(\theta_{o}\right)\right)
$$

Table 5.1 presents the modes (distortion products) of the PMSA according to their types for two different incident angles. Table 5.1 also highlights the cutoff modes in order to clarify that the their orders will be varied in accordance with directions of the incident signals.

Table 5.1: Types of distortion products (modes) for $L=4$ with two different incident angles

\begin{tabular}{c|c|c|c|c|c|c|c}
\hline \hline \multicolumn{7}{c}{ Mode Number } \\
\hline Incident Angle & $-3^{\text {rd }}$ & $-2^{\text {nd }}$ & $-1^{\text {st }}$ & $0^{t h}$ & $1^{\text {st }}$ & $2^{\text {nd }}$ & $3^{\text {rd }}$ \\
\hline $0^{\circ}$ & $\mathrm{E}$ & $\mathbf{C}$ & $\mathrm{P}$ & $\mathrm{P}$ & $\mathrm{P}$ & $\mathbf{C}$ & $\mathrm{E}$ \\
\hline$-30^{\circ}$ & $\mathrm{E}$ & $\mathrm{E}$ & $\mathbf{C}$ & $\mathrm{P}$ & $\mathrm{P}$ & $\mathrm{P}$ & $\mathbf{C}$ \\
\hline E: Evanescent, P: Propagating, C: Cutoff \\
\hline \hline
\end{tabular}

It is worth noting that the total number of propagated modes is similar for both incident angles although some modes are converted from propagation into cutoff and vice versa. Given a separating distance $d$, a number of scatterers $L$, and a relative modulation phase difference $\emptyset_{m}$, we can calculate how many propagating modes exist, confined between angles of the two cutoff modes, in the visible view of the PMSA. The term $\sin \left(\theta_{n}\right)$ should be less than one to ensure having propagating modes. Assuming 
normal incidence again, it can be expressed as

$$
\left|\sin \left(\theta_{n}\right)\right|=\left|\mp n \frac{\emptyset_{m}}{K_{o} d}\right|<1
$$

Therefore, a maximum value for $n$ is

$$
n_{\max }=\frac{K_{o} d}{\emptyset_{m}}
$$

and the total number of possible propagating modes $M$ is

$$
M=2 n_{\max }-1=\frac{2 K_{o} d}{\emptyset_{m}}-1
$$

\subsubsection{Incident Frequency Sensitivity}

Previously, we assumed that a frequency of the incident signals $f_{o}$ used in all equations derived above is constant. If this is not the case, then spatial directions of scattered modes will be no longer the same, thus it is advantageous to find a relationship connecting between the variations in frequencies of incident signals and angles of scattered modes.

Starting with the scattering equation in (5.1), it reduces the time of calculation and facilitates understanding the procedure of the sensitivity derivation. To explicitly include a frequency of the incident signals in the scattering equation, Eq.(5.1) becomes as

$$
\sin \left(\theta_{n}\right)=\sin \left(\theta_{o}\right) \mp n \frac{\emptyset_{m} f_{o}}{2 \pi d c}
$$


The definition of the incident frequency sensitivity is the difference in scattered angles if a frequency of the incident signals varies $\left(\partial \theta_{n} / \partial f_{o}\right)$.

$$
\frac{\partial \theta_{n}}{\partial f_{o}}=\frac{ \pm n \emptyset_{m}}{2 \pi d c \cdot \cos \left(\theta_{n}\right)}
$$

where $\mathrm{c}$ is the light speed in free-space. The scattered angle sensitivity is

$$
\Delta \theta_{n}=\frac{ \pm n \emptyset_{m}}{2 \pi d c \cdot \cos \left(\theta_{n}\right)} \Delta f_{o}
$$

The facts can be deduced from (5.15) are

1. Angles of high order modes are affected more than angles of low order modes.

2. Using larger d reduces the scattered angle sensitivity.

3. Using lower values of phases (i.e., more elements in the PMSA) reduces the sensitivity of scattered angles.

\subsubsection{Retrodirective Modes}

As illustrated before, scattered beams of distortion products are propagating to reach different directions in space according to specific factors governing the PMSA design. One of these scattered beams could propagate back toward a direction of the illuminating source under specific circumstances, as shown in Figure 5.4. Because this is similar to functional operation of retrodirective arrays [8] [151] [79] [159], these modes can be called as retrodirective modes. This happens, when applying $\theta_{n}=\theta_{o}$, and substituting this condition to (5.1), the retrodirective modes are obtained as

$$
n_{\text {retro }}=-\frac{2 K_{o} \sin \left(\theta_{o}\right) d}{\emptyset_{m}}
$$


Assuming $d=\lambda_{o} / 2$ and $\emptyset_{m}=2 \pi / L$ again here as in the previous examples, Eq.(5.16) becomes

$$
n_{\text {retro }}=-L \sin \left(\theta_{o}\right)
$$

For $L=4$, Table 5.1 illustrates the retrodirective modes with different incident angles

Table 5.2: Illustration of the retordirective modes for $L=4$ with five different incident angles

\begin{tabular}{c|c|c|c|c|c}
\hline \hline \multicolumn{7}{c}{ Mode Number } \\
\hline Incident Angle & $-2^{\text {nd }}$ & $-1^{s t}$ & $0^{t h}$ & $1^{\text {st }}$ & $2^{\text {nd }}$ \\
\hline$-30^{\circ}$ & No & No & No & No & Yes \\
\hline$-15^{\circ}$ & No & No & No & Yes & No \\
\hline $0^{\circ}$ & No & No & Yes & No & No \\
\hline $15^{\circ}$ & No & Yes & No & No & No \\
\hline $30^{\circ}$ & Yes & No & No & No & No \\
\hline Yes=retrodirective, No $\neq$ retrodirective \\
\hline \hline
\end{tabular}

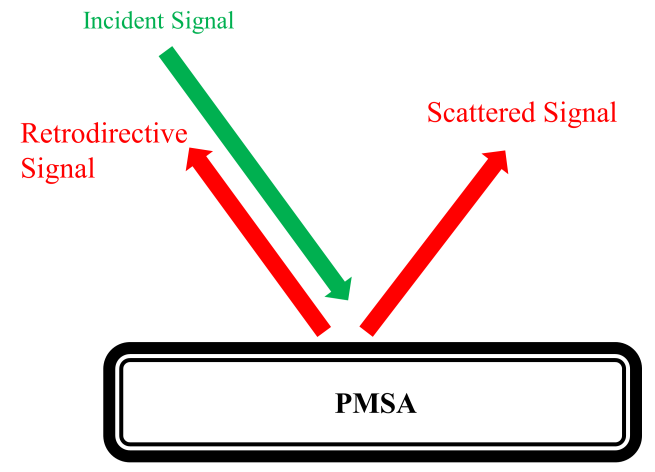

Figure 5.4: Retrodirective modes in the PMSA

As given in Table 5.2, angles of the incident signals play a vital role in determining which mode will act as a retrodirective mode. Also, the retrodirective modes are either up-converted or down-converted in the frequency, whereas conventional retrodirective arrays do not convert frequencies of retrodirective signals. The conventional retrodirective arrays are usually used in wireless systems with security issues, so the property of the frequency conversion with the retrodirective properties make the PMSA more valuable. 


\subsection{The Non-Reciprocity}

In this section, we explore another exciting feature offered by our PMSA being the non-reciprocal functionality. Here, the PMSA acts as a spatial frequency selector. In other words, it passes a specific signal through a particular direction, but the same signal will be blocked in the opposite path or redirect it into a different direction. This feature is quite interesting, so shedding light on it becomes very necessary to enrich the research work with features and potential applications of the presented PMSA. Due to the directionality of phase shift distributions of the modulation sources [20] [161] [85] [160] [46], which advances progressively from left to right or from right to left across the array, the PMSA design is inherently non-reciprocal. The resulting device could be considered as a spatial two-port network, performing tasks of spatial frequency modulation-demodulation (mixing), spatial duplexing, and scattering array (radiation) at the same time, thus operating as a full duplex transceiver. Moreover, the design will be a low profile printed structure, whereas conventional non-reciprocal devices are bulky due to the use of magnets as a core of their parts [86], and hence suffer from integrating with printed printed circuits. This is a promising feature, and is expected to find itself in some distinct applications.

\subsubsection{Half-Duplex Non-Reciprocal Operations}

Assuming a single source with a frequency $w_{o}$ illuminating the PMSA from a particular angle $\theta_{o}$, the signal is up-converted into the 1USB mode and retransmitted at a different angle $\theta_{1}$, where the direction of progressive phase distribution is from right to left as shown in Figure 5.5a. From the General scattering equation (5.1), we obtain

$$
K_{o} \sin \left(\theta_{o}\right)=-K_{1} \sin \left(\theta_{1}\right)-\frac{\emptyset_{m}}{d}
$$


In Figure 5.5b, when the same upconverted signal, $w_{1}=w_{o}+w_{m}$, is applied at an angle $\theta_{1}$, with using the same analysis, the downconverted, $w_{-1}=w_{1}-w_{m}=$ $w_{o}+w_{m}-w_{m}=w_{o}$ and upconverted, $w_{1}=w_{1}+w_{m}=w_{o}+w_{m}+w_{m}=w_{o}+2 w_{m}$ modes are given by

$$
K_{1} \sin \left(\theta_{1}\right)=K_{-1} \sin \left(\theta_{-1^{\prime}}\right)-\frac{\emptyset_{m}}{d}
$$

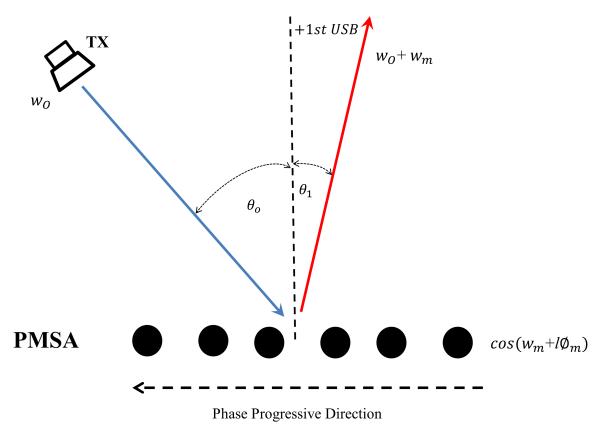

(a)

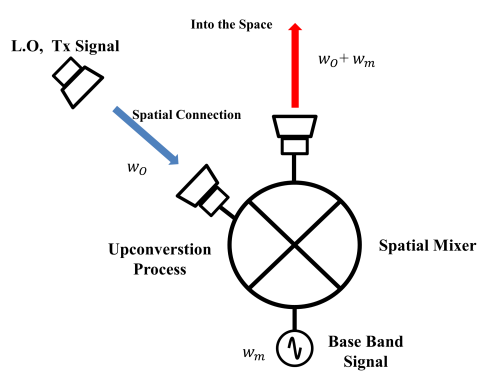

(c)

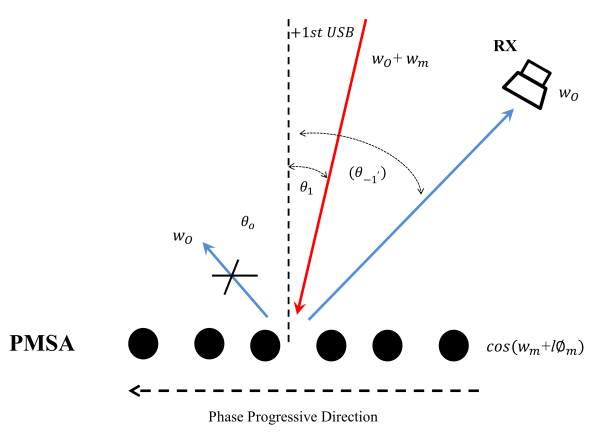

(b)

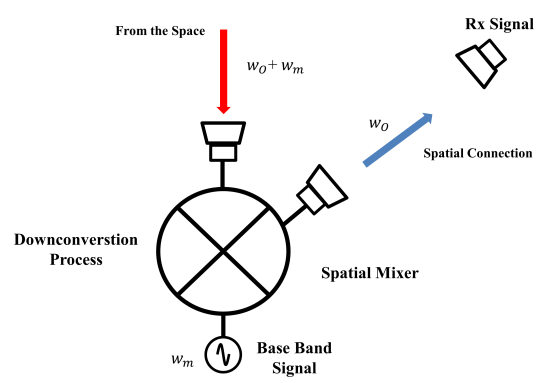

(d)

Figure 5.5: Nonreciprocal half-duplex system a) upconversion part b) downconversion part c) equivalent schematic of the upconversion d) equivalent schematic of the downconversion

$$
K_{1} \sin \left(\theta_{1}\right)=K_{1}^{\prime} \sin \left(\theta_{1^{\prime}}\right)+\frac{\emptyset_{m}}{d}
$$

We add primes on the mode numbers in the last two equations in order to distinguish between the two states of the incident signals. Furthermore, the sign $(-)$ is added before the first part of the right-side of (5.18), but it is not added in (5.19) and (5.20) because the incident signal in the first equation is propagated across the array 
elements from left to right. From Eqs. (5.18), (5.19), and (5.20), we can obtain

$$
K_{-1}^{\prime} \sin \left(\theta_{-1^{\prime}}\right)=K_{1} \sin \left(\theta_{1}\right)+\frac{\emptyset_{m}}{d}=-K_{o} \sin \left(\theta_{o}\right)-\frac{\emptyset_{m}}{d}+\frac{\emptyset_{m}}{d}=-K_{o} \sin \left(\theta_{o}\right)
$$

$$
K_{1}^{\prime} \sin \left(\theta_{1^{\prime}}\right)=K_{1} \sin \left(\theta_{1}\right)-\frac{\emptyset_{m}}{d}=-K_{o} \sin \left(\theta_{o}\right)-\frac{\emptyset_{m}}{d}-\frac{\emptyset_{m}}{d}=-K_{o} \sin \left(\theta_{o}\right)-\frac{2 \emptyset_{m}}{d}
$$

Since $K_{-1}^{\prime}$ is equal to $K_{o}$ as mentioned above, It can be concluded from (5.21) that angles $\theta_{-1}^{\prime}$ and $\theta_{o}$ are not equal, resulting in that the reflected back beam of the signal $\left(w_{-1^{\prime}}=w_{o}\right)$ is not propagating toward the same direction of the incident beam $\left(w_{o}\right)$ in Figure 5.5a. As a result, the PMSA can behave as a two port unidirectional isolator. The ports 1 and 2 are located along the propagation path of the incident beam at $\theta_{o}$ and the backscattered beam at $\theta_{1}$, respectively. Propagation of the illuminating signal from port 1 into port 2 is allowed, while it is prohibited to propagate oppositely from port 2 to port 1 . Although the signal $\left(w_{o}\right)$ is prohibited from reaching port 1 coming from port 2, it will be redirected toward a different direction as mathematically illustrated in (5.21). From a circuit theory point of view, we can say that $S 21 \neq S 12$, and $S 12=0$, which is essential for devices such as circulators. Eq.(5.21) reveals the angle of $\theta_{-1}^{\prime}$ depends on $\emptyset_{m}$ and $\theta_{o}$, which leads to easily control angles of scattered beams while operating in the RF band as we will see in the next section.

From Eq.(5.22) we can see that the PMSA generates another band $\left(w_{o}+2 w_{m}\right)$ reflected back which is possible to reach the incident source again. This frequency band can be completely removed using trivial filtering. Figure 5.5c and 5.5d show the equivalent schematics of non-reciprocal systems when utilizing them as 
spatial upconverter and downconverter mixers, respectively. Moreover, the signal $w_{o}$ in Figure 5.5b reaching the angle $-\theta_{o}$ experiences a downconversion process (demodulation), while the signal $w_{o}$ in Figure 5.5a experiences an upconversion process (modulation) before reaching the angle $\theta_{1}$. These two frequency conversion processes could be combined to come up with a full transceiver. If we re-invoke the same example given above with an incident angle $\theta_{o}=-30^{\circ}$, angles $\theta_{1}$ and $\theta_{-1}^{\prime}$ are $0^{\circ}$ and $30^{\circ}$, respectively. In the upconversation part, a transmitter is located at an angle where the phase distribution of incident signals and the phase distribution of modulation signals at the 1USB component are canceled out by each other, resulting in the up-conversion scattered beam at the broadside $0^{\circ}$. Whereas the phase shift distribution of incident signals at $\theta_{1}=0^{\circ}$ and the phase shift distribution of modulation signals at the 1LSB component are added together, resulting in bending the downconverted scattered beam away from the broadside to reach the angle $\theta_{-1}^{\prime}=30^{\circ}$. Interestingly, if the progressive phase distribution is reversed to start from the opposite end, the same conclusion can be adopted, but angles of upconverted and downconverted components, in principle, are spontaneously switched. Also, these angles can be continuously tuned to make an adaptive (reconfigurable) full transceiver for security issues.

\subsubsection{Full-Duplex Non-Reciprocal Device}

Having discussed operations of both frequency downconversion and upconversion separately, now, we can combine them to create a full duplex system. The structure operating here as a combined antenna-circulator (duplexer)-mixer may be fully understood from equations illustrated above. Figure 5.5 confirms that the 1USB product of the transmitter located at -30 is directed toward the broadside 0 , while the 1LSB of incident angle coming from the broadside $0^{\circ}$ is directed toward the receiver 
located at $30^{\circ}$, knowing that a frequency of the original signal of a transmitter and the 1LSB product of $0^{\circ}$ incident signal are the same.

\subsection{Beamforming of Distortion Products}

As illustrated previously, the PMSA consists of high-speed RF switches (RTSs) and antenna to construct nonlinear elements, mainly responsible for generating distortion products. Each single distortion product is non-coherent with other distortion products and has its particular associated scattered beam, and it is thus easy to control them. The non-coherent nature of distortion products ignites our curiosity if we can exploit and control them separately. One of these exploitations is to control directions of scattered beams associated with each single distortion product. It can be called multi-frequency multi-beam scanning technique what distinguishes our PMSA design from other conventional beamforming techniques.

To steer beam patterns in conventional phased arrays, relative phase shifts must be applied to all elements of an array except the reference element [108]. Integrating phase shifters into a structure is not an easy task as well as increasing the cost. These issues are entirely alleviated with the PMSA. The idea behind leaving the use of phase shifters while maintaining the beamforming functionality is that RF switches are not turned $\mathrm{ON}-\mathrm{OFF}$ at the same time. The process of sequentially turning ON-OFF switches in the time domain is similar to the use of phase shifters in the frequency domain. In other words, an advance or a delay in the time-domain will be a lead or a lag in a phase shift in the frequency-domain. Therefore, the PMSA is inherently able to scan beams of distortion products, leading to that the beamforming functioning of the PMSA combines between the property and application. As a consequence, synthesizing any phase shift value will be an easy task by using a time sequence with different time steps and $\mathrm{ON}-\mathrm{OFF}$ instants. Moreover, directions of time sequences 
also play a vital role in determining and steering beams of distortion products.

The last factor directly affecting values of phases of distortion products is directions of the incident signals, which in turn, changes directions of distortion products beams.

These three factors are repeatedly mentioned before and here again for the sake of completeness. More importantly, as RF switches of the PMSA can be governed electronically, the time sequence (phase modulation) can be readily reprogrammed to select any arbitrary phase shift in real-time, thus providing a continuously tunable (adaptive) PMSA.

\subsubsection{Numerical Examples}

Every distortion component in (4.17), (4.25), and (4.28) generated by scattering arrays has a scattered beam versus $\theta$ and follows a rule of $\sin (L y) / \sin (y)$, where $y$ represents arguments of functions inside brackets in these equations with maximum values being obtained at $y=0$. Peaks occur when the condition in (4.21) is satisfied. Peaks of distortion products are located at different spatial angles and can change their locations if anyone of the three factors mentioned above is varied, leading to steering all distortion products concurrently. This attribute along with the nonreciprocal feature can be considered as essential bricks to build a robust system against interference and intended noise.

In the following, two examples with 4 and 8 elements of uniform linear PMSAs with $d=0.7 \lambda_{o}$ and $0.5 \lambda_{o}$ are simulated using Matlab. In addition to that, the modulation signals employed in the simulations are both single and two tones time sequences to demonstrate that not only harmonics but also intermodulation

distortions IMDs can be controlled and directed into any spatial locations. This capability to work with any number and all types of distortion products is a privilege 
for the PMSA over conventional phased arrays which work only with fundamental frequencies. This step has been taken for being required to predict the overall performance of the PMSA with varying number of tones and with different steps of time sequences. Many factors will be addressed in the simulations to investigate the robustness of the model presented in Chapter 4.

\subsubsection{Example 1: 4-element PMSA with $d=0.7 \lambda_{o}$}

Now consider a simple example of 4-element uniform linear phase-modulated scattering array PMSA. The spacing between elements is $d=0.7 \lambda_{o}$. Here it is assumed that PMSA elements are isotropic; that is, scattering signal amplitude and gain of each scatterer are uniform. Also, we do not take into account the mutual coupling between elements. Transmitting and receiving antennas are placed in the far-field of the PMSA. In the simulation, a fictional TX antenna is placed at some selected spatial locations, and at each location for the Tx antenna, another fictional RX antenna moves angularly from most negative angle, backfire or $-90^{\circ}$, into the most positive angle, endfire or $90^{\circ}$. Scattered powers are detected and recorded at every two degrees. In all simulations, we set modulation and illuminating frequencies as $1 \mathrm{kHz}$ and $12 \mathrm{kHz}$, respectively, because these values are similar to the measurement results after down-conversion as a step to unify the results.

In the first part of the example, we use single-tone (modulation sources), and Eq.(4.17) is implemented in Matlab to illustrate beamforming capabilities of the single-tone PMSA. Results shown in Figure 5.6 represent scattered beams of the PMSA for three different locations of the illuminating signal (fictitious transmitter) which are $\left(-21^{\circ}, 0^{\circ}\right.$ and $\left.21^{\circ}\right)$ as well as when reversing direction of the progressive phase shift distribution of the modulation sources at the incident angle of $0^{\circ}$. It can be seen for example that the scattered beams of the 1USB harmonic appears at three different 
spatial locations $0^{\circ}, 21^{\circ}$ and $-43^{\circ}$ in the visible region of the PMSA for $-21^{\circ}, 0^{\circ}$ and $21^{\circ}$ incident angles, respectively. Moreover, when the incident angles are $21^{\circ}$ and $-21^{\circ}$, the scattering beams of the 1USB and the 1LSB have other beams being grating lobes, emerging at angles $-43^{\circ}$ and $43^{\circ}$, respectively. Appearance of grating lobes of harmonics is due to the same reason in conventional uniform arrays [108]. The possibility of grating lobes occurrence increases as the scattered beams of distortion products move further away from the broadside direction $\theta=0^{\circ}$. This is an inherent problem even in phased arrays and is still there in our PMSA design. The distance $d$ between elements should also follow a rule in (5.23), to avoid grating lobes.

$$
d=\frac{\lambda_{o}}{1+\sin \theta}
$$

where $\lambda_{o}$ is the incident signal wavelength in free-space and $\theta$ is angles scattered beams. Although small spacing distances helps to avoid the occurrence of grating lobes, this could give rise to an increase of mutual coupling between elements. The system performance deteriorates quickly as mutual coupling increases with small separating distances. Thus, moderate distances should be selected as a tradeoff between these two issues. To show effects of large distances on scattered beams of distortion products, the results in Figure $5.6 \mathrm{~b}$ are re-simulated with $d=2 \lambda_{o}$ as shown in Figure 5.7, leading to scattered beams with several peaks. Moreover, we have added small lines in Figure 5.6 to indicate peaks of scattered beams.

Note that as shown in Figure 5.6, amplitudes of scattered beams are not the same due to nature of polynomial representation of distortion products generated in nonlinear components. Also, if we compare between Figure 5.6b and Figure 5.6d, note that directions of scattered beams of lower and upper sidebands distortion products are replaced by each other due to the change in directions of progressive 


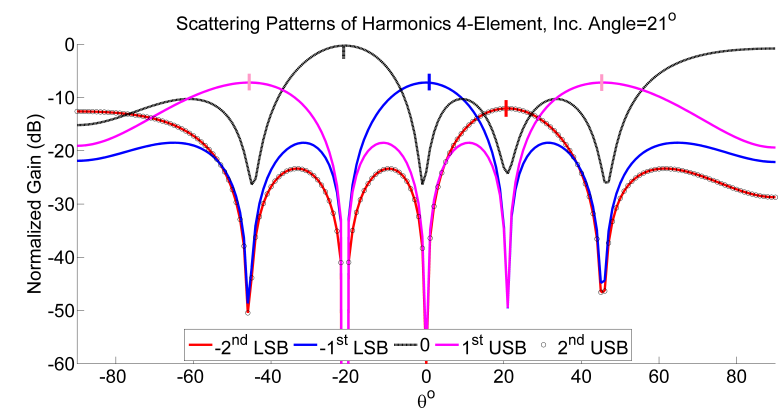

(a)

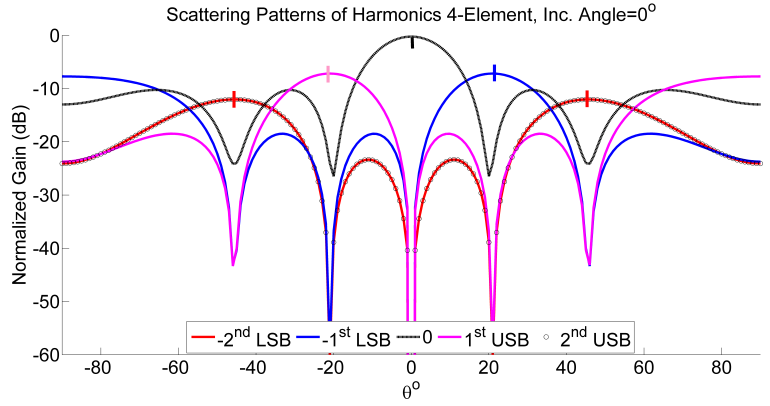

(b)

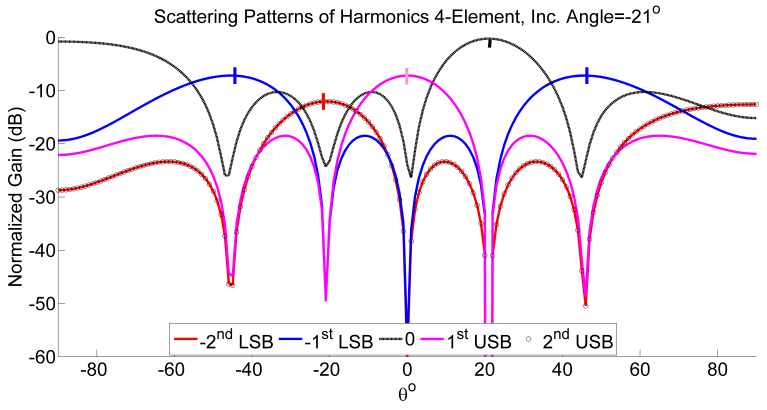

(c)

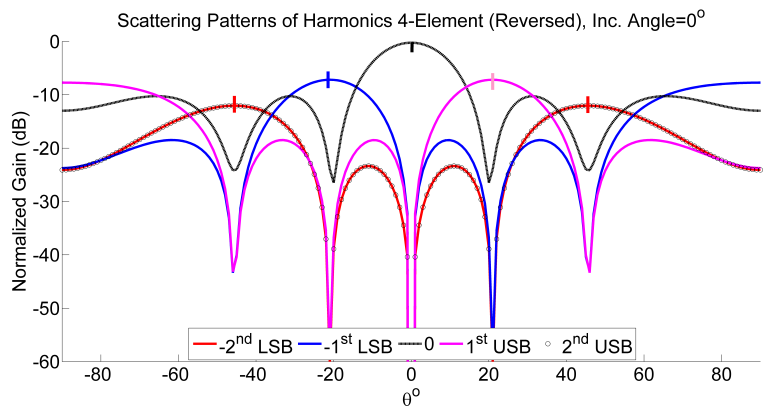

(d)

Figure 5.6: Scattered beams of distortion products for the 4-element PMSA for the incident angles a) $-21^{\circ}$ b) $0^{\circ}$ c) $21^{\circ}$ and d) $0^{\circ}$ reversed-type

phase shift distributions of the modulation sources $\emptyset_{m}$ across array elements. Like conventional phased arrays, scattered beams of distortion products get wider as they 
move away from the broadside direction $\theta=0^{\circ}$. Figure 5.6 shows only the spatial dependence of scattered beams, but not the frequency-dependence. More detailed representations of the example results presented in Figure 5.6 can be obtained if we use 3D plots. Figure 5.8 shows that the 3D plots of scattered beams of distortion products generated in the PMSA are directed into different scattered angles and each beam is tagged with a different frequency determined by the term order of distortion products. To delineate the relationship between frequencies and scattered angles of distortion products, Figure 5.8 also shows the top views of 3D plots.

Beamforming of distortion products can be carried out by changing either direction

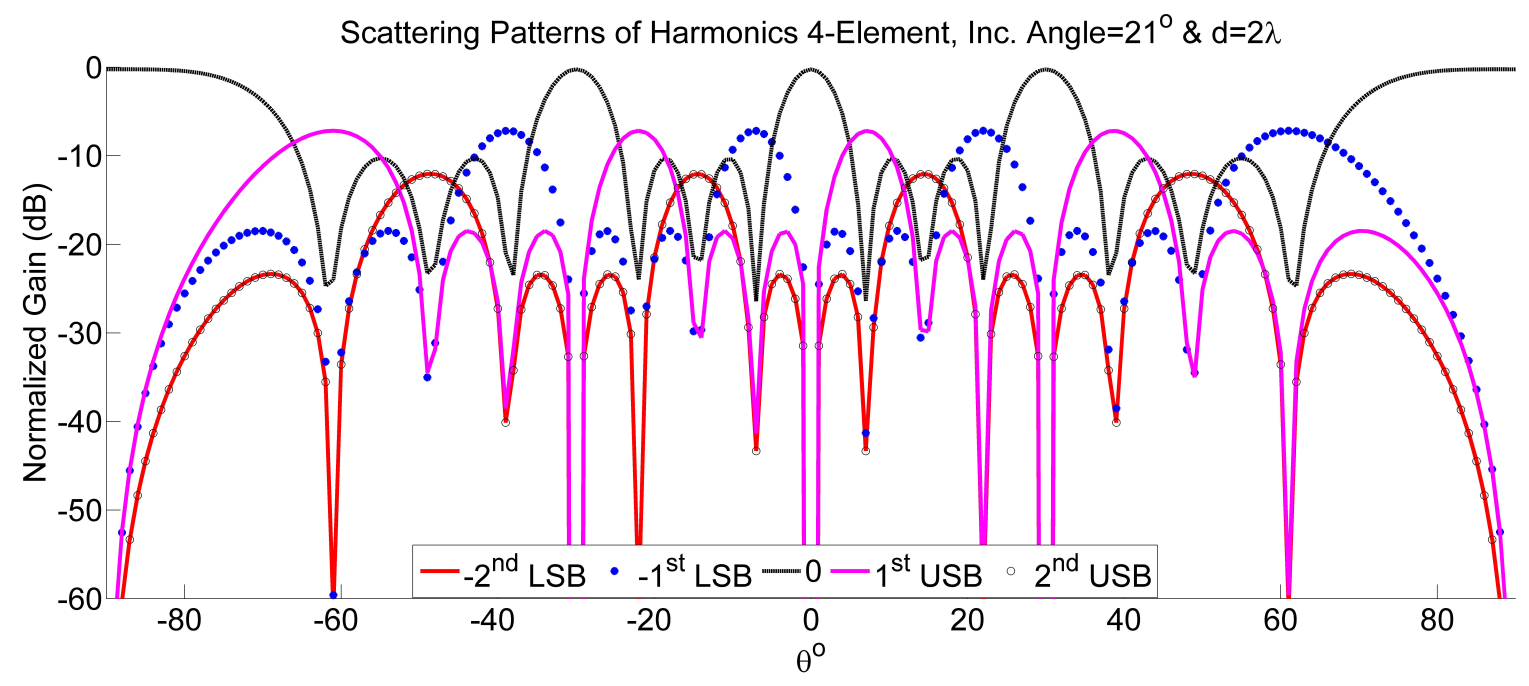

Figure 5.7: Scattered beams of distortion products for the 4-element PMSA with $d=2 \lambda$

of the the incident signals or phase shift distributions of the modulation signals. Because there are plenty of distortion products, we obtain only the 1USB and 1LSB, resulting from the 4-element PMSA as desired distortion products to demonstrate the beamforming capabilities. Figure 5.9a displays scattered beams of the 1USB and 1LSB distortion components when a relative phase shift difference of the modulation signals varies from $60^{\circ}$ to $10^{\circ}$ with a step $10^{\circ}$ for $\theta_{o}=0^{\circ}$. As can be seen, scattered beams approach the specular direction, which is at the broadside $\theta=0^{\circ}$ here, as the 
relative phase shift difference of the modulation sources decreases. As a consequence, when it becomes zero, all scattered beams of distortion products will have the same direction with the fundamental mode to reach the specular direction $-\theta_{o}$. Although scattered beams of higher orders are not shown, we can conclude from (4.22) and Figure 5.9a that as a distortion term number increases, scattered beams approach faster to the broadside direction. Figure 5.9b and 5.9c illustrate scattered beams of distortion products for two different incident angles $0^{\circ}$ and $21^{0}$, respectively, generated in the 4-element PMSA when all elements are switched ON-OFF simultaneously (i.e., no modulation phase shifts). Results do confirm application of the mathematical model presented in Chapter 4. At the first incident angle, all scattered beams of distortion products appear at the broadside $0^{\circ}$ for the $0^{\circ}$ incident angle while the same scattered beams appear at the angle $-21^{\circ}$ for the $21^{\circ}$ incident angle which is like Snell-reflection behavior. In addition to that, we display effects of incident angles on spatial locations of the 1USB and the 1LSB scattered beams, see Figure 5.10a and $5.10 \mathrm{~b}$, respectively. Figure 5.11 presents the potential locations of the 1USB, 1LSB, 2USB, and 2LSB components versus the incident angle and the modulation phase shifts for the 4-element PMSA with $\mathrm{d}=0.7 \lambda_{o}$.

Up to now, all the simulations results presented in the current section are limited to using single-tone modulations sources. Now, we consider the PMSA with two-tone modulation sources as a general example of the multi-tone PMSA. The difference when using two-tone modulation sources is that the scattered beams of distortion products are not only for harmonic distortion HD products but also for intermodulation distortion IMDs products. This increases a number of expected scattered beams of distortion product to make it as a forest of scattered beams. Thus, it is not easy task to deal with all of them. To plot all scattered beams of distortion products, it will be a heavy burden. Thus, we will suffice here with briefly providing 


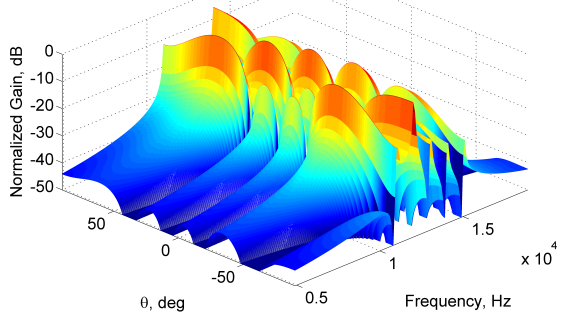

(a)

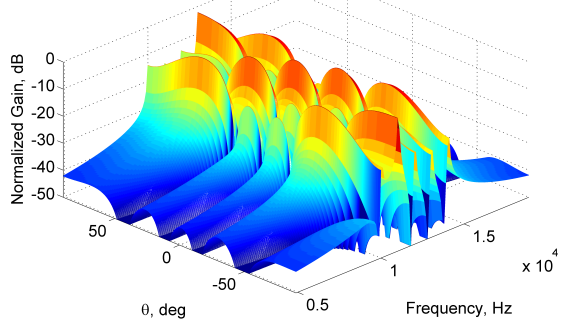

(c)

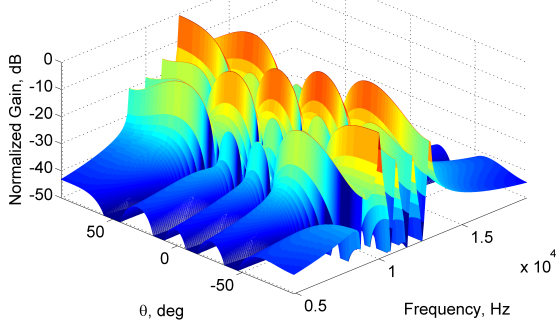

(e)

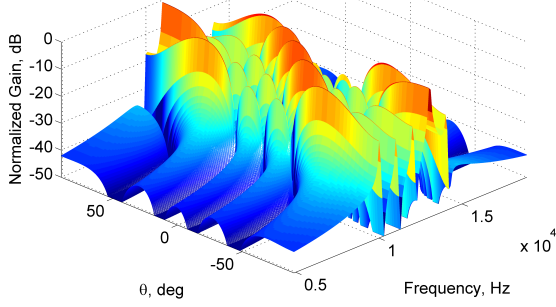

(g)

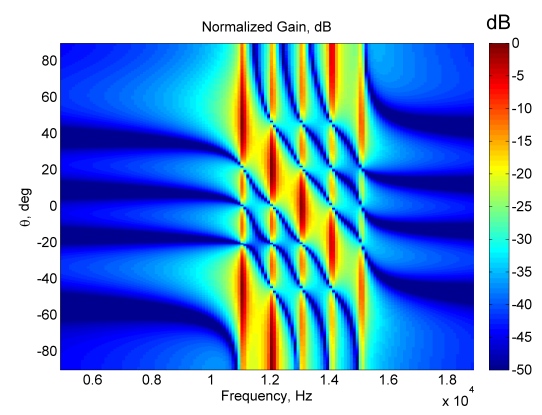

(b)

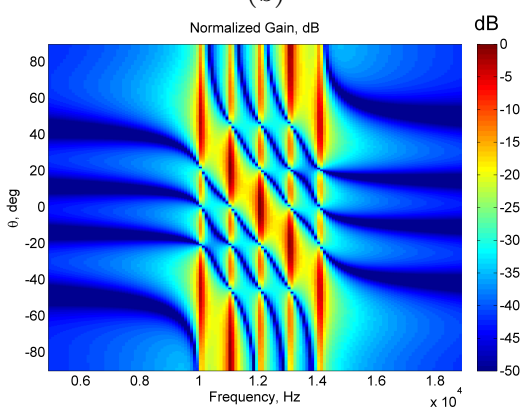

(d)

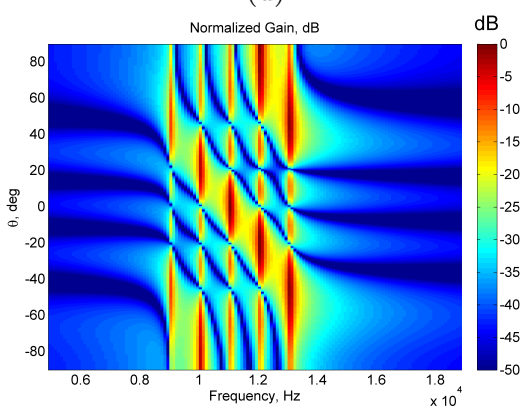

(f)

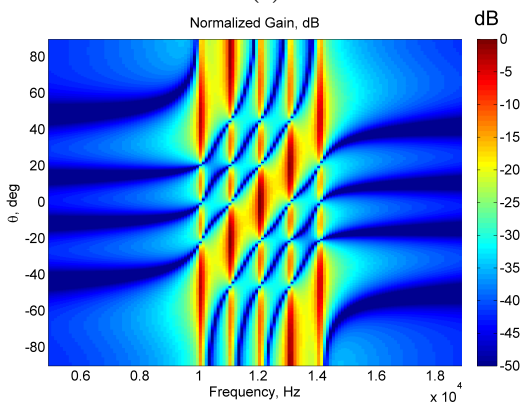

(h)

Figure 5.8: Plot of frequency varying scattered beams versus angle for different incident angles a) $\left.-21^{\circ} 3 \mathrm{D} \mathrm{b}\right)-21^{\circ} 2 \mathrm{D} \mathrm{c)} 0^{\circ} 3 \mathrm{D}$ and d) $0^{\circ} 2 \mathrm{D}$ e) $\left.21^{\circ} 3 \mathrm{D} \mathrm{f)} 21^{\circ} 2 \mathrm{D} \mathrm{g}\right) 0^{\circ} 3 \mathrm{D}$ inverted-type h) $0^{\circ} 2 \mathrm{D}$ inverted-type 


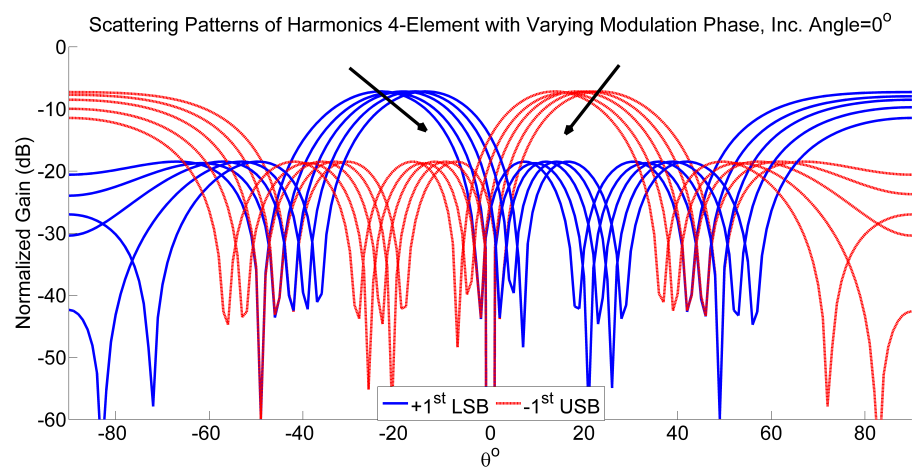

(a)

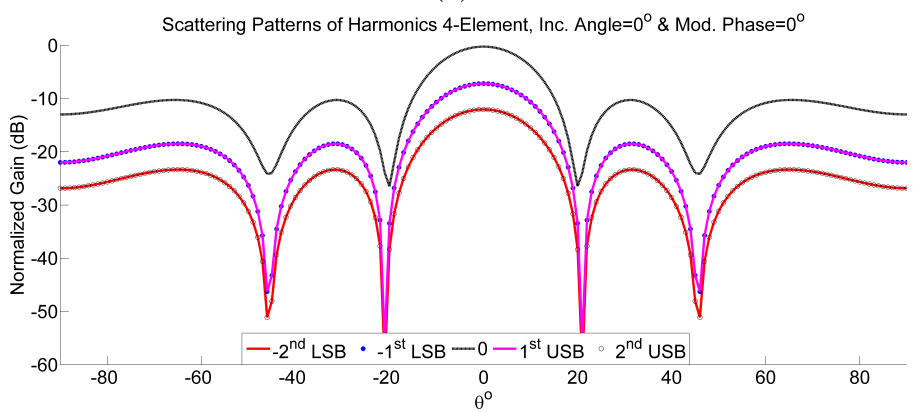

(b)

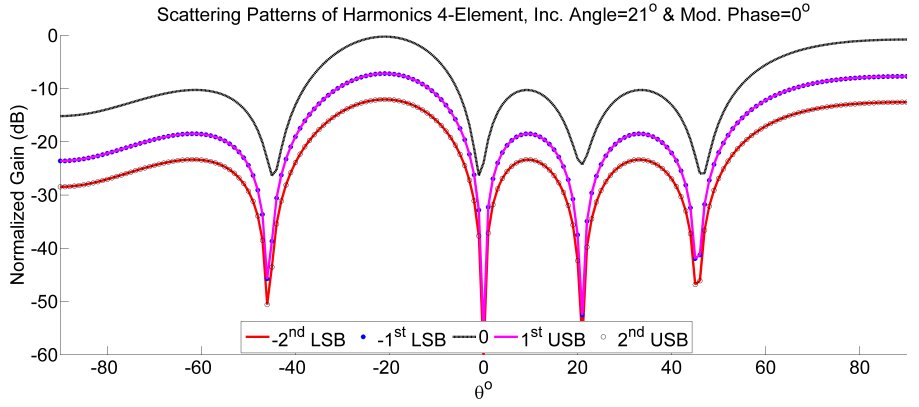

(c)

Figure 5.9: a) Plots of the 1USB and the 1LSB scattered beams of the 4-element PMSA for different modulation phase shifts b) scattered beams of a 4-element PMSA with $\emptyset_{m}=0$ and $\theta_{o}=0^{\circ} \mathrm{c}$ ) scattered beams patterns of a 4-element PMSA with $\emptyset_{m}=0$ and $\theta_{o}=-21^{\circ}$

frequencies and phases of some IMDs and HDs as given in Table 5.3. Frequencies of two tones are $1 \mathrm{kHz}$ and $0.9 \mathrm{kHz}$ while the main frequency is $12 \mathrm{kHz}$.

Many observations can be attained from Table 5.3. First, each distortion product has a different phase combination. Second, symmetrical HDs and IMDs around the main frequency have opposite phases. This means that these distortion products with opposite phases are scattered symmetrical in space. Finally, if phases of the 


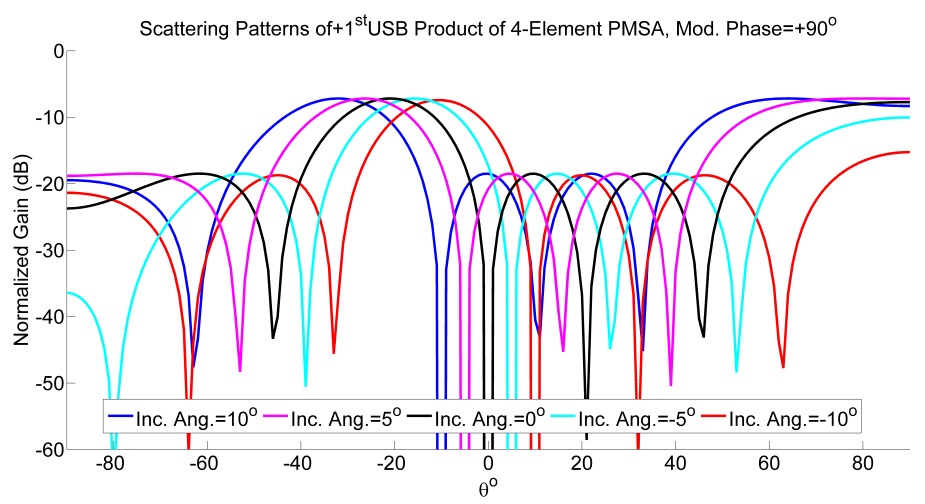

(a)

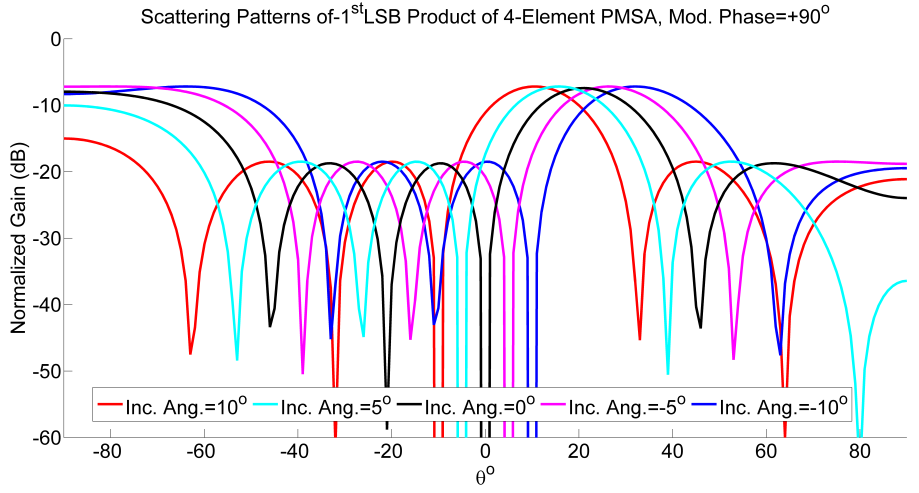

(b)

Figure 5.10: Scattered beams of distortion products for different incident angles a) 1USB b) 1LSB

main tones $w_{m 1}$ and $w_{m 2}$ are similar, some IMDs products given in table like IMD23, IMD12, IMD21, and IMD32 have the same phase and equal to phase of one of the main tones regardless of their different frequencies. Thus, their scattered beams always accompany scattered beams of the first harmonics HD1 and HD2 of the main tones. Moreover, phase of IMD1 is zero or in the correct sense; it will be equal to the inverted phase of the incident signals. Thus, having zero phase shifts for the IMD1 occurs when an angle of the incident signal is zero. Phase of IMD2 is equal to phase of either $w o+2 w_{m 1}$ or $w o+2 w_{m 2}$. The same conclusion can be applied to the negative distortion products which fall on the left side of the main frequency. This description given above is no longer valid if anyone of the main tones has a different phase shift, resulting in that IMD23, IMD12, IMD21, and IMD32 components have different 


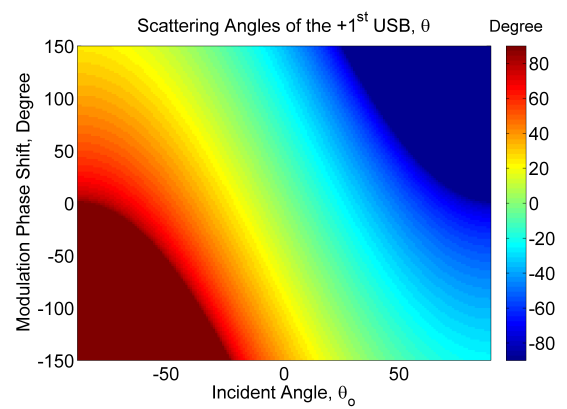

(a)

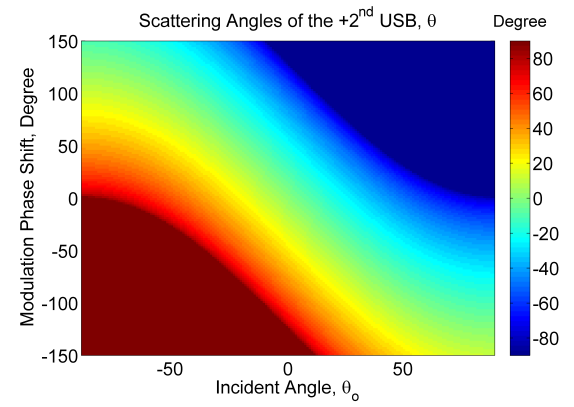

(c)

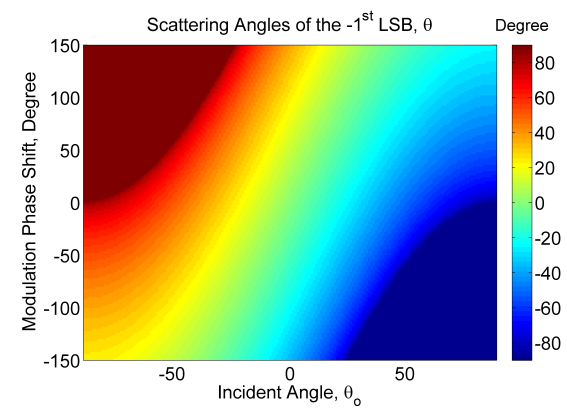

(b)

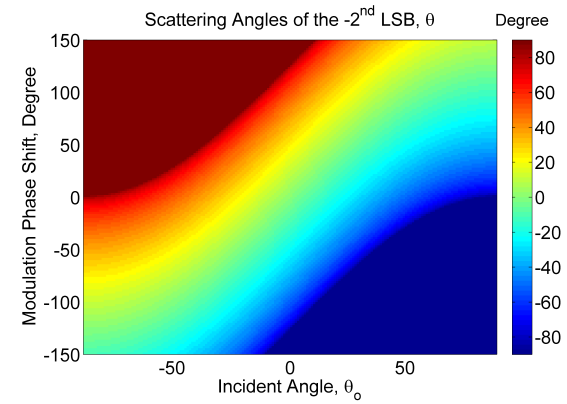

(d)

Figure 5.11: Potential directions of scattered beams of harmonics a) 1USB b) 1LSB c) 2USB and d) $2 \mathrm{LSB}$

phases and their phases will not be the same with the main tones. Consequently, these components will have different spatial locations. If we assume that one of the tones has constant phase while the other is continuously adjusted, IMDs components will have continuously variable phases too. The process of varying phases will make scattered beams of IMDs scannable.

\subsubsection{Example 2: an 8-element PMSA with $d=0.5 \lambda_{o}$}

In the previous example, beamforming capability of distortion products generated in the 4-element PMSA is given in detail with the consideration of several parameters affecting directions of scattered beam directly. Here, we only show a number of scattered beams of distortion products with the increasing number of scatterers. Figure 5.12 shows 3D scattered beams of distortion products of the 8-element PMSA when using the single-tone modulation sources for different incident angles 
Table 5.3: Frequencies and phases of some IMDs and HDs generated in a 4-element PMSA

\begin{tabular}{c|c|c|c}
\hline \hline Distortion-term & Tone-term & frequency & Phase \\
\hline IMD1 & $w_{m 2}-w_{m 1}$ & 12.1 & $\emptyset_{m 2}-\emptyset_{m 1}$ \\
\hline IMD1n & $-w_{m 2}+w_{m 1}$ & 11.9 & $-\emptyset_{m 2}+\emptyset_{m 1}$ \\
\hline IMD23 & $3 w_{m 1}-2 w_{m 2}$ & 12.7 & $3 \emptyset_{m 1}-2 \emptyset_{m 2}$ \\
\hline IMD23n & $-3 w_{m 1}+2 w_{m 2}$ & 11.3 & $-3 \emptyset_{m 1}+2 \emptyset_{m 2}$ \\
\hline IMD12 & $2 w_{m 1}-w_{m 2}$ & 12.8 & $2 \emptyset_{m 1}-\emptyset_{m 2}$ \\
\hline IMD12n & $-w_{m 1}+w_{m 2}$ & 11.2 & $-2 \emptyset_{m 1}+\emptyset_{m 2}$ \\
\hline HD1 & $w_{m 1}$ & 12.9 & $\emptyset_{m 1}$ \\
\hline HD1n & $-w_{m 1}$ & 11.1 & $-\emptyset_{m 1}$ \\
\hline HD2 & $w_{m 2}$ & 13 & $\emptyset_{m 2}$ \\
\hline HD2n & $-w_{m 2}$ & 11 & $-\emptyset_{m 2}$ \\
\hline IMD21 & $2 w_{m 2}-w_{m 1}$ & 13.1 & $2 \emptyset_{m 2}-\emptyset_{m 1}$ \\
\hline IMD21n & $-2 w_{m 2}+w_{m 1}$ & 10.9 & $-2 \emptyset_{m 2}+\emptyset_{m 1}$ \\
\hline IMD32 & $3 w_{m 2}-2 w_{m 1}$ & 13.2 & $3 \emptyset_{m 2}-2 \emptyset_{m 1}$ \\
\hline IMD32n & $-3 w_{m 2}+2 w_{m 1}$ & 10.8 & $-3 \emptyset_{m 2}+2 \emptyset_{m 1}$ \\
\hline IMD2 & $w_{m 2}+w_{m 1}$ & 13.9 & $\emptyset_{m 2}+\emptyset_{m 1}$ \\
\hline IMD2n & $-w_{m 2}-w_{m 1}$ & 10.1 & $-\emptyset_{m 2}-\emptyset_{m 1}$ \\
\hline \hline
\end{tabular}

$-15^{\circ}, 0^{\circ}, 15^{\circ}$, and $30^{\circ}$ along with their top views. The substantial difference between this example and the former one is that more scattered beams of distortion products appear in the visible view of an 8-element PMSA. This validates the discussion presented in Chapter 3 that when a circuit has a higher number of paths, more distortion products can be controlled.

\subsection{The PMSA as an Analysis Tool of Distortion Products in Phased Arrays}

In this section, we exploit the general context of the research in its two perspectives models derived in Chapters 3 and 4 to study and analyze all kinds of distortion products generated in phased arrays. This topic becomes a major issue especially in active phased arrays which need to work in interference-rich surroundings as in expected scenarios of $5 \mathrm{G}$ millimeter-wave networks. Thus, a lot of research has been done to analyze and mitigate effects of distortion products to ensure high data rates between base-stations and users when using beamforming techniques. In addition, antennas, connectors, and transmission-lines are another source of distortions which are called passive intermodulation distortions (PIM) discussed in detail in [55] [179] 


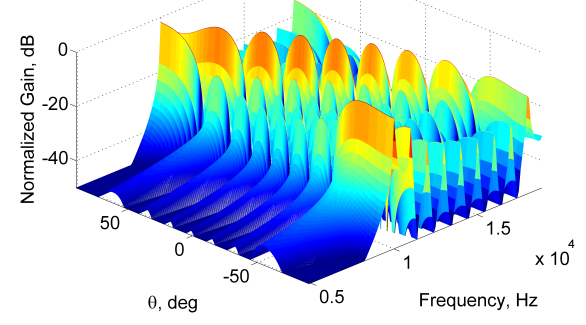

(a)

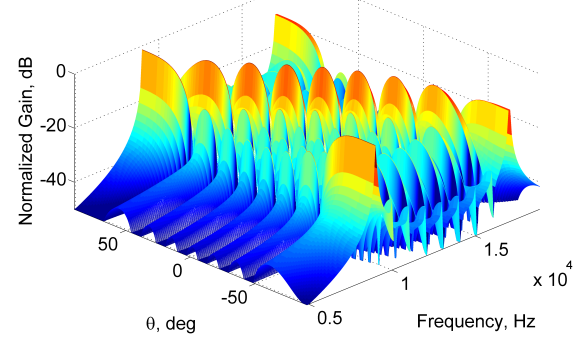

(c)

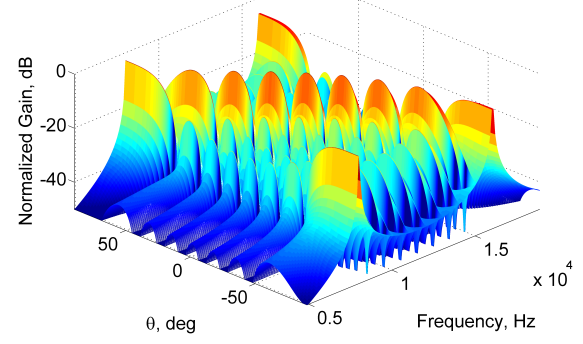

(e)

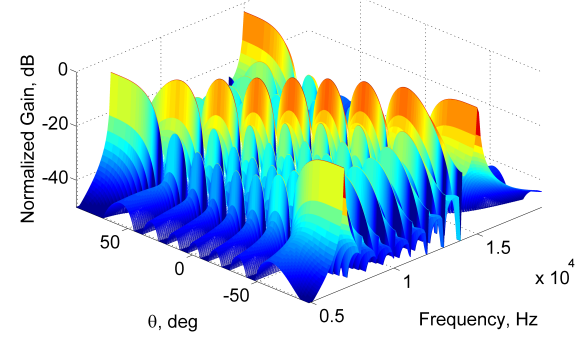

(g)

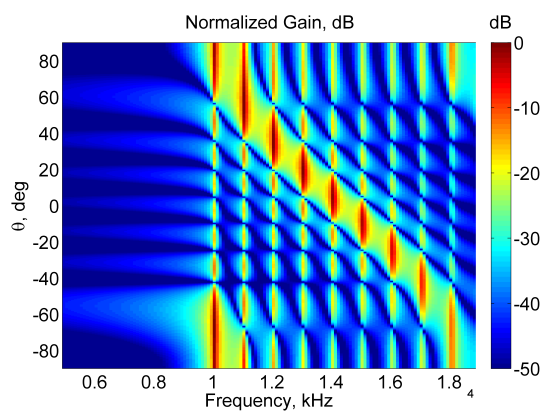

(b)

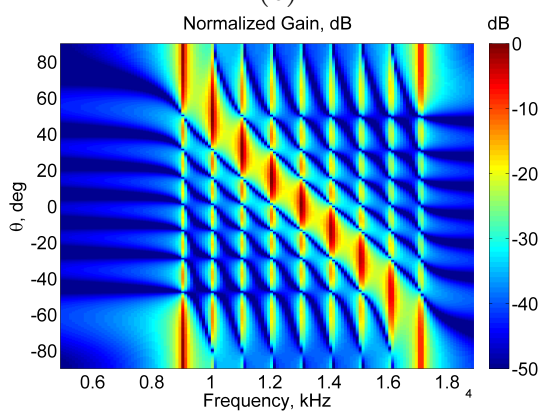

(d)

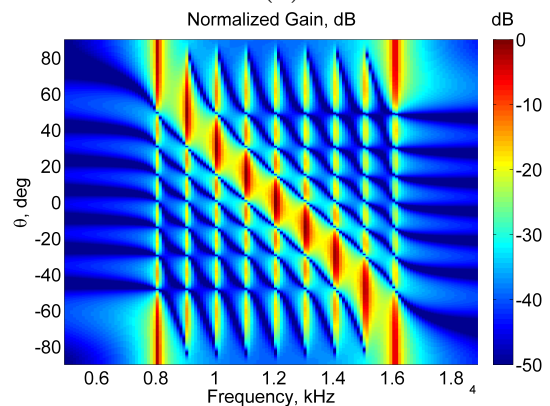

(f)

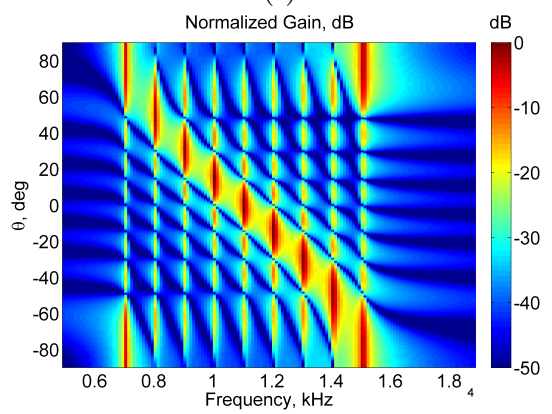

(h)

Figure 5.12: Plot of the frequency varying scattered beams of distortion products versus angle for different incident angles generated in the 8-element PMSA a) $\left.-35^{\circ} 3 \mathrm{D} \mathrm{b}\right)-35^{\circ} 2 \mathrm{D}$ c) $-15^{\circ} 3 \mathrm{D}$ and d) $\left.\left.-15^{\circ} 2 \mathrm{D} \mathrm{e)} 0^{\circ} 3 \mathrm{D} \mathrm{f)} 0^{\circ} 2 \mathrm{D} \mathrm{g}\right) 15^{\circ} 3 \mathrm{D} \mathrm{h}\right) 15^{\circ}$ 
[67] [177] [178]. Although mechanisms of generation of passive and active distortion products are different, they could possibly be treated by the same mathematical models derived before in this research work.

Each signal passes through a variety of active and passive electronic components, transmission lines, and connectors. Responses of these components are not ideal, and each component contributes in distorting signals passing through it. The problem gets worse when more than one signal passes through distorting components at the same time because nonlinear behaviors of components work to mix these signals and present new signals that did not exist before. Some of the new resulting signals, having frequencies that fall within a frequency band of interest, are so harmful, resulting in degradation of the system sensitivity [136]. The topic that is discussed here is highly related to our mathematical models and its importance stems from that both the PMSA and phased arrays have more than one distortion generator, being an array of elements. Thus our mathematical models can be applied to examine distortion products in phased arrays. Distortion products from the point of view of our PMSA are advantageously exploited, and are unwanted in phased arrays, being the main difference between the PMSA and phased arrays.

To ensure high data rate in millimeter-wave $5 \mathrm{G}$ networks between mobile users and base-stations, using single antennas is inadequate, so using steerable beamforming techniques is necessary. Due to high frequencies of $5 \mathrm{G}$ systems, signals experience high attenuation compared to their counterpart in microwave regimes [120]. Designers compensate the high attenuation by integrating an amplifier with each single element (i.e., antenna), and amplifiers are generally considered as the main source of generation of distortion products. In this case, distortion products generated, at each antenna element of the phased array, are passed through a beamforming network to be added at an output of power combiner. These distortion signals may add destructively 
or constructively, relying on a scan angle of the phased array and on directions of arrival of multiple incident interferes [143].

Many efforts have been made before to analyze intermodulation distortion IMD products generated in fixed multi-beam active phased arrays [146] [66] [143]. In [146] [66], authors proposed techniques to reduce the third order intermodulation distortion IMD3. These works considered the systems in the transmit mode with multiple pre-determined fixed beams. The study of a intermodulation distortion products generation in the receive mode with a scanning capability is presented in [143]. Two interferes scenario has been experimentally demonstrated. All research efforts have been focused to analyze distortion products using only the first three terms of the Taylor series because authors thought that this will be sufficient to study effects of IMD3 which most times falls inside the desired bands. However, these analyses will not be highly accurate. Also, in real-world 5G phased arrays, beams must be continuously scanned to track mobile users, so signals coming from other directions can degrade the system performance.

In contrast to all previous research work which was limited to truncated mathematical models, low number of signals, and few RF channels (i.e., antennas), the mathematical model presented in this section is comprehensive. In other words, it can deal with any number of signals (i.e. multi-users or multi-interferer) passing through each RF channel in both the receive and transmit modes, it can deal with an unlimited number of distortion products, it is frequency-independent model covering all frequencies from acoustic band into terahertz band, and it can deal with any number of channels (i.e., the number of antennas in phased arrays or scatterers in the PMSA). All these features make the mathematical models of our PMSA as a robust tool to analyze distortion products generated in arrays. In below, we consider only distortion products generated in phased arrays at the receive-mode for reasons 
clarified later.

\subsubsection{The Mathematical Analysis}

Referring to (4.28), we can treat any number of distortion products resulting from any number of desired signals or interferes. However, the scenario gets worse as either a number of desired signals or interferers increases in conventional phased arrays, leading to a huge number of distortion products. Although distortion products originate from different combinations of negative and positive multiple integers of the main frequencies, some of resulting distortion products have the same frequency with the main signals. Furthermore, the model process will last longer as a number of signals increases, so to ease the calculation process, Eq.(4.28) is truncated to include only the first three terms (i.e., three signals or interferers) to compare our results with ones presented in [143]. Because authors used the arrays under tests in the receive-mode, we utilize our system in the receive-mode too. Thus, we recall (4.28) here

$$
\begin{aligned}
E_{S}\left(\theta, w_{t o t}\right) & =\frac{1}{2} \sum_{k=-\infty}^{\infty} \sum_{m=-\infty}^{\infty} \sum_{s=-\infty}^{\infty} d_{t o t} e^{j\left(\frac{L-1}{2}\left(K_{t o t} d \sin (\theta)-\left(k \emptyset_{m 1}+m \emptyset_{m 2}+s \emptyset_{m 3}+\emptyset_{o}\right)\right)\right)} \\
& \times \frac{\sin \left(\frac{L}{2}\left(K_{t o t} d \sin (\theta)-\left(k \emptyset_{m 1}+m \emptyset_{m 2}+s \emptyset_{m 3}+\emptyset_{o}\right)\right)\right)}{\sin \left(\frac{1}{2}\left(K_{t o t} d \sin (\theta)-\left(k \emptyset_{m 1}+m \emptyset_{m 2}+s \emptyset_{m 3}+\emptyset_{o}\right)\right)\right)} \\
& \times e^{j\left(w_{o}+w_{t o t}\right) t}
\end{aligned}
$$

where $w_{t o t}$ here is equal to $k w_{m 1}+m w_{m 2}+s w_{m 3}$ and $t o t=k+m+s$.

Eq.(5.24) seems only appropriate with the PMSA functioning, so some slight modifications should be carried out to explain well behaviors of distortion products generated in conventional phased arrays. To do so, we need to take a closer look at a generation process of distortion products in phased arrays. The process is quietly 
similar to the generation process of distortion products in the PMSA, but modulation phases will be replaced by phases of signals which are either desired or unwanted induced on array elements. These phases depend mainly on spatial locations of signals in both the receive-mode and the transmit-mode. Although these two processes of distortion products generations are identical in both modes, the analysis of distortion products in the receive-mode is much easier than in the transmit-mode for two reasons

1. Most of distortion products falling outside of frequency bands of the interest are dismissed out by filters placed either at an end of every channel or after the final combiner [143], thus leaving only the most harmful distortion products which are the third and fifth intermodulation distortion products IMD3 and IMD5, thereby reducing the time of analysis.

2. In the transmit-mode, analysis of distortion products is similar to the PMSA except that the modulation signals represent user signals and there is no need to use the incident signals in the mathematical model of the array beamforming.

Therefore, this section sheds light on the scope of analysis of distortion products generated in receive-mode phased arrays. In addition, since the process of distortion products generation similar for both desired and interferers signals, we only mention interferers in our discussion. Figure 5.13 shows an array consisting of multiple elements arranged linearly along the y-axis. The figure reveals a substantial difference between the PMSA and the receive-mode phased array. The PMSA has only antennas and switches, whereas the recieve-mode phased array consists of amplifying units, filter, phase shifters, and a power combiner. Their counterparts in the phased array replace the modulation signals in the PMSA $\left(w_{m 1}, w_{m 2}\right.$, and $\left.w_{m 3}\right)$ by interferers, $\left(w_{1}, w_{2}\right.$, and $\left.w_{3}\right)$. From Figure 5.13, relative phases of interferers can be represented by 
their spatial locations which are according to the ray tracing geometry and distances separating elements [34].

They can be given as

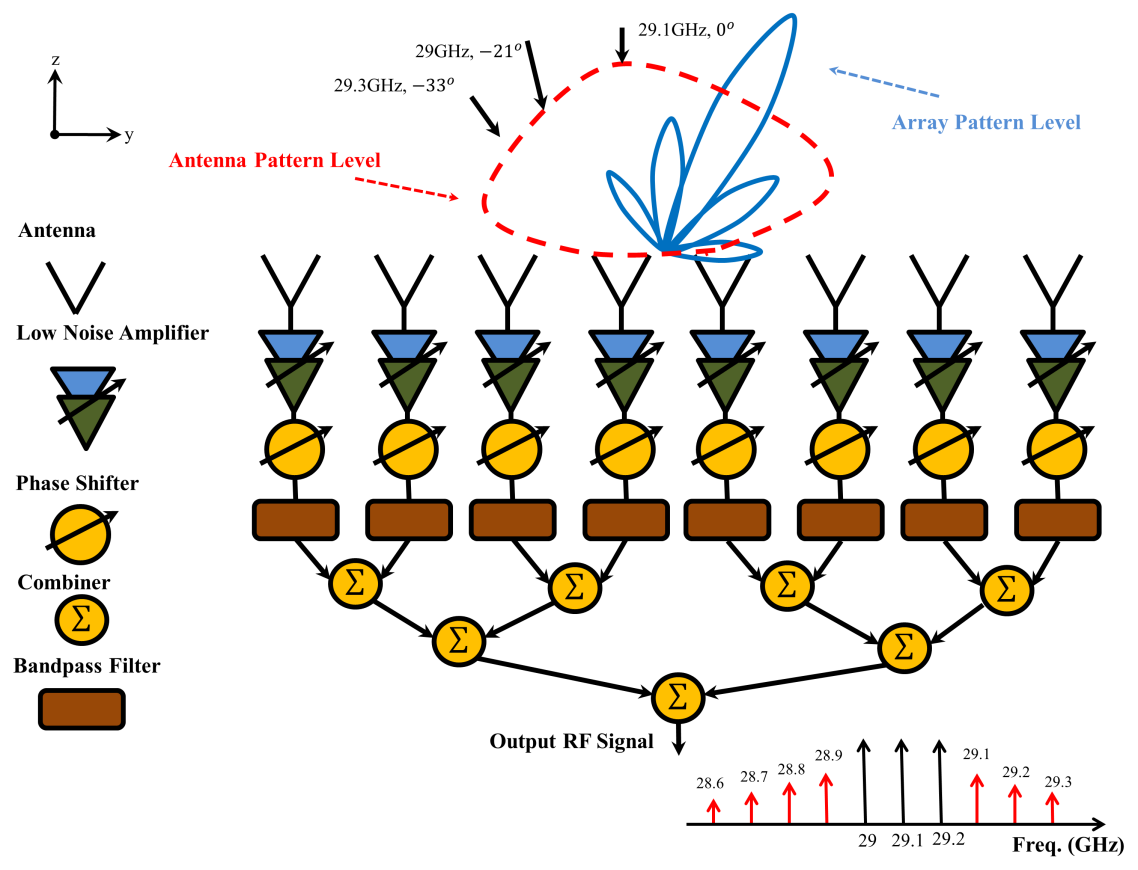

Figure 5.13: Receive-mode phased array with three interferers in the YZ cut-plane incident on each single element in the array

$$
\begin{gathered}
\emptyset_{1}=K_{1} d \sin \left(\theta_{1}\right) \quad \text { for the first interferer } \\
\emptyset_{2}=K_{2} d \sin \left(\theta_{2}\right) \quad \text { for the second interferer } \\
\emptyset_{3}=K_{3} d \sin \left(\theta_{3}\right) \quad \text { for the third interferer }
\end{gathered}
$$

where $K_{1}, K_{2}$, and $K_{3}$, and $d_{1}, d_{2}$, and $d_{3}$ represent the wavenumber of interferes and distances separating elements according into frequencies $f_{1}, f_{2}$, and $f_{3}$, respectively, and $\theta_{1}, \theta_{2}$, and $\theta_{3}$ are the angles of incident interferers, while a true phase shift 
integrated to each channel is given by

$$
\emptyset_{s}=K_{o} d \sin \theta_{s}
$$

to scan the main beam of an array into the desired direction $\theta_{s}$.

$$
\begin{aligned}
E_{S}\left(\theta, w_{i n t}\right) & =\frac{1}{2} \sum_{k=-\infty}^{\infty} \sum_{m=-\infty}^{\infty} \sum_{s=-\infty}^{\infty} d_{t o t} \\
& \times e^{j\left(\frac{L-1}{2}\right)\left(K_{o} d \sin \left(\theta_{s}\right)-k K_{1} d \sin \left(\theta_{1}\right)-m K_{2} d \sin \left(\theta_{2}\right)-s K_{3} d \sin \left(\theta_{3}\right)\right)} \\
& \times \frac{\sin \left(\frac{L}{2}\left(K_{o} d \sin \left(\theta_{s}\right)-k K_{1} d \sin \left(\theta_{1}\right)-m K_{2} d \sin \left(\theta_{2}\right)-s K_{3} d \sin \left(\theta_{3}\right)\right)\right)}{\sin \left(\frac{1}{2}\left(K_{o} d \sin \left(\theta_{s}\right)-k K_{1} d \sin \left(\theta_{1}\right)-m K_{2} d \sin \left(\theta_{2}\right)-s K_{3} d \sin \left(\theta_{3}\right)\right)\right)} \\
& \times e^{j\left(w_{1}+w_{2}+w_{3}\right) t}
\end{aligned}
$$

where $t o t=1+2+3$. It can be noticed that each distortion product term being existed in (5.27) has a phase shift $\emptyset_{s}=K_{o} d \sin \theta_{s}$ added to their particular phases. Thus, all in-band IMDs carry an extra value of the phase $p e^{j \emptyset_{s}}$ introduced by phase shifters regardless of their locations before or after amplifiers [143]. IMDs are then linearly added at the output of an ideal power combiner to generate final array patterns of IMDs. This analysis is that, for LO, RF, or IF phase shifters based phased arrays, IMDs produced in RF frontends are phase shifted and summed at the output of beamforming network. Here, we will not go through more details about RF power signal processing as far as we want to demonstrate the capabilities of the PMSA mathematical models to analyze spatial locations of distortion products in the receive-mode phased arrays.

Also, the model of distortion product characteristics given in (3.68) is exploited here again with trivial modifications to meet requirements of the receive-mode phased 
arrays. The model becomes as

$$
\begin{aligned}
E(t, \theta) & =\left(D \sum _ { l = 1 } ^ { L } \sum _ { k = - \infty } ^ { \infty } \sum _ { m = - \infty } ^ { \infty } \sum _ { s = - \infty } ^ { \infty } \operatorname { c o s } \left(\left(k w_{1}+m w_{2}+s w_{3}\right) t\right.\right. \\
& \left.\left.+(l-1)\left(\emptyset_{r}-k \emptyset_{1}-m \emptyset_{2}-s \emptyset_{3}\right)\right)\right) \times E P(\theta)
\end{aligned}
$$

Now Eqs.(5.27) and (5.28) fulfill the analysis requirements of distortion products in phased arrays for both receive and transmit modes after making few adjustments on the original equations. However, at the receive-mode, the analysis is much easier because most distortion products are filtered out.

Assume a phased array where its main beam is scanned to an angle denoted by $\left(\theta_{s}, 90\right)$ in the YZ cut-plane. Scanning of the main beam is carried out using a relative phase shift difference at the $l-t h$ channel of the phased array given by

$$
\emptyset_{s, l}=-l K_{o} d \sin \theta_{s}
$$

where $K_{o}=2 \pi / w_{o}$ is the wave number at the design frequency. Suppose now that three different interferes arrive at an array aperture from three different directions as illustrated in Figure 5.13, at angles $\theta_{i}, i=1,2,3$ in the YZ cut-plane. Each incident signal induces electrical fields on the phased array antenna elements which in turn produces voltages at input ports of antennas. Voltages induced on the array antenna elements from all interferers have equal amplitudes but different phases. They can be represented mathematically as

$$
\begin{aligned}
& V 1=v a \cos \left(w_{1} t+\emptyset_{1, l}\right) \\
& V 2=v b \cos \left(w_{2} t+\emptyset_{2, l}\right)
\end{aligned}
$$




$$
V 3=v c \cos \left(w_{3} t+\emptyset_{3, l}\right)
$$

where $\emptyset_{i, l}=-l K_{o} d \sin \theta_{i}$.

If frequencies of $V 1, V 2$, and $V 3$ are relatively close to each other, it becomes necessary to illustrate array patterns of the most harmful intermodulation distortion products falling within the original signals which are some of IMD3, IMD5, IMD7, and so on. Although all IMDs exist in the output spectrum of phased arrays, the high order IMDs are inherently low. Thus, in the following, array patterns of IMD3 and IMD5 are only considered. Assuming that signals have the same amplitudes equal to $v$, and $w_{i}, w_{j}, w_{l} \approx w_{o}$ for all $i, j, l \in 1,2,3$. Study each of these IMDs (i.e., IMD3 and IMD5) allows determining apparent directions of patterns of IMDs as illustrated in Table 5.4. From (5.27), array factors of IMD3 components of the receive-mode phased array, at $w_{i}+w_{j}-w_{l}$, where $w_{i} \neq w_{j}$, are given by

$$
\begin{aligned}
& E_{S}\left(\theta_{S}, w_{i}+w_{j}-w_{l}\right) \\
= & D_{i+j-l} \times\left\{\frac{\sin \left(\frac{L}{2} d\left(K_{o} \sin \theta_{s}-K_{i} \sin \theta_{i}-K_{j} \sin \theta_{j}+K_{l} \sin \theta_{l}\right)\right)}{\sin \left(\frac{1}{2} d\left(K_{o} \sin \theta_{s}-K_{i} \sin \theta_{i}-K_{j} \sin \theta_{j}+K_{l} \sin \theta_{l}\right)\right)}\right\} \times e^{+j\left(w_{i}+w_{j}-w_{l}\right) t}
\end{aligned}
$$

If IMD3 components results from two interferers, array factors become according to the component $2 w_{i}-w_{j}$

$$
\begin{aligned}
& E_{S}\left(\theta_{s}, 2 w_{i}-w_{j}\right) \\
& \quad=D_{2 i-j} \times\left\{\frac{\sin \left(\frac{L}{2} d\left(K_{o} \sin \theta_{s}-2 K_{i} \sin \theta_{i}+K_{j} \sin \theta_{j}\right)\right)}{\sin \left(\frac{1}{2} d\left(K_{o} \sin \theta_{s}-2 K_{i} \sin \theta_{i}+K_{j} \sin \theta_{j}\right)\right)}\right\} \times e^{+j\left(2 w_{i}-w_{j}\right) t}
\end{aligned}
$$

Similarly, for the IMD5 components, in all forms $3 w_{i}-w_{j}-w_{l}, 2 w_{i}+w_{j}-2 w_{l}$, and $3 w_{i}-2 w_{j}$, their array factors are given in (5.33), (5.34), and (5.35), respectively, as 


$$
\begin{aligned}
E_{S}\left(\theta_{S}, 3 w_{i}-w_{j}-w_{l}\right) & \\
& =D_{3 i-j-l} \\
& \times\left\{\frac{\sin \left(\frac { L } { 2 } d \left(K_{o} \sin \theta_{s}-3 K_{i} \sin \theta_{i}+K_{j} \sin \theta_{j}+K_{l} \sin \theta_{l}\right.\right.}{\sin \left(\frac { 1 } { 2 } d \left(K_{o} \sin \theta_{s}-3 K_{i} \sin \theta_{i}+K_{j} \sin \theta_{j}+K_{l} \sin \theta_{l}\right.\right.}\right\} \\
& \times e^{+j\left(3 w_{i}-w_{j}-w_{l}\right) t}
\end{aligned}
$$

$$
\begin{aligned}
E_{S}\left(\theta_{S}, 2 w_{i}+w_{j}-2 w_{l}\right) & \\
= & D_{2 i+j-2 l} \\
\times & \left\{\frac{\sin \left(\frac { L } { 2 } d \left(K_{o} \sin \theta_{s}-2 K_{i} \sin \theta_{i}-K_{j} \sin \theta_{j}+2 K_{l} \sin \theta_{l}\right.\right.}{\sin \left(\frac { 1 } { 2 } d \left(K_{o} \sin \theta_{s}-2 K_{i} \sin \theta_{i}-K_{j} \sin \theta_{j}+2 K_{l} \sin \theta_{l}\right.\right.}\right\} \\
\times & e^{+j\left(2 w_{i}+w_{j}-2 w_{l}\right) t}
\end{aligned}
$$$$
E_{S}\left(\theta_{S}, 3 w_{i}-2 w_{j}\right)
$$$$
\begin{aligned}
& =D_{3 i-j-l} \\
& \times\left\{\frac{\sin \left(\frac { L } { 2 } d \left(K_{o} \sin \theta_{s}-3 K_{i} \sin \theta_{i}+2 K_{j} \sin \theta_{j}\right.\right.}{\sin \left(\frac { 1 } { 2 } d \left(K_{o} \sin \theta_{s}-3 K_{i} \sin \theta_{i}+2 K_{j} \sin \theta_{j}\right.\right.}\right\} \\
& \times e^{+j\left(3 w_{i}-2 w_{j}\right) t}
\end{aligned}
$$

Array factors of IMD3 and IMD5 at an array output follow the rule of $\sin (L y) / \sin (y)$ pattern; with a peak given at $y=0$. Peaks of IMDs occur at different angles as shown in Table 5.4. The angles where patterns of IMD3 and IMD5 have peaks are called the apparent angles. As illustrated, the apparent angles of IMDs are not the same 
as their original signals (interferers). The analysis of array factors of IMDs given in (5.31)-(5.35) shows that each single IMD component has a completely different array response than main pattern response of the phased array where it is quite similar to the PMSA when using multi-tone modulation sources. The difference between receive-mode phased array and the PMSA is that in the former one, apparent patterns are constructed inside real circuits while in the latter one, scattered patterns are constructed in space. Phases of varying incident signals on each antenna element in the PMSA, together with the phase shift of distortion products of the modulation signals determine phases of scattered distortion products, while in phased arrays, both arrays own their phase shifts according to a scanning angle $\theta_{s}$ and phases of incident interferers on each element, given in (5.25) and (5.26), make having a different phase for each different IMD component. A phased array pattern appears at an angle $\theta_{s}$, but its IMD patterns appear at angles $\theta_{I M D s}$.

In terms of cancellation, phased arrays do not suppress IMDs products at the output according to the general pattern expressions of IMD3 and IMD5 products versus angle $\theta_{s}$ in (5.31)-(5.35). These assumptions hold true even if interferers reach the phased antenna array aperture at its main pattern nulls. The reason behind this is that nulls in the array pattern are not present at level elements patterns and only exist at the final output port of the beamforming network, as illustrated in Figure 5.13. The apparent angles of all IMDs considered in this section are given in the third column of Table 5.4.

\subsubsection{Simulation Example}

Consider a phased array with eight elements located linearly along y-axis spaced a half free-space wavelength apart at a frequency of the interest $29 \mathrm{GHz}$, being similar to a phased array implemented in [143]. In simulations, elements are isotropic and 
Table 5.4: Apparent angles of IMDs generated from three interferers incident at three different angles $\theta_{i}, \theta_{j}$, and $\theta_{l}$

\begin{tabular}{c|c|c}
\hline \hline IMDs form No. & IMDs Frequency & Apparent angle of IMDs \\
\hline $3^{r d}$ order $\left(1^{\text {st }}\right.$ form $)$ & $w_{i}+w_{j}-w_{l}$, where $w_{i} \neq w_{j}$ & $\theta_{s}=\sin ^{-1}\left\{\sin \theta_{i}+\sin \theta_{j}-\sin \theta_{l}\right\}$ \\
\hline $3^{r d}$ order $\left(2^{\text {nd }}\right.$ form $)$ & $2 w_{i}-w_{j}$ & $\theta_{s}=\sin ^{-1}\left\{2 \sin \theta_{i}-\sin \theta_{j}\right\}$ \\
\hline $5^{\text {th }}$ order $\left(1^{\text {st }}\right.$ form $)$ & $3 w_{i}-w_{j}-w_{l}$, where $w_{i} \neq w_{j}$ & $\theta_{s}=\sin ^{-1}\left\{3 \sin \theta_{i}-\sin \theta_{j}-\sin \theta_{l}\right\}$ \\
\hline $5^{\text {th }}$ order $\left(2^{\text {nd }}\right.$ form $)$ & $2 w_{i}+w_{j}-2 w_{l}$, where $w_{i} \neq w_{j}$ & $\theta_{s}=\sin ^{-1}\left\{2 \sin \theta_{i}+\sin \theta_{j}-2 \sin \theta_{l}\right\}$ \\
\hline $5^{\text {th }}$ order $\left(3^{\text {rd }}\right.$ form $)$ & $3 w_{i}-2 w_{j}$ & $\theta_{s}=\sin ^{-1}\left\{3 \sin \theta_{i}-2 \sin \theta_{j}\right\}$ \\
\hline$\{i, j, l\} \in\{1,2,3\}$ & \\
\hline
\end{tabular}

vertically polarized. A Matlab codes were written to plot radiation patterns and spatial characteristics of different IMDs. In the program, factors like frequency, spacing, array arrangement, number of antennas, and arrival angle of interferers are taken into account. Frequencies of three interferers are $29 \mathrm{GHz}, 29.1 \mathrm{GHz}$, and 29.2GHz incident on the phased array at angles $\theta_{1}=-21^{\circ}, \theta_{2}=0^{\circ}$, and $\theta_{3}=-33^{\circ}$, respectively. These values of incident angles have been selected to regenerate the work results presented in [143] as a step to demonstrate that our second mathematical mode is able to analyze distortion products even those generated by conventional phased arrays.

Incident signals presented in the YZ cut-plane are three with equal intensities. Because a degree of our mathematical model is truncated to include only the third and fifth terms, the total numbers of IMD3 and IMD5 combinations given in Table 5.4 are twenty-four components where the IMD3 has nine components only, and the rest represents the IMD5 components. Table 5.5 presents six frequency combinations for each type (i.e., IMD3 and IMD5), and Figure 5.14 shows their array patterns along with their spatial characteristics of distortion products analyzed at the maximum apparent angles. Peaks of simulated patterns are in good agreement with apparent angles of IMDs given in Table 5.5. It is worth to mention that IMD3 and IMD5 have patterns peaks at the phased array output different from what the main signals have, being independent of incident angles [143] [66] [146]. Also, it can be seen 
from Figure 5.14 that some IMDs components located far away from the normal incident angle have the same amplitudes with IMDs located closely to the normal angle because elements assumed in the simulations are isotropic. Thus, in practical applications, these peaks can be reduced by the element pattern factor $\cos ^{n}(\theta)$. In addition, we present patterns of IMDs products along with the main signals patterns as displayed in Figure 5.14. The slight differences in results compared to [Fig.9 [143]] are because of using two terms in our simulation model which are the third and fifth orders, while being only the third term order in [143]. Some plots in Figure 5.14 have more than one peak because those peaks come from different IMDs components having the same frequency. Hence, for a large number of interferers, IMDs patterns will have more peaks. In other words, as a number of interferes increases, nulls and sidelobes in the IMDs pattern will not exist anymore. In this case, each IMD product results from multiple different frequency combinations, and the power of frequency combinations for each IMD is added constructively at different spatial locations [108] [115] [90]. The level of ripple decreases as a number of interferers increases to beconme a constant response. In Figure 5.15a, we illustrate the resulting IMD3+IMD5 pattern along with patterns of nine interferers having frequencies $28.6,28.7,28.8,28.9,29,29.1,29.2,29.3$, and $29.4 \mathrm{GHz}$ reaching the array at angles of $-21^{\circ}, 0^{\circ},-33^{\circ},-41^{\circ}, 15^{\circ}, 70^{\circ},-55^{\circ}, 10^{\circ}$, and $33^{\circ}$, respectively. As can be seen, a number of peaks increases obviously, and the patterns of main interferers are also distorted because some IMDs combinations have similar frequencies with interferers. However, when all interferers have the same incident angle for example at the broadside, $0^{\mathrm{deg}}$, we can see that all resulting IMDs patterns are similar to array factors of the main interferers, having main lobes at apparent angle $0^{\varnothing}$ as shown in Figure 5.15b. These IMDs will be enhanced too at the beamforming output which in turn reduces sensitivity of a receiver [115] [116] [114]. Thus, IMDs arising from 
nonlinearities impairments of some receiver parts are beamformed. In contrast, when all the interferers given in Figure 5.15a have the same frequency 28.3GHz, this makes the frequencies of resulting IMD3 and IMD5 combinations the same and equal to a frequency of interferers. Each IMD3 or IMD5 component is beamformed into a different direction. However, because all IMDs components and the main interferers have the same frequency, their patterns are added in power, thereby generating an isotropic pattern as shown in Figure 5.15c. In this case, the performance of the array rapidly deteriorates as number of interferers increases since amplifiers in arrays will be forced to work at their saturation points.

To this end, the capability of distortion products analysis in phased arrays utilizing our mathematical models in this dissertation work was demonstrated. It could be, therefore, in future used as an analysis tool of distortion products in arrays.

Table 5.5: IMD3 and IMD5 components for interferers in Figure 5.13

\begin{tabular}{c|c|c|c|c|c|c|c|c|c}
\hline \hline IMD3 & f1 & f2 & f3 & AA & IMD5 & f1 & f2 & f3 & AA \\
\hline 28.8 & $2 \times 29$ & & -29.2 & -9.9 & 28.7 & $2 \times 29$ & 29.1 & $-2 \times 29.2$ & 21.9 \\
\hline 28.9 & 29 & 29.1 & -29.2 & 10.7 & 28.8 & $2 \times 29.1$ & 29 & $-2 \times 29.2$ & 47 \\
\hline 28.9 & $2 \times 29$ & & -29.1 & -45.8 & 29.5 & $3 \times 29.2$ & -29.1 & -29 & Invisible \\
\hline 29.3 & $2 \times 29.2$ & & -29.1 & Invisible & 29.1 & $3 \times 29.1$ & -29.2 & 29 & 64.55 \\
\hline 29.3 & 29.1 & 29.2 & -29 & -10.7 & 28.6 & $3 \times 29$ & & $-2 \times 29.2$ & 0.8 \\
\hline 29.4 & 29.2 & 29.2 & -29 & -47 & 29.4 & $3 \times 29$ & & $-2 \times 29.1$ & Invisible \\
\hline
\end{tabular}

\subsection{The PMSA Based Tunable Spatial Harmonic Generator}

In this section, it may be useful in some applications to change both the frequencies and spatial locations of distortion products. So as not to make the design more complicated, we will only take into account the change in frequencies of the modulation sources while keeping phases constant.

Consider the PMSA with eight isotropic elements placed along the $\mathrm{x}$-axis spaced at a half free-space wavelength apart at the design frequency, and each 


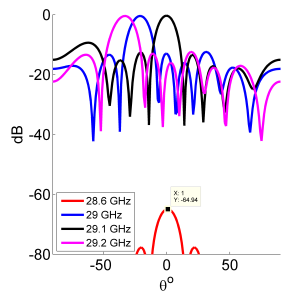

(a)

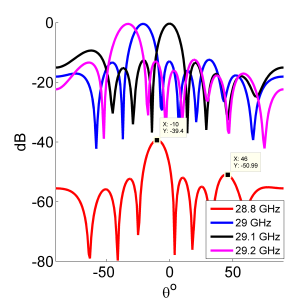

(e)

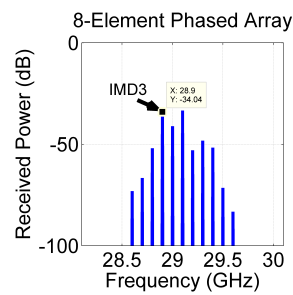

(i)

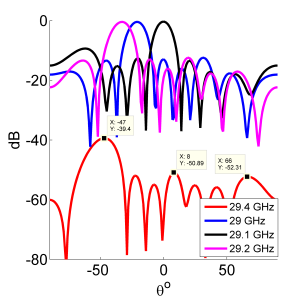

(m)

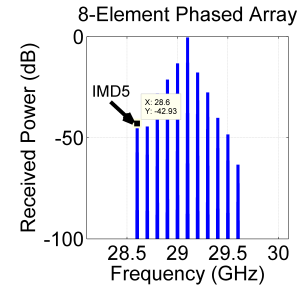

(b)

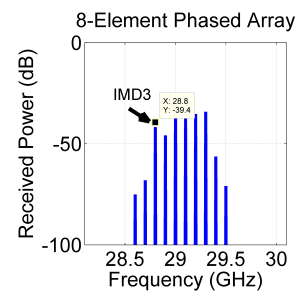

(f)

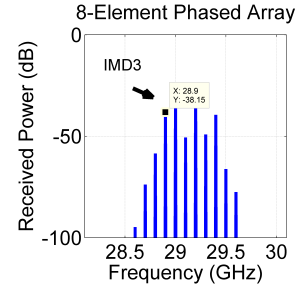

(j)

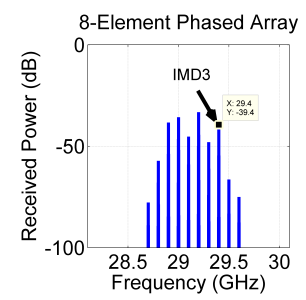

(n)

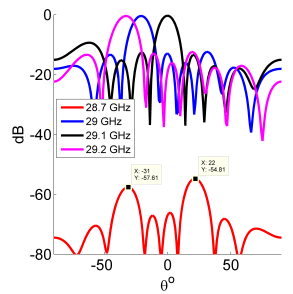

(c)

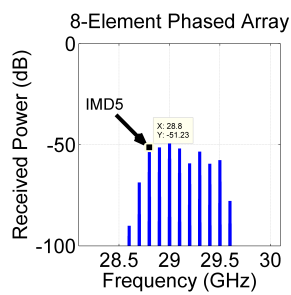

(g)

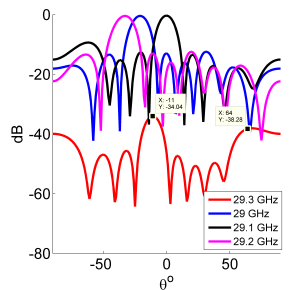

(k)

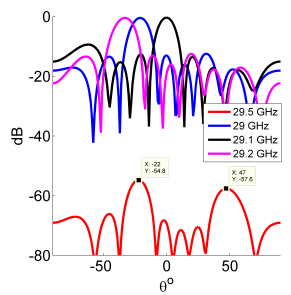

(o)

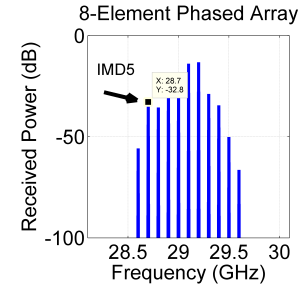

(d)

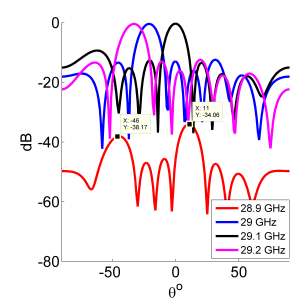

(h)

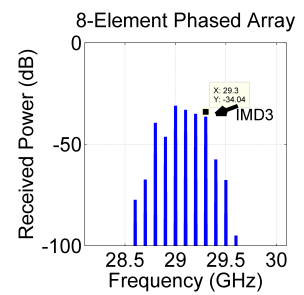

(1)

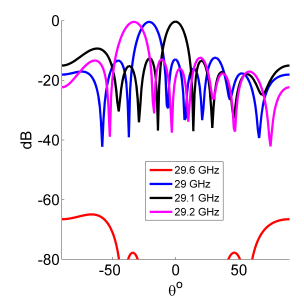

(p)

Figure 5.14: Simulation patterns of IMD3 and IMD5 components along with their distortion products at the output of the phased array in Figure 5.13 a) array pattern of IMD $5=28.6 \mathrm{GHz}$ b) distortion characterstics of IMD $5=28.6 \mathrm{GHz}$ c) array pattern of IMD $5=28.7 \mathrm{GHz}$ d) distortion characterstics of IMD $5=28.7 \mathrm{GHz}$ e) array pattern of $\mathrm{IMD} 3=\mathrm{IMD} 5=28.8 \mathrm{GHz}$ f) distortion characterstics of IMD $3=28.8 \mathrm{GHz}$ g) distortion characterstics of IMD $5=28.8 \mathrm{GHz} \mathrm{h}$ ) array pattern of IMD3 $=$ IMD3 $=28.9 \mathrm{GHz}$ i) distortion characterstics of IMD3 $=28.9 \mathrm{GHz}$ j) distortion characterstics of IMD $3=28.9 \mathrm{GHz} \mathrm{k}$ ) array pattern of IMD $3=29.3 \mathrm{GHz}$ l) distortion characterstics of IMD3 $=29.3 \mathrm{GHz}$ $\mathrm{m}$ ) array pattern of IMD $3=29.4 \mathrm{GHz} \mathrm{n}$ ) distortion characterstics of IMD3 $=29.4 \mathrm{GHz}$ o) array pattern of IMD $5=29.6 \mathrm{GHz}$ ) ) array pattern of IMD $5=29.6 \mathrm{GHz}$

element is modulated by a tunable single-tone modulation source with a relative phase shift difference $45^{\circ}$. A source of the illuminating signals is located at the angle $-15^{\circ}$, thus inducing signals on elements with advanced phase $45^{\circ}$ starting 


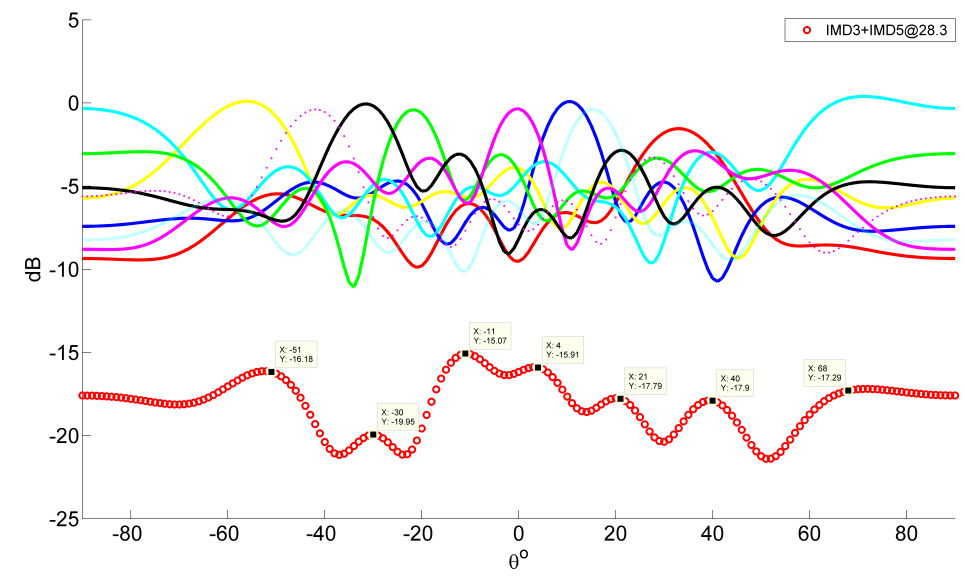

(a)

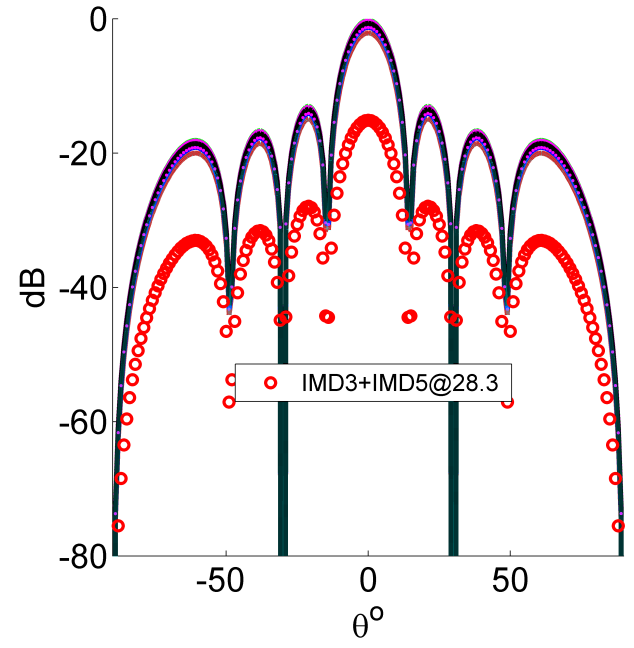

(b)

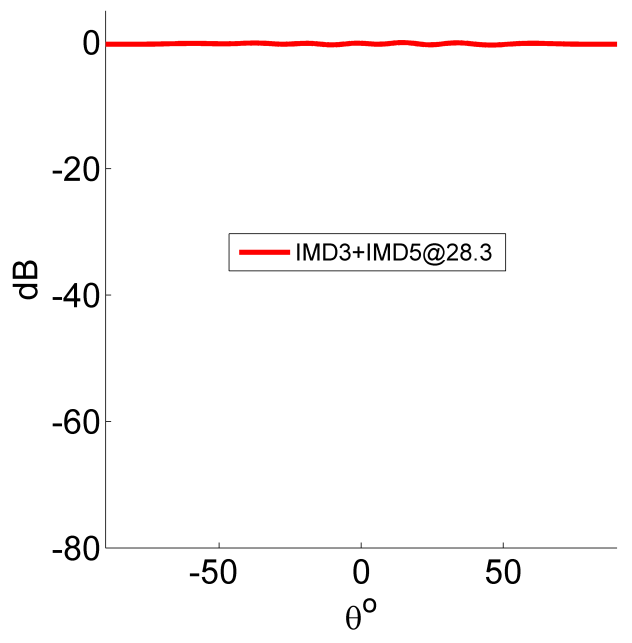

(c)

Figure 5.15: Simulated patterns of IMDs with the main signals when nine interferers are incident on the phased array for a) different frequencies coming from different incident angles b) different frequencies coming from the same incident angles $0^{\circ} \mathrm{c}$ ) same frequencies coming from different incident angles

from left to right. Accordingly, the 1USB harmonic component appears at the broadside direction $0^{\circ}$. Although the frequency of the harmonic component can be continuously tuned, three different frequencies are used in the simulation relying on the modulation sources available in the experimental setup which are 700,899 , and $1004 \mathrm{~Hz}$. Figure 5.16a shows the harmonic characteristics of these three different frequencies, while Figure 5.16b shows the scattered spatial beams of the 1USB 
harmonic components. The scattered beams do not change their spatial locations when a frequency of the modulation sources is changed because they have the same phase shift distributions.

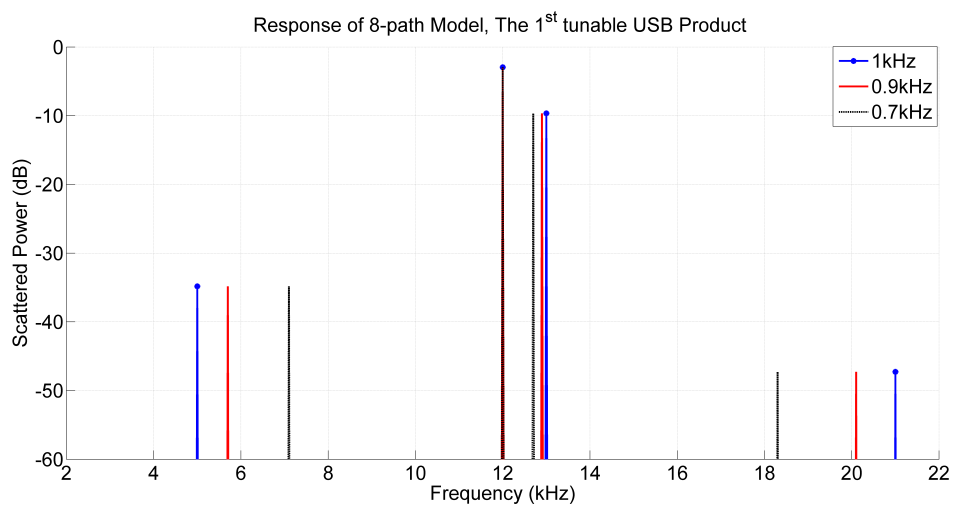

(a)

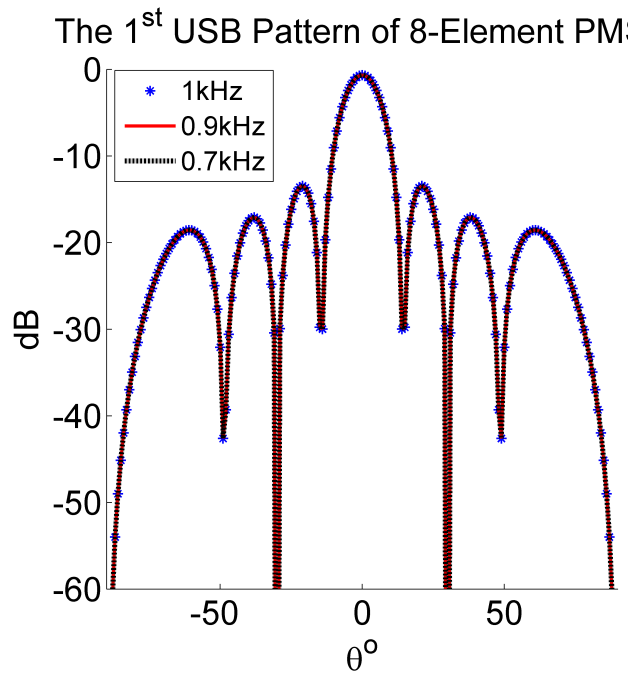

(b)

Figure 5.16: Simulation results of the tunable spatial harmonic generator a) spatial characteristics of the 1USB distortion product b) scattered beams of the 1USB distortion product directed toward angles $0^{\circ}$

\subsection{Direction Finding Based on the PMSA}

In conventional radio frequency direction finding (RDF) techniques, antenna arrays of two or more elements are utilized. Either amplitudes or phases of the received signals at each antenna element are compared and evaluated to determine directions 
of the arrival signals. In both methods, data at the array output should be processed exploiting array signal processing techniques to extract the required information [165] [163] [172]. Because of the heavy burden of signal processing in both of these techniques, some researchers seek to find ways which need low signal processing such as in [65]. Authors exploited a simple structure and harmonic characteristics of time modulated arrays (TMAs) in their proposed design [65] [93] [123] [32] [73]. In [165], authors reported an experimental setup of a 2-element TMA based direction finding. Direction of the incident signals are obtained by inserting an additional coaxial line having a flexible length into the reference antenna working as a phase compensator. The aim of the phase compensator is to cancel the first harmonic in the frequency response of an array output. The compensator has opposite phases compared to phases of unknown RF incident signals induced over antennas. The process of finding the exact opposite phases is similar to search in the phase range of $[0,2 \pi]$.

Similarly, our PMSA design generates harmonics due to non-linear operations and each harmonic generated in the PMSA has some important information including directions of the incident signals. Thus, the PMSA by its nature provides direction finding capabilities because directions of scattered patterns of harmonics are strongly affected by directions of the incident signals as given in (4.24) and demonstrated in the beamforming section in this chapter. Here, we exploit this feature of the PMSA and modify (4.24) for finding unknown directions of the incident signals to be a good potential application. Eq.(4.24) can be rewritten as

$$
\theta_{\text {unknown }}=\arcsin \left(-\sin \theta_{n}-\frac{2 n \pi}{L K d}\right)
$$

where $\theta_{o}$ in (4.24) is replaced by $\theta_{\text {unknown }}$ in (5.36) which represents the unknown direction of the incident signals that can be determined by the aid of spatial angles of the detected scattered beams of harmonics $\theta_{n}$. Figure 5.17 shows the scenario of 
the PMSA based direction finding, consisting of eight elements with $d=0.5 \lambda_{o}$ at the frequency $2.3 \mathrm{GHz}$ and a moving detector that observes scattered harmonics in the PMSA front of view (SFoV). A relative phase shift of the modulation sources between any two successive elements is $45^{\circ}$ starting from right to left.

Although, there are a certain number of scattered beams (i.e., propagating modes

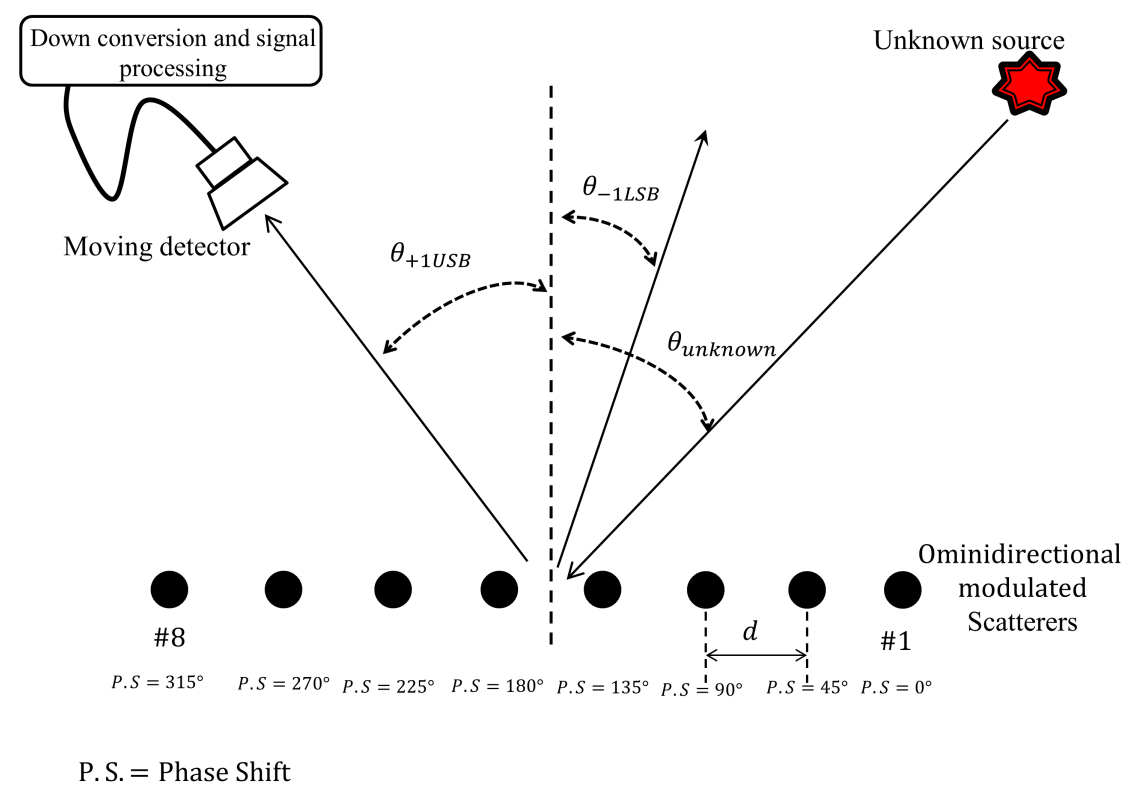

Figure 5.17: Scenario of the direction finding based on the PMSA

of harmonics) and all of them provide information about directions of the incident signals, we select only the first positive and negative distortions products (1LSB and 1USB) to perform the task of direction finding since their scattered beams change almost linearly with that of an incident angle as shown in Figure 5.18. However, neither the 1USB harmonic component nor the 1LSB harmonic component support all incident angles extending from the backfire $-90^{\circ}$ into the endfire $+90^{\circ}$. For example, the scattered beam of 1USB harmonic component generated in Figure 5.17 reaches the backfire $-90^{\circ}$ at the incident angle $48.59^{\circ}$ as illustrated in Figure 5.18, while the scattered beam of 1LSB harmonic component reaches the endfire $+90^{\circ}$ at the incident 
angle $-48.59^{\circ}$. To overcome this deficit, we exploit both 1LSB and 1USB harmonic components to entirely detect all incident signals at any angles in the SFoV of the PMSA.

Consider a single unknown source that changes its location five times and the

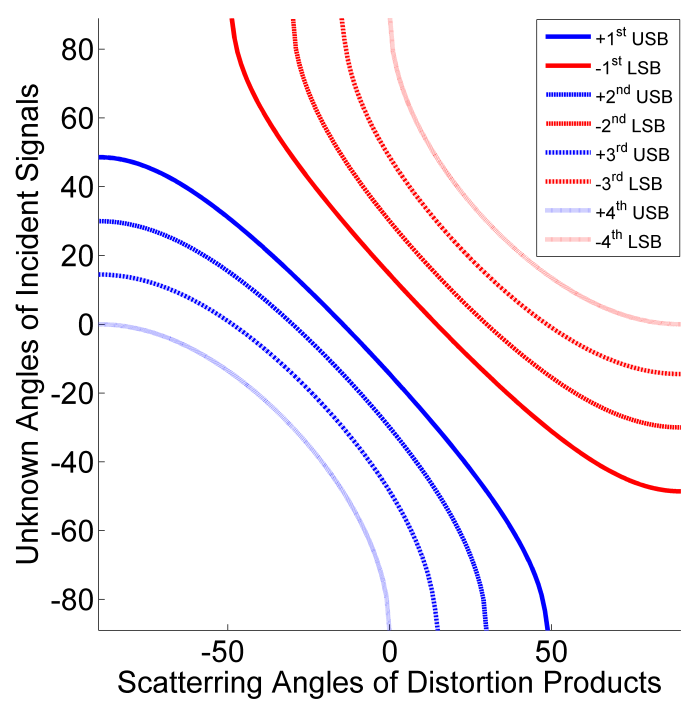

Figure 5.18: Scattering angles of harmonics vs. incident angles of unknown illuminating signals for the 8-element PMSA with $0.5 \lambda$

moving detector finds peaks of the scattered beam of 1USB harmonic component at angles $-65^{\circ},-15^{\circ}$ and $10^{\circ}$, and the peaks of the scattered beam of 1LSB harmonic component at angles $61^{\circ}$ and $84^{\circ}$. The corresponding incident angles of the unknown source according to (5.36) and Figure 5.18 are given in Table 5.6.

Results shown in the Table 5.6 are ideal, but in the practical design, there are some

Table 5.6: Calculation and simulation results

\begin{tabular}{c|c|c}
\hline \hline Harmonic term & Angle of detected scattered beam & Angle of incident signal \\
\hline$+1^{\text {st } U S B}$ & $-65^{\circ}$ & $48^{\circ}$ \\
\hline$+1^{\text {st } U S B}$ & $-15^{o}$ & $0.5^{o}$ \\
\hline$+1^{\text {st } U S B}$ & $10^{\circ}$ & $-25^{o}$ \\
\hline$-1^{\text {st }} L S B$ & $61^{o}$ & $-38.6^{o}$ \\
\hline$-1^{\text {st }} L S B$ & $84^{o}$ & $-48.1^{o}$ \\
\hline \hline
\end{tabular}

limitations which make the PMSA not functioning properly at far angles from the 
broadside direction such as patterns of elements, mutual coupling, and inaccuracies in fabrication. Thus, in the measurement part in Chapter 8, we will use omnidirectional antennas which are quarter wavelength monopoles over ground to provide almost constant radiation patterns in the Azimuth plane in order to mitigate the gap between theoretical and practical results.

To examine the performance of the PMSA system based direction finding with a large number of unknown incident signals, we assume five signals hitting the PMSA from different directions in the far-field of the PMSA. Unknown signals are uncorrelated. Distortion products generated because of each unknown signal hitting the PMSA have different spatial characteristics (i.e., scattered in different directions). Assume that the unknown signals have the same amplitude and different frequencies which are 2.1,2.2, 2.3, 2.4, and $2.5 \mathrm{GHz}$ incident on the PMSA at angles $32^{\circ},-35^{\circ},-4^{\circ}, 11^{\circ}$, and, $3.6^{\circ}$, respectively. Because the spacing $d$ between elements in the PMSA is $0.5 \lambda_{o}$ at the frequency $2.3 \mathrm{GHz}$, scattered angles of distortion products for these five signals when having identical incident angles are not the same. Figure 5.19 considers all these effects of variations in incident frequencies on scattered angles of the 1USB and the 1LSB harmonics.

Similar to principle of the PMSA, the detector should be moved until either the 1USB or the 1LSB harmonic of a certain incident signal is obtained, and all other harmonics of the same incident signal are suppressed at the output of the detector to declare that an angle of the incident signal has been recognized. Spectrums of scattered power of harmonics are recorded at five different spatial locations in order to find the exact angles of five different incident signals as shown in Figure 5.20 with the aid of formula given in (5.36) and Figure 5.19. The scattered signals at the detector output contain all five fundamental components and their harmonics distributed on both their sides at multiple integers of modulation frequency $7 \mathrm{MHz}$. As can be 


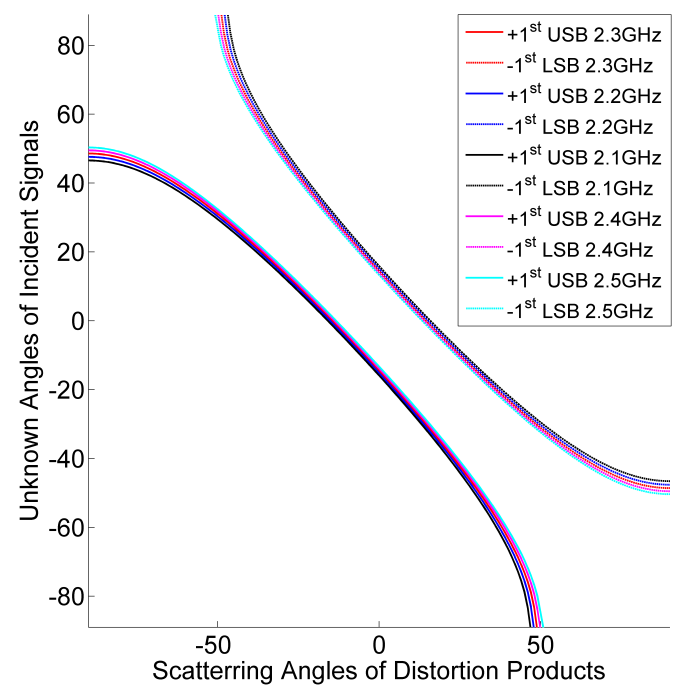

Figure 5.19: Scattering angles of the 1USB and 1LSB harmonics vs. incident angles of the five unknown illuminating signals for the 8-element PMSA with $0.5 \lambda$

seen, scattered responses have shown that only either 1USB or 1LSB harmonics of five different signals can be enhanced at some specific locations. According to basic theories of the PMSA, we can determine angles of the unknown incident signals by using the detected angle of 1USB and 1LSB harmonics with (5.36). Results of the five signals are presented in Table 5.7. Calculation and simulation results are in good agreement. The experimental part of the direction finding utilizing the PMSA will be illustrated in Chapter 8 with a single moving source

Table 5.7: Calculation and simulation results of direction finding for five unknown non-coherent signals

\begin{tabular}{c|c|c|c|c}
\hline \hline Inc. signal no. & Freq(GHz) & DA & H type & UA \\
\hline 1 & 2.3 & $71^{\circ}$ Figure 5.20a & $1 \mathrm{LSB}$ & $-44.1^{\circ}$ \\
\hline 2 & 2.1 & $-53^{\circ}$ Figure 5.20b & $1 \mathrm{USB}$ & $31.7^{\circ}$ \\
\hline 3 & 2.2 & $18^{\circ}$ Figure 5.20c & $1 \mathrm{USB}$ & $-34.8^{\circ}$ \\
\hline 4 & 2.4 & $4^{\circ}$ Figure 5.20d & $1 \mathrm{LSB}$ & $11^{\circ}$ \\
\hline 5 & 2.5 & $-17^{\circ}$ Figure 5.20e & $1 \mathrm{USB}$ & $3.6^{\circ}$ \\
\hline DA=Detected Angles, H= Harmonic UA=Unknown or incident & Angle. \\
\hline
\end{tabular}




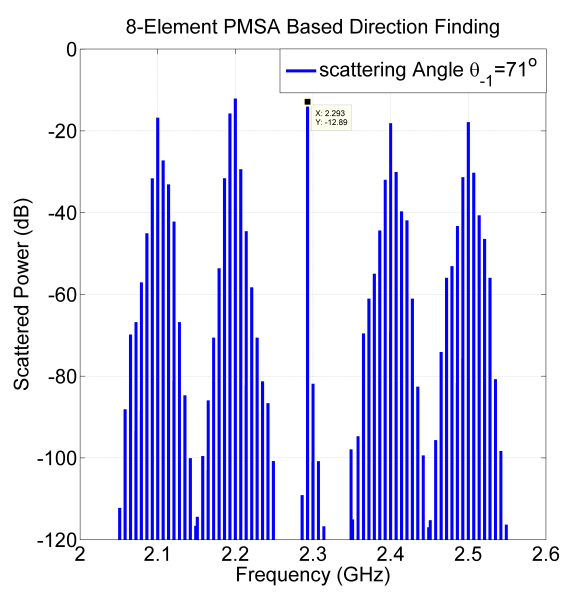

(a)

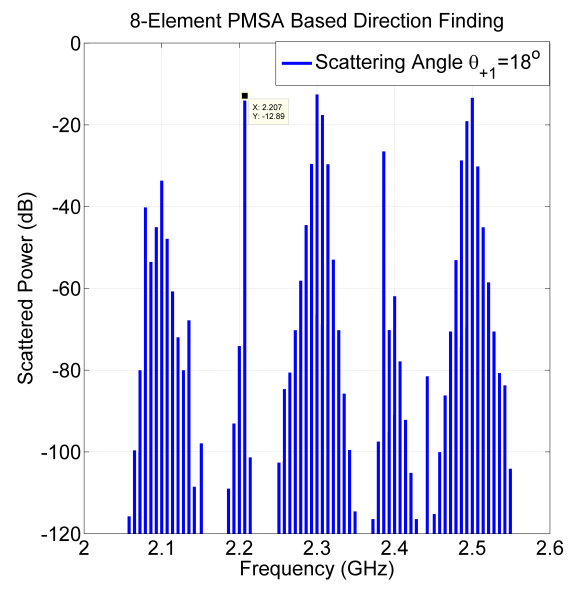

(c)

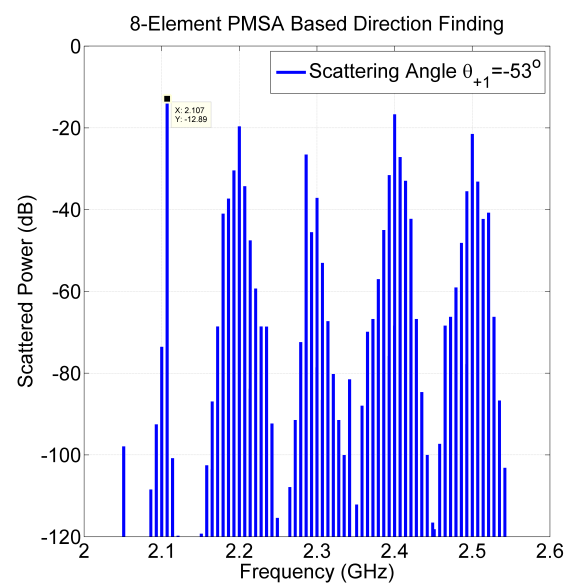

(b)

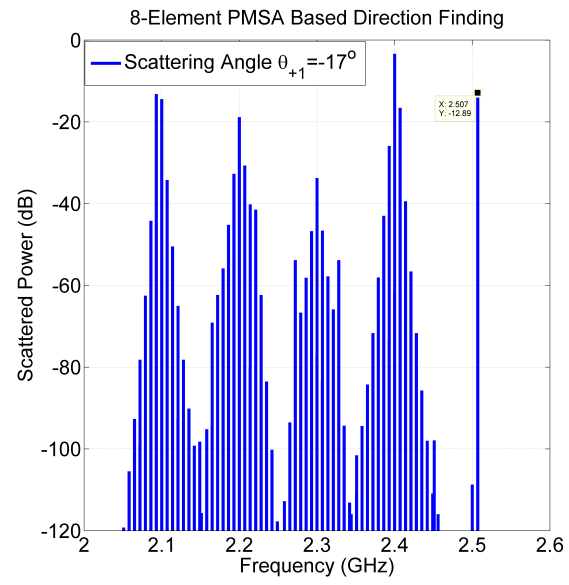

(d)

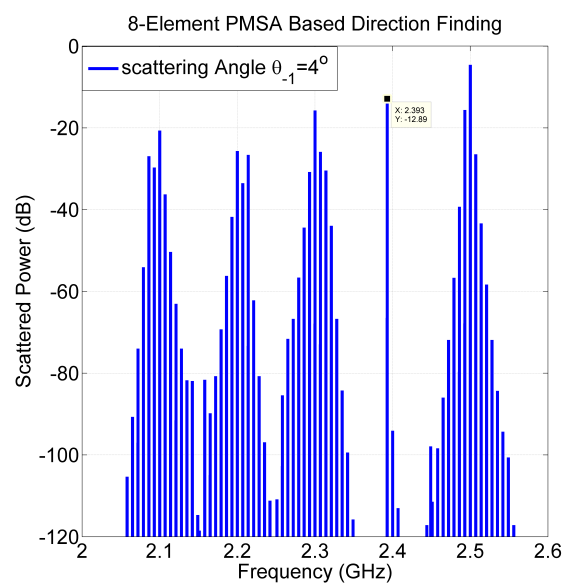

(e)

Figure 5.20: Distortion characteristics at the moving detector output recorded at spatial angles a) $71^{\circ}$ b) $-53^{\circ}$ c) $18^{\circ}$ d) $4^{\circ}$ e) $-17^{\circ}$ 


\section{Chapter 6: Preliminary Works}

\subsection{Introduction}

The main aim of this chapter is to focus on designing and manufacturing the essential parts of the finals prototypes of the PMSA used later in this research measurement work, so it can be considered as a bridge linking between the theoretical parts described in Chapters 3, 4, and 5 and the practical parts that will be described in Chapter 8. The essential parts of the presented design are receive elements, modulators to generate distortion products, and retransmit elements. Although in most cases, the receive and retransmit elements are just a single element performing both functions simultaneously, typically being a single conventional antenna, very few of them are separate. The modulator is merely an electronic switch which is a diode or a transistor controlled by a periodic signal. It is connected to prescribed loads in such a way,as to prohibit the incident signals to reach another port of a circuit, and thus being called reflective-type switch (RTS). Both receive-retransmit elements and modulators are combined in single circuits called modulated scatterers (MSs).

The chapter is organized as follows. Section 6.2, discusses different individual types of antennas. Antennas types are the printed quasi-Yagi dipole, the inset-fed microstrip patch, and the quarter wavelength monopole over a ground. After evaluating them as single elements, they are then assembled in groups to form arrays. In section 6.3, reflective-type switches (RTSs) used as modulators are designed, simulated, fabricated and measured. In this section, influences of design parameters on the overall performance and the strength of scattered signals are illustrated. Finally, section 6.4 describes the design of a phase transformer kit utilizing resistors only. 


\subsection{Design of Antennas for the PMSA}

Antennas are critical parts in all wireless applications including our PMSA. In the PMSA, the antenna is of a twofold importance. On the one hand, it acts as a receive element to then use the received signals in the modulation process. On the other hand, it retransmits the modulated signals again into space. Coverage, range, and number of scattered distortion products which we consider as Key performance metrics of the PMSA system depend strongly on properties of antennas as demonstrated in Chapters 3 and 4 [10]. When using antennas that have wide-beamwidths in arrays, this can extend a coverage of the PMSA (i.e., increasing a number of scattered beams in space), but leads in reduction of the antenna directivity. In contrary, high gain antennas that have narrow beamwidths are preferred in the design of arrays used in the long range communication systems, resulting in limited coverage. These two metrics are undoubtedly contradictory, so using antennas with moderate gains is the only way to overcome these problems. The capacity representing a number of distortion products scattered from the PMSA is influenced by many antenna factors, including antenna bandwidth. Ideally, these factors should be kept as useful as possible. However, the larger aperture is the cost paid with arrays having a large number of elements. Thus, this section provides full details of antenna designs, with the aim of building antenna array designs that meet the PMSA requirements (size, coverage, and range) and are easy to manufacture using the cheap PCB technology.

This section comprises two parts. Three different single types of antennas are designed, simulated, and built in the first part. In the following part, we introduce five different antenna arrays. 


\subsubsection{Single Antenna Design}

\subsubsection{Printed Broadband Yagi dipole Antenna}

To choose an antenna fulfilling the requirements explained above, the broadband quasi-Yagi printed dipole antenna has been adopted as a main element in this research work [134] [31] [99] [95]. A schematic of the antenna, consisting of an active driver, a parasitic director, and a truncated conductor layer acting both as a ground for the feeding line and also as a parasitic reflector for the antenna, is illustrated in Figure 6.1a. There is no need to use an external unbalance-to-balance (BALUN) transformer between arms of the active element of the antenna and a 50ohm microstrip feeding line. The balun is a via-hole microstrip type, integrated between the driver and the reflector on both sides of the substrate as displyed in Figure 6.1a, and it is broadband as described in detail in [31]. Both the printed dipole and most of the balun structure are situated on the backside of the substrate, while the feed-line and a small portion of the balun structure reside on the frontside of the substrate. Lengths of microstrip balun and arms of the dipole driver are about a quarter-wavelength at the design frequency which is $2.3 \mathrm{GHz}$. The via-fed method is used to force the phase difference between two arms of the dipole driver equal to $180^{\circ}$ in order to align directions of current distributions on both arms. Figure $6.1 \mathrm{~b}$ shows the 3D exploded view of the printed quasi-Yagi dipole antenna, Figure 6.1c shows the equivalent circuit of the antenna, and Figure 6.1d shows a direction of the current distribution on the printed dipole antenna. The antenna has been printed on a Rogers 4003C substrate of a dielectric constant 3.55, a tangent loss 0.0027 , and a substrate thickness of 60 mil $(1.52 \mathrm{~mm})$. Table 6.1 presents dimensional parameters of the printed quasi-Yagi dipole antenna, given in Figure 6.1a.

These optimized values have been obtained after several simulations using two 
Table 6.1: Dimensions of the printed quasi-Yagi dipole antenna printed on the Rogers 4003C substrate with a thickness of 60mil, shown in Figure 6.1a

\begin{tabular}{c|c|c|c|c|c|c|c|c|c}
\hline \hline Parameter & $w_{s}$ & $l_{s}$ & $w_{g}$ & $l_{g}$ & $w_{\text {arm }}$ & $l_{\text {arm }}$ & $w_{b f}$ & $l_{b f 1}$ & $l_{b f 2}$ \\
\hline Dimension $(\mathbf{m m})$ & 65 & 70 & 65 & 15 & 6 & 25 & 3.4 & 36 & 6.5 \\
\hline Parameter & $w_{b}$ & $l_{b}$ & $l_{s}$ & $w_{\text {dir }}$ & $l_{\text {dir }}$ & $s$ & $s_{s}$ & $r$ & \\
\hline Dimension $(\mathbf{m m})$ & 6 & 21 & 21 & 6 & 30 & 12 & 1 & 0.5 & \\
\hline \hline
\end{tabular}

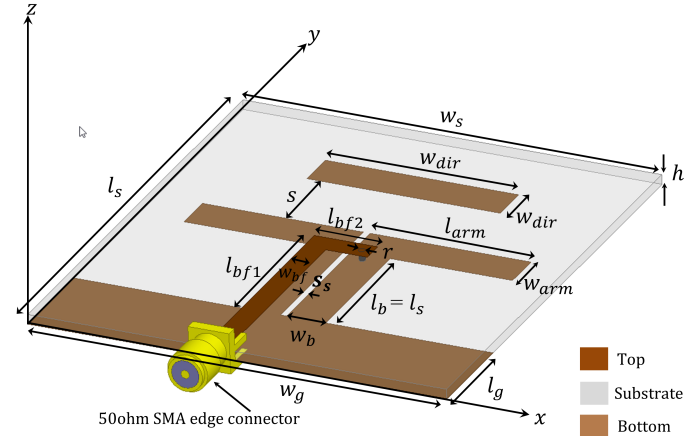

(a)

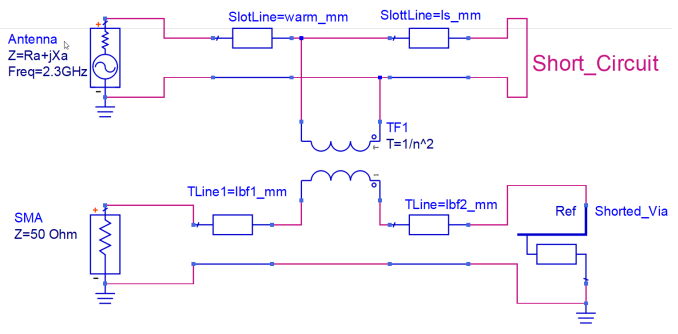

(c)

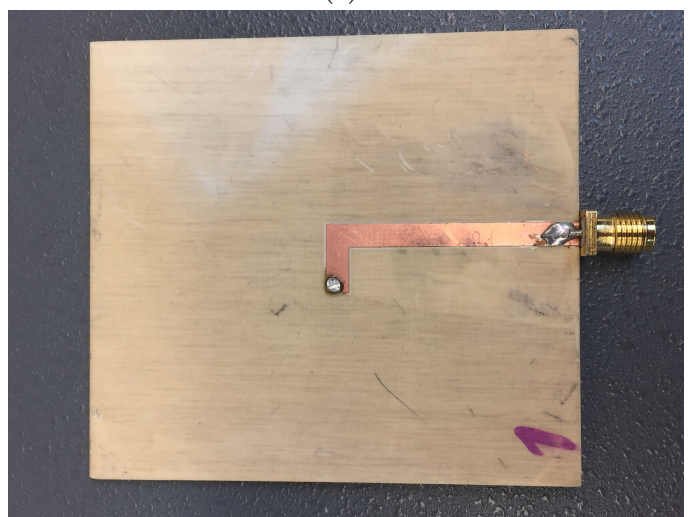

(e)

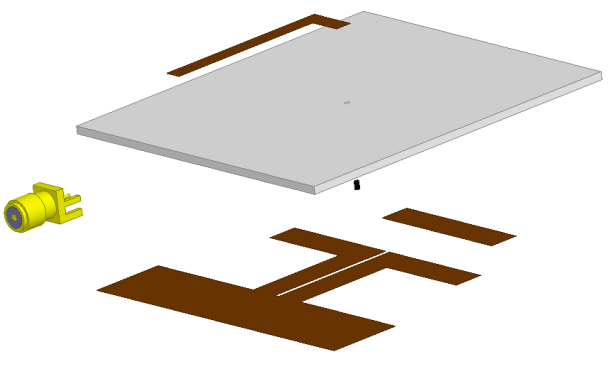

(b)

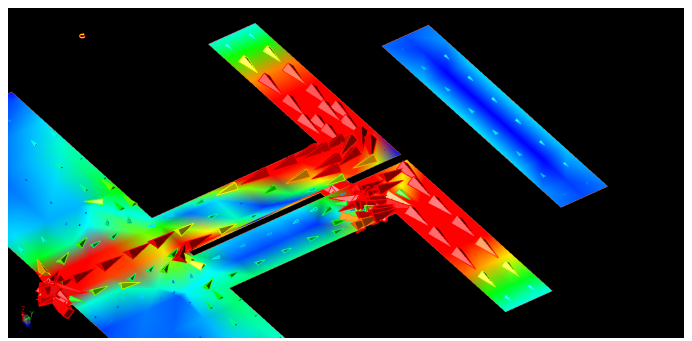

(d)

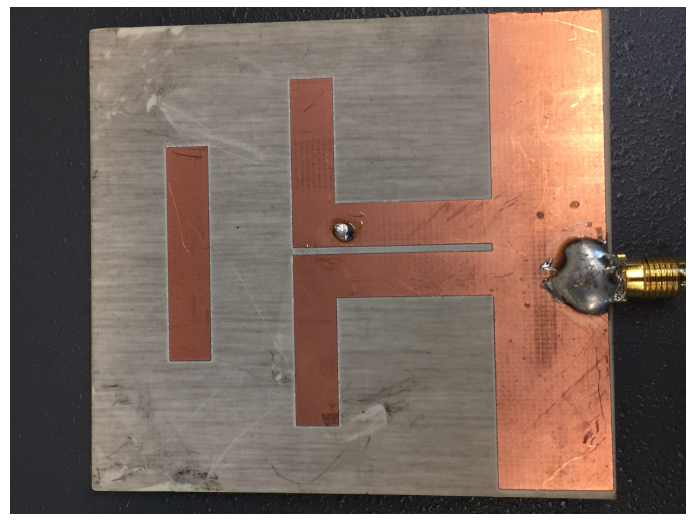

(f)

Figure 6.1: Printed Yagi dipole antenna a) geometry b) 3D exploded view c) equivalent circuit d) current distribution over an antenna aperture at $2.3 \mathrm{GHz}$ e) fabricated top-view f) fabricated bottom-view 


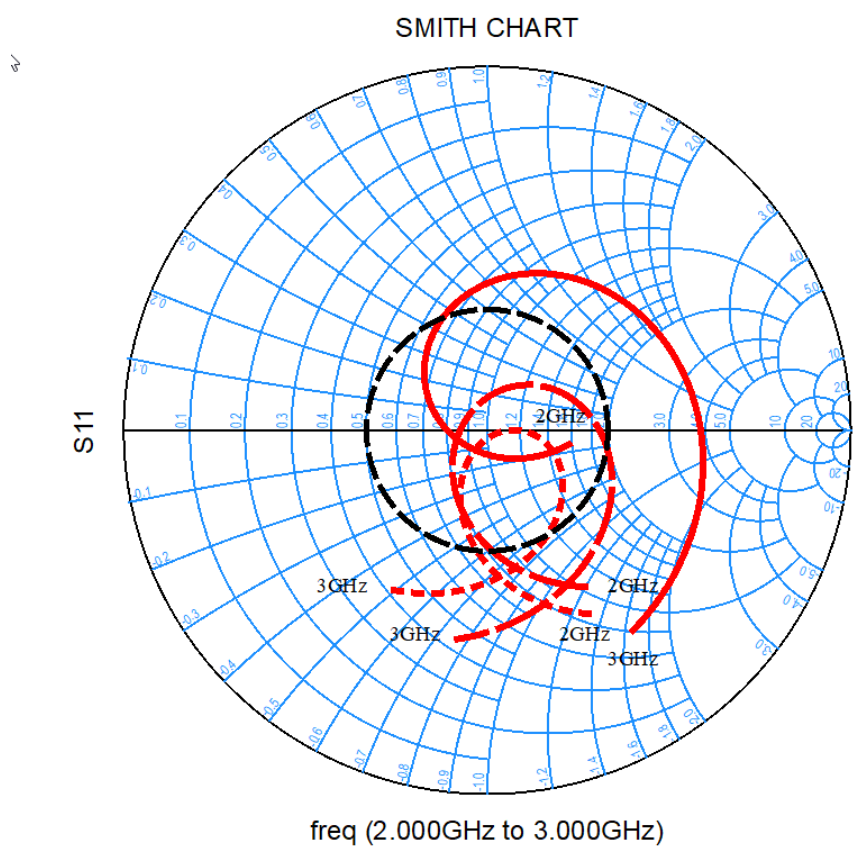

Figure 6.2: Input impedance of the printed Yagi dipole antenna for $l s=11 \mathrm{~mm}$ short dashed red line, $l s=21 \mathrm{~mm}$ long dashed red line, and $l s=31 \mathrm{~mm}$ solid red line

different electromagnetic solvers which are the high frequency structure simulator HFSS from the Ansys Inc. [44] and the advanced system design ADS from Keysight Inc. [2] where the environment of the first simulator uses the finite element method FEM in analyzing structures and the second one uses the method of moment MoM. An input impedance of the antenna is optimized in simulations to be a $50 \mathrm{ohm}$ and so there is no need to use an impedance transformer between the antenna and a 50ohm SMA edge connector. As a consequence, the microstrip transmission-line TL connecting the antenna and the SMA connector is designed to have $50 \mathrm{ohm}$ characteristic impedance with $w_{f}=3.4 \mathrm{~mm}$. The photograph of the top and the bottom of antenna prototype are demonstrated in Figure 6.1e and 6.1f, respectively.

Although the integrated balun acts as a transition unit between the transverse electromagnetic TEM modes in a coaxial cable and quasi-TEM in the printed quasi-Yagi dipole antenna, it also plays a vital role in a mechanism of impedance 
matching. There are several parts in the balun governing the antenna matching. As can be seen, when changing a slot length of the balun $l_{s}$ as $(11 \mathrm{~mm}, 21 \mathrm{~mm}$, and $31 \mathrm{~mm}$ ), the reflection coefficient at an antenna input (S11) on the Smith chart changes dramatically as shown in Figure 6.2. For more details about effects of other balun parts on the matching, interseted readers are referred to [99]. In the design, we select the $l_{s}=21 \mathrm{~mm}$ as a candidate because a good matching result has been attained as shown in Figure 6.2. Figure 6.3 depicts antenna results. Simulation results are obtained by software ADS and HFSS and measured results are obtained by a vector network analyzer VNA at the high radio-frequency test and EMAG Labs. The antenna reflection coefficient $(\mathrm{S} 11)$ is shown in Figure 6.3a. Although there are discrepancies between the simulated and measured results, they are in good agreement. Also, the discrepancy between the measured and simulated results is also to environments being considered ideal in simulations. The measured S11 curve is below $-10 \mathrm{~dB}$ from $2.08 \mathrm{GHz}$ to $2.8 \mathrm{GHz}$. Thanks to the broadband response of the balun [31], the response of antenna is broadband too. The simulated E-cut and H-cut planes, and 3D radiation patterns gains shown in Figure $6.3 \mathrm{~b}$ and 6.3c, respectively. Finally, the AUT absolute gain has been simulated and Figure 6.3d shows the result. The simulated gain is about $4.8 \mathrm{~dB}$ at the design frequency $2.3 \mathrm{GHz}$.

\subsubsection{Inset-fed Microstrip Patch Antenna}

The second type of antennas used in this research work is the inset-fed microstrip patch antenna. This antenna has low profile characteristics, so it is preferred in several applications seeking the integration with PCB boards. It is also chosen here for the same reason. Figure 6.4a shows a schematic of the antenna along with all its optimized dimensions, and Figure 6.4b shows a photograph of the fabricated antenna. Width and length of the inset-fed slot facilitate the impedance matching process by 


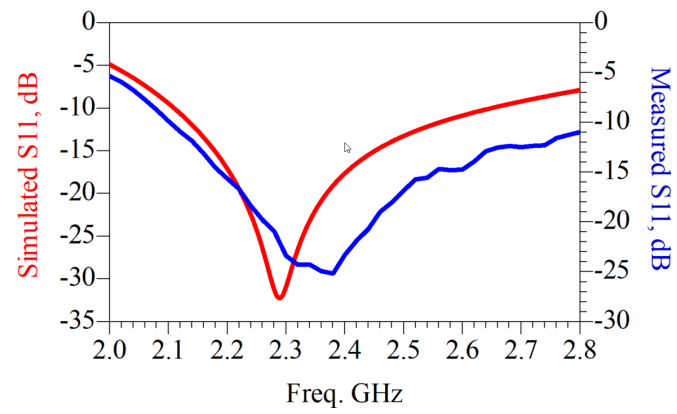

(a)
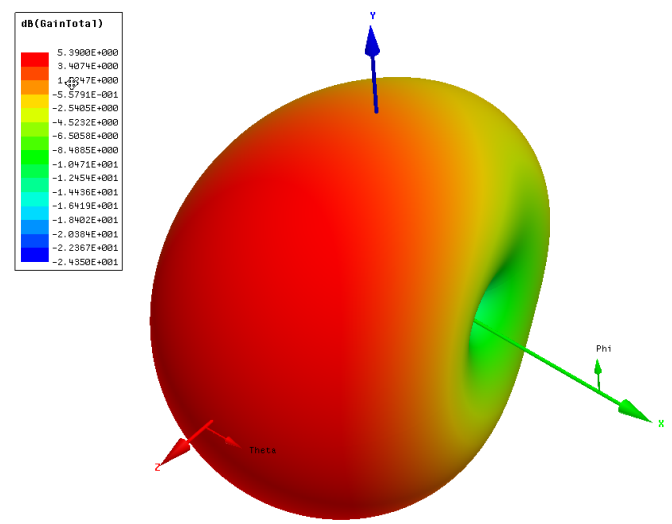

(c)

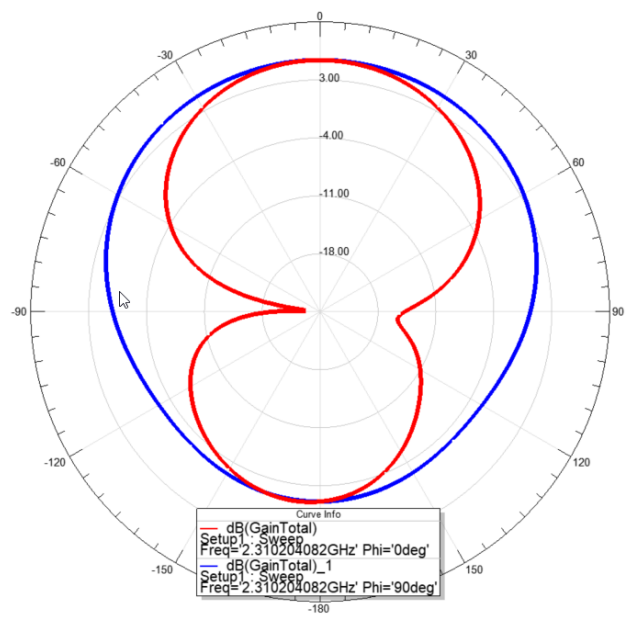

(b)

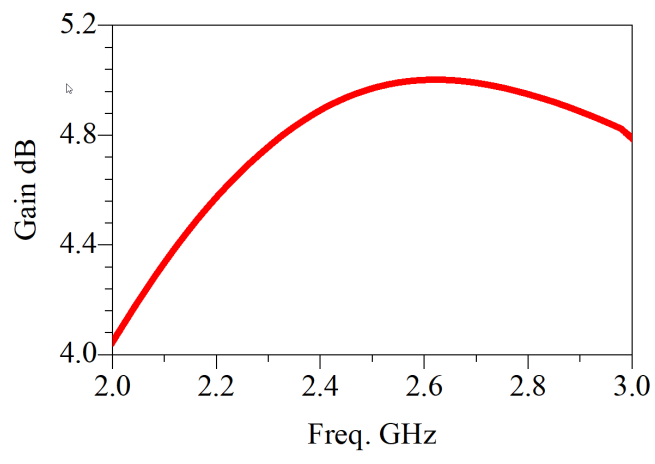

(d)

Figure 6.3: a) Simulated and measured S11 of the printed quasi-Yagi dipole antenna b) simulated E-cut and $\mathrm{H}$-cut planes of the gain radiation pattern at $2.3 \mathrm{GHz}$ c) simulated $3 \mathrm{D}$ gain radiation pattern at $2.3 \mathrm{GHz} \mathrm{d}$ ) simulated gain

increasing degrees of freedom of the design parameters controlling the overall antenna performance [70] [111]. The antenna is linearly polarized, and it is simulated using only the ADS software. The patch antenna is printed on a $60 \mathrm{mil}(1.52 \mathrm{~mm})$ thick Rogers 4003C substrate. The antenna is placed on the xy plane and is directed along the z-axis. The antenna resonates at $2.3 \mathrm{GHz}$ where the simulated $\mathrm{S} 11$ has the minimum value as shown in Figure 6.4c. Figure 6.4d shows real and imaginary parts of the input impedance of the antenna. A vector of the electric field distribution over an antenna aperture is illustrated in Figure 6.4e. As can be noticed, two of aperture 
edges are not radiated because fields distributions are out of phase, while other two edges have in-phase filed distributions, yielding in a constructive interference in space over the antenna aperture. Power transfer from or to the antenna increases as the S11 decreases and the maximum value occurs at $S 11=-\infty$, (i.e., $Z_{\text {in }}=50+j 0 \Omega$ ). Figure 6.4f depicts power transfer vs frequency. The impedance bandwidth is defined over a frequency range where the S11 is less than -10dB. The impedance bandwidth is about $40 \mathrm{MHz}$. Figure $6.5 \mathrm{a}$ and $6.5 \mathrm{~b}$ show the E-plane and H-plane cuts radiation patterns of the antenna, respectively, and Figure $6.5 \mathrm{c}$ shows the $3 \mathrm{D}$ plot of radiation pattern, all at $2.3 \mathrm{GHz}$.

\subsubsection{Monopole Antenna over a Finite Rectangular Ground}

The antennas explained above offer unidirectional radiation patterns as well as having half power beamwidths HPBWs less than $180^{\circ}$ which are around $90^{\circ}$ to $100^{\circ}$, and thus having high gains. Although antennas with these features are highly demanded in some applications, their coverage cannot reach distant angels (i.e., $-90^{\circ}$ and $90^{\circ}$ ). Oppositely, these issues can be overcome if antennas with omnidirectional patterns are utilized. Hence, a monopole antenna over a finite ground is obtained to perform in some measurements as will be shown later in Chapter 8. Figure 6.6 displays a layout of the antenna made of a 3mm brass rod and located over a rectangular copper ground, its exploded view, and a photograph of the fabricated prototype. A SMA connector feeding the antenna is located beneath the ground. The antenna is designed at a frequency of $2.3 \mathrm{GHz}$ with a length $32 \mathrm{~mm}$. Simulated S11 is presented in Figure 6.6d. The 3D-omnidirectional radiation pattern along with an antenna structure is depicted in Figure 6.6e. The main drawback with this antenna is the low gain, leading to a reduction in the range that the antenna can perform in measurements and wireless connection with other antennas. 


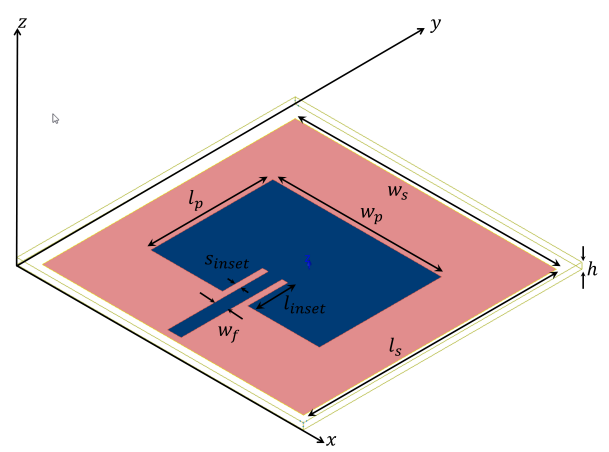

(a)

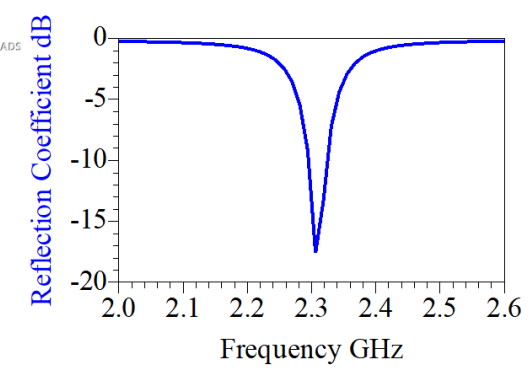

(c)

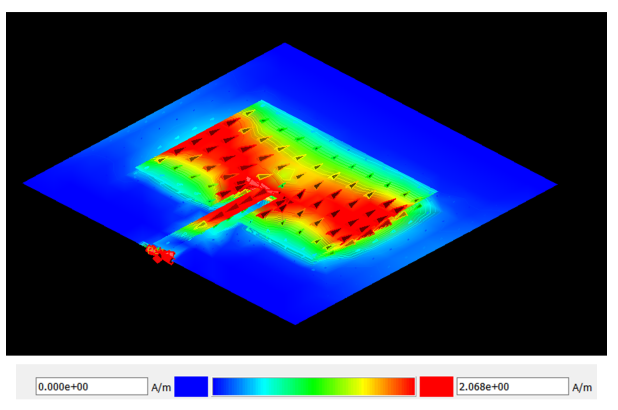

(e)

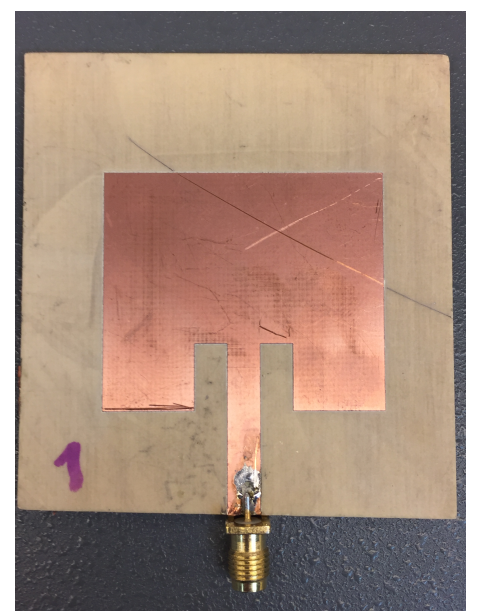

(b)

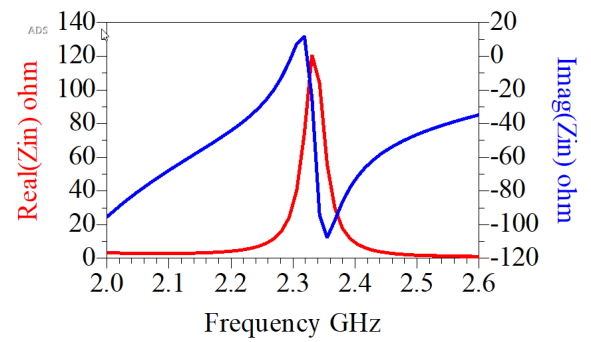

(d)

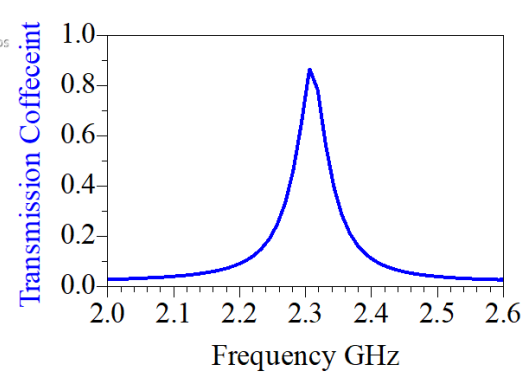

(f)

Figure 6.4: Microstrip patch inset-fed antenna a) geometry $w_{s}=l_{s}=64 \mathrm{~mm} w_{p}=41 \mathrm{~mm} l_{p}=$ $30.25 \mathrm{~mm} w_{f}=3.4 \mathrm{~mm} l_{\text {inset }}=10 \mathrm{~mm}$ and $s_{\text {inset }}=1.4 \mathrm{~mm} \mathrm{~b}$ ) fabricated c) simulated $\mathrm{S} 11 \mathrm{~d}$ ) simulated real and imaginary parts of Zin e) simulated electric field distribution over an antenna aperture f) simulated transmission coefficient

\subsubsection{Conventional Folded Yagi-Uda Antenna}

The last type of antennas used in the research work is the folded Yagi-Uda antenna working at two different frequencies $432 \mathrm{MHz}$ and $2.3 \mathrm{GHz}$, as shown in Figure 6.7. 


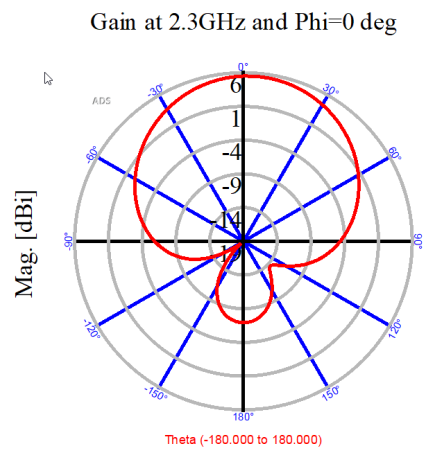

(a)

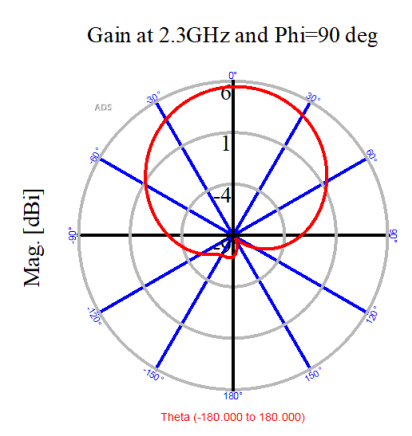

(b)

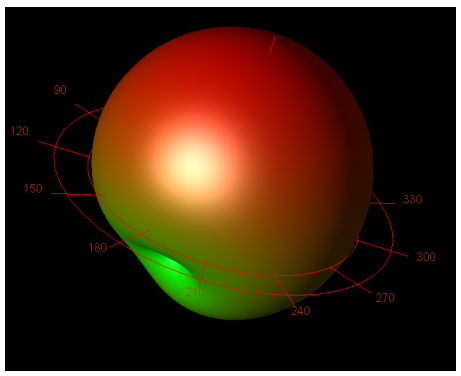

(c)

Figure 6.5: Properties of microstrip patch inset-fed antenna a) E-cut plane radiation pattern b) $\mathrm{H}$-cut plane radiation pattern c) $3 \mathrm{D}$ view of radiation pattern

They are designed and built by Prof. Richard Campbell at Portland State University PSU, ECE department. The first experiments are done with the $432 \mathrm{MHz}$ antennas as will be illustrated later. The antennas working at $2.3 \mathrm{GHz}$ are utilized as transmitting and receiving antennas connected to an illuminating signal generator and a receiver, respectively. These antennas are more common in a different spectrum of applications, so interested readers are referred to [137] [34] for more details about the design.

\subsubsection{Arrays}

Having discussed, designed, and analyzed various antennas in the earlier part, here, different arrangements of arrays using those antennas will be discussed and analyzed. Because our PMSA consists of more than one distortion product generator which means modulated scatterers (MSs) which in turn have antennas in their structures, it becomes imperative to examine the antenna performance when working within an array and also investigate the overall array performance.

As seen in the previous section, the antenna that has the narrower half-power beamwidth (HPBW) has the higher gain (i.e., the gain is inversely proportional to the HPBW), and we will also see here that the array that have the larger aperture length have the higher gain. This rule comes from that the antenna with a larger aperture 


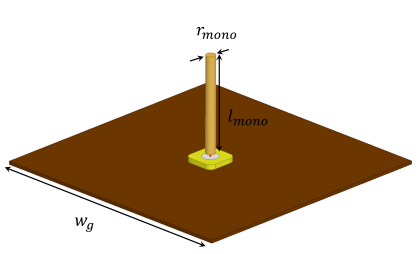

(a)

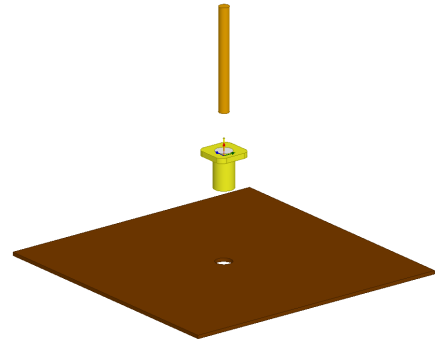

(b)

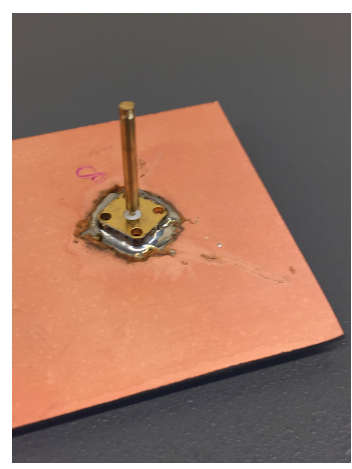

(c)

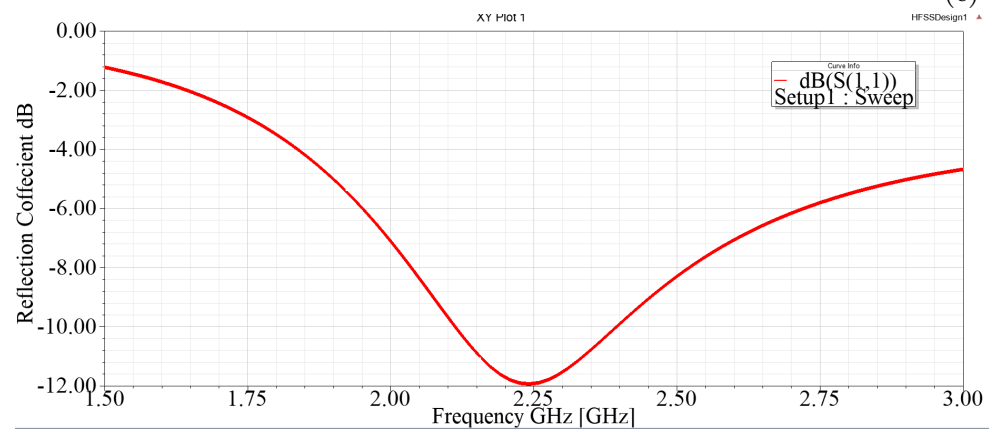

(d)

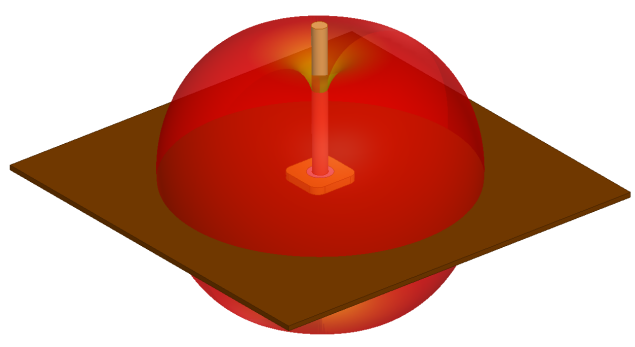

(e)

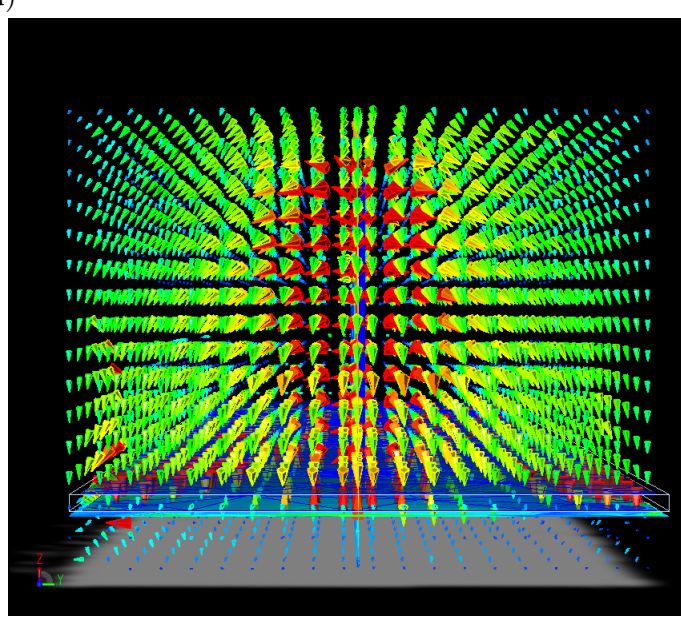

(f)

Figure 6.6: Monopole antenna over a rectangular ground a) 3D geometry b) 3D exploded view c) fabricated d) simulated S11 e) 3D radiation pattern f) 3D representation of electric field distribution

and current distributed over it, results in a narrower far-field radiation beam, high gain, according to the spatial Fourier transform (Green function) [63] [62]. The same is applicable to arrays with larger aperture lengths as well [34] [108].

In the theoretical chapters, we obtained all results utilizing a group of distortion 


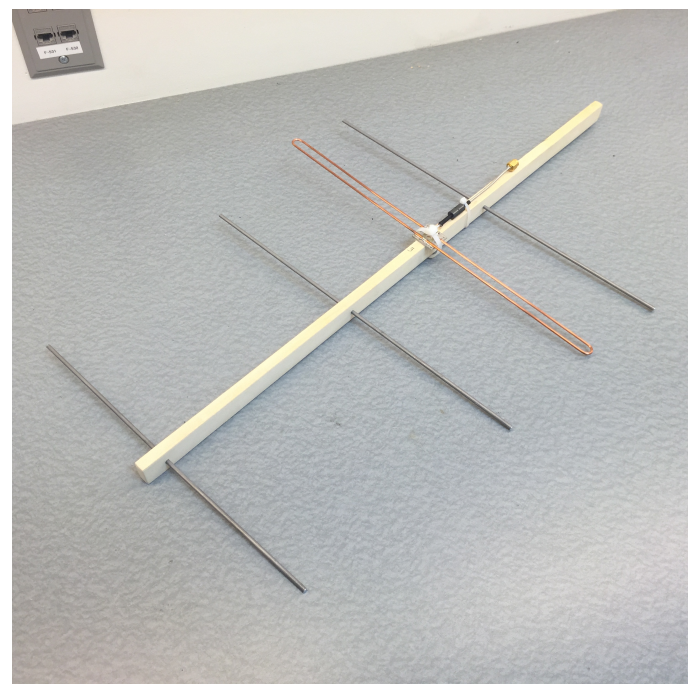

(a)

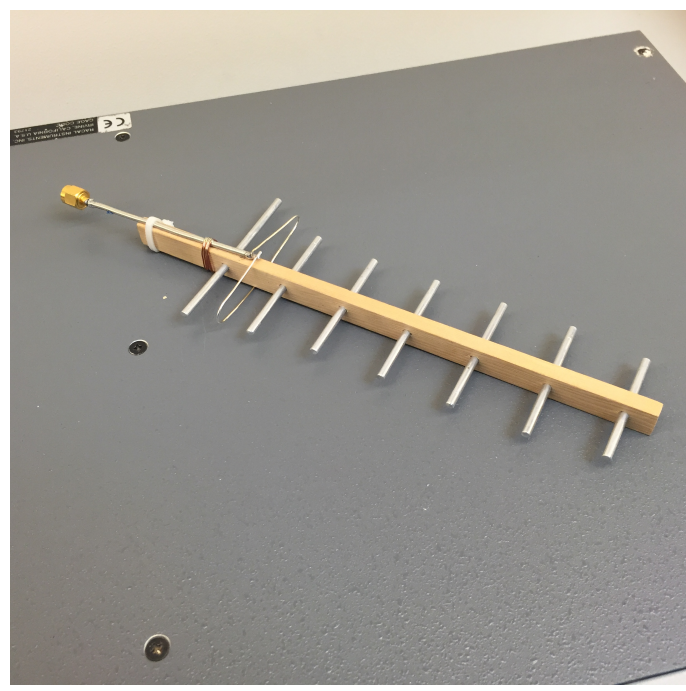

(b)

Figure 6.7: Conventional folded Yagi-Uda antenna working at a frequency of a) $432 \mathrm{MHz}$ b) $2.3 \mathrm{GHz}$

products generators lined up along one axis (i.e., a linear array of modulated scatterers). In addition, we assumed that the generators of distortion products have different phases to fully achieve goals of the PMSA design. Thus, the PMSA scatters the different distortion products into different spatial locations along one axis (one dimension, 1D). The process is somewhat similar to the 1D beamforming mechanism in the conventional phased arrays. However, the distortion products can reach any point in space if we use 2D modulated scattering arrays, but because of the proposed idea introduced for the first time in this research work, we will not go further with 2D modulated scattering arrays and leave it as a future work. Even if we use 2D modulated scattering arrays in the measurements, the relative phase shift difference between elements along one of array dimensions is zero, thereby reducing the analysis of the 2D modulated scattering arrays into the 1D modulated scattering array.

Now, the analysis of $1 \mathrm{D}$ and 2D arrays in the point of view of the phased antenna array theory is similar, but in the latter, single elements are replaced by 1D arrays. The antenna element behaves slightly different inside an array, affected by different 
factors. Therefore, the analysis will include both linear 1D and planar 2D arrays. The arrays will be numerically analyzed using the commercial software HFSS based on the FEM. The overall radiation pattern of an array obtained from the numerical analysis can be factored into two different components: the element pattern EP and the array factor AF. The AF is similar to the overall radiation pattern of an array when all its elements are replaced by isotropic elements. The EP represents the radiation pattern of any single antenna in the array. However, radiation patterns of antennas in the array are not identical due to the electromagnetic mutual coupling between elements [34].

Beam pattern and gain of an array consisting of isotropic elements rely on three different parameters: the total number of antennas in the arrays, spacing between the antennas, and antennas arrangements. Although the main purpose of this section is to analyze arrays used in the measurements, impact of these parameters on the gains and the beam patterns will be explicitly examined.

\subsubsection{Design of Nominated Arrays}

Eight arrays have been designed during this research journey utilizing different types of antennas given above in detail. Table 6.2 provides a brief summary of arrays properties like size, element type, mutual coupling category, spacing, and design frequency. Dimensions and parameters of arrays have been optimized using the full-wave electromagnetic simulator HFSS excluding the first two arrays which their elements already are built by Prof. Richard Campbell. In Figure 6.8, photographs of the first two arrays are presented working at $432 \mathrm{MHz}$. They are used in the first phase of the research experiments and their results were very satisfactory despite some constraints in the measurement environment and facilities as will be described in Chapter 7 . In the second phase, antennas sizes are scaled down to work at $2.3 \mathrm{GHz}$, 
demonstrating the design scalability which is one of the research goals for avoiding or mitigating some of the constraints existing in the first phase of measurements.

The rest of the antenna arrays, in Table 6.2, are designed and analyzed at $2.3 \mathrm{GHz}$.

Table 6.2: Properties of nominated arrays

\begin{tabular}{c|c|c|c|c|c|c}
\hline \hline Array No. & Size & Element & Spacing & Freq. GHz & Fig. No. & MC type \\
\hline Array 1 & $2 \times 1$ & FY & $0.7 \lambda_{o}$ & 0.432 & Figure 6.8a & E \\
\hline Array 2 & $4 \times 1$ & FY & $0.7 \lambda_{o}$ & 0.432 & Figure 6.8b & E \\
\hline Array 3 & $2 \times 1$ & PY & $0.7 \lambda_{o}$ & 2.3 & Figure 6.10 & $\mathrm{H}$ \\
\hline Array 4 & $4 \times 4$ & PY & $0.5 \lambda_{o}$ & 2.3 & Figure 6.11a & Mixed \\
\hline Array 5 & $8 \times 2$ & PY & $0.5 \lambda_{o}$ & 2.3 & Figure 6.12a & Mixed \\
\hline Array 6 & $8 \times 1$ & PY & $0.5-0.7 \lambda_{o}$ & 2.3 & Figure 6.13a & $\mathrm{H}$ \\
\hline Array 7 & $4 \times 1$ & P & $0.7 \lambda_{o}$ & 2.3 & Figure 6.13b & $\mathrm{H}$ \\
\hline Array 8 & $8 \times 1$ & MP & $0.5 \lambda_{o}$ & 2.3 & Figure 6.13c & $\mathrm{H}$ \\
\hline MC:Mutual Coupling Mixed:E-H FY:Folded Yagi PY:Printed Yagi P:Patch MP:Monopole \\
\hline
\end{tabular}

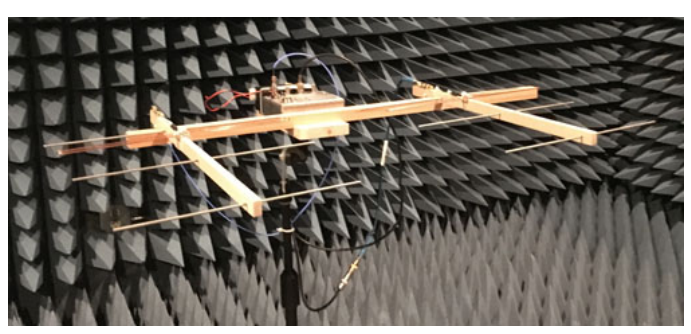

(a)

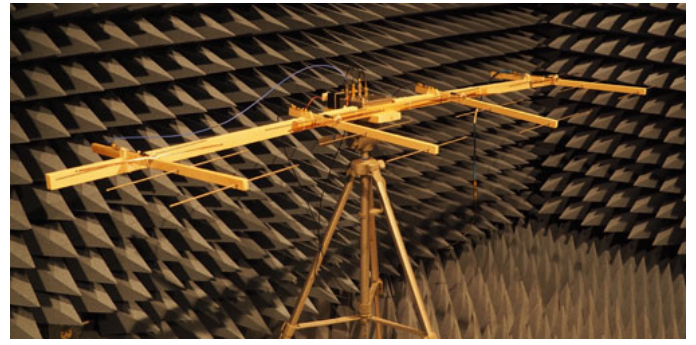

(b)

Figure 6.8: Conventional folded Yagi-Uda antenna arrays working at a frequency of $432 \mathrm{MHz}$ and performing inside the anechoic chamber a) 2-element array b) 4-element array

A performance of an antenna within the array is slightly different than when working standalone. In the standalone case, there is only a single source which is either an incident signal or an applied signal at an antenna input to induce a current, whereas a current induced over an antenna working within an ensemble of antennas comes from different sources [60] [158]. These sources include the main signal and signals emitting from the surrounding antennas. The electromagnetic interaction between elements that changes some antenna properties is an inherent mechanism called the mutual coupling that is known since the advent of antenna arrays in the Second World War WWII, and the study of this topic is still active until now [34]. 
To validate that numerically, the printed quasi Yagi dipole antenna is used in four different scenarios. In the first scenario, a single antenna is analyzed to consider its results as a reference to results of other scenarios. Two antennas are then added along the $\mathrm{x}$-axis, side by side, in the second scenario with a $\lambda_{o} / 2$ distance apart, being $65 \mathrm{~mm}$ at $2.3 \mathrm{GHz}$. The electric field interaction between the two antennas is dominant since it concentrates heavily at the open ends of the antenna active driver. Thus, we can name it as E-mutual coupling or simply E-coupling. When adding these two antennas above each other along the y-axis with a $\lambda_{o} / 2$ distance apart too, the magnetic field interaction is dominant in this case due to its high concentration, so it is called as H-coupling, being the third scenario. In the last scenario, E-coupling and H-coupling two-element arrays are combined to create a two by two array, called mixed E-H coupling array. The E-plane cut radiation patterns, and the reflection coefficients (S11) of the first element in the four scenarios are plotted on Figure 6.9a and 6.9b, respectively. Moreover, Figure 6.9c compares Snn of elements of the array in the fourth scenario where $n=1,2,3,4$. Although all elements in the array analyzed in the HFSS have identical designs, their Snn are not similar because of the mutual coupling, leading us to a vital deduction that elements in the PMSA do not scatter the same power in all directions. The inconsistencies in the amounts of powers transmitted (scattered) from each element in the fourth scenario array are shown in Figure 6.9d, being the transmission coefficient $(\mathrm{T})$. Moreover, radiation patterns of elements follow the same approach. In other words, antennas working within the same array cannot radiate equal power in the same direction as well, and losses due to the variations in radiation patterns are angle-dependent, see Figure 6.9a. Losses will also depend on types and polarization of antennas being utilized (e.g. dipoles, monopoles, patches, etc). Losses due to mismatches in radiation patterns and reflection coefficients (Snn) resulting from the same cause 
being the mutual coupling can be combined in one term called the overall array performance loss. In some cases, variations in reflection coefficients and radiation patterns might result in some array elements having good matching and improved gain in some directions compared to others. Because these directions are typically not the same for different elements in the array (different antennas do not see the same surrounding elements), non-idealities of radiation patterns could be averaged out in large arrays, leading to have a total gain comparable to gains of uniform arrays. However, we may not get the full advantage of the PMSA, since some elements in the PMSA might not be scattering efficiently in some directions. As a consequence, these inconsistencies in powers between elements can be included to other sources of errors as demonstrated in the last section of Chapter 3. As we formerly explained, errors reduce functionality of the PMSA where distortion products are not being completely canceled at predetermined spatial directions.

To this end, we discussed impacts of mutual coupling on the antenna performance. There is a huge number of researches to minimize impacts of the mutual coupling, but the scope of the discussion given above will not shed light on them as much as we want to demonstrate reasons of the non-perfect cancellation of distortion products generated by our PMSA.

Figure 6.10 shows an array consisting of two printed quasi-Yagi dipole antennas stacked above each other as in the second scenario because of having the lowest mutual coupling which leads to mitigating mismatches between the array elements. Figure 6.11a shows a $4 \times 4$ antenna array. The rest of plots in Figure 6.11 show 3D radiation patterns directed into different angles in space with assuming $\phi=0^{\circ}$ in all plots. In addition, an array structure and $3 \mathrm{D}$ radiation patterns of an $8 \times 2$ printed quasi-Yagi antenna array are presented in Figure 6.12. As can be seen in figures, gains of the two arrays have been increased from $4.5 \mathrm{~dB}$ which is a gain of a single 


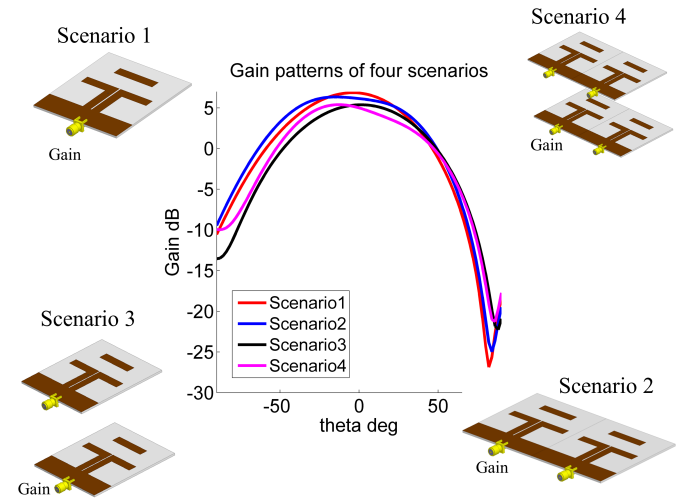

(a)

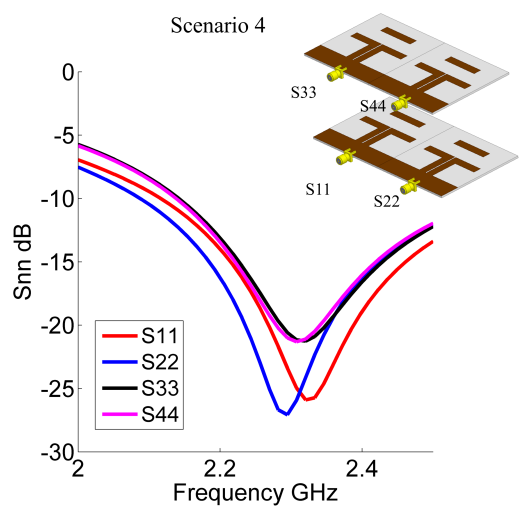

(c)

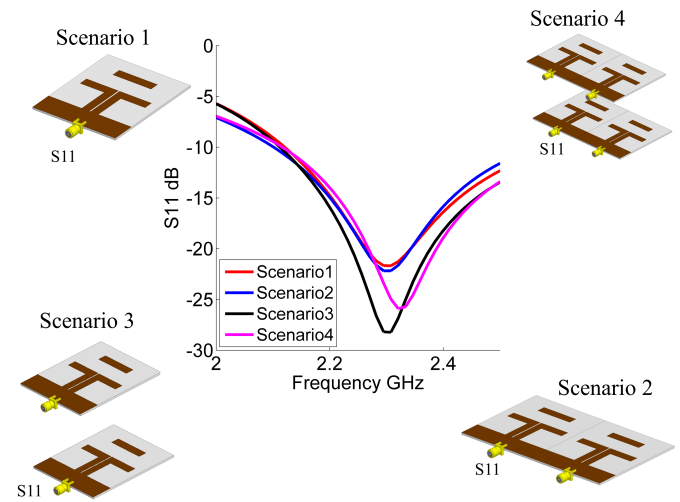

(b)

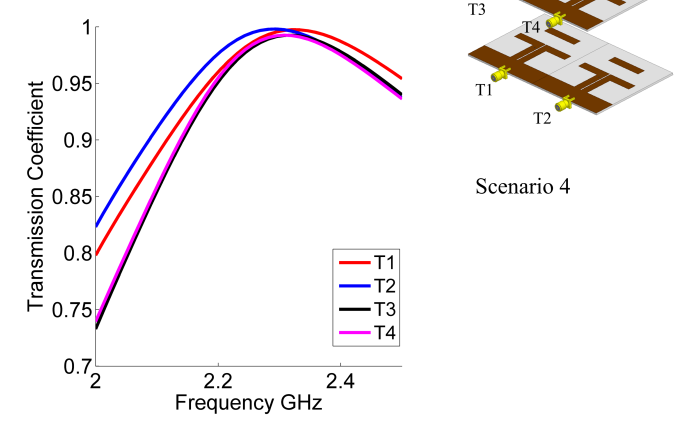

(d)

Figure 6.9: Impact of mutual coupling on antenna properties a) gain radiation patterns of the first element for four different scenarios b) S11 for four different scenarios c) Snn of the fourth scenario only d) Tn of the fourth scenario only

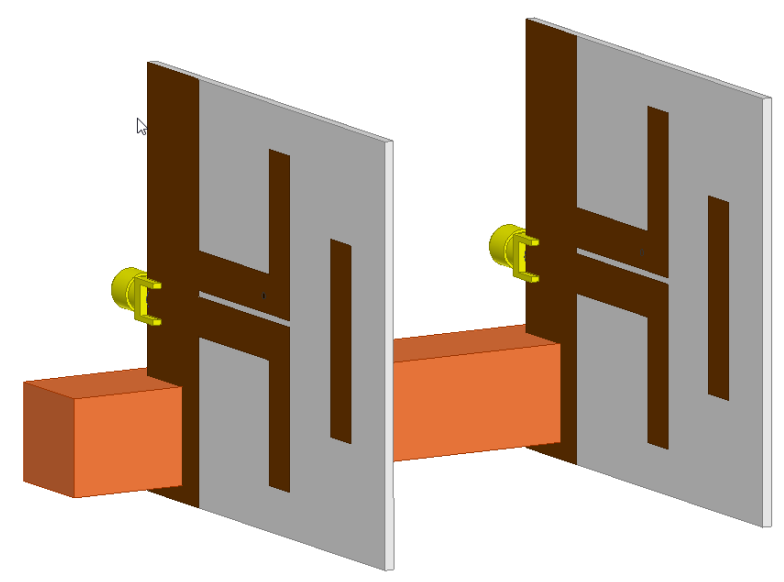

Figure 6.10: Two-element H-coupling type printed quai-Yagi antenna array 


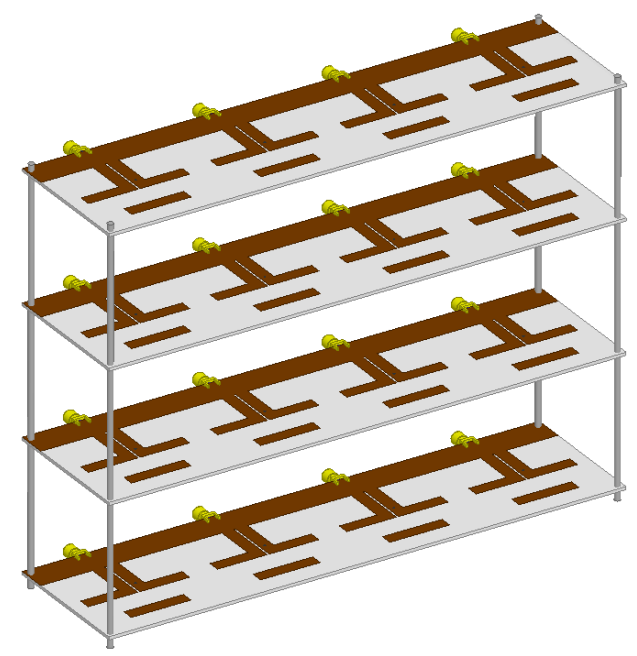

(a)

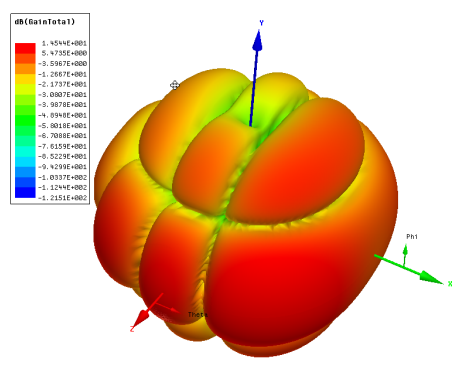

(b)

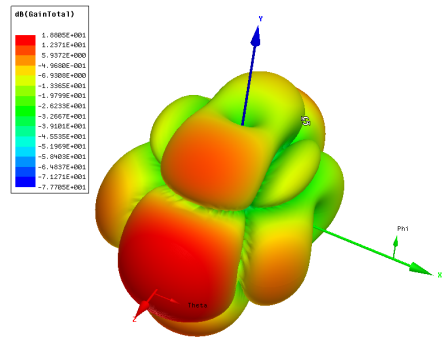

(e)

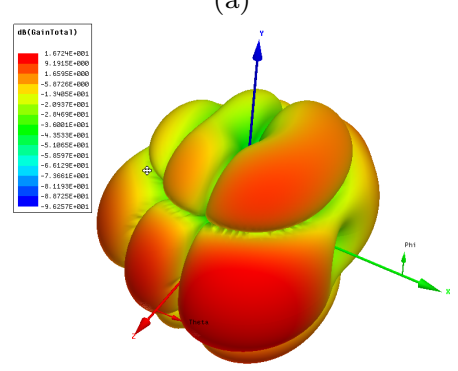

(c)

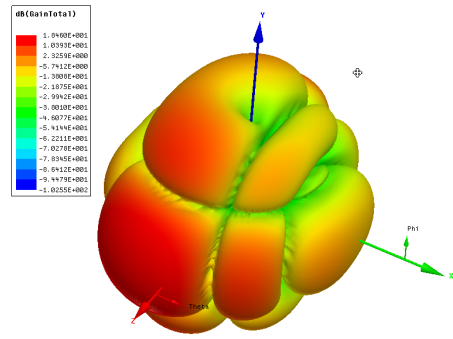

(f)

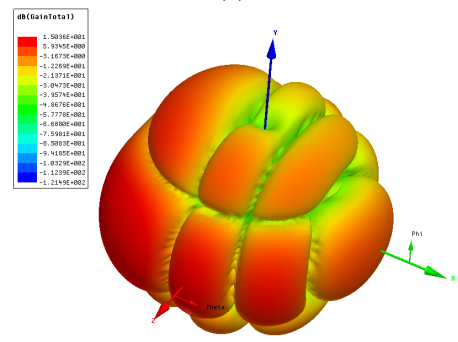

(h)

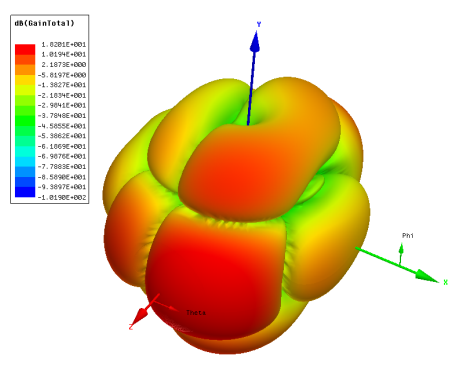

(d)

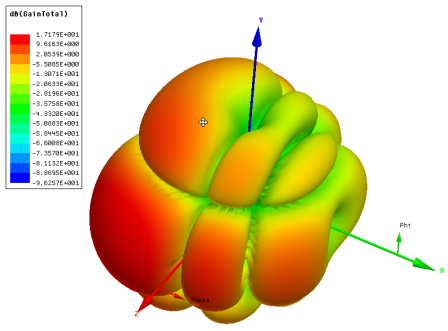

(g)

Figure 6.11: a) A $4 \times 4$ quasi-Yagi printed antenna array and its $3 \mathrm{D}$ radiation patterns directed to b) $\theta=-45^{\circ}, \phi=0^{\circ}$ c) $\theta=-30^{\circ}, \phi=0^{\circ}$ d) $\theta=-15^{\circ}, \phi=0^{\circ}$ e) $\theta=0^{\circ}, \phi=0^{\circ}$ f) $\theta=15^{\circ}, \phi=0^{\circ}$ g) $\left.\theta=30^{\circ}, \phi=0^{\circ} \mathrm{h}\right) \theta=45^{\circ}, \phi=0^{\circ}$ 


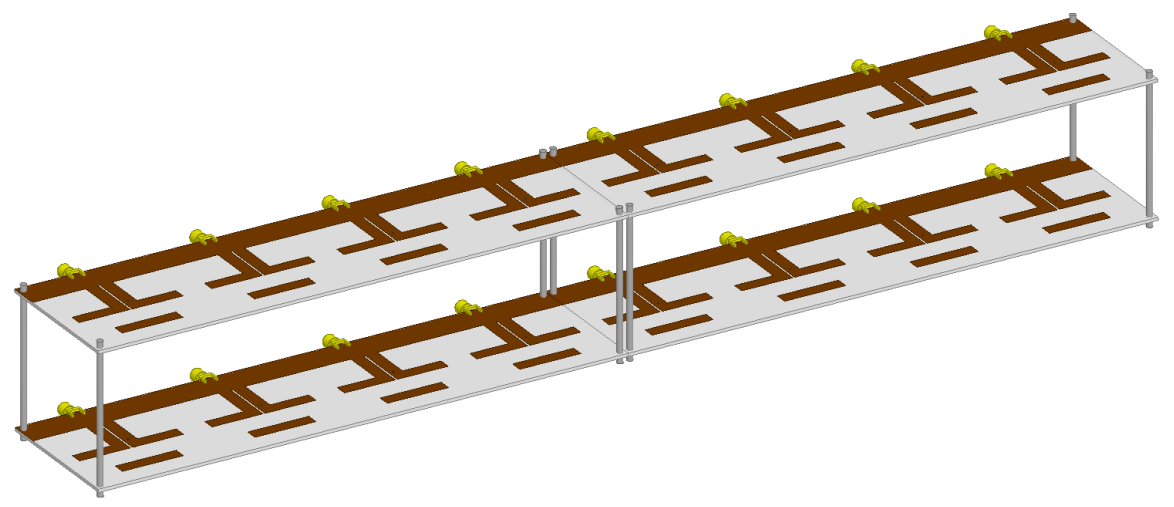

(a)

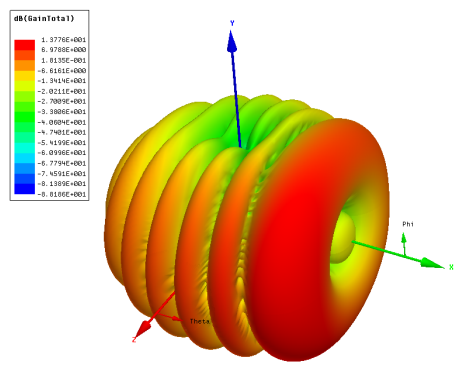

(b)

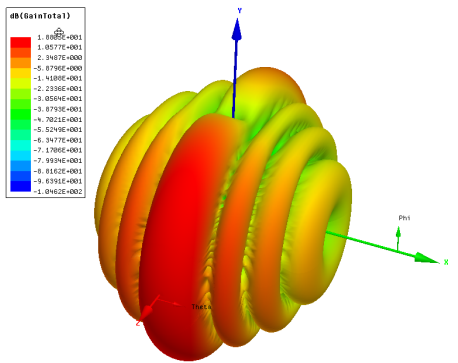

(e)

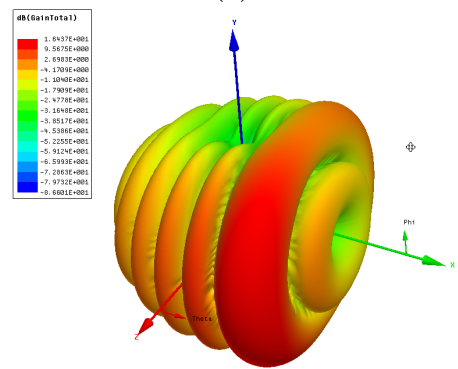

(c)

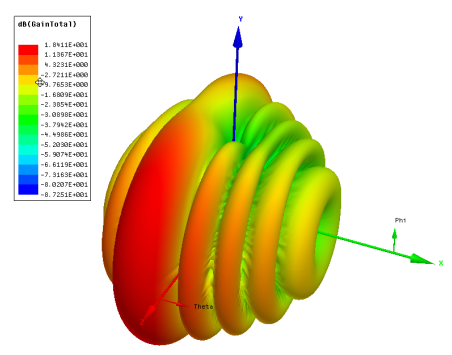

(f)

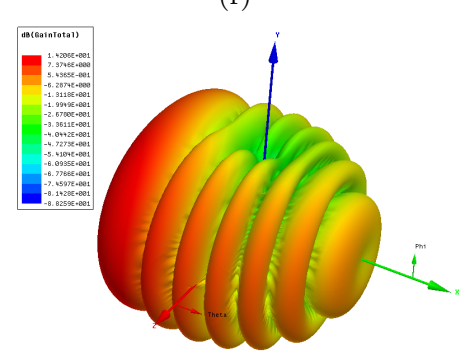

(h)

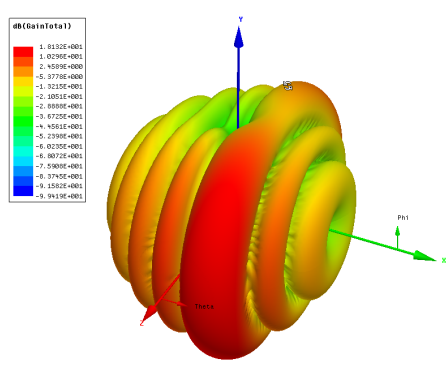

(d)

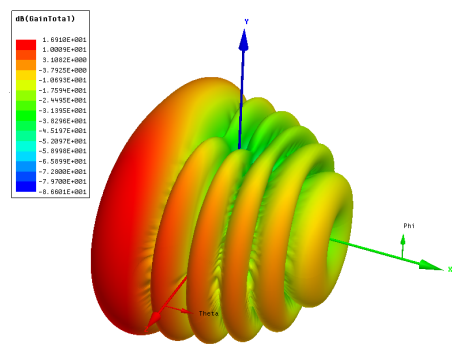

$(\mathrm{g})$

Figure 6.12: a) An $8 \times 2$ quasi-Yagi printed antenna array and its $3 \mathrm{D}$ radiation patterns directed to b) $\theta=-45^{\circ}, \phi=0^{\circ}$ c) $\theta=-30^{\circ}, \phi=0^{\circ}$ d) $\theta=-15^{\circ}, \phi=0^{\circ}$ e) $\theta=0^{\circ}, \phi=0^{\circ}$ f) $\theta=15^{\circ}, \phi=0^{\circ}$ g) $\left.\theta=30^{\circ}, \phi=0^{\circ} \mathrm{h}\right) \theta=45^{\circ}, \phi=0^{\circ}$ 


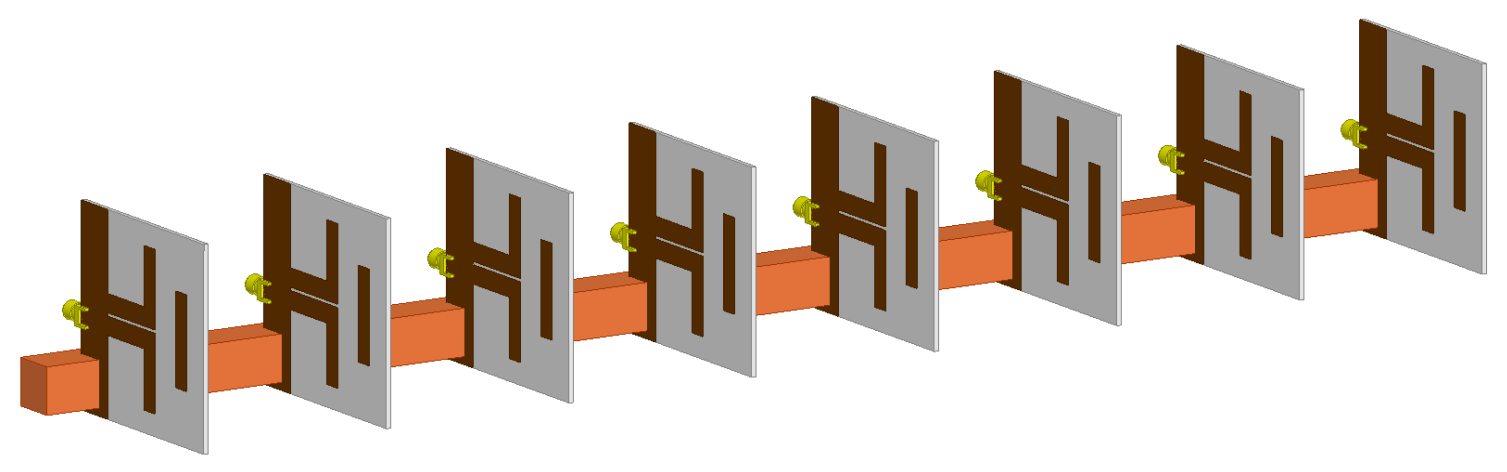

(a)

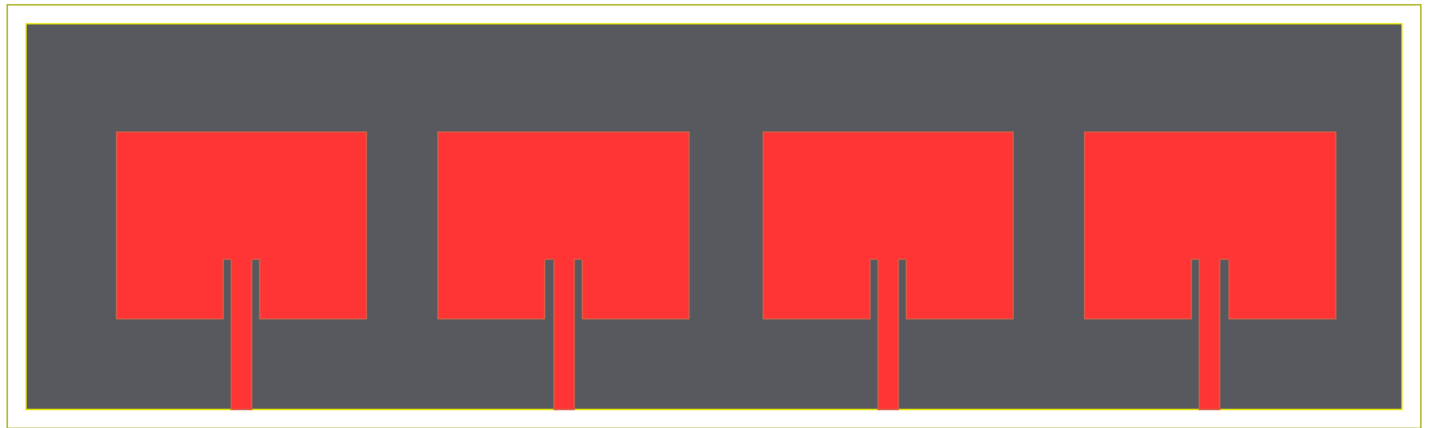

(b)

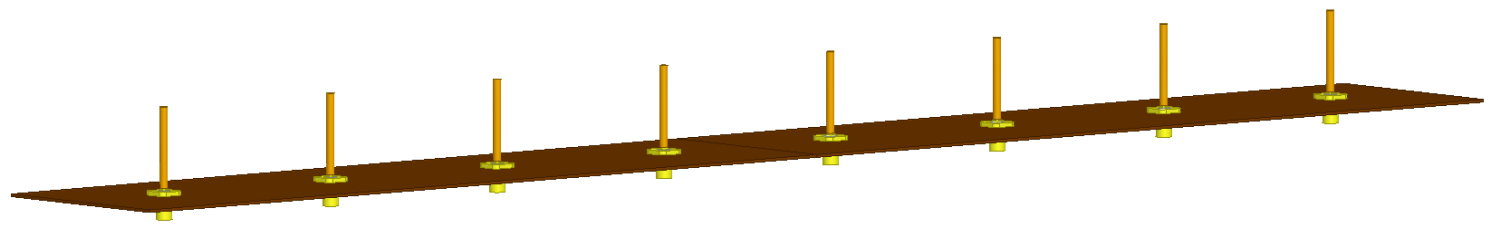

(c)

Figure 6.13: a) An $8 \times 1$ printed quasi-Yagi antenna array b) an $4 \times 1$ microstrip inset-fed patch antenna array c) an $8 \times 1$ quarter-wavelength monoples over ground antenna array

element to $17.5 \mathrm{~dB}$. This is expected because as a number of elements is doubled, the gain is increased by $3 \mathrm{~dB}$. Moreover, gains are reduced if the main beams of radiation patterns are moved away from the broadside direction as a consequence of the beam widening phenomena in phased arrays.

The gain of $8 \times 2$ array is equal to the gain of $4 \times 4$ array since they have the same number of elements. However, the $8 \times 2$ array is preferred in our work over the $4 \times 4$ array because it can control more distortion products in space as will be seen in 
Chapter 8. The common drawback of these two arrays is that their elements have been etched on the same substrate. Thus, distances separating elements cannot be changed. To solve this problem, we built another $8 \times 1$ antenna array using the printed quasi Yagi dipole antenna too, but in this time, elements are fabricated on separate substrates as shown in Figure 6.13a. Here, we can change distances between elements to control the mutual coupling. The aim of this array is to perform in the parametric studies that will be presented Chapter 8 . In addition, we design a $4 \times 1$ microstrip patch and an $8 \times 1$ monopole antenna arrays as demonstrated in Figure $6.13 \mathrm{~b}$ and 6.13c. Because the printed Yagi dipole antenna has higher gain than the monopole antenna, the gain of the $8 \times 1$ printed Yagi dipole array is larger than the gain of the $8 \times 1$ monopole array when, though they have the same aperture length.

From the figures, we can notice that the difference between the arrays used in the PMSA and conventional phased arrays is no need to use transmission lines and combiners/dividers circuits. Instead, signals, which are distortion products, are combined over-the-air, thereby reducing the design complexity.

\subsection{Design of RF Reflective-Type Switch (RF-RTS)}

Not only the antenna is a critical part in our PMSA but also the reflection coefficient modulator plays a vital role in determining the entire response of the PMSA. In our model, the reflection coefficient modulator, in its simple way consisting of an RF switch controlled by a continuous low-frequency sinusoidal signal source and loads with prescribed values, modulates and reflects the RF incident signals to reach the input port again from where they come. The switch is designed to reflect the RF signals when it is either an open or short circuit, so it can be called as a reflective-type switch (RTS). Hereafter, the RTS term will be used instead of the reflection coefficient modulator throughout this section for the sake of simplicity. Because of the RTS 
importance, it is preferred to be implemented simply with good performance. The RTS design task can be carried out with the use of an RF diode since it performs well with low power signals especially if we use low-barrier Schottky diodes. A nonlinear diode with a periodic modulation signal applied to one of its ports is able to produce the two periodic extremes of reflection coefficients $\left|\Gamma_{\text {o.c. }}\right|=-1$ and $\left|\Gamma_{\text {s.c. }}\right|=+1$.

\subsubsection{RTS Power Signal Analysis}

In a general sense, the RTS used in the PMSA design consists of an electronic switch connected to a short circuit load at one of its sides as shown in Figure 6.14a. As can be seen, there are three different signal sources; one of them is a periodic sinusoidal signal source controlling the RTS which is real in this work; the last two sources are being assumed to represent the input and output RF signals induced at one of the RTS ports and arrows distinguish between the incoming and outgoing RF signals. The input RF signal source represents a single-tone signal intended to be modulated and reflected when reaching the RTS, while the output RF source represents a signal with a huge number of tones never reaching the RTS in the case of no mismatch between the RTS and the output RF source. Both the input and output RF sources are not-real, hypothetical sources. The former represents a signal induced at an input port of the scatterer antenna and is given by

$$
v_{\text {ind }}=v_{\text {input }}=V \cos w_{o} t=\sqrt{p_{\text {ind }} Z_{o}}=\sqrt{P_{T} G_{T} G_{\text {sca }}\left(\frac{\lambda_{o}}{4 \pi r_{1}}\right)^{2} Z_{o}} \cos w_{o} t
$$

in which $p_{\text {ind }}$ is the instantaneous power induced over a scatterer antenna, $P_{T}$ is the power sent by an illuminating source, $G_{T}$ denotes the gain of an illuminating source antenna, $G_{s c a}$ is gain of a scatterer antenna, $\lambda_{o}$ is the illuminating-frequency wavelength, $r_{1}$ represents the distance between illuminating source and scatterer, $w_{o}$ 
is frequency of the illuminating signal, and $Z_{o}$ is an input impedance of a scatterer antenna. The input signal computed by the conventional Friis equation in (6.1) goes down to reach the RTS. The RTS, which in turn, reflects the input signal to reach the input port (scatterer antenna) again. When the RTS is open, the input signal does not pass it and is reflected back to reach the input port. The reflected signal under the impact of the open circuit is given by

$$
v_{\text {ref-open }}=v_{\text {input }} \times \Gamma_{\text {o.c. }}, \quad \text { where } \quad \Gamma_{\text {o.c. }}=\frac{z_{\text {o.c. }}-Z_{o}}{z_{\text {o.c. }}+Z_{o}}=1
$$

However, when the RTS is closed, the input signal passes it and is reflected this time by a short circuit load placed on the opposite side of the RTS, being shunt RF capacitors in our adopted RTS. The reflected signal under the impact of the short circuit is given by

$$
v_{\text {ref_short }}=v_{\text {input }} \times \Gamma_{\text {s.c. }}, \quad \text { where } \quad \Gamma_{\text {s.c. }}=\frac{z_{\text {s.c. }}-Z_{o}}{z_{\text {s.c. }}+Z_{o}}=-1
$$

with $z_{\text {o.c. }}$, and $z_{\text {s.c. }}$ being impedances of the short and open circuits of the RTS, respectively. In both states shown in Figure 6.14b, signals reach the input port (scatterer antenna) being launched into space again. This process is periodical because the RTS is governed by a periodical signal. Practically, the passive $\left(\left|\Gamma_{\text {s.c. }}\right|,\left|\Gamma_{\text {o.c. }}\right|\right)$ can be any value between 0 and 1 relying on the mismatch level between the scatterer antenna and the RTS [131].

Consequently, resulting signals from both states are averaged out to represent signals generated by the fictitious RF output source in Figure 6.14a, given by [92]

$$
v_{\text {out }}=v_{\text {ref_open }}-v_{\text {ref-short }}=v_{\text {input }} \times\left|\Gamma_{\text {o.c. }}-\Gamma_{\text {s.c. }}\right|=v_{\text {input }} \times \Delta \Gamma
$$




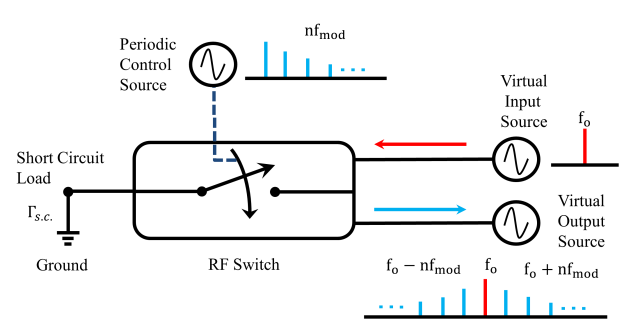

(a)

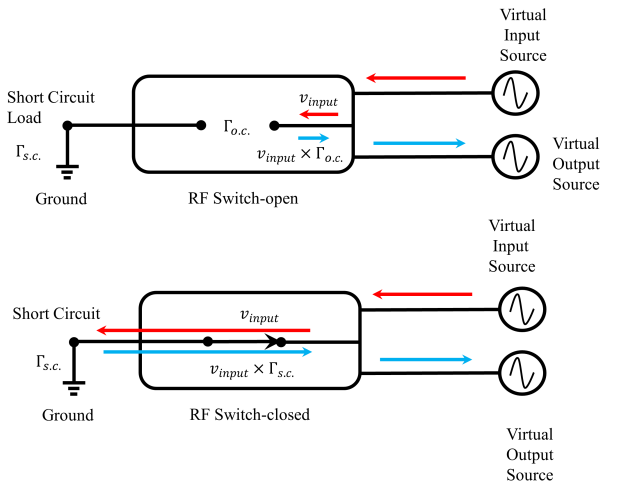

(b)

Figure 6.14: RTS switch a) general schematic b) open-closed states

where $\Delta \Gamma=\left|\Gamma_{\text {o.c. }}-\Gamma_{\text {s.c. }}\right|$ and it is a function with a frequency of the modulation signal controlling the RTS. Thus, $\Delta \Gamma$ can be rewritten as $\Delta \Gamma\left(w_{m o d}\right)$. The periodic $\Delta \Gamma\left(w_{m o d}\right)$ can be approximately written using the Fourier series as

$$
\Delta \Gamma\left(w_{m o d}\right)=\sum_{n=-\infty}^{\infty} \Gamma_{n} \delta\left(w-n w_{m o d}\right)
$$

where $\Gamma_{n}$ are the Fourier coefficients of $\Delta \Gamma\left(w_{m o d}\right)$. Frequency components in (6.5) are distributed about the two terms of $\cos w_{o} t$ in (6.1) which are $1 / 2\left(\delta\left(w-w_{o}\right)+\delta\left(w+w_{o}\right)\right)$. Because the second term is just the image of the first term, it can be ignored. Thus, Eq.(6.4) can be written as

$$
v_{\text {out }}=v_{\text {input }} \times \Delta \Gamma\left(\mathrm{w}_{\text {mod }}\right)=V \cos w_{o} t \times \Delta \Gamma\left(\mathrm{w}_{\text {mod }}\right)=\frac{V}{2} \sum_{n=-\infty}^{\infty} \Gamma_{n} \delta\left(w-\mathrm{w}_{o}+n w_{\text {mod }}\right)
$$

Also, $v_{\text {out }}$ can be expressed in terms of the Friis equation parameters

$$
v_{\text {out }}=\frac{1}{2} \sqrt{P_{T} G_{T} G_{\text {sca }}\left(\frac{\lambda}{4 \pi r_{1}}\right)^{2} Z_{o}} \sum_{n=-\infty}^{\infty} \Gamma_{n} \delta\left(w-w_{o}+n w_{\text {mod }}\right)
$$


As can be seen, $v_{\text {out }}$ consists of an infinite number of signals with frequencies being multiple integers of the control (modulation) frequency distributed on both sides of the RF input signal. $w_{m o d}$ should be much less than $w_{o}, w_{m o d}<<w_{o}$, as illustrated before. Because of the reflected signals from the RTS (i.e., RF output source), power induced over a scatterer antenna, and power captured by a receiver are given by

$$
p_{\text {ind }}=p_{\text {ind }} G_{\text {sca }}=P_{T} G_{T} G_{\text {sca }}^{2}\left(\frac{\lambda}{4 \pi r_{1}}\right)^{2} \frac{1}{4} \sum_{n=-\infty}^{\infty} \Gamma_{n}^{2} \delta\left(w-w_{o}+n w_{m o d}\right)
$$

and

$$
\begin{aligned}
p_{\text {rec }} & =p_{\text {sca }} G_{R}\left(\frac{\lambda}{4 \pi r_{2}}\right)^{2}=P_{T} G_{T} G_{R} G_{\text {sca }}^{2}\left(\frac{\lambda}{4 \pi}\right)^{4}\left(\frac{1}{r_{1} r_{2}}\right)^{2} \\
& \times \frac{1}{4} \sum_{n=-\infty} \infty \Gamma_{n}^{2} \delta\left(w-w_{o}+n w_{\text {mod }}\right)
\end{aligned}
$$

where $r_{2}$ is the distance from a scatterer into the receiver and $G_{R}$ represents gain of the receiver antenna. In our experimental setup, $r_{2}$ is not equal to $r_{1}$ because the TX antenna is located at given locations while the RX antenna is moved circularly around the PMSA in $2^{o}$ steps to record the scattered signals. $\Gamma_{n}^{2}$ in $(6.5)$ is directly proportional to $\left|\Gamma_{\text {o.c. }}-\Gamma_{\text {s.c. }}\right|^{2}$, so if $\left|\Gamma_{\text {o.c. }}-\Gamma_{\text {s.c. }}\right|^{2}$ increases, all terms in $\Gamma_{n}^{2}$ increase too. According to Eqs.(6.8) and (6.9), these powers increase with increasing $\Gamma_{n}^{2}$. The term $\left|\Gamma_{\text {o.c. }}-\Gamma_{\text {s.c. }}\right|$ is called the modulation parameter $\mathrm{M}$ in the field of the RFID research [135] [58] [47] [82] [118]. The parameter M is used here too. Thus, the main purpose of the RTS design is to increase the parameter M as much as possible, leading to an increase in the scattered power. In the derivation presented above, we assumed no mismatches in impedances and polarizations as well as no losses in the RTS circuit. 


\subsubsection{RTS Design}

Having discussed the parameter $\mathrm{M}$ controlling signal strengths reflected from the RTS by switching between two different loads, for example from open-circuit load to short-circuit load, now we should build a RTS switch fulfilling the requirements. Figure 6.15a shows a schematic of the RTS using the software ADS-circuit simulator. In the schematic, a series inductor $L 2$ prohibits the RF signals to reach the driving (control or modulation) source. It should be large enough to behave as an open circuit at the RF design frequency. Two capacitors in parallel are added in shunt. These two capacitors provide a short circuit impedance at the RF design frequency, making the RF signals fully reflected to reach a scatterer antenna again. Also, capacitance should be sufficiently large to provide very small reactance. A $100 \mathrm{Ohm}$ resistor is added between a modulation port and a diode before the inductor to protect the diode from the higher currents.

Some experimental tests have been done before examining entire arrays to figure out impacts of diode types on the performance of a single scatter. Three different types of diodes (HP5200-2835 Schottky, a 1N4152 PN, and HP3080 PIN) are used in the earlier experimental setups. The power of scattered signals from a HP5200-2835 Schottky diode was found to be higher, only about $1.4 \mathrm{~dB}$ and $6 \mathrm{~dB}$ over the powers scattered from scatterers using the 1N4152 PN and the HP3080 PIN diodes, respectively. Thus, a Schottky diode is chosen such that it provides a better response when compared to other diodes as we illustrated in [140].

The cross-section of a low barrier Schottky diode and its corresponding equivalent circuit which was used in the ADS circuit simulator are shown in Figure 6.15b and $6.15 \mathrm{c}$, respectively. The equivalent circuit, consisting of five elements, is commonly used in the analysis of Schottky diodes. $L_{p}$ and $C_{p}$ are the package parasitic inductance and capacitance, respectively. $R_{s}$ is the overall parasitic series resistance, 
including all resistances such as the lead frame resistance, the bondwire resistance, and the bulk resistance of silicon layers. $R_{s}$ dissipates a portion of the RF energy as heat, leading to a reduction in the diode sensitivity. $C_{j}$ is the parasitic junction capacitance, given by

$$
C_{j}=\frac{C_{j o}}{\left(1-\frac{V}{v_{b i}}\right)^{M m}}
$$

where $v_{b i}$ is the built-in potential, $\mathrm{V}$ is the applied voltage which represents a voltage of the control signal, $C_{j o}$ is the zero bias voltage parasitic junction capacitance, and the exponent $M m$ in the denominator depends on the type of doping process which is 0.5 in Schottky diodes. $R_{j}$ is the junction resistance of the diode where the RF signals is transformed into the useful output voltage. It is given by

$$
R_{j}=\frac{8.33 \times 10^{-5} n T}{\left(I_{b}+I_{s}\right)}
$$

The term $n$ denotes the diode ideality factor; $T$ is the physical temperature in Kelvins; $I_{b}$ and $I_{s}$ are the bias and saturation currents. At high bias currents $I_{b}$, $R_{j}$ is approximately zero, and the diode becomes short circuit which allows the RF signals passing through it.

However, when the biasing current $I_{b}$ decreases which is proportional to the forward biasing voltage, $R_{j}$ then increases. Thus, $\Gamma_{\text {s.c. }}$ leaves the short circuit location on the Smith chart, which in turn, reduces the modulation factor M. Oppositely, in the reverse biasing situation, $R_{j}$ becomes higher since $I_{b}$ does not exist, and thus $C_{j}$ becomes dominant. $C_{j}$ also depends on a level of the biasing voltage. In other words, $C_{j}$ increases with the decrease in the reverse biasing voltage, leading to reduction in $\Gamma_{\text {o.c. }}$. As a result, the parameter $\mathrm{M}$ becomes smaller than the expected value. As a result, the scattered power will decrease. Obtaining perfect open and short reflection 
coefficients are impossible due to parasitic elements associated with real-world passive lumped elements and diode packages.

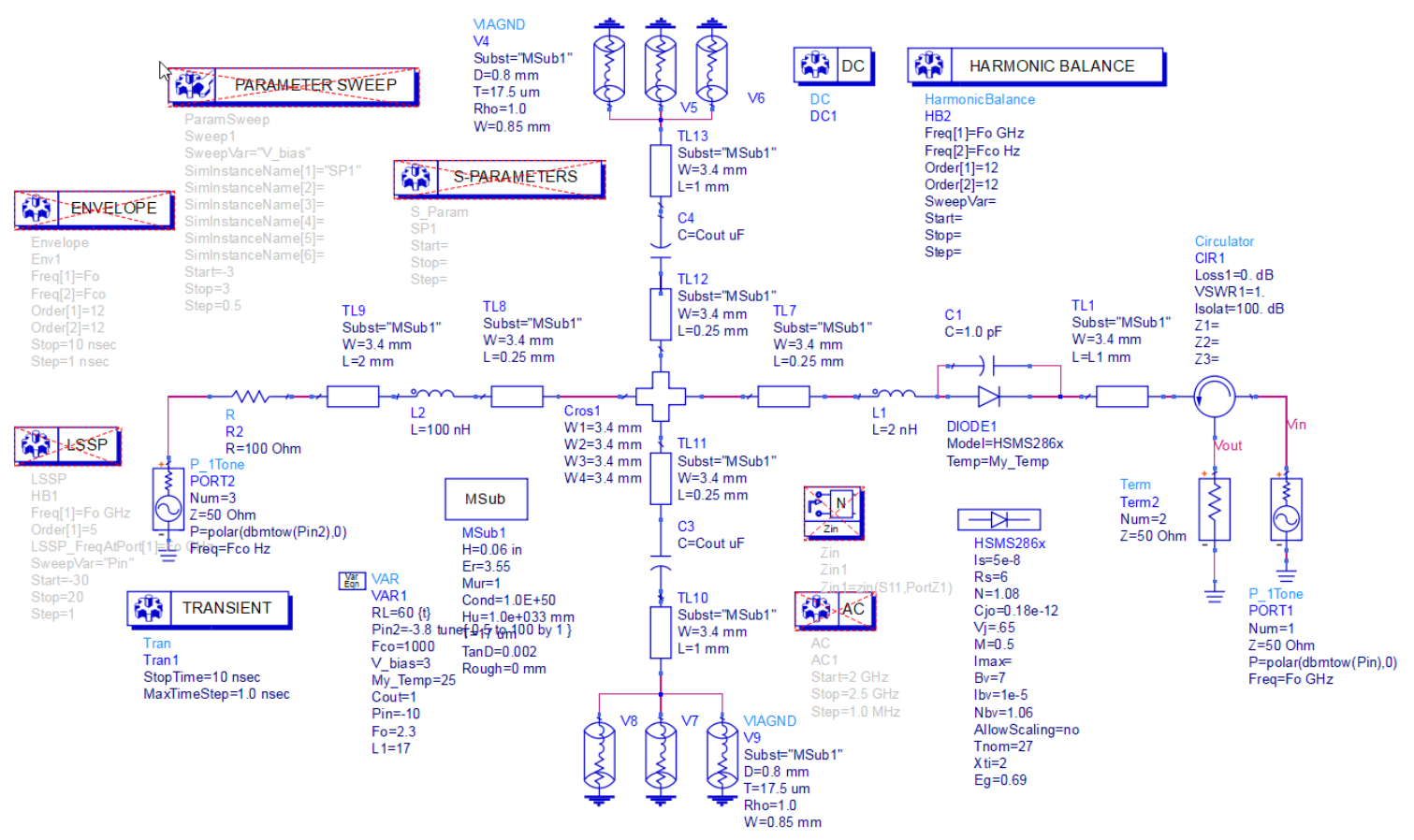

(a)

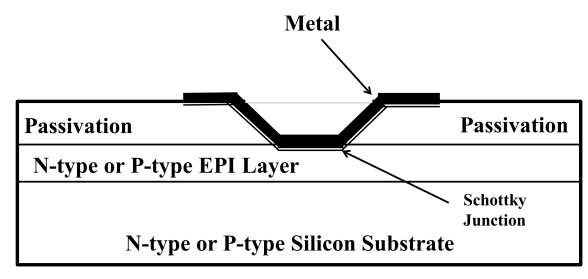

(b)

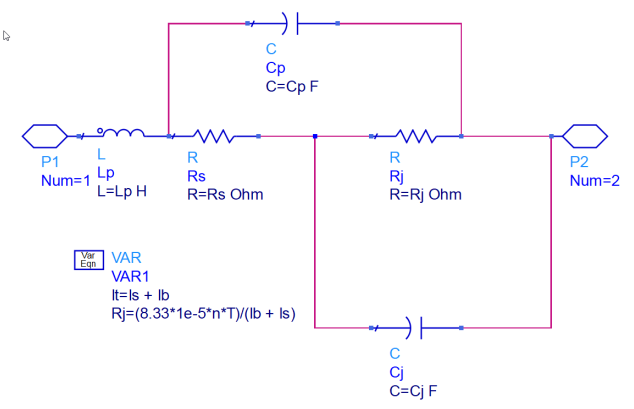

(c)

Figure 6.15: a) RTS schematic design using the ADS b) cross-section of a low barrier Schottky diode c) equivalent circuit of a Schottky diode

The available HP5200-2835 and HSMS2860 low-barrier Schottky diodes are both used in the RTS design. For the sake of simplicity, we focus only on the RTS design using the latter diode due to its frequent use in our scattering arrays, but 
we can also analyze the RTS with the former diode following the same procedure. Because the RTS reflects RF signals in both states (i.e, open and closed), the diode behaves more like a switch than a rectifier. Important simulations being implemented first are the DC sweep and S-parameter to determine the RTS input impedance, see Figure 6.16a. As can be observed, when the diode is forward-biased (i.e., short-circuit), the RTS input impedance approaches the open circuit area on the Smith chart due to a transmission-line inserted between the diode and an input port, rotating the impedance location.

The reflection coefficient of input port travels in a straight line through the 50 ohm impedance match on the Smith chart for the DC sweep from -3v to 3v. The importance of this straight line is to find out effects of biasing voltages on the input impedance which in turn helps us to select proper voltages in order to maximize the reflected power.

Moreover, as shown in Figure 6.16a, the open circuit impedance on the Smith chart are not reachable with low or at least with moderate forward biasing voltages. In contrast to that, the short circuit impedance on the Smith chart are obtained with all reverse biasing voltages applied to the diode anode. The inability to obtain one of the required impedances, because of parasitic effects of the diode and transmission-lines, deteriorates the RTS performance. The modulation factor M will also be lower than its nominated ideal value which is 2 in our ideal RTS. Thus, the factor M has been calculated using simulation results obtained from the ADS as shown in Figure 6.16b for different values of biasing voltage peaks from $\pm 0.5 \mathrm{v}$ to $\pm 3 \mathrm{v}$ with $+0.5 \mathrm{v}$ step throughout a frequency band from $2.1 \mathrm{GHz}$ to $2.5 \mathrm{GHz}$. The factor $\mathrm{M}$ is 1.73 at the $\pm 3 \mathrm{v}$ signal peak which is the highest value being obtained in the simulation, while the minimum value of the factor $\mathrm{M}$ is 1.31 at the $0.5 \mathrm{v}$ signal peak.

Values of all passive lumped components shown on the RTS schematic design are 
obtained after doing several simulations and are then optimally selected, while dimensions of transmission lines are according to $50 \mathrm{ohm}$ characteristic impedance printed on a Rogers 4003C substrate with a dielectric constant 3.55 and thickness $1.52 \mathrm{~mm}$. The main transmission line connects an antenna into a diode switch carrying both the incident and reflected RF signals, but these two signals propagate in opposite directions. In the simulation, a circulator is included to separate between these two signals, and they are overlaid in Figure 6.16c. Notice that the RF input (incident) signal swings between $-100 \mathrm{mv}$ and $+100 \mathrm{mv}$, whereas the RF output (reflected) signal swings between $+83 m \mathrm{v}$ and $+143 m \mathrm{v}$. The former is a pure monotone sinusoidal signal, while the latter, consists of a huge number of tones, being a distorted sinusoidal signal. Their spectral analyses can be seen in Figure 6.16d, being overlaid on the same plot. A $1 \mathrm{kHz}$ single-tone periodic signal switches the RTS, so harmonics can also be seen distributed evenly by multiple integers of $1 \mathrm{kHz}$ on both sides of the main $\mathrm{RF}$ incident signal at a frequency $2.3 \mathrm{GHz}$, confirming that the output signal has more than one tone. The first upper and lower sidebands harmonics are $25 \mathrm{~dB}$ below the reflected fundamental harmonic. Other harmonics are more below. All these results are simulated assuming a $-10 \mathrm{dBm}$ input (incident) signal and $-3.8 \mathrm{dBm}$ modulation signal.

The schematic of the RTS depicted in Figure 6.14a was exported as a Gerber file to a CNC milling machine. A photograph of the RTS layout using SMD components is shown in Figure 6.17a, and Figure 6.17b shows the photograph of another RTS layout built by Prof. Richard Campbell. The vector network analyzer VNA is used to test the RTS board. The measured and simulated results are plotted on the Smith chart for both the forward and reversed biasing under a voltage peak of $1.6 \mathrm{~V}$ as illustrated in Figure 6.17c. The results are not matched well, but they are acceptable. Figure $6.17 \mathrm{~d}$ compares between the simulated and measured modulation factor $M$. 


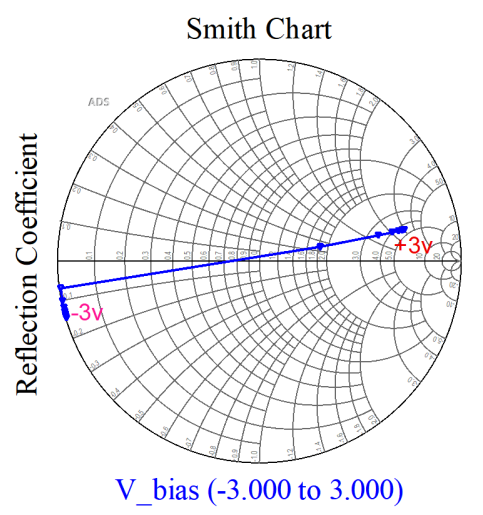

(a)

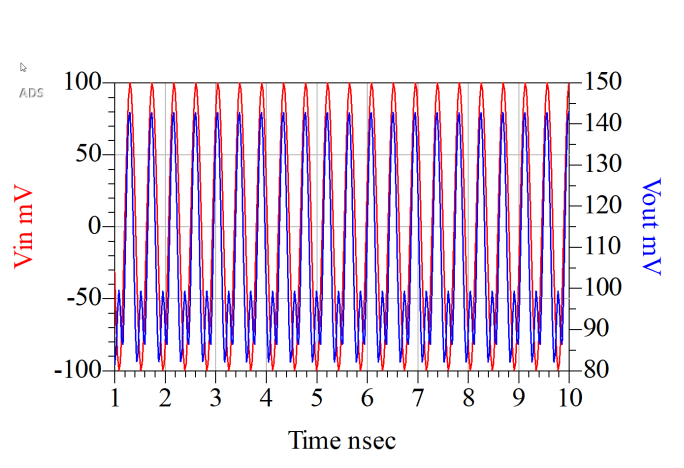

(c)

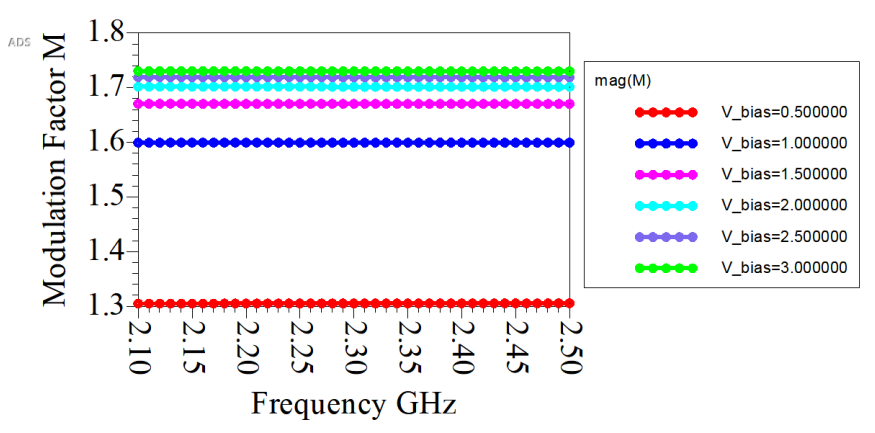

(b)

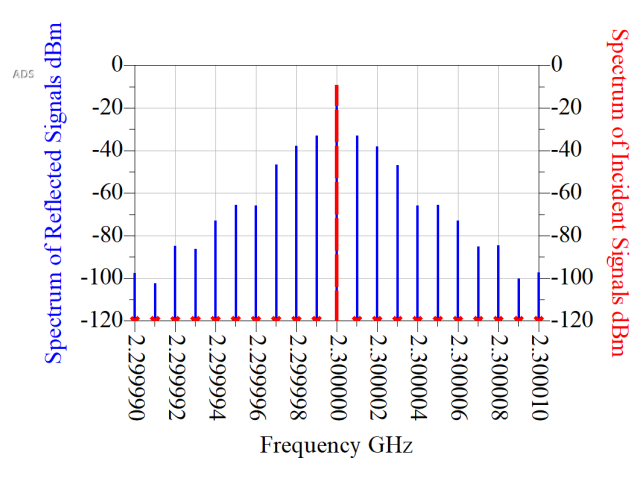

(d)

Figure 6.16: a) Simulated S11 with a DC sweep from -3v to 3v on the Smith chart b) simulated factor $\mathrm{M}$ for different values of modulation voltages $\mathrm{c}$ ) simulated $\mathrm{RF}$ incident and RF reflected signals in the time domain d) simulated RF incident and RF reflected signals in the frequency domain (spectral analysis)

the simulated $M$ is 1.68 at $2.3 \mathrm{GHz}$, whereas the measured $M$ is 1.58 . We think that there are two main reasons behind the discrepancies between the simulated and measured results. The first reason is the parasitic of lumped elements which are not included in the simulations as well as assuming the use of ideal transmission-lines in the simulations, being the second reason. Eventually, testing the RTS response with an absence of incident signals, it is preferred before performing in the entire system. The antenna side port is connected to a digital oscilloscope, and the driving port of the RTS is connected to the modulation source as shown in Figure 6.17g. Although all the fabricated RTS are tested, the measured response of one RTS is provided in 
Figure $6.17 \mathrm{f}$ for the sake of simplicity. It functions as a half wave rectifier as expected. Also, the simulated response of the RTS is provided in Figure 6.17e for the sake of completeness.

\subsection{Multi-Phase Modulation Signal Generator}

After presenting both the antenna arrays and the RTSs (modulators) designs above, we describe here the last part located within the PMSA side being indispensable for the achievement of measurements of the PMSA (device under test-DUT). This part is a multi-port modulation signal generator. Ports generate signals with equal amplitudes and frequencies but with different phases. In the measurements, two different low frequencies modulation signal sources developed for experiments described in [21] are utilized here as shown in Figure 6.18a and 6.18b. The first one is a $700 \mathrm{~Hz}$ single-tone sine-wave source while the second one is a two-tone sine-wave source with frequencies $899 \mathrm{~Hz}$ and $1004 \mathrm{~Hz}$. Each source has four ports, and the relative phase difference between any two successive ports is $90^{\circ}$. Amplitudes of signals in the second source are tunable while being fixed in the first source. Modulation signal sources are tested by using a digital oscilloscope in the Capstone Lab. The measured plots of the waveforms of the modulation sources are given in Figure 6.18. Ideally, amplitudes of modulation signals from different ports of each source must be identical, and a relative phase difference between any two successive ports should also be equal to meet the requirements of the PMSA. However, idealities are never possible in the actual designs because slight variations in values of capacitors, inductors, and resistors of the underlying designs cause errors in amplitudes and phases of the modulation signals. Because these signals are applied to the RTSs in the PMSA, they may cause a serious problem, resulting in degradation in the performance. Thus, it is necessary to find out variations in amplitudes and relative phases of ports of the modulation 

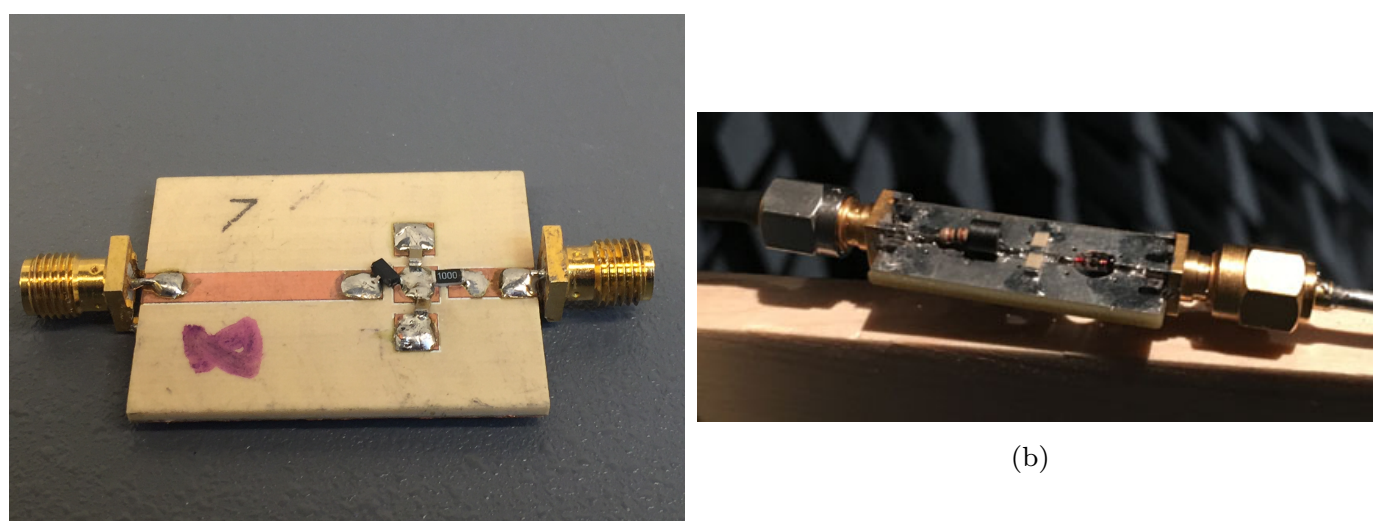

(b)

(a)
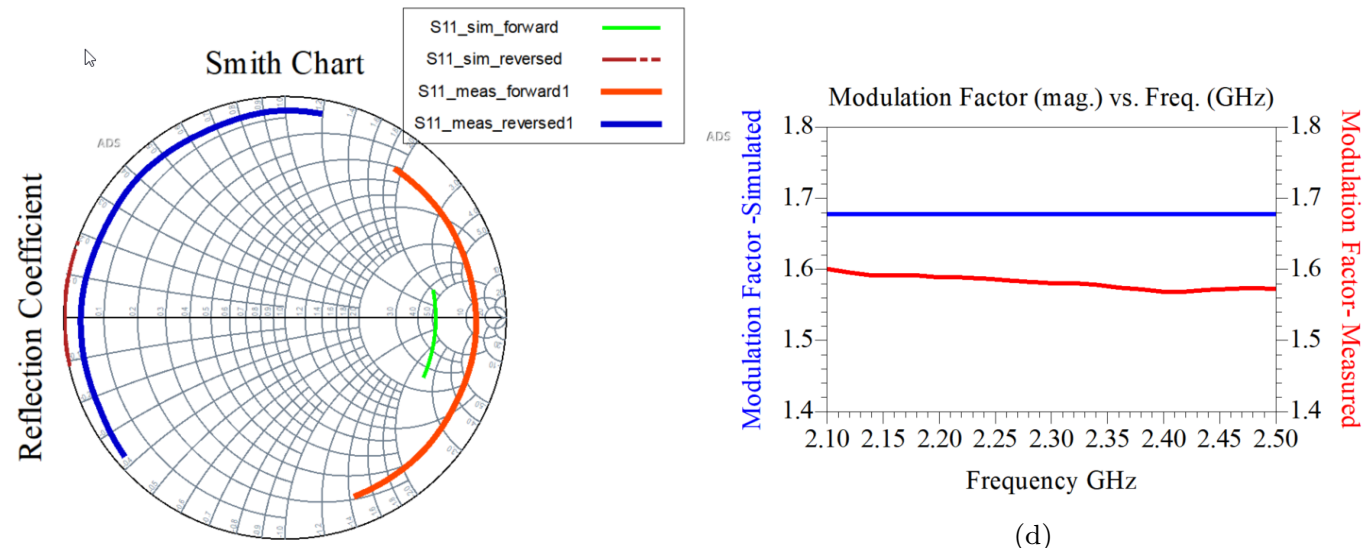

(d)

(c)

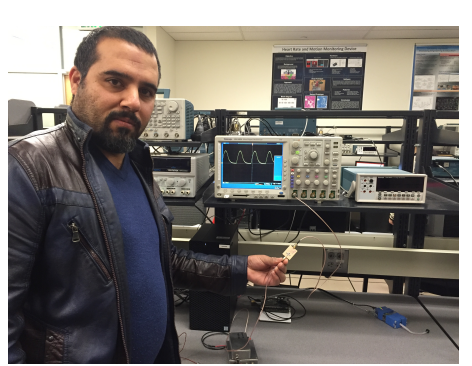

(e)

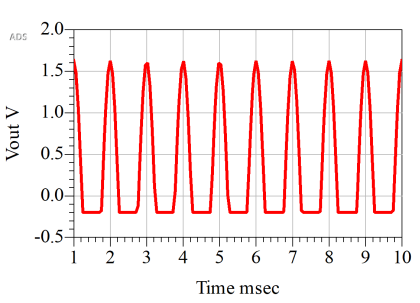

(f)

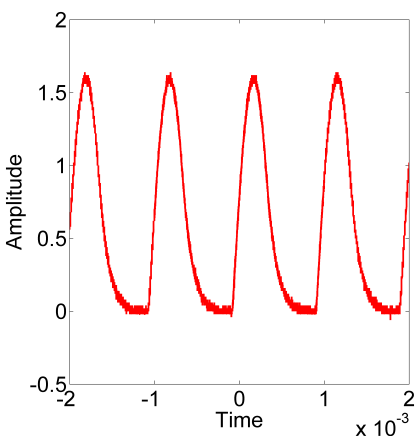

(g)

Figure 6.17: a) Photograph of the RTS prototype using the HSMS2860 Schottky diode b) photograph of the RTS prototype using the HP5200-2835 Schottky diode c) comparison between the simulated and measured S11 of the RTS using HSMS2860 Schottky diode for the reversed and forward biasing on the Smith chart d) comparison between the simulated and measured factor M of RTS using a HSMS2860 Schottky diode e) RTS using HSMS2860 Schottky diode under test connected to a modulating source and a digital oscilloscope f) simulated response of the RTS with single input signal (absence of the RF signals and only presence of the modulation signal) g) measured response of the RTS with single input signal (Modulation signal) 
sources compared to the first port, being considered as a reference port. Again here, we used a digital oscilloscope to take measurements from each port, and are then averaged out to come up with a unique value for each port.

Next, according to arrays with eight elements, a modulation signal source with eight ports is needed as well. Similarly, signals coming from the eight ports should have the same amplitude, but in this time, a relative phase difference between any two successive ports is $45^{\circ}$. To do this with only little effort and low cost, four-to-eight phases transformer kit using resistors only is designed, simulated, fabricated, and tested. The phase transformer kit is based on the basic principles of the resistive voltage divider. As we know, the frequent use of voltage dividers is to change levels of the input voltages at the output port. It simply consists of two resistors confined between an input voltage source and a ground while the point between two resistors denotes the output port. Instead of using reactive elements such as capacitors and inductors as in conventional passive phase shifters, voltage dividers made of resistors only is used to function as a phase shifter as will be demonstrated below. Phases of the transformer kit are divided as (main phases $0^{\circ}, 90^{\circ}, 180^{\circ}$, and $270^{\circ}$ ) and (auxiliary phases $45^{\circ}, 135^{\circ}, 225^{\circ}$, and $\left.315^{\circ}\right)$. The modulation signal source provides the main phases. The auxiliary phases are obtained by combining two voltage dividers using the same load resistor element. A circuit diagram of an auxiliary phase generator is illustrated in Figure 6.19a which also exhibits a phasor diagram of the output and input in Figure 6.19b. The resulting output signal from the auxiliary phase generator when $R_{1}$ and $R_{2}$ are identical is overlaid with input signals on the same plot as given in Figure 6.19c. Assuming both sources have the same frequency, amplitude and phase of a signal obtained at the output port are given by

$$
V_{\text {out } A}=\frac{V_{1} e^{j \emptyset_{1}} R_{2} R_{3}}{R_{1} R_{2}+R_{1} R_{3}+R_{2} R_{3}}+\frac{V_{2} e^{j \emptyset_{2}} R_{1} R_{3}}{R_{1} R_{2}+R_{2} R_{3}+R_{1} R_{3}}
$$




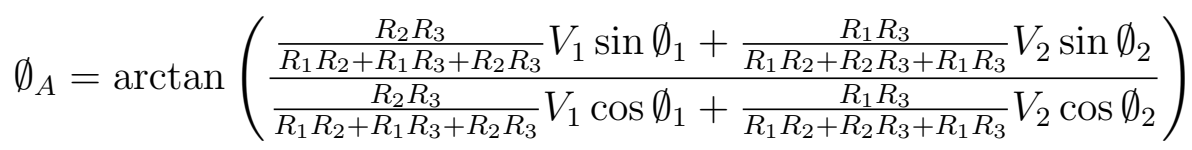

where $V_{1}$ and $V_{2}$ are signals amplitudes of the first and second input sources, respectively. $\emptyset_{1}$ and $\emptyset_{2}$ are signal phases of the first and second input sources, respectively. In equation (6.12) and (6.13), phases are represented by complex forms because they can have any value between $0^{\circ}$ and $360^{\circ}$. Any change in values of factors $\left(V_{1}, V_{2}, \emptyset_{1}, \emptyset_{2}, R_{1}, R_{2}\right.$, and $\left.R_{3}\right)$ leads to a change in phase and amplitude of the output signal. The value of resulting output phase is always confined between values of the input signals phases. To make the above two formulas compatible with our design requirements, some conditions like, $V_{1}=V_{2}=V$ and $R_{1}=R_{2}=R$ are applied, resulting in simplified versions of these two above equations

$$
\begin{aligned}
V_{\text {out } A} & =\frac{V R_{3}}{R+R_{3}+R R_{3}}\left(e^{j \phi_{1}}+e^{j \phi_{2}}\right) \\
\emptyset_{A} & =\arctan \left(\frac{\sin \emptyset_{1}+\sin \theta_{2}}{\cos \emptyset_{1}+\cos \emptyset_{2}}\right)
\end{aligned}
$$

Eq.(6.15) is slightly modified as shown in Figure 6.20 to obtain the correct phase depending on phases of the input signals. Furthermore, because the two signals are added with different phases, a magnitude of the resulting output signal never gets larger than the highest magnitude of either one of the input signals. Output signals with phases $45^{\circ}, 135^{\circ}, 225^{\circ}$, and $315^{\circ}$ can be obtained from input combinations with phases $\left(0^{\circ}, 90^{\circ}\right),\left(90^{\circ}, 180^{\circ}\right),\left(180^{\circ}, 270^{\circ}\right)$, and $\left(270^{\circ}, 0^{\circ}\right)$, respectively, as illustrated in Figure 6.20.

Although all the main and auxiliary phases are attained, their amplitudes are not equal. These issues have been amended using single voltage dividers in paths of the main modulation signals. Any amplitude value can be attained by changing 
values of resistors as shown in Figure 6.19d. Amplitude and phase of a signal obtained at the output port are given by

$$
\begin{gathered}
V_{\text {outB }}=\frac{V_{1} e^{j \emptyset_{1}} 2}{R_{1}+R_{2}} \\
\emptyset_{B}=\emptyset_{1}
\end{gathered}
$$

Here, phase of the output signal is always equal to phase of the input signal. In the design, $V_{\text {outB }}$ must be equal to $V_{\text {out } A}$, as a condition. Figure 6.19 e shows its phasor diagram.

Both types of phase shift generators are combined in one circuit to build a 4-to-8 phase transformer kit. The same schematic is designed by the ADS and LTspice as illustrated in Figure 6.21a and 6.21b, respectively. The 8-phase transformer kit requires a source generating quadrature signals to operate properly. If quadrature signals are not available, and can thus be generated by alternative means, the quadrature polyphase source becomes unnecessary. To take advantages of because no reactive elements are used in the design, the 8-phase transformer kit generates proper phase shifts and almost equal amplitudes at different frequencies and thus the kit response is very wideband at low frequencies. Figure $6.21 \mathrm{c}$ and $6.21 \mathrm{~d}$ show the simulation results of the kit along with the quadrant modulation input signals. Figure $6.22 \mathrm{a}$ and $6.22 \mathrm{~b}$ show photographs of the top and bottom of the kit prototype, and Figure 6.22c shows the measured input and output signals.

However, inconsistencies in values of resistors and errors in magnitudes and phases of the quadrant modulation input signals cause errors in both magnitudes and phases of the output modulation signals of the phase transformer kit. Since these output modulation signals are applied to the RTSs, these errors are multiplied by a distortion product term order $n$, leading to a fast deterioration in a response as a term 


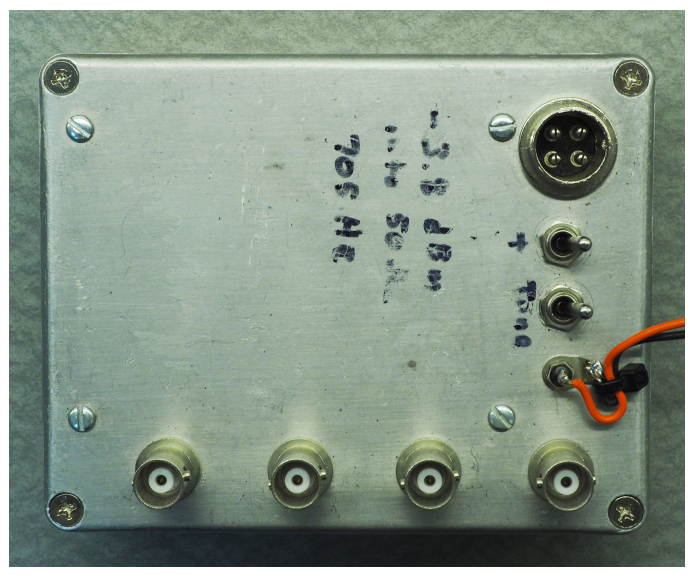

(a)

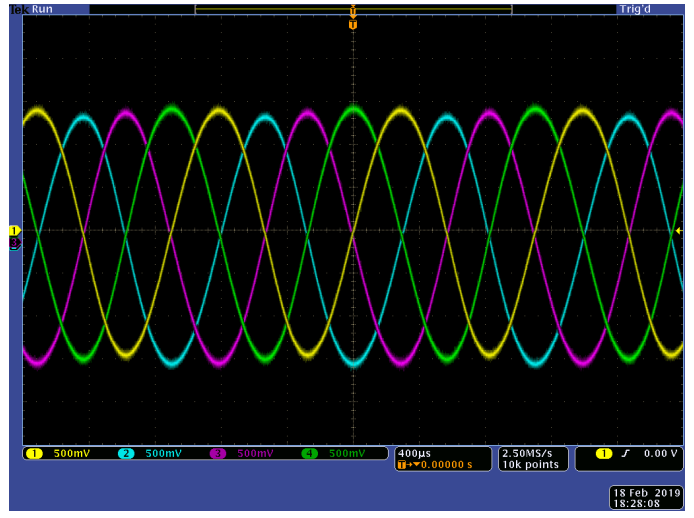

(c)

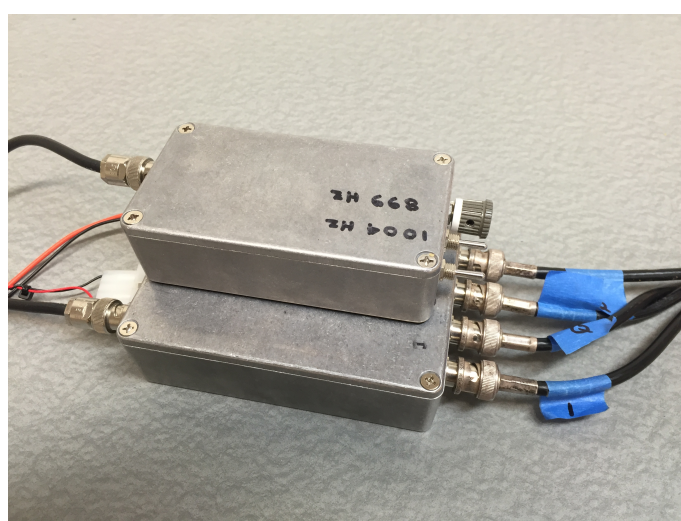

(b)

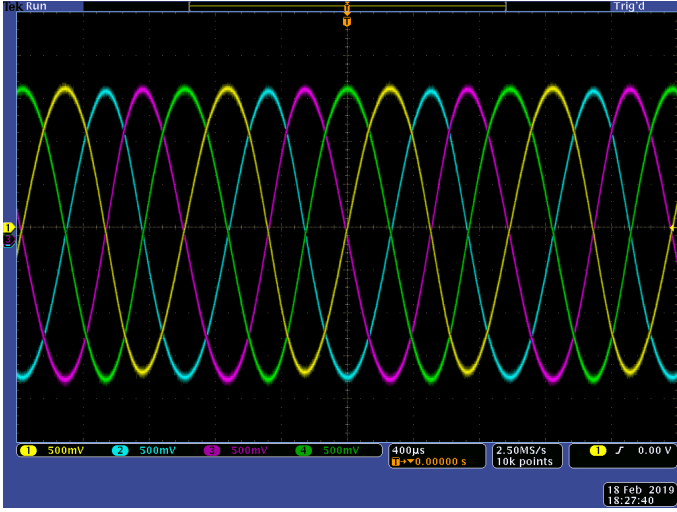

(d)

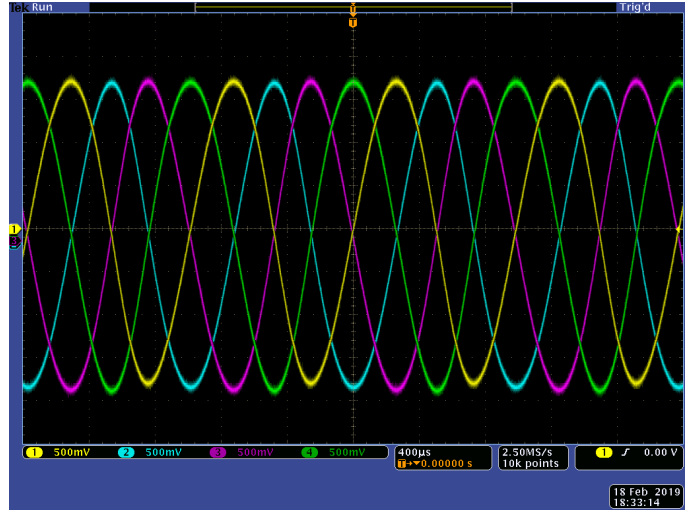

(e)

Figure 6.18: a) $700 \mathrm{~Hz}$ quadrature signal generator b) $899 \mathrm{~Hz}$ and $1004 \mathrm{~Hz}$ quadrature signal generator c) measured waveforms of $700 \mathrm{~Hz}$ quadrature signal generator d) measured waveforms of $899 \mathrm{~Hz}$ quadrature signal generator e) measured waveforms of $1004 \mathrm{~Hz}$ quadrature signal generator

order of distortion products increases. Errors are obtained by taking reads using a digital oscilloscope for each port and are then averaged out as given in Table 6.3 and 


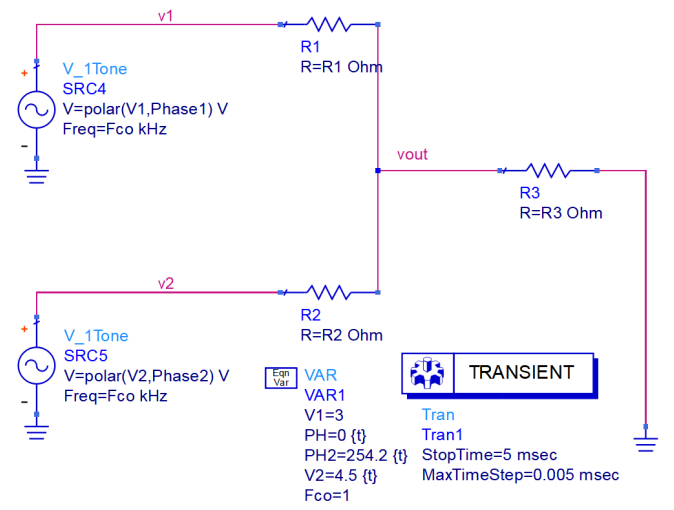

(a)

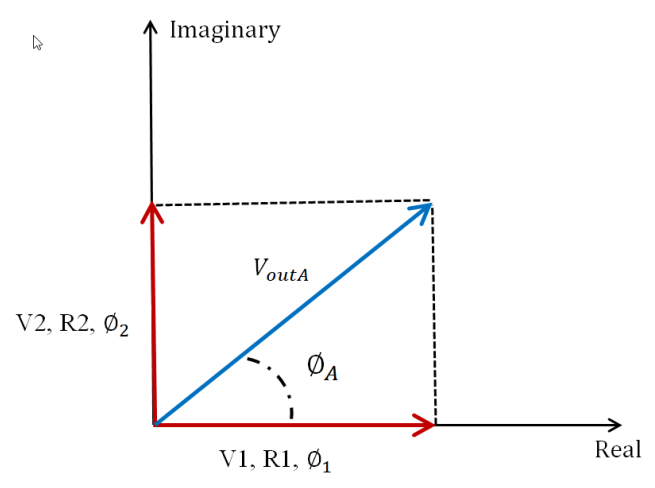

(b)

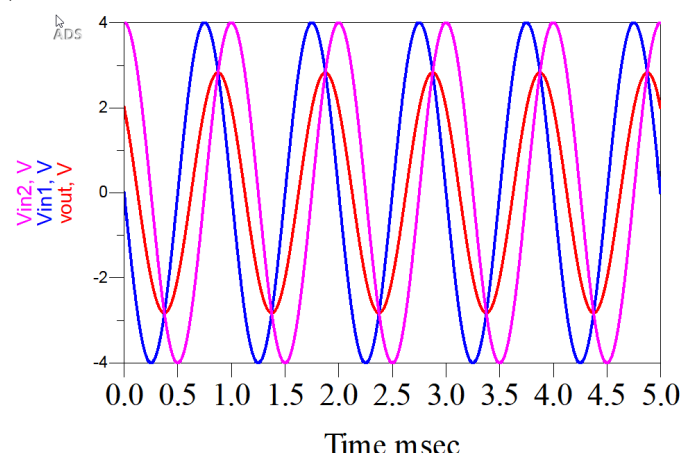

(c)

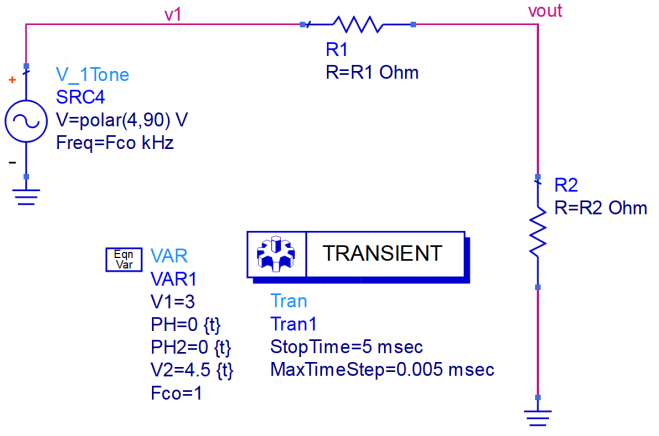

(d)

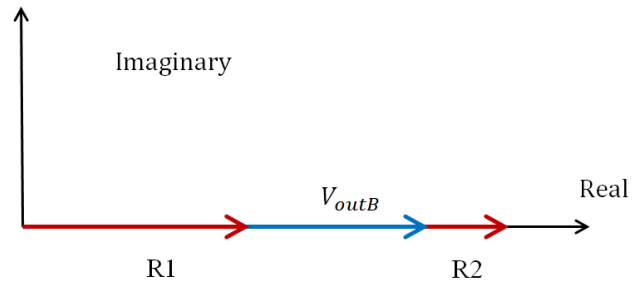

(e)

Figure 6.19: a) Auxiliary phase shift generator type-A b) its phasor diagram c) its input and output waveforms d) main phase shift generator type-B e) its phasor diagram

6.4. These errors will be inserted to the mathematical model presented in chapter 4 to compare with measured results that will be presented in Chapter 8 . 


$$
\begin{aligned}
& 2^{\text {nd }} \text { Quadrant } \quad I m \quad 1^{\text {st }} \text { Quadrant } \\
& \emptyset_{1}=90^{\circ} \quad \uparrow \quad \emptyset_{1}=0^{\circ} \\
& \emptyset_{2}=180^{\circ} \quad \emptyset_{2}=90^{\circ} \\
& \emptyset_{A}=\pi-\emptyset_{A} \quad \emptyset_{A}=\emptyset_{A} \\
& \begin{array}{l|l} 
& \\
\emptyset_{A}=-\pi+\emptyset_{A} & \emptyset_{A}=-\emptyset_{A}
\end{array} \\
& 3^{\text {rd }} \text { Quadrant } \\
& \emptyset_{1}=180^{\circ} \\
& 4^{\text {th }} \text { Quadrant } \\
& \emptyset_{2}=270^{\circ} \\
& \emptyset_{1}=270^{\circ} \\
& \emptyset_{2}=0^{\circ}
\end{aligned}
$$

Figure 6.20: Modified output phase according into the two input phases combination

Table 6.3: Errors in amplitudes and phases of $1004 \mathrm{~Hz}$ modulation signals passing through phase transformer kit

\begin{tabular}{c|c|c|c|c|c|c|c|c}
\hline \hline CH. No. & Amp.min & Amp.max & Mean & Error & P.S.min & P.S.max & Mean & Error \\
\hline 1 & 1.66 & 1.74 & 1.68 & Ref & Ref & Ref & Ref & Ref \\
\hline 2 & 1.22 & 1.72 & 1.67 & 0.01 & 36.51 & 53.81 & 39.52 & -5.48 \\
\hline 3 & 1.66 & 1.72 & 1.68 & 0 & 78.78 & 97.53 & 82.38 & 7.62 \\
\hline 4 & 1.66 & 1.74 & 1.68 & 0 & 122.4 & 132.8 & 126.4 & 9.6 \\
\hline 5 & 1.64 & 1.74 & 1.67 & 0.01 & 169.6 & 175.1 & 172.1 & 7.9 \\
\hline 6 & 1.64 & 1.68 & 1.66 & 0.02 & -141.8 & -133.2 & -137.1 & -2.1 \\
\hline 7 & 1.66 & 1.68 & 1.67 & 0.01 & -92.29 & -85.41 & -88.92 & -0.88 \\
\hline 8 & 1.66 & 1.70 & 1.67 & 0.01 & -49.16 & -40.29 & -44.6 & -0.04 \\
\hline
\end{tabular}

Table 6.4: Errors in amplitudes and phases of $899 \mathrm{~Hz}$ modulation signals passing through phase transformer kit

\begin{tabular}{c|c|c|c|c|c|c|c|c}
\hline \hline CH. No. & Amp.min & Amp.max & Mean & Error & P.S.min & P.S.max & Mean & Error \\
\hline 1 & 1.44 & 1.5 & 1.46 & Ref & Ref & Ref & Ref & Ref \\
\hline 2 & 1.44 & 1.5 & 1.45 & 0.01 & 39.18 & 47.17 & 43.55 & -1.45 \\
\hline 3 & 1.44 & 1.5 & 1.46 & 0 & 81.7 & 87.53 & 84.64 & -5.36 \\
\hline 4 & 1.44 & 1.5 & 1.45 & 0.01 & 126.1 & 131 & 128.4 & -6.6 \\
\hline 5 & 1.44 & 1.5 & 1.45 & 0.01 & 170.3 & 175.3 & 173.1 & -1.9 \\
\hline 6 & 1.42 & 1.48 & 1.44 & 0.02 & -141.6 & -134.7 & -137.7 & -2.7 \\
\hline 7 & 1.44 & 1.48 & 1.44 & 0.02 & -93.35 & -87.36 & -90.25 & 0.25 \\
\hline 8 & 1.44 & 1.46 & 1.44 & 0.02 & -50.02 & -42.12 & -45.31 & 0.31 \\
\hline
\end{tabular}




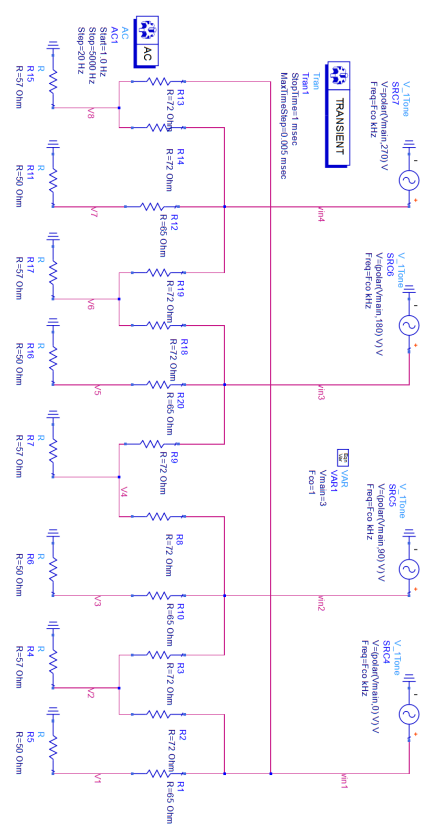

(a)

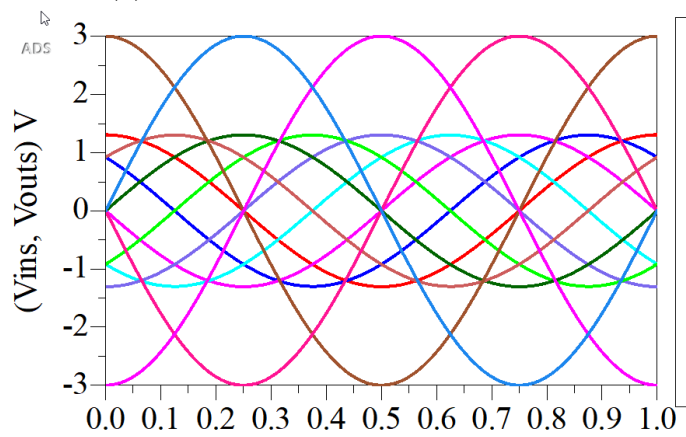

Time msec

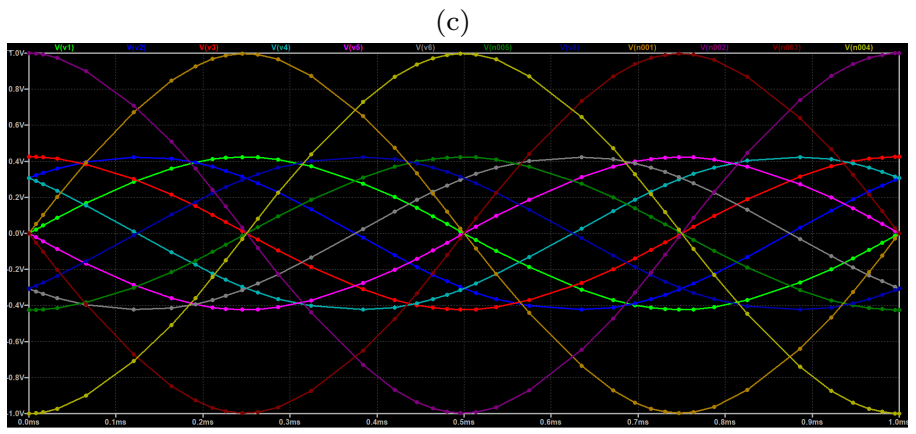

(d)

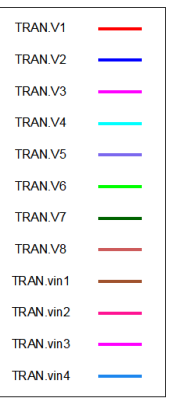

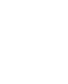

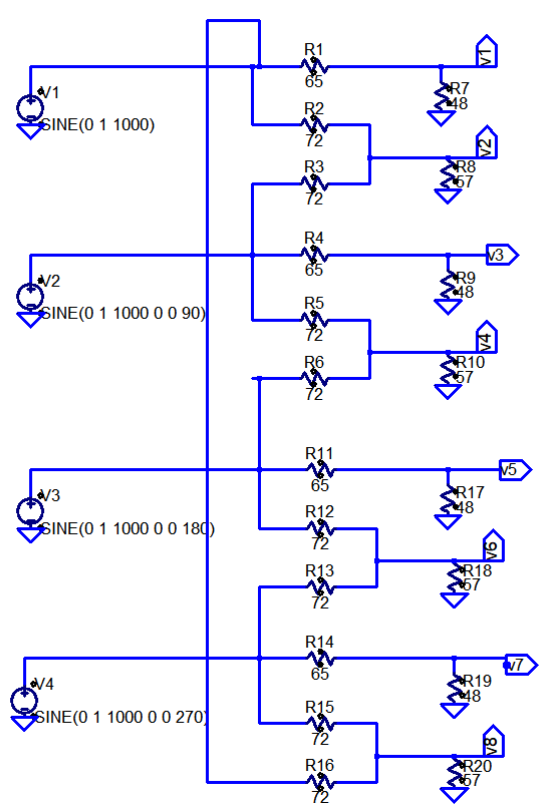

(b) 


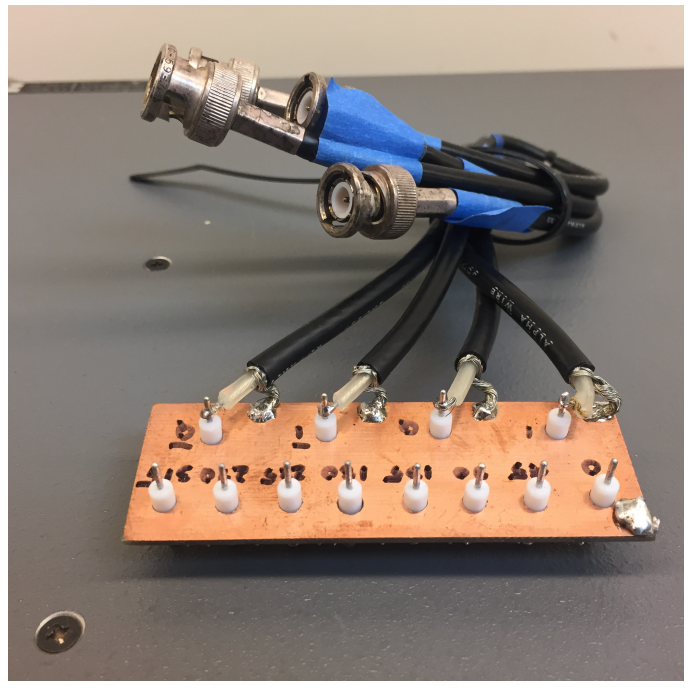

(a)

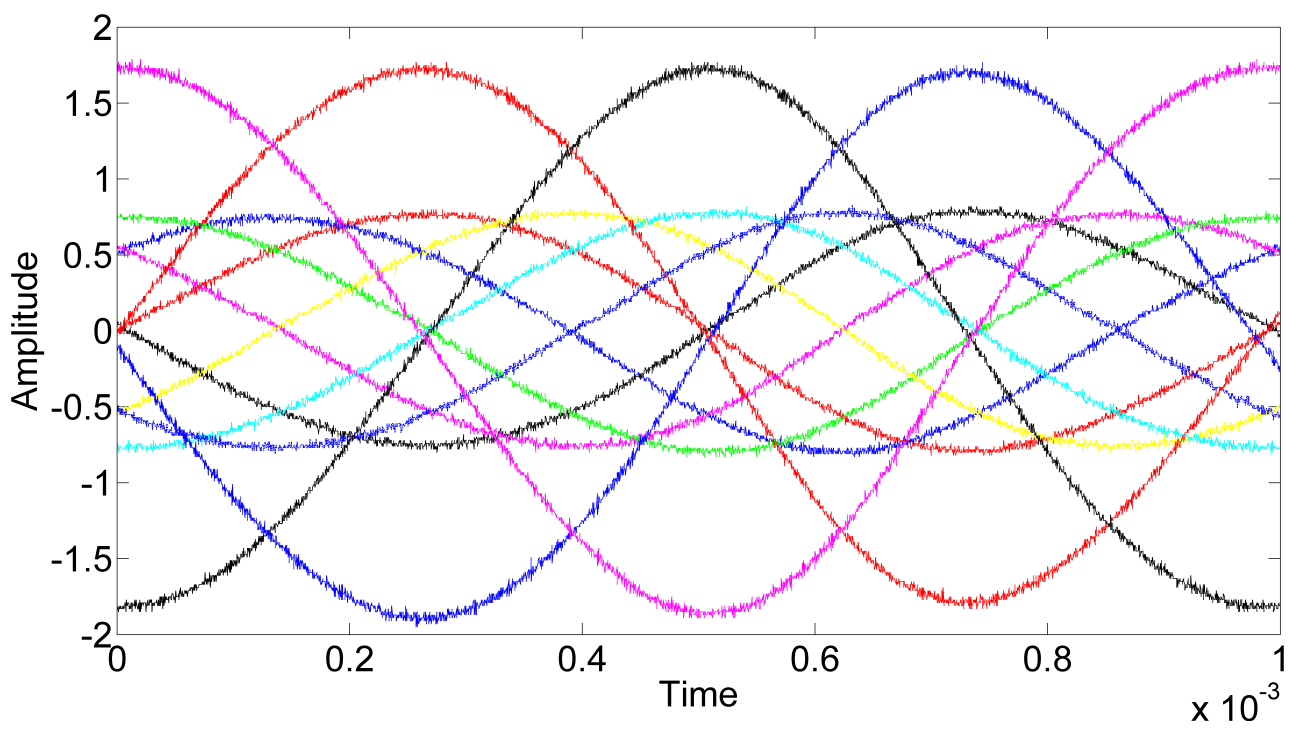

(c)

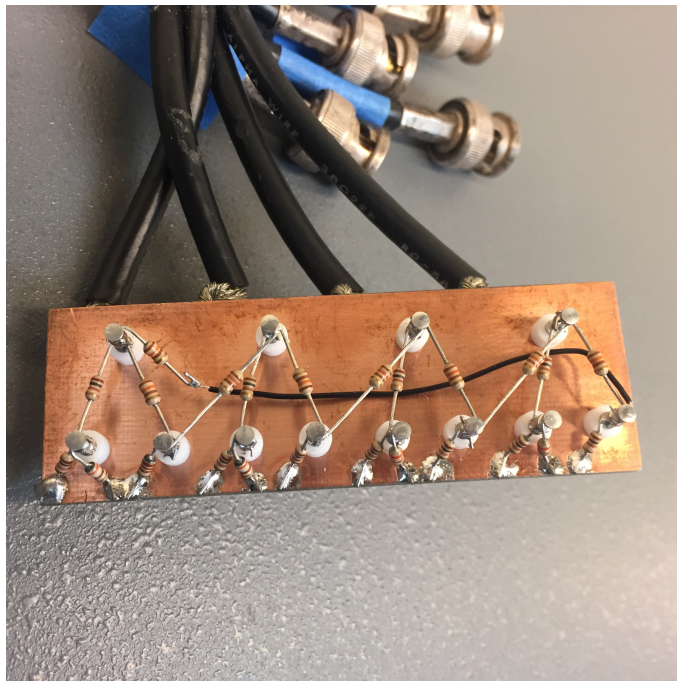

(b)

Figure 6.22: a) Photograph of the phase transformer kit prototype top-view b) photograph of the phase transformer kit prototype bottom-view c) measured input and output waveforms 


\section{Chapter 7: Experimental Setup}

\subsection{Introduction}

In the earlier chapter, detailed designs of different components that will be used to build the whole PMSA were presented. The prototypes were individually designed, analyzed, and tested. In this chapter, we provide thorough details about an environment where the PMSAs are tested and we also present the necessary apparatus to examine capabilities that the PMSA can achieve according to the theoretical parts.

\subsection{Testing Environment}

The proposed structures are characterized in an environment resembling properties of free-space called the anechoic chamber. It is a room shielded by a big metal case. The metal case is covered from the inside by a material absorbing and reducing reflections of electromagnetic signals from walls, ceilings, and floors. Results obtained inside this room is highly precise compared to other test places. Figure 7.1 shows an inside view of the anechoic chamber with an air-jet placed in the middle.

\subsection{Testing Apparatus}

The assembly of hardware components that makes up the testing equipment, presented by the block diagram in Figure 7.2 , is mounted on a mobile table. It consists of two parts. The first part, consisting of an RF signal source, two receive down-converters, a spectrum analyzer, and a signal processing station, lies outside the anechoic chamber as shown in Figure 7.3a. In Figure 7.3b, the second part lied inside the anechoic chamber comprises two antennas along with their cables as well as the device under test DUT (the proposed PMSA). Both signal source Tx and receiver 


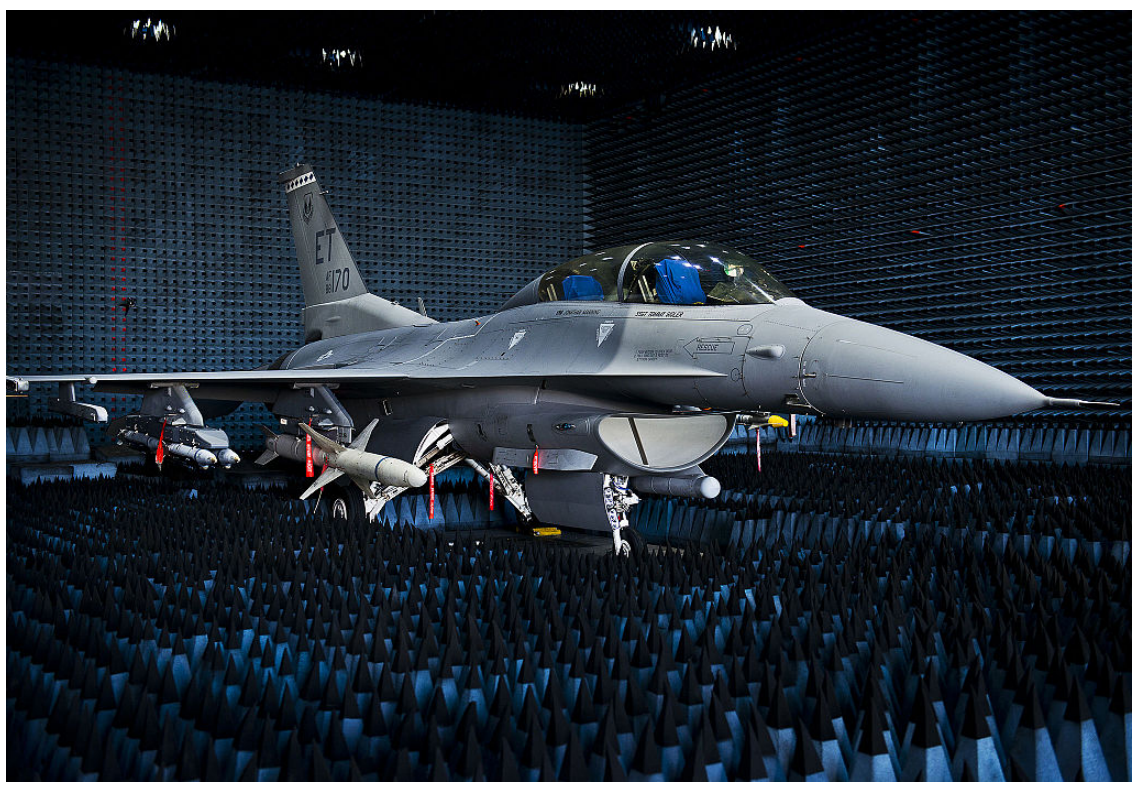

Figure 7.1: Inside view of the anechoic chamber with an air-jet [9]

Rx are narrowband systems working at $2.304 \mathrm{GHz}$, but for the initial experiments, testing apparatus works at $432 \mathrm{MHz}$. Figure 7.4 shows manufactured prototypes of the signal source and the receiver side by side. The signal generated by a Tx source

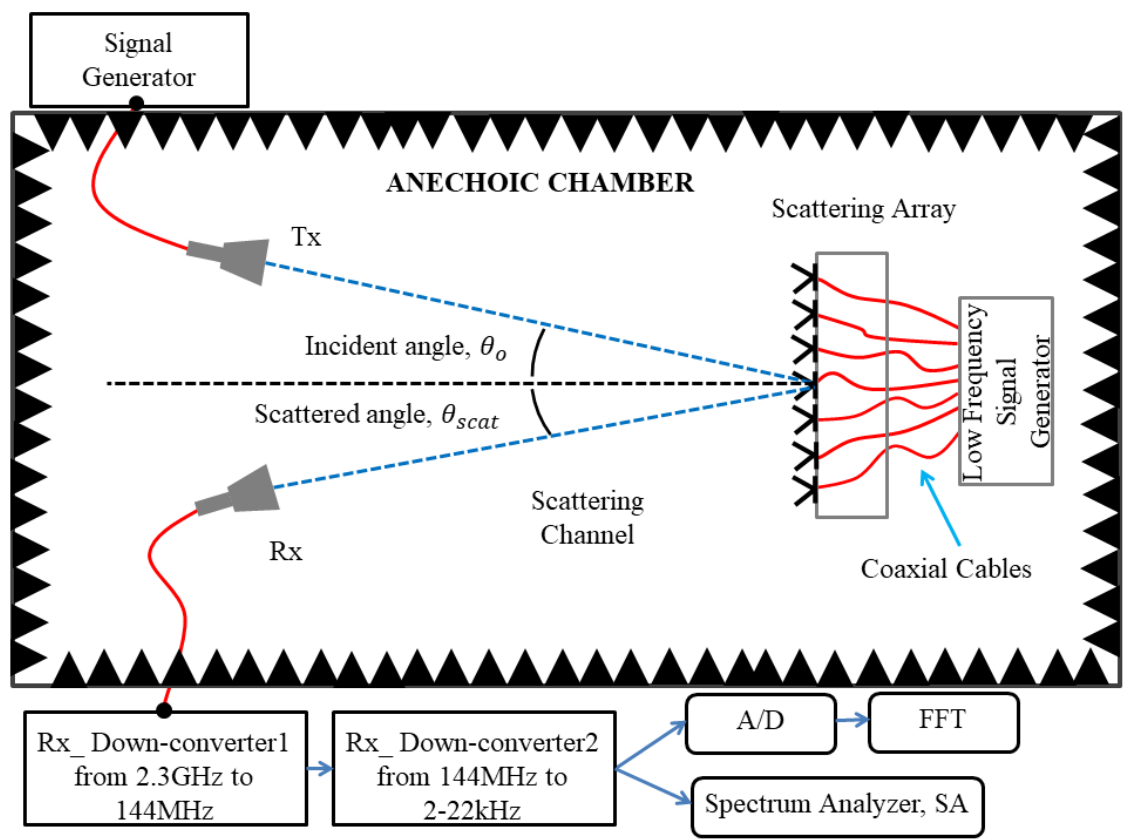

Figure 7.2: Block diagram of the experiemental setup inside and outside the anechoic chamber 


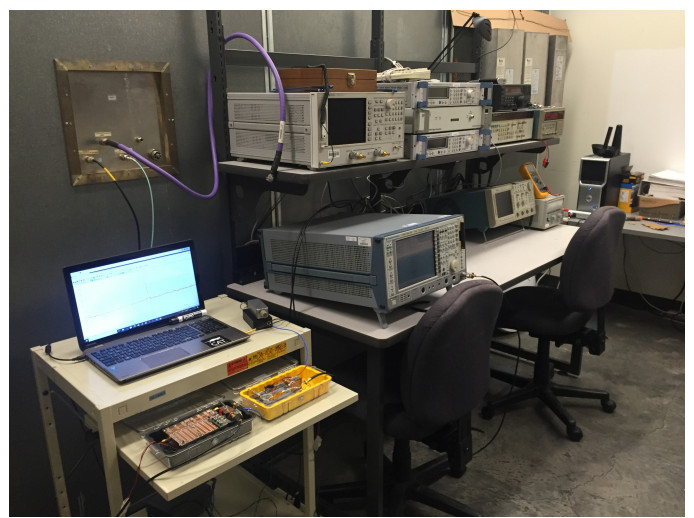

(a)

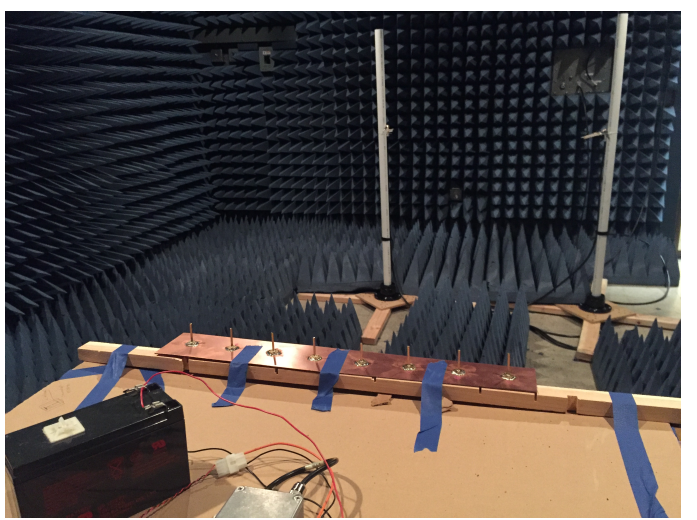

(b)

Figure 7.3: a) Testing apparatus outside the anechoic chamber b) testing apparatus inside the anechoic chamber

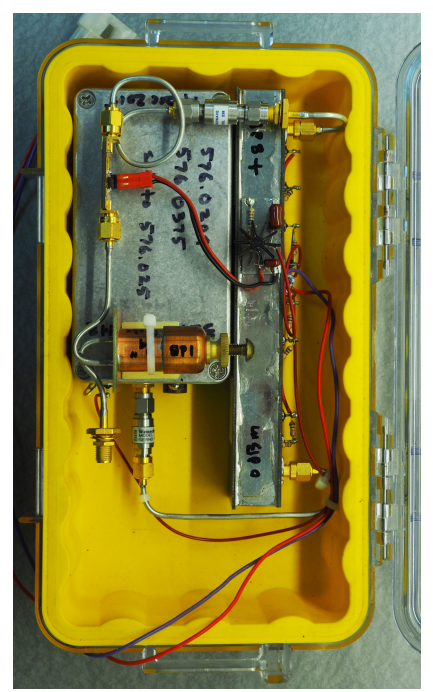

(a)

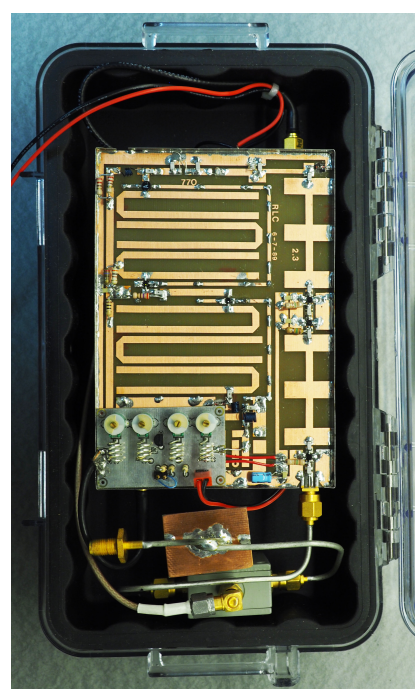

(b)

Figure 7.4: a) In-house signal source working at $2.304 \mathrm{GHz}$ b) in-house receiver working at $2.304 \mathrm{GHz}$

is applied to an $11 \mathrm{~dB}$ gain folded-Yagi Tx antenna where it is located at prescribed location at each time the PMSA performing inside the anechoic chamber. Signals illuminating the PMSA experience to modulation by the RTSs loaded on antennas and driven by the modulation signals. Two different modulation sources are used in measurements. The first modulation source has fixed properties with a frequency of $700 \mathrm{~Hz}$ and output power $-3.8 \mathrm{dBm}$. Furthermore, it has four ports with a $90^{\circ}$ relative phase shift between any two successive ports. In contrast, the second modulation 
source has tunable output powers and two different frequencies. Frequencies are $899 \mathrm{~Hz}$ and $1004 \mathrm{~Hz}$. Also, its four ports have the same relative phases compared to the first modulation source. The modulation sources can work with arrays having no more than four elements at least in one of their dimensions. Arrays with larger dimensions, for example, in the 8-element PMSA, use the 4-to-8 phase transformer kit illustrated in Chapter 6. After modulating the illuminating signals by one modulation source, signals are retransmitted again to reach the receiving antenna which is also a folded-Yagi dipole antenna with gain of $11 \mathrm{~dB}$. In the experimental setups, single-tone and two-tone modualtion sources are utilized in order to show that we can deal not only with harmonics distortions (HDs) but also intermodulations distortions (IMDs). Studying and and analyzing these two types of distortion products are useful especially in $5 \mathrm{G}$ communication systems incorporated with beamforming techniques. The most important fact that we succeeded to characterize the distortion products in space using simple structures (i.e., the PMSA designs).

Scattered signals captured by receiving antenna are first down-converted to $144 \mathrm{MHz}$ and then down-converted again to $12 \mathrm{kHz}$ with a range of $2-22 \mathrm{kHz}$ (audio range). The audio signals are converted from analog to digital using a built-in sound card of the Toshiba-satellite Laptop. The software running on the Laptop used to acquire data is the commercial SigView with a purchased License. A screenshot of the software acquisition interface is displayed in Figure 7.5. Data obtained by the software are post-processed by Matlab using the fast Fourier transform FFT algorithm. Also, data are recorded using a spectrum analyzer SA to plot scattered beams of distortion products.

At each time, the DUT performing inside the anechoic chamber, the Tx antenna is fixed at one location while the $\mathrm{Rx}$ antenna is moved within a specific range of angles in increments of $2^{\circ}$ as shown in Figure 7.6. A range of angles is chosen in 


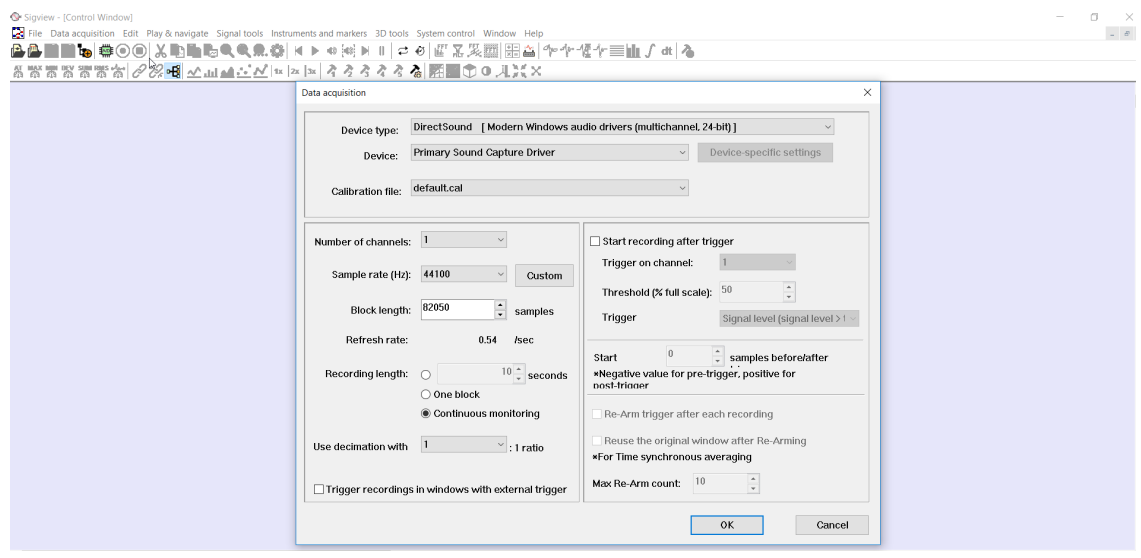

Figure 7.5: Screenshot of the interface panel of the SigView commercial software that used to acquire the measured data

such a way preserving the work in the far-field region, depending on many factors as will be seen later in this chapter. The Rx antenna is rotated such that it makes an angle of $\theta$ with respect to the broadside direction of the DUT across a measurement room (i.e., anechoic chamber). Data taken at every angle has different spatial distortion characteristics as we mentioned before. All DUTs are used in scattering (receiving/retransmitting)-mode where the Tx and Rx antennas are always placed in the far-field of DUTs. However, only the initial experiments working at a frequency of $432 \mathrm{MHz}$ are carried out within the near-field of DUTs due to space limitations of measurement facilities.

In the following experimental setups, we endeavor to prove parametric studies and some potential applications developed in Chapter 5. Three different sets of PMSAs $(8 \times 1$-element PMSA using separate printed quasi-Yagi scatterers, $4 \times$ 1-element PMSA using separate microstrip patch scatterers, and $8 \times 1$-element PMSA using quarter-wavelength monopoles over single ground scatterers) are used. In experiments, the illuminating antenna is placed in different locations to observe its impacts on spatial angular directions of scattered beams of distortion products. The experiments start with the capture of full scattered beams of distortion products for 


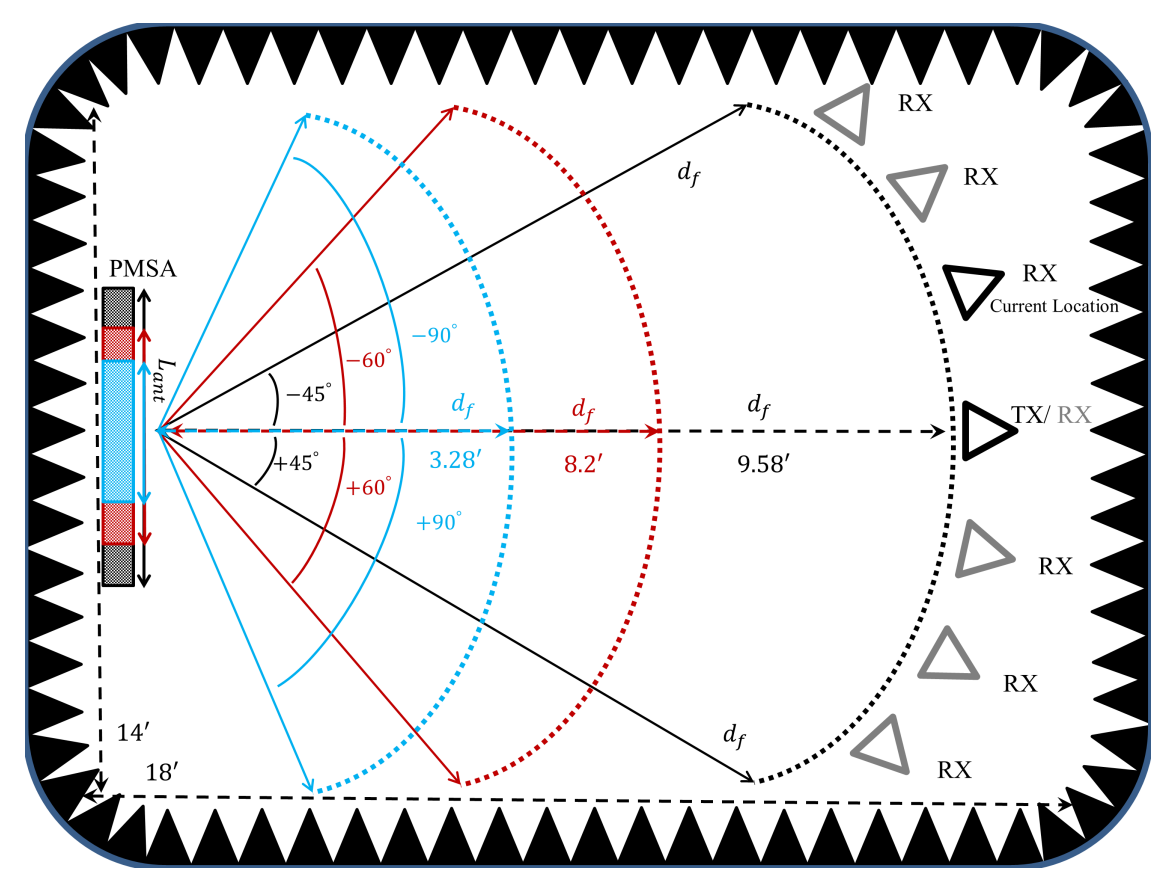

Figure 7.6: Ranges of angles where the measured data are taken depending on the total length of the proposed PMSA and its representative $d_{f}$

different locations of the illuminating antenna and different spacing between elements of the PMSAs and end with finding a direction of the incident signals under the assumption of their unknown directions. Also, a frequency of the modulation sources is changed to prove that scattered beams of distortion products do not change their spatial locations with frequencies.

\subsection{Limitations and constraints}

Because our presented structures consist of arrays of scatterers, they are physically larger than the half-wavelength being emitted by them. Their physical sizes get bigger with the increase of a number of elements. To work within the far-field of structures, distances separating between the Tx antenna to the DUT and from the DUT to the Rx antenna should be at least equal to (7.1) which represents the Fraunhofer distance 
being the limit between the far-field and near-field regions [34].

$$
d_{f}=\frac{2\left(L_{a n t}\right)^{2}}{\lambda_{o}}
$$

where $L_{\text {ant }}$ is a length of the scattering array and $\lambda_{o}$ is the free-space wavelength at a design frequency. For an $L$-element PMSA, $L_{\text {ant }}$ is equal to $(L-1) \times \lambda_{o} / 2$ when $d$ is equal to $\lambda_{o} / 2$. To obtain highly accurate measured results, this rule of thumb should be kept in all measurements except the initial measurements achieved at a frequency of $432 \mathrm{MHz}$. Moreover, we tested several structures such as a single scatterer, a $2 \times 1$-element PMSA, a $4 \times 4$-element PMSA, and an $8 \times 2$-element PMSA and each structure has its particular Fraunhofer distance $d_{f}$ relying on the largest dimension of structure.

Limitation here are that when the PMSA has more elements, for example, the $8 \times 2$-element, the anechoic chamber allows us to cover only angles confined between $\left[-45^{\circ}\right.$ to $\left.+45^{\circ}\right]$ as shown in Figure 7.6 to avoid the near-field problems. When a number of elements become less, a wide range of angles can be covered at the cost of less number of distortion products controlled at a time.

Moreover, one of the constraints faced during measurements is that the $\mathrm{Rx}$ antenna is moved manually for lack of adequate tools for assessing performances of the presented structures such as the controlled autonomous turntable. Also, data were recorded manually for the same reason thereby reducing an accuracy of data obtained from experiments. However, although there are discrepancies between measurement and the simulation results, they are in good agreement as will be seen in Chapter 8 . 


\section{Chapter 8: Experimental Results}

\subsection{Introduction}

In light of the preliminary and promising simulation results obtained in preceding chapters, several experiments were performed in order to examine the feasibility of the presented ideas in an environment resembling an infinitely large room (anechoic chamber) to ensure no signals bouncing off the surrounding walls. As presented in Chapter 7, devices under test (DUTs), and transmit and receive antennas with their own cables are placed inside the anechoic chamber, while all other apparatus are placed outside the anechoic chamber. Results obtained from our PMSAs are presented here with three goals in mind. The first goal is to demonstrate practically that distortion products can be treated (i.e., enhanced or suppressed) in space as the key theme of the presented PMSA. Also, to show the system scalability, two different sets of PMSAs working at frequencies $432 \mathrm{MHz}$ and $2.3 \mathrm{GHz}$ are designed and tested. Results obtained from measurements are compared with the theoretical and simulation results as a step in validating the design concepts. Also, some sources of errors are taken into account to reduce the discrepancies between the measured and simulated results. We believe that these discrepancies may be accounted for, at least in part, by the differences in circumstances and surroundings of every single scatterer in the entire PMSAs. As a consequence, the distortion product rejection ratio DPRR factor is calculated to show effectiveness of the PMSA performance.

Next, we focus on parametric studies utilizing a $8 \times 1$-element PMSA with vertically polarized elements in the arrangement of H-coupling scenario in order to obtain low mutual-coupling between elements. Spatial distortion products and their associated scattered beams, which are radiation patterns in a general sense, alter their 
angular directions in space with the change in values of:

- Distances separating scatterers $d$

- Values and directions of progressive phase distributions of the modulation sources $\pm \emptyset m$

- Directions of the incident signals $\theta o$

Finally, we practically demonstrate some features and potential applications of the PMSA. Retrodirective modes (scattered beams) reflected from the PMSA can be possibly obtained if the illuminating signals, propagating toward an aperture of the PMSA, arrive from particular angles, following the rule given in (5.17). Moreover, we demonstrate the inherent property of beamforming of scattered beams of distortion products using a $8 \times 1$-element PMSA which can also be considered as a potential application. These two features have been implicitly demonstrated in the previous section. However, we present other two separate experimental setups for both a tunable spatial harmonic generator and a direction finding system which are independent from the works presented in the first two parts of this chapter. Both of these setups prove that our PMSA may have a variety of potential applications.

\subsection{Experimental Results of the Phase Modulated Scattering Array (PMSA)}

In this section, we introduce results obtained from two different sets of experiments. In the first set, the PMSAs, performing at a frequency $432 \mathrm{MHz}$ with two different numbers of elements, are tested. Their results are compared to results of single scatterers. We consider these experiments as initials because they are done before developing whole mathematical models that support mechanisms of the PMSA. The PMSA, working at a frequency $2.3 \mathrm{GHz}$ with three different numbers of elements are tested within the second experimental setups. The aim of building two set of PMSAs 
working at two different frequencies which are $432 \mathrm{MHz}$ and $2.3 \mathrm{GHz}$ is substantial for demonstrating that the PMSA is a scalable structure, being able to perform with any frequency (i.e., from acoustic to terahertz bands).

As mentioned in Chapter 7, signals scattered back from the PMSA and captured by a receiver are down-converted into audio range signals via two down-converters. First, the targeted frequency which is either $2.3 \mathrm{GHz}$ or $432 \mathrm{MHz}$ is converted to the VHF band $144 \mathrm{MHz}$, and it is then converted into the audio band $2-22 \mathrm{kHz}$. So the objective behind using the low frequency is twofold: to use the built-in sound cards in laptops and one of widely available commercial software such as SigView for Windows and SigScope for Mac. For these reasons, we used that frequency band in all our simulations in order to compare with measurements. Both of simulations and measurements results are plotted at a frequency range from $2 \mathrm{kHz}$ to $22 \mathrm{kHz}$ and centered at $12 \mathrm{kHz}$ on the $\mathrm{x}$-axis. Amplitudes of simulated and measured results are plotted from $-60 \mathrm{~dB}$ or $-80 \mathrm{~dB}$ to $0 \mathrm{~dB}$ on the $\mathrm{y}$-axis due to the noise floor of the measurement environment. Besides, because of using the modulation signals with very low-frequencies, down-converting received signals into the audio range helps to see obviously distortion products distributed on both sides of the fundamental frequencies distanced by multiple integers of the modulation frequencies.

In the initial experiments with a frequency $432 \mathrm{MHz}$, elements used to construct the PMSAs are conventional folded dipole Yagi antennas. Printed quasi-Yagi dipole antennas working at a frequency $2.3 \mathrm{GHz}$ are used to build scattering arrays for the high frequency experiments. Although diodes used in the RTS designs are manufactured for high-speed RF applications, they perform very well with low modulation frequencies. The modulation frequencies are $700 \mathrm{~Hz}, 899 \mathrm{~Hz}$, and $1004 \mathrm{~Hz}$. In the initial experiments with single-tone modulation sources, the frequency is $700 \mathrm{~Hz}$ while, in the $2.3 \mathrm{GHz}$ experiments, the frequencies are $899 \mathrm{~Hz}$ and $1004 \mathrm{~Hz}$. The 
special feature of interest here is the spatial distortion products are either enhanced or suppressed in space rather than the use of transmission lines, waveguides, and combiners and dividers as in conventional RF communication circuits. This feature may potentially facilitate the use PMSA in very high frequencies such as in terahertz applications, avoiding size limitations and fields perturbation.

\subsubsection{MHz Measurement Results (Initial Experiments)}

A photograph of the single scatterer prototype is shown in Figure 8.1a. It was tested first. An illuminating source working at the frequency $432 \mathrm{MHz}$ connected to an antenna illuminates the scatterer. The distance between the transmitting antenna and the scatterer is about $4 \mathrm{~m}$. The scatterer modulates the illuminating signals with a very low-frequency $700 \mathrm{~Hz}$. The receive antenna is located about $4 \mathrm{~m}$

away from the scatterer too. A distance between the transmit and receive antennas is about $1.5 \mathrm{~m}$ to reduce the direct coupling between them. All antennas in the experiment are conventional folded Yagi type, and they are horizontally polarized. Scattered responses captured by the receive antenna is down-converted into the 0 to $20 \mathrm{kHz}$ baseband as shown in Figure 8.1b. We considered this received response as a reference for next experiments within the ensemble of the initial tests. As can be seen, all harmonics are present and distributed symmetrically about the main frequency. Power of the main frequency is about $-20 \mathrm{~dB}$ while powers of the first upper and lower sidebands (IUSB and 1LSB) harmonics and the second upper and lower sidebands (2USB and 2LSB)harmonics are about $-27 \mathrm{~dB}$ and $-29 \mathrm{~dB}$, respectively. As expected, the modulation sidebands are symmetrical, and harmonics of $700 \mathrm{~Hz}$ out to the $10 \mathrm{th}$ are significant.

To suppress or enhance scattered distortion products in space, the idea originated first is to mimic operation of the conventional image reject mixer. Because the 


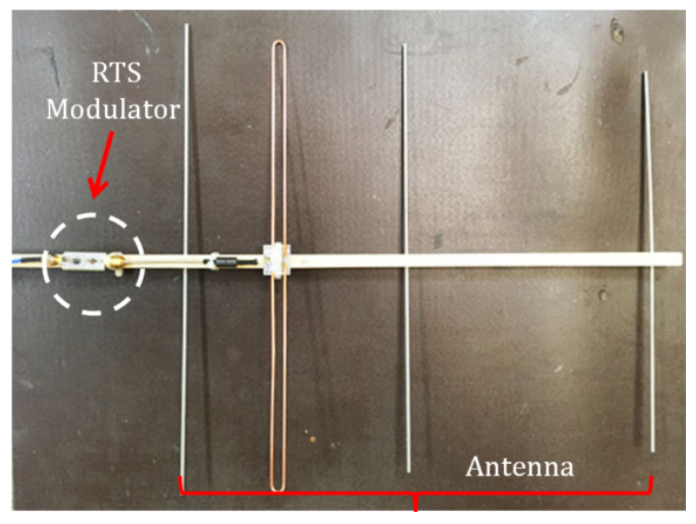

(a)

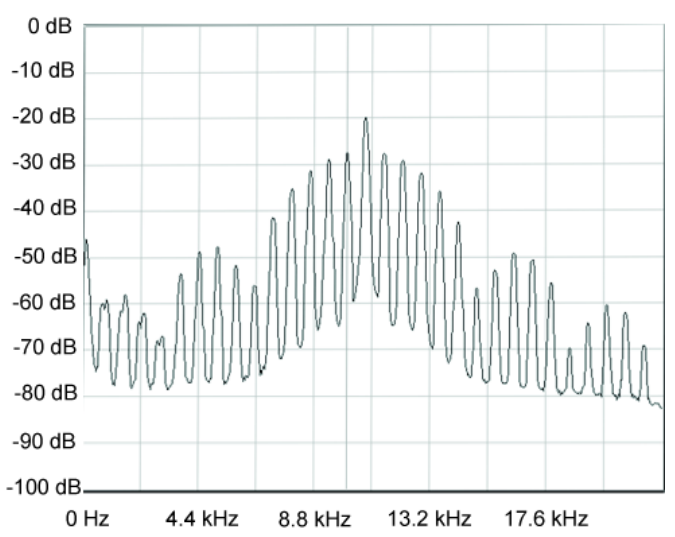

(b)

Figure 8.1: a) $432 \mathrm{MHz}(70 \mathrm{~cm})$ folded dipole Yagi scatterer b) Measured received spectrum of a single scatterer

image-reject mixer has two paths, we used two scatterers to form a 2-element PMSA with a separation distance of $0.7 \lambda_{o}$ as shown in Figure 8.2a. Similar to the image-reject mixer design, phase of the modulation source driving the first scatterer is $0^{\circ}$ and phase of the modulation source driving the second scatterer is $90^{\circ}$ ( $I Q$-arrangement). The illuminating antenna is located at an angle of $\theta_{o}=20^{\circ}$ with respect to the PMSA while the receive antenna is located at an angle of $\theta=0^{\circ}$. According to the angular direction of the illuminating source, the incident signals arrive to the two scatterers at different times, making the $90^{\circ}$ spatial phase shift. Figure $8.2 \mathrm{~b}$ shows the measured received spectrum of the scattered signals. Note that the first lower sideband (1LSB) is enhanced and the first upper sideband (1USB) is suppressed. In addition to that, the third lower sideband (3LSB) is suppressed, and the third upper sideband (3USB) is enhanced. This is expected because third harmonic products are out of phase with their first harmonic counterparts. In contrast, the fifth lower sideband (5LSB) is enhanced, and the fifth upper sideband (5USB) is suppressed because fifth harmonic products are in-phase with their first harmonic counterparts. Suppressed harmonics products are not entirely removed due to different sources of errors, leading to deterioration in the overall performance, which will be discussed in 
the following subsection.

Treatment of harmonics (i.e., suppression or enhancement) occurred in space which validates the theoretical assumptions. However, harmonics are not suppressed forever, but they are redirected into different directions in space. According to these experiment specifications, if the receive antenna is moved to an angle of $\theta=-42^{\circ}$, the suppressed harmonics at the previous location are enhanced, and the enhanced harmonics are suppressed. When the receive antenna is located at an angle of $\theta=0^{\circ}$, the scattered response of the PMSA looks like the response of the conventional USB image-reject mixer, and thus being called spatial USB image-reject mixer. In addition, when moving the receive antenna to be located at an angle of $\theta=-42^{\circ}$, the PMSA behaves like the LSB image-reject mixer.

Furthermore, spatial locations of 1USB and 1LSB harmonics suppression can be easily replaced without changing locations of the illuminating and receive antennas. To do so, we replaced phases of the modulation sources to get ( $Q I$-arrangement). Figure $8.2 \mathrm{c}$ shows the measured received spectrum of scattered signals captured at an angle of $\theta=0^{\circ}$. As shown in the figure, the 1USB harmonic product is enhanced while the 1LSB harmonic product is suppressed. Although these two initial experiments do not follow the rule of thumb of the PMSA because relative phase shift differences between the scatterers must be equal to $360^{\circ}$ divided by a number of total scatterers $L$, the experiments were successfully carried out, and the anticipated results were obtained.

After succeeding in obtaining what we wanted from the first part of the initial experiments, we extended our experimental setup by adding two more scatterers to the previous PMSA. Figure 8.3a shows a 4-element PMSA, performing inside the anechoic chamber. As in the last experiments locations of the illuminating and the receive antennas, distances between elements $d$, and the modulation frequency are 


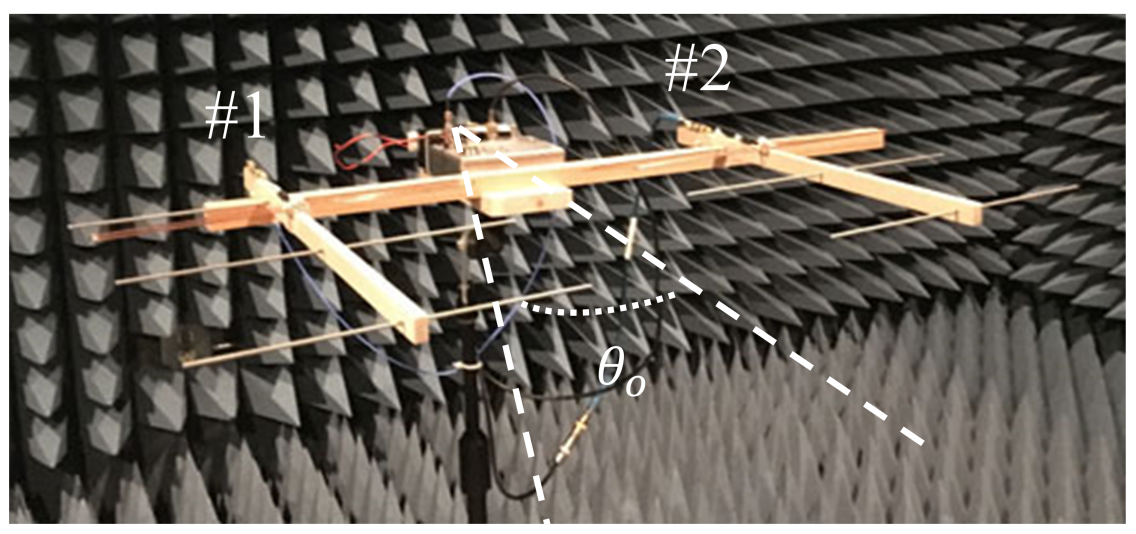

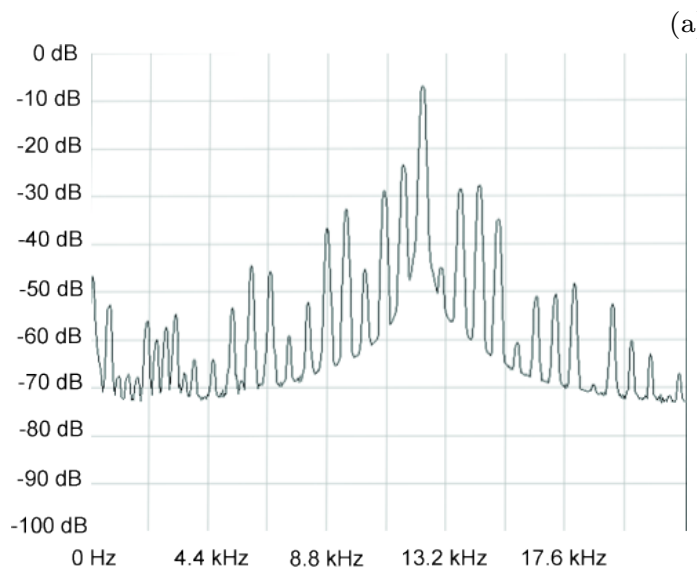

(b)

(a)

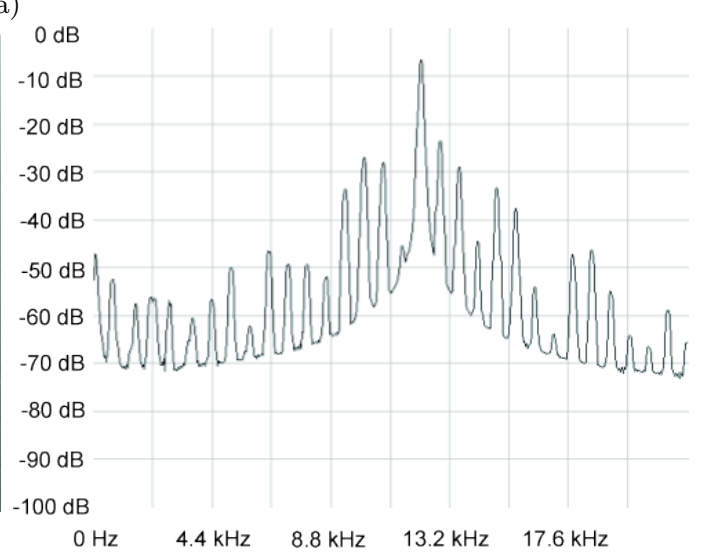

(c)

Figure 8.2: a) 2-element PMSA working at $432 \mathrm{MHz}$ b) measured received spectrum with 1USB suppression c) measured received spectrum with 1LSB suppression

kept the same. The one difference is that the relative phase shift between scatterers follows the PMSA rules (i.e., $360^{\circ}$ divided by the total number of scatterers $L$ ). The RTS modulators associated with each antenna element is switch ON-OFF at $700 \mathrm{~Hz}$ using phases $\emptyset_{1}=0^{\circ}, \emptyset_{2}=90^{\circ}, \emptyset_{3}=180^{\circ}$, and $\emptyset_{4}=270^{\circ}$ where the left-most element in Figure 8.3a is considered as a reference with an $I Q \overline{I Q}$-arrangement. Figure $8.3 \mathrm{~b}$ shows the measured received spectrum of scattered signals. As can 
be seen, more harmonics are suppressed or correctly radiated at different angles. The 1LSB harmonic product is enhanced while the 1USB, 2USB, 2LSB, and 3LSB harmonics products are attenuated. We used the term "attenuated" rather than the term "suppressed" for describing statuses of distortion products which are supposed to be removed entirely according to theoretical calculations because they are still present but with small amplitudes as shown in Figure 8.3b. According to the PMSA theory presented in Chapter 4, the 3USB harmonic component should be suppressed, but it was not. Both of these two issues could be attributed to impacts of the measurement environment because the receive antenna is located inside the near-field region of the PMSA. The far-field measurement is not possible for the frequency $432 \mathrm{MHz}$ with our lab facilities. These effects could be mitigated by focusing or ray-tracing methods. Moreover, to enhance the 1USB harmonic product instead of the 1LSB harmonic product at where the receive antenna is located at an angle of $\theta=0^{\circ}$ and the illuminating antenna is located at an angle of $\theta_{o}=30^{\circ}$, the $\overline{Q I} Q I$-arrangement should be used. The measured received spectrum of scattered signals at a receiver output is shown in Figure 8.3c. According to Chapter 4, phase shift distribution of $I Q \overline{I Q}$-arrangement is called the forward progressive phase shift while a phase shift distribution of $\overline{Q I} Q I$-arrangement is called the backward progressive phase shift. As expected, the more scatterers used in the PMSA, the more harmonics are treated (i.e., canceled or enhanced). Also, it is noteworthy that a harmonic status changes from the suppression to the enhancement once a direction of the progressive phase shift distribution of the modulation sources is reversed. The process of phase reversal does confirm the beamforming property of the PMSA which will be elaborately illustrated by results obtained from the measurements given later. Eventually, our PMSAs designs have been scaled down to work at $2.3 \mathrm{GHz}$ which is the only possible way to resolve impacts of our anechoic chamber on the measurements results. 


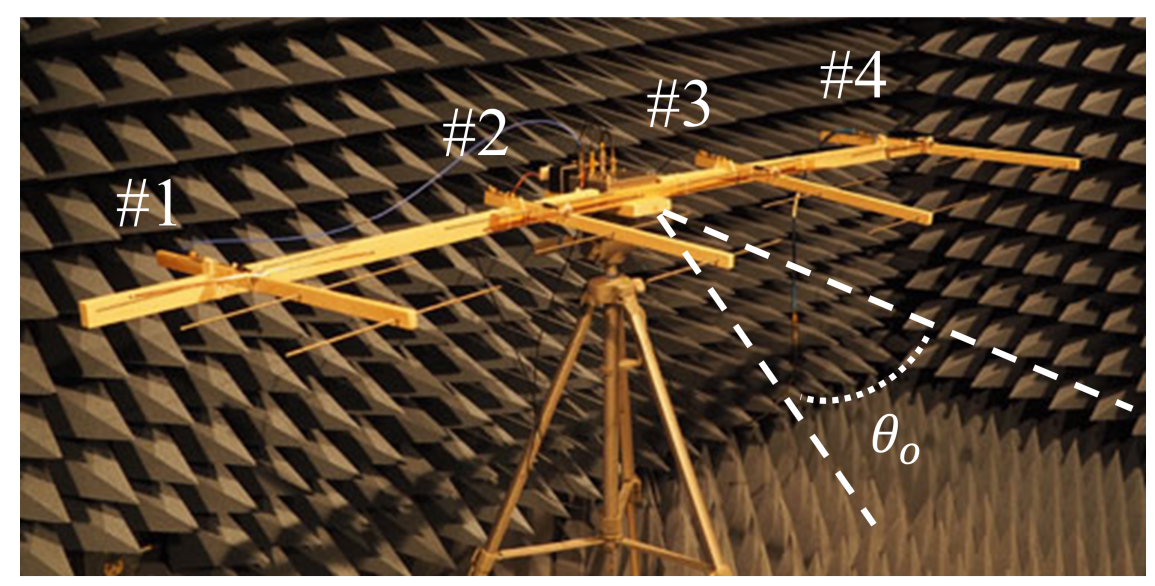

(a)

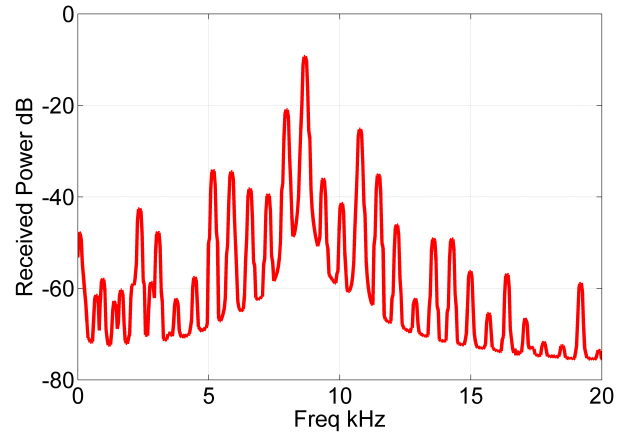

(b)

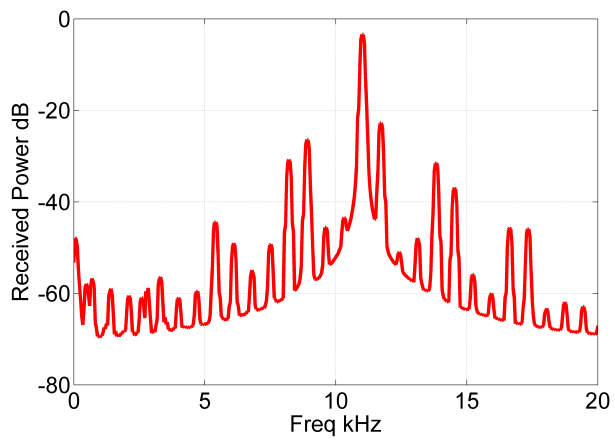

(c)

Figure 8.3: a) 4-Element PMSA working at $432 \mathrm{MHz}$ b) measured received response of scattered signals with $1 \mathrm{USB}, 2 \mathrm{USB}, 2 \mathrm{LSB}$, and $3 \mathrm{LSB}$ suppression c) measured received response of scattered signals with 1LSB, 2LSB, 2USB, and 3USB suppression

\subsection{GHz Measurement Results}

As we mentioned earlier, scaling down PMSA prototypes to work at $2.3 \mathrm{GHz}$ would kill two birds with one stone. The first thing is that inaccuracies in the measured results obtained in the initial experiments because of the size limitation of the anechoic chamber is highly reduced and the second thing is to achieve one of the research goals which is the scalability. Thus, all scatterers designed here work at a frequency $2.3 \mathrm{GHz}$. 
However, the size of the anechoic chamber is not only the source of inaccuracies in results, but its impacts were dominant in the initial experiments. As shown in the Chapter 6, there are different sources of errors, but they could fall under the definition of the term "amplitude and phase errors". Due to the complexity of calculation of errors caused by mutual coupling impacts, those errors are not considered in our mathematical models. The only errors, which are the inconsistencies in amplitudes and phases of the modulation signals given in Chapter 6, are taken into account. This procedure can be tolerated to some extent as will be seen when comparing measured and simulated results.

Even here, experiments are divided into two parts. In the first part, elements of the PMSA are modulated using single-tone modulation sources while two-tone modulation sources are utilized in the second part. In the beginning, three different sizes of the PMSAs which are $2 \times 1,4 \times 4$, and $8 \times 2$ elements PMSAs were performed. Next, the measured results of $8 \times 2$ PMSA was only presented although the $4 \times 4$ PMSA was also tested to avoid a lengthy discussion on findings. The experiments are given as follows:

\subsubsection{Single-tone Experimental Results}

As usual, a single scatterer here, consisting of separate printed quai-Yagig antenna and a HSMS2860 Schottky diode based the RTS modulator connected by a male-to-male SMA connector, was examined first for the sake of comparison with forthcoming results obtained by the $2.3 \mathrm{GHz}$ PMSAs for demonstrating the efficacy of the PMSAs. Before proceeding with measurements of the $2.3 \mathrm{GHz}$ PMSAs, simulation results of the E-cut plane radiation pattern gain of the printed quasi-Yagi dipole antenna is imported from the software HFSS to be manipulated mathematically using the software Matlab to obtain a fitted curve and then added to the proposed models. This 
procedure reduces the discrepancy between simulations and measurements results. Also the conventional radiation pattern gain of the antenna was measured. Figure 8.4 shows the simulated and measured gain patterns along with results of the fitted curve and the function $\cos ^{2.2} \theta$. The fitted curve was used in the mathematical model instead of the simulation result to integrate it easily with the theoretical models.

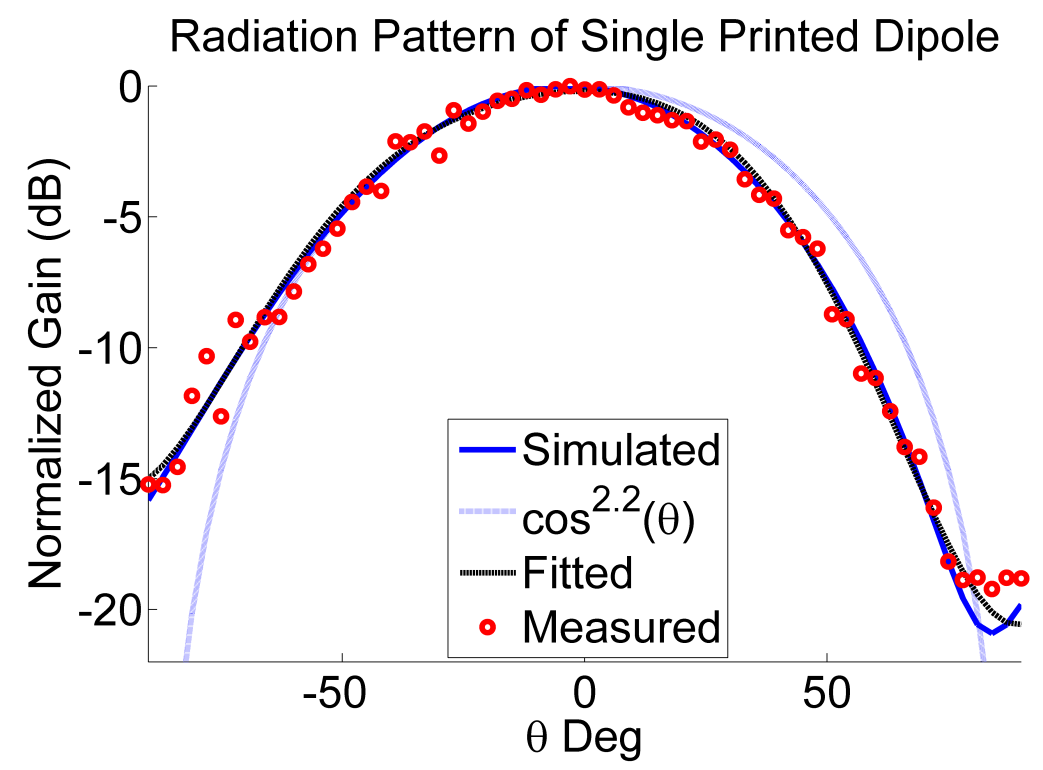

Figure 8.4: E-cut plane radiation pattern gain of the printed quasi-Yagi dipole antenna

A photograph of the single-scatterer prototype working at a frequency $2.3 \mathrm{GHz}$ is shown in Figure 8.5a. As can be seen, the scatterer is horizontally placed. The overall size of the antenna and the RTS modulator together is $65 \times 105 \times 1.52 \mathrm{~mm}^{3}$. The transmitting antenna is placed in front of the DUT (here is the single scatterer) with a separating distance $1 m$ to ensure working in the far-field region of the scatterer and the receive antenna is moved from $\theta=-90^{\circ}$ to $\theta=+90^{\circ}$ with a 2-degree step. Although the data are recorded at several angular locations around the scatterer, we chose just five reads to demonstrate strengths of scattered harmonics (distortion products), being attenuated as we moved from the broadside direction (i.e., no longer being in Line-of-Sight, LoS). Figure 8.5 displays the scattered power of measured and 
simulated received signals at angles of $\theta=-90^{\circ},-45^{\circ}, 0^{\circ}, 45^{\circ}$, and $90^{\circ}$. As expected, harmonics disappear or at least are highly attenuated when the scatterer is directed toward the angles $\theta=-90^{\circ}$ and $\theta=+90^{\circ}$ with respect to the receive antenna because the antenna in the scatterer does not radiate efficiently at these directions as shown in Figure 8.4. Strengths of harmonics increases gradually until reaching to their maximum values at the angle $\theta=0^{\circ}$.

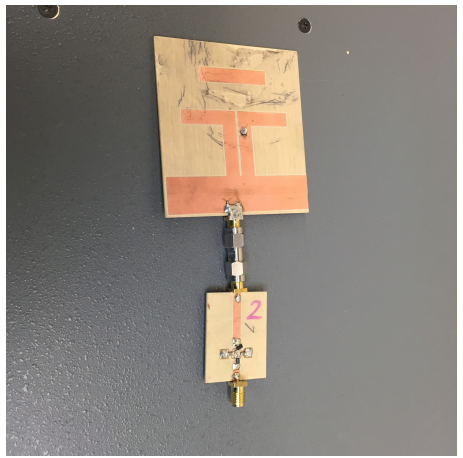

(a)

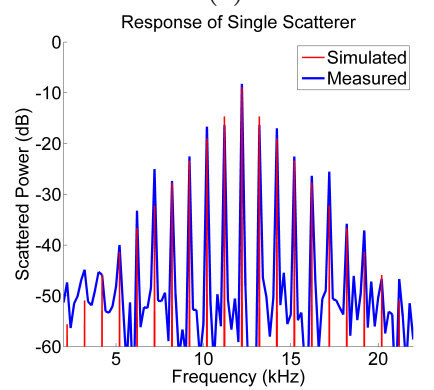

(d)

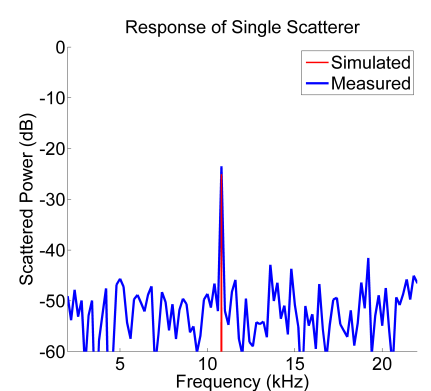

(b)

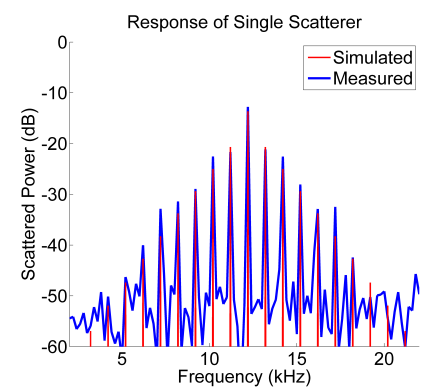

(e)

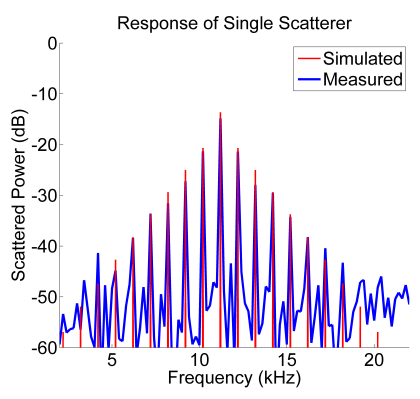

(c)

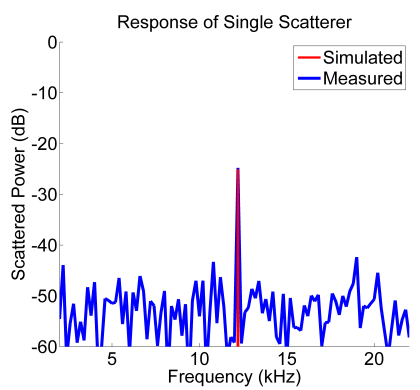

(f)

Figure 8.5: a) $2.3 \mathrm{GHz}$ printed quasi-Yagi scatterer b) received spectrum of scattered signals at $\theta=-90^{\circ} \mathrm{c}$ ) received spectrum of scattered signals at $\theta=-45^{\circ} \mathrm{d}$ ) received spectrum of scattered signals at $\theta=0^{\circ}$ e) received spectrum of scattered signals at $\theta=+45^{\circ}$ f) received spectrum of scattered signals at $\theta=+90^{\circ}$

Also, measured received spectrums of spatially scattered distortion products when the single scatterer is $\mathrm{ON}$ and $\mathrm{OFF}$ are presented in Figure 8.6a. As can be seen, all harmonics distributed about the main frequency in the case of ON are hided in the case of $\mathrm{OFF}$, and the main frequency has two different amplitude values for its ON-OFF states. An amplitude of the main frequency in the former case represents the 
static response produced by different sources as demonstrated in Chapter 2, whereas in the latter case, it is increased by about $7 \mathrm{~dB}$ due to an impact of the signal modulation. Moreover, simulated and measured scattered beams of some selected harmonics are shown in Figure 8.6b and 8.6c, respectively. In Table 8.1, legends given in Figure 8.6b are more clarified, and this clarification may be useful in the subsequent figures of scattered beams.

As can be noticed, all scattered beams of harmonics have almost the same profile shape with respect to a radiation pattern of the conventional printed quasi Yagi antenna. However, these scattered beams of distortion products (harmonics) are independent in their nature because they are not interfered as can be deduced from the results. In addition, most harmonics have almost the same value as we approach to angles of $-90^{\circ}$ and $90^{\circ}$

Table 8.1: Description of harmonic terms

\begin{tabular}{c|c}
\hline \hline Harmonic term & Description \\
\hline 4LSB & $f_{o}-4 \times f_{m}$ \\
\hline 3LSB & $f_{o}-3 \times f_{m}$ \\
\hline 2LSB & $f_{o}-2 \times f_{m}$ \\
\hline 1LSB & $f_{o}-1 \times f_{m}$ \\
\hline 0 & $f_{o}-0 \times f_{m}$ \\
\hline 1USB & $f_{o}+1 \times f_{m}$ \\
\hline 2USB & $f_{o}+2 \times f_{m}$ \\
\hline 3USB & $f_{o}+3 \times f_{m}$ \\
\hline 4USB & $f_{o}+4 \times f_{m}$ \\
\hline \hline
\end{tabular}

Having demonstrated that each distortion product has its particular scattered beam (radiation pattern), handling distortion product and their scattered beams becomes possible if we use more than one scatterer as notified in the initial experiments. Therefore, two scatterers are first placed side by side horizontally and separated by a distance of $d=0.7 \lambda_{o}$ at the design frequency $2.3 \mathrm{GHz}$ with an overall size of $156 \times 70 \times 1.52 \mathrm{~mm}^{3}$ as shown in Figure 8.7. This experimental setup is repeated thrice, and in each round, the $2 \times 1$-element PMSA performs with a different reference element (scatterer) and an angle of the incident signals $\theta_{o}$. 


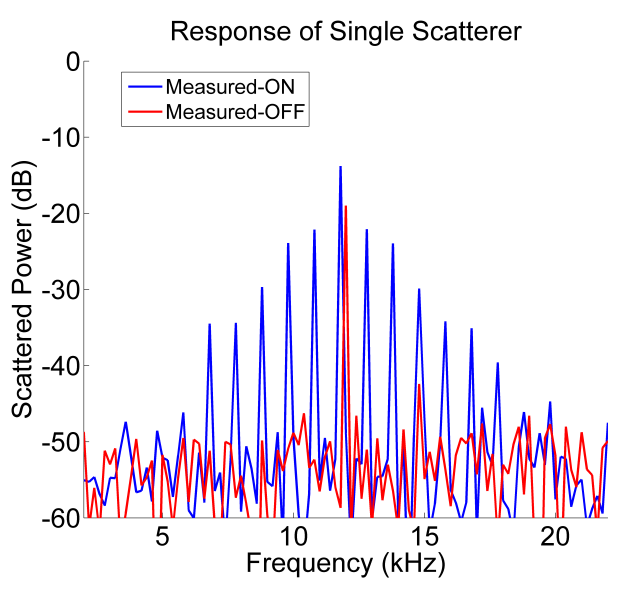

(a)

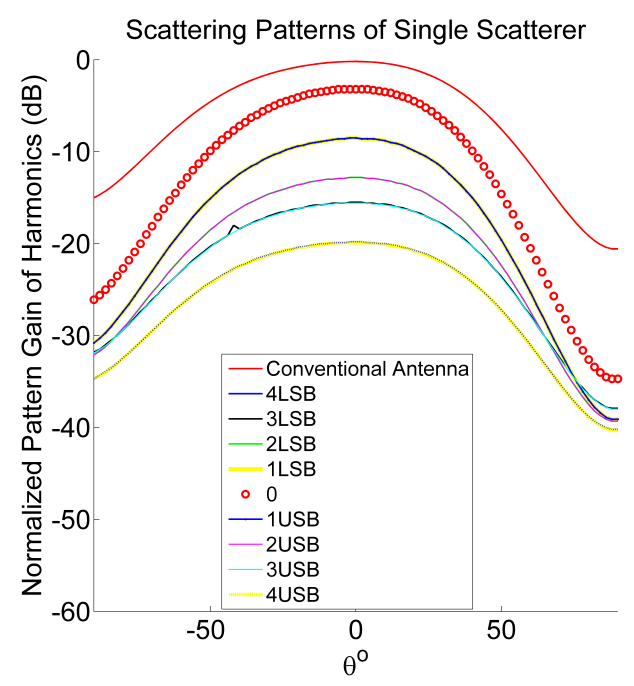

(b)

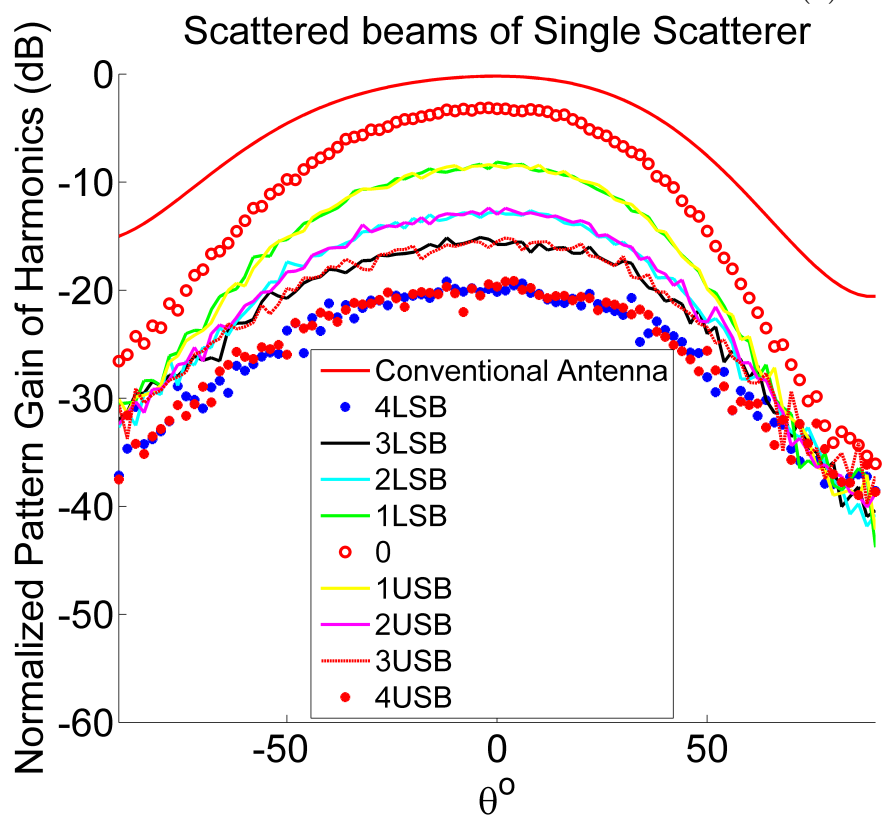

(c)

Figure 8.6: a) Measured received spectrums of scattered signals generated by a single scatterer for both $\mathrm{ON}$ and $\mathrm{OFF}$ states b) simulated scattered beams of some selected harmonics components generated in a single scatterer c) measured scattered beams of some selected harmonics components generated in a single scatterer

In the first and second rounds of measurements, a scatterer element in the left side in

Figure 8.7 is considered as a reference element, and a relative phase shift difference between the two scatterers is $90^{\circ}$. The only difference between these two rounds, the 
illuminating antenna is placed at angles of $\theta_{o}=21^{\circ}$ and $0^{\circ}$, respectively. According to Figure 3.13, the experimental setups in the first and second rounds can be considered as type A and type B circuits, respectively. By analogy, we can predict quite the right directions of scattered beams of distortion products before starting measurements. Moreover, in the third round, the first element on the right side in Figure 8.7 is considered as a reference, and the illuminating antenna is placed at an angle of $\theta_{o}=21^{\circ}$, wherein this round, we obtain the type $\mathrm{C}$ circuit.

In all of these rounds of measurements, the receive antenna is moved from $\theta=-60^{\circ}$ to $\theta=+60^{\circ}$, capturing all scattered signals confined in that range of angles, using both the software SigVeiw and a spectrum analyzer. Both the illuminating and receive antennas are linearly-polarized folded Yagi types working at $2.3 \mathrm{GHz}$. Powers of harmonics are plotted as a function of the current angle of the receive antenna, being the scattered beams as given in Figure 8.8a, 8.8d, and $8.8 \mathrm{~g}$ for the first, second, and third rounds, respectively. In each plot, scattered beams of distortion products (harmonics) are normalized according to the maximum power value for the fundamental harmonic component. As can be seen, the scattered beam of the 3LSB harmonic component always accompanies the scattered beam of the 1USB harmonic component and the scattered beam of the 3USB harmonic component always accompanies the scattered beam of the 1LSB harmonic component. Therefore, their scattered beams have the same angular directions, and the differences in their amplitudes stem from the nature of a harmonic product that means that its amplitude decreases, as a harmonic order increases.

Plots of measured signals captured at angles where scattered beams of distortion products are maximum are shown in Figure 8.8. Each row of sub-figures provides scattered beams of one round and its spatial characteristics of scattered distortion products at two different spatial locations. For the first round, the scattered beam of 
1LSB harmonic component is directed toward an angle of $\theta=0^{\circ}$, and the scattered beam of 1USB harmonic component is directed toward an angle of $\theta=-44^{\circ}$. However, the scattered beam of 1USB beam has another main lobe called grating lobe occurring due to the large distance between scatterers and the main lobe of the scattered beam is directed to reach far angles, as always happens in radiation patterns of conventional phased arrays [34]. Figure 8.8b and 8.8c show measured and simulated received spectrums. Received responses from the first round found at an angle of $\theta=0^{\circ}$ seems like a spectral response of the conventional USB image-reject mixer, and it seems like a spectral response of the conventional LSB image-reject mixer at an angle of $\theta=-44^{\circ}$. Each spectral response has different distortion products characteristics, and it is space-dependent. These two responses can exchange their spatial locations once we select the right scatterer in the PMSA in Figure 8.7 as a reference element, as obtained from results of the third round, see the last row of sub-figures of Figure 8.8). In other words, once we reverse a direction of the progressive phase shift of the modulation sources and keep the illuminating antenna at the same location, received spectrums are exchanged. Also, these two received spectrums with two different harmonic characteristics or in a general sense scattered beams of distortion products can be symmetrically located around the PMSA (the broadside direction) if the illuminating signals reach the PMSA aperture normally as in the second round of measurements. Figure 8.8d, 8.8e, and $8.8 \mathrm{f}$ show scattered beams and their received spectrums of the scattered signals at angles of $\theta_{U S B}=-21^{\circ}$ and $\theta_{L S B}=+21^{\circ}$.

To check the effectiveness of the two-element PMSA, and how it deals with distortion products, we adopt (3.82) to calculate theoretical $D P R R_{n}$ and compare it with the measured one. On this basis, we will deal with distortion products not 


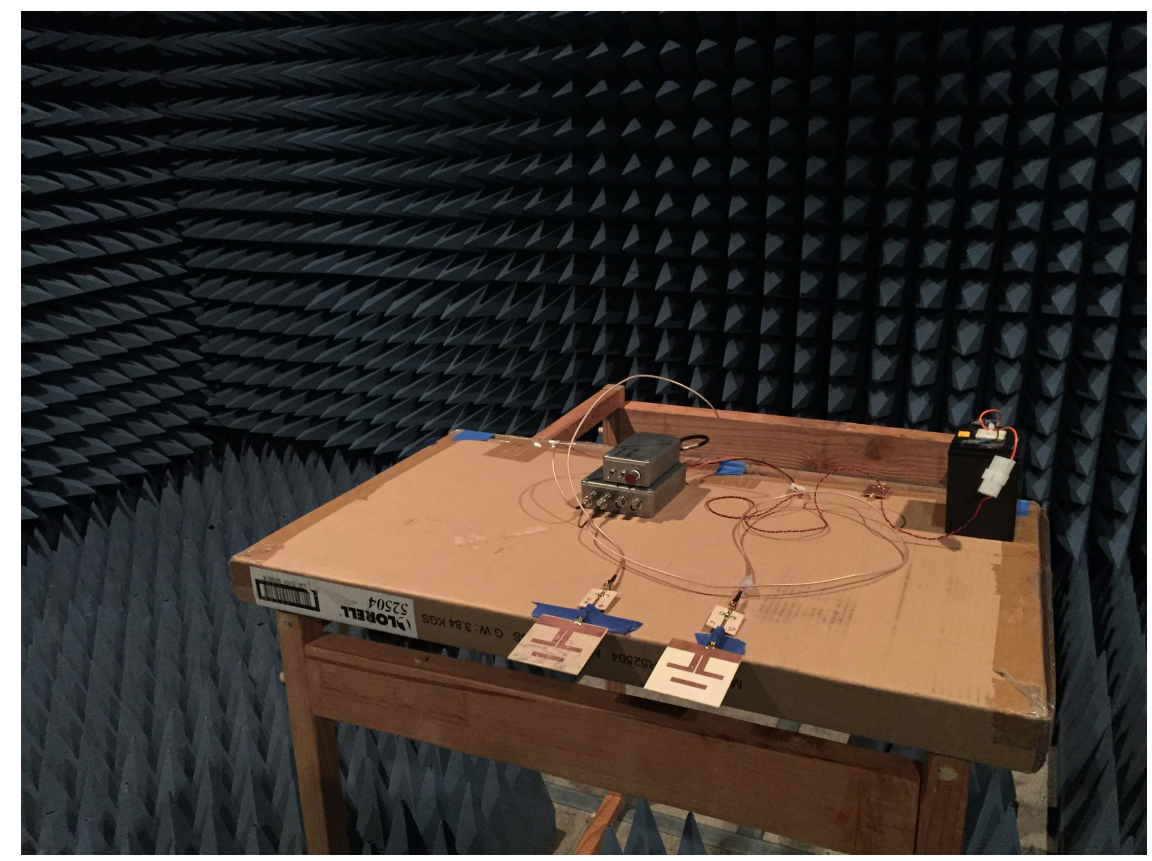

Figure 8.7: 2.3GHz 2-element PMSA using printed quasi-Yagi scatterers

showing up at given locations as canceled distortion products although they emerge at different spatial locations. To do so, received signals of a single scatterer and a $2 \times 1$-element PMSA using the same experimental setup of the first round are overlaid on the same plot as shown in Figure 8.9, where the receive antenna is located at an angle of $\theta=0^{\circ}$. The measured $D P R R_{1}$ of the 1 USB harmonic component is $15 \mathrm{~dB}$ while the theoretical $D P R R_{1}$ of the $1 \mathrm{USB}$ component is $18 \mathrm{~dB}$. The discrepancy in simulated and measured results is because our mathematical model does not include all sources of errors as illustrated before. In contrast, the 1LSB harmonic component of the $2 \times 1$-element PMSA is enhanced by about $6 \mathrm{~dB}$ compared to the response of the single scatterer.

Next, we increased the distance between the two elements to be $d=\lambda_{o}=$ $130 \mathrm{~mm}$ from center to center at the design frequency $2.3 \mathrm{GHz}$ and the incident angle, at this time, becomes $\theta_{o}=+30^{\circ}$. Moreover, the modulation source connected to the first left scatterer in Figure 8.7 has phase of $0^{\circ}$, while the modulation source connected 


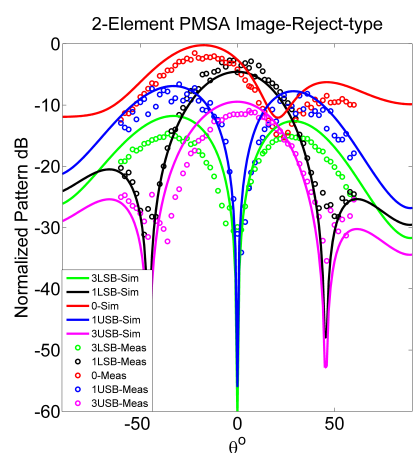

(a)

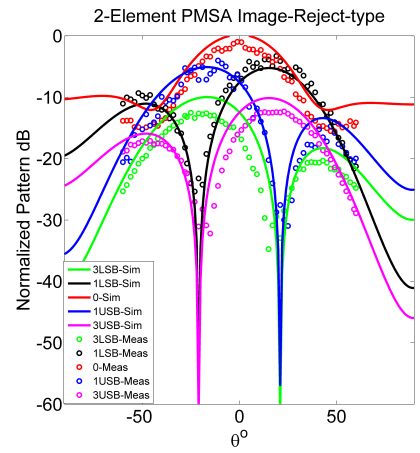

(d)

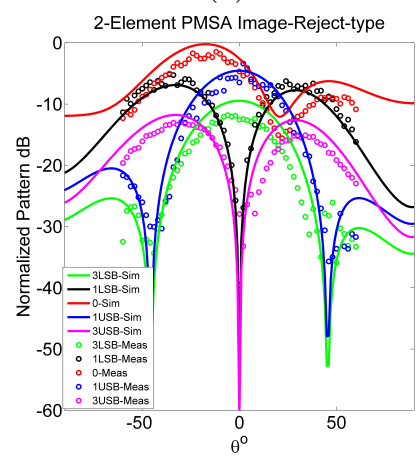

$(\mathrm{g})$

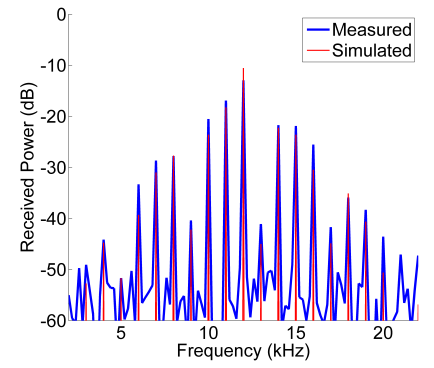

(b)

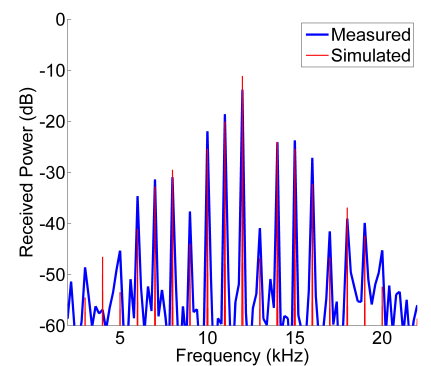

(e)

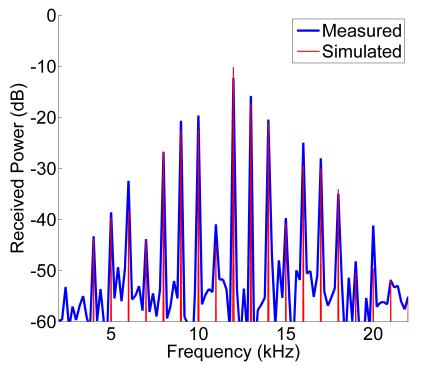

(h)

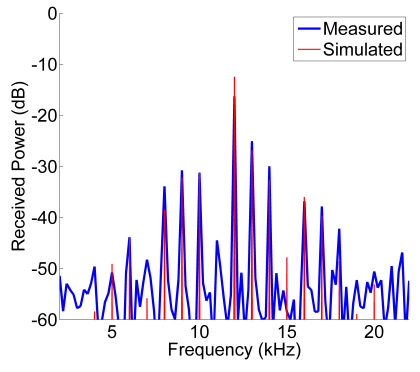

(c)

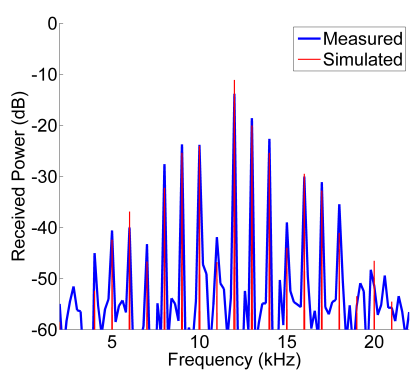

(f)

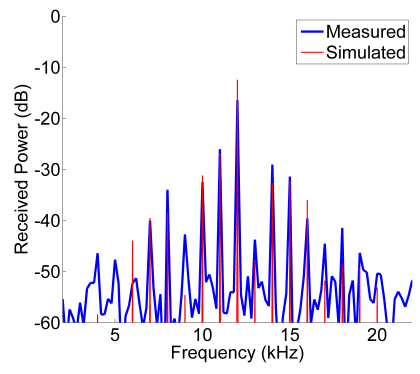

(i)

Figure 8.8: Measured and simulated performances of the 2-element PMSA performing as a spatial image-reject mixer for three different setups a) received scattered beams of harmonics for the first setup b) received spectrum of scattered signals at $\theta=0^{\circ}$ for the first setup c) received spectrum of scattered signals at $\theta=-44^{\circ}$ for the first setup d) received scattered beams of harmonics for the second setup e) received spectrum of scattered signals at $\theta=+21^{\circ}$ for the second setup $\mathrm{f}$ ) received spectrum of scattered signals at $\theta=-21^{\circ}$ for the second setup g) received scattered beams of harmonics for the third setup $h$ ) received spectrum of scattered signals at $\theta=0^{\circ}$ for the third setup i) received spectrum of scattered signals at $\theta=-44^{\circ}$ for the third setup

to the second scatterer has phase of $180^{\circ}$. According to the new illuminating angle, The incident signals reach the two scatterers with a relative phase shift difference 


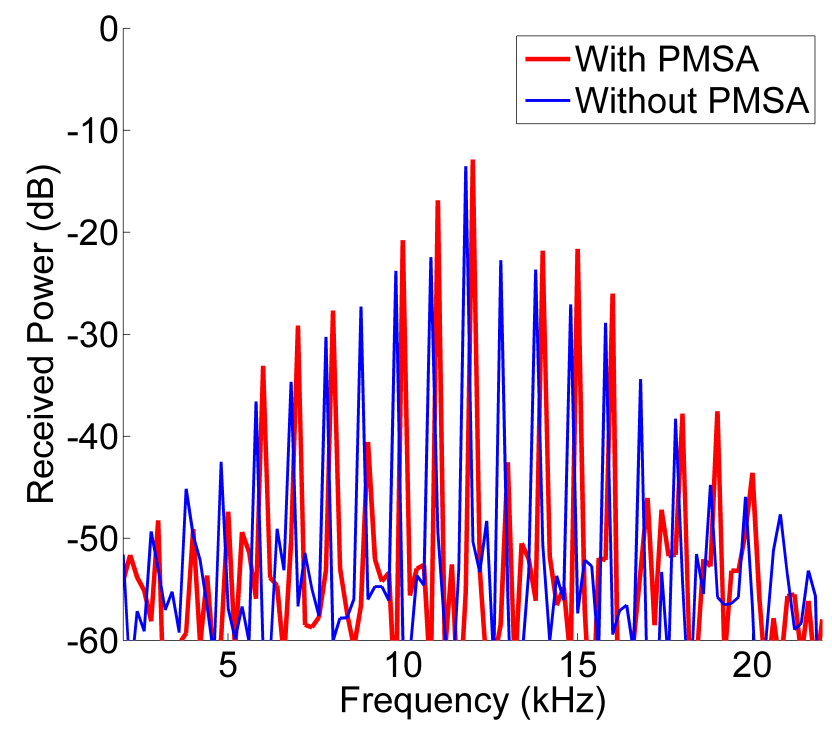

Figure 8.9: A comparison between measured received spectrums of a 2-element PMSA in the first round and a single scatterer captured at an angle $\theta=0^{\circ}$

of $180^{\circ}$. Thus, modulation signals driving and RF illuminating signals induced over each single scatterers have the same phase, but they are out of phase with signals in the other scatterer. A response of a well-known circuit which is the balanced (differential) circuit can be realized. However, here treating signals occurs in space rather than transmission-lines, and dividers or combiners. Scattered beams of some selected harmonics and received spatial characteristics of harmonics at angles of $\theta=$ $0^{\circ}$ and $\theta=-30^{\circ}$ are given in Figure 8.10. The spatial response at an angle of $\theta=0^{\circ}$ has no even harmonics while it does not have odd harmonics at an angle of $\theta=-30^{\circ}$. Both of the spatial responses obey the rule of non-canceled harmonics $=n+p \times L$, where $p=\cdots,-2,-1,0,1,2, \cdots, L=2$, and $n=1$ or 2 for odd or even harmonics cancellation, respectively. Accurately, the measured results show that unwanted harmonics are attenuated by more than $10 \mathrm{~dB}$, but are not completely canceled.

As further investigations, $4 \times 4$ and $8 \times 2$ elements PMSAs shown in Figure 8.11, performing inside the anechoic chamber, are tested for different combinations of 


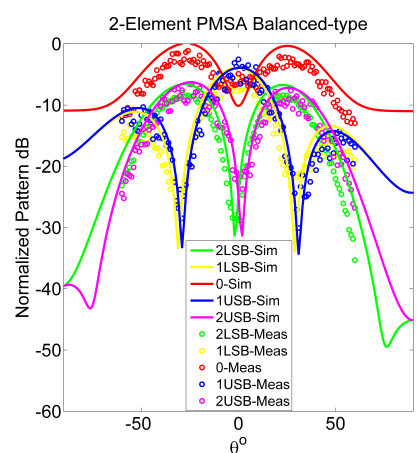

(a)

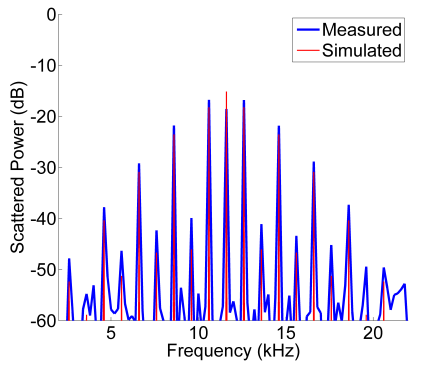

(b)

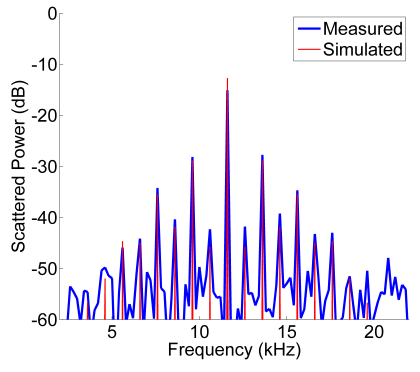

(c)

Figure 8.10: Measured and simulated performances of the 2-element PMSA performing as a spatial balanced-modulator for an illuminating signal $\theta_{o}=+30^{\circ}$ and $d=\lambda_{o}$ a) scattered beams of distortion products (harmonics) b) received spectrum of scattered signals at $\theta=0^{\circ} \mathrm{c}$ ) received spectrum of scattered signals at $\theta=-30^{\circ}$

incident angles and reference elements. Also, Figure 8.11 shows signals scattered by the $8 \times 2$-element PMSA captured by the receive antenna at an arbitrary angle using a spectrum analyzer when the $8 \times 2$-element PMSA (DUT) is OFF and ON and for two bandwidth resolutions.

The $4 \times 4$-element PMSA with an overall size of $260 \times 195 \times 70 \mathrm{~mm}^{3}$ was tested first. It has four sub-arrays and each sub-array, consisting of a $4 \times 1$-element, is printed on the same substrate with a separation distance of $d x=0.5 \lambda_{o}$. The sub-arrays were stacked above each other with a distance separating between any two adjacent layers equal to $d y=0.5 \lambda_{o}$. The modulation source used in this experiment has four ports with phases of $\left(0^{\circ}, 90^{\circ}, 180^{\circ}, 270^{\circ}\right)$, and each port feeds a single column of elements. Because the PMSA here consists of four columns, the horizontal phase shift distribution across the array can be either $\left(0^{\circ}, 90^{\circ}, 180^{\circ}, 270^{\circ}\right)$ or $\left(270^{\circ}, 180^{\circ}, 90^{\circ}, 0^{\circ}\right)$, and a relative phase shift difference between any two successive columns is $90^{\circ}$. Therefore, this PMSA is able to scatter distortion products into different spatial directions only in the horizontal (Azimuth) plane. Although we used the same experimental setup for the 2-element PMSA inside the anechoic chamber, the distances between the DUT $(4 \times 4$-element PMSA $)$ and the transmit and receive 


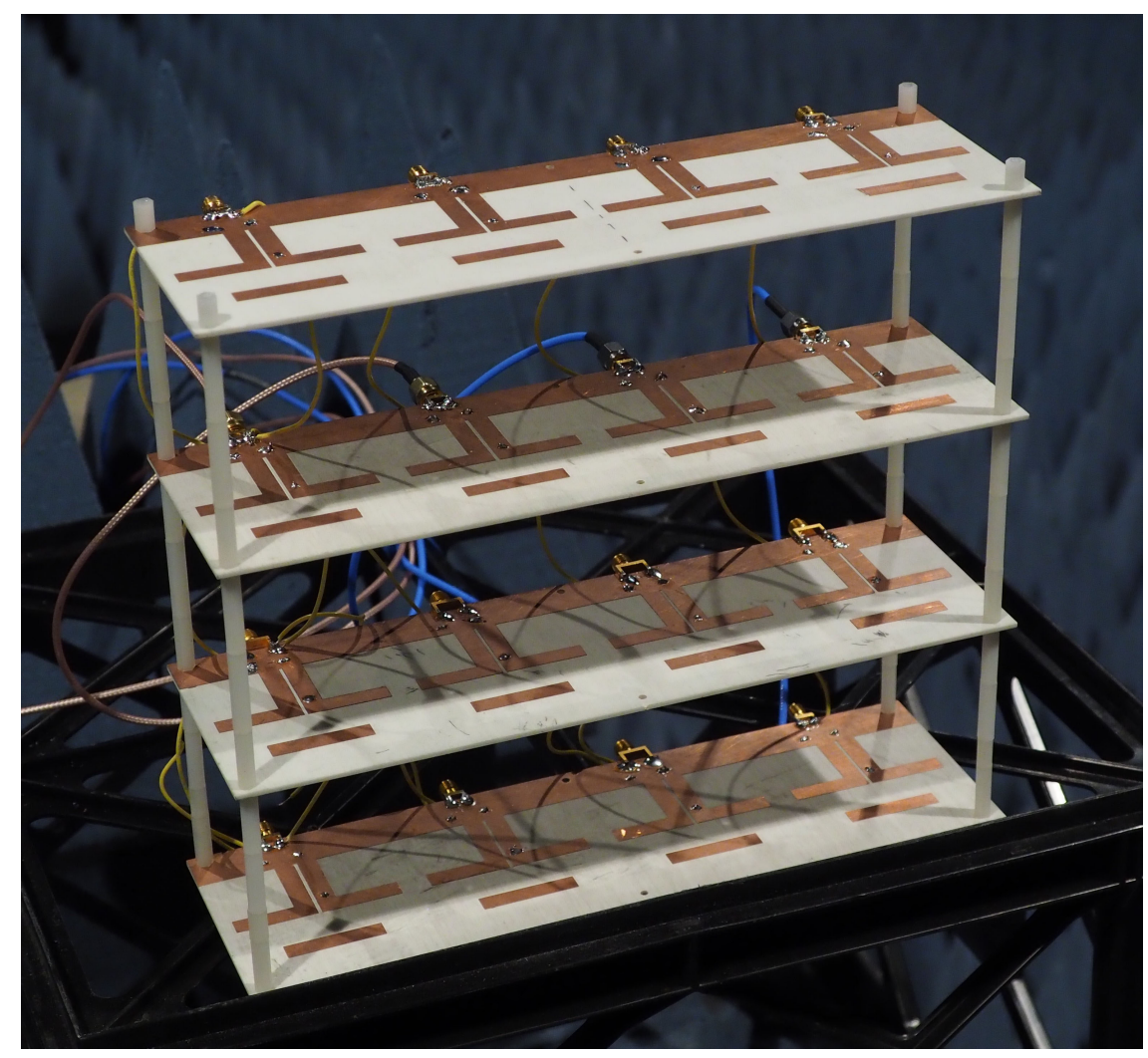

(a)

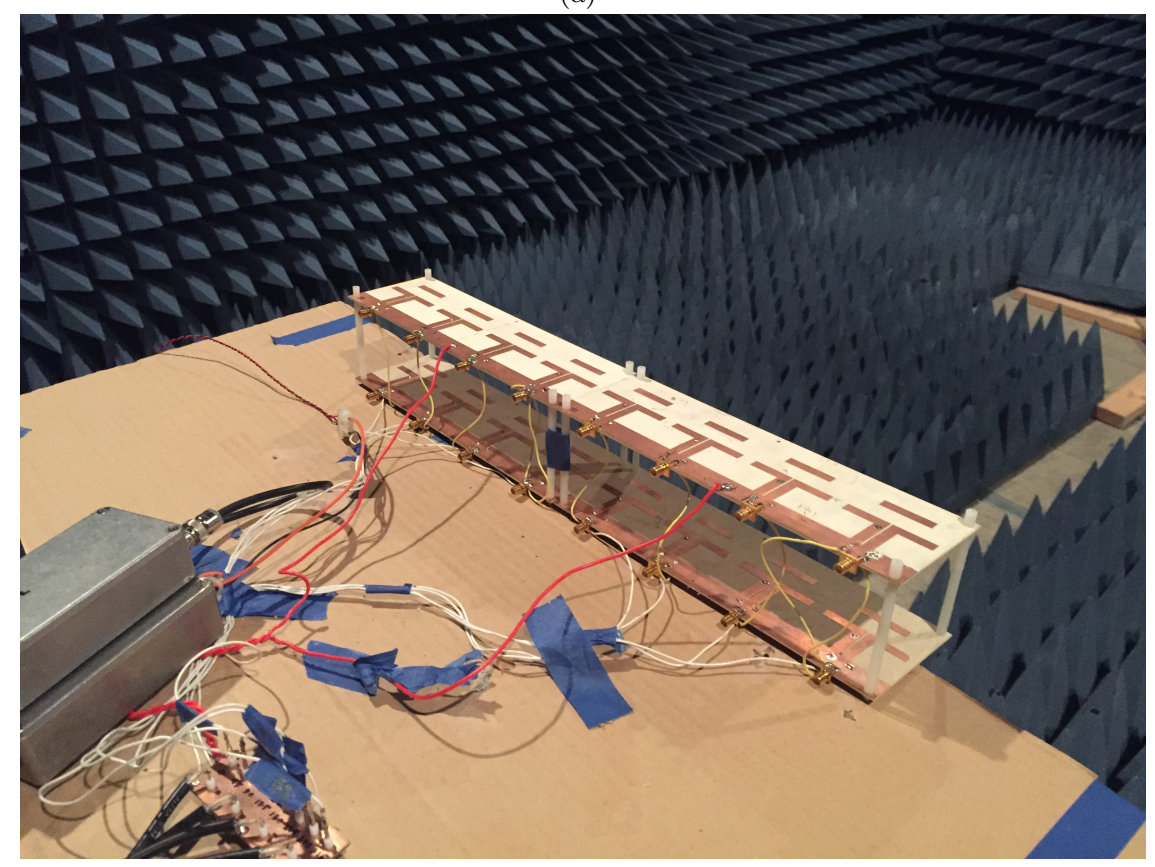

(b)

Figure 8.11: Continued 


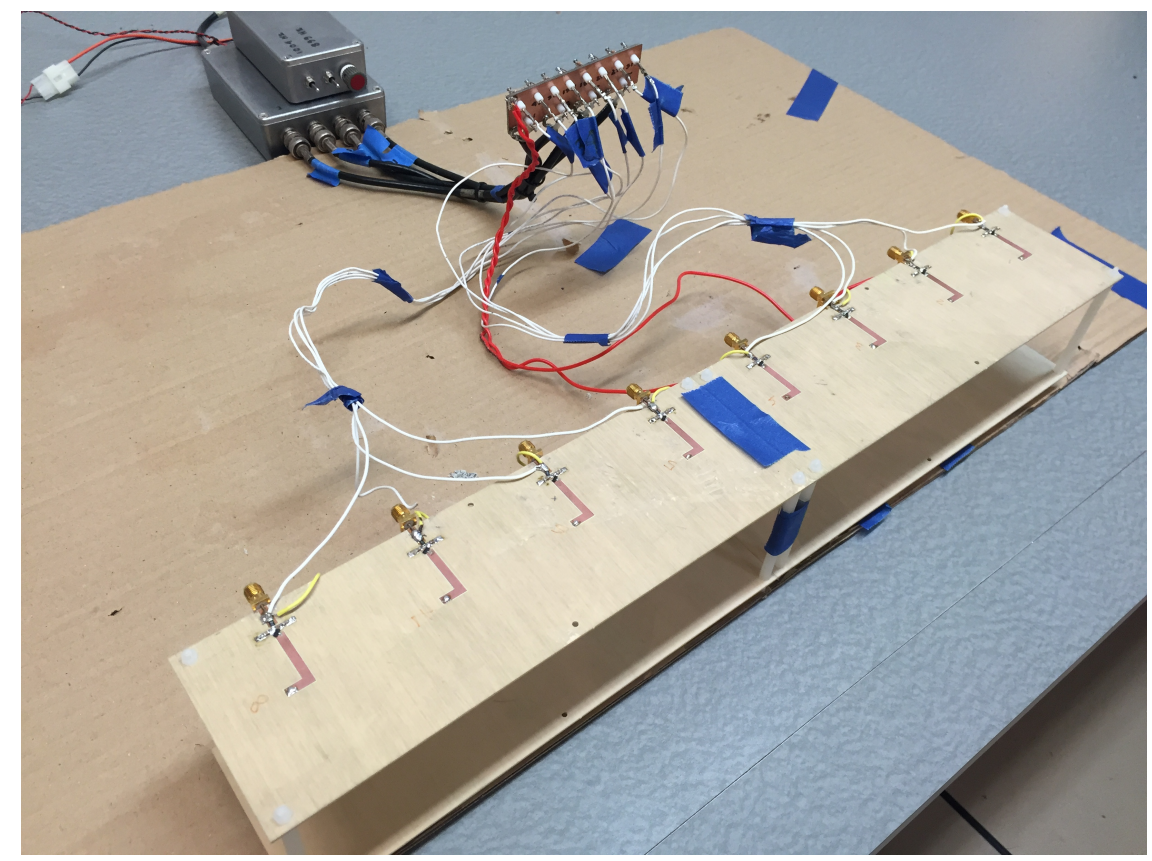

(c)

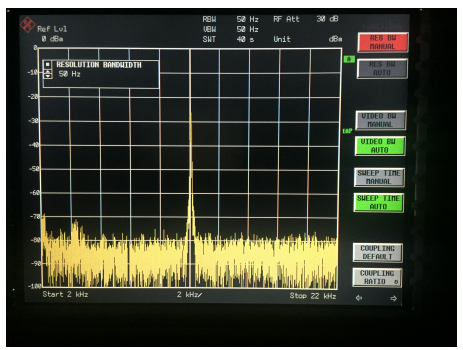

(d)

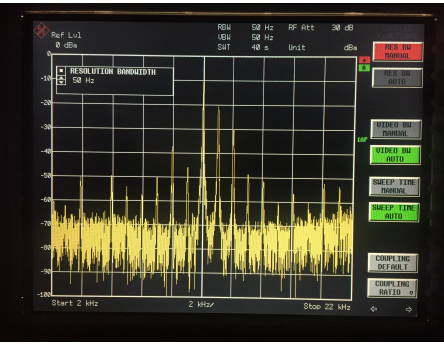

(e)

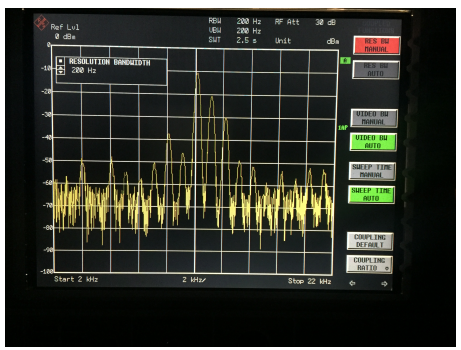

(f)

Figure 8.11: a) Photographs of the $4 \times 4$-element PMSA b) photographs of the $8 \times 2$-element PMSA (bottom view) c) Photographs of the $8 \times 2$-element PMSA (top view) d) received spectrum of scattered signals when the $8 \times 2$-element PMSA is OFF e) received spectrum of scattered signals when the $8 \times 2$-element PMSA is ON (higher bandwidth resolution) $\mathrm{f}$ ) received spectrum of scattered signals when the $8 \times 2$-element PMSA is ON (lower bandwidth resolution)

antennas is increased to $2.5 \mathrm{~m}$ to ensure working within the far-field of the new PMSA.

This new PMSA is tested six times (i.e., six rounds of measurements), and in each round, a different combination of incident angle and reference element is utilized. Moreover, according to Figure 8.11a, the first three combinations share the same direction of the progressive phase shift distribution of $\left(0^{\circ}, 90^{\circ}, 180^{\circ}, 270^{\circ}\right)$ from left to right as well as using the phase shift distribution of $\left(270^{\circ}, 180^{\circ}, 90^{\circ}, 0^{\circ}\right)$ for the last three combinations. In both cases of phase distributions, the illuminating 
antenna was located at angles $\left(+30^{\circ}, 0^{\circ},-30^{\circ}\right)$. For instance, when the illuminating antenna is located at $\theta_{o}=+30^{\circ}$, RF signals reach the PMSA columns at phases $\left(0^{\circ},-90^{\circ},-180^{\circ},-270^{\circ}\right)$ from left to right. Due to the phase inversion of RF signals during the modulation process as we demonstrated before, the retarded phases become advanced. In the first case, a relative phase shift difference of the 1USB distortion component is added to the reversed phases of RF signals induced over antennas, and it becomes $+90^{\circ}+90^{\circ}=+180^{\circ}$, leading to scattering this distortion component into a direction of $-90^{\circ}$ (off-axis). In contrast, a relative phase shift difference of the 1LSB distortion component is subtracted from the reversed phase shift of RF signals, and it becomes $+90^{\circ}-90^{\circ}=0^{\circ}$, leading to scattering this harmonic component into the broadside direction of $0^{\circ}$. However, in the second round, spatial locations of these two distortion components are exchanged. All remaining higher order distortion components which can potentially be treated by the $4 \times 4$-element PMSA follow the same procedure, but phases of the modulation signals are multiplied by a harmonic term number $n$. For example, the resulting phase of the 2LSB distortion component is $2 \times\left(-90^{\circ}\right)+90^{\circ}=-90^{\circ}$, so the PMSA directs this component to the direction of $+30^{\circ}$.

Figure 8.12 shows the measured scattered beams of distortion components obtained from all six rounds of measurements along with their simulated counterparts. Because the anechoic chamber is not wide enough to cover the whole FoV space of the PMSA, the receive antenna was moved from $\theta=-50^{\circ}$ to $\theta=+50^{\circ}$. From the figures, it is hard to tell exactly where maximum lobes of scattered beams are directed due to the several fluctuations in their responses. We believe that the main source of fluctuations is because the receive antenna is manually moved. In each movement, the distance between the PMSA and the receive antenna is probably increased or decreased. As we all know, the RF signal strength is inversely proportional to the 
distance between the receive antennas and DUTs, and thus certainly could lead to fluctuations in the signal strength.

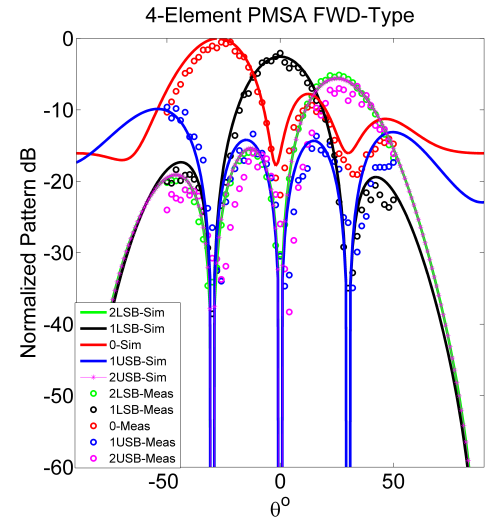

(a)

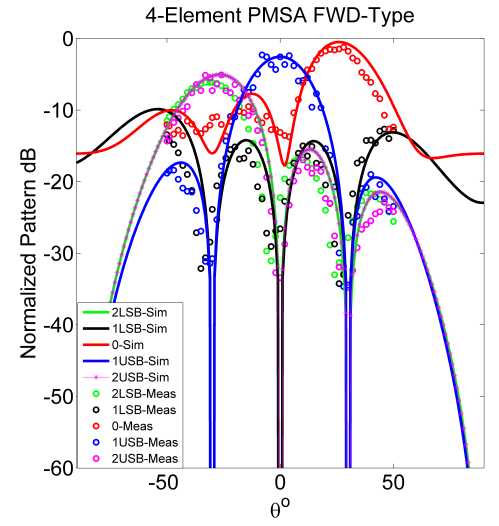

(c)

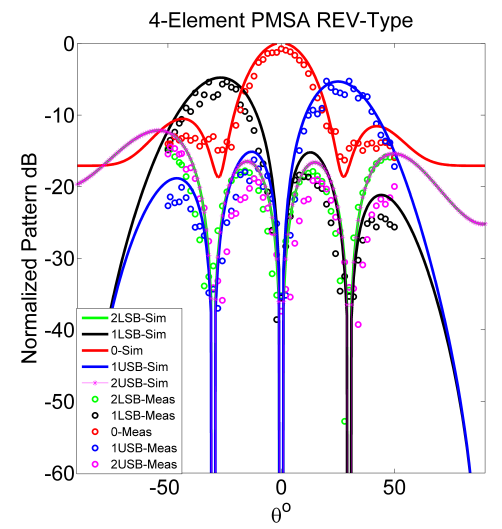

(e)

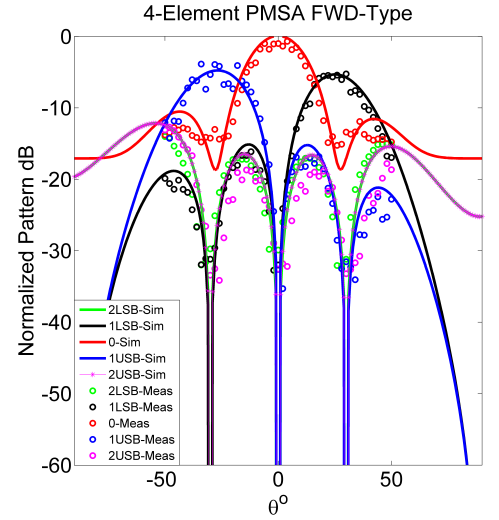

(b)

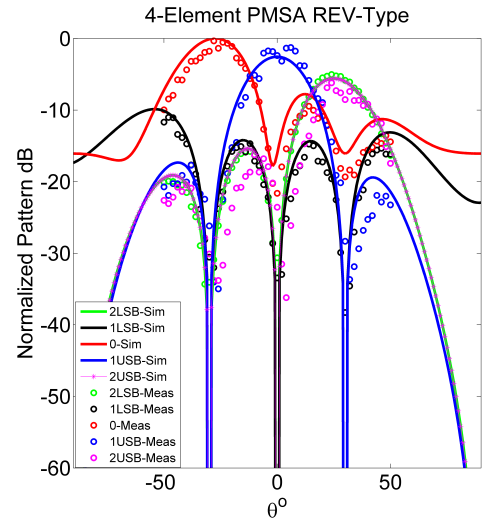

(d)

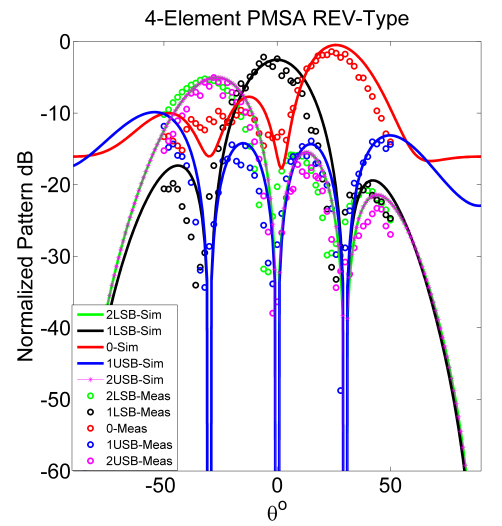

(f)

Figure 8.12: Scattered beams of distortion products generated by the $4 \times 4$-element PMSA from (solid-line simulated and dotted-line measured) a) 1st experimental round b) the 2 nd experimental round c) the 3rd experimental round d) the 4th experimental round e) the 5th experimental round f) the 6th experimental round 
From the first round of measurements, the maximum value of scattered beam of the 1LSB harmonic that is supposed to be at $\theta=0^{\circ}$ is found at $\theta=+2^{\circ}$ with an error of $1.1 \%$ as shown in Figure $8.12 \mathrm{a}$. The maximum value of scattered beam of the fundamental harmonic after subtracting the background impact is found at $\theta=-26^{\circ}$ instead of $\theta=-30^{\circ}$ with an error of $2.2 \%$, and the maximum value of scattered beam of the 2LSB harmonic is found at $\theta=+33^{\circ}$ instead of $\theta=+30^{\circ}$ with an error of $1.6 \%$. As can be seen, the scattered beam of the 1USB harmonic has no main scattered lobe since it is directed to reach $\theta=-90^{\circ}$ and because patterns of individual antennas do not radiate efficiently at this angle, the main lobe is attenuated. Furthermore, sidelobes of the 1LSB harmonic scattered beam are almost -14dB less than their main lobe, while the main lobe of the scattered 2LSB harmonic component is almost $-8 \mathrm{~dB}$ less than the main beam of the fundamental component because the beam is moved away from the broadside direction as well as being a higher order distortion product. For the same reason, the 2LSB harmonic scattered beam becomes wider. It is worth to note that the scattered beams of the 2LSB and 2LUSB distortion products are always directed to the same direction, obeying the non-canceled distortion products rule of the PMSA $=n+p \times L$, where $L=4, p=\ldots,-2,-1,0,1,2, \ldots$ and $n=-2$ or 2. Moreover, scattered beams can change their spatial locations if a direction of progressive phase shift distribution of the modulation sources or a direction of the incident signals is changed as can be seen in the rest of plots in Figure 8.12. Changing locations of scattered beams does confirm that the beamforming is an inherent property of our proposed PMSA.

As can be seen, when one of scattered beams appears at a specific spatial locations, the other beams do not disappear but have small amplitudes. Each spatial direction where one of scattered beams has a maximum value (main lobe) represent a fictitious output port according to Figure 3.13. As in RF conventional communications circuits, 
this fictitious output port passes the desired and unavoidable distortion products and rejects unwanted distortion products. However, unwanted distortion products are attenuated, but not completely canceled due to errors. The measured and simulated received spectrums of scattered signals (i.e., spatial responses of distortion products) are given in Figure 8.13 and 8.14 for the $4 \times 4$-element PMSA. Each row in plots represents one of the six rounds of measurements and measured data are taken at angles of $\theta=+30^{\circ}, 0^{\circ}$, and $-30^{\circ}$. For example, if we take a closer look at the measured results in the first row of Figure 8.13 and the last row of Figure 8.14, the 9LSB, 5LSB, 1LSB, 3USB, and 7USB harmonics are enhanced at an angle of $\theta=0^{\circ}$, and all others are highly attenuated. At an angle of $\theta=+30^{\circ}$, in the first row and at an angle of $\theta=-30^{\circ}$ in the last row, the 6LSB, 2LSB, 2USB, and 6USB harmonics are enhanced, and all others are highly attenuated too. Furthermore, the 8LSB, 4LSB, 0, 4USB, and 8USB harmonics are enhanced at angles of $\theta=-30^{\circ}$ and $\theta=+30^{\circ}$ in the first and last rows, respectively. Most of unwanted harmonics are attenuated by about 20dB. Figure 8.15 shows a comparison between the scattered harmonic responses of the $4 \times 4$-element PMSA and a single scatterer, taken at an angle $\theta=0^{\circ}$. The DPRRs of unwanted distortion products are more than $20 \mathrm{~dB}$ as given in Table 8.2 for all eighteen measurements shown above. Additionally, amplitudes of enhanced harmonics are almost increased by about $12 \mathrm{~dB}$ which is expected because of using an array of scatterers instead of single scatterers. According to Figure 3.13, received responses of the six experimental rounds of measurements, given in Figure 8.13 and 8.14, can be classified as spectral responses of A, F, B, C, E, and D circuits types given in Chapter 3.

The most important fact, that was fulfilled practically inferred from the measured results of the two and four elements PMSAs, is a number of distortion products controlled in each experiment. In other words, we validated practically 
that the more scatterers are used $(L)$, the larger number of distortion products are controlled.

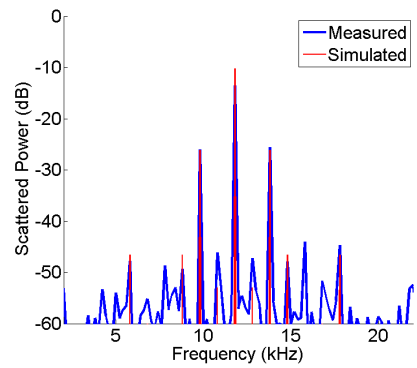

(a)

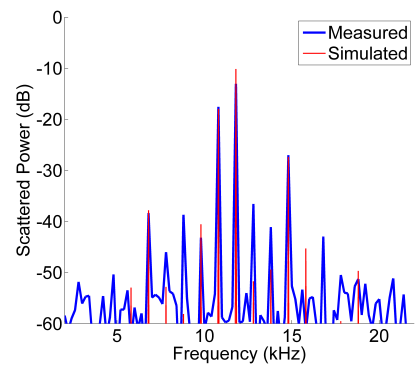

(d)

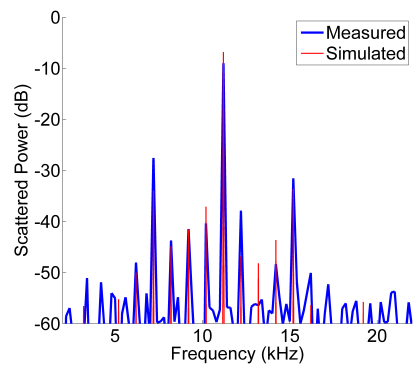

(g)

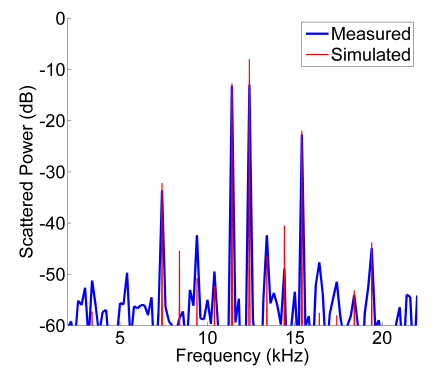

(b)

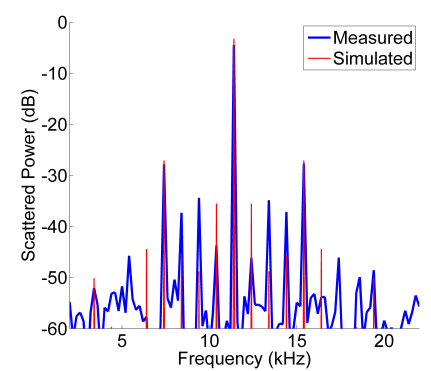

(e)

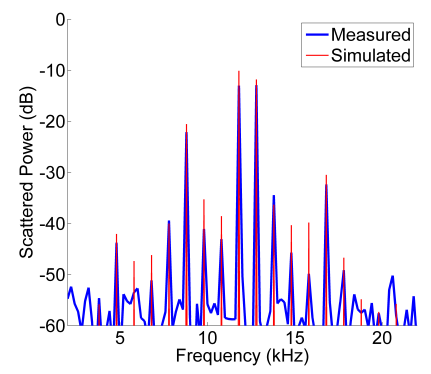

(h)

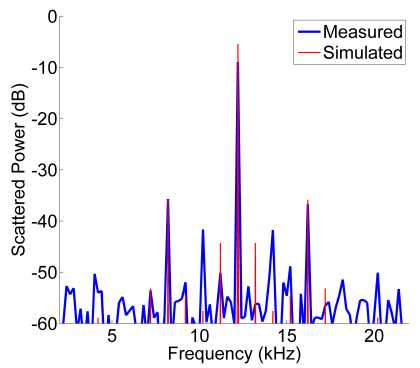

(c)

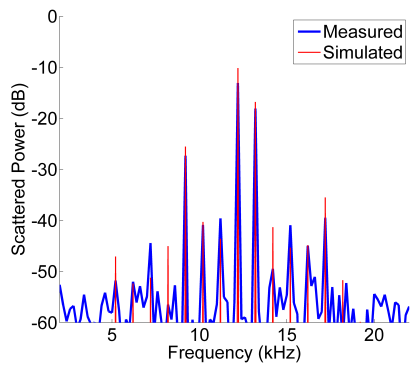

(f)

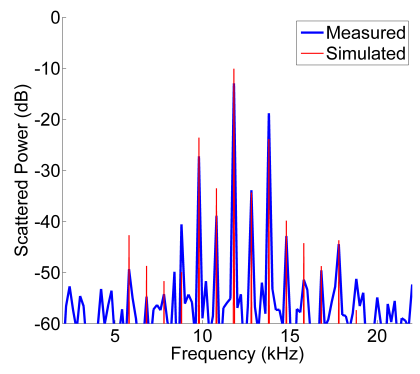

(i)

Figure 8.13: Measured and simulated spectrums of the $4 \times 4$-element PMSA when the first element on the left in Figure 8.11a considered as a reference for the direction of progressive phase shift distribution a) taken at $\theta=+30^{\circ}$ for $\theta_{o}=+30^{\circ}$ b) taken at $\theta=0^{\circ}$ for $\theta_{o}=+30^{\circ}$ c) taken at $\theta=-30^{\circ}$ for $\theta_{o}=+30^{\circ} \mathrm{d}$ ) taken at $\theta=+30^{\circ}$ for $\theta_{o}=0^{\circ}$ e) taken at $\theta=0^{\circ}$ for $\theta_{o}=0^{\circ} \mathrm{f}$ ) taken at $\theta=-30^{\circ}$ for $\theta_{o}=0^{\circ} \mathrm{g}$ ) taken at $\theta=+30^{\circ}$ for $\theta_{o}=-30^{\circ} \mathrm{h}$ ) taken at $\theta=0^{\circ}$ for $\theta_{o}=-30^{\circ}$ i)taken at $\theta=-30^{\circ}$ for $\theta_{o}=-30^{\circ}$

Next, the $8 \times 2$-element PMSA shown in Figure $8.11 \mathrm{~b}$ was performed several rounds with also different combinations of directions of progressive phase shift distributions of the modulation signals and angles of the illuminating sources. The scatterers are horizontally polarized. The whole PMSA consists of two sub-arrays 


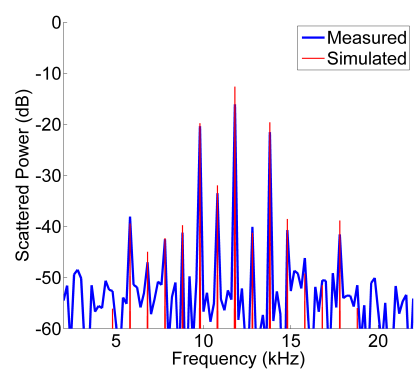

(a)

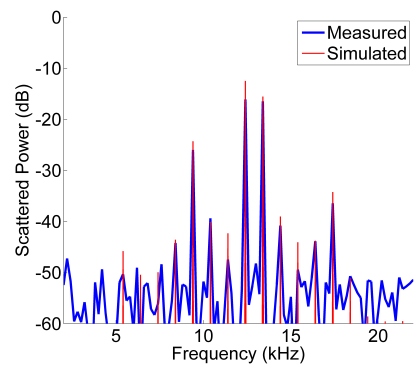

(d)

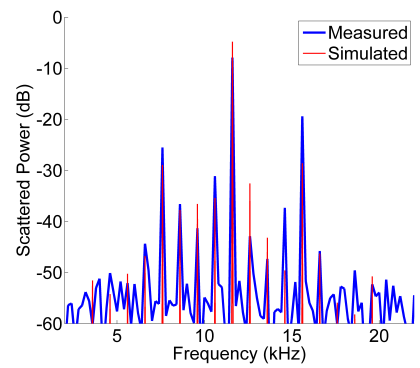

$(\mathrm{g})$

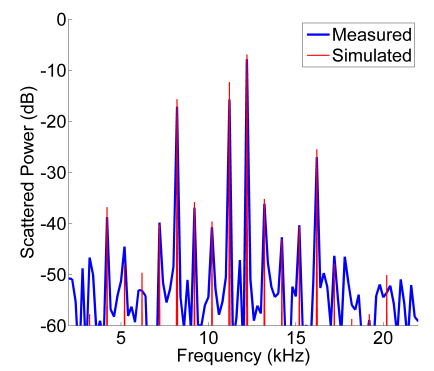

(b)

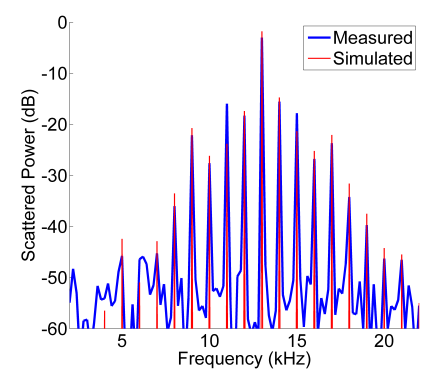

(e)

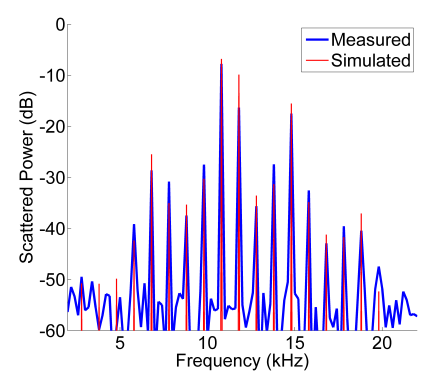

(h)

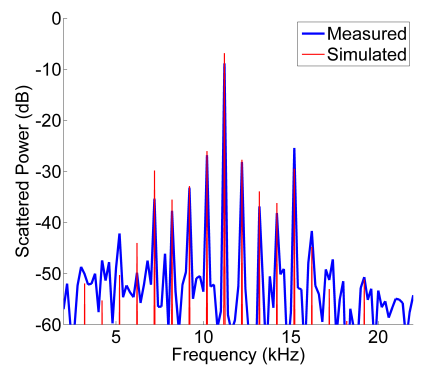

(c)

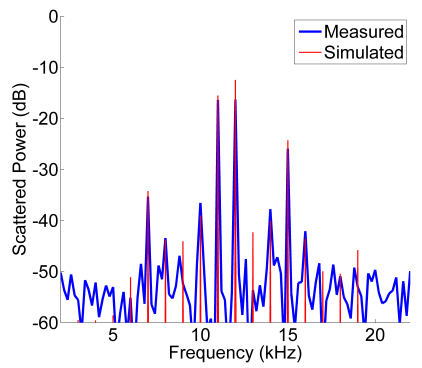

(f)

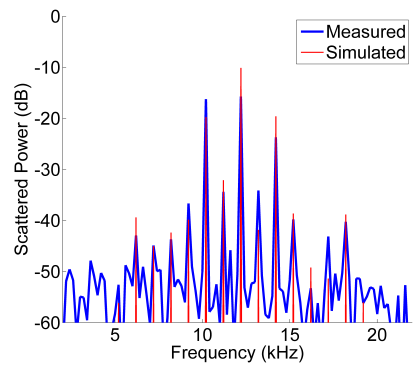

(i)

Figure 8.14: Measured and simulated spectrums of the $4 \times 4$-element PMSA when the first element on the right in Figure 8.11a considered as a reference for the direction of progressive phase shift distribution a) taken at $\theta=+30^{\circ}$ for $\theta_{o}=+30^{\circ}$ b) taken at $\theta=0^{\circ}$ for $\theta_{o}=+30^{\circ} \mathrm{c}$ ) taken at $\theta=-30^{\circ}$ for $\theta_{o}=+30^{\circ} \mathrm{d}$ ) taken at $\theta=+30^{\circ}$ for $\theta_{o}=0^{\circ} \mathrm{e}$ ) taken at $\theta=0^{\circ}$ for $\theta_{o}=0^{\circ} \mathrm{f}$ ) taken at $\theta=-30^{\circ}$ for $\theta_{o}=0^{\circ} \mathrm{g}$ ) taken at $\theta=+30^{\circ}$ for $\theta_{o}=-30^{\circ} \mathrm{h}$ ) taken at $\theta=0^{\circ}$ for $\theta_{o}=-30^{\circ}$ i)taken at $\theta=-30^{\circ}$ for $\theta_{o}=-30^{\circ}$

of $8 \times 1$-element PMSAs separating by a distance $d y=0.5 \lambda_{o}$. In addition, the distance between elements in each single sub-array is $d x=0.5 \lambda_{o}$. The relative phase shift difference between any two adjacent elements is $45^{\circ}$. Also here, the reference element could be either the right most element or the left most element. The phase distribution across the PMSA is $\left(0^{\circ}, 45^{\circ}, 90^{\circ}, 135^{\circ}, 180^{\circ}, 225^{\circ}, 270^{\circ}, 315^{\circ}\right)$, so the 4 -to- 8 phase transformer kit presented in Chapter 6 is used to realize equidistant phases for 
eight modulation signals. The kit is inserted between a 4-port modulation source and the $8 \times 2$-element PMSA (DUT). The distance between the DUT and the illuminating and receive antennas is increased again to be more than $3 \mathrm{~m}$ in order to achieve the measurements in the far-field region of the new PMSA.

In each round, the receive antenna is moved from $\theta=-45^{\circ}$ to $\theta=+45^{\circ}$ with a step of $2^{\circ}$. In the first round, the illuminating antenna is located at $\theta_{o}=+15^{\circ}$, so the incident RF signals arrive at the PMSA columns with a relative phase shift difference of $-45^{\circ}$. Because the PMSA here treats a higher number of distortion products than previous designs, scattered beams of upper and lower sidebands distortion products are presented in separate figures to avoid the difficulty of recognizing traces of scattered beams. Figure 8.16a and 8.16b exhibit a comparison between the simulated and measured scattered beams of the first four of LSB and USB distortion products, respectively. As can be observed, the measured scattered beams agree very well this time with the simulated scattered beams in terms of beamwidths and directions of the main beams of distortion products. All scattered beams are normalized to the maximum value of the scattered beam of the fundamental harmonic (main distortion product) component after subtracting background impacts from it in order to demonstrate that the scattered beams of high order distortion products have lower amplitudes. The scattered beam (specular) of the fundamental harmonic, un-modulated component, is plotted in both figures for the sake of comparison. The specular beam emerges at $\theta=-15^{\circ}$, the scattered beam of the 1LSB harmonic component appears at $\theta=+4^{\circ}$ with error $2.2 \%$, and the scattered beam of the 1USB harmonic component appears $\theta=-32^{\circ}$ with error of $1.1 \%$ compared to the a scattering array front of view (SfoV) which is equal to $180^{\circ}$. Directions of the other scattered beams can be extracted from plots of scattered beams. In addition, scattered beams located behind $\theta= \pm 45^{\circ}$ cannot be obtained due to the limitations 
of measurement facilities. The errors in directions of scattered beams and fluctuations in amplitudes of scattered beams in measured plots belong to a variety of factors such as errors in phases and amplitudes of the modulation signals, mutual coupling, etc. The other plots in Figure 8.16 represent the measured received spectrums of spatially scattered signals along with simulated results at angles of $\theta=-30^{\circ},-15^{\circ}, 0^{\circ},+15^{\circ}$, and $+30^{\circ}$. As can be seen, more distortion products are highly attenuated that, ideally, they should be canceled) and only the desired and unavoidable distortion products are enhanced at given locations. Although we added the errors in Tables (6.2) and (6.3) to the mathematical model, there are slight discrepancies due to the ignorance of some sources of errors. In Figure 8.17, we show the measured and simulated scattered beams of harmonics and the received spectrums of spatially scattered signals when the left most element of the PMSA in Figure 8.11b is considered as a reference element and RF illuminating signals are normally incident on the PMSA aperture. The worst scenario of measured DPRR for the $8 \times 2$-element PMSA is about $16 \mathrm{~dB}$

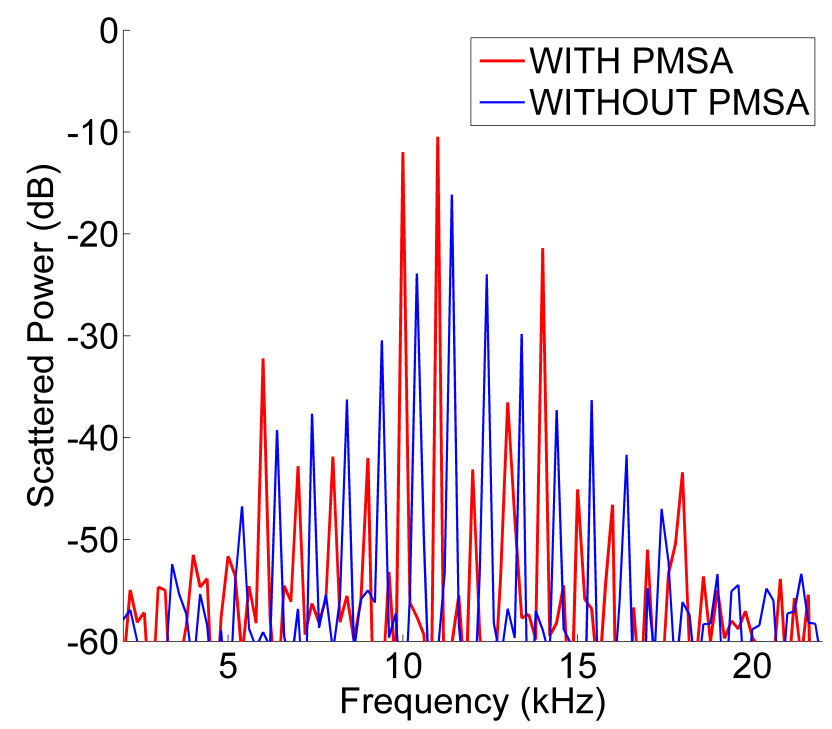

Figure 8.15: A comparison between the measured received spectrums of the $4 \times 4$-element PMSA and a single scatterer 
Table 8.2: DPRRn of distortion products obtained from the six experimental setups of the $4 \times$ 4-element PMSA shown in Figure 8.11a

\begin{tabular}{|c|c|c|c|c|c|c|c|c|}
\hline Round No. & Scattered angles & 3LSB & 2LSB & 1LSB & $\overline{0}$ & 1USB & 2USB & 3USB \\
\hline \multirow{3}{*}{1} & -30 & 26 & 28.5 & 37 & NA & 31 & 26 & 27 \\
\hline & 0 & 25 & 37 & NA & NA & 37.5 & 36 & NA \\
\hline & 30 & 25 & NA & 28 & NA & 26.5 & NA & 27 \\
\hline \multirow{3}{*}{2} & -30 & NA & 26 & 26.5 & NA & $\mathrm{NA}$ & 28 & 26 \\
\hline & 0 & 29 & 27 & 31 & NA & 32 & 26 & 25 \\
\hline & 30 & 28 & NA & 32 & NA & 29 & 26 & NA \\
\hline \multirow{3}{*}{3} & -30 & 31 & $\mathrm{NA}$ & 29 & $\mathrm{NA}$ & 29 & $\mathrm{NA}$ & 30 \\
\hline & 0 & NA & 24 & 27 & NA & NA & 20 & 27 \\
\hline & 26 & 26.5 & 33 & 32 & NA & 31 & 27 & 28 \\
\hline \multirow{3}{*}{4} & -30 & -33 & 26 & 24 & NA & 33.5 & 26.5 & 28 \\
\hline & 0 & NA & 26 & 26 & NA & NA & 23 & 28 \\
\hline & 30 & 30 & NA & 25 & NA & 25 & NA & 33 \\
\hline \multirow{3}{*}{5} & -30 & 34 & 30 & 21 & $\mathrm{NA}$ & $\mathrm{NA}$ & 29 & 36 \\
\hline & 0 & 32 & 29 & 22 & NA & 21.5 & 29 & 31 \\
\hline & 30 & NA & 24 & 33 & NA & NA & 20 & 31 \\
\hline \multirow{3}{*}{6} & -30 & 24 & $\mathrm{NA}$ & 25 & $\mathrm{NA}$ & 26 & $\mathrm{NA}$ & 24 \\
\hline & 0 & 24 & 21 & NA & NA & 30 & 27 & NA \\
\hline & 30 & 34 & 31 & 24 & NA & 25 & 30 & 32 \\
\hline
\end{tabular}

\subsubsection{Two-tone Experimental Results}

The earlier experimental results obtained above using various structures of the PMSA have showed that distortion products which were only harmonic products (HDs) generated by PMSAs were treated in space (i.e., enhancement and cancellation) rather than using conventional means such as transmission lines as inside conventional RF communication circuits. This opens the door to practically prove that not only harmonic products but also intermodulation distortion products (IMDs) can be treated in space. Thus, a generation of IMDs requires driving scatterers by modulation signals having at least two tones. These tones should be added linearly before the modulation process. To fulfill this requirement, we used a modulation source with four ports given in Figure 6.18b. Moreover, the mathematical models presented earlier in this research work have proved that the proposed PMSA is able to deal with modulation signals having an arbitrary number of tones, but the measurements should be carried out to validate these claims. In addition, the encouraging results obtained earlier in this chapter motivate us to do so. Thus, a 


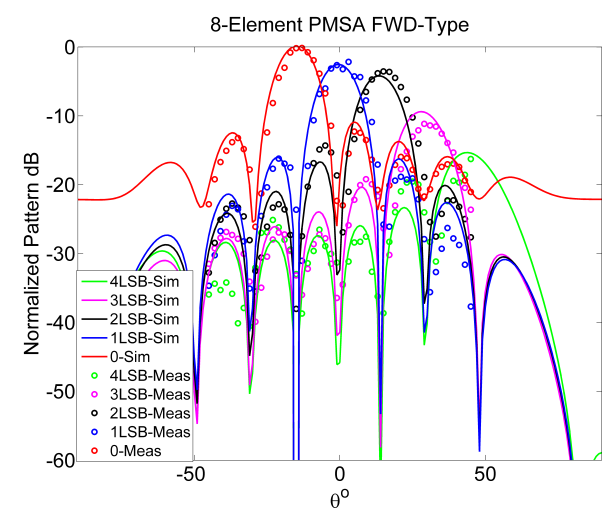

(a)

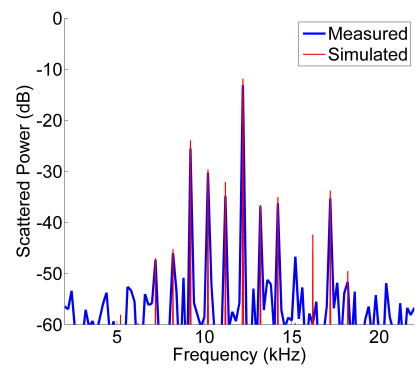

(c)

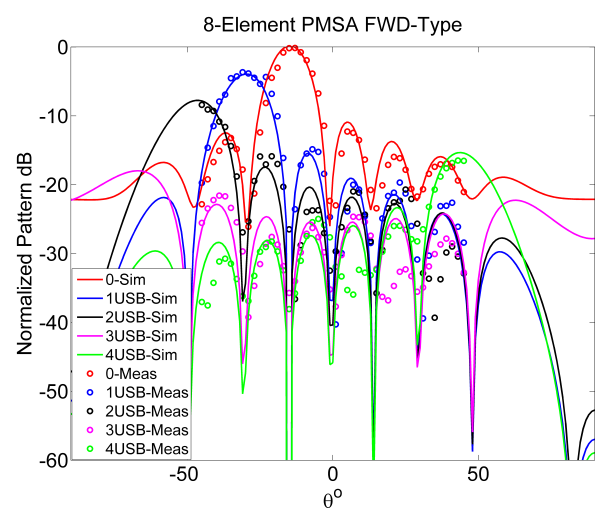

(b)

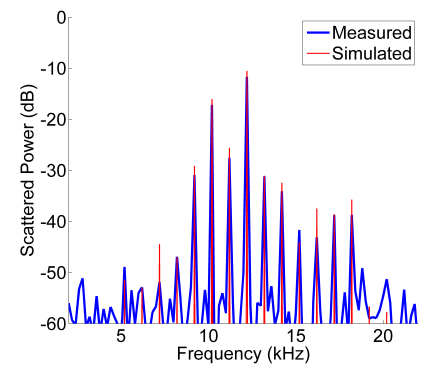

(d)

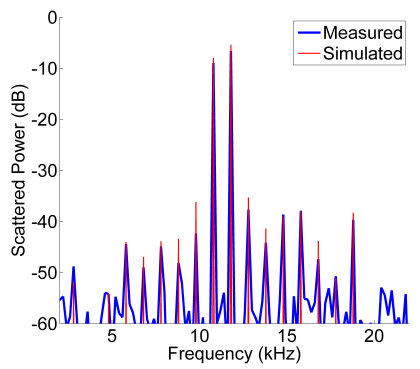

(e)

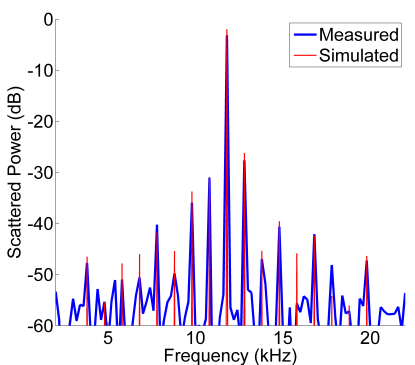

(f)

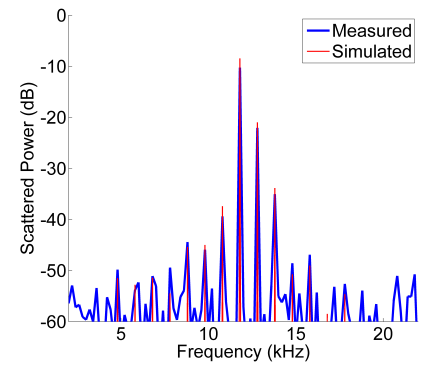

(g)

Figure 8.16: Measured and simulated scattered beams and spectrums of the $8 \times 2$-element PMSA when the first element on the right in Figure $8.11 \mathrm{~b}$ considered as a reference for the direction of progressive phase shift of modulation signals and the illuminating angle $\theta_{o}=+15^{\circ}$ a) scattered beams of LSB distortion products (solid-line simulated and dotted-line measured) b) scattered beams of USB distortion products (solid-line simulated and dotted-line measured) c) received spectrum taken at $\theta=+30^{\circ} \mathrm{d}$ ) received spectrum taken at $\theta=+15^{\circ}$ e) received spectrum taken at $\theta=0^{\circ} \mathrm{f}$ ) received spectrum taken at $\theta=-15^{\circ} \mathrm{g}$ ) received spectrum taken at $\theta=-30^{\circ}$

two-tone modulation source with four ports is utilized with frequencies of $899 \mathrm{~Hz}$ and $1004 \mathrm{~Hz}$. Both tones have identical phases. The measured peak to peak voltages of the first and second tones are 3.38v and 2.96v, respectively, as shown in Figure 6.18. The same PMSAs working at a frequency of $2.3 \mathrm{GHz}$ with sizes of $2 \times 14 \times 4$ and 


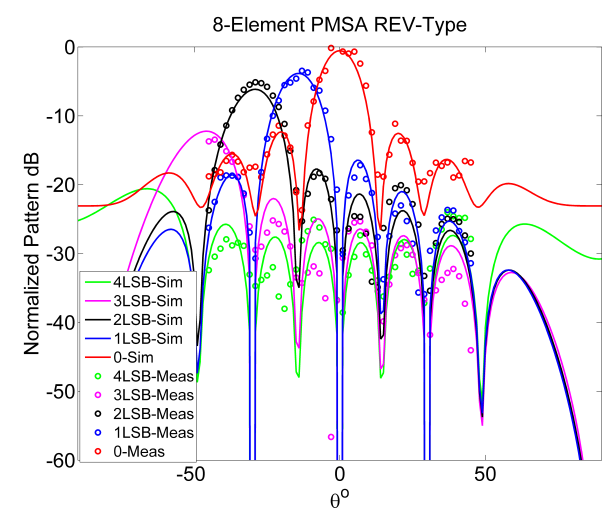

(a)

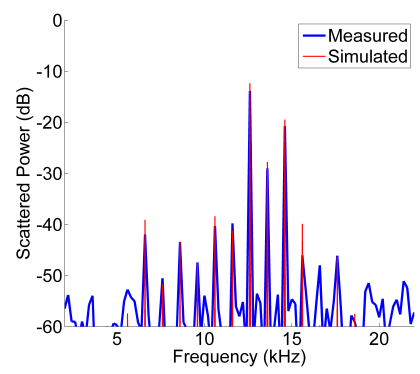

(c)

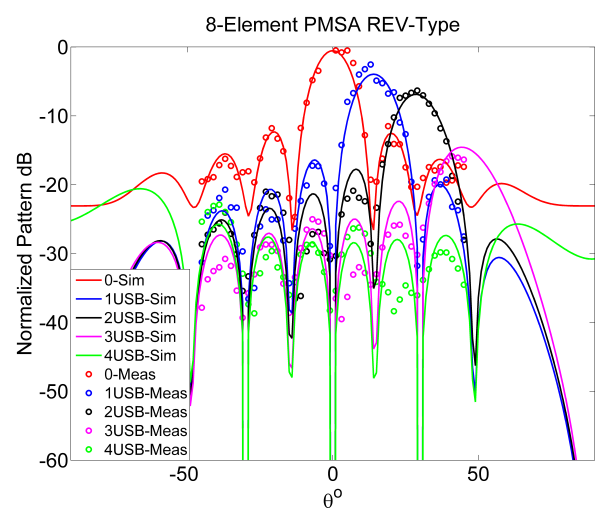

(b)

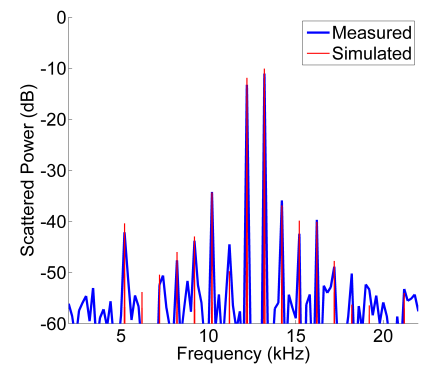

(d)

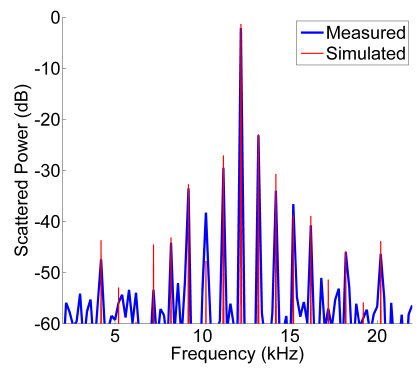

(e)

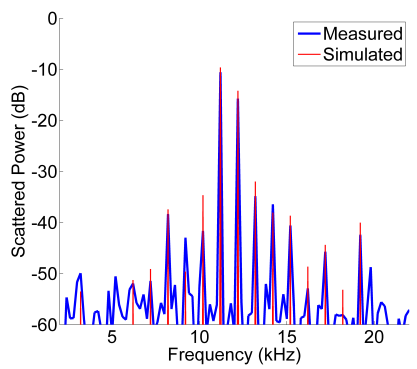

(f)

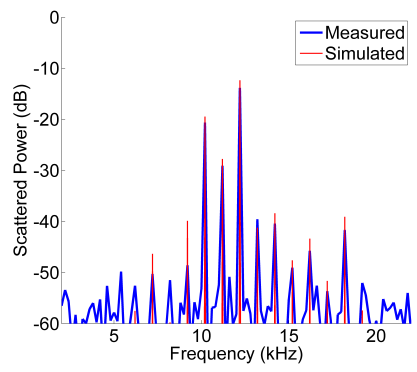

$(\mathrm{g})$

Figure 8.17: Measured and simulated scattered beams and spectrums of the $8 \times 2$-element PMSA when the first element on the left in Figure $8.11 \mathrm{~b}$ considered as a reference for the direction of progressive phase shift and the illuminating angle $\theta_{o}=0^{\circ}$ a) scattered beams of LSB distortion products (solid-line simulated and dotted-line measured) b) scattered beams of USB distortion products (solid-line simulated and dotted-line measured)c) received spectrum taken at $\theta=+30^{\circ}$ d) received spectrum taken at $\theta=+15^{\circ}$ e) received spectrum taken at $\theta=0^{\circ} \mathrm{f}$ ) received spectrum taken at $\theta=-15^{\circ} \mathrm{g}$ ) received spectrum taken at $\theta=-30^{\circ}$

$8 \times 2$ elements which were previously used are performed here again, but results of $2 \times 1$-element and $8 \times 2$-element PMSAs are only presented for the sake of brevity.

First, a $2 \times 1$-element PMSA is tested using the same experimental setup shown in Figure 8.7. A distance $d$ separating elements is $0.7 \lambda_{o}$ and the illuminating antenna 
is placed at an angle of $\theta_{o}=20^{\circ}$ to realize $90^{\circ}$ phase shift difference between $\mathrm{RF}$ signals induced over the two scatterers. Two-tone modulation signals are applied to inputs of scatterers with also $90^{\circ}$ phase shift difference. A response-like of a two-tone USB image reject mixer is obtained at an angle of $\theta=0^{\circ}$ when the right scatterer in Figure 8.7 is a reference, while a response-like of a two-tone LSB image reject mixer is obtained at an angle of $\theta=0^{\circ}$ when the left scatterer becomes a reference. The measured received responses are again down-converted to the frequency band $(2-22 \mathrm{kHz})$ and centered at the frequency $12 \mathrm{kHz}$ which represents the RF illuminating main frequency after the down-conversion. Here, we increased the frequency resolution (FFT size) more than what it was in single-tone experimental measurements in order to avoid missing any details of distortion components. Figure 8.18 shows both of the measured responses of the two-tone image reject mixers as well as the measured response of a $2 \times 1$-element PMSA when $d$ is increased to $\lambda_{o}$ and the illuminating antenna is located at an angle of $\theta_{o}=30^{\circ}$ to realize the two-tone spatial balanced modulator. With these new experimental properties, the phase shift difference between scatterers becomes $180^{\circ}$, and a response-like of the two-tone spatial balanced modulator is acquired at an angle of $\theta=0^{\circ}$ as shown in Figure 8.18c. The difference between received signals obtained from the same $2 \times 1$-element PMSA in Figure 8.8 and 8.18 is that the measured results in the former figure has only HDs distributed around the fundamental frequency while being HDs and IMDs in the latter figure. It is clear from Chapter 3 that when using two-tone modulation signals, there will be HDs and IMDs distributed about the main frequency. In both figure, we tried to remove completely either the 1USB or the 1LSB distortion products relying on a direction of progressive phase shift of the modulation signals, arrival angles of the incident signals, and a location of the receive antenna, but all these removals of undesired distortion products are not entirely carried out because errors in phases 


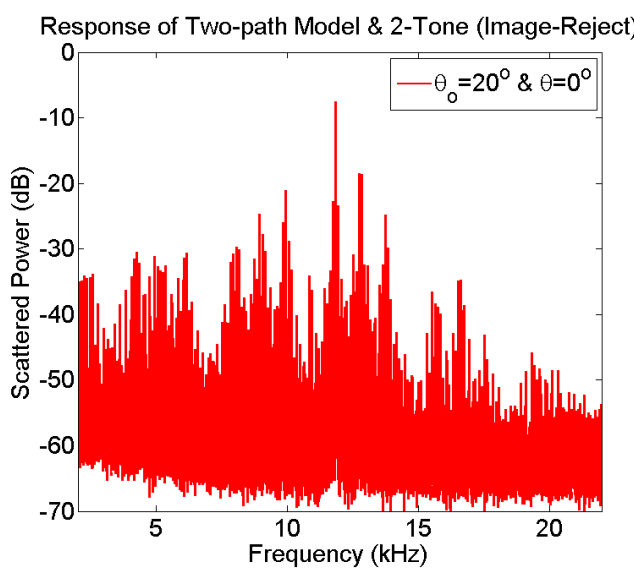

(a)

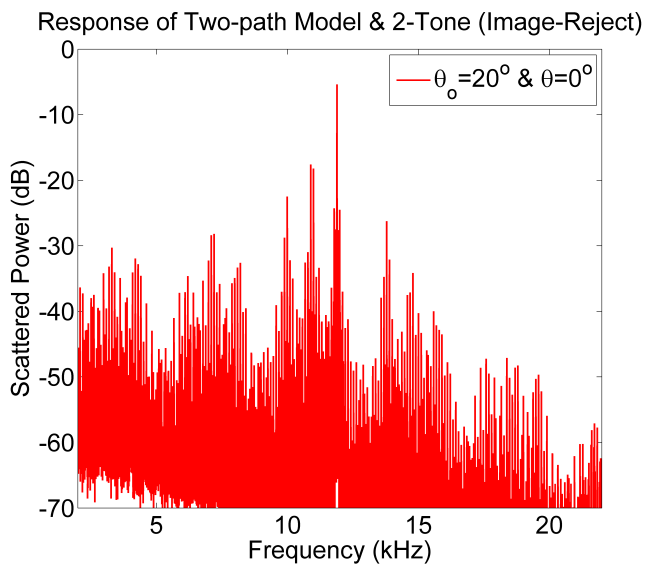

(b)

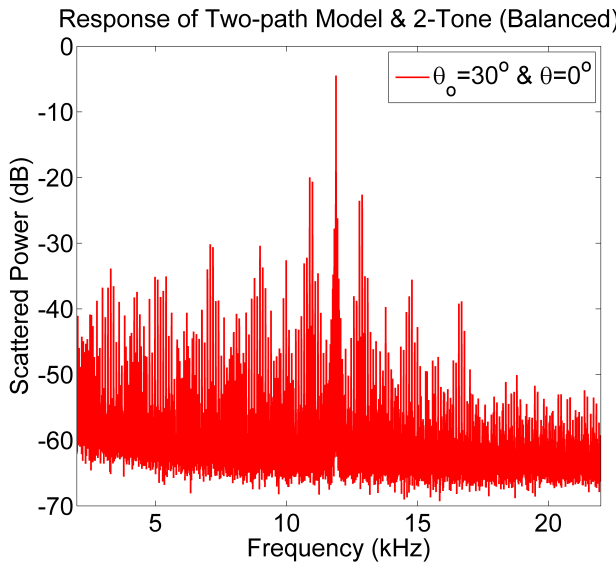

(c)

Figure 8.18: Measured responses taken at an angle of $\theta=0^{0}$ when a $2 \times 1$-element PMSA beahves like a) a two-tone USB image reject mixer b) a two-tone LSB image reject mixer c) a balanced modulator

and amplitudes of modulation signals, mutual coupling, fabrication errors, as well as logistic issues. The most important thing that we should emphasize it is that IMDs standing close to harmonics of adjacent two tones will not be canceled if harmonics are not canceled because the two tones of modulation signals have the same phases at each scatterer. This is practically validated in the measurements and theoretically demonstrated in Chapter 3.

Proceeding with a large-size PMSA, the $8 \times 2$-element PMSA is utilized here again to practically validate that we can handle a large number of IMDs in 
space. The 4-to- 8 phase transformer kit is inserted between the two-tone four-port modulation source and the DUT (i.e., $8 \times 2$-element PMSA). The distance between the transmit-receive antennas on one side and the DUT on the other side is $3.5 \mathrm{~m}$. HDs and IMDs are non-coherent (i.e., have different frequencies) and each single IMD or HD component has its particular scattered beam as will be seen later. As a consequence of using two-tone modulation signals, there will be a huge number of HDs and IMDs components, so that scattered beams of very few of them will be considered. However, received spectrums of scattered signals display all existing distortion products. IMDs generated by scatterers are given by $\left(w_{o} \pm k w_{1} \pm m w_{2}\right)$ where $w_{o}$ is the illuminating frequency, $w_{1}$ is the first-tone frequency, $w_{2}$ is the second-tone frequency, and $k$ and $m$ are integers. Because frequencies of two tones are not far apart only $105 \mathrm{~Hz}$ band in between, most IMDs will be closely distributed on both sides of each two adjacent harmonics of two tones. Every two adjacent harmonics are surrounded by a group of IMDs as will be shown in the measurement results.

Moreover, because two tones of the modulation signals applied to scatterers have the same phase shifts, phase shifts of IMDs will be equal to phases of accompanied harmonics as given by $\left( \pm k \emptyset_{1} \pm m \emptyset_{2}\right)$. For more details, one can be referred to the two-tone analyses in Chapters 3 and 4 . As a result, the HDs and IMDs which have the same phase shifts will have the same spatial directions. Thus, suppressing or enhancing of IMDs can be analyzed in the same means as the suppressing or enhancing of HDs.

Every single output from the phase transformer kit feeds a single column in the $8 \times 2$-element PMSA, and thus the PMSA can spatially distribute HDs and IMDs generated by it only in the azimuth plane. The relative phase shift for each single tone is $+45^{\circ}$. Firstly, the right most element in Figure $8.11 \mathrm{~b}$ is considered as a reference and the $2.3 \mathrm{GHz}$ illuminating antenna is located at $\theta_{o}=15^{\circ}$ with 
respect to the PMSA. The incident signals induced over PMSA elements have $-45^{\circ}$ relative phase shift which is then flipped due to the processes of RTS reflections. Figure 8.19 shows measured results of experimental setup taken at angles of $\theta=0^{\circ}$ and $\theta=-45^{\circ},-30^{\circ},-15^{\circ}, 0^{\circ}, 15^{\circ}, 30^{\circ}, 45^{\circ}$ for a single scatterer and a $8 \times 2$-element PMSA, respectively. As can be seen at an angle of $\theta=0^{\circ}$ in Figure $8.19 \mathrm{e}$, the LSB distortion products of the two tones are enhanced while all other components compared to the measured results of a single scatterer in Figure 8.19a are highly attenuated up to the 9LSB and 7USB distortion products which are enhanced too according to the mathematical formula $k+m=p \times L-1$, where $k+m=-1, L=8$ and $p=-1,0$ and 1 for the 9LSB, 1LSB, and 7USB distortion products, respectively.

It is worth noting that all IMDs with $k+m=-1$, 1LSB like $(-1+0=0-1=$ $-2+1=1-2=-3+2=2-3=-4+3=3-4=\cdots$, etc) are enhanced because they have the same phases as we mentioned before. The measured DPRRs of the 1USB of the two tones are about $26 \mathrm{~dB}$, the measured DPRR of the $I M D_{p} 21$ component is about $24 \mathrm{~dB}$, the measured DPRR of the $I M D_{p} 12$ component is about $25 \mathrm{~dB}$, the measured DPRR of the $I M D_{p} 32$ component is $23 \mathrm{~dB}$, and the measured DPRR of the $I M D_{p} 23$ component is about $16 \mathrm{~dB}$. Theoretically, all DPRRs should be equal to infinite, but because of phase and amplitude errors of the modulations signals given in Tables 6.2 and 6.3, the measured DPRRs have finite values. When moving the receive antenna to $\theta=-30^{\circ}$ as shown in Figure $8.19 \mathrm{c}$, the 1 USBs of the two tones and all associated IMDs such as $(k+m=1=2-1=-1+2=3-2=$ $-2+3=\cdots$, etc) are enhanced while all other distortion components compared to the measured results of a single scatterer in Figure 8.19a are highly attenuated up to the 7LSB and the 9USB distortion products which are enhanced too according to the mathematical formula $k+m=p \times L+1$, where $k+m=1, L=8$ and $p=-1,0$ and 1 for the 7LSB, 1USB, and 9USB distortion products, respectively. The measured 


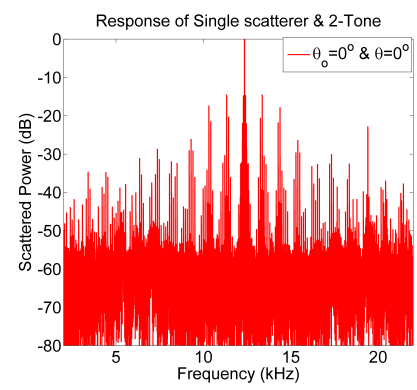

(a)

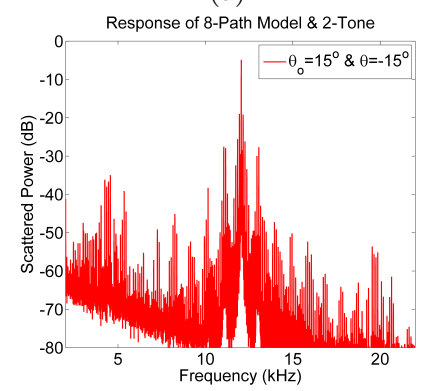

(d)

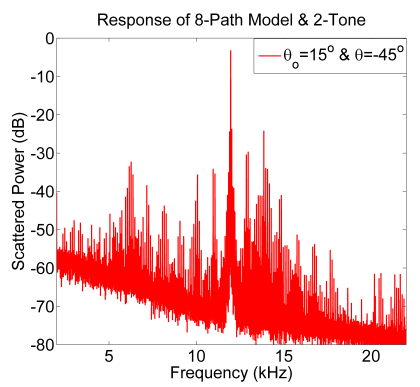

(b)

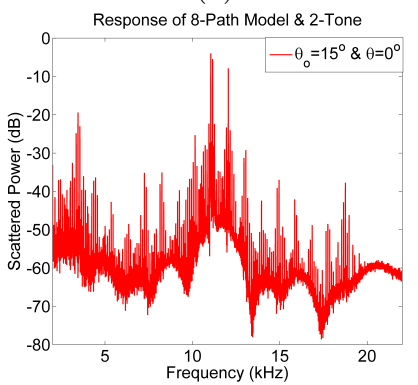

(e)

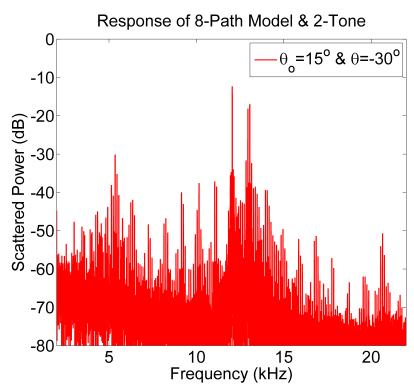

(c)

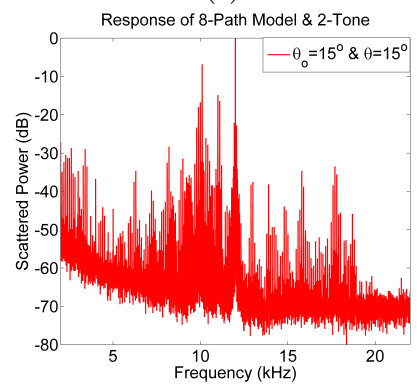

(f)

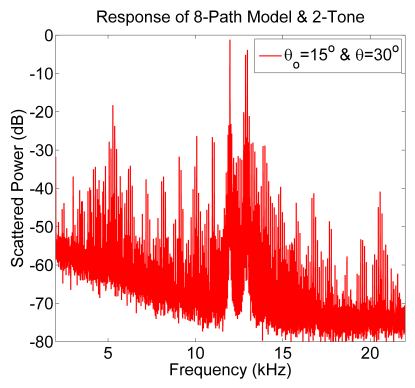

(g)

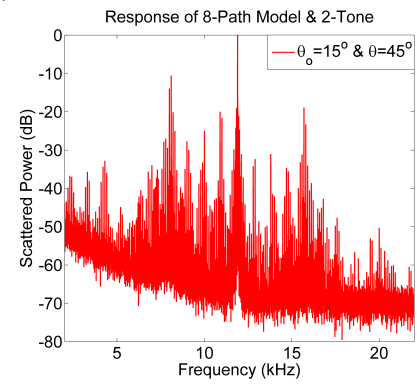

(h)

Figure 8.19: Measured spectrums of a single scatterer and a $8 \times 2$-element PMSA, when the first element on the right in Figure 8.11b considered as a reference for the direction of progressive phase shifts distributions of the two-tone modulation signals and the illuminating signal source located at $\theta_{o}=+15^{\circ}$, taken at a) a single scatterer $\theta=0^{\circ}$ b) $\theta=-45^{\circ}$ c) $\theta=-30^{\circ}$ d) $\theta=-15^{\circ}$ e) $\theta=0^{\circ}$ f) $\theta=+15^{\circ}$ g) $\theta=+30^{\circ}$ h) $\theta=+45^{\circ}$

DPRRs of the 1LSB of the two tones are about $23 \mathrm{~dB}$, the measured DPRR of the $I M D_{n} 21$ component is $28 \mathrm{~dB}$, the measured DPRR of the $I M D_{n} 12$ component is about $28 \mathrm{~dB}$ too, the measured DPRR of the $I M D_{n} 32$ component is about $18 \mathrm{~dB}$, and the measured DPRR of the $I M D_{n} 23$ component is about $16 \mathrm{~dB}$. In Figure $8.19 \mathrm{~b}$ at $\theta=-45^{\circ}$, the 2USB distortion products of the two tones and all associated IMDs such as $(k+m=2=4-2=-2+4=6-4=-4+6=\cdots$, etc $)$ are enhanced while all other components compared to the measured results of a single scatterer in 
Figure 8.19a are highly attenuated up to the 6LSB and 10USB distortion products which are enhanced too according to the mathematical formula $k+m=p \times L+2$, where $k+m=2, L=8$ and $p=-1,0$ and 1 for the 6LSB, 2USB, and 10USB distortion products, respectively. The same explanations can be applied to the rest of the plots in Figure 8.19.

Because there are a huge number of distortion products, it will not be an easy task to plot all their scattered beams. Thus, we select only four IMDs in each side of the main frequency. The selected IMDs are distributed evenly about the first LSB and USB harmonics, and they are given by $(k+m=1$ or -1$)$. All scattered beams of other IMDs can follow their accompanied harmonics in the same rule $(k+m=n$ or $-n$ ) but here $|n|$ is larger than one. Figure 8.20 shows scattered beams of the selected 1LSB and 1USB HDs and IMDs for three different rounds. The first round is when the first right scatterer element in Figure $8.11 \mathrm{~b}$ is considered as a reference, and the illuminating antenna is located at an angle of $\theta_{o}=15^{\circ}$. The second round is when considering the same reference element, and the illuminating antenna is located at an angle of $\theta_{o}=0^{\circ}$. The third round is when the first left scatterer element in Figure $8.11 \mathrm{~b}$ is considered as a reference, and the illuminating antenna is located at an angle of $\theta_{o}=15^{\circ}$. The scattered beams of the USB and LSB distortion products are separated in different plots for the sake of clarity in displaying findings. A scattered beam of the main fundamental frequency component is plotted in all combinations of figures to facilitate the comparison.

Also, there is an important reason for choosing only the IMDs components surrounding the first LSB and USB harmonics of the two tones which is impacts of these IMDs components on performances of receivers in conventional communication systems. Thus, discussing their spatial behaviors is substantial. These IMDs cannot be separated or attenuated by filters unless the main components are attenuated too. 
As can be deduced from the recent figure, HDs and their associated IMDs are added in-phase at the same locations. However, spatial direction of HDs and their associated IMDs can be different if phase of any tone deviates from its given value, resulting in less impacts on the receiver performance. Figure 8.21 shows measured responses of the third round. As can be observed, IMDs change their spatial directions in space once we replace the reference element.

\subsection{Parametric Investigations}

Changing spatial locations of enhanced and suppressed distortion products is one of the interesting properties of our presented work. As we mentioned before that there are several factors governing spatial locations of distortion products, we choose only $\theta_{o}$ and $d$ to carry out tasks of parametric investigations because it is easy to vary them during experimental operations. Another parameter which is a relative phase shift difference of the modulations signals is not selected because of the apparatus limitations (i.e., modulation sources have fixed properties such as frequency and phase). These two parameters subject to change for each single round of experimental measurements in order to demonstrate the flexibility of the PMSA.

However, the PMSAs working at $2.3 \mathrm{GHz}$ presented above are built on the same substrates and they have fixed distances between elements, so that we built another array which has eight separate scatterers to give us the freedom to choose different values for $d$. The parameter $d$ should be in the range of allowed values which is mostly between $0.4 \lambda_{o}$ and $\lambda$. Figure 8.22 shows the new PMSA consisting of eight elements fabricated on different boards using the same substrate which is the Rogers $4003 \mathrm{c}$ with thickness $1.52 \mathrm{~mm}$, a dielectric constant 3.55 , and a loss tangent 0.0021 . As seen in the earlier experiments, directions of scattered beams depend critically upon directions of the incident signals, but in all previous experiments, these directions of the incident 


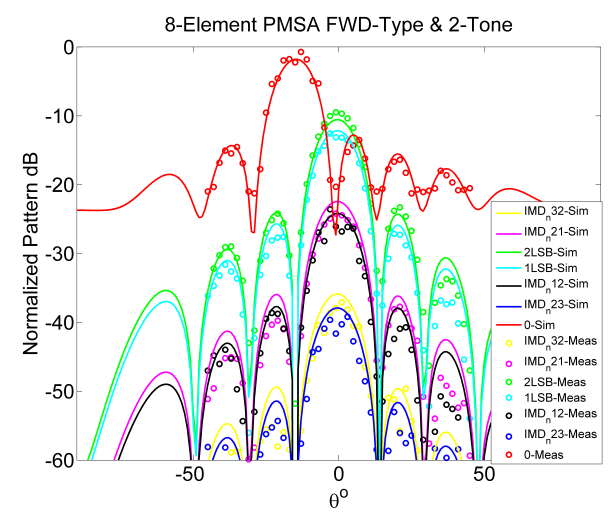

(a)

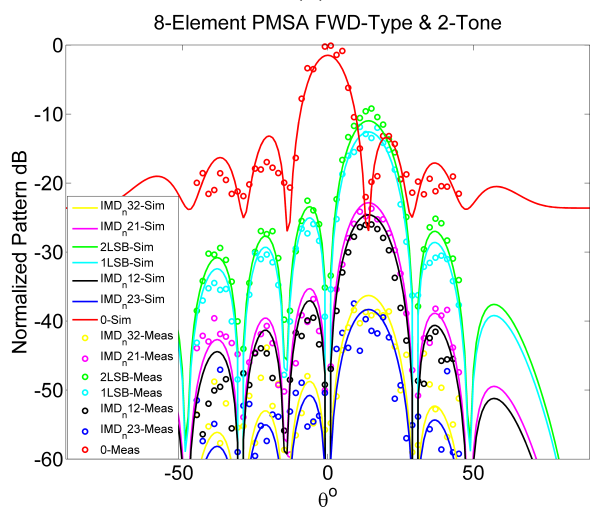

(c)

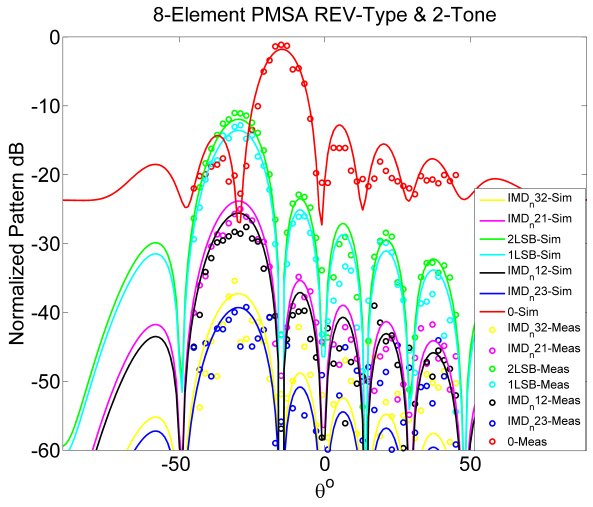

(e)

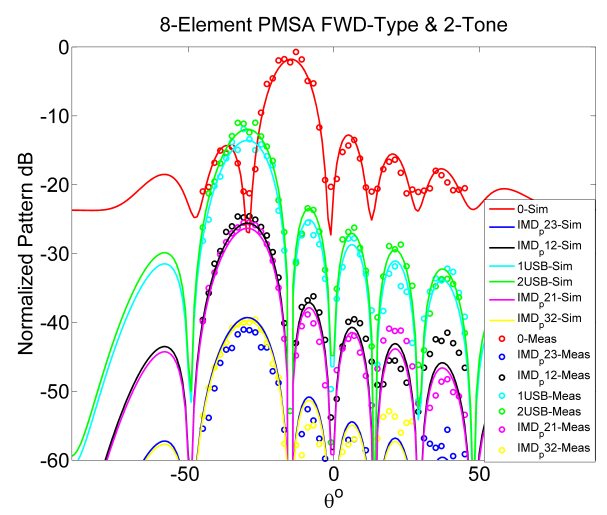

(b)

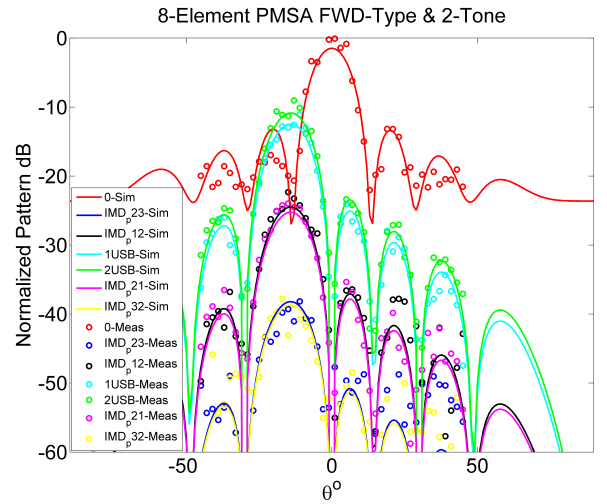

(d)

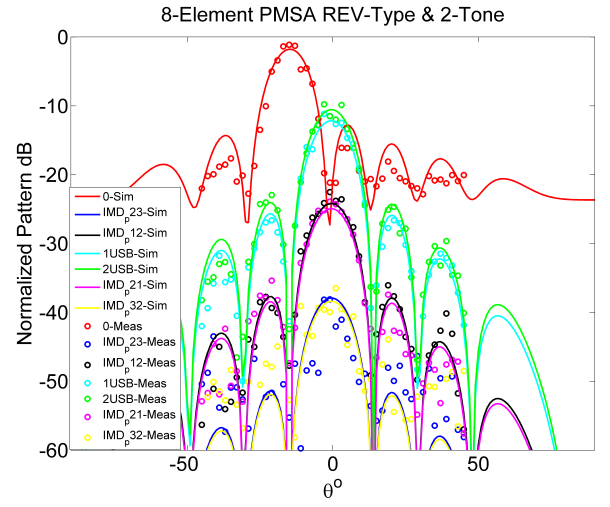

(f)

Figure 8.20: Measured and simulated scattered beams of IMDs and HDs generated by the $8 \times$ 2-element PMSA a) scattered beams of LSBs (1st setup) b) scattered beams of USBs (1st setup) c) scattered beams of LSBs (2nd setup)d) scattered beams of USBs (2nd setup) e) scattered beams of LSBs (3rd setup) f) scattered beams of USBs (3rd setup)

signals were given at specific values. Here, $\theta_{o}$ will be changed arbitrarily and scattered beams of distortion products will be monitored and plotted with respect to the new 


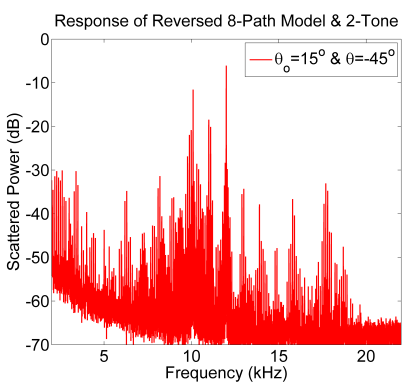

(a)

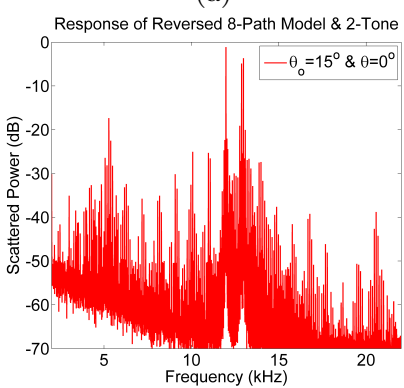

(d)

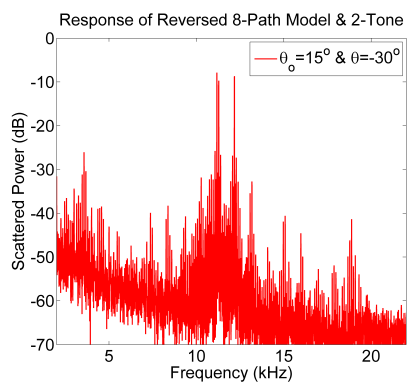

(b)

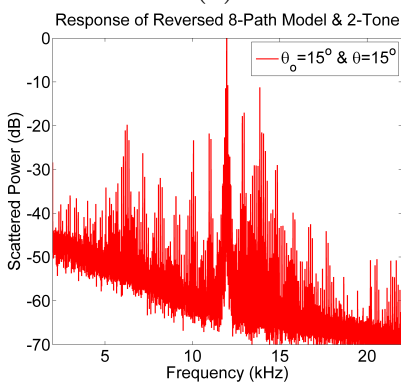

(e)

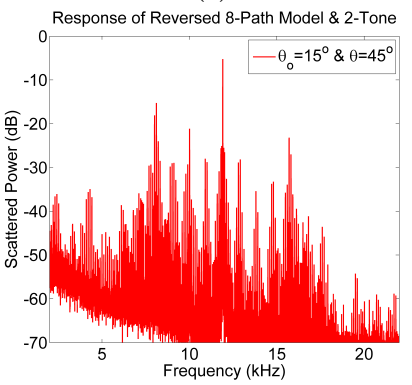

(g)

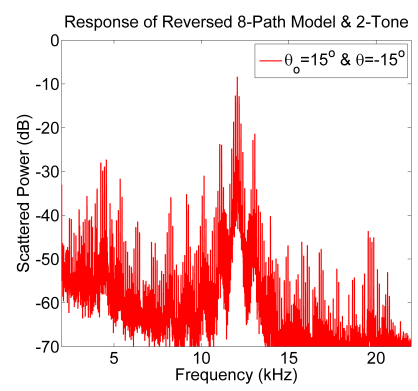

(c)

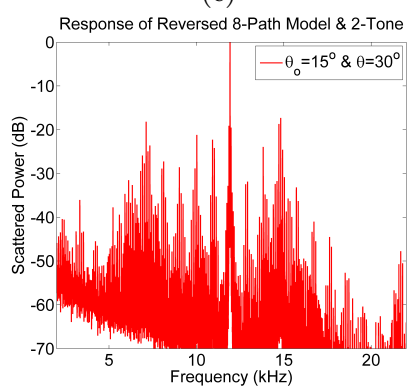

(f)

Figure 8.21: Measured spectrums of the $8 \times 2$-element PMSA, when the first element on the left in Figure $8.11 \mathrm{~b}$ considered as a reference for the direction of progressive phase shifts of the two-tone modulation signals and the illuminating signal source located at $\theta_{o}=+15^{\circ}$, taken at a) $\theta=-45^{\circ}$ b) $\theta=-30^{\circ}$ c) $\theta=-15^{\circ}$ d) $\theta=0^{\circ}$ e) $\theta=+15^{\circ}$ f) $\theta=+30^{\circ}$ g) $\theta=+45^{\circ}$

changes in the incident angles. In the second part of investigations, some elements of the $8 \times 1$-element PMSA up to 3 are turned-off (deactivated) during the operation to observe their impacts on the overall performance and determine the tolerance of the PMSA if at least one of its elements fails. 


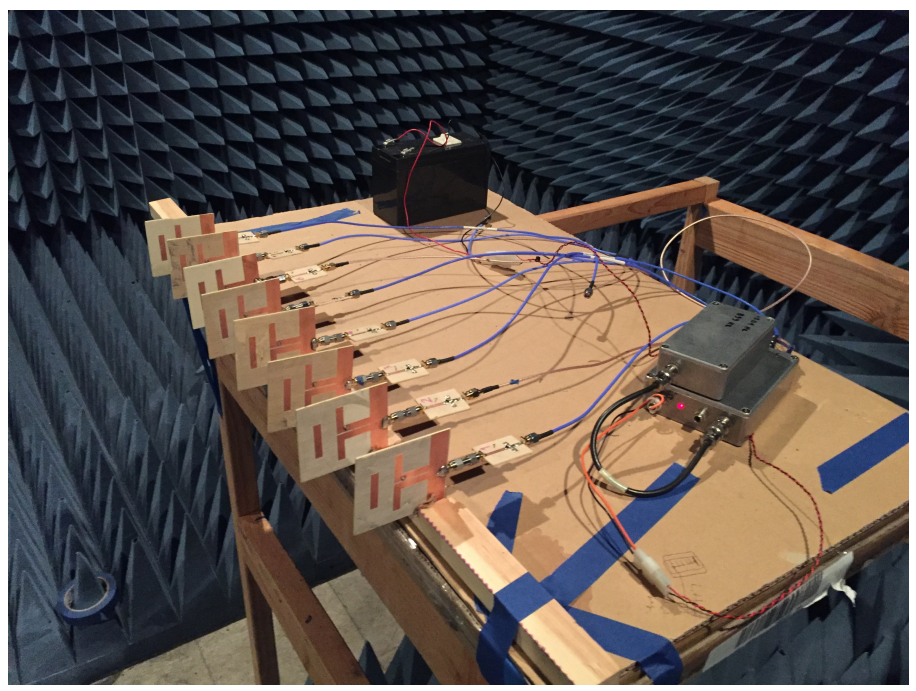

Figure 8.22: A photograph of the $8 \times 1$-element PMSA prototype performing inside the anechoic chamber

\subsubsection{Investigating the Effects of Varying $\theta_{o}$ and $d$ on the Overall Performance}

Because everything occurs in space (i.e., enhancement and cancellation of distortion products) under rules imposed by standard parameters of the PMSA, here, it is reasonable to show that our proposed system does still work well if one of these parameters is broken. This means that the parameters mentioned in the introduction of this section become variable during every single experimental round. Plots of spatial characteristics of distortion products are angle-dependent, but this is not explicitly shown. Therefore, these plots become useless. As an alternative, plots of scattered beams of distortion products are only provided here due to their explicit angle-dependence. In addition, distortion beam scanning is one of the salient features of the PMSA because it provides adequate information about spatial locations of enhancement and suppression of distortion products in space, and it can be considered as a potential application for the PMSA.

The initial parameters of the $8 \times 1$-element PMSA shown in Figure 8.22 are that $d=65 \mathrm{~mm}\left(\lambda_{o} / 2\right)$ at $2.3 \mathrm{GHz}, \theta_{o}=15^{\circ}$, the relative phase difference $=45^{\circ}$, and the 
first left element in Figure 8.22 is considered as a reference. First, the distance $d$ is fixed at a given value, whereas we select three values for $\theta_{o}$ which are $5^{\circ}, 15^{\circ}$, and $25^{\circ}$. Because the PMSA generates and scatters several distortion products, we consider a few of them which are the 2LSB, 1LSB, 1USB, and 2USB distortion products for the sake of simplicity. Simulated scattered beams using the mathematical model given in Chapter 4 integrated with real-world radiation patterns of individual elements (i.e., antennas) used in the $8 \times 1$-element PMSA along with measured scattered beams are presented. As can be seen in Figure 8.23, angles of the incident signals play vital roles in determining angles of scattered beams of distortion products. The measured data was taken from $\theta=-45^{\circ}$ to $\theta=+45^{\circ}$ because of the anechoic chamber space limitations. As a result, when $\theta_{o}$ becomes $25^{\circ}$, a peak of scattered beam of the 2USB distortion product cannot be captured. Also, a scattered beam of the 1USB distortion product is pulled away from the broadside direction with the increase of the incident angle. As for the 2LSB distortion product, its scattered beam moves toward the broadside direction. That is expected according to the theory and this also means that more high order distortions of the lower sideband (LSB) distortion products can appear in the front of view of the PMSA depending on the size of the incident angle until reaching the critical angle $\theta_{c}$.

In addition, when $\theta_{o}$ is $15^{\circ}$, a scattered beam of the 2LSB distortion product is directed to reach a direction of the incident signals, being a retrodirective beam, and a scattered beam of the 1LSB distortion product becomes almost a retrodirective beam at the incident angle $\theta_{o}=10^{\circ}$. A scattered beam of the 1LSB distortion product crosses the broadside direction coming from right to left with the increase in the incident angle too. All amplitudes of scattered beams of distortion products are normalized with respect to the maximum value of scattered power of the 1LSB distortion product to maintain the differences between amplitudes of distortion 
products.

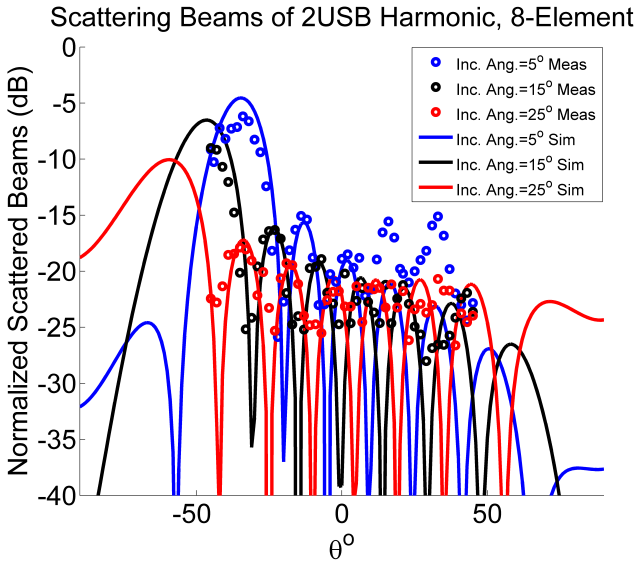

(a)

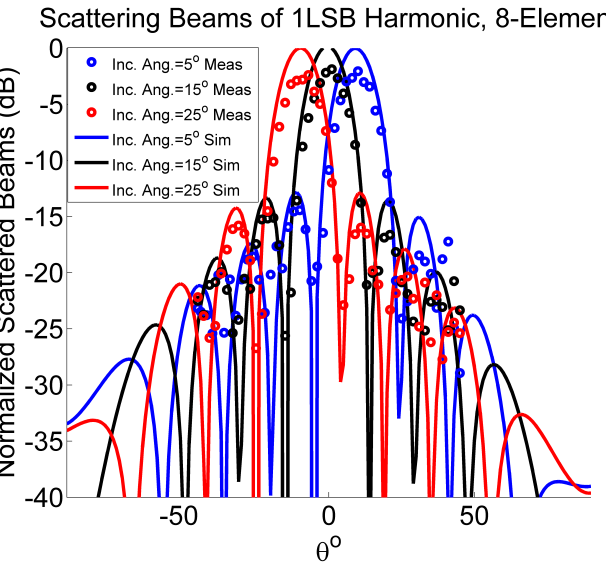

(c)

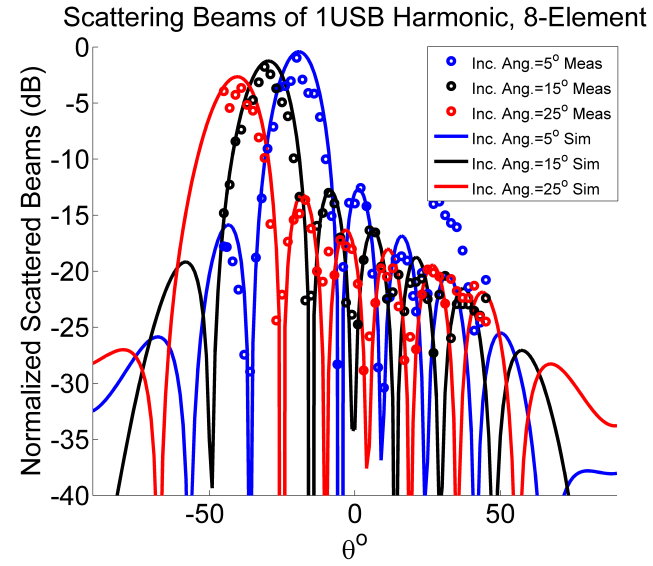

(b)

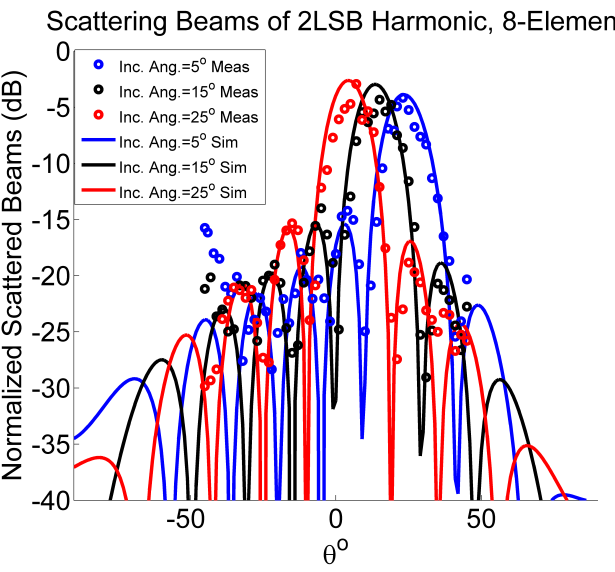

(d)

Figure 8.23: Scattered beams of distortion products generated by the $8 \times 1$-element PMSA with fixed $d$ and varying $\theta_{o}$ of a) the 2USB b) the 1USB c) the 1LSB d) the 2LSB, (solid-line simulated, dotted-line measured)

We can conclude that the beamforming property of the proposed PMSA is validated practically by changing just an angle of the illuminating signals. This process does not need to use complicated phase shifters to carry out the beamfroming task so that it can be potentially considered one of the proposed design benefits.

Next, $d$ is changed from $0.5 \lambda_{o}$ to $0.9 \lambda_{o}$ with a $0.2 \lambda_{o}$ step while $\theta_{o}$ is changed for only keeping a relative phase shift difference of the RF incident signals induced on PMSA elements constant $45^{\circ}$. Two important observations have been witnessed 
and shown in Figure 8.24 during the measurements with the increase of $d$. The first observation is angular locations of scattered beams of distortion products which means that scattered beams of all distortion products tend to reach the broadside direction (i.e., $\theta=0^{\circ}$ ) with the increase of $d$. Scattered beams of the 2USB, 2LSB, and $1 \mathrm{USB}$ distortion products move from almost $\theta=-50^{\circ}$ to $\theta=-30^{\circ}, \theta=+15^{\circ}$ to $\theta=+5^{\circ}$, and $\theta=-30^{\circ}$ to $\theta=-17.5^{\circ}$, respectively, as $d$ increases from $0.5 \lambda_{o}$ to $0.9 \lambda_{o}$, whereas a scattered beam of the 1LSB distortion product keeps its location because it is already located in the broadside direction. The tendency of scattered beams of distortion products to reach the broadside direction with the increase of $d$ can be adopted to increase a number of scattered beams from arrays having the same number of scatterers but at the expense of appearance of grating lobes.

In the second observation, scattered beams of distortion products become narrower as $d$ increases similar to what happens for radiation patterns of conventional arrays when their aperture lengths increase. For example, the HPBW of scattered beam of the $1 \mathrm{LSB}$ distortion product is $18^{\circ}, 12.5^{\circ}$, and $10^{\circ}$ for $d=0.5 \lambda_{o}, 0.7 \lambda_{o}$, and $0.9 \lambda_{o}$, respectively. However, the HPBWs of other scattered beams are wider because they are not located at the broadside direction.

\subsubsection{Investigations of the Failure of Scatterers on the Overall Performance of the PMSA}

Again, the $8 \times 1$-element PMSA with $d=0.5 \lambda_{o}$ and $\theta_{o}=15^{\circ}$ shown in Figure 8.22 is utilized to demonstrate fault tolerance behavior of the PMSA when some elements fail. The modulation signals driving scatterers supposed to fail during the operation are turned-off, emulating failures of elements. Figure 8.25 consists of three rows of sub-figures, and each row displays the measured received spectrum of scattered signals captured at $\theta=0^{\circ}$ when a specific number of scatterers stops working in 


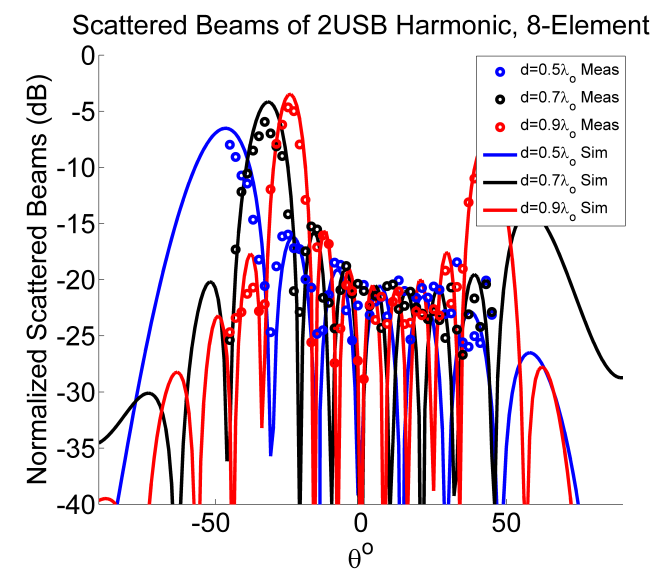

(a)

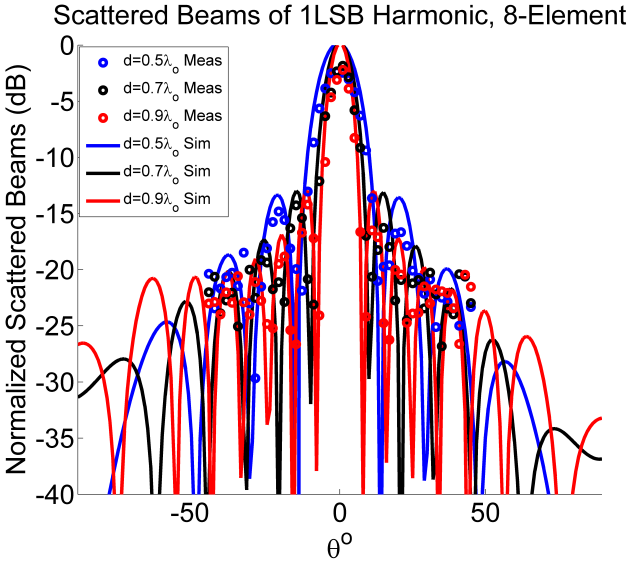

(c)

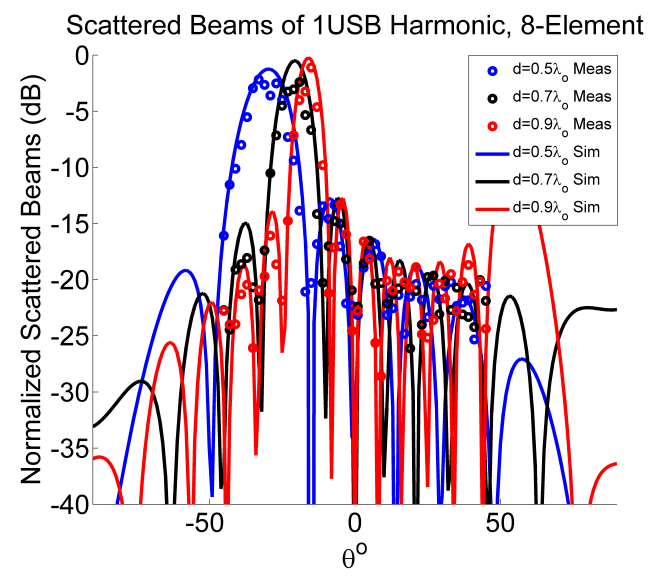

(b)

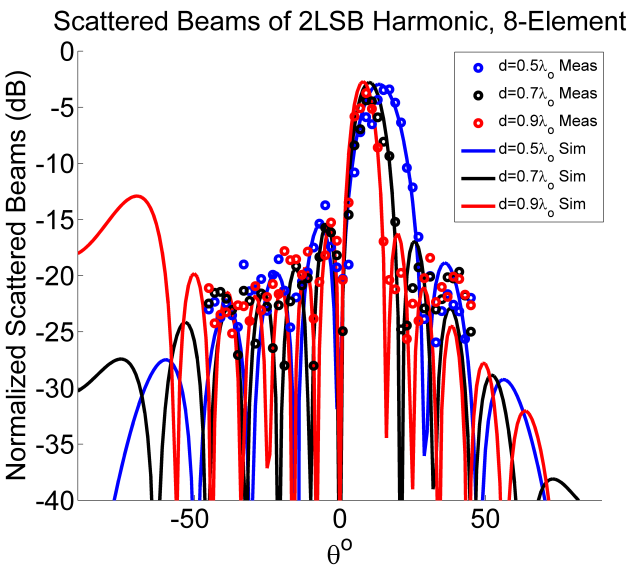

(d)

Figure 8.24: Scattered beams of distortion products generated by the $8 \times 1$-element PMSA with varying $d$ of a) the 2USB b) the 1USB c) the 1LSB d) the 2LSB, (solid-line simulated, dotted-line measured)

the PMSA. For this array, at $\theta=0^{\circ}$, the 1LSB distortion product is enhanced. In the first row, when one element fails, powers of other distortion products increase. However, the 1LSB distortion product is still dominant, and the measured responses can clearly state that the performance of the PMSA is still acceptable. The second row shows when two scatterers stop working simultaneously. These two scatterers may or may not be adjacent. Also, the PMSA performance is still acceptable to some extent despite the rise in powers of other distortion products. In the following row, a number of scatterers assumed to fail at the same time are three. The measured 
received spectrums scattered from the $8 \times 1$-element PMSA become more chaotic.

The measured results do confirm that the PMSA performance is still good if one or two scatterers do not work.

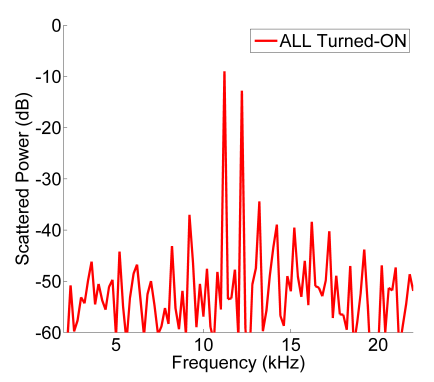

(a)

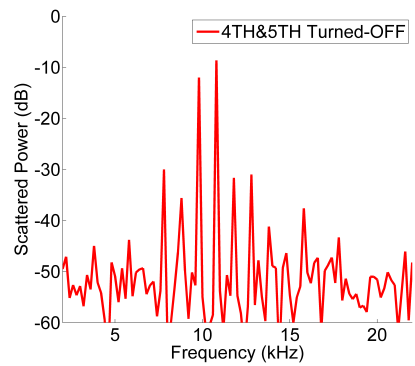

(d)

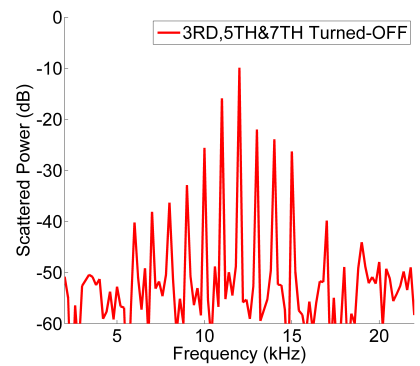

$(\mathrm{g})$

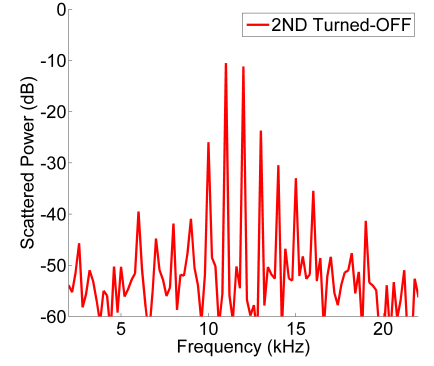

(b)

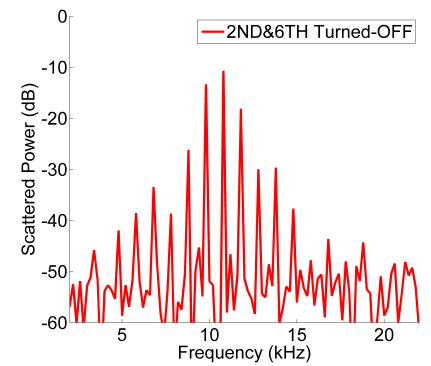

(e)

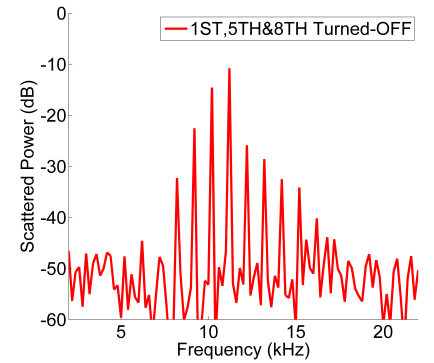

(h)

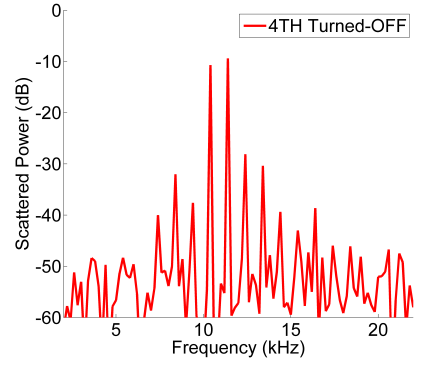

(c)

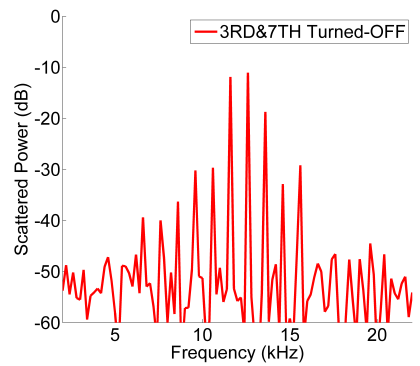

(f)

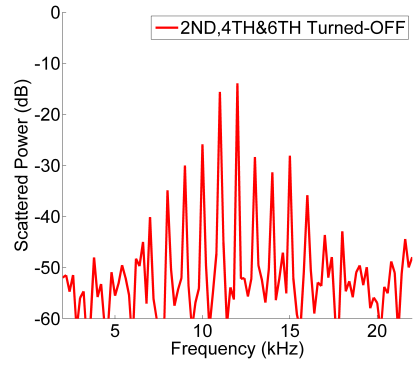

(i)

Figure 8.25: Measure received spectrums of scattered distortion products when some elements of the $8 \times 1$ PMSA turned-off a) all elements turned-on b) 2 nd c) 4 th d) 4 th +5 th e) 2 nd +6 th f) 3 rd +7 th g) $3 \mathrm{rd}+5$ th +7 th h) $1 \mathrm{st}+5$ th +8 th i) $2 \mathrm{nd}+4 \mathrm{th}+6$ th

Scattered beams of the first four distortion products (2LSB, 1LSB, 1USB, and 2USB) are plotted in Figure 8.26. In Figure 8.26a, we considered only the fourth scatterer as a failed element. The failure of a single element does not show any effects on the main lobes of scattered beams of distortion products, and it only affects sidelobes. The relative difference between the main lobes and the first sidelobes is 


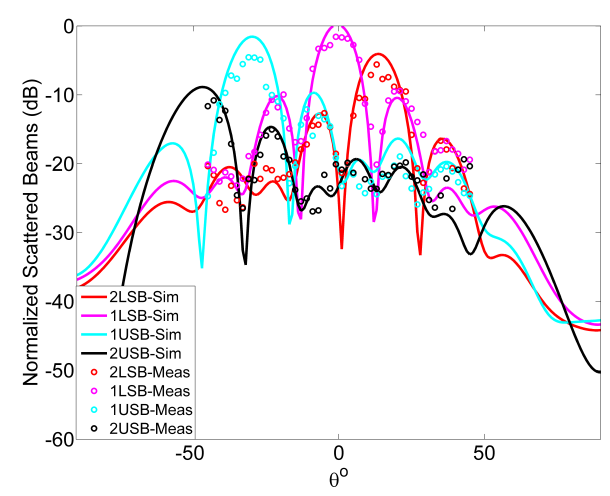

(a)

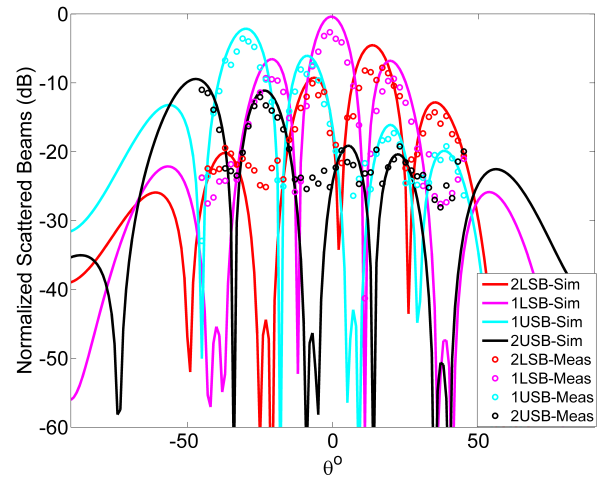

(b)

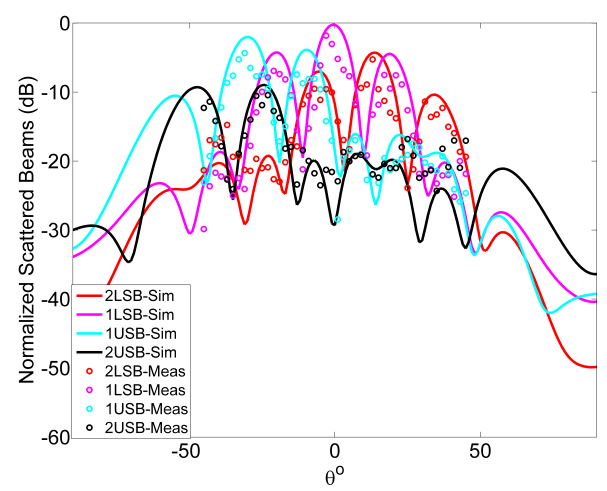

(c)

Figure 8.26: Scattered beams of distortion products (2LSB, 1LSB, 1USB, and 2USB) when a number of elements in the $8 \times 1$ PMSA turned-off a) single element b) two elements c) three elements (solid-line simulated, dotted-line measured)

decreased to be about $10 \mathrm{~dB}$. Also, it can be seen that levels of sidelobes increase with the increase in number of failed elements as shown in Figure 8.26b and 8.26c. For two failed elements, the difference between the main and side lobes becomes almost $7 \mathrm{~dB}$, while it is about $4 \mathrm{~dB}$ for three failed elements. As we said before, a response of the two failed elements is tolerable to some extent because we can still distinguish between the main lobes and sidelobes of scattered beams of distortion products. However, the situation gets worse with the three failed elements, and the overall performance of the PMSA has been severely degraded. The scattered beam of the 2USB distortion product has two main lobes separating by $20^{\circ}$. The scattered beam of the 1USB distortion product also has two main lobes, but one of them has less amplitude by 
only $2 \mathrm{~dB}$. The deterioration in the scattered beam of the 2LSB distortion product is similar to the scattered beam of the 1USB distortion product. The only beam that could be functioning but less than normal is the scattered beam of the 1LSB distortion product.

\subsection{Potential Applications of the Proposed Phase-Modulated Scattering Array (PMSA)}

\subsubsection{Tunable Spatial Distortion Product Generator}

Here also, the $8 \times 1$-element PMSA shown in Figure 8.22 is used to demonstrate that spatial characteristics of distortion products are not changed if we change the modulation frequency provided the phase shift distribution of the modulation signals is kept the same. Three different frequencies of modulation signals are used at different times in this experimental setup which are $700 \mathrm{~Hz}, 899 \mathrm{~Hz}$, and $1004 \mathrm{~Hz}$. In addition, the $d$ and $\theta_{o}$ are chosen as $\lambda_{o} / 2$ and $15^{\circ}$, respectively. Measured spatial distortion product responses are taken at an angle of $\theta=0^{\circ}$ and the 1LSB distortion component is enhanced at that angle because the first left element in Figure 8.22 is chosen as a reference for the phase shift distribution. Figure 8.27 shows the measured results. Results reveal that frequencies of spatial distortion products can be tuned continuously without altering their spatial locations. The 1LSB distortion product has different amplitudes as shown in the figure because the modulation signals used in the experiment have different amplitudes.

\subsubsection{PMSA based Direction Finding}

An experimental phase-modulated scattering array (PMSA) was designed and built utilizing eight quarter-wave monopoles over a conductor ground and eight

separate RTS modulators operating at $2.3 \mathrm{GHz}$ as shown in Figure 8.28. The 


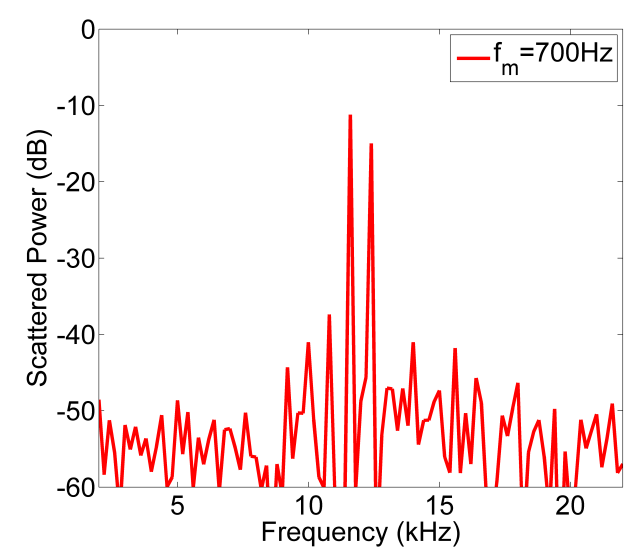

(a)

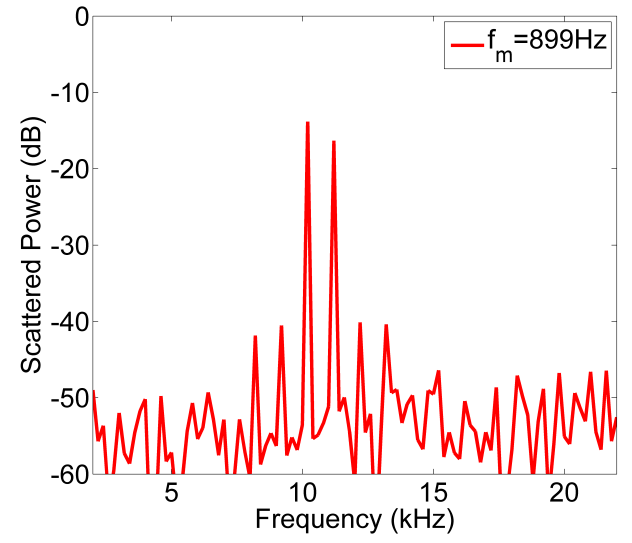

(b)

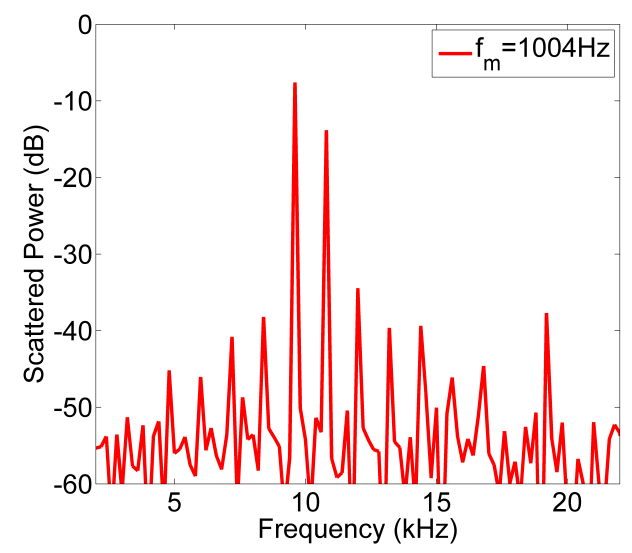

(c)

Figure 8.27: Measured results of the frequency tunable PMSA captured at an angle of $\theta=0^{\circ}$ for a) $700 \mathrm{~Hz}$ b) $899 \mathrm{~Hz}$ c) $1004 \mathrm{~Hz}$

experimental measurements were carried out inside the anechoic chamber with the same illuminating and receive antennas used in the previous tests. The $1004 \mathrm{~Hz}$ modulation signals drive the RTS modulators. We moved the illuminating antenna from $-40^{\circ}$ to $40^{\circ}$ with a $10^{\circ}$ step. Scattered signals are also down-converted. For every single step, we optimized angular locations of the receive antenna to read exact locations of the first upper and lower sidebands (1LSB, 1USB). The reason to use two different distortion products is to cover all angles required to expect existence of the incident (unknown) signals. The optimized angular locations of the 1LSB and 1USB distortion products are plugged in (5.37) to calculate the exact locations of the 
incident (unknown) RF source. The measured results are displayed in Figure 8.29 along with the theoretical results. As can be seen, errors between the measured and theoretical results are less than $5^{\circ}$. The errors may belong for several aspects as follows:

1. Errors in amplitudes and phases of modulations signals

\section{The mutual coupling between the scatterers}

3. Errors resulting from moving the receive antenna and the PMSA manually

The promising results obtained in the measurements open the door for more exploitation in the future works using the high-level of signal processing to make everything autonomous without the need to move the receive antenna to find the exact angular directions of the incident signals, exploiting the spatial characteristics of distortion products to extract the required information about directions of the incident signals.

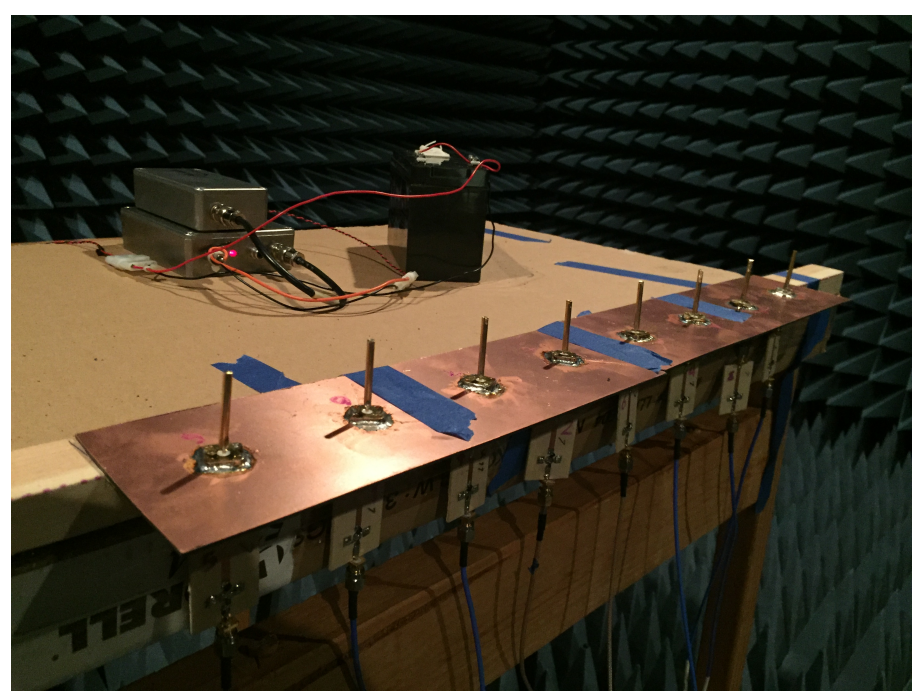

Figure 8.28: A photograph of the $8 \times 1$-element PMSA prototype using quarter-wave monopoles antennas, performing inside the anechoic chamber 


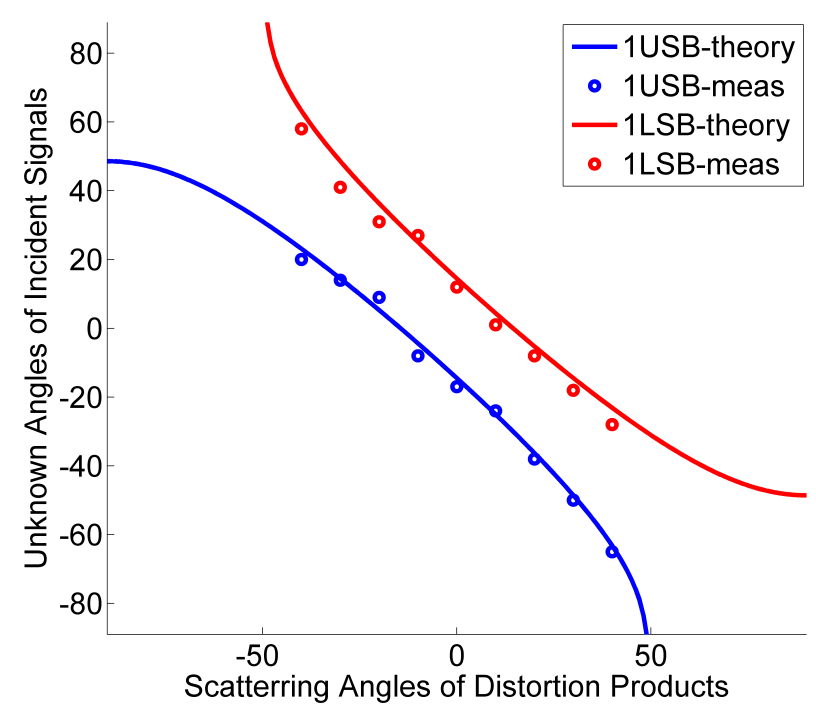

Figure 8.29: Measured results along with theoretical results of the angles of the incident RF signals versus the captured angles of the 1LSB and 1USB distortion products 


\section{Chapter 9: Conclusion and Future Work}

\subsection{Conclusion}

In this research work, suppression and enhancement of distortion products occurred in space was demonstrated for the first time using the modulated scattering technique (MST). We assumed non-conventional ways rather than using physical RF components such as transmission lines, waveguides, filters, phase shifters, power dividers, and combiners to handle distortion products. It is an array of identical scattering elements working simultaneously and together called the phase-modulated scattering arrays (PMSAs). Although arrays of scattering elements are used in a variety of applications such as microwave imaging, RFID, IoT sensors, these applications exploit only single distortion products generated by scatterers to discriminate between the incident and scattered signals. No literature has been reported regarding the use of an array of scatterers to enhance or suppress several distortion products at the same time in space. Motivated by the potential of filling this missing part, we developed a structure able to generate distortion products and treat them in space. Thus, our PMSA structure is a simple design because our scattering elements are not connected, thereby no need for a feeding network. Basically, the PMSA is a topology similar to that of conventional phased arrays, but elements are antennas associated with electronic switches. By turning switches ON-OFF at different times, the PMSA can treat (i.e., enhance or suppress) distortion products which either harmonics distortions HDs or intermodulation distortions IMDs in space.

Chapter 2 presented the concepts of modulated scattering techniques (MST) in the first part. Simple explanations about mono-static and bi-static MST systems were given. Then, mathematical procedures were provided to show factors that affect 
the overall performance of a scatterer and a general process of distortion products generation and how they propagate were also provided. Mathematical procedures divided scattered signals into two types, which are static and dynamic. The MST performance depends totally on the latter type. Next, a generic representation for reflection coefficients plotted on the Smith chart was introduced. In the second part of Chapter 2, principles of array signal processing based phased antenna array were explained for one and two dimensions problems. Moreover, we stated some arrays features such as steering phases, grating lobes, and array weightings briefly. Eventually, the chapter introduced the most fundamental theory of image-reject mixer operation.

Chapter 3 introduced the first mathematical model developed in this research work. The polyphase multipath technique used in different receiving and transmitting communication circuits because it is able to cancel a large number of distortion products without the need to use high-performance filters was the basis of our first mathematical model. Every single scatterer was represented by a single path in the adopted technique. Nonlinear distortion products generated in each track are combined constructively or destructively at the fictitious output port. To this end, the proposed model was able to detect signals at the output if values of phase shifters in the proposed model are appropriately chosen. This was true with communication circuits because they utilize fixed phase shifters, but in our proposed systems values of phase shifters depends on, distances between scatterers, angles of the illuminating signals, and relative difference value and direction of progressive phase shift distribution of the modulation signals. If one of these parameters, especially arrival angles of the illuminating signals change, the mathematical model based on the conventional polyphase multipath technique becomes useless. Thus, we have overcome this deficit by adding some extra terms being able to correct phase shift 
deviations. Moreover, errors terms in phases and amplitudes of the modulation and illuminating signals have been added to the ideal modified model to come up with more realistic model describing well distortion products generated and scattered by our PMSA. Eventually, after all modifications that were made, the mathematical model can define exact spatial locations of suppression and enhancement of distortion products, resulting in a robust mathematical model based on communication signal processing.

In Chapter 4, because components of distortion products which are either HD or IMD have different resulting phases and frequencies, they can be treated individually and simultaneously. Also, each single distortion component has replicas generated from other scatterers working within the same ensemble, so they have their scattered beams (radiation patterns in a general sense). Therefore, we developed the second mathematical model based on the phased antenna array theory. The resulting mathematical model of scattered beams of distortion products developed in the chapter consists of an infinite number of terms, but radiation pattern equations of phased arrays consist of only one term. However, not all of scattered beams terms in the PMSA carry real power as we indicated earlier in this research work. This considers the fundamental difference between radiation pattern equations of phased arrays and scattered beams equations of the PMSAs. Moreover, each scattered beam directed to a different spatial location is tagged with a different frequency, and thus realizing a spatial-diversity to frequency-diversity transformation. Meanwhile, the mathematical model of scattered beams of distortion products was extended to include both backscattering and forward-scattering parts.

Chapter 5 discussed the most important features and some potential applications of the presented PMSA. They have been introduced as follows:

- The first feature described was diffraction grating-like behavior. Fortunately, 288 
the generalized equation, called the scattering equation of distortion products (modes) developed in Chapter 5 was very similar to an equation of diffraction grating devices. It was also developed for single-tone, two-tone, and multi-tone modulation signals. According to the scattering equation, our proposed system can reconfigure its parameters easily to change directions of scattered modes. Meanwhile, the general scattering equation was used to derive other equations for differentiating between propagating modes carrying real power and evanescent modes carrying reactive power, a total number of propagating modes, retrodirective modes, and frequency sensitivity.

- The second salient feature illustrated was nonreciprocity. Because a progressive phase shift distribution of the modulation sources driving scatterers is increased gradually in one direction, the PMSA is inherently nonreciprocal. This feature could make the PMSA used in different applications because it can isolate incoming from outgoing signals spatially and temporally.

- We also demonstrated the beamforming property. Scattered beams of distortion products can change their spatial location relying on many parameters. Once we changed a reference element for phase shift distributions, an arrival angle of incident signals, and spaces between scatterers, we succeeded to scan scattered beams of distortion products. Two examples were given with four and eight scatterers to validate the beamforming process theoretically. The most important thing that should be noted is that we succeeded to scan scattered beams of distortion products without using real phase shifters. It is worth noting that our PMSA is a simple and low-cost design.

- Because our system consists of an array of nonlinear elements, it was useful to exploit its developed mathematical models to study and investigate behaviors 
of distortion products generated in active arrays and MIMO systems. The study of distortion products in active arrays becomes a hot research topic nowadays because $5 \mathrm{G}$ systems use active arrays instead of single elements as in previous generations of communication systems. We made slight modifications to our proposed mathematical models to be compatible with requirements of active arrays. An example was explained following the same example as in [143] to demonstrate effectiveness of our mathematical models in analyzing distortion products. The results obtained from our mathematical models were more accurate compared to [143] because our system can deal with infinite terms for underlying equations, but authors just truncated their model to the third term. Furthermore, our mathematical models succeeded to show spatial distortion characteristics of scattered signals at specific locations that were not provided in [143]. Eventually, we demonstrated other two examples by assuming nine signals hitting a receive-mode active array at the same time. In the first example, the nine signals arrived from the same direction but having different frequencies. Apparent patterns of distortion products are the same with patterns of the main signals. However, in the second example, we used nine signals having the same frequency but coming from different directions. In this case, we found that all resulting IMD3 and IMD5 have the same frequency, so their patterns are added in power in space to create isotropic response

- We implemented a theoretical setup to demonstrate that spatial distortion characteristics of scattered signals are not changed if we change the modulation frequency provided that we keep the same value of relative phase shift difference of the modulation signals and a reference element. We demonstrated that by using three different frequencies $700 \mathrm{~Hz}, 899 \mathrm{~Hz}$, and $1004 \mathrm{~Hz}$, and we found that spatial locations of scattered beams of the 1USB distortion product for these 
three frequencies were not different.

- The last section in Chapter 5 was the direction finding. Because the scattered signals rely on directions of the incident signals, this encouraged us to derive an equation to extract information about directions of the incident signals once we know directions of the scattered signals. Moreover, because there are a lot of distortion products, we exploited only the first LSB and USB distortion products. Incident signals were assumed coming from different directions, and simulated results successfully recognized directions of the incident signals.

In Chapter 6, we presented our initial hardware work. Three different single antennas were designed, analyzed, simulated, fabricated, and tested. Antennas were printed quasi-Yagi dipole, inset-fed microstrip patch, and a quarter wavelength monopole over a ground. Simulation and measured results were in good agreement. Then, different types of arrays were simulated using the single antennas developed in the first section of Chapter 6. Also, mutual coupling effects on performances of antennas working within the same array were shown because our proposed system needs all scatterers to be identical in their operations. Results showed that elements located in different locations in an array radiate slightly different powers. However, differences in powers were small, so problems with these challenges were tolerated. Next, a reflective-type switch RTS was designed using the software ADS. A Schottky diode was the electronic part performing as a switch and was responsible for generating distortion products. After simulating the RTS, it was fabricated and tested. The last section in the chapter was dedicated to design, build, and test a 4-to-8 phase transformer kit using only resistors.

Chapter 7 introduced the experimental environment where the PMSAs tested. It also presented the required apparatus and some facilities limitations. 
Chapter 8 was fully dedicated to show how the experimental results agree with theory. We started with results obtained from two different PMSAs working at a frequency $432 \mathrm{MHz}$. The measured results were compared to results of a single scatterer. Afterward, we moved to test PMSAs working at a higher frequency which was 2.3GHz. Single element, $2 \times 1$-element PMSA, $4 \times 4$-element PMSA, and $8 \times 2$-element PMSA were first tested using single-tone modulation sources. Results showed that more distortion products could be controlled in case of PMSAs with large sizes. Spatial distortion characteristics of scattered signals were exhibited as well as their scattered beams for different combinations of directions of progressive phase shifts distributions of the modulation sources and arrival angles of incident signals. Next, two-tone modulation signals were used to see effects of IMDs. Scattered beams of few IMDs were plotted too. Afterward, some parameters were changed during every single round of experimental setups to demonstrate their effects on the overall performances. Also, failures of elements were emulated by stopped driving some RTSs during operations to check a system tolerance against element failures. Results were acceptable to some extent when one or two elements failed. More than two elements failed at the same time, results are more distorted. Eventually, two different applications were carried out. The first one was the tunable spatial frequency generator and the second one was the direction finding.

\section{$9.2 \quad$ Accomplished Objectives}

Here we show a list that demonstrates all objectives achieved in our research work.

1. Two different mathematical models based on communication signal processing and phased antenna array theory were developed in Chapters 3 and 4 . The first mathematical model was able to recognize and define spatial characteristics of distortion products at any point in space, while the second mathematical model 
was able to define scattered beams of distortion products.

2. Sub-mathematical models describing some interesting features and potentials applications of the PMSA using the mathematical models developed in the first objective were presented. All sub-models were simulated using the software Matlab. The results were satisfied.

3. Different types of antennas, arrays, RTS modulators, and phase transformers were implemented.

4. Extensive experimental measurements were made to validate the two theoretical models where different sizes of the PMSAs were tested inside the anechioc chamber. In this part, cancellation and enhancement of distortion products using single-tone and two-tone modulation sources were validated practically as the main theme of the research work. In addition to that, some parametric studies were carried out by changing directions of the incident signals or spaces between elements to show that the beamforming can be realized without using phase shifters. Elements failure, tunable spatial harmonic generator, and direction finding were also demonstrated practically. All results obtained from measurements were compared to theoretical ones and they were in good agreement.

\subsection{Future Work}

In this research work, we developed the most important underlying mathematical models required to describe the mechanism of suppression and enhancements of distortion products in space using simple structures of nonlinear active arrays. The results show that the PMSAs are in their initial development stage and future investigations in fields of theories, applications, design frequencies, manufacturing, 
and performance need to be carried out to further exploring our PMSA. Therefore for future investigations and developments, it would be very beneficial to find out unknown properties and applications of the phase-modulated scattering array (PMSA) presented in this dissertation. This leads to coming up with new electromagnetic structures. Below is a list of expected scenarios to be studied.

- In chapters 3 and 4 , two different mathematical models were derived and developed. However, they assume that our proposed system is one dimensional. Thus, these two models can be extended to include 2D-PMSAs.

- In chapter 5, we explained and mathematically illustrated the nonreciprocity property of the PMSA, but we could not demonstrate that practically. Thus, building an experimental setup to validate this property will be one of our future works.

- The proposed system can steer scattered beams of distortion products, so integrating adaptive beamforming algorithms to control electronic switches makes the proposed systems adaptive and robust.

- Building modulation sources that have tunable phase shifts to design a reconfigurable PMSA.

- Another interesting direction is to build a linear to circular polarization converter and a polarization rotator using an array of scatterers.

- It is also to design spatial power dividers, isolation, mixers, and modulators.

- It could be used to build a full autonomous direction finding systems.

- Forward/backward scattering systems with two different frequencies is another direction of future studies. 
- Using frequency doublers instead of using conventional elements (scatterers) of the PMSA is one of possible interesting research directions.

- Design and building incident linear polarized signals into scattered orbital angular momentum (OAM)-carrying signals converters using the PMSAs is another future study 


\section{Bibliography}

[1] Mohamed A Abou-Khousa, Sergey Kharkovsky, and Reza Zoughi. Novel near-field millimeter-wave differential probe using a loaded modulated aperture. IEEE Transactions on Instrumentation and Measurement, 58(5):1273-1282, 2009.

[2] ADS ADVANCED SYSTEM DESIGN https://www.keysight.com. Keysight corp., santa rosa, california, 2018.

[3] Ertugrul Aksoy and Erkan Afacan. Calculation of sideband power radiation in time-modulated arrays with asymmetrically positioned pulses. IEEE Antennas and Wireless Propagation Letters, 11:133-136, 2012.

[4] Francesco Amato and Gregory D Durgin. Signal-to-noise ratio measurements for iot communications with quantum tunneling reflectors. In 2016 IEEE 3rd World Forum on Internet of Things (WF-IoT), pages 383-388. IEEE, 2016.

[5] Francesco Amato, Christopher W Peterson, Muhammad B Akbar, and Gregory D Durgin. Long range and low powered rfid tags with tunnel diode. In RFID Technology and Applications (RFID-TA), 2015 IEEE International Conference on, pages 182-187. IEEE, 2015.

[6] Francesco Amato, Christopher W Peterson, Brian P Degnan, and Gregory D Durgin. Tunneling rfid tags for long-range and low-power microwave applications. IEEE Journal of Radio Frequency Identification, 2(2):93-103, 2018. 
[7] Francesco Amato, Hakki M Torun, and Gregory D Durgin. Beyond the limits of classic backscattering communications: A quantum tunneling rfid tag. In 2017 IEEE International Conference on RFID (RFID), pages 20-25. IEEE, 2017.

[8] S Andre and D Leonard. An active retrodirective array for satellite communications. IEEE Transactions on Antennas and Propagation, 12(2):181-186, 1964.

[9] Anechoic chamber. Anechoic chamber - Wikipedia, the free encyclopedia, 2014. [Online; accessed 29-January-2019].

[10] Omar Mohammed Bakr. A scalable and cost effective architecture for high gain beamforming antennas. PhD thesis, UC Berkeley, 2010.

[11] William C Barott and Braham Himed. Time-modulated array pattern for sidelobe blanking in spectrometry and radar. IEEE Antennas and Wireless Propagation Letters, 13:1015-1018, 2014.

[12] D Berry, $\mathrm{R}$ Malech, and $\mathrm{W}$ Kennedy. The reflectarray antenna. IEEE Transactions on Antennas and Propagation, 11(6):645-651, 1963.

[13] Grzegorz Bogdan, Yevhen Yashchyshyn, and Miłosz Jarzynka. Time-modulated antenna array with lossless switching network. IEEE Antennas and Wireless Propagation Letters, 15:1827-1830, 2016.

[14] J-C Bolomey, Barry J Cown, GEORGES Fine, LUIS Jofre, Masoud Mostafavi, D Picard, JP Estrada, PG Friederich, and FL Cain. Rapid near-field antenna testing via arrays of modulated scattering probes. IEEE Transactions on Antennas and Propagation, 36(6):804-814, 1988. 
[15] Jean Charles Bolomey, Santiago Capdevila, Lluis Jofre, and Jordi Romeu. Electromagnetic modeling of rfid-modulated scattering mechanism. application to tag performance evaluation. Proceedings of the IEEE, 98(9):1555-1569, 2010.

[16] Jean-Charles Bolomey and Fred E Gardiol. Engineering applications of the modulated scatterer technique. Artech House, 2001.

[17] HW Briscoe and PL Fleck. Data recording and processing for the experimental large aperture seismic array. Proceedings of the IEEE, 53(12):1852-1859, 1965.

[18] Finn Bryn. Optimum signal processing of three-dimensional arrays operating on gaussian signals and noise. The Journal of the Acoustical Society of America, 34(3):289-297, 1962.

[19] Lorena Cabria, José Angel García, Antonio Tazón, and Angel Mediavilla. Nonlinear distortion reduction in active arrays taking advantage of their spatial power-combining properties. International Journal of RF and Microwave Computer-Aided Engineering: Co-sponsored by the Center for Advanced Manufacturing and Packaging of Microwave, Optical, and Digital Electronics (CAMPmode) at the University of Colorado at Boulder, 16(1):24-33, 2006.

[20] Christophe Caloz, Andrea Alù, Sergei Tretyakov, Dimitrios Sounas, Karim Achouri, and Zoé-Lise Deck-Léger. Electromagnetic nonreciprocity. Physical Review Applied, 10(4):047001, 2018.

[21] Richard L Campbell. A novel high frequency single-sideband transmitter using constant-envelope modulation. In 1998 IEEE MTT-S International Microwave Symposium Digest (Cat. No. 98CH36192), volume 2, pages 1121-1124. IEEE, 1998. 
[22] HI Cantu, VF Fusco, and S Simms. Microwave reflection amplifier for detection and tagging applications. IET microwaves, antennas $\&$ propagation, 2(2):115-119, 2008.

[23] S. Capdevila, L. Jofre, J. Romeu, and J. C. Bolomey. Passive rfid based sensing. In 2011 IEEE International Conference on RFID-Technologies and Applications, pages 507-512, Sep. 2011.

[24] Santiago Capdevila, Lluís Jofre, Jordi Romeu, and Jean Charles Bolomey. Multi-loaded modulated scatterer technique for sensing applications. IEEE Transactions on Instrumentation and Measurement, 62(4):794-805, 2013.

[25] Jack Capon, Roy J Greenfield, and Richard T Lacoss. Design seismic arrays for efficient on-line beamforming. Technical report, MASSACHUSETTS INST OF TECH LEXINGTON LINCOLN LAB, 1967.

[26] Jack Capon, Richard T Lacoss, et al. Off-line signal processing results for the large aperture seismic array. Technical report, MASSACHUSETTS INST OF TECH LEXINGTON LINCOLN LAB, 1966.

[27] Jin Chang and Shaohui Quan. Design of long range rfid tag in x-band based on modulated scattering technique. In 2016 IEEE International Conference on Ubiquitous Wireless Broadband (ICUWB), pages 1-4. IEEE, 2016.

[28] Kai Chang, Robert A York, Peter S Hall, and Tatsuo Itoh. Active integrated antennas. IEEE transactions on microwave theory and techniques, 50(3):937-944, 2002.

[29] Philippe Charas. Method of and apparatus for filtering intermodulation products in a radiocommunication system, January 19 1999. US Patent $5,862,459$. 
[30] Chunhee Cho, Xiaohua Yi, Dan Li, Yang Wang, and Manos M Tentzeris. Passive wireless frequency doubling antenna sensor for strain and crack sensing. IEEE Sensors Journal, 16(14):5725-5733, 2016.

[31] Huey-Ru Chuang and Liang-Chen Kuo. 3-d fdtd design analysis of a 2.4-ghz polarization-diversity printed dipole antenna with integrated balun and polarization-switching circuit for wlan and wireless communication applications. IEEE Transactions on microwave theory and techniques, 51(2):374-381, 2003.

[32] Ben Clark and James A Flint. Accuracy of harmonic analysis techniques for direction of arrival estimation using time modulated arrays. 2018.

[33] Carlos Collado, Jordi Mateu, Oscar Menéndez, and Juan M O’Callaghan. Nonlinear distortion in a 8-pole quasi-elliptic bandpass hts filter for cdma system. IEEE transactions on applied superconductivity, 15(2):992-995, 2005.

[34] Balanis Constantine et al. Antenna theory: analysis and design. MICROSTRIP ANTENNAS, third edition, John wiley $\&$ sons, 2005.

[35] Steve C Cripps. Advanced techniques in RF power amplifier design. Artech House, 2002.

[36] AL Cullen and JC Parr. A new perturbation method for measuring microwave fields in free space. Proceedings of the IEE-Part B: Radio and Electronic Engineering, 102(6):836-844, 1955.

[37] Spyridon-Nektarios Daskalakis, Stylianos D Assimonis, Eleftherios Kampianakis, and Aggelos Bletsas. Soil moisture scatter radio networking with low power. IEEE Transactions on Microwave Theory and Techniques, 64(7):2338-2346, 2016. 
[38] Spyridon Nektarios Daskalakis, John Kimionis, Ana Collado, George Goussetis, Manos M Tentzeris, and Apostolos Georgiadis. Ambient backscatterers using fm broadcasting for low cost and low power wireless applications. IEEE Transactions on Microwave Theory and Techniques, 65(12):5251-5262, 2017.

[39] Daniel M Dobkin. The RF in RFID: UHF RFID in practice. Newnes, 2012.

[40] D Domanin, Umberto Gatti, Piero Malcovati, and Franco Maloberti. A multipath polyphase digital-to-analog converter for software radio transmission systems. In 2000 IEEE International Symposium on Circuits and Systems. Emerging Technologies for the 21st Century. Proceedings (IEEE Cat No. 00CH36353), volume 2, pages 361-364. IEEE, 2000.

[41] M Donelli. Design of long-range, powerless rfid sensor at 10 ghz. Electronics Letters, 49(20):1277-1278, 2013.

[42] Massimo Donelli. A 24ghz environmental sensor based on the modulated scattering technique (mst). In 2014 IEEE Conference on Antenna Measurements \&3 Applications (CAMA), pages 1-3. IEEE, 2014.

[43] Massimo Donelli. Guidelines for the design and optimization of wireless sensors based on the modulated scattering technique. IEEE Transactions on Instrumentation and Measurement, 63(7):1824-1833, 2014.

[44] HFSS ELECTRONIC DESKTOP ANSYS https://www.ansys.com. Ansys corp., pittsburgh, pa, 2018.

[45] Neal R Erickson, Ronald M Grosslein, Ronna B Erickson, and Sander Weinreb. A cryogenic focal plane array for 85-115 ghz using mmic preamplifiers. IEEE Transactions on Microwave Theory and Techniques, 47(12):2212-2219, 1999. 
[46] Nicholas A Estep, Dimitrios L Sounas, Jason Soric, and Andrea Alù. Magnetic-free non-reciprocity and isolation based on parametrically modulated coupled-resonator loops. Nature Physics, 10(12):923, 2014.

[47] Adrian N Farr and Ian J Forster. Reflecting modulator circuit comprising a negative impedance amplifier, May 16 2006. US Patent 7,046,957.

[48] Farhad Farzami, Seiran Khaledian, Besma Smida, and Danilo Erricolo. Reconfigurable dual-band bidirectional reflection amplifier with applications in van atta array. IEEE Transactions on Microwave Theory and Techniques, 65(11):4198-4207, 2017.

[49] Farhad Farzami, Seiran Khaledian, Besma Smida, and Danilo Erricolo. Ultra-low power reflection amplifier using tunnel diode for rfid applications. In 2017 IEEE International Symposium on Antennas and Propagation 83 USNC/URSI National Radio Science Meeting, pages 2511-2512. IEEE, 2017.

[50] Javier Fondevila, JC Bregains, Francisco Ares, and Eduardo Moreno. Optimizing uniformly excited linear arrays through time modulation. IEEE Antennas and Wireless Propagation Letters, 3(1):298-301, 2004.

[51] Ann Franchois, Alain Joisel, Christian Pichot, and J-C Bolomey. Quantitative microwave imaging with a 2.45-ghz planar microwave camera. IEEE Transactions on Medical Imaging, 17(4):550-561, 1998.

[52] R Garbacz and J Eberle. The measurement of time-quadrature components of a scattered field. In WESCON/60 Conference Record, volume 4, pages 131-138. IEEE, 1960.

[53] Mohammad Tayeb Ahmad Ghasr. Real-time and portable microwave imaging system. 2009. 
[54] Paul F Goldsmith, C-T Hsieh, GR Huguenin, J Kapitzky, and EL Moore. Focal plane imaging systems for millimeter wavelengths. IEEE transactions on microwave theory and techniques, 41(10):1664-1675, 1993.

[55] Viatcheslav Golikov, Sami Hienonen, and Pertti Vainikainen. Passive intermodulation distortion measurements in mobile communication antennas. In Vehicular Technology Conference, 2001. VTC 2001 Fall. IEEE VTS 54th, volume 4, pages 2623-2625. IEEE, 2001.

[56] R Green. Scattering from conjugate-matched antennas. IEEE Transactions on Antennas and Propagation, 14(1):17-21, 1966.

[57] RB Green. Relationships between antennas as scatterers and radiators. IEEE Trans. on Antennas and Prop, 14(1):17-21, 1966.

[58] Joshua D Griffin and Gregory D Durgin. Complete link budgets for backscatter-radio and rfid systems. IEEE Antennas and Propagation Magazine, $51(2): 11-25,2009$.

[59] Yu-Chun Guo, Xiao-Wei Shi, and Lei Chen. Retrodirective array technology. Progress In Electromagnetics Research, 5:153-167, 2008.

[60] I Gupta and A Ksienski. Effect of mutual coupling on the performance of adaptive arrays. IEEE Transactions on Antennas and Propagation, 31(5):785-791, 1983.

[61] Y Hadad, DL Sounas, and A Alu. Space-time gradient metasurfaces. Physical Review B, 92(10):100304, 2015.

[62] Robert C Hansen. Array pattern control and synthesis. Proceedings of the IEEE, 80(1):141-151, 1992. 
[63] Robert C Hansen. Phased array antennas, volume 213. John Wiley \& Sons, 2009 .

[64] R Harrington. Electromagnetic scattering by antennas. IEEE Transactions on Antennas and Propagation, 11(5):595-596, 1963.

[65] Chong He, Xianling Liang, Zhaojin Li, Junping Geng, and Ronghong Jin. Direction finding by time-modulated array with harmonic characteristic analysis. IEEE Antennas and Wireless Propagation Letters, 14:642-645, 2015.

[66] Chris Hemmi. Pattern characteristics of harmonic and intermodulation products in broadband active transmit arrays. IEEE Transactions on Antennas and Propagation, 50(6):858-865, 2002.

[67] Justin Henrie, Andrew Christianson, and William J Chappell. Engineered passive nonlinearities for broadband passive intermodulation distortion mitigation. IEEE microwave and wireless components letters, 19(10):614-616, 2009 .

[68] Sami Hienonen et al. Studies on microwave antennas: passive intermodulation distortion in antenna structures and design of microstrip antenna elements. Helsinki University of Technology, 2005.

[69] Ming-Kuei Hu. On measurements of microwave e and $\mathrm{h}$ field distributions by using modulated scattering methods. IRE Trans. Microw. Theory Tech, 8(3):295-300, 1960.

[70] Ying $\mathrm{Hu}$, David $\mathrm{R}$ Jackson, Jeffery $\mathrm{T}$ Williams, Stuart A Long, and Varada Rajan Komanduri. Characterization of the input impedance of the inset-fed rectangular microstrip antenna. IEEE Transactions on Antennas and Propagation, 56(10):3314-3318, 2008. 
[71] KEIGO IIZUKA. A photoconductive probe for measuring electromagnetic fields. Technical report, HARVARD UNIV CAMBRIDGE MASS CRUFT LAB, 1962.

[72] Sandeep Inampudi, Mohammad Mahdi Salary, Samad Jafar-Zanjani, and Hossein Mosallaei. Rigorous space-time coupled-wave analysis for patterned surfaces with temporal permittivity modulation. Optical Materials Express, 9(1):162-182, 2019.

[73] Ronghong Jin, Jingfeng Chen, Chong He, Xianling Liang, Weiren Zhu, and Junping Geng. Direction finding using time modulated array. In 2017 Sixth Asia-Pacific Conference on Antennas and Propagation (APCAP), pages 1-3. IEEE, 2017.

[74] KG Johannsen and J Hintze. Intermodulation in multiple fixed beam distributed amplifier phased array antenna systems. International journal of satellite communications, 9(3):155-165, 1991.

[75] Klaus G Johannsen. Scan beam antenna intermodulation improvement due to spatial dispersion. IEEE transactions on aerospace and electronic systems, (4):543-557, 1987.

[76] R Justice and V Rumsey. Measurement of electric field distributions. IRE Transactions on Antennas and Propagation, 3(4):177-180, 1955.

[77] Takana Kaho, Tadao Nakagawa, and Katsuhiko Araki. Reduction of intermodulation distortion in active phased array antenna systems using a distortion controller. In Microwave Symposium Digest, 2002 IEEE MTT-S International, volume 2, pages 781-784. IEEE, 2002. 
[78] Takana Kaho, Tadao Nakagawa, Katsuhiko Araki, and Kohji Horikawa. Carrier power to intermodulation-distortion power-ratio-increasing technique in active phased-array antenna systems. IEEE transactions on microwave theory and techniques, 50(12):2987-2994, 2002.

[79] Mohammed Kalaagi and Divitha Seetharamdoo. Retrodirective metasurface operating simultaneously at multiple incident angles. 2018.

[80] Seiran Khaledian, Farhad Farzami, Danilo Erricolo, and Besma Smida. A full-duplex bidirectional amplifier with low dc power consumption using tunnel diodes. IEEE Microwave and Wireless Components Letters, 27(12):1125-1127, 2017.

[81] Himanshu Khatri, Prasad S Gudem, and Lawrence E Larson. Distortion in current commutating passive cmos downconversion mixers. IEEE Transactions on Microwave Theory and Techniques, 57(11):2671-2681, 2009.

[82] John Kimionis, Apostolos Georgiadis, Ana Collado, and Manos M Tentzeris. Enhancement of rf tag backscatter efficiency with low-power reflection amplifiers. IEEE Transactions on Microwave Theory and Techniques, 62(12):3562-3571, 2014.

[83] Dr klaus Finkenzeller and RFID Handbook. Fundamentals and applications in contactless smart cards and identification. April 2003 P, 187.

[84] Eric AM Klumperink, Rameswor Shrestha, Eisse Mensink, Vincent J Arkesteijn, and Bram Nauta. Cognitive radios for dynamic spectrum access-polyphase multipath radio circuits for dynamic spectrum access. IEEE Communications Magazine, 45(5), 2007. 
[85] Toshiro Kodera, Dimitrios L Sounas, and Christophe Caloz. Nonreciprocal magnetless crlh leaky-wave antenna based on a ring metamaterial structure. IEEE Antennas and Wireless Propagation Letters, 10:1551-1554, 2011.

[86] Vikram Bidare Krishnamurthy, Kyung Wook Paik, Mario Ghezzo, William Paul Kornrumpf, and Eric Joseph Wildi. Fabrication of compact magnetic circulator components in microwave packages using high density interconnections, July 7 1998. US Patent $5,776,275$.

[87] Daniel Gregory Kuester. Passive binary-modulated backscatter in microwave networks with applications to rfid. 2013.

[88] W Kummer, A Villeneuve, T Fong, and F Terrio. Ultra-low sidelobes from time-modulated arrays. IEEE Transactions on Antennas and Propagation, 11(6):633-639, 1963.

[89] Kaneyuki Kurokawa. Power waves and the scattering matrix. IEEE transactions on microwave theory and techniques, 13(2):194-202, 1965.

[90] Erik G Larsson and Liesbet Van der Perre. Out-of-band radiation from antenna arrays clarified. arXiv preprint arXiv:1802.02475, 2018.

[91] Antonio Lazaro, Javier Lorenzo, Ramon Villarino, and David Girbau. Backscatter transponder based on frequency selective surface for fmcw radar applications. Radioengineering, 23(2):632-641, 2014.

[92] Antonio Lazaro, Angel Ramos, David Girbau, and Ramon Villarino. A novel uwb rfid tag using active frequency selective surface. IEEE transactions on antennas and propagation, 61(3):1155-1165, 2013. 
[93] Sangjoon Lee, Hyungoo Yoon, and Byung-jun Jang. Real-time direction-finding system using time-modulated array and usrp. In Ubiquitous and Future Networks (ICUFN), 2017 Ninth International Conference on, pages 794-796. IEEE, 2017.

[94] Thomas H Lee. The design of CMOS radio-frequency integrated circuits. Cambridge university press, 2003.

[95] Kevin MKH Leong, Yongxi Qian, and Tatsuo Itoh. Surface wave enhanced broadband planar antenna for wireless applications. IEEE Microwave and wireless components letters, 11(2):62-64, 2001.

[96] Gang Li, Shiwen Yang, Yikai Chen, and Zaiping Nie. A hybrid analog-digital adaptive beamforming in time-modulated linear arrays. Electromagnetics, 30(4):356-364, 2010.

[97] Gang Li, Shiwen Yang, and Zaiping Nie. Adaptive beamforming in time modulated antenna arrays based on beamspace data. In 2009 Asia Pacific Microwave Conference, pages 743-746. IEEE, 2009.

[98] Gang Li, Shiwen Yang, and Zaiping Nie. Direction of arrival estimation in time modulated linear arrays with unidirectional phase center motion. IEEE Transactions on Antennas and Propagation, 58(4):1105-1111, 2010.

[99] RongLin Li, Terence Wu, Bo Pan, Kyutae Lim, Joy Laskar, and Manos M Tentzeris. Equivalent-circuit analysis of a broadband printed dipole with adjusted integrated balun and an array for base station applications. IEEE Transactions on Antennas and Propagation, 57(7):2180-2184, 2009.

[100] VV Liepa and TBA Senior. Modification to the scattering behavior of a sphere by reactive loading. Proceedings of the IEEE, 53(8):1004-1011, 1965. 
[101] Jenshan Lin and Tatsuo Itoh. Active integrated antennas. IEEE Transactions on Microwave Theory and Techniques, 42(12):2186-2194, 1994.

[102] Mingkai Liu, David A Powell, Yair Zarate, and Ilya V Shadrivov. Huygens metadevices for parametric waves. Physical Review X, 8(3):031077, 2018.

[103] Qing Huo Liu, Zhong Qing Zhang, Tonghui T Wang, JA Bryan, Gary A Ybarra, Loren W Nolte, and William T Joines. Active microwave imaging. i. 2-d forward and inverse scattering methods. IEEE Transactions on Microwave Theory and Techniques, 50(1):123-133, 2002.

[104] Erwin G Loewen and Evgeny Popov. Diffraction gratings and applications. CRC Press, 1997.

[105] Sergey L Loyka. The influence of electromagnetic environment on operation of active array antennas: Analysis and simulation techniques. IEEE Antennas and Propagation Magazine, 41(6):23-39, 1999.

[106] SL Loyka. Characteristics of a receiving intermodulation channel of active array antennas. International Journal of Electronics, 80(4):595-602, 1996.

[107] Stephen A Maas. The RF and microwave circuit design cookbook. Artech House, 1998.

[108] Robert J Mailloux. Phased array antenna handbook. Artech house, 2017.

[109] SK Mandal, Rowdra Ghatak, and GK Mahanti. Minimization of side lobe level and side band radiation of a uniformly excited time modulated linear antenna array by using artificial bee colony algorithm. In 2011 IEEE Symposium on Industrial Electronics and Applications, pages 247-250. IEEE, 2011. 
[110] Roberto Maneiro-Catoira, Julio Brégains, José A García-Naya, and Luis Castedo. Time modulated arrays: From their origin to their utilization in wireless communication systems. Sensors, 17(3):590, 2017.

[111] MA Matin and AI Sayeed. A design rule for inset-fed rectangular microstrip patch antenna. WSEAS Transactions on Communications, 9(1):63-72, 2010.

[112] Hamidreza Memarzadeh-Tehran, Jean-Jacques Laurin, and Raman Kashyap. Optically modulated probe for precision near-field measurements. IEEE Transactions on Instrumentation and Measurement, 59(10):2755-2762, 2010.

[113] Eisse Mensink, Eric AM Klumperink, and Bram Nauta. Distortion cancellation by polyphase multipath circuits. IEEE Transactions on Circuits and Systems I: Regular Papers, 52(9):1785-1794, 2005.

[114] Christopher Mollén, Ulf Gustavsson, Thomas Eriksson, and Erik G Larsson. Out-of-band radiation measure for mimo arrays with beamformed transmission. In Communications (ICC), 2016 IEEE International Conference on, pages 1-6. IEEE, 2016.

[115] Christopher Mollén, Ulf Gustavsson, Thomas Eriksson, and Erik G Larsson. Spatial characteristics of distortion radiated from antenna arrays with transceiver nonlinearities. IEEE Transactions on Wireless Communications, 17(10):6663-6679, 2018.

[116] Christopher Mollén, Erik G Larsson, and Thomas Eriksson. Waveforms for the massive mimo downlink: Amplifier efficiency, distortion, and performance. IEEE Transactions on Communications, 64(12):5050-5063, 2016.

[117] JP Balthasar Mueller, Noah A Rubin, Robert C Devlin, Benedikt Groever, and Federico Capasso. Metasurface polarization optics: independent phase 
control of arbitrary orthogonal states of polarization. Physical review letters, 118(11):113901, 2017.

[118] Pavel V Nikitin and KVS Rao. Performance limitations of passive uhf rfid systems. In 2006 IEEE Antennas and Propagation Society International Symposium, pages 1011-1014. IEEE, 2006.

[119] Pavel V Nikitin, KVS Rao, and Roberto D Martinez. Differential res of rfid tag. Electronics Letters, 43(8):431-432, 2007.

[120] Ali M Niknejad and Hossein Hashemi. mm-Wave silicon technology: $60 \mathrm{GHz}$ and beyond. Springer Science \& Business Media, 2008.

[121] E Nordholt and Durk Van Willigen. A new approach to active antenna design. IEEE Transactions on Antennas and Propagation, 28(6):904-910, 1980.

[122] Stephen O'brien, Hanmin Zhao, and Jo S Major Jr. Optical semiconductor device with diffraction grating structure, July 20 1999. US Patent 5,926,493.

[123] Alan O’Donnell, William Clark, Joseph Ernst, and Robert McGwier. Analysis of modulated signals for direction finding using time modulated arrays. In Radar Conference (RadarConf), 2016 IEEE, pages 1-5. IEEE, 2016.

[124] Sophocles J Orfanidis. Introduction to signal processing. Prentice-Hall, Inc., 1995.

[125] Matteo Pastorino. Stochastic optimization methods applied to microwave imaging: A review. IEEE Transactions on Antennas and Propagation, $55(3): 538-548,2007$.

[126] Jose Carlos Pedro and Nuno Borges Carvalho. Intermodulation distortion in microwave and wireless circuits. Artech House, 2003. 
[127] Brett Pigon. Predicting nonlinear behavior in receive phased array antennas. In Military Communications Conference, 2009. MILCOM 2009. IEEE, pages 1-7. IEEE, 2009.

[128] Lorenzo Poli, Toshifumi Moriyama, and Paolo Rocca. Pulse splitting for harmonic beamforming in time-modulated linear arrays. International Journal of Antennas and Propagation, 2014, 2014.

[129] Lorenzo Poli, Paolo Rocca, Luca Manica, and Andrea Massa. Handling sideband radiations in time-modulated arrays through particle swarm optimization. IEEE Transactions on Antennas and Propagation, 58(4):1408-1411, 2010.

[130] C Pon. Retrodirective array using the heterodyne technique. IEEE Transactions on Antennas and Propagation, 12(2):176-180, 1964.

[131] David M Pozar. Microwave engineering. John Wiley \& Sons, 2009.

[132] DM Pozar and TA Metzler. Analysis of a reflectarray antenna using microstrip patches of variable size. Electronics Letters, 29(8):657-658, 1993.

[133] Frank Press. The need for fundamental research in seismology: A summary of the report of the panel on seismic improvement. Eos, Transactions American Geophysical Union, 40(3):212-221, 1959.

[134] Yongxi Qian, William R Deal, Noriaki Kaneda, and Tatsuo Itoh. Microstrip-fed quasi-yagi antenna with broadband characteristics. Electronics Letters, 34(23):2194-2196, 1998.

[135] KVS Rao and PV Nikitin. Theory and measurement of backscattering from rfid tags. IEEE Antennas and Propagation Magazine, 48(6):212-218, 2006. 
[136] Behzad Razavi and Razavi Behzad. RF microelectronics, volume 2. Prentice Hall New Jersey, 1998.

[137] Campbell Richard. Square four aerials. pages 60-64, 2017.

[138] Jack H Richmond. A modulated scattering technique for measurement of field distributions. IRE Transactions on Microwave Theory and Techniques, $3(4): 13-15,1955$.

[139] Paolo Rocca, Giacomo Oliveri, Robert J Mailloux, and Andrea Massa. Unconventional phased array architectures and design methodologiesa review. Proceedings of the IEEE, 104(3):544-560, 2016.

[140] Madeleine Roche, Richard Campbell, and Nasr Alkhafaji. Uhf array element using a reflection coefficient modulator. In 2018 IEEE/MTT-S International Microwave Symposium-IMS, pages 446-449. IEEE, 2018.

[141] Wonil Roh, Ji-Yun Seol, Jeongho Park, Byunghwan Lee, Jaekon Lee, Yungsoo Kim, Jaeweon Cho, Kyungwhoon Cheun, and Farshid Aryanfar. Millimeter-wave beamforming as an enabling technology for $5 \mathrm{~g}$ cellular communications: Theoretical feasibility and prototype results. IEEE communications magazine, 52(2):106-113, 2014.

[142] Bhaskara Rupakula, Ahmed Nafe, Samet Zihir, Yaochen Wang, Tsu-Wei Lin, and Gabriel Rebeiz. 63.5-65.5-ghz transmit/receive phased-array communication link with $0.5-2 \mathrm{gb} / \mathrm{s}$ at $100-800 \mathrm{~m}$ and \pm 50 scan angles. IEEE Transactions on Microwave Theory and Techniques, 66(9):4108-4120, 2018.

[143] Bhaskara Rupakula and Gabriel M Rebeiz. Third-order intermodulation effects and system sensitivity degradation in receive-mode $5 \mathrm{~g}$ phased arrays in the 
presence of multiple interferers. IEEE Transactions on Microwave Theory and Techniques, (99):1-16, 2018.

[144] Mohammad Mahdi Salary, Samad Jafar-Zanjani, and Hossein Mosallaei. Electrically tunable harmonics in time-modulated metasurfaces for wavefront engineering. New Journal of Physics, 20(12):123023, 2018.

[145] Mohammad Mahdi Salary, Samad Jafar-Zanjani, and Hossein Mosallaei. Time-varying metamaterials based on graphene-wrapped microwires: Modeling and potential applications. Physical Review B, 97(11):115421, 2018.

[146] W Sandrin. Spatial distribution of intermodulation products in active phased array antennas. IEEE Transactions on Antennas and Propagation, 21(6):864-868, 1973.

[147] Willy Sansen. Distortion in elementary transistor circuits. IEEE Transactions on Circuits and Systems II: Analog and Digital Signal Processing, 46(3):315-325, 1999 .

[148] Willy Sansen. Distortion in elementary transistor circuits. In Analog Design Essentials, pages 519-565. Springer, 2006.

[149] H Shanks. A new technique for electronic scanning. IRE Transactions on Antennas and Propagation, 9(2):162-166, 1961.

[150] HE Shanks and RW Bickmore. Four-dimensional electromagnetic radiators. Canadian Journal of Physics, 37(3):263-275, 1959.

[151] E Sharp and M Diab. Van atta reflector array. IRE Transactions on Antennas and Propagation, 8(4):436-438, 1960. 
[152] Aleksey Shitvov, Dmitry Zelenchuk, and Alexander Schuchinsky. Carrier-power dependence of passive intermodulation products in printed lines. In 2009 Loughborough Antennas $\&$ Propagation Conference, pages 177-180. IEEE, 2009.

[153] Rameswor Shrestha, Eric AM Klumperink, Eisse Mensink, Gerard JM Wienk, and Bram Nauta. A polyphase multipath technique for software-defined radio transmitters. IEEE journal of solid-state circuits, 41(12):2681-2692, 2006.

[154] Dimitrios L Sounas and Andrea Alù. Non-reciprocal photonics based on time modulation. Nature Photonics, 11(12):774, 2017.

[155] Harry Stockman. Communication by means of reflected power. Proceedings of the IRE, 36(10):1196-1204, 1948.

[156] B Strait and D Cheng. Measurement of microwave magnetic diffraction fields. IRE Transactions on Antennas and Propagation, 10(5):643-645, 1962.

[157] Saqib Subhan, Eric AM Klumperink, and Bram Nauta. Towards suppression of all harmonics in a polyphase multipath transmitter. In Circuits and Systems (ISCAS), 2011 IEEE International Symposium on, pages 2185-2188. IEEE, 2011.

[158] Thomas Svantesson and Anders Ranheim. Mutual coupling effects on the capacity of multielement antenna systems. In Acoustics, Speech, and Signal Processing, 2001. Proceedings.(ICASSP'01). 2001 IEEE International Conference on, volume 4, pages 2485-2488. IEEE, 2001.

[159] Cheng Tao, Mohammad Memarian, Yasuo Morimoto, and Tatsuo Itoh. Non-periodic metasurfaces for blazing and beam splitting. In Microwave Conference (APMC), 2016 Asia-Pacific, pages 1-4. IEEE, 2016. 
[160] Sajjad Taravati and Christophe Caloz. Space-time modulated nonreciprocal mixing, amplifying and scanning leaky-wave antenna system. In Antennas and Propagation 83 USNC/URSI National Radio Science Meeting, 2015 IEEE International Symposium on, pages 639-640. IEEE, 2015.

[161] Sajjad Taravati, Bakhtiar A Khan, Shulabh Gupta, Karim Achouri, and Christophe Caloz. Nonreciprocal nongyrotropic magnetless metasurface. IEEE Transactions on Antennas and Propagation, 65(7):3589-3597, 2017.

[162] Aleksandar Tasic, Junxiong Deng, and Zhang Jin. Multi-mode low noise amplifier with transformer source degeneration, January 24 2012. US Patent $8,102,213$.

[163] A Tennant and B Chambers. A two-element time-modulated array with direction-finding properties. IEEE Antennas and Wireless Propagation Letters, 6:64-65, 2007.

[164] A Tennant and B Chambers. Control of the harmonic radiation patterns of time-modulated antenna arrays. In 2008 IEEE Antennas and Propagation Society International Symposium, pages 1-4. IEEE, 2008.

[165] Alan Tennant. Experimental two-element time-modulated direction finding array. IEEE Transactions on Antennas and Propagation, 58(3):986-988, 2010.

[166] Manolis T Terrovitis and Robert G Meyer. Intermodulation distortion in current-commutating cmos mixers. IEEE Journal of Solid-State Circuits, 35(10):1461-1473, 2000.

[167] Stewart Thomas and Matthew S Reynolds. Qam backscatter for passive uhf rfid tags. In 2010 IEEE International Conference on RFID (IEEE RFID 2010), pages 210-214. IEEE, 2010. 
[168] Stewart J Thomas, Travis Deyle, Reid Harrison, and Matthew S Reynolds. Rich-media tags: Battery-free wireless multichannel digital audio and image transmission with uhf rfid techniques. In RFID (RFID), 2013 IEEE International Conference on, pages 231-236. IEEE, 2013.

[169] Stewart J Thomas, Reid R Harrison, Anthony Leonardo, and Matthew S Reynolds. A battery-free multichannel digital neural/emg telemetry system for flying insects. IEEE transactions on biomedical circuits and systems, $6(5): 424-436,2012$.

[170] Stewart J Thomas, Eric Wheeler, Jochen Teizer, and Matthew S Reynolds. Quadrature amplitude modulated backscatter in passive and semipassive uhf rfid systems. IEEE Transactions on Microwave Theory and Techniques, 60(4):1175-1182, 2012.

[171] Stewart Jennings Thomas. Modulated backscatter for low-power high-bandwidth communication. PhD thesis, 2013.

[172] Y Tong and A Tennant. Simultaneous control of sidelobe level and harmonic beam steering in time-modulated linear arrays. Electronics letters, 46(3):201-202, 2010.

[173] Yizhen Tong and Alan Tennant. A two-channel time modulated linear array with adaptive beamforming. IEEE Transactions on Antennas and Propagation, 60(1):141-147, 2012.

[174] Ayhan Vural, D Cheng, and B Strait. Measurement of diffraction fields of finite cones by a scattering technique using light modulation. IEEE Transactions on Antennas and Propagation, 11(2):200-201, 1963. 
[175] Ayhan M Vural and David K Cheng. A light-modulated scattering technique for diffraction field measurements. Radio Sci. J. Res, 68:355-362, 1964.

[176] Benny Walther, Christian Helgert, Carsten Rockstuhl, Frank Setzpfandt, Falk Eilenberger, Ernst-Bernhard Kley, Falk Lederer, Andreas Tünnermann, and Thomas Pertsch. Spatial and spectral light shaping with metamaterials. Advanced Materials, 24(47):6300-6304, 2012.

[177] David Weinstein. Passive intermodulation distortion in connectors, cable and cable assemblies. Amphenol application note, 2001.

[178] Jonathan R Wilkerson, Kevin G Gard, and Michael B Steer. Electro-thermal passive intermodulation distortion in microwave attenuators. In Microwave Conference, 2006. 36th European, pages 157-160. IEEE, 2006.

[179] Jonathan R Wilkerson, Peter G Lam, Kevin G Gard, and Michael B Steer. Distributed passive intermodulation distortion on transmission lines. IEEE Transactions on Microwave Theory and Techniques, 59(5):1190-1205, 2011.

[180] Shiwen Yang, Yeow Beng Gan, and Anyong Qing. Sideband suppression in time-modulated linear arrays by the differential evolution algorithm. IEEE Antennas and Wireless Propagation Letters, 1:173-175, 2002.

[181] Shiwen Yang, Yeow Beng Gan, Anyong Qing, and Peng Khiang Tan. Design of a uniform amplitude time modulated linear array with optimized time sequences. IEEE Transactions on Antennas and Propagation, 53(7):2337-2339, 2005.

[182] Jaehyok Yi, Youngoo Yang, Myungkyu Park, Wonwoo Kang, and Bumman Kim. Analog predistortion linearizer for high-power $\mathrm{rf}$ amplifiers. IEEE Transactions on Microwave Theory and Techniques, 48(12):2709-2713, 2000. 
[183] T Yoshitake, S Tahara, and S Suzuki. Intermodulation distortion measurements of a microstrip band-pass filter made from double-sided yba2cu3o x films. Applied physics letters, 67(26):3963-3965, 1995.

[184] Quanjiang Zhu, Shiwen Yang, Ruilin Yao, and Zaiping Nie. Gain improvement in time-modulated linear arrays using spdt switches. IEEE Antennas and Wireless Propagation Letters, 11:994-997, 2012.

[185] Quanjiang Zhu, Shiwen Yang, Li Zheng, and Zaiping Nie. Design of a low sidelobe time modulated linear array with uniform amplitude and sub-sectional optimized time steps. IEEE Transactions on Antennas and Propagation, 60(9):4436-4439, 2012. 


\section{Appendix A: Nonlinear Circuits}

A behavior of a nonlinear circuit shown in Figure A.1 can be represented mathematically by

$$
y(t)=a_{o}+a_{1} x(t)+a_{2} x^{2}(t)+a_{3} x^{3}(t)+\cdots+a_{n} x^{n}(t)+\cdots=\sum_{n=0}^{\infty} a_{n} x^{n}(t)
$$

The coefficients $a_{o}$ and $a_{1}$ represent the DC-offset and the linear terms in the systems, respectively, while $a_{2}, a_{3}, a_{4}, \cdots$ are nonlinear components and they become dominant as power of an input signal increases.

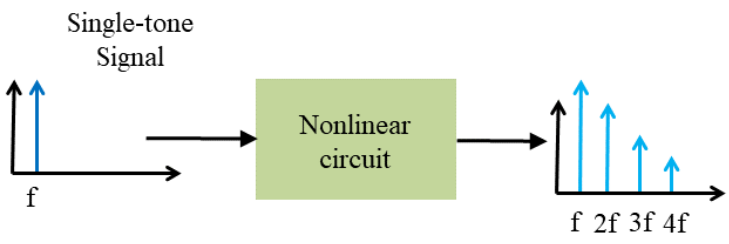

Two-tone Signal

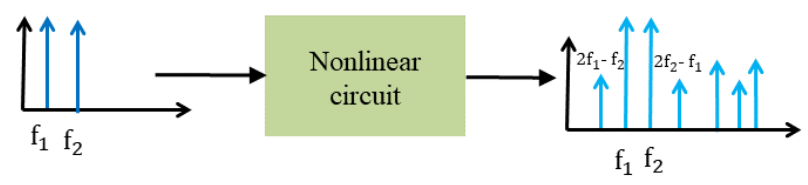

Figure A.1: Nonlinear Circuits representations

\section{A.1 Single-tone Analysis}

If we consider a single-tone signal as an input signal and it is $x(t)=A \cos \left(w t+\varphi_{i n}\right)$, then there is an infinite number of resulting harmonics at an output port of a circuit 
in Figure A.1. The output equation can be written as

$$
\begin{aligned}
y(t)= & a_{o}+a_{1} A \cos \left(w t+\varphi_{i n}\right),+a_{2} A^{2} \cos ^{2}\left(w t+\varphi_{i n}\right)+a_{3} A^{3} \cos ^{3}\left(w t+\varphi_{i n}\right)+ \\
& a_{4} A^{4} \cos ^{4}\left(w t+\varphi_{i n}\right)+\cdots=\sum_{n=0}^{\infty} a_{n} A^{n} \cos ^{n}\left(w t+\varphi_{i n}\right)
\end{aligned}
$$

The first six terms are

$$
\begin{gathered}
a_{0} \\
a_{1} x(t)=a_{1} A \cos \left(w t+\varphi_{i n}\right) \\
a_{2} x^{2}(t)=a_{2} A^{2} \cos ^{2}\left(w t+\varphi_{i n}\right)=\frac{1}{2} a_{2} A^{2}\left(1+\cos 2\left(w t+\varphi_{i n}\right)\right) \\
a_{3} x^{3}(t)=a_{3} A^{3} \cos ^{3}\left(w t+\varphi_{i n}\right)=\frac{3}{4} a_{3} A^{3} \cos \left(w t+\varphi_{i n}\right)+\frac{1}{4} a_{3} A^{3} \cos 3\left(w t+\varphi_{i n}\right) \\
a_{4} x^{4}(t)=\frac{3}{8} a_{4} A^{4}+\frac{a_{4} A^{4}}{2} \cos \left(2 w t+2 \varphi_{i n}\right)+\frac{1}{8} a_{4} A^{4} \cos \left(4 w t+4 \varphi_{i n}\right) \\
a_{5} x^{5}(t)=\frac{10 a_{5} A^{5}}{16} \cos \left(w t+\varphi_{i n}\right)+\frac{5 a_{5} A^{5}}{16} \cos \left(3 w t+3 \varphi_{i n}\right)+\frac{a_{5} A^{5}}{16} \cos \left(5 w t+5 \varphi_{i n}\right)
\end{gathered}
$$

After placing all terms together, the resulting output equation is

$$
\begin{aligned}
y(t)= & b_{o}+b_{1} \cos \left(w t+\varphi_{i n}\right)+b_{2} \cos \left(2\left(w t+\varphi_{i n}\right)\right)+b_{3} \cos \left(3\left(w t+\varphi_{i n}\right)\right)+\cdots= \\
& \sum_{n=0}^{\infty} b_{n} \cos \left(n\left(w t+\varphi_{i n}\right)\right)
\end{aligned}
$$

where $b_{n}$ represent Taylor series constants. It can be clearly noticed, (B.3), that a phase of the output signal at the nth harmonic product is multiplied by a harmonic number $\mathrm{n}$. In other words, if we have an $n w t$ term, an $n \varphi_{i n}$ will be produced. 


\section{Appendix B: Three Paths Polyphase Circuit}

This appendix presents a mathematical presentation that prove the mechanism of harmonic cancellation using a three path polyphase technique. A general schematic of a circuit was presented in Chapter 3, but it is also recalled here for the sake of completeness.

As shown in Figure B.1, three signals, having the same frequency and different

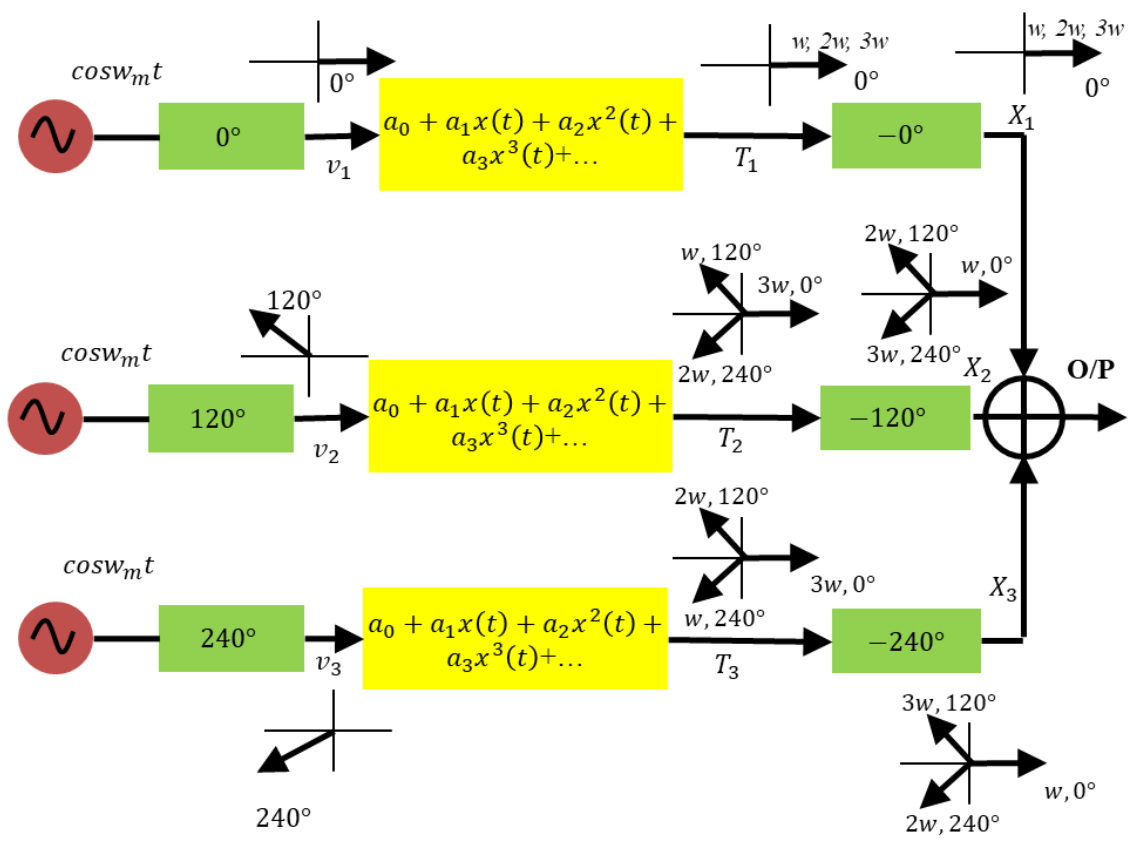

Figure B.1: Three-path polyphase circuit

phases, are applied at inputs of paths. Mathematically, they can be expressed as

$$
\begin{gathered}
v 1=A \cos \left(w_{m} t\right) \\
v 2=A \cos \left(w_{m} t+120\right) \\
v 3=A \cos \left(w_{m} t+240\right)
\end{gathered}
$$


These three signals are applied to inputs of nonlinear circuits. Outputs of nonlinear circuits are given by

$$
\begin{aligned}
T_{1} & =\left(a_{o}+\frac{a_{2} A^{2}}{2}\right)+\left(a_{1}+\frac{3 a_{3} A^{3}}{4}\right) \cos \left(w_{m} t\right)+\frac{a_{2} A^{2}}{2} \cos \left(2 w_{m} t\right) \\
+ & \frac{a_{3} A^{3}}{4} \cos \left(3 w_{m} t\right)+\cdots \\
T_{2}= & \left(a_{o}+\frac{a_{2} A^{2}}{2}\right)+\left(a_{1}+\frac{3 a_{3} A^{3}}{4}\right) \cos \left(w_{m} t+120\right) \\
& +\frac{a_{2} A^{2}}{2} \cos \left(2 w_{m} t+240\right)+\frac{a_{3} A^{3}}{4} \cos \left(3 w_{m} t+360\right)+\cdots \\
T_{3} & =\left(a_{o}+\frac{a_{2} A^{2}}{2}\right)+\left(a_{1}+\frac{3 a_{3} A^{3}}{4}\right) \cos \left(w_{m} t+240\right) \\
& +\frac{a_{2} A^{2}}{2} \cos \left(2 w_{m} t+480\right)+\frac{a_{3} A^{3}}{4} \cos \left(3 w_{m} t+720\right)+\cdots
\end{aligned}
$$

Output signals of nonlinear circuits are passed through other phase shifters having opposite signs with respect to values of the initial phase shifts of the main input signals given in (B.1), (B.2), and (B.3). Output signals from the second phase shifters are given by

$$
\begin{aligned}
X_{1} & =\left(a_{o}+\frac{a_{2} A^{2}}{2}\right)+\left(a_{1}+\frac{3 a_{3} A^{3}}{4}\right) \cos \left(w_{m} t\right)+\frac{a_{2} A^{2}}{2} \cos \left(2 w_{m} t\right) \\
& +\frac{a_{3} A^{3}}{4} \cos \left(3 w_{m} t\right)+\cdots
\end{aligned}
$$




$$
\begin{aligned}
X_{2} & =\left(a_{o}+\frac{a_{2} A^{2}}{2}\right)+\left(a_{1}+\frac{3 a_{3} A^{3}}{4}\right) \cos \left(w_{m} t\right)+\frac{a_{2} A^{2}}{2} \cos \left(2 w_{m} t+120\right) \\
& +\frac{a_{3} A^{3}}{4} \cos \left(3 w_{m} t+240\right)+\cdots \\
X_{3} & =\left(a_{o}+\frac{a_{2} A^{2}}{2}\right)+\left(a_{1}+\frac{3 a_{3} A^{3}}{4}\right) \cos \left(w_{m} t\right)+\frac{a_{2} A^{2}}{2} \cos \left(2 w_{m} t+240\right) \\
& +\frac{a_{3} A^{3}}{4} \cos \left(3 w_{m} t+120\right)+\cdots
\end{aligned}
$$

Signals in (B.7), (B.8), and (B.9) are added at the final output, resulting in

$$
O P=X_{1}+X_{2}+X_{3}=\left(a_{0}+\frac{a_{2} A^{2}}{2}\right)+\left(a_{1} A+\frac{3 a_{3} A^{3}}{4}\right) \cos \left(w_{m} t\right)+\frac{a_{4} A^{4}}{4} \cos \left(4 w_{m} t\right)+\ldots
$$

Hence

$$
\begin{aligned}
& \cos 2 w_{m} t+\cos \left(2 w_{m} t+120\right)+\cos \left(2 w_{m} t+240\right)=\sum_{i=1}^{3} \cos \left(2 w_{m} t+(i-1) \varphi\right)=0 \\
& \cos 3 w_{m} t+\cos \left(3 w_{m} t+240\right)+\cos \left(2 w_{m} t+120\right)=\sum_{i=1}^{3} \cos \left(3 w_{m} t+2(i-1) \varphi\right)=0
\end{aligned}
$$

and so on.

As can be seen in (B.10) and (B.11), the 2nd and 3rd harmonics are canceled, because they have balanced phase shifts across paths. However, from peroidic nature of nonlinear circuits and phases with $2 \pi$, harmonics of the 5 th, 6th, 8 th, 9 th, $\cdots$, are also canceled. 